2. is

4

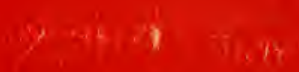

papers in

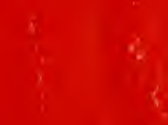

is

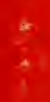

MICROBIAL GENETICS bacteria and bacteriol viruses

selected by JOSHIJA LEDERBERG

tot-

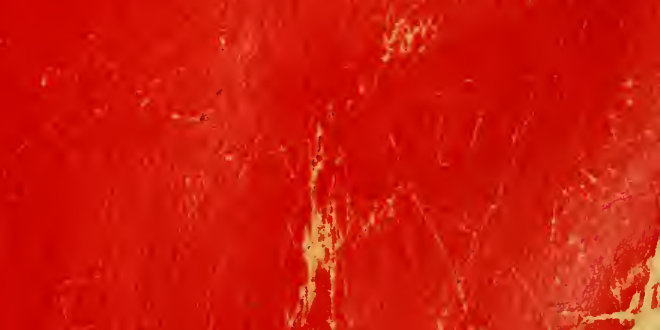




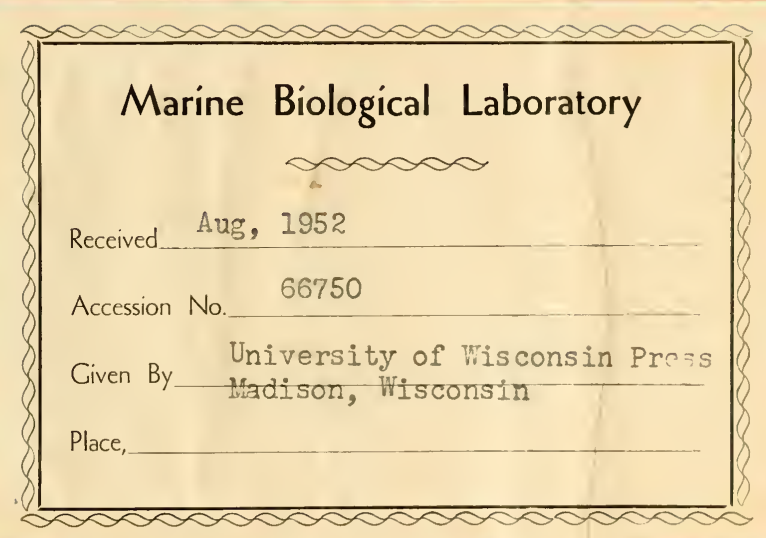




MICROBIAL GENETICS 



\section{papers in}

\section{MICROBIAL GENETICS}

bacteria and bacterial viruses

selected by JOSHUA LEDERBERG

UNIVERSITY OF WISCONSIN PRESS - MADISON - 1952 
COPYRIGHT 1951 BY THE REGENTS OF THE UNIVERSITY OF WISCONSIN SECOND PRINTING, 1952 PRINTED IN THE UNITED STATES OF AMERICA BY CUSHING-MALI.OY, INC. - ANN ARBOR, MICHIGAN 


\section{PREFACE}

THE FIRST purpose of this book is to supplement a lecture course on microbial genetics with original readings in a form most readily available to the student. At most universities, the papers included in this collection will be represented by single copies of the original journals. Thus, it is usually difficult for any number of students to read the papers at all, and impossible for anyone (including the instructor) to read and perhaps annotate them at leisure. This volume is not intended to include all the reading on this subject which should be required for a course. I hope, however, that it will make available most of the papers requiring the most general or the most intensive study.

The scope of the collection must obviously have some limits in size and cost. These have led to the restriction to bacteria and bacterial viruses, although consideration of fungi, protozoa, algae, and viruses is essential to a well-rounded course, even to one organized, as at Wisconsin, primarily for students majoring in bacteriology. Within this field, there are a great many important papers which have necessarily been omitted. No apologies need be offered for a selection which must be largely arbitrary. The papers selected were those which seemed to illustrate best certain concepts and methodologies for the benefit of a course in microbial genetics. In some choices, weight had to be given to other circumstances such as the length and availability of alternative papers. It must be emphasized that these selections do not reflect historical priority or scientific worth as such. A bibliographic list is included to help remedy the unavoidable deficiencies of the collection.

This book could not have been produced without the magnanimous cooperation of the publishers and copyright holders of the original papers, to whom specific acknowledgment is made elsewhere. Special thanks are due to the authors who have been very helpful, especially in providing scarce reprints for use as copy. I must also record an obligation to my colleagues who did their best to advise me on the proper scope and contents of this collection.

Madison, April I, I95I 



\section{CONTENTS}

Introduction BY JoSHUA LEDERBERG

ix

Mutations of Bacteria from Virus Sensitivity to Virus Resistance, BY S. E. Luria and M. Delbrück

The Distribution of the Numbers of Mutants in Bacterial

Populations, By D. E. Lea And C. A. Coulson

Origin of Bacterial Variants, By Howard B. Newcombe

Delayed Phenotypic Expression of Spontaneous Mutations in Escherichia coli, By Howard B. Newcombe

Origin of Bacterial Resistance to Antibiotics, BY M. Demerec

Chloromycetin Resistance in $E$. coli, a Case of Quantitative Inheritance in Bacteria, by L. L. Cavalli and G. A. Maccacaro

Studies on Nutritionally Deficient Bacterial Mutants Isolated by Means of Penicillin, By Bernard D. Davis

The Demonstration of Non-specific Components in Salmonella paratyphi A by Induced Variation, BY D. W. BRU NER AND P. R. EDwards

The Natural Occurrence of Phase 2 of Salmonella paratyphi A, by P. R. Edwards, L. A. Barnes, and Mary C. Babcock

Changes Induced in the O Antigens of Salmonella, BY D. W. BRUNER AND P. R. EDWARDS

Mutations in Escherichia coli Induced by Chemical Agents, By Evelyn M. Witkin

The Effect of Metabolites upon Growth and Variation of Brucella abortus, by Robert J. Goodlow, Leonard A. Mika, And Werner BRAUN

Gene Recombination and Linked Segregations in Escherichia coli, BY JoSHUA LEDERBERG

Aberrant Heterozygotes in Escherichia coli, BY JoshuA LederberG 
Cytological Observations on Bact. coli, Proteus vulgaris and Various Aerobic Spore-Forming Bacteria with Special Reference to the Nuclear Structures, вy C. F. RoBinow

Studies on the Chemical Nature of the Substance Inducing Transformation of Pneumococcal Types, by Oswald T. Avery, Colin M. MacLeod, and Maclyn McCarty

Induced Lysogenicity and Mutation of Bacteriophage within Lysogenic Bacteria, By F. M. Burnet and Dora Lush

Mutations of Bacterial Viruses Affecting Their Host Range, BY S. E. Luria

Genetic Recombination between Host-Range and Plaque-Type Mutants of Bacteriophage in Single Bacterial Cells, By A. D. Hershey AND Raquel Rotman

Genetic Recombinations Leading to Production of Active Bacteriophage from Ultraviolet Inactivated Bacteriophage Particles, BY S. E. LURIA and R. Dulbecco

Bibliography 
Mutation and adaptation. - The problem of organic adaptation in bacteria has been resolved into two points of view which are closely analogous to "neoDarwinian" and "Lamarckian" concepts of organic evolution, respectively. The first holds that adaptive changes occur spontaneously, as sporadic mutations, not in any specific relationship to environmental conditions, and that natural selection functions to fix the best adapted genotypes. The Lamarckian viewpoint suggests that the adaptive mutation is itself directed by the environment, and that natural selection plays a subsidiary role. Many of the papers reprinted show experiments which support the spontaneous mutation hypothesis $(1,3,5)$. One (6) deals with the end result of bacterial adaptation to chloromycetin and also argues for spontaneous mutations with accumulative effect by separating the components by recombination. There is no representative here of the alternative point of view which is defended strongly by Hinshelwood and his associates $(28,67,70)$. In considering this problem it is essential to keep in mind physiological adaptations which are nonheritable, but whose interplay with heritable changes may trap the unwary (97, 120, 45-Lwoff). General discussions of this problem are included in most of the review articles $(32,54$, $81,82,87,12$ \&, 129).

Mutations for resistance to bacteriophage had been considered some years before Luria and Delbrück's paper (1), notably by Burnet (34). This author drew the same conclusions concerning the spontaneity of the resistance mutations, mainly through his ability to pick out resistant mutants by their colonial morphology without the use of phage as a selective agent. Much of the current work on $E$. coli phages $(43,44,21)$ involves a set of 7 virus strains described as $\mathrm{T}_{\mathrm{I}}$ through $\mathrm{T}_{7}$. Mutation rates for resistance to these phages have been recorded, together with useful information on cross-resistance patterns (48).

The physiological study of mutant characters is known as phenogenetics, in contrast to formal or cryptogenetics which deals directly with the mechanism of hereditary transmission. One would reasonably expect that a gene mutation would require a period of time to work its effects on the phenotype or outward behavior of the organism. This lag in bacterial mutation effects (phenotypic or phenomic lag) was first noticed directly with phage-resistance mutations

The citations in the Introduction refer to the Bibliography, pp. 299-303. 
induced by radiations ( 45 -Demerec). Later, Newcombe postulated a similar lag for spontaneous mutations to account for discrepancies in the estimation of mutation rates by different methods, according to whether they counted mutant individuals or mutant clones (4). In both of these instances, however, the interpretation is complicated by the cytological structure of the bacterial cell (15) which speaks for the concurrence of several nuclei (and therefore of several replicate gene sets) in each cell. Recessive mutations such as phage or drug resistance would require for their phenotypic expression that mutant and nonmutant nuclei be segregated, so that there would also be a segregation lag in the genesis of the mutant phenotype $(103,14,47)$. The description of phenomic lag in the development of nutritionally sufficient "back-mutations" induced by ultra-violet light (UV) (7) avoids this difficulty, for these mutations are presumably dominant, and would not require segregation for their expression. A final caution which must be entered is that we cannot be entirely certain that the mutation-producing event within the cell is coincidental with the experimental irradiation (mutation delay hypothesis) $(26,49)$.

Mutant characters.-Something is known of the phenogenetics of phage resistance. The extraction of sensitive and resistant cultures results in preparations differing in the ability to neutralize the phage "in vitro." This argues strongly for the concept that phage sensitivity is correlated with the presence of a specific receptor substance $(44,61)$. Another metabolic correlate of phage resistance is less explicable. In certain specific combinations of bacterium and phage, the resistance mutation simultaneously leads to new nutritional requirements, (of ten for tryptophane or proline). Unfortunately, this correlation has not been found in strains which can be studied by recombination, so that it is not certain whether a simple genetic change causes the two effects, $(23,139$, 45-Luria).

Phage resistant mutations play such a large part in genetic study because of the ease with which they can be selected out of large populations, simply by adding suspensions of the virus under appropriate conditions. This facility is shared by antibiotics and other antibacterials as selective agents. The advantage that the "drug" is not another biological system whose variability must be watched is counterbalanced by the fact that resistance to most chemicals is a quantitative rather than an all-or-none phenomenon, and that a great many genes may be involved. The probable reason for this is that antibiotics interfere with key metabolic steps that are linked to a wide diversity of other reactions, so that any one of a number of biochemical changes may influence the growth response to an inhibitor. The initial reaction between phages and cells is, on the other hand, directly associated with the presence of a receptor substance with a high order of specificity.

Current interest in drug-resistance mutations is largely motivated by their importance in limiting the effectiveness of chemotherapy (95), but they have provided interesting material for more theoretical genetic studies as well. In 
most cases, resistance may be developed in a series of individually small steps, speaking for the cumulative action of a number of gene mutations as postulated above. The evidence for this comes primarily from kinetic studies of increasing resistance under selection $(5,107,135)$. It has been confirmed by direct crossing experiments involving chloromycetin resistant mutants of $E$. coli (6). Progeny of crosses between a sensitive and a fully-adapted (many-step) resistant parent showed a segregation of many intermediate grades of resistance, representing the reassortment of the sensitive and resistant genes in a variety of combinations.

Streptomycin-resistance shows some unique features in contrast to the agents just summarized. In many species, a mutation conferring full resistance occurs at a rate, about $10^{-10}$ per fission, which overshadows the smaller step mutations characteristic of resistance to other agents $(5,28,100,135)$. This low rate is perhaps the smallest mutation rate to be accurately measured in any organism. A more striking oddity is the mutation which over-adapts the cell to streptomycin so that the resistant mutant is dependent upon streptomycin for growth $(95,100)$. The function of streptomycin is possibly to regulate an over-expanded enzyme system, analogous to the precedent of a sulfonamidedependent mutant of Neurospora (141).

Amino acids and vitamins are not commonly thought of as antibiotics but it has long been known that they sometimes interfere with, rather than promote, bacterial growth $(78,111,117-$ Snell, 140). The very ubiquity of these compounds imparts a special interest to them as possible natural regulators. Of equal interest is the correlation between sensitivity to amino acids and virulence in Salmonella and Brucella (12, 109) which may reflect a hitherto unsuspected general principle.

It is likely that a biochemical basis will be found for the effects of all types of mutations (29), but microorganisms have been especially prolific in the production of mutants with overt effects on metabolism. For this reason, microbial and biochemical genetics are intimately associated and often confused. Gene mutations affecting anabolic processes are usually detected as nutritional or auxotrophic mutants, whose growth depends upon an external supply of the missing metabolite. Auxotrophs have been used for the exploration of many biosynthetic pathways, which are remarkably similar in bacteria, molds, and mammals. Davis' paper (7) outlines this methodology, which is based on the pioneer investigations of Tatum, Beadle, and other workers on the production and characterization of auxotrophic mutants in fungi (30, 69, 117-Tatum) and in bacteria $(63,126,127,112)$. Mutations leading to catabolic defects have been especially useful in bacterial work, both as genetic markers and in the analysis of fermentation pathways (53).

Mutations to auxotrophy do not lend themselves to quantitative estimation of rates, despite more or less efficient selective methods for their isolation (88). On the other hand, mutations from auxotrophy to the nutritionally wild type or prototrophic state are suitable for selective counting methods, but due care 
must be taken to minimize residual growth of auxotrophic inocula at the expense of growth factors carried over with the cells or resident as impurities in the test medium ( 114,116$)$.

Serological variation is of key importance in medical bacteriology, but its genetic study is barely under way. The work of Kauffmann and of Edwards and Bruner on Salmonella illustrates the provocative information now at hand $(8,9,10,71)$. A compendium of the serological variation of bacteria generally would be inappropriate here, but the subject has been treated exhaustively in excellent reviews and books $\left(66,36,54,3^{2}\right)$.

Given two alternative forms of a gene-say $a$ and $A$, each of which mutates into the other at a definite rate-it is easy to show that an equilibrium will eventually be established such that the ratio of $a: A$ will be equal to the ratio of the mutation rates to the respective conditions (42). Most mutation rates are so low (of the order of one per million or billion cell divisions) that mutational equilibria would take too long for human observation, even if the necessary constancy of the environment were possible. Mutations involving the antigenic structure of the flagella of Salmonella have, however, been found to have unusually high rates (accounting for the readiness with which they have been found), and Stocker (124) has described mutational equilibrium as approached from inocula of either form.

Induced mutations.-Since Muller's announcement in 1928 that X-rays would induce mutations in fruitflies, an extensive segment of genetic research has concerned the discovery of mutagenic agents and the conditions of their effect. Higher organisms like Drosophila and maize are indispensable in the finer analysis of the cytological basis of induced genetic alterations, but microorganisms are very useful tools in the screening of new agents for mutagenic activity, and in the study of the gross quantitative aspects of such activity. The same types of mutants already mentioned as best enumerated on a selective basis are particularly useful here. Dose-response data have been published for mutations induced by X-rays $(113,45)$ and by UV $(49,104)$ but their interpretation, especially for UV, is far from simple.

It has been thought that radiations induced mutations by direct photochemical processes, i.e., that the gene itself might be activated by the absorption of a quantum of UV, or by collision with a secondary electron following an $\mathrm{X}$-ray quantum absorption, $(60,80)$. Some revision of this concept is now necessary on the basis of recent research. The effectiveness of X-rays is potentiated by the presence of oxygen, and there may be a tenfold difference between the doses required for a given effect in oxygen as against an inert atmosphere (47). This argues for a radiochemical intermediate, possibly some free radical (peroxide?) which depends upon oxygen for its production under the influence of X-radiation.

Owing to the powerful penetrability of $\mathrm{X}$-radiation, it has been used in Drosophila studies more extensively than UV, which penetrates through living material so poorly that there are serious experimental difficulties in its ap- 
plication in the genetics of plants and animals. The small size of bacterial cells ideally suits them for experiments with this agent, and UV is probably the most convenient and widely used mutagenic treatment in microbial experiments. The analysis of UV-effects has been stimulated by the discovery of photoreactivation $(72,55)$. A number of workers have found that a treatment of various types of cells (actinomycete spores, bacteria, bacteriophage, mold spores, Arbacia eggs) with visible light partially cancels both the lethal and the mutation-inducing effects of a previous dose of UV $(56,73,104,105,134)$. This might suggest that a light-sensitive substance is produced by UV, but the possible nature of this substance and its locus within the cell are obscure. Bacteriophage inactivated with $\mathrm{UV}$ is photoreactivated by visible light only following its absorption into sensitive cells. The effect of UV in inhibiting enzyme synthesis is also subject to photoreactivation (125) but no systems simpler than intact cells have been shown to give such an effect.

Chemical mutagens.-A byproduct of research on chemical warfare agents during World War II was the realization of the possibility of mutagenic activity of chemicals. The nitrogen and sulfur mustards $(\beta$-chloro-alkylamines and sulfides) have been studied especially extensively, and found to be potent mutagens for all organisms studied. In general, their effects are similar to those of X-rays and UV, but there are differences in details $(26)$. The similarity of effects is made the more pronounced by the fact that mutants that are relatively resistant to "mustard" can be selected in E. coli, strain B, and these mutants also show augmented resistance to UV and X-ray. $\left(33,13^{8}\right)$.

Active programs are under way in several laboratories to screen compounds for mutagenic activity, with bacteria prominent among the test organisms. The methodology of such a program, and some of the precautions needed to justify a positive conclusion, are illustrated in Witkin's paper (11). There is a wealth of further literature on this subject, and it is likely to remain an active field. There is so far no rational basis which can be used to predict the activity of new compounds, and substances as diverse as formaldehyde, acriflavine, urethane, caffeine, hydrogen peroxide, and manganous ion are credibly reported as active in one or another system $(26,49,58,65,47)$. In general, reagents with a high reactivity for labile organic $\mathrm{H}$ groups, e.g., formaldehyde, organic peroxides, acyl halides and sulfates, ethylene oxide, acetic anhydride, and diazomethane are probably mutagenic (86) but this does not account for the mutagenicity of such chemically inactive compounds as caffeine or urethane.

Much more careful work is needed in this field, but so far there is no convincing evidence for any appreciable specificity in the mutagenic capacities of any of these chemicals or physical treatments. Each of them induces a wide diversity of mutations (as far as this has been investigated), and in gcneral, the results of, e.g., X-ray treatment, would not be readily distinguishable from that of formaldehyde. On the other hand, it is possible that different gene forms may differ in the frequency with which they will respond to different 
mutagenic treatments; but if there is any specificity, it has so far been of a second order. In this respect, induced resemble spontaneous mutations, whence some genes may mutate more frequently than others, but not in such a way that the environment can be said to direct the mutation of a specific gene preferentially to the exclusion of others.

Spontaneous mutation.-We may return at this point to the mechanism of "spontaneous mutation," keeping in mind that the study of experimentally controlled variables on mutation erases the distinction between spontaneous and induced. So long as the concept connoted by these terms is kept clearly in mind as one distinct from that of directed mutation, there need be no confusion.

Evidence bearing on the relationship between growth and mutation is especially paradoxical, for mutations to phage resistance, for instance, apparently do not accumulate in a resting culture ( 1 ). On the other hand, cultures whose growth is regulated in a special steady-state, controlled-flow culture vessel ("chemostat") mutate at nearly constant rates per unit time, whether the cells are proliferating slowly or rapidly (I06). One interpretation is that spontaneous mutations are due not to intramolecular accidents or reproductive errors, but rather to the action of intracellular chemical mutagens formed by metabolic processes. In this connection it is worth noting that formaldehyde and hydrogen peroxide are both fairly common metabolic intermediates, and that two other mutagens, caffeine and allyl isothiocyanate (mustard oil), are also natural products. Information on the effect of temperature changes on mutation rate would be especially valuable if it could be dissected from effects on growth or metabolism. Under conditions of steady growth a temperature increment of $10^{\circ}$ accelerated mutations in $E$. coli by a factor of about 2, and a similar increment is reported in other systems $\left(8_{5}\right)$.

Bacterial populations.-The necessity for thinking of bacterial cultures always in terms of populations, which may have genotypically diverse components, can scarcely be over-emphasized. The process reviewed to this point, mutation, is the fundamental source of genetic variation, but in view of the smallness of spontaneous mutation rates, it is obvious that the occasional change of a cell from one genetic condition to another can make little impression upon the composition of bacterial populations. The forces that determine which genetic types will predominate in bacterial cultures are the subject of population dynamics. In diploid sexual organisms, population genetics is greatly complicated by recombination and by the concealment of genetic variation in the heterozygous condition, so that the most drastic culling may have to be carried out for a great many generations to have a marked effect on the relative frequency of different gene forms. Selection in bacteria is, as a rule, more straightforward, as shown, for example, by the quantitative isolation of phageresistant mutants by a single application of the virus. The physiological interactions of bacteria in dense cultures lead to less trivial problems in population dynamics. Such interaction may involve an obvious competition for nutrients, 


\section{INTRODUCTION}

or the production of auto- or trans-inhibitory metabolites. An especially clearcut example of the latter in an economically important organism is reprinted here (12). The intricate interrelationships of the production of and sensitivity to species-specific antibiotics ("colicins") have been thoroughly analyzed for $E$. coli by Fredericq (59). One of the most exhaustive analyses of selection dynamics in bacteria will serve best to illustrate the complexity of populational interactions in which several distinct effects may be superimposed (115). A number of populational interactions have been described with a less complete analysis $(138,136,85)$.

Population complexities may also arise when more than one mutation occurs, so that the population consists of several categories of genotypes. One example has been cited already in the stepwise development of resistance by the cumulative effect of serial mutations. A second has been described so recently that its implications are still under discussion. In independent experimental work, three groups of investigators noticed a perplexing sequence of cycles in bacterial populations under conditions of continuous or reiterated culture (25, 106, 124). "Marker" mutant cells increased in proportion as mutations accumulated, but instead of increasing indefinitely, their ratio was subject to sporadic downward shifts. The same interpretation was independently formulated for each case: an adaptive mutation increasing the fit of the bacteria to their rather artificial in vitro environment. Owing to the overwhelming preponderance of the cells not carrying the marker, the adaptive mutation will usually occur in an unmarked cell, the descendants of which will then displace the rest of the population, markers and all. After the changeover, marker mutations accumulate again until a possible second changeover takes place to complete another cycle. It is not predicted that this process could alter the ultimate equilibrium of cell types under mutation "pressure," but that it would alter the short-term course of cultures in which marker mutation pressures outweigh selection effects is apparent. Such adaptive mutations undoubtedly serve as models for evolutionary specialization; as the streptomycindependent mutation cited earlier shows most strikingly, genetic adaptations are often quite specific for the immediate environment. This type of specialization undoubtedly accounts for the often noted loss of virulence encountered frequently among pathogenic bacteria maintained on artificial culture media.

Interclonal variation: Sexual recombination.-The preceding discussion of bacterial populations has supposed that each bacterial cell is genetically isolated from its partners, i.e., that reproduction is exclusively vegetative or clonal. A large body of evidence is now at hand, however, which shows that this picture is incomplete, and that some account must be taken of interclonal processes. One such process was discovered in 1946 by Tatum and Lederberg $(45,84,128)$ and described as genetic recombination, for the experiments are based on the selective isolation of genetic factor combinations from mixtures of different kinds of mutant cells. The conditions under which these genetic exchanges take place, and the patterns in which they result, led these authors 


\section{JOSHUA LEDERBERG}

to conclude that recombination in E. coli, strain $\mathrm{K}-12$, results from a sexual process ( 13 ). This conclusion is based upon evidence which is, to date, entirely negative, indirect, or genetic. It is substantiated, however, by single-cell studies on heterozygous diploid cultures (14, 142), and by a direct demonstration of the nonfiltrability of the agents of recombination (40). A variety of selective techniques can be used for the isolation of recombinants from strain K-I 2 of E. coli. In addition to the selection of prototrophs from mixtures of auxotrophs which is most commonly used, one may select for dually resistant recombinants from mixtures of cells each resistant to one inhibitor (83). A combination of these methods has been profitably employed to detect new strains crossable to K-12; a number were found, some with many properties differentiating them from each other. Developments in the author's laboratory to the date of this writing have been summarized (47-Lederberg).

Recombination in strain $\mathrm{K}-12$ has been applied by a number of other workers for various problems ( 102 ). The genetics of resistance to chloromycetin (6) and to streptomycin have been contrasted $(46$, I0I). The very occurrence of factorial segregation shows, of course, that bacterial enzymes are not to be identified with the genes required for their formation (98). In fact, it is controversial whether the patterns of gene-enzyme interrelationships in bacteria support the "one gene-one enzyme" proposed as a generalization from the properties of auxotrophs in Neurospora (69, 47-Lederberg, Horowitz, Bonner).

Transformations.-The history of "type transformation" is of ten regarded as dating from Griffith's classical experiment on pneumococcal types in 1928 (64), but this work is antedated by a confusing array of studies which date at least as far back as the controversy over the etiology of typhus fever. When the rickettsial etiology of this disease was finally established, many workers suggested that the serological reaction of Proteus OX-19 with rickettsial antibodies (Weil-Felix reaction) was due to a transformation of non-reactive Proteus by rickettsial products, and gave the name "paragglutination" to induced serological variations such as were supposed to be involved here. In support of this hypothesis, (to which no credit is now given) many workers reported that the serological reaction of $E$. coli and other enteric bacteria could be modified by cultivation in filtrates, extracts, or lysates of serologically distinct cultures (reviewed in 81 ). Griffith's demonstration was, however, the first which proved to be readily reproducible by other workers, and remains a proper starting point for modern discussions of transformation. His paper is unfortunately too lengthy to be appropriate for reprinting here, but his observations, and those of other workers leading to the isolation and characterization of the active principle responsible for the pneumococcus transformation are summarized in the paper reprinted here (16).

The genetic interpretations of this transformation are currently a subject of lively discussion. Its description as a specific induced mutation is probably less fruitful than as a transfer of hereditary material from one cell to another (47-Taylor). The main questions which still have to be answered include 


\section{INTRODUCIION}

a) the further physical and chemical characterization of the agent, b) its genetic complexity (that is, whether it involves single characters, the full hereditary material of the pneumococcus, or something intermediate), and c) its cytological relationships to nuclear or extranuclear structures in these cells. The current approaches to these problems are summarized in a number of papers and reviews, $(27,91,93, \mathrm{I} 3 \mathrm{O}, \mathrm{I} 3 \mathrm{I})$.

In addition to the volume of older literature which must be supposed to carry some grain among the chaff, there are a number of more recent reports of transformations in various organisms. These include E. coli (31), Hemophilus influenzae (22), Shigella paradysenteriae (133), Alkaligenes radiobacter (39), and staphylococcus $\left(3^{8}\right)$. Discussion of these and other transformations should take into account complications which might arise from bacterial life cycles more complex than usually regarded.

This presentation has emphasized the contrasting features rather than the similarities of the phenomena described as genetic recombination vs. transformation. Both phenomena tend to the same genetic result: the elaboration of the cells whose hereditary traits are derived from more than one parent. When more is known of the morphological basis of "sexual" genetic recombination on one hand, and of the genetic properties of transformations, on the other, a more profitable synthesis of these contrasting concepts may issue.

The recrudescence of interest in bacterial cytology has been largely independent of, though contemporary with the development of bacterial genetics documented in this book (77). The geneticist notes at least two fields where cytological information is indispensable to him: a) the form and behavior of the bacterial nucleus, and b) the possible existence of complex "life cycles." Reliable information on both these subjects is relatively meagre, but the evidence of at least the existence of nuclei in bacterial cells is relatively convincing. Robinow's paper reprinted here (15) may be taken as one point of departure for the more recent work on this subject $(4 \mathrm{I}, 74)$. With the improvement of techniques, we may look forward to rapid progress in the establishment of the details of the nuclear cycle at cell division, segregation, etc. $(99,118,92$, 122,47 ).

The expression "life cycle" has come to carry many connotations in bacteriology which hinder a careful discussion of the often conflicting observations of generations of bacteriologists. At least one type of cycle is indisputable, the formation of highly resistant endospores by many bacteria. Whether endosporogenesis has any genetic significance is controversial. At the least, they may be supposed to represent the stage at which the presence of only one nucleus per cell (in contrast to the two, four, or more, characteristic of most rods) is the most likely (see 54), and on this basis may be particularly useful for certain types of genetic experiments. More than one student of the mode of nuclear segregation into the endospore has proposed a sequence of nuclear fusion and meiotic reduction (i.e., autogamy). However, the cytological figures are not easily interpreted, especially in view of the pitfalls of logical recon- 


\section{JOSHUA LEDERBERG}

struction of fixed cells, and in this form autogamy is a blind alley without the possibility of genetic novelty, and therefore not amenable to genetic test, (74).

The genetic significance of another cycle, the "L-forms," is equally obscure. It is expected that this problem will be clarified in the very near future to an extent that makes a detailed discussion of its present status here premature. The reader is referred to provocative discussions published elsewhere $\left(5^{0}, 5^{1}, 5^{2}\right.$, $75,76,132,123)$ These studies reopen the possibility of the existence of diminutive forms of many bacteria which may be capable of passing through ordinary bacterial filters. These forms may also be highly resistant (like the endospores) to heat and antiseptics. Since they also may have special nutritional requirements, they may remain dormant in ordinary bacteriological media which support the normal bacterial form. If these observations are confirmed, the filtrable L-forms might well play a role in some of the transformation experiments cited earlier. However, the transforming agent would consist not of a single genetic factor transferred from one cell to another, but of a diminutive form of one cell which germinates under the influence of another. The analogy between the diminutive forms and the gametes of other plants and animals is obvious on a physical plane; the possibility that they may play a similar function in bacterial biology is a provocative one which will require careful study.

One of the reasons for including papers on viruses and bacteria in the same volume is the necessity for considering both the host and the "parasite" in any physiological studies of their association. Furthermore, it is becoming apparent that a great many bacterial cultures carry bacteriophages in a symbiotic relationship ("lysogenicity") so that what are taken to be bacteriological investigations have an unavoidable virological component. A second reason is that the phage-bacterium complex may be profitably regarded as a unit for comparison with other cellular systerns which carry extranuclear hereditary components ( 57,119$)$. Burnet's paper illustrates this very clearly ( 17 ).

It might be worth pointing out also the formal analogy between the transfer of a latent virus from a lysogenic to a sensitive bacterium and the transfer of the capsular attributes from smooth to rough bacteria in the pneumococcus transformation. Whether this analogy is more than superficial only time will tell. It is becoming clear, however, that there is a continuous gradation of properties between systems of cytoplasmic heredity such as the chloroplasts of green plants, and the metabolic granules of yeast, and infective associations such as the "killer" factors in Paramecium, rickettsia in the Arthropoda, and viruses in plants and mammals. In many instances at both extremes, "disinfection" or cure is possible by the use of appropriate drugs (streptomycin for chloroplasts, acriflavine for yeast granules) $(47,57)$.

Burnet's paper also illustrates one of the earliest and clearest cases of mutation in a bacteriophage. In a later study, Luria described host range mutations in phages which restore the virus' ability to attack bacteria which had mutated to resistance to the previous form of the virus (18). This process of compensatory 
mutations on the part of bacterium and virus evidently can sometimes be repeated over a great many cycles, and is of some economic importance in the acetone fermentation industry. These mutations in bacteriophage have been used as the basis for further genetic studies which have revealed the occurrence of genetic recombination among viruses (as well as in bacteria) $(19,20)$. Two lines of attack are exemplified in the papers here. One uses patent markers, such as host range and plaque morphology. For the other, recombination among lethal mutations induced by $\mathrm{UV}$ is used to explain multiplicity reactivation, or the cooperation of several particles damaged by UV to initiate phage growth, in contrast to the ineffectiveness of individual damaged particles.

This work has naturally stimulated attempts to show similar phenomena in animal viruses. At present these are represented by reports by Burnet and Lind (37) of probable recombination in the influenza virus, and similar investigations are under way in other laboratories. Plant and animal viruses are likely to be more difficult to study from this point of view, chiefly because of technical difficulties in initiating infections with single virus particles $(35,62,79)$.

Genetic study of bacteria and viruses is closely interwoven with the most general problems of their biology; this is not surprising, for the same has happened in other areas of biology. But it is to be hoped that genetics will be regarded not as a unique or isolated part of bacteriological study, but as an element of all teaching and research in microbiology. 

THE PAPERS 



\title{
MUTATIONS OF BACTERIA FROM VIRUS SENSITIVITY TO VIRUS RESISTANCE ${ }^{1,2}$
}

\author{
S. E. LURIA ${ }^{3}$ AND M. DELBRÜCK \\ Indiana University, Bloomington, Indiana, and \\ Vanderbill University, Nashville, Tennessee
}

Received May 29, 1943

\section{INTRODUCTION}

W HEN a pure bacterial culture is attacked by a bacterial virus, the culture will clear after a few hours due to destruction of the sensitive cells by the virus. However, after further incubation for a few hours, or sometimes days, the culture will often become turbid again, due to the growth of a bacterial variant which is resistant to the action of the virus. This variant can be isolated and freed from the virus and will in many cases retain its resistance to the action of the virus even if subcultured through many generations in the absence of the virus. While the sensitive strain adsorbed the virus readily, the resistant variant will generally not show any affinity to it.

The resistant bacterial variants appear readily in cultures grown from a single cell. They were, therefore, certainly not present when the culture was started. Their resistance is generally rather specific. It does not extend to viruses that are found to differ by other criteria from the strain in whose presence the resistant culture developed. The variant may differ from the original strain in morphological or metabolic characteristics, or in serological type or in colony type. Most often, however, no such correlated changes are apparent, and the variant may be distinguished from the original strain only by its resistance to the inciting strain of virus.

The nature of these variants and the manner in which they originate have been discussed by many authors, and numerous attempts have been made to correlate the phenomenon with other instances of bacterial variation.

The net effect of the addition of virus consists of the appearance of a variant strain, characterized by a new stable character-namely, resistance to the inciting virus. The situation has often been expressed by saying that bacterial viruses are powerful "dissociating agents." While this expression summarizes adequately the net effect, it must not be taken to imply anything about the mechanism by which the result is brought about. A moment's reflection will show that there are greatly differing mechanisms which might produce the same end result.

D'Herelle (1926) and many other investigators believed that the virus by direct action induced the resistant variants. GRATIA (I92I), BURNET (I929), and others, on the other hand, believed that the resistant bacterial variants are produced by mutation in the culture prior to the addition of virus. The

1 Theory by M. D., experiments by S. E. L.

2 Aided by grants from the Dazian Foundation for Medical Research and from the ROCKEFELLER FOUNDATION.

Fellow of the Guggenheim Foundation. 
virus merely brings the variants into prominence by eliminating all sensitive bacteria.

Neither of these views seems to have been rigorously proved in any single instance. BURNET'S (1929) work on isolations of colonies, morphologically distinguishable prior to the addition of virus, which proved resistant to the virus comes nearest to this goal. His results appear to support the mutation hypothesis for colony variants. It may seem peculiar that this simple and important question should not have been settled long ago, but a close analysis of the problem in hand will show that a decision can only be reached by a more subtle quantitative study than has hitherto been applied in this field of research.

Let us begin by restating the basic experimental finding.

A bacterial culture is grown from a single cell. At a certain moment the culture is plated with virus in excess. Upon incubation, one finds that a very small fraction of the bacteria survived the attack of the virus, as indicated by the development of a small number of resistant colonies, consisting of bacteria which do not even adsorb the virus.

Let us focus our attention on the first generation of the resistant variantthat is, on those bacteria which survive immediately after the virus has been added. These survivors we may call the "original variants." We know that these bacteria and their offspring are resistant to the virus. We may formulate three alternative hypotheses regarding them.

a. Hypothesis of mutation to immunity. The original variants were resistant before the virus was added, and, like their offspring, did not even adsorb it. On this hypothesis the virus did not interact at all with the original variants, the origin of which must be ascribed to "mutations" that occur quite independently of the virus. Naming such hereditary changes "mutations" of course does not imply a detailed similarity with any of the classes of mutations that have been analyzed in terms of genes for higher organisms. The similarity may be merely a formal one.

b. Hypothesis of acquired immunity. The original variants interacted with the virus, but survived the attack. We may then inquire into the predisposing cause which effected the survival of these bacteria in contradistinction to the succumbing ones. The predisposing cause may be hereditary or random. Accordingly we arrive at two alternative hypotheses-namely,

$\mathrm{b}_{1}$. Hypothesis of acquired immunity of hereditarily predisposed individuals. The original variants originated by mutations occurring independently of the presence of virus. When the virus is added, the variants will interact with it, but they will survive the interaction, just as there may be families which are hereditarily predisposed to survive an otherwise fatal virus infection. Since we know that the offspring of the original variants do not adsorb the virus, we must further assume that the infection caused this additional hereditary change.

b2. Hypothesis of acquired immunity-hereditary after infection. The original variants are predisposed to survival by random physiological variations in size, age, etc. of the bacteria, or maybe even by random variations in the 
point of attack of the virus on the bacterium. After survival of such random individuals, however, we must assume that their offspring are hereditarily immune, since they do not even adsorb the virus.

These alternative hypotheses may be grouped by first considering the origin of the hereditary difference. Do the original variants trace back to mutations which occur independently of the virus, such that these bacteria belong to a few clones, or do they represent a random sample of the entire bacterial population? The first alternative may then be subdivided further, according to whether the original variants do or do not interact with the virus. Disregarding for the moment this subdivision, we may formulate two hypotheses:

I. First hypothesis (mutation): There is a finite probability for any bacterium to mutate during its life time from "sensitive" to "resistant." Every offspring of such a mutant will be resistant, unless reverse mutation occurs. The term "resistant" means here that the bacterium will not be killed if exposed to virus, and the possibility of its interaction with virus is left open.

2. Second hypothesis (acquired hereditary immunity): There is a small finite probability for any bacterium to survive an attack by the virus. Survival of an infection confers immunity not only to the individual but also to its offspring. The probability of survival in the first instance does not run in clones. If we find that a bacterium survives an attack, we cannot from this information infer that close relatives of it, other than descendants, are likely to survive the attack.

The last statement contains the essential difference between the two hypotheses. On the mutation hypothesis, the mutation to resistance may occur any time prior to the addition of virus. The culture therefore will contain "clones of resistant bacteria" of various sizes, whereas on the hypothesis of acquired immunity the bacteria which survive an attack by the virus will be a random sample of the culture.

For the discussion of the experimental possibility of distinction between these two hypotheses, it is important to keep in mind that the offspring of a tested bacterium which survives is resistant on either hypothesis. Repeated tests on a bacterium at different times, or on a bacterium and on its offspring, could therefore give no information of help in deciding the present issue. Thus, one has to resort to less direct methods. Two main differences may be derived from the hypotheses:

First, if the individual cells of a very large number of microcolonies, each containing only a few bacteria, were examined for resistance, a pronounced correlation between the types found in a single colony would be expected on the mutation hypothesis, while a random distribution of resistants would be expected on the hypothesis of acquired hereditary immunity. This experiment, however, is not practicable, both on account of the difficulty of manipulation and on account of the small proportion of resistant bacteria.

Second, on the hypothesis of resistance due to mutation, the proportion of resistant bacteria should increase with time, in a growing culture, as new mutants constantly add to their ranks. 
In contrast to this increase in the proportion of resistants on the mutation hypothesis, a constant proportion of resistants may be expected on the hypothesis of acquired hereditary immunity, as long as the physiological conditions of the culture do not change. To test this point, accurate determinations of the proportion of resistant bacteria in a growing culture and in successive subcultures are required. In the attempt to determine accurately the proportion of resistant bacteria, great variations of the proportions were found, and results did not seem to be reproducible from day to day.

Eventually, it was realized that these fluctuations were not due to any uncontrolled conditions of our experiments, but that, on the contrary, large fluctuations are a necessary consequence of the mutation hypothesis and that the quantitative study of the fluctuations may serve to test the hypothesis.

The present paper will be concerned with the theoretical analysis of the probability distribution of the number of resistant bacteria to be expected on either hypothesis and with experiments from which this distribution may be inferred.

While the theory is here applied to a very special case, it will be apparent that the problem is a general one, encountered in any case of mutation in uniparental populations. It is the belief of the authors that the quantitative study of bacterial variation, which until now has made such little progress, has been hampered by the apparent lack of reproducibility of results, which, as we shall show, lies in the very nature of the problem and is an essential element for its analysis. It is our hope that this study may encourage the resumption of quantitative work on other problems of bacterial variation.

\section{THEORY}

The aim of the theory is the analysis of the probability distributions of the number of resistant bacteria to be expected on the hypothesis of acquired immunity and on the hypothesis of mutation.

The basic assumption of the hypothesis of acquired hereditary immunity is the assumption of a fixed small chance for each bacterium to survive an attack by the virus. In this case we may therefore expect a binomial distribution of the number of resistant bacteria, or, in cases where the chance of survival is small, a Poisson distribution.

The basic assumption of the mutation hypothesis is the assumption of a fixed small chance per time unit for each bacterium to undergo a mutation to resistance. The assumption of a fixed chance per time unit is reasonable only for bacteria in an identical state. Actually the chance may vary in some manner during the life cycle of each bacterium and may also vary when the physiological conditions of the culture vary, particularly when growth slows down on account of crowding of the culture. With regard to the first of these variations, the assumed chance represents the average chance per time unit, averaged over the life cycle of a bacterium. With regard to the second variation, it seems reasonable to assume that the chance is proportional to the growth rate of the bacteria. We will then obtain the same results as on the simple assump- 
tion of a fixed chance per time unit, if we agree to measure time in units of division cycles of the bacteria, or any proportional unit.

We shall choose as time unit the average division time of the bacteria, divided by $\ln 2$, so that the number $\mathrm{N}_{\mathrm{t}}$ of bacteria in a growing culture as function of time $t$ follows the equations

$$
\mathrm{dN}_{\mathrm{t}} / \mathrm{dt}=\mathrm{N}_{\mathrm{t}} \text {, and } \mathrm{N}_{\mathrm{t}}=\mathrm{N}_{\mathrm{o}} \mathrm{e}^{\mathrm{t}} .
$$

We may then define the chance of mutation for each bacterium during the time element dt as

$$
\text { adt, }
$$

so that a is the chance of mutation per bacterium per time unit, or the "mutation rate."

If a bacterium is capable of different mutations, each of which results in resistance, the mutation rate here considered will be the sum of the mutation rates associated with each of the different mutations.

The number $\mathrm{dm}$ of mutations which occur in a growing culture during a time interval $\mathrm{dt}$ is then equal to this chance (2) multiplied by the number of bacteria, ${ }^{4}$ or

$$
\mathrm{dm}=\operatorname{adtN},
$$

and from this equation the number $m$ of mutations which occur during any finite time interval may be found by integration to be

$$
\mathrm{m}=\mathrm{a}\left(\mathrm{N}_{\mathrm{t}}-\mathrm{N}_{0}\right)
$$

or, in words, to be equal to the chance of mutation per bacterium per time unit multiplied by the increase in the number of bacteria.

The bacteria which mutate during any time element $\mathrm{dt}$ form a random sample of the bacteria present at that time. For small mutation rates, their number will therefore be distributed according to Poisson's law. Since the mutations occuring in different time intervals are quite independent from each other, the distribution of all mutations will also be according to Poisson's law.

This prediction cannot be verified directly, because what we observe, when we count the number of resistant bacteria in a culture, is not the number of mutations which have occurred, but the number of resistant bacteria which have arisen by multiplication of those which mutated, the amount of multiplication depending on how far back the mutation occurred.

If, however, the premise of the mutation hypothesis can be proved by other means, the prediction of a Poisson distribution of the number of mutations

- We assume that the number of resistant bacteria is at all times small in comparison with the total number of bacteria. If this condition is not fulfilled, the total number of bacteria in this equation has to be replaced by the number of sensitive bacteria. The subsequent theoretical developments will then become a little more complicated. For the case studied in the experimental part of this paper the condition is fulfilled. 
may be used to determine the mutation rate. It is only necessary to determine the fraction of cultures showing no mutation in a large series of similar cultures. This fraction $\mathrm{p}_{0}$, according to theory, should be:

$$
\mathrm{p}_{0}=\mathrm{e}^{-\mathrm{m}} \text {. }
$$

From this equation the average number $m$ of mutations may be calculated, and hence the mutation rate a from equation (4).

Let us now turn to the discussion of the distribution of the number of resistant bacteria.

The average number of resistant bacteria is easily obtained by noting that this number increases on two accounts-namely, first on account of new mutations, second on account of the growth of resistant bacteria from previous mutations. During a time element dt the increase on the first account will be, by equation (3): $\operatorname{adt} N_{t} . N_{t}$, the number of bacteria present at time $t$, is given by equation ( $\mathrm{I}$ ). The increase on the second account will depend on the growth rate of the resistant bacteria. In the simple case, which we shall treat here, this growth rate is the same as that of the sensitive bacteria, and the increment on this account is $\rho \mathrm{dt}$, where $\rho$ is the average number of resistant bacteria present at time t. We have then as the total rate of increase of the average number of resistant bacteria $\mathrm{d} \rho / \mathrm{dt}=\mathrm{aN}_{\mathrm{t}}+\rho$ and upon integration

$$
\rho=\operatorname{taN}_{\mathrm{t}}
$$

if we assume that at time zero the culture contained no resistant bacteria.

It will be seen that the average number of resistant bacteria increases more rapidly than the total number of bacteria. Indeed the fraction of resistant bacteria in the culture increases proportionally to time. This, as pointed out in the introduction, is a distinguishing feature of the mutation hypothesis but unfortunately, as will be seen in the sequel, is not susceptible to experimental verification due to statistical fluctuations.

The resistant bacteria in any culture may be grouped, for the purpose of this analysis, into clones, taking together all those which derive from the same mutation. We may say that the culture contains clones of various age and size, calling "age" of a clone the time since its parent mutation occurred and "size" of a clone the number of bacteria in a clone at the time of observation. It is clear that size and age of a clone determine each other. If, in particular, we make the simplifying hypothesis that the resistant bacteria grow as fast as the normal sensitive strain, the relation between size and age will be expressed by equation ( $\mathrm{I}$ ), with appropriate meaning given to the symbols.

The relation implies that the size of a clone increases exponentially with its age. On the other hand, the frequency with which clones of different ages may be encountered in any culture must decrease exponentially with age, according to equations (3) and (I).

Combining these two results-namely, that clone size increases exponentially with clone age and that frequency of clones of different age decreases exponentially with clone age-we see that the two factors cancel when the 
average number of bacteria belonging to clones of one age group is considered. In other words, at the time of observation we shall have, on the average, as many resistant bacteria stemming from mutations which occurred during the first generation after the culture was started as stemming from mutations which occurred during the last generation before observation, or during any other single generation.

On the other hand, for small mutation rates it is very improbable that any mutation will occur during the early generations of a single or of a limited number of experimental cultures. It follows that the average number of resistant bacteria derived from a limited number of experimental cultures will, probably, be considerably smaller than the theoretical value given by equation (6), and, improbably, the experimental value will be much larger than the theoretical value. The situation is similar to the operation of a (fair) slot machine, where the average return from a limited number of plays is probably considerably less than the input, and improbably, when the jackpot is hit, the return is much bigger than the input.

This result characterizes the distribution of the number of resistant bacteria as a distribution with a long and significant tail of rare cases of high numbers of resistant bacteria, and therefore as a distribution with an abnormally high variance. This variance will be calculated below.

For such distributions the averages derived from limited numbers of samples yield very poor estimates of the true averages. Somewhat better estimates of the averages may in such cases be obtained by omitting, in the calculation of the theoretical averages, the contribution to these averages of those events which probably will not occur in any of our limited number of samples. We may do this, in the integration leading to equation (6), by putting the lower limit of integration not at time zero, when the cultures were started, but at a certain time $t_{0}$, prior to which mutations were not likely to occur in any of our experimental cultures. We then obtain as a likely average $\mathrm{r}$ of the number of resistant bacteria in a limited number of samples, instead of equation (6),

$$
\mathrm{r}=\left(\mathrm{t}-\mathrm{t}_{0}\right) \mathrm{aN}_{\mathrm{t}} \text {. }
$$

It now remains to choose an appropriate value for the time interval $t-t_{0}$.

For this purpose we return to equation (4), in which it was stated that the average number of mutations which occur in a culture is equal to the mutation rate multiplied by the increase of the number of bacteria. Let us then choose $t_{0}$ such that up to that time just one mutation occurred, on the average, in a group of $\mathrm{C}$ similar cultures, or

$$
I=\mathrm{aC}\left(\mathrm{N}_{\mathrm{t}_{0}}-\mathrm{N}_{0}\right) \text {. }
$$

In this equation we may neglect $\mathrm{N}_{0}$, the number of bacteria in each inoculum, in comparison with $\mathrm{N}_{t_{0}}$, the number of bacteria in each culture at the critical time $t_{0}$. We may also express $\mathrm{N}_{t_{0}}$ in terms of $\mathrm{N}_{\mathrm{t}}$, the number of bacteria at the time of observation, applying equation $(\mathrm{I})$ :

$$
N_{t_{0}}=N_{t} e^{-\left(t-t_{0}\right)} \text {. }
$$


We thus obtain

$$
\mathrm{t}-\mathrm{t}_{0}=\ln \left(\mathrm{N}_{\mathrm{t}} \mathrm{Ca}\right) \text {. }
$$

Equations (6a) and (7) may be combined to eliminate $t-t_{0}$ and to yield a relation between the observable quantities $r$ and $N_{t}$ on the one hand and the mutation rate a on the other hand, to be determined by this equation:

$$
\mathbf{r}=\mathrm{aN}_{\mathrm{t}} \ln \left(\mathrm{N}_{\mathrm{t}} \mathrm{Ca}\right) \text {. }
$$

This simple transcendental equation determining a may be solved by any standard numerical method. In figure $I$, the relation between $r$ and $a N_{t}$ is plotted for several values of $\mathrm{C}$.

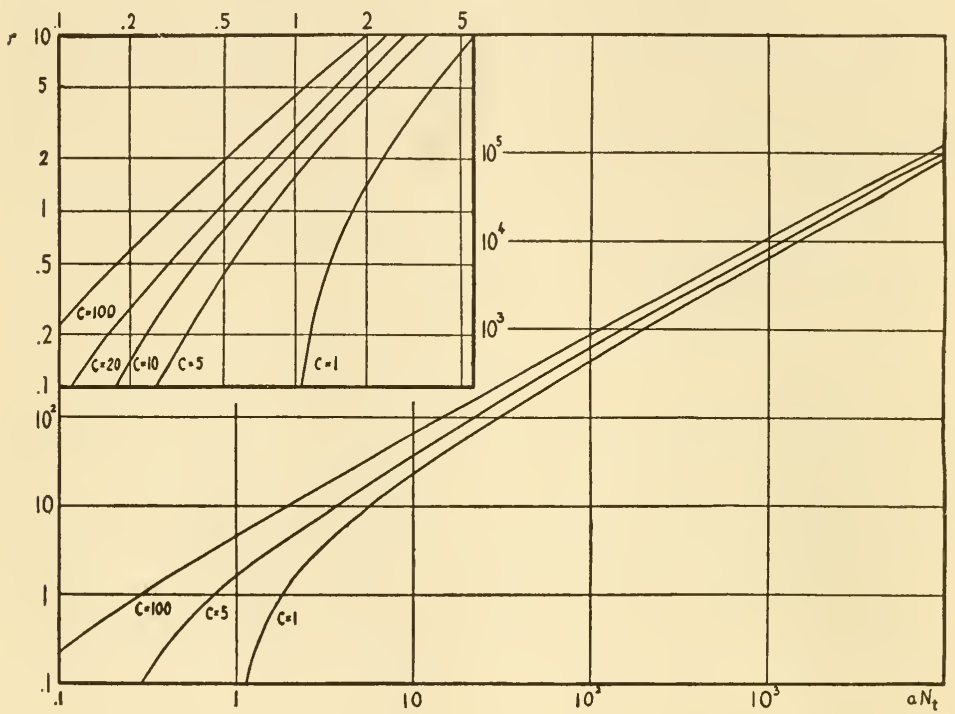

Figure 1.-The value of $\mathrm{aN}_{\mathrm{t}}$ as a function of $\mathrm{r}$ for various values of $\mathrm{C}$. The upper left hand part of the figure gives the curves for low values of $\mathrm{aN}_{\mathrm{t}}$ and of $\mathrm{r}$ on a larger scale. See text.

Estimates of a obtained from equation (8) will be too high if in any of the experimental cultures a mutation happened to occur prior to time $t_{0}$. From the definition of $t_{0}$ it will be seen that this can be expected to happen in little more than half of the cases.

While we have thus obtained a relation permitting an estimate of the mutation rate from the observation of a limited number of cultures, this relation is in no way a test of the correctness of the underlying assumptions and, in particular, is not a test of the mutation hypothesis itself. In order to find such tests of the correctness of the assumption we must derive further quantitative relations concerning the distribution of the number of resistant bacteria and compare them with experimental results. 
Since we have seen that the mutation hypothesis, in contrast to the hypothesis of acquired immunity, predicts a distribution of the number of resistant bacteria with a long tail of high numbers of resistant bacteria, the determination of the variance of the distribution should be helpful in differentiating between the two hypotheses. We may here again determine first the true variance-that is, the variance of the complete distribution-and second the likely variance in a limited number of cultures, by omitting those cases which are not likely to occur in a limited number of cultures.

The variance may be calculated in a simple manner by considering separately the variances of the partial distributions of resistant bacteria, each partial distribution comprising the resistant bacteria belonging to clones of one age group. The distribution of the total number of resistant bacteria is the resultant of the superposition of these independent partial distributions.

Each partial distribution is due to the mutations which occurred during a certain time interval $\mathrm{d} \tau$, extending from $(\mathrm{t}-\tau)$ to $(\mathrm{t}-\tau+\mathrm{d} \tau)$. The average number of mutations which occurred during this interval is, according to equation (3),

$$
\mathrm{dm}=\mathrm{aN}_{\tau} \mathrm{d} \tau=\mathrm{aN}_{\mathrm{t}} \mathrm{e}^{-\tau} \mathrm{d} \tau .
$$

These mutations will be distributed according to Poisson's law, so that the variance of each of these distributions is equal to the mean of the distribution. We are however not interested in the distribution of the number of mutations but in the distribution of the number of resistant bacteria which stem from these mutations at the time of observation-that is, after the time interval $\tau$. Each original mutant has then grown into a clone of size $\mathrm{e}^{\tau}$. The distribution of the resistant bacteria stemming from mutations occurred in the time interval $\mathrm{d} \tau$ has therefore an average value which is $\mathrm{e}^{\tau}$ times greater than the average number of mutations, and a variance which is $\mathrm{e}^{2 \tau}$ times greater than the variance of the number of mutations. Thus we find for the average number of resistant bacteria:

$$
\mathrm{d} \rho=\mathrm{aN}_{\mathrm{t}} \mathrm{d} \tau
$$

and for the variance of this number

$$
\operatorname{var}_{\mathrm{d} \rho}=\mathrm{aN}_{\mathrm{t}} \mathrm{e}^{\tau} \mathrm{d} \tau
$$

From this variance of the partial distribution, the variance of the distribution of all resistant bacteria may be found simply by integrating over the appropriate time interval - that is, either from time $t$ to time $\circ(\tau$ from $\circ$ to $t)$, if the true variance is wanted, or from time $t$ to time $t_{0}\left(\tau\right.$ from $\circ$ to $\left.t-t_{0}\right)$, if the likely variance in a limited number of cultures is wanted. In the first case we obtain:

$$
\operatorname{var}_{\rho}=\mathrm{aN}_{\mathrm{t}}\left(\mathrm{e}^{\mathrm{t}}-\mathrm{I}\right)
$$

In the second case we obtain:

$$
\operatorname{var}_{r}=a N_{t}\left[e^{\left(t-t_{0}\right)}-I\right] .
$$


Substituting here the previously found value of $\left(t-t_{0}\right)$ and neglecting the second term in the brackets, we obtain:

$$
\operatorname{var}_{\mathrm{r}}=\mathrm{Ca}^{2} \mathrm{~N}_{\mathrm{t}}^{2} \text {. }
$$

Comparing this value of the likely variance with the value of the likely average, from equation (8), we see that the ratio of the standard deviation to the average is:

$$
\sqrt{\operatorname{var}_{r}} / r=\sqrt{\mathrm{C}} / \ln \left(\mathrm{N}_{\mathrm{t}} \mathrm{Ca}\right) .
$$

It is seen that this ratio depends on the logarithm of the miutation rate and will consequently be only a little smaller for mutation rates many thousand times greater than those considered in the experiments reported in this paper.

In the beginning of this theoretical discussion we pointed out that the hypothesis of acquired immunity leads to the prediction of a distribution of the number of resistant bacteria according to Poisson's law, and therefore to the prediction of a variance equal to the average. On the other hand, if we compare the average, equation (8), with the variance, equation (I I), (not, as above, with the square root of the variance), we obtain

$$
\operatorname{var}_{\mathbf{r}}=\mathrm{rN}_{\mathrm{t}} \mathrm{Ca} / \ln \left(\mathrm{N}_{\mathrm{t}} \mathrm{Ca}\right) .
$$

Equation (I 2a) shows that the likely ratio between variance and average is much gater than unity on the hypothesis of mutation, if $\left(\mathrm{N}_{\mathrm{t}} \mathrm{Ca}\right)$, the total number of mutations which occurred in our cultures, is large compared to unity. ${ }^{5}$

It is possible to carry the analysis still further and to evaluate the higher moments of the distribution function of the number of resistant bacteria, or even the distribution function itself. The moments are comparatively easy to obtain, while the calculation of the distribution function involves considerable

${ }^{5}$ In some of the experiments reported in the present paper we did not determine the tota 1 number of resistant bacteria in each culture, but the number contained in a small sample from each culture. In these cases the variance of the distribution of the number of resistant bacteria will be slightly increased by the sampling error. The proper procedure is here first to find the average number of resistant bacteria per culture by multiplying the average per sample by the ratio

$$
\frac{\text { volume of culture }}{\text { volume of sample; }}
$$

second, to evaluate the mutation rate with the help of equation (8); third, to figure the likely variance for the cultures by equation $(\mathrm{r} I)$; fourth, to divide this variance by the square of the ratio $\left(r_{3}\right)$ to obtain that part of the variance in the samples which is due to the chance distribution of the mutations. The experimental variance should be greater than this value, on account of the sampling variance. The sampling variance is in all our cases only a small correction to the total variance, and it is sufficient to use its upper limit, that of the Poisson distribution, in our calculations. Consequently, when comparing the experimental with the calculated values, we first subtract from the experimental value the sampling variance, which we take to be equal to the average number of resistant bacteria. 
mathematical difficulties. An approximation to the beginning of the distribution function-that is, to its values for small numbers of resistant bacteriamay be obtained by grouping mutations according to the bacterial generation during which they occurred. For instance, the probability of obtaining seven resistant bacteria may be broken down into the sum of the following alternative events: (a) seven mutations during the last generation; (b) three mutations during the last generation and two mutations one generation back; (c) three mutations during the last generation and one mutation two generations back; (d) one mutation during the last generation and three mutations one generation back; (e) one mutation during the last generation, one mutation one generation back and one mutation two generations back.

The probability of each of these events depends only on the mutation rate and on the final number of bacteria.

The grouping of mutations according to the bacterial generation during which they occurred, and the assumption that the bacteria increase in simple geometric progression, simplify the calculation sufficiently to permit numerical computation. On the other hand, the classes with two, four, eight, etc., mutants are artificially favored by this procedure, so that a somewhat uneven distribution results, with too high values for two, four, eight, etc., resistant bacteria (see fig. 2).

\section{MATERIAL AND METHODS}

The material used for our experimental study consisted of a bacterial virus $\alpha$ and of its host, Escherichia coli B (DELBRÜCK and LURIA I942). Secondary cultures after apparently complete lysis of B by virus $\alpha$ show up within a few hours from the time of clearing. They consist of cells which are resistant to the action of virus $\alpha$, but sensitive to a series of other viruses active on B. The resistant cells breed true and can be established easily as pure cultures. No trace of virus could be found in any pure culture of the resistant bacteria studied in this paper. The resistant strains are therefore to be considered as non-lysogenic.

Tests were made to see whether the resistance to virus $\alpha$ was a stable character of the resistant strains. In the first place, it was found that virus $\alpha$ is not appreciably adsorbed by any of the resistant strains. In the second place, when a certain amount of virus $\alpha$ is mixed with a growing culture of a resistant strain, no measurable increase of the titer of virus $\alpha$ occurs over a period of several hours. This is a very sensitive test for the occurrence of sensitive bacteria, and its negative result for all resistant strains shows that reversion to sensitivity must be a very rare event.

Morphologically at least two types of colonies of resistant bacteria may be distinguished. The first type of colony is similar to the type produced by the sensitive strain both in size and in the character of the surface and of the edge. The second type of colony is much smaller and translucent. The difference in colony type is maintained in subcultures. Microscopically the bacteria from these two types of colonies are indistinguishable. They also do not differ 
from each other or from the sensitive strain in their fermentation reactions on common sugars and in the characteristics of their growth curves in nutrient broth. In particular, the lag periods, the division times during the logarithmic phase of growth, and the maximum titers attained are identical for the sensitive strain and for the two variants. Both variants, therefore, fulfill the requirements for the applicability of the theory developed above.

In the presentation of our experimental results we have lumped the counts of the two types of colonies together, because: (I) theoretically, this is equivalent to summing the corresponding mutation rates; (2) experimentally, we are not certain whether each of these types does not actually comprise a diversity of variants; (3) experimentally, no correlation appeared to exist between the occurrence of these variants, which shows the independence of the causes of their occurrence.

Cultures of $\mathrm{B}$ were grown either in nutrient broth (containing .5 percent $\mathrm{NaCl}$ ) or in an asparagin-glucose synthetic medium. In the latter, the division time during the logarithmic phase of growth was 35 minutes, as compared with 19 minutes in broth. In synthetic medium, the acidity increased during the time of incubation from $\mathrm{pH} 7$ to $\mathrm{pH} 5$.

In cultures of strain $\mathrm{B}$, between $1 \mathrm{O}^{-8}$ and $1 \mathrm{O}^{-5}$ of the bacteria are found usually to give colonies resistant to the action of virus $\alpha$ when samples of such cultures are plated with large amounts of virus. In order to be reasonably certain that the resistant bacteria found in the test had not been introduced into the test culture with the initial inoculum, the test cultures were always started with very small inocula, containing between 50 and 500 bacteria from a growing culture. Thus any resistant bacterium found at the moment of testing (when the culture contains between $10^{8}$ and $5 \times 10^{9}$ bacteria/cc) must be an offspring of one of the sensitive bacteria of the inoculum.

All platings were made on nutrient agar plates. The plating experiments for counting the number of resistant bacteria in a liquid culture of the sensitive strain were done by plating either a portion or the entire culture with a large amount of virus $\alpha$. The virus was plated first, and spread over the entire surface of the agar. A few minutes later the bacterial suspension to be tested was spread over the central part of the plate, leaving a margin of at least one centimeter. Thus all bacteria were surrounded by large numbers of virus particles.

Microscopic examination of plates seeded in this manner showed that lysis takes place very quickly; only bacteria which at the time of plating were in the process of division may sometimes complete the division. The resistant colonies which appear after incubation are therefore due to resistant bacterial cells present at the time of plating.

The total number of bacteria present in the culture to be tested was determined by colony counts in the usual manner.

The resistant colonies of the large type appear after I2-I6 hours of incubation, the colonies of the small type appear after 18-24 hours, and never reach half the size of the former ones. Counts were usually made after 24 and 48 hours. 
EXPERIMENTAL

\section{A Test of the Reliability of the Plating Method}

In our experiments we wanted to study the fluctuations of the numbers of resistant bacteria found in cultures of sensitive bacteria. It was therefore necessary to show first that the method of testing did not involve any unrecognized variables, which caused the number of resistant colonies to vary from plate to plate or from sample to sample.

Therefore, parallel platings were made using a series of samples from the same bacterial culture. If our plating method is reliable, fluctuations should in this arrangement be due to random sampling only, and the variance from a series of such samples should be equal to the mean.

Table I gives the results of three such experiments. It.will be seen that in

TABLE I

The number of resistant bacteria in different samples from the same culture.

\begin{tabular}{|c|c|c|c|}
\hline SAMPLE NO. & $\begin{array}{c}\text { EXP. NO. IOa } \\
\text { RESISTANT COLONIES }\end{array}$ & $\begin{array}{c}\text { EXP. NO. II } \\
\text { RESISTANT COLONIES }\end{array}$ & $\begin{array}{c}\text { EXP. NO. } 3 \\
\text { RESISTANT COLONIES }\end{array}$ \\
\hline I & 14 & 46 & 4 \\
\hline 2 & I5 & 56 & 2 \\
\hline 3 & 13 & $5^{2}$ & 2 \\
\hline 4 & $2 \mathrm{I}$ & 48 & I \\
\hline 5 & I 5 & 65 & 5 \\
\hline 6 & 14 & 44 & 2 \\
\hline 7 & 26 & 49 & 4 \\
\hline 8 & 16 & $5 \mathrm{I}$ & 2 \\
\hline 9 & 20 & 56 & 4 \\
\hline 10 & 13 & 47 & 7 \\
\hline mean & I6.7 & $5 \mathrm{I} .4$ & $3 \cdot 3$ \\
\hline variance & 15 & 27 & 3.8 \\
\hline$x^{2}$ & 9 & $5 \cdot 3$ & 12 \\
\hline$\hat{\mathrm{P}}$ & .4 & .8 & .2 \\
\hline
\end{tabular}

all three cases variance and mean agree as well as may be expected. There is therefore no reason to assume that the method of sampling or plating introduces any fluctuations into our results besides the sampling error.

\section{Fluctuations of the Number of Resistant Bacteria in Samples from a Series of Similar Cultures}

As pointed out in the introduction and in the theoretical part, the hypothesis of acquired immunity and the hypothesis of mutation lead to radically different predictions regarding the distribution of the number of resistant bacteria in a series of similar cultures. The hypothesis of acquired immunity predicts a variance equal to the average, as in sampling, while the mutation hypothesis predicts a much greater variance.

Series of five to roo cultures were set up in parallel with small equal inocula, and were grown until maximum titer was reached. Three kinds of cultures 
were used-namely: (I) I0.0 cc aerated broth cultures; (2) .2 cc broth cultures; (3).2 cc synthetic medium cultures.

The results of all tests for the number of resistant bacteria are summarized in table 2 and table 3 .

\section{TABLE 2}

The number of resistant bacteria in series of similar cultures.

\begin{tabular}{|c|c|c|c|c|c|c|c|c|}
\hline EXPERTMENT NO. & $\mathbf{I}$ & IO & II & I5 & I6 & 17 & $21 \mathrm{a}$ & $2 \mathrm{Ib}$ \\
\hline Number of cultures & 9 & 8 & 10 & 10 & 20 & I 2 & I9 & 5 \\
\hline Volume of cultures, cc & 10.0 & 10.0 & 10.0 & 10.0 & $.2 *$ & $.2 *$ & .2 & 10.0 \\
\hline Volume of samples, cc & .05 & .05 & .05 & .05 & .08 & .08 & .05 & .05 \\
\hline \multicolumn{9}{|l|}{ Culture No. } \\
\hline I & 10 & 29 & 30 & 6 & I & I & 0 & $3^{8}$ \\
\hline 2 & I8 & 41 & ro & 5 & $\circ$ & 0 & $\circ$ & 28 \\
\hline 3 & I 25 & 17 & 40 & IO & 3 & 0 & 0 & 35 \\
\hline 4 & Io & 20 & 45 & 8 & 0 & 7 & 0 & 107 \\
\hline 5 & I 4 & $3 \mathbf{I}$ & $\mathbf{1} 83$ & 24 & $\circ$ & ○ & 8 & 13 \\
\hline 6 & 27 & 30 & 12 & 13 & 5 & 303 & $\mathbf{I}$ & \\
\hline 7 & 3 & 7 & 173 & 165 & $\circ$ & 0 & $\circ$ & \\
\hline 8 & 17 & I 7 & 23 & 15 & 5 & 0 & I & \\
\hline 9 & 17 & & 57 & 6 & 0 & 3 & 0 & \\
\hline Io & & & $5 I$ & 10 & 6 & 48 & 15 & \\
\hline II & & & & & 107 & $\mathbf{I}$ & 0 & \\
\hline 12 & & & & & ○ & 4 & 0 & \\
\hline 13 & & & & & $\circ$ & & I9 & \\
\hline$I_{4}$ & & & & & 0 & & 0 & \\
\hline 15 & & & & & $\mathbf{I}$ & & $\circ$ & \\
\hline I 6 & & & & & o & & 17 & \\
\hline 17 & & & & & $\circ$ & & II & \\
\hline I 8 & & & & & 64 & & $\circ$ & \\
\hline I9 & & & & & 0 & & 0 & \\
\hline 20 & & & & & 35 & & & \\
\hline Average per sample & 26.8 & 23.8 & 62 & 26.2 & I I. 35 & 30 & 3.8 & 48.2 \\
\hline Variance (corrected for & & & & & & & & \\
\hline sampling) & I 217 & 84 & 3498 & 2178 & 694 & 6620 & 40.8 & $\operatorname{II} 71$ \\
\hline Average per culture & 5360 & 4760 & 12400 & 5240 & 28.4 & 75 & I5.I & 8440 \\
\hline Bacteria per culture & $3.4 \times 10^{10}$ & $4 \times 10^{10}$ & $4 \times 10^{20}$ & $2.9 \times 10^{10}$ & $5.6 \times 10^{8}$ & $5 \times 10^{8}$ & I. I $\times 10^{8}$ & $3.2 \times 10^{10}$ \\
\hline Mutation rate & $1.8 \times 10^{-8}$ & $1.4 \times 10^{-8}$ & $4.1 \times 10^{-8}$ & $2.1 \times 10^{-8}$ & I. I $\times 1 \mathrm{rO}^{-8}$ & $3.0 \times 10^{-3}$ & $3.3 \times 10^{-8}$ & $3.0 \times 10^{-8}$ \\
\hline Standard deviation $\{\exp$. & I. 3 & .39 & .95 & I. 8 & $2 \cdot 3$ & $2 \cdot 7$ & I. 7 & .71 \\
\hline Average calc. & .35 & .33 & .33 & $\cdot 37$ & .94 & .67 & I.04 & .26 \\
\hline
\end{tabular}

- Cultures in synthetic medium.

It will be seen that in every experiment the fluctuation of the numbers of resistant bacteria is tremendously higher than could be accounted for by the sampling errors, in striking contrast to the results of plating from the same culture (see table I) and in conflict with the expectations from the hypothesis of acquired immunity.

We want to see next whether these results fit the expectations from the hypothesis of mutation. We must therefore compare the experimental results with the relations developed in the theoretical part, keeping in mind that the theory con

First we can compare, according to equation (I 2), the experimental and the calculated values of the ratio between the standard deviation and the average of the numbers of resistant bacteria. These ratios are included in tables 2 and 3. It is seen that the experimental and theoretical values are reasonably close. 
However, in all but one case the experimental ratio is greater than the value calculated from the theory - that is, the variability is even greater than predicted.

TABLE 3

Distribution of the numbers of resistant bacteria in series of similar cultures.

\begin{tabular}{|c|c|c|c|c|}
\hline EXPERIMENT NO. & \multicolumn{2}{|c|}{22} & \multicolumn{2}{|c|}{23} \\
\hline $\begin{array}{l}\text { Number of cultures } \\
\text { Volume of cultures, cc } \\
\text { Volume of samples, } \mathrm{cc}\end{array}$ & \multicolumn{2}{|c|}{$\begin{array}{l}100 \\
.2^{*} \\
.05\end{array}$} & 87 & $\begin{array}{l}2^{*} \\
2\end{array}$ \\
\hline & 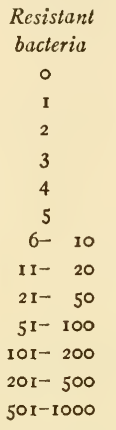 & $\begin{array}{c}\text { Number of } \\
\text { cultures } \\
57 \\
20 \\
5 \\
2 \\
3 \\
1 \\
7 \\
2 \\
2 \\
0 \\
\circ \\
0 \\
1\end{array}$ & $\begin{array}{c}\text { Resistant } \\
\text { bacteria } \\
0 \\
\text { I } \\
2 \\
3 \\
4 \\
5 \\
6-\quad 10 \\
11-20 \\
2 I-50 \\
51-100 \\
101-200 \\
20 I-500 \\
501-1000\end{array}$ & $\begin{array}{c}\text { Number of } \\
\text { cultures } \\
29 \\
17 \\
4 \\
3 \\
3 \\
2 \\
5 \\
6 \\
7 \\
5 \\
2 \\
4 \\
0\end{array}$ \\
\hline $\begin{array}{l}\text { Average per sample } \\
\text { Variance (corrected for sampling) } \\
\text { Average per culture } \\
\text { Bacteria per culture } \\
\text { Mutation rate } \\
\frac{\text { Standard deviation }}{\text { Average }}\left\{\begin{array}{l}\text { exp. } \\
\text { calc. }\end{array}\right.\end{array}$ & $\begin{array}{r}10 \\
6270 \\
40 \\
2.8 \times \\
2.3 \times \\
7 \\
1\end{array}$ & $\begin{array}{l}4^{8} \\
10^{8} \\
10^{-8} \\
8 \\
5\end{array}$ & $\begin{array}{r}64 \\
2.4 \\
2.3\end{array}$ & $\begin{array}{l}.6 \\
.6 \\
<10^{8} \\
<10^{-8} \\
8\end{array}$ \\
\hline
\end{tabular}

* Cultures in synthetic medium.

A part of this discrepancy may be accounted for by the fact that the time $t_{0}$, mutations occurring prior to which were disregarded by the theory, was chosen in such a manner that on the average one mutation would occur prior to time $t_{0}$. This mutation, if it occurs, will of course tend to increase the variance, and in some of the experiments the high value of the experimental variance can be traced directly to one exceptional culture in which a mutation had evidently occurred several generations prior to time $t_{0}$. Unfortunately, there is no general criterion by which one might eliminate such cultures from the statistical analysis, because, in a culture with an exceptionally high count of resistant bacteria, these do not necessarily stem from one exceptionally early mutation, but may also be due to an exceptionally large number of mutations after time $t_{0}$.

There may also be other reasons why the observed variances are higher than the expected ones. First of all, the simplifying assumption that the mutation 
rate per bacterial generation is independent of the physiological state of the bacteria may be too simple. If the mutation rate is higher for actively growing bacteria than for bacteria near the saturation limit of the cultures, early mutations and big clone sizes will be favored, and therefore higher variations of the numbers of resistant bacteria can be expected. Second, the assumption of a sudden transition from sensitivity to resistance may also be too simple. It is conceivable that the character "resistance to virus" may not fully develop in the bacterial cell in which the mutation occurs, but only in its offspring, after one or more generations. However, if this were the case, cultures with only one or two resistant bacteria should be relatively rare. The last experiment listed in table 3 , in which the entire cultures were plated, shows a rather high proportion of cultures with only one resistant bacterium. This seems to show that the

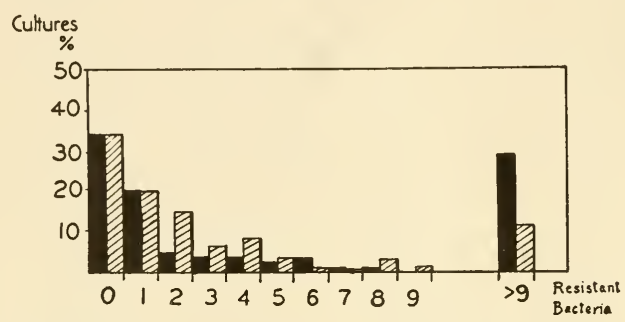

FIGURE 2.-Experimental (Experiment No. 23) and calculated distributions of the numbers of resistant bacteria in a series of similar cultures. Solid columns: experimental. Cross-hatched columns: calculated.

character "resistance to virus" in general does come to expression in the bacterial cell in which the corresponding mutation occurred, as assumed by the theory.

Another way of comparing the experimental results with the theory is to compare the experimental distribution of resistant bacteria with the approximate distribution calculated by the method outlined at the end of the theoretical part. The theoretical distribution has to be calculated from the average number of mutations per culture given by equation (5). Only experiments where the whole culture is tested can therefore be used for such a comparison. This method tests the fitting of the expectations for small numbers of resistant bacteria, in contrast to the comparison of the standard deviations, which involves predominantly the cultures with high numbers of resistant bacteria.

Figure 2 shows the experimental and calculated distributions for Experiment No. 23; the cultures with more than nine resistant bacteria are lumped together in one class, since the distribution has not been calculated for values higher than nine.

It is seen that the fitting for small values is satisfactory. In particular, the 
number of cultures with one resistant bacterium very closely fits the expectation. The classes with two, four, eight, etc., resistant bacteria are bound to be favored in the theoretical distribution, as explained in the theoretical part.

The results shown in figure 2 also confirm the assumption that the discrepancy between experimental and calculated standard deviations must be due to an excess of cultures with large numbers of resistant bacteria.

Summing up the evidence, we may say that the experiments show clearly that the resistant bacteria appear in similar cultures not as random samples but in groups of varying sizes, indicating a correlating cause for such grouping, and that the assumption of genetic relatedness of the bacteria of such groups offers the simplest explanation for them.

\section{Mutation Rate}

As pointed out in the theoretical part of this paper, mutation rates may be estimated from the experiments by two essentially different methods. The first method makes use of the fact that the number of mutations in a series of similar cultures should be distributed in accordance with Poisson's law; the average number of mutations per culture is calculated from the proportion of cultures containing no resistant bacteria at the moment of the test, according to equation (5).

There are two technical difficulties involved in the application of this method. In the first place, rather large numbers of cultures have to be handled and conditions have to be chosen so that the proportion of resistant bacteria is neither too small nor too large. In the second place, the entire cultures have to be tested, which means, in our method of testing, that cultures of rather small volume have to be used and great care must be taken to plate as nearly as possible the entire culture.

Experiment No. 23 (see table 3) permits an estimate of the mutation rate by this method. Out of 87 cultures, no resistant bacteria were found in 29 cultures, a proportion of .33 . From equation (5) we calculate therefore that the average number of mutations per culture in this experiment was I.IO. Since the total number of bacteria per culture was $2.4 \times 10^{8}$, we obtain as the mutation rate, from equation (4),

$$
\begin{aligned}
\mathrm{a} & =.47 \times 10^{-8} \text { mutations per bacterium per time unit } \\
& =.32 \times 10^{-8} \text { mutations per bacterium per division cycle. }
\end{aligned}
$$

This calculation makes use exclusively of the proportion of cultures containing no resistant bacteria. It is therefore inefficient in its use of the information gathered in the experiment.

The second method makes use of the average number of resistant bacteria per culture. The relation of this average number with the mutation rate was discussed in the theoretical part of this paper and was found to be expressed by equation (8). The mutation rates calculated by this method for each experiment are collected in table 4 . 
Values of mutation rate from different experiments.

\begin{tabular}{|c|c|c|c|}
\hline EXPERIMENT NO. & NUMBER OF CULTURES & VOLUME OF CULTURES & MUTATION RATE \\
\hline & & & Mutations per bacterium \\
\hline I & 9 & $\begin{array}{c}c c \\
10.0\end{array}$ & $\begin{array}{c}\text { per lime unit } \\
1.8 \times 10^{-8}\end{array}$ \\
\hline 10 & 8 & 10.0 & $1.4 \times 10^{-8}$ \\
\hline II & 10 & 10.0 & $4.1 \times 10^{-8}$ \\
\hline I5 & Io & 10.0 & $2.1 \times 10^{-8}$ \\
\hline I6 & 20 & $.2^{*}$ & I. $1 \times 10^{-8}$ \\
\hline 17 & 12 & $.2^{*}$ & $3.0 \times 10^{-8}$ \\
\hline $2 \mathrm{ra}$ & 19 & .2 & $3.3 \times 10^{-8}$ \\
\hline $2 \mathrm{Ib}$ & 5 & 10.0 & $3.0 \times 10^{-8}$ \\
\hline 22 & 100 & $.2^{*}$ & $2.3 \times 10^{-8}$ \\
\hline 23 & 87 & $.2^{*}$ & $2.4 \times 10^{-8}$ \\
\hline Average & & & $2.45 \times 10^{-8}$ \\
\hline
\end{tabular}

* Cultures in synthetic medium.

It will be seen that the values of the mutation rate obtained by the second method are all higher than the value found by the first method. This discrepancy may be traced back to the same cause as the discrepancy between the calculated and observed values of the standard deviation of the numbers of resistant bacteria. This, we found, was due to an excess of early mutations, giving rise to big clones of resistant bacteria. These big clones do not affect the mutation rate calculated by the first method, but they do affect the results of the second method, which is based on the average number of resistant bacteria.

One sees in table 4 that the mutation rate calculated by the second method does not vary greatly from experiment to experiment. In particular, it will be noted that there is no significant difference between the values obtained from cultures in broth and from cultures in synthetic medium, notwithstanding the considerable difference of metabolic activity and of growth rate of the bacteria in these two media. This shows that the simple assumption of a fixed small chance of mutation per physiological time unit is vindicated by the results. It may also be noted in table 4 that there is no significant difference between the mutation rates obtained from ro cc cultures and those obtained from .2 cc cultures, or between the experiments with many and those with few cultures. The variability of the value of the mutation rate seems to be solely due to the peculiar probability distribution of the number of resistant bacteria in series of similar cultures predicted by the mutation theory.

At this point an experiment may be mentioned by which it was desired to find out whether or not mutations occur in a culture after the bacteria have ceased growing. A culture was grown to saturation and was then tested repeatedly for resistant bacteria and for total number of bacteria over several 
days. The proportion of resistant bacteria did not change, even when the sensitive bacteria began to die, showing that the resistant bacteria have the same death rate in aging cultures as the sensitive bacteria.

\section{DISCUSSION}

We consider the above results as proof that in our case the resistance to virus is due to a heritable change of the bacterial cell which occurs independently of the action of the virus. It remains to be seen whether or not this is the general rule. There is reason to suspect that the mechanism is more complex in cases where the resistant culture develops only several days after lysis of the sensitive bacteria.

The proportion of mutant organisms in a culture and the mutation rate are far smaller in our case than in other studied cases of heritable bacterial variation. The possibility of investigation of such rare mutations is in our case merely the result of the method of detecting the mutant organisms. In other cases, the variants are detected by changes in the colony type which is produced by the mutant organism, either in the pigmentation or in the character of the surface or the edge of the colony. Often, colonies of intermediate character occur, and it is difficult to decide whether they are mixed colonies or stem from bacteria with intermediate character. This is particularly true of cases where the mutation rate is high and where reverse mutation occurs. Fairly high mutation rates, however, are a prerequisite of any study of colony variants, since the number of colonies that can be examined is limited by practical reasons.

The study of mutations causing virus resistance is free of these difficulties. The segregation of the mutant from the normal organisms occurs in the onecell stage by elimination of the normal individuals, and the character of the colony which develops from a mutant organism is of secondary importance. Owing to the total elimination of the normal individuals, the number of organisms which may be examined is very much higher than for any other method; more than $10^{8}$ bacteria may be tested on a single plate. Since the mutations to virus resistance are of ten associated with other significant characters, the method may well assume importance with regard to the general problems of bacterial variation.

It must not be supposed that the peculiar statistical difficulties encountered in our case are restricted to cases of very low mutation rates. The essential condition for the occurrence of the peculiar distribution studied in the theoretical part of this paper is the following: the initial number of bacteria in a culture must be so small that the number of mutations which occur during the first division cycle of the bacteria is a small number. This will always be true, however great the mutation rate, if one studies cultures containing initially a small number of organisms.

In a series of very interesting studies of the color variants of Serratia marcescens, Bunting (1940a, I940b, i 942; Bunting and Ingraham 1942) succeeded to some extent in obviating the statistical difficulties by always using 
inocula of about 100,000 bacteria. In some of her cases this number was sufficiently high to result in numerous mutations during the first division cycle of the bacteria. In other cases the number was apparently not high enough, since the author reports troublesome variations of the fractions of variants in successive subcultures. In those cases where the size of the inocula was high enough, the author succeeded in deriving reproducible values for the mutation rates from the study of single cultures, followed through numerous subcultures. In these cases it is sufficient to apply the equations of the theory referring to the average numbers of mutants as a function of time. It is clear, however, that this method is applicable only in cases of mutation rates of at least $10^{-4}$ per bacterium per division cycle.

In our case, as in many others, the virus resistant variants do not exhibit any striking correlated physiological changes. There is therefore little opportunity for an inquiry into the nature of the physiological changes responsible for the resistance to virus. Since the offspring of the mutant bacteria, when isolated after the test, are unable to synthesize the surface elements to which the virus is specifically adsorbed in the sensitive strain, one might suppose that this loss is a direct effect of the mutation. However, it is also conceivable that the loss occurs upon contact with virus, since it is detected only after such contact (hypothesis $b_{1}$ ). In some of the cases studied by BURNET (I929), where the mutational change to resistance is correlated with a change of phase, from smooth to rough or vice versa, the change of the surface structure must be a direct result of the mutation, since the mutant colonies may be picked up prior to the resistance test and, when tested, exhibit the typical change of affinity of the surface structure. These findings make it more probable that the loss of surface affinity to virus is a direct effect of the mutation.

The alteration of specific surface structures due to genetic change is a phenomenon of the widest occurrence. The genetic factors determining the antigenic properties of erythrocytes are well known. There is evidence (WEBSTER i937; Holmes 1938; Stevenson, Schultz, and Clark 1939) that resistance or sensitivity to virus in plants and animals is correlated with, or even dependent on, genetic changes, possibly affecting the antigenic make-up of the cellular surface. The proof that resistance to a bacterial virus may be traced to a specific genetic change may assume importance, therefore, with regard to the general problems of virus sensitivity and virus resistance.

\section{SUMMARY}

The distribution of the numbers of virus resistant bacteria in series of similar cultures of a virus-sensitive strain has been analyzed theoretically on the basis of two current hypotheses concerning the origin of the resistant bacteria.

The distribution has been studied experimentally and has been found to conform with the conclusions drawn from the hypothesis that the resistant bacteria arise by mutations of sensitive cells independently of the action of virus.

The mutation rate has been determined experimentally. 


\section{LITERATURE CITED}

Buntinc, M. I., I940a A description of some color variants produced by Serratia marcescens, strain 274. J. Bact. 40: 57-68.

I940b The production of stable populations of color variants of Serratia marcescens \#274 in rapidly growing cultures. J. Bact. 40: 69-8I.

1942 Factors affecting the distribution of color variants in aging broth cultures of Serratia marcescens \#274. J. Bact. 43: 593-606.

Buntinc, M. I., and L. J. IngRahaM, I942 The distribution of color variants in aging broth cultures of Serratia marcescens \#2 74. J. Bact. 43: 585-591.

BURNET, F. M., 1929 Smooth-rough variation in bacteria in its relation to bacteriophage. J. Path. Bact. 32: r $^{-4} 42$.

DeLbRÜcK, M., and S. E. LURIA, I942 Interference between bacterial viruses. I. Arch. Biochem. I : II I-I4I.

Gratia, A., I92I Studies on the d'Herelle phenomenon. J. Exp. Med. 34: II $5^{-1} 3^{1}$.

D'Herelle, F., I926 The Bacteriophage and Its Behavior. Baltimore: Williams and Wilkins.

Holmes, F. O., 1938 Inheritance of resistance to tobacco-mosaic disease in tobacco. Phytopathology 28: $553-56 \mathrm{I}$ :

Stevenson, F. J., E. S. Schultz, and C. F. Clark, 1939 Inheritance of immunity from virus X (latent mosaic) in the potato. Phytopathology 29: $362-365$.

WEBSTER, L. T., I 937 Inheritance of resistance of mice to enteric bacterial and neurotropic virus infections. J. Exp. Med. 65: 26r-286. 


\title{
THE DISTRIBUTION OF THE NUMBERS OF MUTAN'TS IN BACTERIAL POPULATIONS
}

\author{
By D. E. LEA,* Department of Radiotherapeutics and Strangeways Laboratory, Cambridge \\ AND C. A. COULSON, Wheatstone Physics Laboratory, King's College, London
}

\section{INTRODUCTION}

Luria \& Delbruck (1943) have shown that if a culture of some hundreds or thousands of millions of Bacterium coli, grown from a single cell, is plated out on a nutrient medium impregnated with a bacteriophage to which the strain of coli is sensitive, the vast majority of the bacteria are lysed, but a few give rise to colonies. These colonies contain only bacteria resistant to the bacteriophage, and give rise only to resistant bacteria on further subcultivation. Evidently hereditary variations or mutations can occur in bacteria. Numerous other examples are known of mutations in bacteria, affecting fermentation reactions (e.g. Lewis, 1934), resistance to chemicals (e.g. Stewart, 1947), to antibiotics (e.g. Demerec, 1945), or to radiation (Witkin, 1946).

The demonstration of phage-resistant mutants necessarily involves the exposing of the bacteria to the phage, and it is not immediately obvious whether the mutation to phage resistance occurs spontaneously during the growth of the culture, and is merely made apparent by subsequently testing with phage, or whether the mutation is induced by the phage and does not occur until the bacteria are brought into contact with phage. Most experiments on bacterial variation have left open the two alternatives of spontaneous mutation on the one hand, and induced mutation or adaptation on the other, and the interpretation adopted has usually been determined by the previous training of the individual worker rather than by any compelling evidence provided by the experiments.

Luria and Delbruck, however, in their paper, described a method by which a decision between the two alternative explanations may be reached, and concluded that the acquirement of resistance to phage is a spontaneous mutation which occurs during the growth of the culture and prior to its treatment with phage. Demerec (1945) and Witkin (1946) have applied the same method to mutants resistant to penicillin and to $\mathrm{X}$-rays respectively, and have concluded that these changes also are spontaneous mutations occurring independently of the penicillin or of the radiation respectively.

The principle of Luria and Delbruck's test is as follows. A culture of (say) $10^{9}$ bacteria is divided into (say) ten equal portions which are separately tested for phage-resistant organisms by plating out on a phage-impregnated medium. A small number is found in each of the ten portions, and the numbers are found experimentally to be distributed with a variance approximately equal to the mean. This result is not surprising on either hypothesis. On the spontaneous mutation theory, we suppose that mutations to phage resistance occurred from time to time during the growth of the culture. All the bacteria produced by subsequent divisions of a mutant bacterium were similarly phage resistant. Thus the culture of $10^{9}$ bacteria contained a certain number of phage-resistant bacteria,

* [Note by C. A. C. A few days before Dr Lea's untimely death in June 1947, the manuseript and the calculations reported here had just been completed. It was Dr Lea's intention to make further experiments more suitable to a test of the theory outlined in this paper. These experiments cannot now be made, but it has been thought wise to publish the theory and numerical tables because of their value to other investigators.]

[Reprinted by permission of the Cambrictge University Press from Journal of Ginftics

$49:$ (3) 264-285, December, 1949] 
being either bacteria which had recently undergone mutation, or bacteria derived from the division of mutants which arose earlier in the growth of the culture. When the culture was divided into ten equal portions the phage-resistant organisms were distributed at random between the ten portions. We may expect, therefore, the numbers in the different portions to fall in a multinomial distribution with variance nearly equal to the mean.

On the adaptation or induced mutation theory, it is supposed that no phage-resistant bacteria arose during the growth of the culture. The ten portions, at the time of plating out, each contained $10^{8}$ normal bacteria and no resistant bacteria. On being brought into contact with the phage most were lysed, but a few were able to adapt themselves to the phage (or the phage-induced mutations in them). The probability of this process is very small, but was presumably the same for all the bacteria. On this theory, therefore, we expect the number of resistant colonies on the ten parallel plates to be distributed in a Poisson distribution with variance equal to the mean. Either theory is thus capable of accounting for the experimental variance, and this experiment alone does not make possible a decision between the two theories.

A second experiment is now made in which (say) ten cultures, of (say) $10^{8}$ bacteria are tested for phage-resistant organisms. On the adaptation or induced-mutation theory this experiment is not essentially different from the preceding one, and we again expect the numbers of phage-resistant colonies on the ten test plates to be distributed in a Poisson distribution with variance equal to the mean. For, on this theory, the phage-resistant mutants do not appear until the bacteria are plated out on the phage-impregnated medium, and there can be no relevant difference between a culture of $10^{9}$ bacteria divided into ten equal portions, and ten separately grown cultures of $10^{8}$ bacteria.

In practice a very different result is obtained: the distribution obtained is much wider than in the former experiment, and has a variance many times-perhaps fifty timesthe mean.

On the spontaneous mutation hypothesis a very wide distribution of the number of phage-resistant bacteria in parallel cultures is to be expected. The reason is that not only do the parallel cultures differ in the numbers of mutations which have occurred, hut also, and much more importantly, they differ in the stages at which the mutations occurred. If a mutation occurs towards the end of the growth of a culture, it will give rise to one phage-resistant organism, but if it occurs early in the growth, say when the culture is only one-hundredth of its final size, it will give rise to a large number of phage-resistant organisms. Thus even in cultures in which equal numbers of mutations have occurred, the numbers of phage-resistant organisms will usually be widely different.

It is evident, therefore, that the hypothesis that spontaneous mutation to phage resistance occurs during the growth of the culture before it is brought into contact with the phage is in qualitative agreement with the experimental result, while the alternative hypothesis of mutation induced by the phage, or adaptation of the bacterium to the phage, is not. Luria and Delbruck's method thus provides, for the first time, a clear means of distinguishing between the two hypotheses.

As left by Luria and Delbruck, the method is a qualitative one, since they do not derive the shape of the distribution to be expected on the spontaneous mutation theory. They do derive expressions for the mean and variance of the distribution, but as they point out, on account of the extreme skewness of the distribution, the mean and variance are very 
inefficient statistics for estimating the parameters of the distribution from experimental results, or for testing the agreement of experiment and theory.

The purpose of the present paper is to extend Luria and Delbruck's method by calculating the form of the distribution of numbers of mutants in parallel cultures to be expected on the spontaneous mutation theory, so making the test of the applicability of the spontaneous mutation theory a quantitative test. Statistically efficient methods of deducing the mutation rate from experimental observations are also discussed.

\section{The DISTRIBUtion}

\section{First method}

During the active growth of a culture, the number of organisms increases as an exponential function of the time, and may be represented as

$$
n=e^{\beta t} \text {, }
$$

there being one organism at time $t=0$.

Thus

$$
d n=\beta n d t .
$$

If $\alpha$ is the mutation rate, defined by the relation that $\alpha d t$ is the probability that an individual phage-sensitive organism shall undergo mutation to phage resistance in time $d t$, $n \alpha d t$ is the mean number of mutations which occur in time $d t$. (Strictly, since $n$ is the total number of organisms, we should subtract from $n$ in this formula the number of resistant organisms, but in practice the number of mutant organisms in a culture is a minute fraction of the total number.) Hence the mean number $(m)$ of mutations which will have occurred in the culture by the time it has grown to size $n$ at time $t$ is

$$
\int_{0}^{t} n \alpha d t=\frac{\alpha}{\beta} \int_{1}^{n} d n=\frac{\alpha}{\beta}(n-1) .
$$

Since at all relevant times $n$ much exceeds unity, we may write

$$
m=\frac{\alpha}{\beta} n
$$

for the mean number of mutations in the culture by the time it has attained size $n$. The mean number of mutations which occur while the culture grows from $n_{1}$ to $n_{2}$ organisms is evidently

$$
m\left(\frac{n_{2}-n_{1}}{n}\right) .
$$

If it should happen that the mutation rate $\alpha$ and the growth rate $\beta$ are equally affected by factors such as nutritional conditions and density of population which affect $\beta$, then the mutation rate per generation, though not per unit time, will be independent of these factors, and $\alpha / \beta$, which may be regarded as the probability of mutation per division, will be constant even though $\alpha$ and $\beta$ are not. Under these conditions equations (3) and (4) can be derived without the assumption of exponential growth.

We must distinguish between the number of mutations which have occurred, and the number of mutants, the latter being derived not only by mutation of the normal bacteria but also by division of bacteria which have suffered mutation earlier. If $r(r \geqslant m)$ is the number of mutants in the culture at a given time, and if we assume that the division rate of the mutant is the same as that of the normal bacteria, then $r \beta d t=r d n / n$ is the probability 
that one of the mutants shall divide in time $d t$. Similarly, $(r-1) d n / n$ is the probability that one of the mutants shall divide in time $d t$ in a culture containing $(r-1)$ mutants.

Let $p_{r}$ (a function of $n$ ) be the probability that a culture of $n$ bacteria grown from a single bacterium at $t=0$ shall have $r$ mutants (i.e. of a large number of cultures of $n$ bacteria, a proportion $p_{r}$ of cultures will have $r$ mutants).

Consider the proportion $p_{r}+\frac{d p_{r}}{d n} d n$ of cultures which, at time $t+d t$, when the culture size is $n+d n$, have $r$ mutants. These will be derived from:

(a) cultures which, at time $t$, had $r-1$ mutants and in which a mutation occurred in the interval $d t$;

(b) cultures which, at time $t$, had $r-1$ mutants and in which a mutant divided in the interval $d t$

(c) cultures which, at time $t$, had $r$ mutants and in which neither mutation nor division of a mutant occurred in the interval $d t$;

providing that the interval $d t$ is small enough. For $(\alpha / \beta) d n$ and $r d n / n$ to be much less than unity, the possibility of more than one of the rare events mutation and division of a mutant in the interval $d t$ can be neglected. We see, therefore, that

$$
p_{r}+\frac{d p_{r}}{d n} d n=p_{r-\mathbf{1}}\left\{\frac{\alpha}{\beta} d n+(r-1) \frac{d n}{n}\right\}+p_{r}\left\{1-\frac{\alpha}{\beta} d n-r \frac{d n}{n}\right\},
$$

so that

$$
\frac{d p_{r}}{d n}+\frac{\alpha}{\beta} p_{r}+\frac{r}{n} p_{r}=p_{r-1}\left(\frac{\alpha}{\beta}+\frac{r-1}{n}\right) .
$$

Making, from (3), the substitution $m=(\alpha / \beta) n$, we have

$$
\frac{d p_{r}}{d m}+p_{r}+\frac{r}{m} p_{r}=p_{r-1}\left(1+\frac{r-1}{m}\right) .
$$

Multiplying by the integrating factor $e^{m}$ we have

$$
\frac{d q_{r}}{d m}+\frac{r}{m} q_{r}=q_{r-1}\left(1+\frac{r-1}{m}\right), \quad \text { where } q_{r}=e^{m} p_{r} .
$$

Now $m$ is the mean number of mutations which have occurred in a culture by the time it contains $n$ bacteria. Therefore $e^{-m}$, the first term of the Poisson distribution, is the probability $p_{0}$ that no mutation shall have occurred. Thus $q_{0}=e^{m} p_{0}=1$ for all values of $m$. Evidently initially, when $m=0, q_{r}=0$ for all $r>0$.

Starting from $q_{0}=1$ we can calculate $q_{1}, q_{2}, q_{3}$, etc. in succession from the differential equation (6). Thus:

$$
\begin{array}{ll}
\frac{d q_{1}}{d m}+\frac{1}{m} q_{1}=1, & \text { whence } q_{1}=\frac{1}{2} m ; \\
\frac{d q_{2}}{d m}+\frac{2}{m} q_{2}=\frac{1}{2} m\left(1+\frac{1}{m}\right), & \text { whence } q_{2}=\frac{1}{6} m+\frac{1}{8} m^{2} ; \\
\frac{d q_{3}}{d m}+\frac{3}{m} q_{3}=\left(\frac{1}{6} m+\frac{1}{8} m^{2}\right)\left(1+\frac{2}{m}\right), & \text { whence } q_{3}=\frac{1}{12} m+\frac{1}{12} m^{2}+\frac{1}{48} m^{3} .
\end{array}
$$

Evidently $q_{r}$ is a polynomial in $m$, with powers ranging from 1 to $r$.

Writing

$$
q_{r}=\sum_{j=1}^{r} C_{j, r} \frac{m^{j}}{j !}=C_{1, r} m+C_{2, r} \frac{m^{2}}{2 !}+\ldots+C_{r, r} \frac{m^{r}}{r !}
$$


we have

$$
\frac{d q_{r}}{d m}=\sum_{j=1}^{r} C_{j, r} \frac{m^{j-1}}{(j-1) !} .
$$

Inserting (7) and (8) in (6), and equating coefficients of $m^{j-1} / j$ !, we have

$$
(j+r) C_{j, r}=j C_{j-1, r-1}+(r-1) C_{j, r-1} .
$$

With this recurrence relation a table of $C_{j, r}$ may be drawn up. Such a table, for values of $r \leqslant 10$, is given in the Appendix.

Evidently

$$
C_{1, r}=\frac{1}{r(r+1)} ; \quad C_{r, r}=2^{-r} .
$$

From (6) and (7), $p_{0}=e^{-m}$, and for $r \geqslant 1$

$$
p_{r}=\sum_{j=1}^{r} C_{j, r}\left(e^{-m} \frac{m^{j}}{j !}\right)=C_{1, r}\left(e^{-m} m\right)+C_{2, r}\left(e^{-m} \frac{m^{2}}{2 !}\right)+\ldots+C_{r . r}\left(e^{-m} \frac{m^{r}}{r !}\right) .
$$

A generating function for $p_{r}$

Define a function

$$
f(x, m)=q_{0}+q_{1} x+q_{2} x^{2}+\ldots=\sum_{r=0}^{\infty} q_{r} x^{r} .
$$

We have

$$
\frac{\partial f}{\partial x}=\Sigma r x^{r-1} q_{r} \text { and } \frac{\partial f}{\partial m}=\Sigma x^{r} \frac{d q_{r}}{d m} .
$$

Multiplying equation (6) by $x^{r}$ and summing for all $r$ we have

$$
\Sigma x^{r} \frac{d q_{r}}{d m}+\frac{x}{m} \Sigma r x^{r-1} q_{r}=x \Sigma x^{r-1} q_{r-1}+\frac{x^{2}}{m} \Sigma(r-1) x^{r-2} q_{r-1},
$$

or, using (13),

$$
\frac{\partial f}{\partial m}+\frac{x}{m} \frac{\partial f}{\partial x}=x f+\frac{x^{2}}{m} \frac{\partial f}{\partial x}
$$

whence

$$
\frac{\partial \phi}{\partial m}+\frac{x}{m}(1-x) \frac{\partial \phi}{\partial x}=x
$$

where* $\phi=\log f$. This equation is satisfied by

providing

$$
\phi(x, m)=m \psi(x),
$$

i.e.

$$
\begin{gathered}
\psi+x(1-x) \psi^{\prime}=x, \\
\psi^{\prime}+\frac{\psi}{x(1-x)}=\frac{1}{1-x} .
\end{gathered}
$$

Multiplying by $x /(1-x)$ and integrating

$$
\frac{x}{1-x} \psi=\frac{1}{1-x}+\log (1-x)-1,
$$

the integration constant -1 being introduced since when $x=0, f=q_{0}=1$, so that $\phi=0$ and so $\psi=0$. Thus

$$
\psi=1+\frac{1-x}{x} \log (1-x)=\frac{x}{1.2}+\frac{x^{2}}{2.3}+\frac{x^{3}}{3.4}+\ldots,
$$

whence

$$
f=e^{m \psi}=e^{m}(1-x)^{m(1-x) x},
$$

so that $p_{r}=e^{-m} q_{r}$ is the coefficient of $x^{r}$ in the expansion in ascending powers of $x$ of

$$
(1-x)^{m(1-x) / x} \quad \text { or of } e^{-m} \exp \left[m\left(\frac{x}{1.2}+\frac{x^{2}}{2.3}+\ldots\right)\right] \text {, }
$$

\footnotetext{
* $\log$ means natural logarithm to base $e=2 \cdot 718 \ldots$ throughout this paper.
} 
i.e. of

$$
e^{-m}+\left(\frac{x}{1.2}+\frac{x^{2}}{2.3}+\ldots\right) e^{-m} \frac{m}{1 !}+\left(\frac{x}{1.2}+\frac{x^{2}}{2.3}+\ldots\right)^{2} e^{-m} \frac{m^{2}}{2 !}+\ldots
$$

Comparing with (11), it is evident that $C_{j, r}$ is the coefficient of $x^{r}$ in the expansion of

$$
\left(\frac{x}{1.2}+\frac{x^{2}}{2.3}+\ldots\right)^{j}
$$

It will be shown later that $C_{j, r}$ is the probability that a culture in which exactly $j$ mutations have occurred shall contain $r$ mutants. If we define

$$
D_{j, r}=\sum_{r=j}^{r} C_{j, r}
$$

$D_{j, r}$ is the probability that a culture in which exactly $j$ mutations have occurred shall have $\leqslant r$ mutants. Summing equation (9) over all $r$ between $j$ and $r$ leads to the following recurrence relation between the $D_{j, r}$,

or

$$
\begin{aligned}
& (r+j) C_{j, r}=j\left(D_{j-1, r-1}-D_{j, r-1}\right), \\
& (r+j) D_{j, r}=r D_{j, r-1}+j D_{j-1, r-1} .
\end{aligned}
$$

Equation (18) is useful as an arithmetical check during the computation of the $C_{j, r}$ by means of equation (9).

It is sometimes convenient to discuss $P_{r}$ defined as

$$
P_{r}=\sum_{r=0}^{r} p_{r}
$$

$P_{r}$ being the probability that a culture shall contain $0,1,2, \ldots$, or $r$ mutants, i.e. any number of mutants up to $r$. $P_{0}=e^{-m} ; P_{r}=1$ for all $r$ at $m=0$. The following equations can be readily deduced by summing (5) and (11) over all $r$ between 0 and $r$ :

$$
\begin{gathered}
\frac{d P_{r}}{d m}+P_{r}\left(1+\frac{r}{m}\right)=P_{r-1}\left(1+\frac{r}{m}\right), \\
P_{r}=e^{-m}+\sum_{j=1}^{r} D_{j, r}\left(e^{-m} \frac{m^{j}}{j !}\right) .
\end{gathered}
$$

\section{Second method}

An alternative method of calculating $p_{r}$, the probability of a culture having $r$ mutants, is instructive. A mutant appearing by mutation any time after the culture has passed the size $\frac{1}{2} n$ will not have time to divide by the time the culture size reaches $n$, and will therefore contribute 1 to the final complement of mutants. A mutant appearing by mutation during the period in which the culture grows from $\frac{1}{4} n$ to $\frac{1}{2} n$ will have time to divide once only and will therefore contribute 2 mutants to the final complement. A mutant appearing by mutation in the period in which the culture grows from $\frac{1}{8} n$ to $\frac{1}{4} n$ will have time to divide twice only and will contribute 4 mutants to the final complement, and so on.

Confining attention for the moment to all those cultures in which exactly one mutation occurs during the growth of the culture from one organism to $n$ organisms, in one-half of the cultures the mutation will occur while the culture is growing from $\frac{1}{2} n$ to $n$, in one-quarter of the cultures the mutation will occur while the culture is growing from $\frac{1}{4} n$ to $\frac{1}{2} n$, and so on (compare equation (4)). Or, in other words, in one-half of these cultures the mutation occurs at such a time that it gives rise finally to 1 mutant, in one-quarter of the cultures 
the mutation occurs at such a time that it gives rise finally to 2 mutants, in one-eighth at such a time that it gives rise to 4 mutants, and so on.

According to this argument, a mutation is necessarily represented, by the time the culture has reached size $n$, by a clone of 1 or 2 or 4 or 8 , etc., organisms, there being no intermediate numbers. This would be so if divisions in a clone were synchronous. It is probably true that clones of 3,5 or 7 cells will be less common than clones of 2,4 or 8 cells (cp. Adolph \& Bayne-Jones, 1932), but rather than make the extreme assumption that only integral powers of 2 are to be considered it is probably preferable to neglect this fact and to assume that the frequency of clones of different sizes is a smooth function of clone size.

A clone which, by time $t$, contains $\nu$ mutants will have originated when the number of bacteria in the culture was about $n / \nu$. We thus replace the subdivision of the growth of the culture into generations by subdivision into intervals in which the population increased from $\frac{1}{2} n$ to $n$, from $\frac{1}{3} n$ to $\frac{1}{2} n$, from $\frac{1}{4} n$ to $\frac{1}{3} n$, and so on, and suppose that a mutation which occurred while the population increased from $n /(\nu+1)$ to $n / v$ is, by the time the population has grown to $n$, represented by a clone of $\nu$ mutants. Now, of those cultures in which exactly one mutation has occurred, the proportion in which the mutation occurred while the culture grew from $n /(\nu+1)$ to $n / \nu$ is $\left(\frac{1}{\nu}-\frac{1}{\nu+1}\right)=\frac{1}{\nu(\nu+1)}$ (cp. equation (4)). This may be represented as the coefficient of $x^{\nu}$ in the generating function

$$
\frac{x}{1.2}+\frac{x^{2}}{2.3}+\ldots+\frac{x^{\nu}}{\nu(\nu+1)}+\ldots
$$

Considering now all those cultures in which exactly $j$ independent mutations occurred, the fraction of cultures in which the final number of mutants is $v$ is, from (23), evidently the coefficient of $x^{\nu}$ in the expansion of

$$
\left(\frac{x}{1.2}+\frac{x^{2}}{2.3}+\frac{x^{3}}{3.4}+\ldots\right)^{j}
$$

Now if $m$ is the mean number of mutations per culture, the proportion of cultures in which exactly $j$ mutations occurs is $e^{-m} \frac{m^{j}}{j !}$. Thus the proportion of all cultures in which the final number of mutants is $r$ is the coefficient of $x^{r}$ in the expansion of

$$
\sum_{j=0}^{\infty}\left(\frac{x}{1.2}+\frac{x^{2}}{2.3}+\frac{x^{3}}{3.4}+\ldots\right)^{j} e^{-m} \cdot \frac{m^{j}}{j !} .
$$

Thus $p_{r}$, the probability of a culture having $r$ mutants, $m$ being the mean number of mutations per culture, is the coefficient of $x^{r}$ in the expansion of

$$
e^{-m} \exp \left[m\left(\frac{x}{1.2}+\frac{x^{2}}{2.3}+\ldots\right)\right]
$$

in agreement with equation (15).

\section{Arithmetical procedure}

By means of the recurrence relation (9) and the boundary values (10) a table of values of $C_{j, r}$ has been computed for all (integral) values of $j$ from 1 to 36 and of $r$ from 1 to 64 , subject to $r \geqslant j$. Equation (18) was employed as a check on the arithmetic at $j=63 . C_{j, r}$ 
is the probability that a culture in which exactly $j$ mutations have occurred shall contain $r$ mutants. In practice when comparing the theoretical and experimental distributions the distributions will always be grouped. For economy of space, therefore, we do not publish the full table of $C_{j, r}$ but give in Table 1 grouped values for $r=1,2,3,4,5-8,9-16$, $17-32,33-64$ and $>64$.

Table 1. $C_{j, r}$

$C_{j, r}$ is the probability that a culture in which exactly $j$ mutations have occurred shall have $r$ mutants $(r \geqslant j)$. For values of $r$ greater than 2, the values of $C_{j, r}$ have been grouped. Thus the numbers in the column headed ' $17-32$ ' are values of $\sum_{r=17}^{r=32} C_{j, r}$. See also the Appendix for certain other values of $C_{j}, r$.

\begin{tabular}{|c|c|c|c|c|c|c|c|c|}
\hline$j \backslash r$ & 1 & 2 & $3-4$ & $5-8$ & $9-16$ & $17-32$ & $33-64$ & $>64$ \\
\hline 1 & $0 \cdot 5000$ & $0 \cdot 1667$ & $0 \cdot 1333$ & 0.0889 & $0 \cdot 0523$ & $0 \cdot 0285$ & $0 \cdot 0149$ & 0.0154 \\
\hline 2 & - & $0 \cdot 2500$ & $0 \cdot 2778$ & $0 \cdot 2118$ & 0.1272 & $0 \cdot 0671$ & $0 \cdot 0335$ & 0.0325 \\
\hline 3 & - & - & $0 \cdot 2500$ & $0 \cdot 3100$ & $0 \cdot 2161$ & $0 \cdot 1161$ & 0.0563 & 0.0515 \\
\hline 4 & - & - & 0.0625 & $0 \cdot 3093$ & $0 \cdot 2986$ & $0 \cdot 1735$ & 0.0834 & 0.0726 \\
\hline 5 & - & - & - & $0 \cdot 2060$ & $0 \cdot 3479$ & $0 \cdot 2353$ & $0 \cdot 1149$ & 0.0960 \\
\hline 6 & - & 一 & - & 0.0885 & $0 \cdot 3445$ & $0 \cdot 2949$ & $0 \cdot 1504$ & 0.1216 \\
\hline 7 & - & - & - & 0.0260 & $0 \cdot 2907$ & $0 \cdot 3441$ & 0.1894 & $0 \cdot 1497$ \\
\hline 8 & - & - & - & $0 \cdot 0039$ & $0 \cdot 2100$ & $0 \cdot 3753$ & $0 \cdot 2305$ & $0 \cdot 1804$ \\
\hline 9 & - & - & - & - & $0 \cdot 1312$ & $0 \cdot 3830$ & $0 \cdot 2721$ & $0 \cdot 2137$ \\
\hline 10 & - & - & - & - & 0.0718 & $0 \cdot 3665$ & 0.3122 & $0 \cdot 2495$ \\
\hline 11 & 一 & - & - & - & 0.0342 & $0 \cdot 3294$ & $0 \cdot 3486$ & 0.2878 \\
\hline 12 & - & - & - & - & 0.0138 & $0 \cdot 2790$ & $0 \cdot 3789$ & $0 \cdot 3284$ \\
\hline 13 & - & - & - & - & 0.0045 & $0 \cdot 2.234$ & $0 \cdot 4010$ & 0.3711 \\
\hline 14 & - & - & - & - & 0.0011 & $0 \cdot 1698$ & $0 \cdot 4135$ & 0.4157 \\
\hline 15 & - & - & - & - & 0.0002 & $0 \cdot 1228$ & 0.4155 & $0 \cdot 4616$ \\
\hline 16 & - & - & - & 一 & 0.0000 & $0 \cdot 0846$ & 0.4071 & $0 \cdot 5084$ \\
\hline 17 & - & - & 一 & - & - & $0 \cdot 0553$ & $0 \cdot 3891$ & 0.5555 \\
\hline 18 & - & - & - & - & - & 0.0343 & $0 \cdot 3633$ & $0 \cdot 6024$ \\
\hline 19 & - & - & - & - & - & 0.0200 & $0 \cdot 3315$ & $0 \cdot 6485$ \\
\hline 20 & - & - & - & - & - & 0.0109 & $0 \cdot 2961$ & $0 \cdot 6930$ \\
\hline 21 & - & - & - & - & - & 0.0055 & $0 \cdot 2591$ & 0.7354 \\
\hline 22 & - & - & 一 & 一 & - & 0.0026 & $0 \cdot 222 \cdot 2$ & $0 \cdot 7752$ \\
\hline 23 & - & - & 一 & 一 & - & 0.0011 & 0.1871 & 0.8118 \\
\hline 24 & - & - & - & - & - & $0 \cdot 0004$ & $0 \cdot 1546$ & $0 \cdot 84.50$ \\
\hline 25 & - & - & 一 & - & - & $0 \cdot 0001$ & $0 \cdot 1254$ & 0.8745 \\
\hline 26 & - & 一 & - & 一 & - & $0 \cdot 0000$ & $0 \cdot 0998$ & 0.9001 \\
\hline 27 & - & - & - & 一 & - & - & $0 \cdot 0779$ & $0 \cdot 922: 21$ \\
\hline 28 & - & - & - & - & - & - & 0.0596 & 0.9404 \\
\hline 29 & - & - & - & 一 & - & - & 0.0447 & 0.9553 \\
\hline 30 & - & - & - & - & - & - & 0.0327 & 0.9673 \\
\hline 31 & 一 & - & - & 一 & - & - & 0.0234 & 0.9766 \\
\hline 32 & - & - & - & 一 & - & - & 0.0164 & 0.9836 \\
\hline 33 & $\ldots$ & - & 一 & 一 & - & - & 0.0111 & 0.9889 \\
\hline 34 & - & - & - & - & 一 & - & 0.0074 & 0.9926 \\
\hline 35 & - & - & - & - & - & - & $0 \cdot 0048$ & 0.9952 \\
\hline 36 & - & - & - & 一 & - & - & $0 \cdot 0030$ & 0.9970 \\
\hline
\end{tabular}

$p_{r}$ is the probability that a culture of such a size that the mean number of mutations is $m$ shall contain $r$ mutants. $p_{0}=e^{-m}$ and for $r \geqslant 1, p_{r}=\sum_{j=1}^{r} C_{j, r}\left(e^{-m} \frac{m^{j}}{j !}\right)$. For any given value of $m, p_{r}$ can be calculated with the aid of the table of $C_{j, r}$, for the same groupings of $r$. The calculation is facilitated if a table of Poisson coefficients $e^{-m} \frac{m^{j}}{j !}$ is available (Molina, 1942). In Table 2 values of $p_{r}$ are given for a number of values of $m$ from 0.05 to 15 .

The figures in Tables 1,2 and 4 are liable to occasional rounding errors of one unit in the last decimal place.

Journ. of Genetics 49 


\section{LIMITING FORM OF DISTRIBUTION FOR LARGE NUMBERS}

Table 2 provides the means of testing the agreement between theoretical and experimental distributions in experiments in which the mean number of mutations per culture is 15 or fewer, and in which a minority of the cultures have more than 64 mutants. To extend Tables 1 and 2 by use of the recurrence relation (9) to cover experiments in which the mean number of mutations per culture considerably exceeds 15 , and to subdivide the class

Table 2. $p_{r}$

$p_{r}$ is the probability that a culture shall have $r$ mutants, the average number of mutations which have occurred per culture being $m$. For values of $r$ greater than 2, the values of $p_{r}$ have been grouped. Thus the numbers in the column headed ' $17-32$ ' are values of $\sum_{r=17}^{r=32} p_{r}$.

\begin{tabular}{|c|c|c|c|c|c|}
\hline$m \backslash r$ & 0 & 1 & 2 & $3-4$ & $5-8$ \\
\hline 0.05 & 0.9512 & 0.0283 & 0.0082 & 0.0067 & 0.004 \\
\hline $0 \cdot 10$ & 0.9048 & 0.0452 & 0.0162 & 0.0134 & 0.009 \\
\hline $0 \cdot 15$ & 0.8607 & 0.0646 & 0.0239 & 0.0200 & 0.013 \\
\hline $0 \cdot 20$ & 0.8187 & 0.0819 & 0.0314 & 0.0267 & 0.018 \\
\hline $0 \cdot 25$ & $0 \cdot 7788$ & 0.0974 & 0.0385 & 0.0332 & 0.02 \\
\hline $0 \cdot 30$ & 0.7408 & $0 \cdot 1111$ & 0.0454 & 0.0397 & 0.027 \\
\hline $0 \cdot 35$ & 0.7047 & $0 \cdot 1233$ & 0.0519 & 0.0462 & 0.032 \\
\hline 0.40 & $0 \cdot 6703$ & $0 \cdot 1341$ & 0.0581 & 0.0525 & 0.03 \\
\hline 0.45 & $0 \cdot 6376$ & $0 \cdot 1435$ & 0.0640 & 0.0587 & 0.04 \\
\hline 0.50 & $0 \cdot 6065$ & $0 \cdot 1516$ & 0.0695 & 0.0648 & 0.04 \\
\hline 0.55 & 0.5769 & 0.1587 & 0.0747 & 0.0707 & 0.05 \\
\hline $0 \cdot 60$ & 0.5488 & $0 \cdot 1646$ & 0.0796 & 0.0765 & \\
\hline 0.65 & 0.5220 & 0.1697 & 0.0841 & 0.0821 & 0.06 \\
\hline $0 \cdot 70$ & 0.4966 & $0 \cdot 1738$ & 0.0884 & 0.0876 & 0.06 \\
\hline 0.75 & 0.4724 & 0.1771 & 0.0923 & 0.0928 & 0.07 \\
\hline 0.80 & 0.4493 & $0 \cdot 1797$ & 0.0959 & 0.0979 & 0.07 \\
\hline 0.85 & 0.4274 & $0 \cdot 1817$ & 0.0992 & $0 \cdot 1028$ & 0.08 \\
\hline 0.90 & 0.4066 & 0.1830 & $0 \cdot 1022$ & $0 \cdot 1076$ & 0.08 \\
\hline 0.95 & $0 \cdot 3867$ & $0 \cdot 1837$ & $0 \cdot 1049$ & 0.1121 & 0.09 \\
\hline 1.0 & $0 \cdot 3679$ & 0.1839 & $0 \cdot 1073$ & $0 \cdot 1164$ & 0.09 \\
\hline $1 \cdot 2$ & 0.3012 & $0 \cdot 1807$ & $0 \cdot 1145$ & $0 \cdot 1317$ & 0.11 \\
\hline $1 \cdot 4$ & $0 \cdot 2466$ & $0 \cdot 1726$ & $0 \cdot 1180$ & $0 \cdot 1438$ & $0 \cdot 13$ \\
\hline $1 \cdot 6$ & $0 \cdot 2019$ & $0 \cdot 1615$ & 0.1184 & $0 \cdot 1528$ & $0 \cdot 14$ \\
\hline $1 \cdot 8$ & $0 \cdot 1653$ & $0 \cdot 1488$ & 0.1165 & $0 \cdot 1587$ & $0 \cdot 16$ \\
\hline $2 \cdot 0$ & $0 \cdot 1353$ & $0 \cdot 1353$ & 0.1128 & $0 \cdot 1620$ & 0.17 \\
\hline $2 \cdot 2$ & $0 \cdot 1108$ & $0 \cdot 1219$ & 0.1077 & $0 \cdot 1629$ & 0.18 \\
\hline $2 \cdot 4$ & 0.0907 & $0 \cdot 1089$ & $0 \cdot 1016$ & 0.1617 & 0.19 \\
\hline $2 \cdot 6$ & 0.0743 & $0 \cdot 0966$ & 0.0949 & $0 \cdot 1587$ & 0.19 \\
\hline $2 \cdot 8$ & 0.0608 & 0.0851 & 0.0880 & $0 \cdot 1543$ & $0 \cdot 20$ \\
\hline $3 \cdot 0$ & 0.0498 & 0.0747 & 0.0809 & 0.1487 & \\
\hline $3 \cdot 2$ & 0.0408 & 0.0652 & 0.0739 & 0.1421 & $0 \cdot 20$ \\
\hline $3 \cdot 4$ & 0.0334 & 0.0567 & 0.0671 & $0 \cdot 1350$ & $0 \cdot 20$ \\
\hline $3 \cdot 6$ & 0.0273 & 0.0492 & $0 \cdot 0607$ & 0.1274 & 0.20 \\
\hline $3 \cdot 8$ & 0.0224 & 0.0425 & 0.0545 & 0.1195 & $0 \cdot 20$ \\
\hline $4 \cdot 0$ & $0 \cdot 0183$ & $0 \cdot 0366$ & 0.0488 & 0.1115 & $0 \cdot 20$ \\
\hline $4 \cdot 2$ & 0.0150 & 0.0315 & 0.0436 & $0 \cdot 1036$ & $0 \cdot 196$ \\
\hline $4 \cdot 4$ & 0.0123 & $0 \cdot 0270$ & 0.0387 & 0.0958 & \\
\hline $4 \cdot 6$ & 0.0101 & 0.0231 & 0.0343 & 0.0882 & 0.18 \\
\hline $4 \cdot 8$ & $0 \cdot 0082$ & 0.0198 & 0.0303 & 0.0809 & 0.17 \\
\hline 5 & $0 \cdot 0067$ & 0.0168 & 0.0267 & 0.0739 & $0 \cdot 170$ \\
\hline 6 & 0.0025 & 0.0074 & 0.0136 & 0.0451 & 0.13 \\
\hline 7 & 0.0009 & 0.0032 & 0.0066 & 0.0258 & 0.093 \\
\hline 8 & 0.0003 & 0.0013 & 0.0031 & 0.0141 & 0.06 \\
\hline 9 & 0.0001 & 0.0006 & 0.0014 & 0.0074 & $0 \cdot 04$ \\
\hline 10 & 0.0000 & $0 \cdot 0002$ & $0 \cdot 0006$ & 0.0038 & 0.02 \\
\hline 11 & (). 0000 & $0 \cdot 0001$ & $0 \cdot 0003$ & 0.0019 & 0.01 \\
\hline 12 & 0.0000 & 0.0000 & 0.0001 & 0.0009 & 0.00 \\
\hline 13 & 0.0000 & 0.0000 & 0.0001 & 0.0004 & 0.00 \\
\hline 14 & 0.0000 & 0.0000 & 0.0000 & 0.0002 & 0.00 \\
\hline 15 & 0.0000 & $0 \cdot 0000$ & 0.0000 & 0.0001 & 0.001 \\
\hline
\end{tabular}

\begin{tabular}{|c|c|c|c|}
\hline $9-16$ & $17-32$ & $33-64$ & $>64$ \\
\hline 0.0026 & 0.0014 & 0.0008 & 0.0008 \\
\hline 0.0053 & 0.0029 & 0.0015 & 0.0015 \\
\hline 0.0081 & 0.0044 & 0.0023 & 0.0023 \\
\hline 0.0109 & $0 \cdot 0059$ & 0.0031 & 0.0031 \\
\hline 0.0138 & 0.0074 & 0.0038 & 0.0039 \\
\hline 0.0167 & 0.0090 & 0.0046 & 0.0047 \\
\hline 0.0196 & 0.0106 & 0.0055 & 0.0055 \\
\hline 0.0226 & 0.0122 & $0 \cdot 0063$ & 0.0063 \\
\hline 0.0257 & 0.0139 & 0.0071 & 0.0071 \\
\hline 0.0288 & 0.0155 & 0.0079 & 0.0079 \\
\hline 0.0319 & 0.0172 & 0.0088 & 0.0087 \\
\hline 0.0351 & 0.0189 & 0.0096 & $0 \cdot 0096$ \\
\hline 0.0383 & 0.0207 & 0.0105 & 0.0104 \\
\hline 0.0415 & 0.0224 & 0.0114 & 0.0112 \\
\hline $0 \cdot 0448$ & 0.0242 & 0.0123 & 0.0120 \\
\hline 0.0482 & 0.0260 & 0.0132 & 0.0129 \\
\hline 0.0515 & 0.0279 & 0.0141 & 0.0137 \\
\hline 0.0549 & 0.0297 & 0.0150 & 0.0146 \\
\hline 0.0583 & $0 \cdot 0316$ & 0.0159 & 0.0154 \\
\hline 0.0617 & 0.0335 & 0.0168 & 0.0163 \\
\hline 0.0757 & 0.0414 & 0.0207 & 0.0198 \\
\hline 0.0899 & 0.0496 & 0.0247 & 0.0233 \\
\hline $0 \cdot 1041$ & 0.0581 & $0 \cdot 0288$ & 0.0270 \\
\hline $0 \cdot 1184$ & 0.0670 & 00332 & 0.0307 \\
\hline 0.1324 & 0.0761 & 0.0377 & 0.0345 \\
\hline 0.1462 & 0.0855 & 0.0423 & 0.0383 \\
\hline 0.1595 & 0.0951 & 0.0471 & 0.0423 \\
\hline $0 \cdot 1723$ & $0 \cdot 1049$ & 0.0521 & 0.0464 \\
\hline $0 \cdot 1844$ & $0 \cdot 1149$ & 0.0573 & 0.0505 \\
\hline 0.1958 & $0 \cdot 1249$ & 0.0626 & 0.0547 \\
\hline $0 \cdot 2063$ & 0.1351 & 0.0680 & $0 \cdot 0590$ \\
\hline $0 \cdot 2159$ & $0 \cdot 1452$ & 0.0736 & 0.0634 \\
\hline 0.2246 & $0 \cdot 1554$ & 0.0794 & 0.0679 \\
\hline $0 \cdot 2324$ & 0.1655 & 0.0853 & 0.0725 \\
\hline 0.2391 & $0 \cdot 1755$ & 0.0913 & 0.0771 \\
\hline $0 \cdot 2447$ & $0 \cdot 1854$ & 0.0975 & 0.0819 \\
\hline $0 \cdot 2493$ & 0.1951 & $0 \cdot 1038$ & 0.0867 \\
\hline $0 \cdot 2529$ & $0 \cdot 2046$ & $0 \cdot 1102$ & 0.0917 \\
\hline $0 \cdot 2555$ & 0.2139 & $0 \cdot 1167$ & 0.0967 \\
\hline 0.2571 & 0.2228 & 0.1234 & 0.1018 \\
\hline $0 \cdot 2516$ & 0.2620 & 0.1577 & 0.1289 \\
\hline $0 \cdot 2285$ & $0 \cdot 2898$ & 0.1932 & 0.1584 \\
\hline $0 \cdot 1953$ & $0 \cdot 3043$ & 0.2283 & 0.1903 \\
\hline $0 \cdot 1586$ & $0 \cdot 3056$ & 0.2615 & $0 \cdot 2244$ \\
\hline $0 \cdot 1233$ & 0.2950 & 0.2913 & $0 \cdot 2608$ \\
\hline 0.0923 & 0.2751 & 0.3165 & $0 \cdot 2991$ \\
\hline 0.0668 & $0 \cdot 2486$ & $0 \cdot 3359$ & 0.3391 \\
\hline 0.0469 & 0.2185 & $0 \cdot 3489$ & $0 \cdot 3805$ \\
\hline $0 \cdot 0320$ & 0.1871 & 0.3552 & 0.4228 \\
\hline 0.0214 & 0.1564 & 0.3549 & $0 \cdot 4657$ \\
\hline
\end{tabular}


$>64$ mutants into further classes, e.g. 65-128, 129-256, 257-512 mutants, etc., would involve an impracticable amount of arithmetic. An attempt was therefore made to find asymptotic formulae for $p_{r}$ or $P_{r}$ valid for large values of $m$. We have not succeeded in finding explicit formulae, but have obtained some information on the form of the function.

If we consider $P_{r}$ as a continuous function of the two variables $r$ and $m$, then for values of $r \gg 1$ we have approximately $P_{r}-P_{r-1}=\partial P_{r} / \partial r$. Thus equation (21) approximates to

$$
\begin{gathered}
\frac{\partial P_{r}}{\partial m}+\left(1+\frac{r}{m}\right) \frac{\partial P_{r}}{\partial r}=0, \\
P_{r}=F\left(\frac{r}{m}-\log m\right),
\end{gathered}
$$

where $F$ is any function.

In Fig. 1 we have plotted $P_{r}$ (derived from Table 2, i.e. based on the recurrence relation) against $(r / m-\log m)$ for $r=8,16,32$ and 64 , using five values of $m$ (viz. 4, 6, 8, 13, 15)

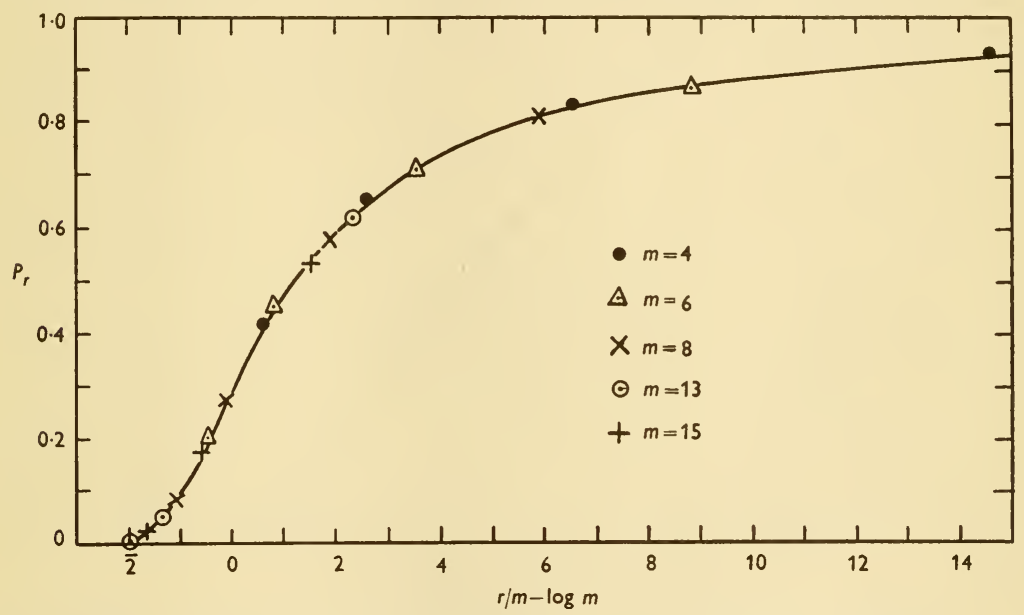

Fig. 1. $P_{r}$, for different $r$ and $m$, is a function of $r / m-\log m$. The points are plotted for $r=8,16,32$ and 64 , and with $m=4,6,8,13$ and 15 .

selected so that the twenty points are conveniently spaced. It is seen that the points lie quite well on a single curve, showing that these values of $r$ are large enough for equation (24) to be a satisfactorily close approximation to equation (21). The smooth curve in Fig. 1 is thus a graph of the function $F$ which enters into equation (25).

For any given value of $m,(r / m-\log m)$ is evidently distributed in a skew distribution about a median $1 \cdot 24$. We have found by trial that the derived variate $(r / m-\log m+4 \cdot 5)^{-1}$ is distributed in a distribution rather closely approximating to a Gaussian distribution of standard deviation $0 \cdot 086$. This is shown by the closeness with which the points in Fig. 2 lie on a straight line. The points in Fig. 2 are derived from those of Fig. 1 by transforming the ordinates to probits, defined by the relation $\frac{1}{\sqrt{(2 \pi)}} \int_{-\infty}^{y-5} e^{-\frac{1}{2} y^{2}} d y=P_{r}$, where $y$ is the probit corresponding to $P_{r}$. (Tables of probits are given in Fisher \& Yates, 1938.) Also, 
the abscissae are transformed to values of $(r / m-\log m+4 \cdot 5)^{-1}$. Fig. 2 shows that, approximately,

$$
x=\left(\frac{1}{r / m-\log m+4 \cdot 5}-0 \cdot 174\right) / 0 \cdot 086=\left(\frac{11 \cdot 6}{r / m-\log m+4 \cdot 5}-2 \cdot 02\right)
$$

is a normal deviate.

We conclude, in this semi-empirical manner, that when the spontaneous mutation theory is to be compared with experiments falling outside the scope of Table 2 (i.e. experiments in which cultures containing more than 64 mutants are frequent), it will be satisfactory for practical purposes to suppose $x=\left(\frac{11 \cdot 6}{r / m-\log m+4 \cdot 5}-2 \cdot 02\right)$ to be normally

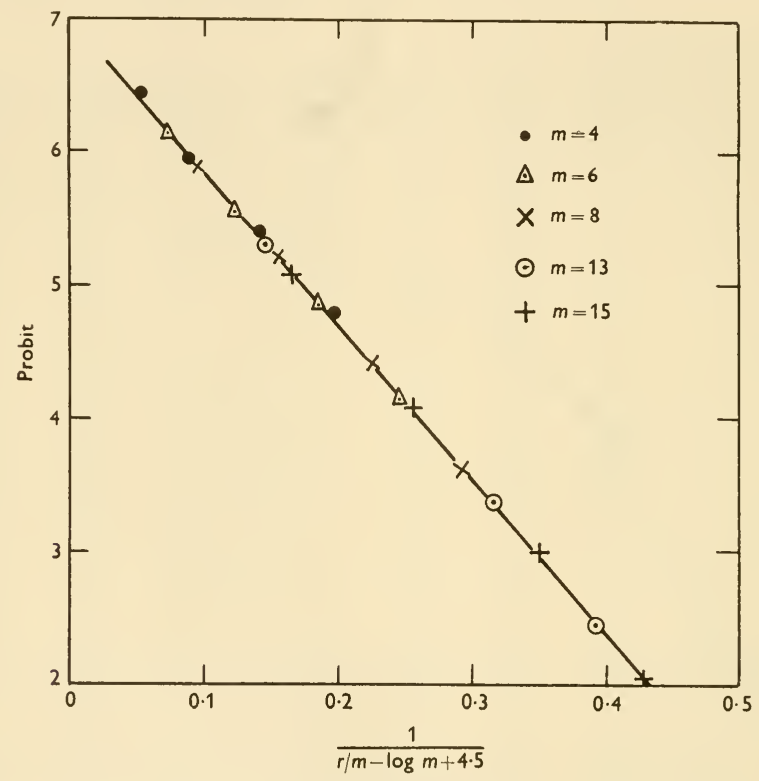

Fig. 2. $1 /(r / m-\log m+4 \cdot 5)$ is distributed in an approximately normal distribution. The points are plotted for $r=8,16,32$ and 64 , and with $m=4,6,8,13$ and 15 .

distributed with unit variance about the value $0 . r$ is the number of mutants in an individual culture, $m$ is the mean number of mutations per culture in the batch of parallel cultures.

The median of the distribution satisfies the relation

$$
\frac{11 \cdot 6}{r / m-\log m+4 \cdot 5}-2 \cdot 02=0 \text {, i.e. at the median } \quad r / m-\log m=1 \cdot 24 .
$$

This equation provides a means of making a first estimate of $m$ from the count of the number $(r)$ of mutants in the median culture of the batch.

The quartiles of the distribution (i.e. values of $r$ making $P_{r}=0.25$ or 0.75 ) satisfy the relations:

$$
\text { at quartiles: } \quad r / m-\log m=-0 \cdot 2 \text { and } \quad r / m-\log m=4 \cdot 1 \text {, }
$$


which relations may be used as a first test of whether the spread of an experimental distribution is comparable with the theoretical spread.

All the relations in this section are approximations, to be used only when dealing with experiments which lie outside the scope of Table 2. The approximation should not be used for the extreme ends of the distribution, e.g. for values of $P_{r}$ exceeding $0 \cdot 95$ or less than $0 \cdot 05$.

\section{THE ESTIMATION OF MUTATION RATE FROM EXPERIMENTAL OBSERVATIONS}

\section{$m$ from the mean number of mutants per culture}

As shown by Luria and Delbruck, the mean and variance of the distribution can be simply calculated, without knowing the distribution $p_{r}$, as follows:

While the culture grows from $n_{1}$ to $n_{1}+d n_{1}$, the mean number of mutations will be $(m / n) d n_{1}$ (cp. equation (4)), the actual number being distributed in a Poisson distribution about this mean with variance also $(m / n) d n_{1}$ (since the variance of a Poisson distribution is equal to the mean). The contribution to the final number of mutants (when the culture size is $n$ ) will be $n / n_{1}$ mutants for each mutation. Thus the contribution to the final number of mutants will be distributed about a mean $\frac{n}{n_{1}} \frac{m}{n} d n_{1}$ with a variance $\left(\frac{n}{n_{1}}\right)^{2} \frac{m}{n} d n_{1}$. Thus the mean of the required distribution is

$$
\bar{r}=\int_{1}^{n} \frac{n}{n_{1}} \frac{m}{n} d n_{1}=m \log n
$$

and the variance of an individual determination of $r$ will be

$$
\sigma^{2}=\int_{1}^{n}\left(\frac{n}{n_{1}}\right)^{2} \frac{m}{n} d n_{1} \doteqdot m n *
$$

We can confirm that our distribution $p_{r}$ yields the same mean and variance. The mean is

$$
\bar{r}=\Sigma r p_{r} \text {. }
$$

Since $p_{r}$ is the coefficient of $x^{r}$ in the expansion of $e^{-m} \exp \left[m\left(\frac{x}{1.2}+\frac{x^{2}}{2.3}+\ldots\right)\right]$ (equation (15)), and since $q_{r}=e^{m} p_{r}$, we have

$$
\Sigma q_{r} x^{r}=\exp \left[m\left(\frac{x}{1.2}+\frac{x^{2}}{2.3}+\ldots\right)\right]
$$

Differentiating

$$
\Sigma r x^{r-1} q_{r}=m\left(\frac{1}{2}+\frac{x}{3}+\frac{x^{2}}{4}+\ldots\right) \exp \left[m\left(\frac{x}{1.2}+\frac{x^{2}}{2.3}+\ldots\right)\right] .
$$

Inserting $x=1$, and putting $\frac{1}{2}+\frac{1}{3}+\ldots+\frac{1}{n} \doteqdot \log n$ and $\frac{1}{1.2}+\frac{1}{2.3}+\ldots \doteqdot 1$, we have

$$
\bar{r}=\Sigma r p_{r}=e^{-m} \Sigma r q_{r} \doteqdot m \log n .
$$

Again, multiplying (32) by $x^{2}$ and differentiating,

$\Sigma r(r+1) x^{r} q_{r}=m\left\{\left(x+x^{2}+x^{3}+\ldots\right)+m\left(\frac{x^{2}}{2}+\frac{x^{3}}{3}+\ldots\right)\left(\frac{1}{2}+\frac{x}{3}+\ldots\right)\right\} \exp \left[m\left(\frac{x}{1.2}+\frac{x^{2}}{2.3}+\ldots\right)\right]$.

Inserting $x=1$,

$$
\begin{gathered}
\sum r(r+1) q_{r} \doteqdot m e^{m}\left(n+m \log ^{2} n\right) \\
\sum r(r+1) p_{r} \doteqdot m n+m^{2} \log ^{2} n
\end{gathered}
$$

* But see Appendix : the correct value is $\sigma^{2} \div 2 m n$. 
Now the variance

$$
\begin{aligned}
\sigma^{2} & =\Sigma(r-\bar{r})^{2} p_{r}=\Sigma r^{2} p_{r}-2 \bar{r} \Sigma r p_{r}+\bar{r}^{2} \Sigma p_{r} \\
& =\Sigma r(r+1) p_{r}-\bar{r}-\bar{r}^{2} .
\end{aligned}
$$

Thus $\sigma^{2} \doteqdot m n-m \log n$, i.e. $\sigma^{2} \doteqdot m n$ since $n \gg 1$.

Since $\bar{r}=m \log n$, a possible method of determining $m$ (and hence the mutation rate) experimentally would be to divide by $\log n$ the mean number of mutants per culture experimentally determined in a batch of $N$ parallel cultures. However, on examination it appears that the precision of the estimate of $m$ given by this method does not increase with increase of $N$. For it is evident that the total numbers of mutants in batches of $N$ parallel cultures each of size $n$ will be distributed (from batch to batch) in much the same way as the numbers of mutants in parallel cultures of size $n N$. The mean number of mutations in a culture of size $n N$ will be $m N$ (since the mean number of mutations is proportional to the size of the culture, cp. equation (3)), and hence by application of (30) the variance of the number of mutations in cultures of size $n N$ is $m N . n N=m n N^{2}$. Thus the total number of mutants in a batch of $N$ cultures of size $n$ is distributed from batch to batch with a variance $m n N^{2}$. A fraction $1 / N$ of this total number (i.e. the mean number per culture derived from a count of $N$ cultures) is therefore distributed with variance $m n$.

Thus we see that the variance of the mean number $\bar{r}$ of mutants in $N$ cultures is no smaller than the variance of the number of mutants in an individual culture, which shows that however many cultures are averaged, no improvement in precision is obtained over the use of a single culture selected at random. Consequently, the mean number of mutants per culture is an extremely inefficient statistic from which to calculate the mutation rate. If, nevertheless, this method of estimating $m$ is employed, the variance $\left(\sigma_{m}^{2}\right)$ of the estimate of $m$ will (from (29) and (30)) be

$$
\frac{m n}{(\log n)^{2}} \text {, }
$$

independent of the number $N$ of cultures averaged.

\section{$m$ from proportion of cultures without mutants}

In view of the unsuitability of $\bar{r}$ as a means of estimating $m$ from numerical data, Luria and Delbruck proposed its estimation by equating $e^{-m}$ to the proportion of cultures experimentally determined to be without mutants. In a batch of $N$ parallel cultures, in which the mean number of mutations is $m$ per culture, the expected number of cultures without mutation is $N e^{-m}$, the actual number being distributed about this mean in a binomial distribution having a variance $N e^{-m}\left(1-e^{-m}\right)$. Thus the variance of the estimate $e^{-m}$ is $e^{-m}\left(1-e^{-m}\right) / N$. Since $\frac{d m}{d\left(e^{-m}\right)}=-e^{m}$, the corresponding estimate of $m$ has a variance $\left(\sigma_{m}^{2}\right)$ which is $e^{2 m}$ times as great, i.e.

$$
\sigma_{m}^{2}=\frac{e^{m}-1}{N}
$$

Thus the standard error $\left(\sigma_{m}\right)$ in the estimate of $m$ is given by

$$
\left(\frac{\sigma_{m}}{m}\right)^{2}=\frac{1}{N}\left(\frac{e^{m}-1}{m^{2}}\right)
$$

$\sigma_{m} / m$ thus varies with $m$. It has a minimal value when $m=1.594$, when a fraction 0.2032 of cultures have no mutants. At $m=1 \cdot 594,\left(\sigma_{m} / m\right)^{2}$ takes the value 1.544/N. At small or 
large values of $m$ (e.g. when the fraction of cultures without mutants exceeds 0.9 or is less than 0.01$)$, the value of $\left(\sigma_{m} / m\right)$ is much increased. Fig. $3 A$ shows graphically $\frac{\sigma_{m}}{m} \sqrt{ } N$ as a function of $m$.

The low precision at small values of $m$ is to be attributed simply to the fact that an experiment in which the great majority of the cultures have no mutants does not provide much precise information about the mutation rate. The reduced precision at high values of $m$ is, however, to be ascribed to the fact that this method of determining $m$ does not

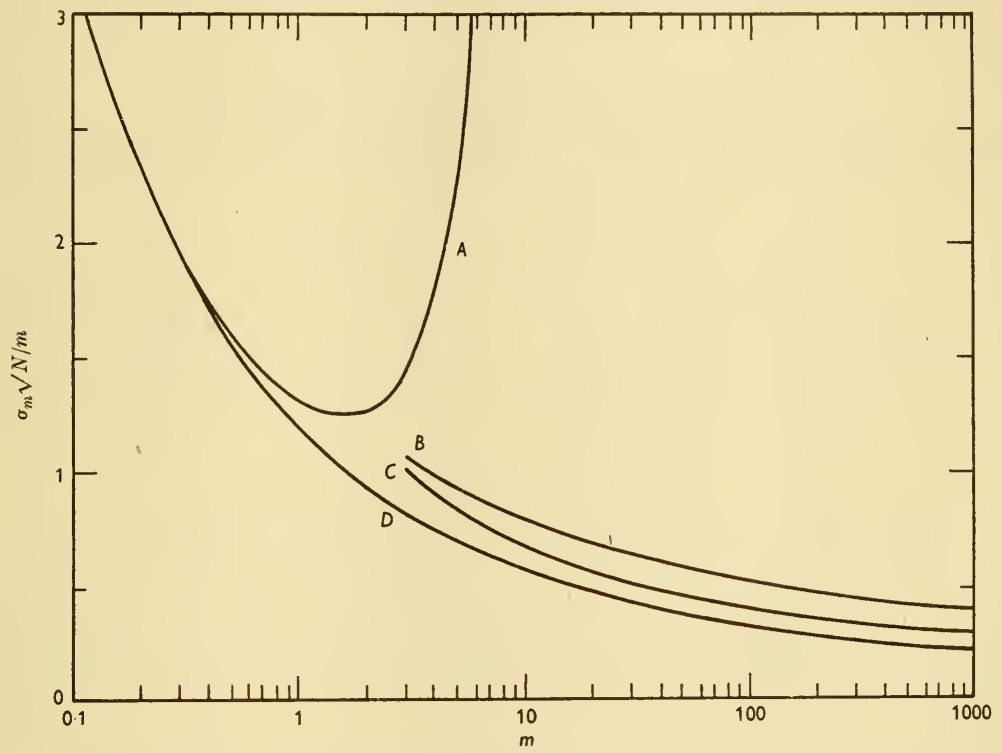

Fig. 3. The precision of the estimate of $m$ derived by various methods: $A$, the method of the proportion of cultures without mutants; $B$, the inethod of the median; $C$, the method of $S[x]=0 ; D$, the method of maximal likelihood.

make full use of the experimental data, and in these cases more suitable methods, which we shall describe, enable a more precise estimate of $m$ to be made from the same data.

\section{$m$ from the median}

When the mutation rate is to be deduced from an experiment in which all, or nearly all, the cultures had mutants, so that the method just discussed is inapplicable, a very convenient method is to deduce $m$ from the median of the distribution. The counts of the numbers of mutants in $N$ parallel cultures are arranged in ascending order, and the middle one selected. The count in this culture is an estimate of $r_{0}$, the median of the distribution of $r$. Since we know that (approximately) the derived variate

$$
x=\left(\frac{a}{r / m-\log m+b}-c\right) \quad \text { with } a=11 \cdot 6, b=4 \cdot 5, c=2 \cdot 02
$$


is normally distributed about median 0 , it follows that

$$
\frac{r_{0}}{m}-\log m=\frac{a}{c}-b=1 \cdot 24
$$

This equation enables an estimate of $m$ to be made from an experimentally determined value of $r_{0}$. With its aid Table 3 has been constructed, which enables $m$ to be obtained for any value of $r_{0}$ up to 4400 . While the derivation of $m$ from the median is not the most efficient way of utilizing the experimental data from a statistical standpoint, it is the quickest satisfactory method, and is useful for making a preliminary estimate even if a more elaborate method is to be employed in making the final estimate.

\section{Table 3. Preliminary estimation of $m$ from median value of $r$}

Thus if the middle culture of the series has 50 mutants, interpolation in the table between $r_{0}=49 \cdot 2$ and $r_{0}=55.8$ gives $r_{0} / m=3 \cdot 81$, so that $m=50 / 3 \cdot 81=13 \cdot 1$. This is the mean number of mutations per culture.

\begin{tabular}{|c|c|c|c|c|c|c|c|}
\hline$r_{0}$ & $r_{0} / m$ & $r_{0}$ & $r_{0} / m$ & $r_{0}$ & $r_{0} / m$ & $r_{0}$ & $r_{0} / m$ \\
\hline $1 \cdot 4$ & $1 \cdot 3$ & $15 \cdot 3$ & $2 \cdot 9$ & 117 & $4 \cdot 5$ & 787 & $6 \cdot 1$ \\
\hline 1.6 & 1.4 & $17 \cdot 4$ & $3 \cdot 0$ & 132 & $4 \cdot 6$ & 884 & $6 \cdot 2$ \\
\hline $1 \cdot 9$ & $1 \cdot 5$ & $19 \cdot 9$ & $3 \cdot 1$ & 150 & $4 \cdot 7$ & 993 & $6 \cdot 3$ \\
\hline $2 \cdot 3$ & $1 \cdot 6$ & $2 \cdot 2 \cdot 7$ & $3 \cdot 2$ & 169 & 4.8 & 1115 & $6 \cdot 4$ \\
\hline $2 \cdot 7$ & 1.7 & 25.9 & $3 \cdot 3$ & 190 & $4 \cdot 9$ & 1251 & 6.5 \\
\hline $3 \cdot 2$ & $1 \cdot 8$ & $29 \cdot 5$ & $3 \cdot 4$ & 215 & $5 \cdot 0$ & 1404 & $6 \cdot 6$ \\
\hline $3 \cdot 7$ & 1.9 & $33 \cdot 5$ & $3 \cdot 5$ & 242 & $5 \cdot 1$ & 1575 & 6.7 \\
\hline $4 \cdot 3$ & $2 \cdot 0$ & $38 \cdot 1$ & $3 \cdot 6$ & 273 & $\therefore 2$ & 1767 & $6 \cdot 8$ \\
\hline .50 & $2 \cdot 1$ & $43 \cdot 3$ & $3 \cdot 7$ & 307 & $5 \cdot 3$ & 1981 & 6.9 \\
\hline$\pi 7$ & $2 \cdot 2$ & $49 \cdot 2$ & $3 \cdot 8$ & 346 & $5 \cdot 4$ & 2221 & \\
\hline $6 \cdot 6$ & $2 \cdot 3$ & 55.8 & $3 \cdot 9$ & 389 & $5 \cdot 5$ & 2490 & $7 \cdot 1$ \\
\hline $7 \cdot 7$ & $2 \cdot 4$ & $63 \cdot 2$ & $4 \cdot 0$ & 438 & $5 \cdot 6$ & 2791 & 12 \\
\hline 8.8 & 2.5 & $71 \cdot 6$ & $4 \cdot I$ & 493 & $5 \cdot 7$ & 3127 & $7 \cdot 3$ \\
\hline $10 \cdot 1$ & $2 \cdot 6$ & $81 \cdot 1$ & $4 \cdot 2$ & 554 & $5 \cdot 8$ & 3503 & \\
\hline $11 \cdot 6$ & $2 \cdot 7$ & 91.7 & $4 \cdot 3$ & 623 & $5 \cdot 9$ & 3924 & 7.5 \\
\hline $13 \cdot 3$ & $2 \cdot 8$ & 104 & $4 \cdot 4$ & 700 & $6 \cdot 0$ & 439.5 & \\
\hline
\end{tabular}

The precision of an estimate of $m$ made in this way from counts of $N$ cultures may be determined by calculating $\sigma_{m} / m$. We shall make use of the approximate result that $x$ is distributed in a normal distribution with unit variance. The probability of $x$ lying between $x$ and $x+d x$ is $\frac{1}{\sqrt{(2 \pi)}} e^{-\frac{1}{2} x^{2}} d x$. The probability of its lying between 0 and $x$ is $\frac{1}{\sqrt{(2 \pi)}} \int_{0}^{x} e^{-\frac{1}{2} x^{2}} d x$, and for observations in the neighbourhood of the median $\left(x^{2} \ll 1\right)$ we may write this as $\frac{x}{\sqrt{ }(2 \pi)}$. Thus $\left(\frac{1}{2}+\frac{x}{\sqrt{ }(2 \pi)}\right)$ is the probability of getting an observation $\leqslant x$, and $\left(\frac{1}{2}-\frac{x}{\sqrt{1}(2 \pi)}\right)$ is the probability of getting an observation $\geqslant x$. Thus the probability that, of $N=2 s+1$ observations, $s$ shall be $\leqslant x, s$ shall be $\geqslant x$, and one shall be between $x$ and $x+d x$ is (providing $x$ is in the neighbourhood of the median)

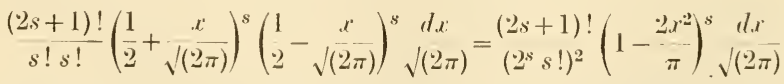

$$
\begin{aligned}
& \doteqdot \frac{(2 s+1) !}{\sqrt{(2 \pi)\left(2^{s} s !\right)^{2}}} \exp \left(-\frac{2 s x^{2}}{\pi}\right) d x \doteqdot\left[2 \pi\left(\frac{\pi}{4 s}\right)\right]^{-1} \exp \left(\frac{-\frac{1}{2} x^{2}}{\pi / 4 s}\right)
\end{aligned}
$$

for $s \gg 1$. Thus the median value of a set of $N=2 s+1$ values of $x$ is distributed about $x=0$ with variance $\frac{\pi}{4 s} \div \frac{\pi}{2 N}$. 
It follows that if equation (37) is used to deduce from an experimentally determined median value $r_{0}$ an estimate of $m$, then this estimate will be subject to a variance $\frac{\pi}{2 N} /\left(\frac{\partial x}{\partial m}\right)_{0}^{2}$, the suffix 0 denoting evaluation at the median. Differentiating (36),

$$
\frac{\partial x}{\partial m}=\frac{(x+c)^{2}(1-b+\log m)}{a m}+\frac{x+c}{m}=(x+c)^{2} \frac{d}{m}+\frac{x+c}{m},
$$

where $d=(1-b+\log m) / a$. Hence at the median $x=0,\left(\frac{\partial x}{\partial m}\right)_{0}=\frac{c}{m}(c d+1)$. Thus the variance $\sigma_{m}^{2}$ of the estimate of $m$ derived from the median is given by

$$
\left(\frac{\sigma_{m}}{m}\right)^{2}=\frac{1}{N} \frac{\frac{1}{2} \pi\left(a / c^{2}\right)^{2}}{(1+a / c-b+\log m)^{2}}=\frac{1}{N} \frac{\frac{1}{2} \pi}{c^{2}(c d+1)^{2}},
$$

or, inserting the values of $a, b, c$ from (36),

$$
\left(\frac{\sigma_{m}}{m}\right)^{2}=\frac{1}{N} \frac{12 \cdot 70}{(2 \cdot 24+\log m)^{2}} .
$$

Fig. $3 B$ is a plot of $\left(\frac{\sigma_{m}}{m}\right) \sqrt{ } N$ against $m$ as given by (41). Having used Table 3 to make an estimate of $m$ from the observation of the median value of $r$, Fig. $3 B$ is consulted to obtain the standard deviation to be ascribed to the estimate of $m$.

$$
m \text { from } S[x]=0
$$

An alternative method of estimating $m$ from experiments in which all or nearly all of the cultures have mutants is the following. Since $x$ is distributed approximately normally about the value $x=0$, the mean value of $x$ is zero. An estimate of $m$ from a set of $N$ observations can therefore be made by finding that value of $m$ which makes

$$
S[x]=S\left[\frac{a}{r / m-\log m+b}-c\right]=0,
$$

the summation being over the $N$ experimental observations. In using this method a first estimate of $m$ is made by the median method. Inserting this value of $m$ into (36), each experimental value of $r$ is converted into a value of $x$, and the sum $S[x]$ formed. A series of adjacent values of $m$ are then tried, and the value of $m$ which makes $S[x]=0$ found (e.g. by plotting $S[x]$ against $m$ ).

The estimate of $m$ obtained in this way is a little more precise than that based on the median. The mean $S[x] / N$ of a batch of $N$ independent values of $x$ will be distributed (from batch to batch) with variance $1 / N$ about a mean zero. Suppose that its value for a particular batch is $\delta$, so that

$$
S[x]=N \delta .
$$

If $m+\delta_{m}$ is the estimate of $m$ derived from this particular batch $\left(\delta_{m}\right.$ being the deviation between the estimated and true values of $m$ ),

$$
S\left[x+\frac{\partial x}{\partial m} \delta_{m}\right]=0 .
$$


Thus $\delta_{m} \frac{S[\partial x / \partial m]}{N}=-\delta$ or approximately $\delta_{m} E\left[\frac{\partial x}{\partial m}\right]=-\delta$, where we have replaced the mean value of $\partial x / \partial m$ for the set of $N$ observations by the expectation $E\left[\frac{\partial x}{\partial m}\right]$ of $\frac{\partial x}{\partial m}$.

Now from (39) we have

$$
\frac{\partial x}{\partial m}=\frac{1}{m}\left\{x^{2} d+x(2 c d+1)+c^{2} d+c\right\},
$$

and $x$ being normally distributed with unit variance about mean zero,

$$
E\left[x^{2}\right]=1 \text { and } E[x]=0 .
$$

Thus

$$
E\left[\frac{\partial x}{\partial m}\right]=\frac{1}{m}\left\{d\left(1+c^{2}\right)+c\right\}
$$

and so

$$
\delta_{m}=\frac{-m \delta}{d\left(1+c^{2}\right)+c} \text {. }
$$

The variance of $\delta$ from batch to batch being $1 / N$, we obtain for the variance $\left(\sigma_{m}{ }^{2}\right)$ of $m$ the relation

$$
\left(\frac{\sigma_{m}}{m}\right)^{2}=\frac{1}{N} \overline{\left\{d\left(1+c^{2}\right)+c\right\}^{2}},
$$

with $a=11 \cdot 6, b=4 \cdot 5, c=2 \cdot 02, d=(1-b+\log m) / a$.

A plot of $\frac{\sigma_{m}}{m} \sqrt{ } N$ against $m$ as computed by this formula is given in Fig. $3 C$. Having derived $m$ by the method described in this section, the standard deviation to be ascribed to it is read from Fig. $3 C$.

\section{Maximal likelihood method: large counts}

None of the methods we have so far described is fully efficient statistically. At the expense of somewhat more laborious computation a fully efficient estimate of the mutation rate may be made by employing the method of maximal likelihood. We give two solutions: one for experiments which fall within the range of Tables 1 and 2, i.e. in which most of the cultures have fewer than 64 mutants, which is set out in the next section, and one for experiments falling outside the range of Tables 1 and 2 , and for which the approximation that $x$ is a normal deviate is employed, which is set out in the present section.

The probability that the number of mutants shall lie between $r$ and $r+d r$ is given approximately (for $r$ not too small) as

$$
\frac{1}{\sqrt{(2 \pi)}} e^{-\frac{1}{2} x^{2}} d x=\frac{1}{\sqrt{(2 \pi)}} e^{-\frac{1}{2} x^{2}} \frac{\partial x}{\partial r} d r=f d r \quad \text { (say). }
$$

Thus

$$
\frac{d}{d m} \log f=\frac{1}{f} \frac{d f}{d m}=-x \frac{\partial x}{\partial m}+\frac{\partial^{2} x}{\partial r \partial m} / \frac{\partial x}{\partial r} .
$$

Now

$$
x=\left(\frac{a}{r / m-\log m+b}-c\right) \quad \text { with } a=11 \cdot 6, b=4 \cdot 5, c=2 \cdot 02,
$$

and by differentiating we find

$$
\frac{\partial x}{\partial m}=(x+c)^{2} \frac{d}{m}+\frac{x+c}{m} ; \quad \frac{\partial^{2} x}{\partial m \partial r} / \frac{\partial x}{\partial r}=2(x+c) \frac{d}{m}+\frac{1}{m},
$$


where

Thus

$$
d=(1-b+\log m) / a \text {. }
$$

Now $L$, the $\log$ likelihood, is (apart from irrelevant terms) $S[\log f]$, the summation being for the $N$ observations of $r$, and the maximal likelihood condition is

$$
0=\frac{d L}{d m}=S\left[\frac{1}{f} \frac{d f}{d m}\right]
$$

i.e.

$$
S\left[x(x+c)^{2} d+x(x+c)-2(x+c) d-1\right]=0 .
$$

The routine for applying this method is as follows. Employing the preliminary estimate of $m$ given by the median method, (48) is used to calculate $d$, and then (46) is used to calculate a value of $x$ from each of the $N$ experimental observations of $r$. For each of these $N$ values of $x$ the expression

$$
x(x+c)^{2} d+x(x+c)-2(x+c) d-1
$$

is evaluated and the $N$ quantities added. The sum is similarly evaluated for several adjacent values of $m$, and by plotting against $m$ (or otherwise) the value of $m$ which satisfies $(50)$ is deduced.

The variance to be attached to the maximal likelihood estimate of a parameter $m$ is given by Fisher's formula (cp. e.g. Fisher, 1938)

$$
\sigma_{m}^{2}=\frac{1}{N i}
$$

where $i=E\left[\left(\frac{1}{f} \frac{d f}{d m}\right)^{2}\right]$ is the expectation of $\left(\frac{1}{f} \frac{d f}{d m}\right)^{2}$. Hence, using (49),

$$
\begin{aligned}
i m^{2} & =E\left[\left\{x^{3} d+x^{2}(2 c d+1)+x\left(c^{2} d+c-2 d\right)-(2 c d+1)\right\}^{2}\right] \\
& =d^{2} E\left[x^{6}\right]+\left(6 c^{2} d^{2}+6 c d-4 d^{2}+1\right) E\left[x^{4}\right] \\
& +\left(c^{4} d^{2}+2 c^{3} d-12 c^{2} d^{2}-12 c d+4 d^{2}+c^{2}-2\right) E\left[x^{2}\right] \\
& +\left(4 c^{2} d^{2}+4 c d+1\right) \\
& + \text { terms involving odd powers of } x .
\end{aligned}
$$

Now it is readily shown that $x$ being distributed normally about zero with unit variance, $E\left[x^{n}\right]$ vanishes for odd $n$, and

$$
E\left[x^{0}\right]=E\left[x^{2}\right]=1, \quad E\left[x^{4}\right]=3, \quad E\left[x^{6}\right]=15 .
$$

Inserting these values in (52) we obtain

$$
i m^{2}=d^{2}\left(c^{4}+10 c^{2}+7\right)+d\left(2 c^{3}+10 c\right)+\left(c^{2}+2\right)
$$

so that

$$
\left(\frac{\sigma_{m}}{m}\right)^{2}=\frac{1}{N} \frac{1}{d^{2}\left(c^{4}+10 c^{2}+7\right)+d\left(2 c^{3}+10 c\right)+\left(c^{2}+2\right)},
$$

with $a=11 \cdot 6, b=4 \cdot 5, c=2 \cdot 02, d=(1-b+\log m) / a$.

The part of Fig. $3 D$ to the right of $m=10$ is a plot of $\left(\frac{\sigma_{m}}{m}\right) \sqrt{ } N$ against $m$. Having determined the maximal likelihood estimate of $m$, as described in this section, the standard deviation to attach to it is read off from Fig. $3 D$. 


\section{Maximal likelihood method: smaller counts}

In this section we describe the method of arriving at the maximal likelihood estimate of $m$ from an experiment falling within the scope of Tables 1 and 2 ; i.e. one in which the majority of cultures have fewer than 64 mutants.

$p_{r}$ is the probability of a culture having $r$ mutants. The log likelihood of a set of $N$ values of $r$ is (apart from irrelevant terms)

$$
L=S\left[\log p_{r}\right],
$$

$S$ denoting summation over the $N$ experimental values of $r$.

The maximal likelihood value of $m$ is that satisfying

$$
0=\frac{d L}{d m}=S\left[\frac{1}{p_{r}} \frac{d p_{r}}{d m}\right]
$$

Now from equation (11)

$$
p_{\boldsymbol{r}}=\sum_{j=1}^{\infty} C_{j, r} e^{-m} \frac{m^{j}}{j !}, \quad \frac{d p_{r}}{d m}=\sum_{j=1}^{\infty} C_{j, r} e^{-m}\left(\frac{m^{j-1}}{(j-1) !}-\frac{m^{j}}{j !}\right),
$$

so that

$$
\frac{1}{p_{r}} \frac{d p_{r}}{d m}=\frac{t_{r}-p_{r}}{p_{r}}
$$

where

$$
t_{r}=\sum_{j=1}^{\infty} C_{j, r}\left(e^{-m} \frac{m^{j-1}}{(j-1) !}\right)
$$

Thus the maximal likelihood estimate of $m$ is that satisfying

$$
S\left[\frac{t_{r}-p_{r}}{p_{r}}\right]=0
$$

$t_{r}$ has been computed for a range of values of $m$ exactly as described earlier for $p_{r}$, and in Table 4 values of $\left(t_{r}-p_{r}\right) / p_{r}$ are listed for a range of values of $m$ and for the same grouped ranges of $r$ as were used previously.

The method of estimating $m$ is therefore the following. A preliminary estimate of $m$ is obtained either by the median method or by equating $e^{-m}$ to the proportion of cultures without mutants. Table 4 is entered at the value of $m$ nearest to this preliminary estimate, and a value of $\left(t_{r}-p_{r}\right) / p_{r}$ read off for each of the $N$ experimental values of $r$. The $N$ values are summed. The procedure is repeated for several adjacent values of $m$, and thence (graphically or otherwise) the value of $m$ inferred which would make $S\left[\frac{t_{r}-p_{r}}{p_{r}}\right]=0$.

The variance of this maximal likelihood estimate of $m$ is given by the relation

Thus

$$
\begin{gathered}
\sigma_{m}^{2}=\frac{1}{N i}, \quad \text { where } \quad i=\sum_{r=0}^{\infty} \frac{1}{p_{r}}\left(\frac{d p_{r}}{d m}\right)^{2}=\Sigma \frac{\left(t_{r}-p_{r}\right)^{2}}{p_{r}} . \\
\left(\frac{\sigma_{m}}{m}\right)^{2}=\frac{1}{N} \frac{1}{m^{2} \sum \frac{\left(t_{r}-p_{r}\right)^{2}}{p_{r}}} .
\end{gathered}
$$

$\Sigma$ here means summation over all values of $r$ from 0 to infinity, and is to be distinguished from $S$, meaning summation over the $N$ experimental observations. 
Table 4. $\frac{t_{r}-p_{r}}{p_{r}}$ and $\frac{\sigma_{m}}{m} \sqrt{ } N$

This table is used in estimating mutation rate by the maximal likelihood method.

$\frac{t_{0}-p_{0}}{p_{0}}=-1$ for all values of $m$.

\begin{tabular}{|c|c|c|c|c|c|c|c|c|c|}
\hline & & & & & & & & & \\
\hline$m \backslash r$ & 1 & 2 & $3-4$ & $5-8$ & $9-16$ & $17-32$ & $33-64$ & $>64$ & $\sigma_{m} \sqrt{ } N / m$ \\
\hline 0.05 & $19 \cdot 000$ & $19 \cdot 723$ & $20 \cdot 019$ & $20 \cdot 179$ & $20 \cdot 211$ & $20 \cdot 176$ & $20 \cdot 124$ & $20 \cdot 057$ & $4 \cdot 527$ \\
\hline $0 \cdot 10$ & $9 \cdot 000$ & $9 \cdot 698$ & $9 \cdot 998$ & $10 \cdot 166$ & $10 \cdot 206$ & $10 \cdot 174$ & $10 \cdot 124$ & $10 \cdot 057$ & $3 \cdot 239$ \\
\hline $0 \cdot 15$ & $5 \cdot 667$ & 6.341 & $6 \cdot 644$ & $6 \cdot 821$ & $6 \cdot 868$ & $6 \cdot 839$ & $6 \cdot 790$ & $6 \cdot 723$ & $2 \cdot 674$ \\
\hline $0 \cdot 20$ & $4 \cdot 000$ & $4 \cdot 652$ & $4 \cdot 957$ & $5 \cdot 142$ & $5 \cdot 196$ & $5 \cdot 171$ & $5 \cdot 123$ & $5 \cdot 057$ & $2 \cdot 341$ \\
\hline $0 \cdot 25$ & 3.000 & $3 \cdot 632$ & 3.939 & $4 \cdot 130$ & $4 \cdot 191$ & $4 \cdot 170$ & $4 \cdot 123$ & $4 \cdot 057$ & $2 \cdot 116$ \\
\hline $0 \cdot 30$ & $2 \cdot 333$ & $2 \cdot 946$ & 3.254 & 3.452 & $3 \cdot 519$ & $3 \cdot 501$ & $3 \cdot 456$ & $3 \cdot 390$ & 1.951 \\
\hline $0 \cdot 35$ & $1 \cdot 857$ & $2 \cdot 451$ & $2 \cdot 760$ & 2.964 & 3.038 & $3 \cdot 023$ & $2 \cdot 979$ & 2.914 & 1.824 \\
\hline $0 \cdot 40$ & 1.500 & $2 \cdot 077$ & $2 \cdot 386$ & $2 \cdot 596$ & $2 \cdot 676$ & $2 \cdot 665$ & & & 1.722 \\
\hline 0.45 & $1 \cdot 222$ & 1.783 & $2 \cdot 092$ & $2 \cdot 307$ & $2 \cdot 393$ & $2 \cdot 386$ & $2 \cdot 344$ & $2 \cdot 279$ & 1.638 \\
\hline 0.50 & 1.000 & 1.545 & $1 \cdot 855$ & $2 \cdot 075$ & $2 \cdot 166$ & $2 \cdot 162$ & $2 \cdot 121$ & $2 \cdot 057$ & 1.588 \\
\hline 0.55 & $0 \cdot 818$ & $1 \cdot 349$ & $1 \cdot 658$ & 1.882 & 1.979 & 1.978 & $1 \cdot 939$ & $1 \cdot 875$ & 1.507 \\
\hline $0 \cdot 60$ & $0 \cdot 667$ & $1 \cdot 184$ & $1 \cdot 492$ & $1 \cdot 720$ & $1 \cdot 822$ & 1.825 & & & $1 \cdot 455$ \\
\hline $0 \cdot 65$ & 0.538 & $1 \cdot 043$ & $1 \cdot 350$ & $1 \cdot 582$ & $1 \cdot 689$ & $1 \cdot 695$ & 1.658 & 1.595 & $1 \cdot 409$ \\
\hline 0.70 & 0.429 & 0.920 & $1 \cdot 227$ & 1.462 & $1 \cdot 574$ & 1.584 & 1.548 & $1 \cdot 485$ & $1 \cdot 368$ \\
\hline 0.75 & 0.333 & $0 \cdot 813$ & $1 \cdot 119$ & $1 \cdot 357$ & 1.474 & 1.487 & 1.452 & $1 \cdot 390$ & $1 \cdot 332$ \\
\hline $0 \cdot 80$ & $0 \cdot 250$ & 0.719 & $1 \cdot 023$ & $1 \cdot 264$ & $1 \cdot 386$ & $1 \cdot 402$ & $1 \cdot 369$ & $1 \cdot 307$ & $1 \cdot 299$ \\
\hline 0.85 & $0 \cdot 176$ & 0.634 & 0.937 & $1 \cdot 181$ & $1 \cdot 308$ & $1 \cdot 327$ & $1 \cdot 295$ & $1 \cdot 233$ & $1 \cdot 269$ \\
\hline 0.90 & 0.111 & 0.559 & $0 \cdot 860$ & $1 \cdot 107$ & $1 \cdot 237$ & $1 \cdot 259$ & $1 \cdot 229$ & $1 \cdot 168$ & $1 \cdot 242$ \\
\hline 0.95 & 0.053 & $0 \cdot 491$ & 0.790 & $1 \cdot 039$ & $1 \cdot 174$ & $1 \cdot 199$ & $1 \cdot 170$ & $1 \cdot 109$ & $1 \cdot 217$ \\
\hline $1 \cdot 0$ & 0.000 & 0.429 & 0.727 & 0.978 & $1 \cdot 117$ & $1 \cdot 145$ & $1 \cdot 117$ & 1.057 & $1 \cdot 194$ \\
\hline $1 \cdot 2$ & -0.167 & 0.228 & 0.520 & 0.777 & 0.931 & 0.971 & 0.948 & 0.890 & $1 \cdot 118$ \\
\hline $1 \cdot 4$ & -0.286 & 0.080 & 0.364 & $0 \cdot 627$ & 0.793 & 0.845 & 0.828 & 0.771 & 1.059 \\
\hline $1 \cdot 6$ & -0.375 & -0.034 & $0 \cdot 243$ & 0.508 & 0.686 & 0.749 & $0 \cdot 736$ & $0 \cdot 682$ & $1 \cdot 012$ \\
\hline 1.8 & -0.444 & -0.125 & $0 \cdot 144$ & 0.411 & 0.599 & 0.672 & 0.665 & 0.612 & 0.973 \\
\hline $2 \cdot 0$ & -0.500 & $-0 \cdot 200$ & 0.063 & 0.330 & 0.526 & $0 \cdot 609$ & $0 \cdot 608$ & 0.557 & $0 \cdot 940$ \\
\hline $2 \cdot 2$ & -0.545 & -0.262 & -0.007 & $0 \cdot 260$ & 0.464 & 0.556 & 0.560 & 0.511 & 0.913 \\
\hline $2 \cdot 4$ & -0.583 & -0.315 & -0.066 & $0 \cdot 200$ & 0.409 & 0.510 & 0.520 & 0.473 & $0 \cdot 888$ \\
\hline $2 \cdot 6$ & $-0 \cdot 615$ & -0.361 & -0.119 & $0 \cdot 147$ & 0.362 & 0.471 & 0.486 & 0.441 & 0.867 \\
\hline $2 \cdot 8$ & -0.643 & -0.401 & -0.164 & 0.099 & 0.319 & 0.436 & 0.456 & 0.413 & $0 \cdot 848$ \\
\hline $3 \cdot 0$ & -0.667 & -0.436 & -0.205 & $0 \cdot 057$ & $0 \cdot 280$ & 0.404 & 0.430 & $0 \cdot 390$ & 0.831 \\
\hline $3 \cdot 2$ & -0.688 & -0.467 & -0.242 & 0.018 & $0 \cdot 245$ & $0 \cdot 376$ & 0.407 & $0 \cdot 369$ & 0.816 \\
\hline $3 \cdot 4$ & -0.706 & -0.495 & -0.275 & -0.017 & $0 \cdot 213$ & 0.350 & $0 \cdot 386$ & $0 \cdot 350$ & 0.802 \\
\hline $3 \cdot 6$ & -0.722 & -0.520 & -0.305 & -0.050 & 0.183 & $0 \cdot 326$ & $0 \cdot 367$ & $0 \cdot 334$ & 0.789 \\
\hline $3 \cdot 8$ & -0.737 & -0.542 & -0.333 & -0.080 & $0 \cdot 155$ & $0 \cdot 304$ & $0 \cdot 350$ & $0 \cdot 319$ & 0.777 \\
\hline $4 \cdot 0$ & -0.750 & -0.563 & -0.358 & -0.107 & $0 \cdot 129$ & $0 \cdot 284$ & $0 \cdot 334$ & $0 \cdot 305$ & $0 \cdot 766$ \\
\hline $4 \cdot 2$ & -0.762 & -0.581 & -0.381 & -0.133 & $0 \cdot 105$ & 0.264 & 0.320 & $0 \cdot 293$ & 0.756 \\
\hline $4 \cdot 4$ & -0.773 & -0.598 & -0.402 & -0.157 & 0.082 & $0 \cdot 246$ & $0 \cdot 306$ & $0 \cdot 282$ & 0.747 \\
\hline $4 \cdot 6$ & -0.783 & -0.614 & -0.422 & $-0 \cdot 179$ & 0.061 & 0.229 & 0.293 & $0 \cdot 272$ & 0.738 \\
\hline $4 \cdot 8$ & -0.792 & -0.629 & -0.441 & $-0 \cdot 200$ & 0.041 & $0 \cdot 213$ & $0 \cdot 282$ & $0 \cdot 263$ & $0 \cdot 730$ \\
\hline $5 \cdot 0$ & -0.800 & -0.642 & -0.458 & -0.220 & 0.021 & $0 \cdot 197$ & 0.271 & $0 \cdot 254$ & 0.722 \\
\hline 6 & -0.833 & -0.697 & -0.530 & -0.304 & -0.062 & $0 \cdot 130$ & $0 \cdot 223$ & $0 \cdot 219$ & 0.690 \\
\hline 7 & -0.857 & -0.737 & -0.584 & $-0 \cdot 369$ & -0.128 & 0.073 & $0 \cdot 184$ & $0 \cdot 194$ & 0.665 \\
\hline 8 & -0.875 & -0.768 & $-0 \cdot 626$ & $-0 \cdot 422$ & -0.184 & 0.025 & 0.151 & $0 \cdot 174$ & $0 \cdot 644$ \\
\hline 9 & -0.889 & -0.792 & -0.660 & -0.465 & -0.231 & -0.016 & 0.121 & 0.157 & 0.627 \\
\hline 10 & -0.900 & -0.812 & -0.689 & -0.502 & -0.272 & -0.053 & 0.095 & $0 \cdot 143$ & 0.613 \\
\hline 11 & -0.909 & -0.828 & -0.712 & -0.534 & -0.307 & -0.086 & 0.071 & $0 \cdot 131$ & $0 \cdot 600$ \\
\hline 12 & -0.917 & -0.842 & $-0 \cdot 733$ & -0.561 & -0.339 & -0.116 & 0.048 & $0 \cdot 120$ & $0 \cdot 589$ \\
\hline 13 & -0.923 & -0.853 & -0.750 & -0.586 & -0.367 & $-0 \cdot 143$ & 0.028 & 0.110 & 0.580 \\
\hline 14 & -0.929 & -0.863 & -0.765 & -0.607 & -0.393 & -0.167 & 0.008 & 0.101 & 0.571 \\
\hline 15 & -0.933 & -0.872 & -0.779 & -0.626 & -0.416 & -0.190 & -0.010 & 0.092 & 0.564 \\
\hline
\end{tabular}


In the final column of Table 4 we have tabulated

$$
\frac{1}{m \sqrt{\left(\Sigma \frac{\left(t_{r}-p_{r}\right)^{2}}{p_{r}}\right)}}=\frac{\sigma_{m}}{m} \sqrt{ } \text {. }
$$

Having determined the maximal likelihood estimate of $m$ as just described, the value of $\sigma_{m} \sqrt{ } / N / m$ is read off from the last column of Table 4. These values of $\sigma_{m} \sqrt{ } N / m$ have been used in plotting the part of Fig. $3 D$ to the left of $m=10$. Between $m=3$ and $m=15$, the values of $\sigma_{m} \sqrt{ } / \mathrm{m}$ calculated from (58) and from (53) agree satisfactorily.

\section{Summary}

Statistical calculations are made of the distribution numbers of mutants in a culture of bacteria in which the number of mutants increases on account both of new mutations and of division of old mutants. In this way the largely qualitative conclusions of Luria and Delbruck are extended and placed on a firm quantitative basis. The results of these calculations, which enable the mutation rate to be inferred from experiments with parallel cultures, are presented in the form of tables. Statistically efficient methods of using these tables are discussed.

\section{REFERENCES}

Adolph, E. F. \& Bayne-Jones, S. (1932). J. Cell. Comp. Physiol. 1, 409.

Demerec, M. (1945). Proc. Nat. Acad. Sci., Wash., 31, 16.

Fisher, R. A. (1938). Statistical Theory of Estimations. Calcutta University Readership lectures.

Fisher, R. A. \& Yates, F. (1938). Statistical Tables for Biologists, Agriculturalists and Medical Research. Edinburgh: Oliver and Boyd.

Lewis, I. M. (1934). J. Bact. 28, 619.

Lurta, S. E. \& Delbruck, M. (1943). Genetics, 28, 491.

Molina, E. C. D. (1942). Poisson's Exponential Binomial Limit Tables. New York: Van Nostrand. Stewart, F. M. (1947). J. Hyg., Camb., 45, 28.

Witkin, E. M. (1946). Proc. Nat. Acad. Sci., Wash., 32, 59. 


\section{APPENDIX. (By C.A.C.)}

(1) It has been suggested that a table of the individual coefficients $C_{j, r}$ introduced in equation (7), and which give the expansion of $q_{r}$ in powers of $m$, might be useful. Such a tabJe, for $r \leqslant 10$, is shown below.

\begin{tabular}{|c|c|c|c|c|c|c|c|c|c|c|}
\hline \multicolumn{11}{|c|}{$\begin{array}{c}\text { Table of } C_{j, r} \\
\text {. }\end{array}$} \\
\hline$r \backslash j$ & 1 & 2 & 3 & 4 & 5 & 6 & 7 & 8 & 9 & 10 \\
\hline 1 & $\frac{1}{2}$ & & & & & & & & & \\
\hline 2 & $\frac{1}{6}$ & $\frac{1}{4}$ & & & & & & & & \\
\hline 3 & $\frac{1}{12}$ & $\frac{1}{8}$ & $\frac{1}{8}$ & & & & & & & \\
\hline 4 & $\frac{1}{2} \overline{0}$ & $\frac{1}{8}$ & $\frac{1}{8}$ & $\frac{1}{16}$ & & & & & & \\
\hline 5 & $\frac{1}{30}$ & $\frac{7}{80}$ & $\frac{5}{48}$ & $\frac{1}{12}$ & $\frac{1}{32}$ & & & & & \\
\hline 6 & $\frac{1}{42}$ & $\frac{41}{720}$ & $\frac{181}{2180}$ & $\frac{1}{12}$ & $\frac{5}{8 B}$ & $\frac{-1}{64}$ & & & & \\
\hline 7 & $\frac{1}{58}$ & $\frac{108}{2520}$ & $\frac{87}{1440}$ & $\frac{41}{340}$ & $\frac{35}{576}$ & $\frac{1}{32}$ & $\frac{1}{128}$ & & & \\
\hline 8 & $\frac{1}{72}$ & $\frac{853}{25200}$ & $\frac{551}{10080}$ & $\frac{173}{2582}$ & $\frac{107}{1728}$ & $\frac{1}{24}$ & $\frac{7}{384}$ & $\frac{1}{256}$ & & \\
\hline 9 & $\frac{1}{80}$ & $\frac{18}{700}$ & $\frac{13578}{302400}$ & $\frac{\frac{1313}{22880}}{2 y^{2}}$ & $\frac{307}{5184}$ & $\frac{203}{4320}$ & $\frac{7}{256}$ & $\frac{1}{86}$ & $\frac{1}{512}$ & \\
\hline 10 & $\frac{1}{110}$ & $\frac{1678}{75800}$ & $\frac{251}{8720}$ & $\frac{7587}{151200}$ & $\frac{5889}{108864}$ & $\frac{1881}{34580}$ & $\frac{1186 \theta}{3+580}$ & $\frac{5}{288}$ & $\frac{3}{512}$ & $\frac{1}{1024}$ \\
\hline
\end{tabular}

(2) It should perhaps be pointed out that the replacement in (3) of $n-1$ by $n$ is an approximation whose effect is quite negligible provided that $r \ll n$, as occurs in all experiments. In fact, even for $r$ of the order of $n^{\frac{1}{2}}$, the values of $q_{r}$ are seriously in error. As a result of this, and of the fact that it allows $r$ to exceed $n$ (which is manifestly impossible since $r$ is the number of mutants and $n$ is the total number of bacteria), the generating function (15) actually gives an infinite value for all the moments. These two difficulties have been removed in a development of this theory, to be published by $\mathrm{Mr}$ D. G. Kendall, of Oxford. But unfortunately his more strictly correct generating function cannot be expanded with any ease to determine the $q_{r}$. Except for large $r$ or small $n$, however, it differs insignificantly from our (15).

(3) Mr Kendall has kindly pointed out to me that the argument in (31) and (32), which was copied from Luria and Delbruck, is not quite valid. For in (32) the complete series $\frac{1}{2}+\frac{x}{3}+\frac{x^{2}}{4}+\ldots$ is not convergent when $x=1$, and in order to get an expression for the mean value and the variance it was necessary artificially to curtail this series by truncating it at its term $x^{n} / n$. This device is not a valid procedure, and it appears that although there is no change in the mean $\bar{r}$, the variance $\sigma^{2}$ of an individual determination of $r$ requires to be multiplied by 2 , so that the correct relation $\sigma^{2} \div 2 m n$. 


\section{Origin of Bacterial Variants}

NUMERous bacterial variants are known which will grow in environments unfavourable to the parent strain, and to explain their occurrence two conflicting hypotheses have been advanced. The first assumes that the particular environment produces the observed change in some of the bacteria exposed to it, whereas the second assumes that the variants arise spontaneously during growth under normal conditions, the part played by the adverse environment being purely selective. These are known respectively as the 'adaptation' and the 'spontaneous mutation' hypo. theses.

In order to discriminate between them an experimental approach (the 'fluctuation test') was developed by Luria and Delbrück in $1943^{1}$. This test has been applied to a number of variants, some of them in widely separated strains of bacteria (see Table 1). and in each case the conclusion reached has been that the variant arose by spontaneous mutation. The validity of the fluctuation test has not been challenged. at least so far as I am aware; but on the other hand

Tabie 1. Bacterial variations shown by means of the fluctuation test to arise through spontaneous mutation

\begin{tabular}{|l|l|l|}
\hline \multicolumn{1}{|c|}{ Organism } & \multicolumn{1}{c|}{ Variation to } & Reference \\
\hline F. coli & phage T1 resistance & $1,6,5$ \\
& phage T3-T7 resistance & 6 \\
& radiation resistance & 7 \\
histidine independence & 8 \\
streptomycin resistance & 9,10 \\
ataph. aureus & penicillin resistance & 11 \\
& sulphathiazole resistance & 12 \\
Clostridium septicum & atreptomycin resistance & 13 \\
Haracil independence & 14 \\
Eberthella typhosa & streptomycin resistance & 15 \\
See also data from : & tryptophan independence & 16 \\
E. coli-mutabile & iactose fermentation & 17 \\
Salmonella & fermentation variants & 18 \\
\hline
\end{tabular}

it has gained only limited recognition (see ref. 2). In part this may be due to the statistical and essen. tially indirect nature of the argument on which it is based, and if so the more direct experimental evidence described below would seem to be of value.

Bacteria of Escherichia coli strain $B / r$ susceptible to phage $T 1$ were plated on agar and incubated until a limited population increase had taken place. On alternate plates the bacteria were redistributed over the surface of the agar by spreading with 0.1 c.c. of sterile saline. All were then sprayed with phage $T 1$, and counts made of the colonies of resistant survivors 
which developed after further inoubation. (For details of the spray technique, see refs. $3,4,5$.)

On the adaptation hypothesis, the bacteria present at the end of the initial growth-period would all be phage-susceptible, and spreading would serve only to redistribute the members of a homogeneous population. No striking differences in colony count between spread and unspread plates would therefore be expected.

On the alternative (spontaneous mutation) hypothesis, both susceptible and resistant cells would be present at the end of the initial growth-period wherever a sufficient end-population had been reached. Further, mutations taking place a generation or moro before the cessation of growth would each be repre. sented by a minute cluster of resistant cells all descended from the one original mutant. Where the arrangement of the bacteria is left undisturbed, a cluster would give rise to a single resistant colony after the application of phage ; but where the becteria are redistributed over the surface of the agar by spreading, a colony would develop from each resistant cell. Higher counts would thus be expected from the spread then from the unspread plates. This is, in fact, what was found (see Table 2), the difference being as much as fifty-fold where the and population was highest.

Table 2. Resistant coluules developiug after syraying agar plate cultures of $E$. coli with phage $T 1$, showing the effect of redisterbution by spreading prior to spraying

\begin{tabular}{|c|c|c|c|c|}
\hline Incub. (hr.) & 3 & 4 & 5 & 6 \\
\hline $\begin{array}{l}\text { Bact. plated } \\
\text { End No. bact. } \\
\text { Factor Increase }\end{array}$ & $\begin{array}{c}5.1 \times 10^{4} \\
1.7 \times 10^{6}\end{array}$ & $\begin{array}{c}5.1 \times 10^{4} \\
2.3 \times 10^{7} \\
480\end{array}$ & $\begin{array}{c}5 \cdot 1 \times 10^{6} \\
2.6 \times 10^{6} \\
5,100\end{array}$ & $\begin{array}{c}5.1 \times 10^{4} \\
2.8 \times 10^{0} \\
54,800\end{array}$ \\
\hline $\begin{array}{r}\text { Resist. colonies } \\
\text { replicatt test } 1 \\
2 \\
3 \\
4 \\
5 \\
6\end{array}$ & $\begin{array}{cl}\text { unsp. } & \text { sp. } \\
0 & 0 \\
0 & 0 \\
0 & 1 \\
0 & 0 \\
0 & 0 \\
0 & 1\end{array}$ & $\begin{array}{cl}\text { unsp. } & \text { gp. } \\
0 & 0 \\
3 & 0 \\
0 & 6 \\
2 & 0 \\
1 & 0 \\
2 & 2\end{array}$ & $\begin{array}{cr}\text { unsp. } & \text { 8p. } \\
5 & 194 \\
3 & 14 \\
4 & 16 \\
8 & 13 \\
2 & 4 \\
6 & 112\end{array}$ & $\begin{array}{cr}\text { unsp. } & \text { sp. } \\
46 & 2,254 \\
25 & 1,434 \\
45 & 3,294 \\
49 & 3,719 \\
26 & 1,538 \\
49 & 399\end{array}$ \\
\hline Total & 2 & 8 & $28 \quad 353$ & $240 \quad 12,638$ \\
\hline
\end{tabular}

- Estimated by washing and assay ; averages of three independent determinatlons. unsp. - unspread; sp. - spread.

It might be suggested that the bacteria are less likely to become 'adapted' when crowded togethor in the developing colonies. If this were true, the proportion of bacteria becoming 'adapted' should bo least where microcolony size is greatest. Table '2 shows that the average number of bacteria per microcolony at the time of spraying rose with increasing period of incubation from 33 up to 54,900 , but that thore was no corresponding decline in the proportion

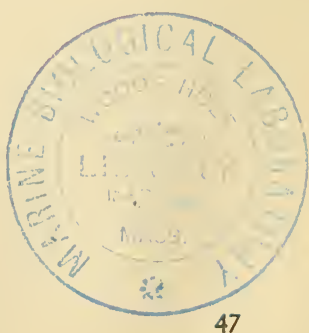


of resistant colonies to bacteria sprayed. Thus, crowding cannot account for the lower colony counts from unspread plates.

This experiment, therefore, confirms the conclusion drawn from the fluctuation test, namely, that phageresistant variants arise by spontaneous change prior to contact with phage.

Atumic Energy Project,

\section{Huward B. Newcombe}

National Research Council (Canada),

Chalk River, Ontario. Jan. 7.

${ }^{1}$ Luria, S. E., and Delbrück, M., Genetic8, 28, 491 (1943).

'Hinshelwood, C. N., "The Chemical Kinetics of the Bacterial Cell' (Oxford University Press, London, 1946).

- Demerec, M., Proc. Nat. Acad. Sci., U.S., 32, 36 (1946).

- Beale, G. H., J. Gen. Microbiol., 2, 131 (1948).

3 Newcombe, H. B., Genetics, 33, 447 (1948).

- Demerec, M., and Fano, U., Genetic8, 30, 119 (1945).

' Witkin, E. M., Genetics, 32, 221 (1947).

- Ryan, F. J. Cold Spring Harbor Symposia on Quantitative Blology, 11, 215 (1946); Proc. Nat. Acad. Sci., U.S., 34, 426 (1948).

- scott, G. W. (in preparaticn).

${ }^{10}$ Newcombe, H. B., and Hawirko, R. (in preparation).

11 Demerec, M., Proc. Nat. Acad. Sci., U.S., 31, 16 (1945); Aun. Missouri Botan. Garden, 32, 131 (1945).

13 Oakberg, E. F., and Luria, S. E., Genetics, 32, 249 (1947).

1s Demerec, M., J. Bact., 58, 63 (1948).

10 Ryan, F. J., et al., Proc. Nat. Acad. Sci., U.S., 32, 261 (1946)

${ }^{18}$ Alexander, H. E., and Leldy, G. J. Exp. Med., 85, 607 (1947).

${ }^{18}$ Curcho, M. de la G., J. Bact., 58, 374 (1948).

${ }^{17}$ Lewis, I. M., J. Bact., 28, 619 (1934).

18 Kristensen, M., Acta Path. Microbiol., Scand., 17, 193 (1940). 


\title{
DELAYED PHENOTYPIC EXPRESSION OF SPONTANEOUS MUTATIONS IN ESCHERICHIA COLI*
}

\author{
HOWARD B. NEWCOMBE ${ }^{1}$ \\ Carnegie Institution, Cold Spring Harbor, N. Y.
}

Received April 24, 1948

\section{INTRODUCTION}

$T$ HE quantitative study of mutations occurring at low rates requires an organism that can be grown conveniently in large numbers, and mutations that can be readily detected. Furthermore, where information is desired concerning the effect of a gene change shortly after its occurrence, it is necessary to be able to examine the phenotype of the organism immediately following the change and at intervals thereafter.

Bacteria are superior to higher organisms for these purposes, since (1) large populations can be handled, (2) there are numerous mutants that can be readily detected and counted, and (3) individual organisms result from each cell division, enabling the phenotype to be determined at any time after a gene change. It was the purpose of this investigation to discover the rate of spontaneous mutation of the bacterium Escherichia coli, strain $B / r$, from sensitivity to resistance to the phage $T 1$, and also, by indirect means, to determine the interval between time of occurrence of the mutation and phenotypic expression.

The reasons for investigating spontaneous mutation rate and time of phenotypic expression are as follows:

Two previously developed methods of estimating mutation rate in bacteria have yielded discrepant results (LURIA and DELBRÜCK 1943). This discrepancy has been ascribed to an error in the assumptions on which one of the methods is based; but, which assumption and which method is in error is not known.

One of the methods rests on the assumption that a gene mutation expresses itself immediately in the individual cell in which it occurs; and one of the possible interpretations of the discrepancy is that this is not the case, but that on the contrary one or more generations of growth are required before the mutation is expressed.

Delayed phenotypic expression, or "cytoplasmic lag" as it has been termed, has been observed in Paramecium (see SonNEBORN 1947); and if something of this nature occurs also in bacteria it is important from the standpoint of understanding gene action.

* The cost of the accompanying tables has been paid by the Galton and MENDEL MEMORIAL FUND.

1 Present address: Biological and Medical Research Branch, National Research Council, Atomic Energy Project, Chalk River, Ontario, Canada.

[Reprinted by permission from Genftics $33: 447-476$, September, 1948] 


\section{Methods of Previous Workers}

The early experiments of LURIA and DELBRÜCK (1943) referred to above will now be considered in detail.

To find out the number of phage-resistant mutants in a phage sensitive liquid culture, the whole culture if its population size is small, or a sample of it if it is large, is spread on agar together with the particular phage under consideration. On incubation, all bacteria that are sensitive to the phage are lysed, leaving only the resistant mutants. Each such mutant will eventually form a separate colony; and from the number of colonies the number of mutants in the liquid culture, or the sample, can be estimated.

By using this teclınique it is possible to determine the rate of mutation to phage resistance. As stated, two methods have been devised by LuRIA and DELBRÜCK (1943). The experimental procedure in both of the methods is to grow a series of similar liquid cultures from small inocula, and to determine the numbers of resistant bacteria in each, as well as in the average population. The two estimates are derived from these primary data, the first using the proportion of cultures in which resistant mutants have appeared, and the second using the average number of resistant mutants per culture.

These methods are of course only strictly applicable where it is possible to eliminate the original type without affecting the mutant type, and where there are no selective differentials between mutant and original types.

In their experiments these test cultures were started with small inocula ( 50 to 500 bacteria from a growing culture of $E$. coli, strain $B$ ) and were grown to saturation either in broth or in synthetic medium. (The volume of the cultures was $10 \mathrm{cc}$ in some experiments and $0.2 \mathrm{cc}$ in others, the final numbers of bacteria being of the order of $3 \times 10^{1 n}$ and $3 \times 10^{8}$ respectively.) At the end of growth, samples of the cultures - or in some cases whole cultures-were tested to determine the numbers of bacteria resistant to the phage $T 1$.

The cultures from which the inocula were taken contained between 1 and 1,000 resistant individuals per $10^{8}$ total bacteria. Thus, the chance of introducing a resistant bacterium in to the test cultures via the inoculum was small and in the rare event of one being introduced the fact would be indicated by an excessive proportion of resistant bacteria in the fully grown test culture. In practice, any resistant bacteria found at the time of testing-that is, after the cultures are fully grown-will therefore be the mutant offspring of one of the sensitive bacteria in the inoculum.

An estimate of mutation rate per bacterium per division cycle can be obtained if the numbers of mutations, and the average number of cell divisions, occurring in a series of cultures are known. The latter may be calculated from the final population in the series and the former from the proportion of cultures containing no mutants. The greatest accuracy is obtained when this proportion is neither too large nor too small, and the method cannot be used if every culture contains a mutant. Since the proportion of cultures having no mutants is a function of the number of cell devisions in a culture, it may be adjusted by altering the volume of medium, $0.2 \mathrm{cc}$ being the amount used 
in the experiments under consideration. To determine this proportion it is of course necessary to test whole cultures, as distinct from samples.

This method will be known as method 1 throughout the present paper. It should be noted that it is based upon an estimate of the number of resistant clones developing in the series (this estimate being obtained from the proportion of cultures in which no resistant mutants have developed), and that it takes no account of the numbers of individuals in these clones at the end of the growth. For the purpose of this paper, the term "mutant clone" will refer to those individuals carrying genetic factors for phage resistance which have a common origin in a single mutation. Within a mutant clone individuals which are phenotypically resistant to phage will be collectively termed a "resistant clone." A culture may contain one or more mutant clones of varying age.

The second method of LURIA and DELBRÜCK uses the average number of resistant bacteria in a series of similar cultures and calculates mutation rate from this value, the average population, and the number of cultures. The number of mutants arising during the growth of a culture is of course, on the average, a function of the mutation rate. But there are very large variations in the number of mutants present in different cultures grown under identical conditions, these being due to chance variations in the time of occurrence of the mutations. Thus the occasional occurrence of a mutation early in the growth of a culture, at a time when the population is small, will result in a much higher than average number of mutants in that culture. Because of these statistical fluctuations, mutation rate cannot be calculated from the number of mutants in a single culture started from a small inoculum. It can, however, be estimated from the average number of mutants in a series of similar cultures; and the mathematical details of the method have been worked out by LURIA and DELBRÜCK (1943). This method will be known as method 2 throughout the present paper. It differs from method 1, which utilizes the number of resistant clones occurring in liquid cultures, in that it takes into consideration the number of resistant individuals. Furthermore, in the event of any change in the mutation rate during growth, method 2 would give an average of the mutation rates obtaining in each of the generations during which mutations had occurred-equal weight being given to the early generations when the population and the number of mutations occurring were small, and to the later generations when both these values had increased. In contrast to this, in method 1 changes in the mutation rate during growth would give an estimate strongly biased in favor of the rate obtaining during the later period when the population and the absolute number of mutations occurring was large. Moreover, a delay in the phenotypic appearance of a mutation would reduce the rate as estimated by method 1 , because recent mutations would not be detectable. The rate as estimated by method 2 would be affected less, since early mutations, which have a greater number of generations in which to become phenotypically resistant, are represented by larger numbers of descendants than are the later mutations. The possibility that mutation rate is 
not constant throughout growth, and the possibility that phenotypic resistance does not appear for one or more generations after mutation to resistance has taken place, will now be considered.

A striking and unexpected finding of the LURIA and DELBRÜCK experiments was that the rates estimated by these two methods differed by a considerable factor, that from method 1 (utilizing the number of resistant clones developing in liquid cultures) being lower than that from method 2 (utilizing the average number of resistant individuals per culture). The averages of their estimates are $.32 \times 10^{-8}$ and $2.4 \times 10^{-8}$ per bacterium per division cycle, respectively. This difference has since been confirmed by I)EMEREC and Fano (1945), who have in addition shown that it is not peculiar to experiments using $T 1$ but is also true of rates of mutation to resistance to other phages (T3, T4, T5, T6, and T7) when estimated by methods 1 and 2 .

A statistical bias in method 2 (which gives the high estimate) may contribute to the discrepancy, but the work of LURIA and DELBRÜCK suggests that its contribution is small. (For a discussion of this point the reader is referred to the original publication. Also, a method of estimating mutation rate, which avoids this source of error will be considered in a later section of the present paper.)

Since the two methods will give the same estimate if, and only if, (1) the rate of mutation is constant throughout growth and (2) the occurrence of a mutation gives rise to a phenotypic mutant without delay, it was concluded that one of these two conditions did not obtain. There was no critical evidence to indicate which one, however, since the discrepant estimates could be explained by assuming either a high mutation rate during the greater part of the growth period, dropping during the last few divisions, or a delay of one or more generations between mutation and phenotypic expression.

LURIA and DELBRÜCK did not favor the latter interpretation, since a fixed delay of one or more generations before the development of phenotypic resistance would mean that mutant clones would number two or more individuals at the time when phenotypic resistance appeared. It was therefore assumed that cultures with just one resistant individual would be rare if there were a delay; and these, instead of being rare, had been observed in considerable numbers (see LURIA 1946). It has been pointed out, on the other hand, that if some lines of descent within the clone were to develop resistance earlier than others, mutation plus delay could give rise to cultures having only one phenotypically resistant individual (SONNEBORN 1946). Thus the assumption of a delay is permissible provided it is also assumed that phenotypic expression is earlier in some lines of descent than in others within the same mutant clone.

Two alternative possibilities therefore exist: (1) that of a relatively high mutation rate during all but the last few generations, and (2) that of a variable delay in phenotypic expression. The possible significance of the second of these two alternatives should be considered. If there is a delay in phenotypic expression, then some cultures which showed no phenotypically resistant bacteria would contain mutants that could not be detected. Also, the end number of resistant bacteria in a culture would represent only part of the genetic mutants 
present, some not having developed resistance by the time growth stopped. Thus the mutation rates calculated by methods 1 and 2 would both be underestimated, and the extent of the underestimate would depend upon the magnitude of the delay.

The information available so far sets no limit on the suspected delay or its variability within clones of mutants, and it is even possible that an extreme situation exists in which both are considerable. If this is true, mutation rate is greatly underestimated by the methods outlined.

It should be noted at this point that a delay similar to that suspected in the case of spontaneous mutations does in fact occur in irradiated material (Demerec 1946; and Demerec and Latarjet 1946). Although there is at present no certainty that spontaneous and induced mutations behave in precisely the same manner, it is of interest to consider the nature of the delay in the one case in which it has been established, that is, in induced mutations.

To determine the time of appearance of induced mutations, Demerec irradiated bacteria in liquid suspension, using ultraviolet radiation in some experiments and X-rays in others. These treated bacteria were spread on agar, incubated for varying periods of time to permit cell reproduction, sprayed with phage $T 1$, and incubated again until colonies appeared. The spraying caused all susceptible bacteria to be infected and lysed; but where a mutation to resistance had occurred and had been expressed phenotypically, the resulting bacteria continued to grow after phaging, giving rise to one visible colony for each such mutation.

Irradiation caused an enormous increase in the number of resistant clones that appeared during growth, over the number that appeared during the same number of generations in untreated bacteria. The delay between irradiation and phenotypic change was such that less than one percent of the induced mutants appeared prior to the first division, 50 percent appeared after about five divisions, and some did not appear until after 11 or 12 divisions.

These observations give some support to the possibility that there is also a delay in the expression of spontaneous mutations.

The experiments described here were designed to distinguish between this and the alternative possibility of a change in the rate of mutation during growth. They show that there is in fact a delay, and that it is the cause of the discrepant estimates of rate obtained by using the LURIA and DEBRÜCK methods 1 and 2. Some indication of the extent and variability of the delay has been sought, and an attempt has been made to obtain a more accurate estimate of mutation rate.

\section{MATERIALS}

The bacterium Escherichia coli strain $B / r$ was used in these investigations. This is a mutant derived from strain $B$ (WITKIN 1946, 1947), and is more resistant than $B$ to the action of radiations.

The mutations studied are those resulting in resistance to phage $T 1$. There are at least two different categories of mutant: those resistant to $T 1$ but not to any other of the known phages, and those resistant to phages $T 1$ and 
T5. These mutant categories are designated $B / r / 1$ and $B / r / 1,5$ respectively. Within each there occurs a number of morphologically distinguishable colony forms, and it is possible that these represent a number of mutations of dissimilar origin; but in this study no attempt has been made to distinguish between the various types of mutation that give rise to resistance to $T 1$.

DISCREPANT ESTIMATES OF MUTATION RATE FROM NUMBERS OF RESISTANT CLONES (METHOD 1) AND OF RESISTANT INDIVIDUALS (METHOD 2)

In view of the possibility that $B / r$ may $\operatorname{differ}$ from $B$ in the rate with which it mutates, rates for $B / r$ were determined by each of the LURIA and DELBRÜCK methods, using phage $T 1$.

Eight separate experiments were carried out, and for each experiment 25 broth cultures of $0.2 \mathrm{cc}$ were grown. Small inocula were used, and the cultures were incubated for 18 hours, by which time growth had stopped.

The inocula contained approximately ten bacteria per culture in four of the experiments, and approximately $10^{4}$ in the other four. These numbers were small enough so that the chance carry-over of a mutant in the inoculum would be readily detected.

Method 1 was used to calculate mutation rate, a, from the proportion of cultures having no resistant bacteria, $\mathrm{P}_{0}$, and the average number of bacteria at the end of growth, $\mathrm{N}$, using the formula:

$$
\mathrm{a}=-(\ln 2)\left(\ln \mathrm{P}_{0}\right) / \mathrm{N} \text {. }
$$

The above formula is derived from formulas (4) and (5) of LuRIA and DeLBRÜCK (1943), ln being the natural logarithm.

Method 2 was used to calculate mutation rate, a, from the average number of resistant bacteria per culture, $r$, the average number of bacteria at the end of growth, $\mathrm{N}$, and the number of cultures, $\mathrm{C}$, using the formula:

$$
\mathrm{r}=(\mathrm{aN} / \ln 2) \ln (\mathrm{CaN} / \ln 2) \text {. }
$$

This is derived from formula (8) of LuRIA and DELBRÜCK.

The natural logarithm of 2 appears in these formulas because the mutation rate refers to the rate per bacterium per division cycle, as distinct from the rate per bacterial division. The significance of this distinction is best visualized by using a concrete example. If a population of $10^{8}$ bacteria passes through one division cycle and one mutation takes place, the number of bacterial divisions is $10^{8}$ and the mutation rate per bacterial division is $1 \times 10^{-8}$. The mean population throughout the cycle, however, is $10^{8} / \ln 2$, so that the rate per bacterium per division cycle is $\ln 2 \times 10^{-8}$, which is $.693 \times 10^{-8}$.

The first of these two methods of expressing mutation rate would be applicable if mutation took place only at the time of cell division, and affected just one of the offspring. The second would be applicable if mutability were continuous throughout the division cycle. In the absence of information on this point the choice is arbitrary, and since the latter method has been used by previous authors its use is continued in this paper to facilitate comparisons. 
These considerations are of course based on the assumption that each bacterium divides, an assumption which will be discussed later in the paper.

Methods 1 and 2 have been used with strain $B / r$ in order to determine (a) whether, as with strain $B$, estimates obtained by method 1 are lower than those obtained by method 2, and (b) whether the estimates from these two methods are the same for $B / r$ as for $B$.

The data from these experiments and the estimated mutation rates are given in table 1 . Those obtained using method 1 average $.40 \times 10^{-8}$ and, those using method 2 average $3.6 \times 10^{-8}$.

It will be seen from table 9 -in which the results of previous workers, using strain $B$, have been quoted-that the discrepancy between the estimates of mutation rate given by the two methods as applied to $B / r$ is similar to the discrepancy using strain $B$. It is also evident that the estimate of mutation rate for strains $B / r$ and $B$ are similar.

\section{THE ELIMINATION OF A POSSIBLE UPWARD BIAS IN METHOD 2 BY THE USE OF LARGE INOCULA (METHOD 3)}

The formula for calculating mutation rate from the average number of mutants per culture (method 2) disregards the early divisions, when the population is small and it is unlikely that a mutation will occur. The divisions which enter into the calculation are those occurring after an arbitrary time, this time being chosen so that on the average one mutation will occur prior to it in the whole series of cultures. LURIA and DELBRÜCK point out that the chance occurrence of this early mutation might account for part of the discrepancy between the estimates of rate obtained with the two methods. For a detailed discussion of this point the reader is referred to their paper.

It was therefore necessary to arrive at an estimate which, like that obtained by method 2, would utilize the number of resistant bacteria arising during growth in liquid culture, but which would not be biased by the chance occurrence of early mutations. This was done by growing the test cultures from inocula of sufficient size to ensure that an appreciable number of mutations would take place during the first division. Since much of the statistical fluctuation in end numbers of resistant bacteria is thus eliminated, mutation rates may be estimated from single cultures. (An experiment similar to this has been proposed by SHAPIRO 1946.)

The method can be used only if the proportion of resistant bacteria in the inoculum is small, since otherwise the relatively small increase due to mutation during growth could not be accurately determined. To serve as inocula, therefore, cultures containing very small proportions of resistant bacteria were chosen.

Five $50 \mathrm{cc}$ and five $300 \mathrm{cc}$ aerated cultures were grown from inocula of $2.6 \times 10^{9}$ and $2.1 \times 10^{8}$ bacteria, respectively. Synthetic medium, the M-9 of ANDERSon (1946), was used because the bacteria can be grown to a higher number per unit volume in it than in broth. In two scparate sets of experiments growth resulted in increases in the numbers of individuals of approximately a hundredfold and three thousandfold, respectively. 


\section{TABLE 1}

Estimates of mutation rate of $B / r$ to resistance to phage $T 1$, using the methods of LURIA and DELBRUCK (1943) (methods 1 and 2 in the present paper), calculating from the number of cultures with no resistant bacteria, and from the average number of resistant bacteria per culture, respectively, in series of similar cultures started from small inocula.

\begin{tabular}{lcccccccc}
\hline \multicolumn{1}{c}{ EXPERIMENT } & A & B & C & D & E & F & G & H \\
\hline Inoculum (no. of bact.) & 10 & 10 & 10 & 10 & $10^{4}$ & $10^{4}$ & $10^{4}$ & $10^{4}$ \\
Number of cultures & 25 & 25 & 25 & 25 & 25 & 25 & 25 & 25 \\
Vol. of cultures, cc & 0.2 & 0.2 & 0.2 & 0.2 & 0.2 & 0.2 & 0.2 & 0.2 \\
\hline
\end{tabular}

Culture Number

Number of Resistant Bacteria

\begin{tabular}{rrrrrrrrr}
1 & 140 & 2 & 2 & 1 & 1 & 1 & 134 & 4 \\
2 & 0 & 41 & 57 & 5 & 9 & 21 & 18 & 1 \\
3 & 0 & 45 & 1 & 1 & 2 & 34 & 34 & 0 \\
4 & 43 & 11 & 6 & 4 & 0 & 287 & 380 & 6 \\
5 & 60 & 8 & 1 & 4 & 242 & 46 & 3 & 149 \\
6 & 13 & 1 & 1 & 71 & 2 & 33 & 15 & 176 \\
7 & 48 & 30 & 1 & 5 & 12 & 23 & 2 & 31 \\
8 & 3 & 1 & 2 & 12 & 0 & 3 & 6 & 1 \\
9 & 4 & 1 & 14 & 1 & 2 & 102 & 4 & 0 \\
10 & 36 & 0 & 0 & 1 & 2 & 84 & 2 & 48 \\
11 & 55 & 11 & 3 & 49 & 11 & 148 & 5 & 0 \\
12 & 447 & 0 & 139 & 9 & 29 & 0 & 18 & 45 \\
13 & 1 & 1 & 40 & 0 & 17 & 120 & 0 & 3 \\
14 & 9 & 7 & 142 & 0 & 42 & 10 & 33 & 2 \\
15 & 27 & 1 & 1 & 1 & 7 & 4 & 725 & 134 \\
16 & 14 & 4 & 154 & 1 & 15 & 0 & 1 & 25 \\
17 & 30 & 6 & 3 & 60 & 14 & 0 & 5 & 34 \\
18 & 160 & 15 & 4 & 37 & 45 & 13 & 0 & 14 \\
19 & 231 & 9 & 1 & 1 & 110 & 0 & 0 & 131 \\
20 & 35 & 0 & 158 & 7 & 32 & 376 & 118 & 22 \\
21 & 37 & 1 & 5 & 44 & 0 & 1 & 1 & 60 \\
22 & 0 & 21 & 1 & 18 & 32 & 133 & 15 & 0 \\
23 & 1 & 0 & 0 & 0 & 8 & 4 & 0 & 158 \\
24 & 8 & 27 & 2 & 12 & 36 & 42 & 151 & 6 \\
25 & 3 & 221 & 0 & 79 & 0 & 4 & 9 & 263 \\
\hline
\end{tabular}

Method 1

Cult. with no resist. $b$.

Bact. per cult., $\times 10^{8}$

$\begin{array}{llllllll}3 & 4 & 3 & 3 & 4 & 4 & 4 & 4 \\ 3.1 & 4.6 & 2.5 & 2.8 & 4.2 & 3.7 & 3.2 & 3.8 \\ .42 & .28 & .59 & .53 & .31 & .34 & .40 & .33\end{array}$

Mutation rate, $\times 10^{-8}$

Method 2

Av. resist. b. per cult.

Mutation rate, $\times 10^{-8}$

$\begin{array}{lll}56.1 & 18.5 & 29.5\end{array}$

16.9

26.

$\begin{array}{lll}59.5 & 68.6 & 52.6\end{array}$

$\begin{array}{llllllll}5.1 & 1.5 & 3.8 & 2.1 & 2.1 & 4.5 & 5.8 & 3.9\end{array}$

Assays were made of total bacteria and of numbers of resistant individuals in the inocula and in the fully grown test cultures. These four values are designated $N_{1}, r_{1}, N_{2}$ and $r_{2}$, respectively. For the sake of accuracy, five or ten independent assays were made in each case. The number of generations of growth, $g$, was determined for each of the cultures from the values $N_{1}$ and $N_{2}$. 
Where the mutation rate per bacterium per division cycle is a, and the rate per bacterial division is a/(ln 2), the proportion of mutant bacteria in a culture will rise during growth by a fixed increment of a/2(ln2) per generation, provided the inoculum is of sufficient size so that there are no appreciable statistical fluctuations in the numbers of mutations occurring in the first division. Thus mutation rate can be obtained from the formula

$$
a=2(\ln 2)\left(r_{2} / N_{2}-r_{1} / N_{1}\right) / g \text {. }
$$

TABLE 2

Mutation rates of $B / r$ to resistance to phage $T 1$, calculated from the increase in the proportion of resistant bacteria in cultures grown from large inocula (method 3 of this paper). Short grouth period.

\begin{tabular}{|c|c|c|c|c|c|c|}
\hline TEST CULTURE & A & B & $\mathrm{C}$ & $\mathrm{D}$ & $\mathrm{E}$ & INOCULUM \\
\hline Replicate assays & 10 & 10 & 10 & 10 & 10 & 10 \\
\hline Inoculum, bact., $\times 10^{8}$ & 26 & 26 & 26 & 26 & 26 & - \\
\hline Vol. of culture, cc & 50 & 50 & 50 & 50 & 50 & - \\
\hline Incr. in no. of bact. & $114 \times$ & $75 \times$ & $112 \times$ & $135 \times$ & $46 \times$ & - \\
\hline Resist. bact., $\times 10^{-8}$ & 19.6 & 20.8 & 24.4 & 20.9 & 15.4 & 7.0 \\
\hline Standard deviation & 3.7 & 4.7 & 2.6 & 3.5 & 2.7 & 1.3 \\
\hline Generations growth & 6.8 & 6.2 & 6.8 & 7.1 & 5.5 & - \\
\hline Incr. resist. b., $\times 10^{-8}$ & 12.6 & 13.8 & 17.4 & 13.9 & 8.5 & - \\
\hline Mutation rate, $\times 10^{-8}$ & 2.6 & 3.1 & 3.5 & 2.7 & 2.1 & - \\
\hline
\end{tabular}

TABLE 3

Mutation rates of $B / r$ to resistance to phage $T 1$, calculated from the increase in the proportion of resistant bacteria in cultures grown from large inocula (method 3 of this paper). Long growth period.

\begin{tabular}{|c|c|c|c|c|c|c|}
\hline TEST CULTURE & $\mathrm{F}$ & G & $\mathrm{H}$ & I & $\mathrm{J}$ & INOCULUM \\
\hline Replicate assays & 5 & 5 & 5 & 5 & 5 & 10 \\
\hline Inoculum, bact., $\times 10^{8}$ & 2.1 & 2.1 & 2.1 & 2.1 & 2.1 & - \\
\hline Vol. of culture, cc & 300 & 300 & 300 & 300 & 300 & - \\
\hline Incr. in no. of bact. & $3600 \times$ & $2910 \times$ & $2780 \times$ & $2770 \times$ & $3470 \times$ & - \\
\hline Resist. bact. $\times 10^{-8}$ & 37.3 & 30.3 & 298 & 42.4 & 24.7 & 4.4 \\
\hline Standard deviation & 6.9 & 3.9 & 3.5 & 6.2 & 5.6 & 1.1 \\
\hline Generations growth & 11.8 & 11.5 & 11.5 & 11.5 & 11.8 & - \\
\hline Incr, resist, b, $\times 10^{-8}$ & 32.9 & 25.9 & 25.4 & 38.0 & 20.3 & - \\
\hline Mutation rate, $\times 10^{-8}$ & 3.9 & 3.1 & 3.0 & 4.6 & 2.4 & - \\
\hline
\end{tabular}

The data from two sets of experiments (involving 6 generations and 11 generations of growth) are given in tables 2 and 3, together with the mutation rates obtained. These rates average $2.8 \times 10^{-8}$ and $3.4 \times 10^{-8}$, respectively-values which are not appreciably different from each other, or from those obtained by method 2, using the average number of resistant bacteria per culture in cultures started with small inocula.

Since there is no question of a statistical bias in calculating mutation rate by method 3 , the agreement may be interpreted as confirming the higher 
value obtained by means of method 2 . This is important, inasmuch as there has been no estimate of the magnitude of the bias in method 2, or of the extent to which the discrepancy between methods 1 and 2 is a product of it.

The above experiments demonstrate that this discrepancy is due to biological rather than to statistical causes.

There are two reasons for measuring mutation rate over periods of 6 and of 11 generations. First, had it been measured over the shorter period only, it would be possible for the results to be biased by a high mutation rate during the first generation or so. The obtaining of similar estimates of rate from both a short and a long period, however, eliminates this as an appreciable source of error.

Second, formula (3) assumes that the mutants multiply at the same rate as the parent strain. That this assumption is approximately true is indicated by certain experiments of of LURIA and DELBRÜCK and later of DEMEREC and FANo. Somewhat more critical evidence however is obtainable from experiments such as the above in which close agreement between estimates of mutation rate from short and from long periods of growth indicate that there is no appreciable bias arising from differential increase during logarithmic growth. A mathematical demonstration of this will be found in a paper by SHAPIRo (1946).

For present purposes it is sufficient to state the argument in general terms. From formula (3) it is evident that mutation acting alone causes the proportion of mutants $\mathrm{r} / \mathrm{N}$ to rise arithmetically with each successive generation. If, however, the mutants were to increase more (or less) rapidly than the parent strain there would be superimposed upon this an exponential increase (or decrease) in $\mathrm{r} / \mathrm{N}$, and estimates of rate obtained using formula (3) would tend to rise (or fall) correspondingly with increasing periods of exponential growth. Thus it is clear that close agreement between estimates of mutation rate over periods of 6 and 11 generations constitutes evidence that the high estimates obtained using methods 2 and 3 are not the result of a differential favoring the mutant during periods of logarithmic growth.

It would be quite possible to supplement the above evidence on the relative rates of increase of mutant and non-mutant strains, by preparing mixed cultures and determining the change in proportion which takes place as the result of competitive growth, as in the above mentioned experiments of LURIA and DelbrüCK, and Demerec and FAno. However the results of such experiments are not entirely critical in the case of the spontaneously occurring mutants, since the mutant strain must first be selected by growing in the presence of phage, and then must be freed from all phage particles by suspending a resistant colony in liquid, streaking the suspension on agar, and incubating until visible colonies are formed, the process being repeated a number of times. Such prolonged growth offers considerable opportunity for further mutation and selection, and it is impossible to be certain that the strain which is finally obtained will not have changed with regard to its ability to compete with the non-mutant.

This difficulty may be avoided, however, by the use of radiation induced 
mutants of a similar kind, since these occur much more frequently, and cultures containing mutants and non-mutants in suitable proportions for competitive growth experiments can be obtained without the necessity for first isolating the mutant strain. Evidence has been obtained in this manner in connection with a separate study (NEwcombe and ScotT) which will be published later. Six independent mutant clones were tested over prolonged periods of growth (18 to 20 generations) in competition with the corresponding non-mutant strain. In some cases the proportion of mutants was found to remain unchanged and in others to have declined slightly, the factors of change ranging from $1.20 \pm 40$ down to $0.16 \pm .05$. In no case did the proportion of mutants increase significantly during growth.

Thus, provided one assumes that the radiation induced mutants are identical to those occurring spontaneously, evidence from this source is in agreement with the above conclusion that the high estimates of mutation rate from methods 2 and 3 cannot be due to a differential favoring the mutants during the period of rapid growth.

These experiments do not eliminate, however, the possibility that during the approach to saturation, when the environment has altered considerably, there may be conditions favoring the mutant strain. An increase in the proportion of mutants during this period of approximately six times, would be sufficient to produce the observed high estimates of mutation rate arrived at from the numbers of individual resistant bacteria.

In order to test this possibility, an experiment was designed in which the conditions associated with the approach to saturation could act repeatedly on a bacterial population passing through approximately five to six generations, thus accentuating any selection.

Five replicate test cultures, each containing in the first instance one cc of broth, were incubated over a period of three days, during which time the amount of liquid medium was doubled at regular intervals and finally brought up to fifty cc. If the approach to saturation in the test cultures used in connection with methods 2 and 3 is accompanied by a six-fold increase in the proportion of mutants, one would expect from the above treatment an increase of much more than six times, in addition to any mutants which arose from spontaneous mutation. Also, estimates of mutation rate obtained from these cultures using method 3 , should be very much greater than the corresponding estimates from normal test cultures.

Such estimates of mutation rate from the above experiment are presented in table 4 . It will be seen that these are not appreciably increased by keeping the cultures under conditions approaching saturation throughout the whole of the growth period.

In evaluating these data it will be noted that all assays are of numbers of viable bacteria, and that if there is appreciable death due to the conditions of growth, the number of cell generations will be underestimated. This problem is considered in detail in a later section, where it will be shown that the effect of undetected cell death is to increase the estimate of mutation rate obtained, to a value above that of the true rate. Thus the experiment is weighted against 
the argument, and the results can be considered as critical evidence that there are no appreciable differentials favoring the mutants during the latter part of the growth of a culture.

The conclusions from the experiments discussed above are applicable to differentials, both of division and of survival, and eliminate all possibility that the widely different estimates of mutation rate obtained using method 1

TABLE 4

Mutation rates of $B / r$ to resistance to phage $T 1$, calculated from the increase in the proportions of resistant bacteria in cultures grown from large inocula (method 3 of this paper). Entire growth under conditions approaching saturation.

\begin{tabular}{lcccccc}
\hline \multicolumn{1}{c}{ TEST CULTURE } & $\mathrm{K}$ & $\mathrm{L}$ & $\mathrm{M}$ & $\mathrm{N}$ & $\mathrm{O}$ & INOCULUM \\
\hline Replicate assays & \multicolumn{1}{c}{5} & 5 & \multicolumn{1}{c}{5} & \multicolumn{1}{c}{5} & 5 & 5 \\
Inoculum, bact., $\times 10^{8}$ & 29.1 & 29.1 & 29.1 & 29.1 & 29.1 & - \\
Initial vol. of cult., cc & 1 & 1 & 1 & 1 & 1 & - \\
Final vol. of cult., cc & 50 & 50 & 50 & 50 & 50 & - \\
Incr. in no. of bact. & $33 \times$ & $24 \times$ & $48 \times$ & $60 \times$ & $46 \times$ & - \\
Resist. bact., $\times 10^{-8}$ & 15.9 & 15.5 & 17.2 & 18.7 & 21.6 & 5.0 \\
Standard deviation & 2.0 & 2.9 & 1.3 & 1.2 & 1.8 & 0.7 \\
\hline Generations growth & 5.1 & 4.6 & 5.6 & 5.9 & 5.5 & - \\
Incr. resist. b., $\times 10^{-8}$ & 10.9 & 10.5 & 12.2 & 13.7 & 16.6 & - \\
Mutation rate, $\times 10^{-8}$ & 4.3 & 4.6 & 4.4 & 4.6 & 6.5 & - \\
\hline
\end{tabular}

on the one hand, and methods 2 and 3 on the other, are due to such differentials.

Method 3 thus provides critical confirmation of the estimates obtained by method 2 .

VARIATIONS IN MUTATION RATE WHICH WOULD BE REQUIRFD TO EXPLAIN THE DISCREPANT ESTIMATES FROM METHODS 1 AND 2

It thus seems certain that the high estimates of mutation rate from the numbers of resistant individuals are not the product of an upward bias, and we may now turn to the possibility that the low estimates from the numbers of resistant clones are due to a downward bias. Two alternative possibilities have been suggested which would account for these lower values: (1) a change to a low rate of mutation during the later part of growth, and (2) a delay in the phenotypic expression of a mutation.

The problem of distinguishing between these will be simplified if we consider the first and determine the time at which the supposed transition would have to occur, and whether this time is related to the number of generations from resting stage or to the approach to saturation.

To determine whether the supposed transition would be a function of the number of generations from resting stage the data of table 1 may be used. Half the cultures were inoculated with 10 and half with 10,000 bacteria, and 
these passed through 25 and 15 generations respectively (the relevant data are given in table 5). The mutation rates calculated by methods 1 and 2

TABLE 5

Generations between resting stage and the approximate time of the first mutation in series of liquid cultures started from widely different inocula showing that mutation rate is independent of this variable over the range of 6 to 18 generations. (Data from table 1.)

\begin{tabular}{lcccccccc}
\hline \multicolumn{1}{c}{ EXPERIMENT } & $\mathrm{A}$ & $\mathrm{B}$ & $\mathrm{C}$ & $\mathrm{D}$ & $\mathrm{E}$ & $\mathrm{F}$ & $\mathrm{G}$ & $\mathrm{H}$ \\
\hline Inoculum (no. of bact.) & 10 & 10 & 10 & 10 & $10^{4}$ & $10^{4}$ & $10^{4}$ & $10^{4}$ \\
End no. of bact. $\times 10^{8}$ & 3.1 & 4.6 & 2.4 & 2.8 & 4.2 & 3.7 & 3.2 & 3.8 \\
Generations growth & 24.9 & 25.5 & 24.6 & 24.7 & 15.4 & 15.2 & 15.0 & 15.8 \\
\hline Max. resist. b. in series & 447 & 221 & 158 & 79 & 242 & 376 & 725 & 263 \\
Gen. after first mutation & 8.8 & 7.8 & 7.3 & 6.4 & 8.0 & 8.6 & 9.5 & 8.1 \\
\hline Gen. to first mutation & 16.1 & 17.7 & 17.3 & 18.3 & 7.4 & 6.6 & 5.5 & 7.7 \\
Mut. rate (method 1), $\times 10^{-8}$ & .42 & .28 & .59 & .53 & .31 & .34 & .40 & .33 \\
Mut. rate (method 2), $\times 10^{-8}$ & 5.1 & 1.5 & 3.8 & 2.1 & 2.1 & 4.5 & 5.8 & 3.9 \\
\hline
\end{tabular}

showed no effect due to the difference in number of generations from resting stage. Thus the time of the supposed transitions would have to be a function of the approach to saturation, and not of the number of generations from resting stage. This means that mutation rate would have to be of the order of $3 \times 10^{-8}$ during the whole of the logarithmic growth phase, dropping to something like $.4 \times 10^{-8}$ during the last few divisions.

It will be shown later that there are apparent variations in mutation rate during early growth, but that they are associated with the first few divisions after resting stage and occur too early to have any bearing on the immediate problem.

It is thus evident that the possible effect of a low mutation rate during the later part of growth can be eliminated by confining one's tests to the period of rapid growth; and critical evidence for or against a possible delay can be obtained from estimates of mutation rate based on the number of resistant clones appearing during rapid growth.

\section{EVIDENCE FOR A DELAY BETWEEN MUTATION AND PHENOTYPIC EXPRESSION (METHOD 4)}

It now remains to determine the rate of appearance of resistant clones during rapid growth, when no approach to saturation is involved.

If the rate of appearance of resistant clones is high $\left(3 \times 10^{-8}\right)$, the discrepancy between methods 1 and 2 can be interpreted without assuming a delay between mutation and phenotypic expression. If, on the other hand, rate of appearance of resistant clones is low $\left(.4 \times 10^{-8}\right)$, then from the previous evidence the number of individuals in an average resistant clone must be greater than expected on the basis of the number of generations passed through after 
its first appearance. This would be interpreted as indicating that the mutant clone had its origin one or more divisions prior to its becoming phenotypically detectable.

One obvious alternative to this interpretation should be mentioned, namely that the excess numbers of mutants are due to more rapid division in these than in the parent strain. This is rendered unlikely however by the evidence of DEMEREC and FANo (1945) that these mutants do not divide more rapidly, and in addition more critical evidence against the possibility has been obtained from the experiments described under method 3.

The experimental procedure was essentially that used by Demerec (1946) in his work on mutation rates in E. coli following irradiation. Bacteria are grown on agar for varying periods of time, sprayed with phage, and incubated until colonies appear. Mutations occurring during growth, and gaining phenotypic expression, will give rise to resistant clones. Since individual bacteria cannot move about on the agar, the members of a clone are confined to a particular locality. These resistant clones survive the application of phage, and eventually form colonies of visible size. Thus each mutation which gives rise to phage resistance is in the end represented by one colony.

An estimate of mutation rate is obtained by dividing the number of resistant clones appearing in a given period by the number of bacterial divisions times $1 /$ ln 2. Thus, if $R_{1}$ and $R_{2}$ are the numbers of resistant clones present at times 1 and 2 , the number of resistant clones arising during the interval between times 1 and 2 is $R_{2}-R_{1}$. Similarly, if $N_{1}$ and $N_{2}$ are the numbers of bacteria present at times 1 and 2 respectively, the increase during the interval will be $\mathrm{N}_{2}-\mathrm{N}_{1}$. Since each division of a bacterium increases the total number by one, this value is equal to the number of bacterial divisions during the period. Mutation rate per bacterium per division cycle, a, will therefore be obtained from the formula:

$$
a=(\ln 2)\left(R_{2}-R_{1}\right) /\left(N_{2}-N_{1}\right) .
$$

Since the values of $R_{1}, R_{2}, N_{1}$ and $N_{2}$ represent viable cells only, an assumption is involved, namely that all bacteria divide. It will be shown later that this assumption is approximately correct for the early stages of logarithmic growth, and it is assumed that no appreciable increase in the propertion of cells which fail to divide, takes place untii the phase of declining growth rate is approached. In these experiments precautions were taken to ensure that growth is limited to the period of exponential increase.

It will also be shown that the effect of the presence of cells which do not divide further, will be to increase the estimated rate of mutation. The present experiments can therefore be considered critical if the estimates of mutation rate obtained using method 4 are found to be low relative to those obtained using methods 2 and 3 .

Estimates of the values of $R_{1}, R_{2}, N_{1}$, and $N_{2}$ are obtained as follows. Four plates are inoculated with a suitable number of bacteria, two being incubated until time 1 and two until time 2. One plate from each incubation period is sprayed with phage and then incubated further. The numbers of colonies 
developing on these plates represent the numbers of resistant clones present at the time of spraying, that is, $R_{1}$ and $R_{2}$, respectively. The remaining plate from each incubation period is washed with ten cc of normal saline and the numbers of bacteria present $\left(\mathrm{N}_{1}\right.$ and $\mathrm{N}_{2}$, respectively) determined by colony counts.

Where time 1 is the time of plating the bacteria, and no divisions can have taken place, $\mathrm{N}_{1}$ is determined in a more direct manner. Instead of plating and then washing off the bacteria plated, an equivalent quantity of the culture from which the inoculum was taken is diluted and colony counts made.

An estimate of mutation rate is thus obtained from four plates. In all experiments these four plates were replicated several times, and a corresponding number of independent estimates of rate obtained. These independent estimates have been averaged, and the standard deviations calculated.

In all experiments in which growth was determined by the use of duplicate plates, care was taken to ensure the same amount of growth on both plates. All plates were warmed in the incubator before plating the bacteria, and when removed for plating were kept warm on a thermostatically controlled warm table until returned to the incubator. All platings and removals from the incubator followed an accurately timed schedule. The temperature in the incubator was kept as uniform as possible by circulating the air rapidly with fans. At the end of incubation, growth was stopped abruptly by chilling plates in contact with the cold metal of a refrigerator freezing unit. This chilling did not affect the survival of the bacteria or the phenotypic expression of the mutants.

Additional precautions were required with respect to (1) the choice of cultures from which to inoculate the plates, (2) the number of bacteria plated, and (3) the amount of phage applied by spraying.

(1) When relatively large numbers (of the order of $10^{8}$ ) of bacteria are plated, there will be a certain number of resistant cells in the inoculum. These resistant cells have occurred by mutation during the growth of the culture from which the inoculum is taken. Cultures vary widely in the number of mutants present at the end of growth, and in these experiments the number present in the inoculum $\left(R_{1}\right)$ was determined by spraying with phage immediately after the bacteria had been plated. Where the number is excessive it is apt to obscure the increase in resistant clones resulting from growth, or to render the determination of the increase less accurate. For this reason, cultures having an excessive number of resistant cells were not used as inocula. The cultures that were used contained from 5 to 50 resistant bacteria per $10^{8}$ sensitive.

There is no evidence that this selection biased the results, since mutation rates obtained using these inocula were the same regardless of the number of resistant bacteria present.

(2) The size of the inoculum was adjusted so that the end number of bacteria on the plate would be approximately $2 \times 10^{9}$. With end numbers of less than this the number of resistant colonies was reduced, and at the same time the accuracy of the method. With excessively large end numbers of bacteria there is a reduction in the apparent mutation rate. The precise interpretation 
of the phenomenon is uncertain. In the absence of evidence to the contrary it has been assumed that mutations do occur at the normal rate, but that they fail to develop visible colonies owing to the presence of large numbers of sensitive bacteria that are not lysed by the phage - a situation which occurs if phage is applied after bacterial growth has passed the logarithmic phase.

Tests showed that this apparent reduction in mutation rate occurred only if the end number of bacteria exceeded $5 \times 10^{9}$.

(3) It is known that bacteria which are infected during rapid growth have a latent period of 13 minutes - at the end of which time they burst, liberating on the average 180 phage particles (DELBRÜCK and LURIA 1942). If some of the bacteria fail to be infected at the time of spraying, it is unlikely that they will escape infection once lysis of the others starts. Any uninfected individuals would, on the average, pass through somewhat less than one division during the 13-minute latent period. Thus, with a large proportion of the bacteria uninfected at the time of spraying, a somewhat less than twofold increase in population would be expected before all the bacteria became infected, and the apparent mutation rate from such an experiment would be increased proportionally. Where all but a small proportion of the bacteria are infected at the time of spraying, the apparent mutation rate would not be appreciably greater than in the case of 100 percent infection.

In the present experiments the number of phage particles applied was equal to the number of bacteria, or slightly in excess. Large excesses were not used since these involve long periods of spraying, with resultant wetting of the surface of the agar and a tendency for the bacteria to be moved about by the moisture. Larger numbers of phage particles have been used, however, by BEALE (1948), who concentrated the phage by centrifuging. The estimates of mutation rate that he obtained in this manner do not differ appreciably from those obtained in the present experiments, and it may be assumed that the quantities used in the latter were adequate.

By the method described in this section it was possible to determine the rate with which resistant clones appear during the period of rapid growth from the resting stage onward.

The results of four separate experiments, each with eight independent replicates, are given in table 6 . In these experiments the bacteria were grown over a period of approximately eight generations; and the mutation rate obtained is that for the whole growth period.

It will be seen that the average of estimates of rate from all experiments is low $\left(.59 \times 10^{-8}\right)$ and is in close agreement with the low estimates from method 1 (average $\left..40 \times 10^{-8}\right)$. As pointed out earlier, this constitutes evidence that resistant clones arise from mutations occurring one or more generations prior to their first becoming detectable.

It should be mentioned at this point that subsequent experiments, described in the next section and summarized in figure 1 , have enabled mutation rate to be calculated from a tenfold increase in bacterial titer onward. This eliminates the contribution of the first few divisions, during which an excessive number of 
TABLE 6

M utation rate of $B / r$ to resistance to phage $T 1$, estimated from the number of resistant clones appearing during bacterial multiplication on agar (method 4 of this paper).

\begin{tabular}{lcccc}
\hline \multicolumn{1}{c}{ EXPERIMENT } & $\mathrm{A}$ & $\mathrm{B}$ & $\mathrm{C}$ & $\mathrm{D}$ \\
\hline Inoculum (no. of bact.), $\times 10^{8}$ & .115 & .119 & .106 & .055 \\
Resist. b. in inoc., $\times 10^{-8}$ & 30.2 & 30.2 & 10.0 & 38.0 \\
Incr. in no. of bact. & $264 \times$ & $250 \times$ & $345 \times$ & $317 \times$ \\
Generations of growth & 8.1 & 8.0 & 8.5 & 8.3 \\
\hline \multicolumn{1}{c}{ Replicates } & & Mutation Rate, $\times 10^{-8 *}$ & \\
1 & .49 & .54 & .48 & .53 \\
2 & .47 & .61 & .69 & .38 \\
3 & .56 & .70 & .57 & .49 \\
4 & .42 & .49 & .59 & .71 \\
5 & .70 & .54 & .89 & .50 \\
7 & .69 & .46 & .44 & .52 \\
8 & .45 & .51 & .69 & .63 \\
& .53 & .66 & 1.04 & .89 \\
\hline Av. mutation rate, $\times 10^{-8}$ & .54 & .57 & .67 & .58 \\
Standard deviation & .098 & .078 & .205 & .156 \\
\hline IIut. rate from $10 \times$ increase & .45 & .48 & .60 & .52 \\
onward, $\times 10^{-8} \dagger$ & & & & \\
\hline
\end{tabular}

* Each replicate mutation rate is calculated from an independent single-plate estimate of each of the following four values: (1) number of bacteria in the inoculum, (2) end number of bacteria, (3) number of resistant bacteria in the inoculum, and (4) end number of resistant clones, using formula 4 of this paper.

$\dagger$ This mutation rate is calculated from a tenfold increase onward using the information in figure 1 and the method outlined in the section on method 4. It is a more accurate estimate of the rate of formation of resistant clones during logarithmic growth, since the bias from the high early rate of appearance of resistant clones is removed.

resistant clones appears, and reduces the average rate obtained from these experiments from $.59 \times 10^{-8}$ to $.51 \times 10^{-8}$.

\section{RATE OF APPEARANCE OF RESISTANT MICROCOLONIES DURING THE EARLY DIVISIONS ON A SOLID MEDIUM}

Where there is a delay between mutation and phenotypic expression such that the first phenotypically resistant individual appears $n$ generations after the mutation has occurred, there will be $2^{\mathrm{n}}$ bacteria in the mutant clone when it first becomes detectable. When a mutation occurs, a delay of $\mathrm{n}$ generations in the time of its appearance will correspond to a $2^{\mathrm{n}}$-fold increase in the population. The apparent number of bacterial divisions in which the mutation has occurred will then be $2^{\mathrm{n}}$ times the true number, and when an estimate is made from the number of detectable mutant clones, the apparent rate will be reduced to $\frac{1}{2} \mathrm{n}$ of the true value. 
From the interpretation here adopted, it follows that a sample from a culture will contain some mutant individuals which are not yet phenotypically resistant. When such a sample is spread on agar and allowed to grow (as in method 4) these hidden mutants develop phenotypic resistance during the first few divisions and thus give rise to resistant microcolonies. Since a mutant clone may contain a number of these hidden mutants, and since these are dispersed over the surface of the agar, a number of resistant microcolonies can

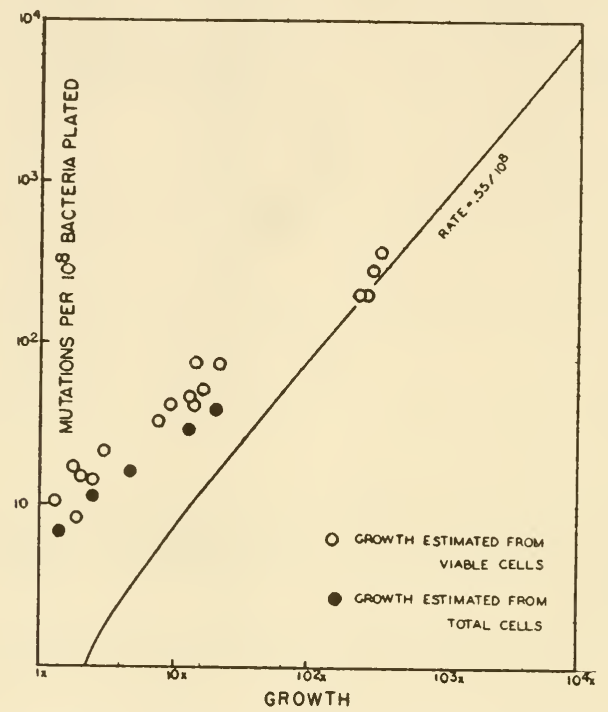

Fig. 1.-Numbers of mutations to phage (TI) resistance arising during bacterial multiplication, as estimated from the numbers of resistant microcolonies present after varying periods of growth.

result from a single mutation prior to plating. This is of course not true for mutations occurring after plating, because the products of these are confined to one locality.

Thus the rate of appearance of resistant microcolonies during the early divisions would be expected to be high, approaching the true mutation rate; and the rate of appearance during later divisions would be expected to be low, approaching $2^{\mathrm{n}}$ times the true mutation rate.

Evidence has been obtained on this point, using method 4 and plotting the numbers of resistant clones arising during varying periods of incubation $\left(R_{2}-R_{1}\right.$, where time 1 is the time of plating) against growth in terms of factor increase in the number of bacteria $\left(\mathrm{N}_{2} / \mathrm{N}_{1}\right)$. The absolute increase in resistant clones with growth depends, of course, upon the original number of bacteria plated, and where data are obtained from a number of separate experiments they are comparable only if expressed in terms of the number of individuals in 
the inoculum. It is therefore convenient, when plotting a curve using data from a number of experiments, to express the increase in terms of resistant clones per $10^{8}$ bacteria in the inoculum. This has been done in figure 1 , where $\left(R_{2}-R_{1}\right) / N_{1} \times 10^{8}$ is plotted against growth $\left(N_{2} / N_{1}\right)$, time 1 being the time of plating. Each point shown in figure 1 was obtained by averaging at least eight independent estimates of increase in number of resistant clones and of growth.

Two experimental procedures for estimating growth were used in this case. One was that mentioned earlier, in which bacteria are washed from the plate and the number determined by dilution and assay. The other was by direct count of the numbers of bacteria in the developing microcolonies.

For the latter purpose small numbers of bacteria were plated on agar, and the plates were incubated at the same time and for the same period as those that were to be sprayed with phage. These growth assay plates were then chilled to stop division and examined under a high-power dry objective. The numbers of bacteria in 50 microcolonies were counted and averaged. The method gave accurate results up to an increase in number of bacteria of approximately 64 times.

In figure 1, points obtained by calculating growth by means of assay of bacteria washed from duplicate plates are shown as hollow dots. Those for which growth was calculated from the average number of bacteria per microcolony are shown as solid dots.

Something similar to the expected high early rate of appearance of resistant clones is observed in these results. In figure 1 the numbers of resistant clones appearing during growth are plotted on a logarithmic grid against growth expressed in terms of factor increase in numbers of bacteria. For comparison, a curve is drawn showing the expected numbers - assuming a rate of $.55 \times 10^{-8}$ and immediate phenotypic expression. The experimental curve does show a high early rate, declining as growth proceeds. This is qualitatively what would be expected where there is a delay, though the rate is actually higher than expected.

The increase in resistant clones when material is sprayed with phage after a twofold increase in population is approximately 12 per $10^{8}$ bacteria plated, which represents an apparent mutation rate of $8.3 \times 10^{-8}$; whereas the rate expected during the first division is the same as that obtained from liquid cultures, that is, something between 2.8 and $3.4 \times 10^{-8}$.

Furthermore, by extrapolating the curve backward it would appear that about seven per $10^{8}$ bacteria become resistant during the lag phase before any division has taken place.

This point has been studied by incubating bacteria with a known lag phase of 70 minutes for 30,60 , and 90 minutes on agar and then spraying with phage. The results are given in table 7 , and show an appreciable increase in resistant colonies from plates sprayed just before the onset of the first division and during the very early part of the division.

The points just considered are not directly related to the main issue. The important contribution of these experiments is to show that the rate of appearance of resistant clones declines during the first few divisions on agar. 
A METHOD OF REDUCING THE DOWNWARD BIAS IN METHOD 2 DUE TO THE DELAY IN PHENOTYPIC EXPRESSION (METHOD 5)

As mentioned in the introduction, a delay between mutation and phenotypic expression must be variable within a mutant clone, expression occurring in some lines of descent earlier than in others. This leaves open the questions of the extent of the delay, the nature of the variation, and the rate of gene muta-

TABLE 7

Increase in number of bacteria resistant to phage T1 during early growth, using strain B/r grow'n on agar, and spraying with phage during the lag phase and early part of the first division.

\begin{tabular}{llll}
\hline \multicolumn{1}{c}{ EXPERIMENT } & $\mathrm{A}$ & $\mathrm{B}$ & $\mathrm{C}$ \\
\hline Replicate plates & 10 & 10 & 10 \\
Bact. in inoculum, $\times 10^{8}$ & 11.0 & 11.0 & 11.0 \\
Resist. bact. in inoc., per $10^{8}$ & 10.1 & 10.1 & 10.1 \\
Incubation, minutes & 30 & 60 & 90 \\
\hline Incr. in no. of bact. & $1.0 \times$ & $1.0 \times$ & $1.3 \times$ \\
New resist. bact., per $10^{8}$ & 0.1 & 5.8 & 10.7 \\
Standard deviation & 1.89 & 1.87 & 2.05 \\
\hline
\end{tabular}

tion, the last being to a greater or smaller degree underestimated by the methods dealt with so far. Method 5 is designed to obtain a less biased estimate of mutation rate, and, from this, some idea of the exteni of the delay.

With a variable delay it would be expected that mutant clones arising from mutations early in the growth of a culture would contain a higher proportion of phenotypically resistant cells than would younger mutant clones, and that a higher and less biased estimate of mutation rate would be obtained if it could be calculated from these older clones alone.

To do this the following method has been devised. In a series of similar test cultures a few of the cultures contain many times the average number of resistant cells, because of the chance occurrence in these cultures of an early mutation. In such cases the precise numbers of resistant bacteria from the earliest mutation and from subsequent mutations cannot be determined directly. The probable number from later mutations, however, is approximately equal to the mean number in the whole series. This is, incidentally, a very slight overestimate, and a more precise approach will be considered later.

Using this method, the probable number of resistant bacteria descended from the earliest mutation in a series of cultures is obtained by subtracting the mean number of resistant bacteria per culture, $r$, from the highest number occurring in any one of the cultures, h. At the probable time of occurrence of this mutation the population in the culture would have been $\mathrm{N} /(\mathrm{h}-\mathrm{r})$, where $\mathrm{N}$ is the end population; and the population in the whole series of cultures would have been $\mathrm{CN} /(\mathrm{h}-\mathrm{r})$, where $\mathrm{C}$ is the number of cultures in the series. Thus the first mutation in the series occurred when the population was $\mathrm{CN} /(\mathrm{h}-\mathrm{r})$, and since the inocula were small the number of bacterial divisions 
giving rise to this population would also be $\mathrm{CN} /(\mathrm{h}-\mathrm{r})$. The mutation rate, a, when one mutation occurs in this number of bacterial divisions is of course given by the formula:

$$
\mathrm{a}=(\ln 2)(\mathrm{h}-\mathrm{r}) / \mathrm{CN} .
$$

Mutation rate is very slightly underestimated by this formula, since $r$ is an overcorrection for the probable number of resistant cells from mutations subsequent to the first. A more precise correction would be obtained by averaging the number of resistant cells per culture, exclusive of those from the first mutation in the series. This complication has not been introduced, however, since the gain in accuracy would not be appreciable.

The rates calculated in this manner may be considered as approaching the true rate if most of the members of these older clones have become phenotypically resistant. If only a small proportion have become resistant, this rate is still an underestimate, although it would be closer to the true rate of gene mutation than the estimates using method 2. This method will be known as method 5 .

The rates obtained in this manner, using the data from table 1, are presented in table 8 , together with the values required for the calculations. Data of

TABLE 8

Mutation rate of $B / r$ to resistance to phage $T 1$, calculated from the maximum number of resistan bacteria in any one culture of a series, using method 5 of this paper, and the data in table 1.

\begin{tabular}{|c|c|c|c|c|c|c|c|c|}
\hline EXPERIMENT & A & B & $\mathrm{C}$ & D & E & F & G & $\mathrm{H}$ \\
\hline Number of cultures & 25 & 25 & 25 & 25 & 25 & 25 & 25 & 25 \\
\hline Bact. per cult., $\times 10^{8}$ & 3.1 & 4.6 & 2.5 & 2.8 & 4.2 & 3.7 & 3.2 & 3.8 \\
\hline Av. resist. b. per cult. & 56.1 & 18.5 & 29.5 & 16.9 & 26.8 & 59.5 & 68.6 & 52.6 \\
\hline Mar & 447 & 221 & 158 & 79 & 242 & 287 & 725 & 263 \\
\hline Mutation rate, $\times 10^{-8}$ & 3.6 & 1.3 & 1.3 & .83 & 1.5 & 2.3 & 5.7 & \\
\hline
\end{tabular}

Luria and Delbrück, and Demerec and Fano, obtained using phage T1, have been treated similarly, and a detailed comparison is made in table 9 of rates from methods 1,2 , and 5 , using information from all sources. For the sake of convenience, the rates obtained by methods 2 and 5 have been expressed in the last two columns of the table in terms of the smaller value from method 1 , as ratios-rate (2)/rate (1), and rate (5)/rate (1).

Taking into consideration all available information on mutation to resistance to phage $T 1$, rates from methods 2 and 5 differ only slightly and are between four and nine times the rates from method 1.

The application of method 5 has also been extended to the data obtained by DEMEREC and $\mathrm{F}_{\text {ANO }}$ using phages other than $T 1$; and averages of mutation rates from methods 1,2 , and 5 , and of the ratios rate (2)/rate (1), and rate $(5) /$ rate (1), have been worked out for all available data on strains $B$ and $B / r$ and phages $T 1, T 3, T 4, T 5, T 6$, and $T 7$. They are given in table 9 . 
The purpose of these calculations was to determine whether mutation rate when estimated from the older mutant clones only is higher than when estimated from the average numbers of resistant bacteria per culture. If the delay is sufficiently variable and of sufficient magnitude, it would be expected that

TABLE 9

Mutation rates of strains $B$ and $B / r$ to resistance to phage $T 1$, from series of similar liquid cultures started with small inocula, using methods 1,2 , and 5 and calculating from data of LURIA and DELBRÜCK (1943, table 3, experiment 23), DEMEREC and FANO (1945, table 4), and the present paper (tables 1 and 8). All the experiments which are suitable for the application of the three methods have been used.

\begin{tabular}{|c|c|c|c|c|c|c|c|}
\hline EXPER. & STRAIN & CULTURES & $\begin{array}{l}\text { RATE (1) } \\
\times 10^{-8}\end{array}$ & $\begin{array}{c}\text { RATE (2) } \\
\times 10^{-8}\end{array}$ & $\begin{array}{l}\text { RATE (5) } \\
\times 10^{-8}\end{array}$ & $\begin{array}{r}\text { RATIO } \\
(2) /(1)\end{array}$ & $\begin{array}{r}\text { RATIO } \\
(5) /(1)\end{array}$ \\
\hline \multicolumn{8}{|c|}{ LURIA and DELBRÜCK } \\
\hline A & B & 87 & .32 & 2.4 & 1.5 & 7.5 & 4.4 \\
\hline \multicolumn{8}{|c|}{ DEMEREC and FANO } \\
\hline A & B & 53 & .63 & 1.4 & 1.8 & 2.2 & 2.9 \\
\hline B & & 53 & .33 & 2.3 & 6.1 & 7.0 & 18.5 \\
\hline $\mathrm{C}$ & & 17 & 1.15 & 3.9 & 9.2 & 3.4 & 7.9 \\
\hline $\mathrm{D}$ & & 25 & .71 & 3.1 & 4.1 & 4.4 & 5.8 \\
\hline $\mathrm{E}$ & & 19 & .66 & 3.2 & 3.0 & 4.8 & 4.5 \\
\hline $\mathrm{F}$ & & 38 & 1.26 & 6.1 & 7.8 & 4.7 & 6.2 \\
\hline G & & 45 & .24 & 0.8 & 1.3 & 3.3 & 5.4 \\
\hline $\mathrm{H}$ & & 44 & .63 & 3.2 & 6.0 & 5.7 & 9.6 \\
\hline I & & 45 & .31 & 2.0 & 2.9 & 6.4 & 9.4 \\
\hline \multirow[t]{2}{*}{$\mathrm{J}$} & & 45 & .92 & 1.3 & 1.3 & 1.4 & 1.4 \\
\hline & & Average & .68 & 2.7 & 4.4 & 4.3 & 7.2 \\
\hline \multicolumn{8}{|c|}{ Present investigation } \\
\hline A & $B / r$ & 25 & .42 & 5.1 & 3.6 & 12.2 & 8.6 \\
\hline B & & 25 & .28 & 1.5 & 1.3 & 5.4 & 4.4 \\
\hline C & & 25 & .59 & 3.8 & 1.3 & 6.6 & 2.1 \\
\hline $\mathrm{D}$ & & 25 & .53 & 2.1 & 8.3 & 4.0 & 1.6 \\
\hline $\mathrm{E}$ & & 25 & .31 & 2.1 & 1.5 & 6.8 & 4.7 \\
\hline $\mathbf{F}$ & & 25 & .34 & 4.5 & 2.3 & 13.2 & 6.7 \\
\hline G & & 25 & .40 & 5.8 & 5.7 & 14.5 & 13.8 \\
\hline \multirow{2}{*}{\multicolumn{2}{|c|}{$\mathrm{H}$}} & 25 & .33 & 3.9 & 1.5 & 11.8 & 4.6 \\
\hline & & Average & .40 & 3.6 & 2.3 & 9.3 & 5.8. \\
\hline
\end{tabular}

in these older clones a higher proportion of the mutants would have become phenotypically resistant.

Not all experiments show a difference between estimates of rate from the average resistant bacteria (method 2) and estimates from the highest number of resistant bacteria, of which the majority are presumably descended from an early mutation (method 5). Examining data from all sources, the estimates from method 5 and from method 2 do not differ significantly. 
With the exception of the mutations to $B / 3,4$ and $B / 3,4,7$, estimates from method 5 are of the order of six to seven times those from method 1 , and the lower ratios in the case of these exceptions are in all probability due to the high proportion of slow-growing $B / 3,4$, and $B / 3,4,7$ mutants-Demerec and $\mathrm{F}_{\mathrm{ANO}}$ having shown in their experiments on competitive growth that these mutants grow much more slowly than the parent strain $B$.

A crude average of the ratio rate (5)/rate (1) from mutations other than those to $B / 3,4$, and $B / 3,4,7$ shows that estimates obtained by method 5

TABLE 10

Mean mutation rates of strains $B$ and $B / r$ to phage resistance, estimated by methods 1,2 , and 5 ; data of LURIA and DELBRÜCK, DEMEREC and FANO, and the present investigation (table 9 ), all from series of similar liquid cultures started with small inocula.

\begin{tabular}{|c|c|c|c|c|c|c|c|c|c|}
\hline PHAGE & STRAIN & $\begin{array}{l}\text { RESISTANT } \\
\text { BACTERIA }\end{array}$ & $\begin{array}{l}\text { NO. OF } \\
\text { EXPER. } \\
\text { AVER- } \\
\text { AGED }\end{array}$ & $\begin{array}{c}\text { AVER. } \\
\text { RATE } \\
(1) \\
\times 10^{-8}\end{array}$ & $\begin{array}{c}\text { AVER. } \\
\text { RATE } \\
(2) \\
\times 10^{-8}\end{array}$ & $\begin{array}{c}\text { AVER. } \\
\text { RATE } \\
(5) \\
\times 10^{-8}\end{array}$ & $\begin{array}{c}\text { AVER. } \\
\text { RATIO } \\
(2) /(1)\end{array}$ & $\begin{array}{c}\text { AVER. } \\
\text { RATIO } \\
(5) /(1)\end{array}$ & SOURCE \\
\hline$T 1$ & $B / r$ & $B / r / 1,5 ; B / r / 1$ & 8 & .40 & 3.6 & 2.2 & 9.3 & 5.8 & table 9 \\
\hline$T 1$ & $B$ & $B / 1,5 ; B / 1$ & 1 & .32 & 2.4 & 1.5 & 7.5 & 4.4 & L. \& D. \\
\hline$T 1$ & $B$ & $B / 1,5 ; B / 1$ & 10 & .68 & 2.7 & 4.4 & 4.3 & 7.1 & D. \& F. \\
\hline$T 5$ & $B$ & $B / 1,5$ & $5^{*}$ & .38 & 1.5 & 2.6 & 4.3 & 7.5 & D. \& F. \\
\hline$T 6$ & $B$ & $B / 6$ & 3 & 5.0 & 30.8 & 42.0 & 5.3 & 7.1 & D. \& F. \\
\hline$T 3$ & $B$ & $B / 3,4,7 ; B / 3,4$ & 7 & 5.1 & 10.0 & 12.5 & 2.3 & 2.9 & D. \& F. \\
\hline$T 4$ & $B$ & $B / 3,4,7 ; B / 3,4$ & 3 & 5.5 & 12.9 & 34.8 & 2.7 & 5.8 & D. \& F. \\
\hline$T 7$ & $B$ & $B / 3,4,7$ & 3 & 4.4 & 12.2 & 9.6 & 2.8 & 2.4 & D. \& F. \\
\hline
\end{tabular}

* Note: One experiment containing an exceptional culture with 20,000 resistant bacteria has been omitted from these averages.

are approximately 6.4 times those obtained by method 1 . The significance of this ratio in relation to the extent and variability of the delay between mutation and phenotypic expression will be considered in the discussion.

THE INFLUENCE OF DIVISION MORTALITY UPON ESTIMATES

OF MUTATION RATE

All the methods of estimating mutation rate are subject to a small error because some of the bacteria resulting from a division fail to divide again (WILson 1922). The number of mutations occurring during a division is thus underestimated, and the number of divisions, where calculated from numbers of viable bacteria, is also underestimated.

It might be assumed that the apparent variations in the mutation rate during the early part of the growth cycle are due to corresponding changes in the mortality associated with division. That this is not the case will be demonstrated; and corrections to be applied to mutation rates which have been based upon the assumption of unity survival will be considered.

Where growth has been estimated from the numbers of viable bacteria, a correction may be obtained in the following manner. Let $\mathrm{S}$ be the chance of 
survival of one of the bacteria resulting from a division. In a population of $\mathrm{N}$ viable bacteria, one division will raise the number from $\mathrm{N}$ to $2 \mathrm{NS}$ viable individuals, and, where $\mathrm{A}$ is the true rate of mutation, will result in the production of ANS/In 2 viable mutant individuals. If the increase in population is determined from assays of viable bacteria, the apparent mutation rate, a, during this division will be $(\ln 2)(\mathrm{ANS}) /(\ln 2)(2 \mathrm{NS}-\mathrm{N})$, which reduces to AS/ $(2 S-1)$. The true mutation rate may thus be obtained from the apparent rate by the formula:

$$
A=a(2 S-1) / S .
$$

The relationship between the true mutation rate and an apparent rate, $a_{1}$, obtained by utilizing the increase in total population as distinct from viable population, may be derived in a similar manner and is expressed by the formula:

$$
\mathrm{A}=\mathrm{a}_{1} / \mathrm{S} \text {. }
$$

Where estimates of mutation rate have been made in both ways, that is, using both viable population increase and total population increase, the value of $\mathrm{S}$ may be determined from the apparent mutation rates a and $\mathrm{a}_{1}$. The formula for this as obtained from formulas (6) and (7) by simultaneous solution and is:

$$
\mathrm{S}=\left(\mathrm{a}+\mathrm{a}_{1}\right) / 2 \mathrm{a} .
$$

The true mutation rate also may be obtained from the values a and $a_{1}$, using equation (8) and substituting for S. This yields the formula:

$$
A=2 a \cdot a_{1}\left(a+a_{1}\right) \text {. }
$$

These formulae may be applied to the data from figure 1 in order to determine whether the high apparent mutation rate during the first few divisions could be due to a low survival. It will be noted that points on figure 1 obtained by estimating growth from viable numbers of bacteria do not differ greatly from those obtained by estimating growth from total bacteria. This suggests that mortality due to division is not excessive, and we may calculate the chance of survival of the individual products of one division, by using formula (8). Thus, over the period from a twofold to a tenfold increase in population, the rates estimated from the two curves would be approximately $2.6 \times 10^{-8}$ and $1.4 \times 10^{-8}$, respectively. From these two values the calculated survival is 0.75 . Therefore the high rate of appearance of resistant clones during the early divisions on a solid medium discussed earlier cannot be due to an excessive division mortality.

It should also be noted that the estimate of rate which would have been obtained over this period of growth by either method, had survival been unity, is given by formula (9) and is $1.9 \times 10^{-8}$. This latter figure, however, has little bearing on the present discussion, as it is in any case intermediate between the estimates of rate obtained from numbers of resistant clones and from numbers of resistant individuals. 
It is realized that this early low division mortality may not persist throughout the whole of the growth cycle but may rise as the population in a culture approaches the saturation density. In order to minimize the possibility of this effect, the end population of cultures grown on agar (method 4) was not allowed to reach that period at which growth rate begins to decline (approximately $5 \times 10^{9}$ bacteria on a 100 millimeter plate of broth agar). If, despite these precautions, there was an increase in division mortality, then as pointed out earlier, the estimates of mutation rate obtained would be somewhat greater than the true rate. It will be noted that this would tend to weight the data obtained by method 4 against the interpretation arrived at. We may therefore conclude that errors from this source do not affect the main conclusions.

\section{DISCUSSION}

From the experiments described, it is apparent that those methods which take into account only the number of resistant clones developing in a given period, and not the number of resistant individuals in these clones, give a low estimate of the mutation rate (see table 11 for summary).

Method 1 falls in this category, since it is based upon the proportion of cultures in which no phenotypic mutants have appeared-this being a function of the total number of phenotypically resistant clones in the series of culturesand is independent of the size of the clone.

Method 4 also falls in this category, since the members of a mutant clone are confined to one spot on the agar and after one member has become resistant others may change in the same manner without being detected. Method 4, which uses the number of resistant clones appearing during rapid growth, provides a necessary check on method 1, which uses the number of resistant clones developing during growth to saturation, and which would be strongly biased if resistant clones appeared at a different rate during the few divisions prior to the cessation of growth. The estimates of rate obtained by these two methods are, within the limits of experimental error, similar, and in the present experiments - using resistance of strain $B / r$ to the phage $T 1-$ average approximately $0.5 \times 10^{-8}$.

Methods that are based on the increase in numbers of resistant individuals developing during growth, however, as distinct from the numbers of resistant clones, produce much higher estimates of mutation rate. The following fall in this category: method 2, which uses the average number of resistant bacteria in a series of similar cultures started with small inocula; method 3 , which uses the number of resistant bacteria in a single culture started with a large inoculum; and method 5 , which uses the highest number of resistant bacteria in any one culture of a series.

Method 3, which is based on a relatively direct calculation, provides a necessary check on method 2, which is subject to a statistical bias. Similarly, method 5 , which is based on the early mutations, provides a check on method 2 , which would be biased if the delay in developing phenotypic resistance were excessive in an appreciable proportion of the lines of descent within a mutant clone. Method 3 is also of use in this connection in the one experiment in which 
TABLE 11

Averages of mutation rates for strains $B$ and $B / r$ to resistance to phage $T 1$ obtained by methods 1 to 5. Summary of calculations using data, or mutation rates already calculated, from the present investigation, LuRIA and Delbrück (1943), Demerec and Fano (1945), and BeAle (1948).

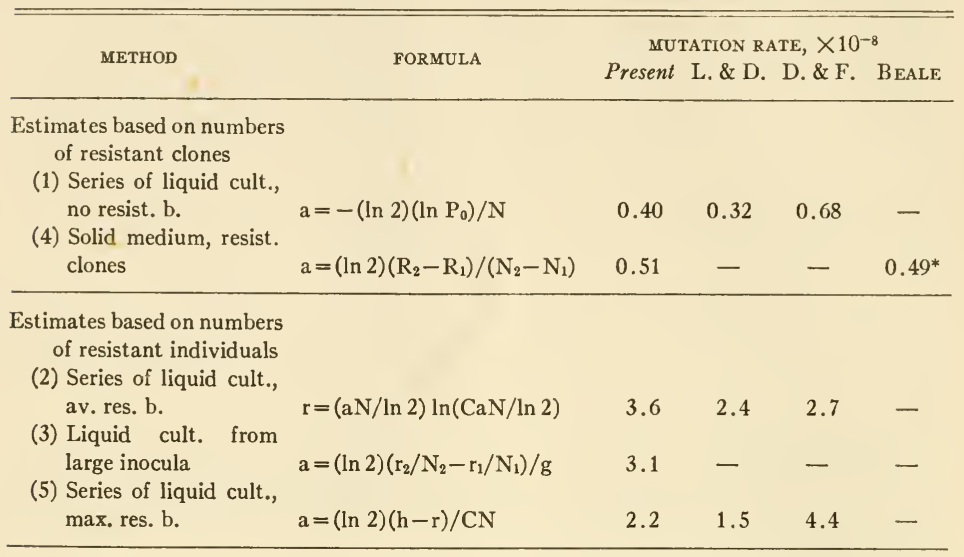

Early growth

(4a) Solid med., res. clones, first div.

$a=(\ln 2)\left(R_{2}-R_{1}\right) /\left(N_{2}-N_{1}\right)$

8.3

Symbols:

a, mutation rate per bacterium per division cycle.

$r$, average number of resistant bacteria per culture.

$h$, highest number of resistant bacteria in any one culture of a series.

$\mathrm{N}$, average number of bacteria per culture; $\mathrm{N}_{1}$ and $\mathrm{N}_{2}$, average number at times 1 and 2 respectively.

$\mathrm{C}$, number of cultures.

$R$, average number of resistant clones per plate; $R_{1}$ and $R_{2}$, average numbers at times 1 and 2 respectively.

$\mathrm{g}$, number of cell generations.

$\mathrm{P}_{0}$, proportion of test cultures in which there are no resistant mutants.

* Note: the value $.7 \times 10^{-8}$ bacterial divisions obtained by BEALE has been converted to the rate per bacterium per division cycle by multiplying by $\ln 2(=.693)$.

the growth period is long, and in which the majority of the resistant individuals are from mutations occurring many generations previous to the cessation of growth.

The estimates of rate obtained by these methods are all considerably greater than those which took into consideration only the numbers of resistant clones and not the numbers of resistant individuals, and differ only slightly among themselves. In the present experiments they average approximately $3 \times 10^{-8}$.

It is thus evident that the numbers of resistant individuals in a mutant clone must be greater than would be expected on the basis of the time of phenotypic appearance of that clone. One simple interpretation of this-namely, 
that the growth rate is higher in the mutant clones than in the parent strainis ruled out by the results obtained using method 3 . The only plausible interpretation, therefore, seems to be that a mutation to resistance is not phenotypically expressed until after a number of divisions have taken place.

Two possibilities exist with regard to such a delay: (1) that after a fixed period of one or more generations all of the offspring of the original mutant become phenotypically resistant at the same time, and (2) that the delay is variable within the mutant clone, some lines of descent becoming resistant earlier than others. As pointed out by LURIA (1946), the presence of cultures having only one resistant bacterium eliminates the first of these. Thus the delay must be variable within the mutant clones, and a single individual may develop phenotypic resistance in the first instance, being followed later by other members of the clone.

As pointed out, the presence of phenotypically susceptible individuals in the younger mutant clones, and the occurrence of changes in these to phenotypic resistance, results in a high apparent mutation rate when the members of the clone are dispersed and the changes are thus rendered detectable in each individual, and in a low apparent mutation rate when the members of a clone are grouped together and only the first change to phenotypic resistance is detectable. In the early growth on agar a situation exists in which the members of mutant clones in the inoculum are dispersed over the plate but the products of subsequent divisions of individual bacteria are grouped together. In such circumstances the apparent mutation rate would be expected to be high during the first division, as in liquid cultures, and to decline during subsequent generations to the apparent rate obtained when only the numbers of resistant clones are considered. This, in fact, has been observed and lends support to the conclusions reached.

In this connection, however, it should be noted that the rate of appearance of resistant bacteria during the first division on agar following a resting stage is higher than expected, by a factor of approximately three or four. This does not affect the main line of reasoning, although it is of interest in itself and appears to be well substantiated by the data.

Attempts to interpret this as due to the bacteria's failing to adsorb phage during the early stages of growth after a resting stage have not been successful, since one would have to suppose complete failure of adsorption over a period of at least two generations.

Two possibilities remain: (1) that of a genuine high rate of mutation during this division, together with physiological conditions favoring immediate phenotypic expression, since otherwise the products of these mutations would not be detectable; and (2) that of physiological conditions favoring phenotypic expression of mutants which had not hitherto become resistant. So far, no methods have been devised to distinguish between these two possibilities.

Thus the rate of appearance of resistant clones during early growth on agar is in agreement with the concept of a delayed phenotypic expression, although an additional phenomenon appears to be involved.

The size of a mutant clone at the time of appearance of the first resistant in- 
dividual may be obtained by comparing the estimates of mutation rate from method 1, utilizing the number of resistant clones developing in liquid cultures, with those from method 5 , utilizing the number of resistant individuals in old mutant clones. Table 9 may be used for this purpose, and the ratio rate (5)/ rate (1) considered. If one omits the data on mutations to $B / 3,4$ and $B / 3,4$, 7 , because of the bias resulting from the known low growth rate of these mutants (DEMEREC and FANO 1945, table 7), the ratios in the cases of the remaining mutations appear to be similar. A crude average of these is 6.4. This indicates that there are approximately six or seven individuals in a mutant clone at the time of appearance of the first resistant individual; that is, that the delay between mutation and phenotypic expression in at least one member of the resulting clone is of the order of 2.7 generations.

The extent of the delay in individual lines of descent within a mutant clone other than the first one to become resistant, cannot be determined directly. However, the absence of any striking difference between the estimates of mutation rate obtained by methods 2 and 5 suggests that the delay is not excessive; and the similar values, $2.8 \times 10^{-8}$ and $3.4 \times 10^{-8}$, obtained by method 3 with growth periods of six and eleven generations respectively, indicate that no very large proportion of a mutant clone changes to phenotypic resistance after six generations from the time of the mutation.

The possibility that the delay in expression of irradiation-induced changes is of the same origin as that observed in spontaneous mutations should be considered at this point, although a positive answer cannot be given, since strictly comparable studies of the induced changes have not yet been carried out, and, in particular, nothing is known of the numbers of resistant individuals developing in the late-appearing resistant clones resulting from irradiation.

If irradiation results in the immediate induction of gene changes similar to those occurring spontaneously, and the delay is one of phenotypic expression similar to that in untreated material, then the numbers of resistant individuals in the late-appearing resistant clones should rise rapidly after the clones first become detectable, because of susceptible members becoming phenotypically resistant, and should eventually approach the numbers in the resistant clones that appeared soon after treatment. Variation in the time of occurrence of phenotypic expression in different lines of the same mutant clone would also be expected in irradiated material.

Until information of this nature is obtained it is not possible to say with certainty that the observed delays in appearance of induced and spontaneous mutations are of the same origin. In the absence of more detailed information, one can only speculate on the basis of the variation between clones. Since in untreated material there is no striking difference between the apparent mutation rates as determined over periods of six and eleven generations (method 3 ), it would seem that six generations is adequate both for phenotypic expression in most of the mutants within a clone and also for expression in at least one individual in most mutant clones. In irradiated material an appreciable proportion of the resistant clones do not appear until after the sixth generation; 
and it is therefore tentatively suggested that the two delays are not of the same origin.

It should also be noted that a delay in phenotypic expression has been observed in Paramecium following hybridization (Kimball 1937; SonNeborN 1943; SonNEBORN and LyNCH 1934) and has been termed "cytoplasmic lag." This phenomenon is at least superficially similar to that described in the present work, in that "phenotypic expression ... commonly appears first at slightly different times in different lines of descent within the clone"(SonNEBORN 1947). The extent of the delay appears to vary in Paramecium with the nature of the change, and in two instances of alteration in type there is no detectable delay. (These are the alterations from mating type I to mating type II in variety 1 , and from mating type $\mathrm{V}$ to mating type $\mathrm{VI}$ in variety 3 . For a discussion of this, see SONNEBORN 1947.) It will therefore be of interest to determine whether a similar diversity in the delay exists in bacteria, since the apparent differences in the mutations so far studied, all of which mutations are to resistance to one or another of the phages, are relatively small and can be explained as due to known differences in the growth rates of the mutants. Until more information of this nature is available it is not profitable to speculate further about whether "cytoplasmic lag" in protozoa and "delayed phenotypic expression" in bacteria have a common basis.

\section{CONCLUSIONS}

Evidence of a delay in the phenotypic expression of spontaneous bacterial mutations to phage resistance, using $E$. coli, strain $B / r$, has been obtained. This evidence comes from a comparison of the rate of appearance of phenotypically resistant clones during bacterial multiplication with the rate of gene mutation, the latter being estimated from the numbers of resistant individuals arising during similar periods of growth.

The rate of appearance of resistant clones per bacterium per division cycle is approximately $0.5 \times 10^{-8}$, whereas the numbers of resistant individuals correspond to a much higher rate of mutation, a rate of approximately $3 \times 10^{-8}$.

Since it is known that the mutants do not divide more rapidly than the parent strain, the excess number of individuals composing a mutant clone indicates that the clone was formed sometime prior to its becoming phenotypically detectable. Thus there appears to be a delay between mutation and phenotypic expression.

The possibilities of alternative interpretations of the data of previous workers on the basis of a statistical bias in one of the methods, and of a change in the mutation rate during the later part of growth, have been eliminated by using methods which are not susceptible to these sources of error.

A delay in phenotypic expression would be expected to result in a high rate of appearance of resistant clones during the first few divisions on agar, declining to the value $0.5 \times 10^{-8}$ with further growth. This has been observed, although an additional phenomenon appears to be present which results in the appearance of a higher-than-expected number of resistant clones during the 
first division. This phenomenon, although of interest in itself, does not affect the main conclusions.

It is estimated that phenotypic expression occurs in at least one member of a mutant clone approximately two or three generations after the mutation, and that in many cases it affects at first only one individual, others of the mutants becoming resistant during subsequent divisions.

Expression does not appear to be delayed in any appreciable proportion of a clone beyond approximately six generations after the mutation.

\section{ACKNOWLEDGEMENTS}

I wish to thank Dr. M. Demerec for much helpful advice and criticism, Dr. B. McClintock for valuable discussion of the work during its progress and DR. S. E. LuRIA for a number of very useful comments on the manuscript.

\section{LITERATURE CITED}

ANDEkSON, E. H., 1946 Growth requirements of virus-resistant mutants of Escherichia coli strain "B." Proc. Nat. Acad. Sci. 32: 120-128.

Beale, G. H., 1948 J. Gen. Microbiol. in press.

DeLBRÜcK, M., and LURIA, S. E., 1942 Interference between bacterial viruses I. Arch.Biochem. 1: $111-141$.

DEMEREC, M., 1946 Induced mutations and possible mechanisms of transmission of heredity in Escherichia coli. Proc. Nat. Acad. Sci. 32: 36-46.

DEMEREC, M., and FANo, U., 1945 Bacteriophage-resistant mutants in Escherichia coli. Genetics 30: 119-136.

Demerec, M., and Latarjet, R., 1946 Mutations in bacteria induced by radiation. Cold Spring Harbor Symp. Quant. Biol. 11 : 38-50.

Krmball, R. F., 1937 The inheritance of sex at apomixis in Paramecium aurelia. Proc. Nat. Acad. Sci. 23 : $469-474$.

LURIA, S. E., 1946 Spontaneous bacterial mutations to resistance to antibacterial agents. Cold Spring Harbor Symp. Quant. Biol. 11: 130-137.

LURIA, S. E., and DELBRÜCK, M., 1943 Mutations of bacteria from virus sensitivity to virus resistance. Genetics 28: 491-511.

Shapiro, A., 1946 The kinetics of growth and mutation in bacteria. Cold Spring Harbor Symp. Quart. Biol. 11: 228-235.

SonNEBorn, T. M., 1943 Development and inheritance of serological characters in variety one of Paramecium aurelia. Genetics 28: 90.

1946 Discussion of paper by LuRIA, in Cold Spring Harbor Symp. Quant. Biol. 11: 138.

1947 Recent advances in the genetics of Paramecium and Euplotes. Advances in Genetics 1: 263-358.

Sonneborn, T. M., and Lynch, R. S., 1934 Hybridization and segregation in Paramecium aurelia. J. Exp. Zool. 67: 1-72.

WiLson, G. S., 1922 The proportion of viable bacteria in young cultures with especial reference to the technique employed in counting. J. Bact. 7: 405-446.

Witkin, E. M., 1946 Inherited differences in sensitivity to radiation in Escherichia coli. Proc. Nat. Acad. Sci. 32: 59-68.

1947 Genetics of resistance to radiation in Escherichia coli. Genetics 32: 221-248. 


\title{
ORIGIN OF BACTERIAL RESISTANCE TO ANTIBIOTICS ${ }^{1}$
}

\author{
M. DEMEREC
}

Department of Genetics, Carnegie Institution of Washington, Cold Spring Harbor, New York

Received for publication March 29, 1948

In this brief review of the problem of the genetic aspects of the origin of bacterial resistance to antibiotics, I intend to discuss mainly work done in my laboratory. I shall (1) offer evidence that bacterial resistance to penicillin and streptomycin is not induced by these compounds but originates spontaneously through genetic changes comparable to gene mutations; (2) describe the resistance patterns observed in experiments with penicillin and streptomycin; and (3) outline a possible mechanism responsible for resistance, and for the differences between the resistance patterns observed with penicillin and those observed with streptomycin.

All experiments were done in vitro. Penicillin was tested with Staphylococcus aureus, strain NRRL 313 (Demerec, 1945a), and streptomycin was tested with the same strain of $S$. aureus and with Escherichia coli, strain B. The streptomycin was obtained from Chas. Pfizer \& Co., New York, at a time when this compound was still very scarce, and I wish here to acknowledge their generosity. In all the experiments bacteria were first grown in broth cultures without any penicillin or streptomycin; these were used only in the tests for resistance, made by growing bacteria on broth agar plates containing various concentrations of one or the other antibiotic.

\section{SURVIVAL CURVES}

Figures 1 and 2 show the behavior of our strain of $S$. aureus when plated on nutrient agar plates to which specified amounts of penicillin (figure 1) or streptomycin (figure 2) had been added. It is evident from these curves that on low concentrations of either antibiotic all bacteria survived and formed colonies. Threshold concentrations are indicated on the curves by sharp breaks, the numbers of survivors decreasing very rapidly with increase of concentrations. The slope of the curves is very steep at concentrations near the threshold, but levels out as concentrations become increasingly higher. These curves show that we were dealing with mixed populations of types sensitive to antibiotics and types more or less resistant. Sensitive bacteria made up by far the largest portion of the populations.

The two curves are very similar in the region of lower concentrations. A striking difference is evident, however, in the region of higher concentrations; on the medium containing streptomycin, survivors continued to appear even at the highest concentration used in the experiments.

${ }^{1}$ Substance of a paper delivered at the Symposium on Antibiotics at the Chicago meeting of Section $\mathrm{N}$ of the American Association for the Advancement of Science, December 30, 1947.

[Reprinted by permission of The Williams \& Wilkins Company from Journal of BACTERIOLOGY 56 : (1) 63-74, July, 1948] 
Tests of survivors confirmed the conclusion indicated by the survival curves. Strains isolated from the colonies of plates having high concentrations of either penicillin or streptomycin proved to be more resistant to the respective compound and grew on concentrations at least as high as those of the plates from which they

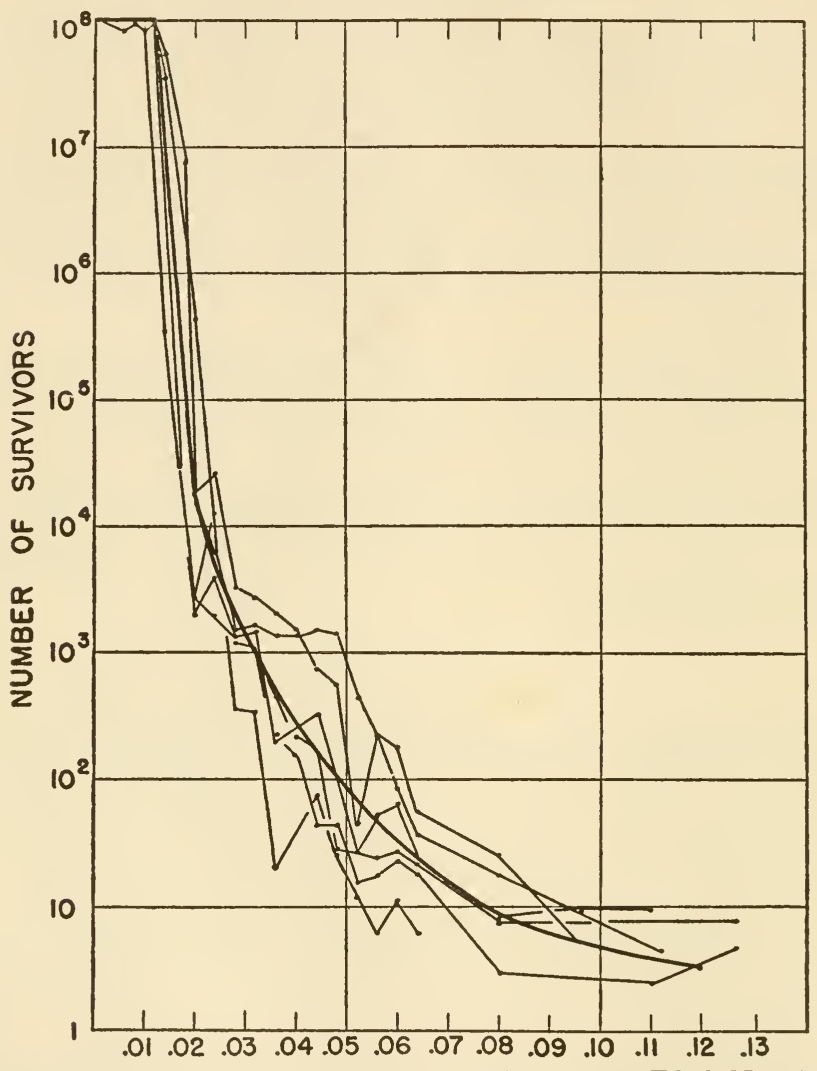

PENICILLIN CONCENTRATION: OXFORD UNITS PER ML

Figure 1. Survival curves for Staphylococcus aureus plated on nutrient agar containing various concentrations of penicillin. The six light curves represent results of six independont experiments, and the heavy curve represents the average of these experiments.

had been isolated. It was found that resistance to one of these antibiotics is independent of resistance to the other; that is to say, strains with increased resistance to penicillin are still sensitive to streptomycin, and vice versa. It seems unnecessary to discuss in detail the by now well-established fact that strains that once become resistant as a rule continue so. 
ORIGIN OF RESISTANCE

The numerous experiments made in gathering the data for the survival curves showed clearly that in large populations of bacteria there were always some individuals more resistant to the antibiotics than others. Since we used very small

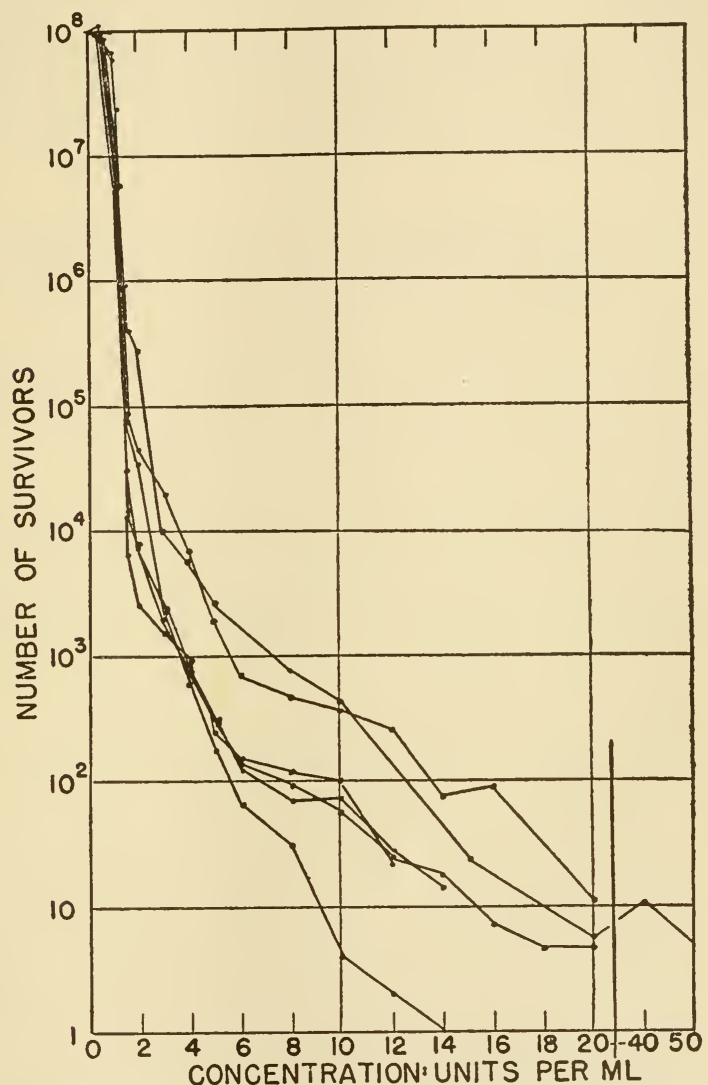

Figure 2. Survival curves for Staphylococcus aureus plated on nutrient agar containing various concentrations of streptomycin.

inocula (50 to 300 bacteria), the proportion of resistant bacteria was too small to account for their presence by assuming that they came about through division of one or more resistant individuals that may have been present in the inoculum. Therefore the resistant individuals must have originated in the experimental cultures. Two alternative possibilities were considered with respect to the 
mechanism of this origin: (1) that resistance was induced by some interaction between the antibiotic and the bacteria when they were together on the plate; and (2) that it originated independently of the antibiotic, by mutation, the antibiotic acting only as a selective agent in the isolation of mutants by destruction of sensitive bacteria.

A relatively simple method was available for distinguishing experimentally between these two possibilities. It was devised by Luria and Delbrück (1943) in a study of the origin of bacterial resistance to bacteriophages, and was adapted for work with antibiotics in our study of the origin of resistance to penicillin (Demerec, 1945a). Following is a brief description of the method as used in our experiments.

From a single culture of bacteria, small inocula ( 50 to 300 bacteria) were taken and used to start 21 or more independent broth cultures. These were incubated for 24 hours, or until growth had reached the saturation point. During incubation the number of bacteria (in experiments using $E$. coli or $S$. aureus) increased to about $2 \times 10^{8}$ per $\mathrm{ml}$. From the same culture that served as the source of the inocula, 10 samples of bacteria of the same size as the inocula were plated on a culture medium containing the same concentration of antibiotic as was used in the tests, in order to determine if any resistant bacteria were present in these samples. The concentration of the antibiotic had to be high enough so that there were no survivors among the small number of bacteria plated-in other words, that there were no bacteria resistant to that concentration in the inocula used to start cultures. It followed, then, that all resistant bacteria found in full-grown cultures had necessarily originated in these cultures during the period when the number of bacteria increased from 50 to 300 to about $2 \times 10^{8}$ per ml.

Next, from 20 of the broth cultures, samples of $0.1 \mathrm{ml}$ were taken and plated on petri dishes containing the same concentration of the antibiotic $(0.064$ units of penicillin, or 5 units of streptomycin per $\mathrm{ml}$ of medium). Fifteen $0.1-\mathrm{ml}$ samples were plated from the twenty-first tube. Thus two sets of plates were obtained: one set of 20 in which each plate had bacteria from a different culture, and another set of 15 all having bacteria from the same culture. The plates were incubated for $\mathbf{2 4}$ hours, or for a longer period if the growth of colonies was slow. After incubation the number of colonies on each plate was determined. These colonies represented resistant bacteria that had been present in the sample plated.

On both sets of plates in this test the experimental conditions were similar, like numbers of bacteria (about $2 \times 10^{7}$ ) having been plated onto nutrient agar containing identical concentrations of antibiotic. Therefore, if resistance were induced through interaction between the bacteria and the antibiotic when they were in contact with each other, approximately similar numbers of resistant bacteria would presumably be obtained on all the plates, regardless of the origin of the bacterial samples; the variation between plates should not exceed random variability. In the event that the origin of resistance is mutational, on the other hand, similar numbers of resistant colonies would be obtained only among the platings taken from the same culture, since these represented repeated tests of 
the same mixture of resistant and sensitive bacteria. Among the samples from separate cultures, if mutations occur at random, a large number of resistant colonies would be obtained from cultures in which mutation happened to occur early in the growth of the culture, and a small number of resistant colonies from cultures in which mutation happened to occur late, provided the growth rate of resistant bacteria is not appreciably different from that of normal ones. If resistance originates by mutation, then, the variation in numbers of resistant bacteria would be much greater between samples taken from separate cultures than between samples taken from the same culture.

TABLE 1

$N$ umber of bacteria ( $E$. coli) resistant to a concentration of 5 units of streptomycin per $m l$ of agar medium in samples taken from a series of independent cultures and similar samples taken from a single culture which assayed $1.3 \times 10^{8}$ bacteria per $\mathrm{ml}$

\begin{tabular}{|c|c|c|c|c|c|c|c|}
\hline \multicolumn{4}{|c|}{ SAMPLES FROM INDEPENDENT CULTURES } & \multicolumn{4}{|c|}{ SAMPLES FROM SINGLE CULTURE } \\
\hline Culture no. & $\begin{array}{l}\text { No. of } \\
\text { resistant } \\
\text { bacteria }\end{array}$ & Culture no. & $\begin{array}{l}\text { No. of resistant } \\
\text { bacteria }\end{array}$ & Sample no. & $\begin{array}{l}\text { No. of } \\
\text { resistant } \\
\text { bacteria }\end{array}$ & Sample no. & $\begin{array}{l}\text { No. of resistant } \\
\text { bacteria }\end{array}$ \\
\hline 1 & 67 & 11 & 56 & 1 & 142 & 11 & 110 \\
\hline 2 & 159 & 12 & 91 & 2 & 155 & 12 & 125 \\
\hline 3 & 135 & 13 & 123 & 3 & 132 & 13 & 135 \\
\hline 4 & 291 & 14 & 97 & 4 & 123 & 14 & 121 \\
\hline 5 & 75 & 15 & 48 & 5 & 140 & 15 & 112 \\
\hline 6 & 117 & 16 & 52 & 6 & 146 & & \\
\hline 7 & 73 & 17 & 54 & 7 & 141 & & \\
\hline 8 & 129 & 18 & 89 & 8 & 137 & & \\
\hline 9 & 86 & 19 & 111 & 9 & 128 & & \\
\hline 10 & 101 & 20 & 164 & 10 & 121 & & \\
\hline \multirow{4}{*}{\multicolumn{3}{|c|}{$\begin{array}{l}\text { A verage. } \ldots \ldots \ldots \ldots \ldots \ldots \\
\text { Variance. } \ldots \ldots \ldots \ldots \ldots \\
\text { Chi-square. } \ldots \ldots \ldots \ldots \ldots \\
\mathrm{P} \text { much less than } \ldots \ldots \ldots \ldots\end{array}$}} & 105.9 & \multicolumn{3}{|c|}{ Average. . . . . . . . . . . . } & .. 131.2 \\
\hline & & & 2913.9 & \multicolumn{4}{|c|}{ Variance.............. 151.1} \\
\hline & & & 550.3 & \multicolumn{4}{|c|}{ Chi-square . . . . . . . . . . 17.3} \\
\hline & & & 0.001 & \multicolumn{4}{|c|}{$\mathbf{P} \ldots \ldots \ldots \ldots \ldots \ldots$} \\
\hline
\end{tabular}

Table 1 shows the results of such an experiment with $E$. coli and streptomycin. In addition to the tests represented in the table, the concentration of bacteria was determined in 11 cultures, including the one from which the 15 samples were taken. The average number of bacteria in 10 cultures was $2.2 \times 10^{8}$ per ml, with extreme variants of 1.9 and 2.3 , and the average number in the eleventh was $2.1 \times 10^{8}$ per $\mathrm{ml}$. Thus the variation in numbers of bacteria among the different cultures was so small that it could have introduced only negligible differences between the numbers of resistant colonies observed on different plates. It is evident from table 1 that the variation in number of resistant colonies was considerably greater among platings from independent cultures than among platings from a single culture. The extreme variants of independent cultures were 48 and 291, the average 106, the variance 2,914, chi-square 550, and the probability that this variation was due to chance is insignificant. On the other 
hand, the variation in number of resistant colonies among platings of samples taken from one culture was very small; and the probability that this variation was due to chance is 26 per 100 trials. Very similar results were obtained in experiments using $S$. aureus and penicillin (Demerec, 1945a).

These results, then, favor the assumption that resistance to certain concentrations of penicillin or streptomycin originates through mutation, and that resistant bacteria may be found in any large population, the proportion depending on the mutation rate.

Oakberg and Luria (1947) reached identical conclusions after experimenting with $S$. aureus and sodium sulfathiazole. This suggests that mutations may be generally responsible for the origin of resistance that is transmitted to the offspring of the individuals that acquire it.

\section{RESISTANCE STEPS}

A very interesting feature of bacterial resistance to antibiotics is the stepwise increase in degree of resistance that can be brought about by selection. This feature is particularly well expressed in penicillin resistance. Figure 3 reproduces curves from an earlier paper (Demerec, 1945a) showing the effect of selection on the increase in resistance of $S$. aureus to penicillin. The first is the survival curve of the stock culture. At a concentration of 0.15 units per ml there were no survivors, but at a concentration of 0.12 units about 4 per $10^{8}$ bacteria lived. Firststep resistant strains were isolated from stock culture bacteria surviving sublethal concentrations. The second curve of figure 3 is a typical survival curve of such first-step resistant strains. Some individuals of these strains survived concentrations up to about 0.2 units. When first-step resistant strains were grown on sublethal concentrations of penicillin, second-step resistant strains were isolated from the survivors. A typical second-step survival curve is shown third on figure 3 . Third-and fourth-step resistant strains were obtained in similar manner.

It is of interest to note that the building up of resistance is more rapid with each selection step. Thus, with our strain of S. aureus, a concentration of 0.15 units was sufficient to eliminate all bacteria of the original strain, but a concentration of about 0.2 units was required to eliminate all bacteria of the first-step resistant strain, and concentrations of about 0.4 units for the second-step, 1 unit for the third-step, and 7 units for the fourth-step. The fifth-step strain was for all practical purposes completely resistant to penicillin, since it was not affected by a concentration of 250 units per $\mathrm{ml}$. With each step the increase in resistance appeared to be exponential.

Whereas the building up of resistance to penicillin followed a definite pattern, resistance to streptomycin showed a considerable degree of variability. Among first-step resistant strains-that is, among strains isolated from colonies of the original strain that survived sublethal doses of streptomycin-there were some that were only slightly more resistant than the original strain, some that were almost completely resistant, and some that fell between these two extremes (figure 4). It has been found that the variability in degree of resistance among 
first-step penicillin-resistant strains (Demerec, 1945b) is slight as compared with the variability observed among first-step streptomycin-resistant strains.

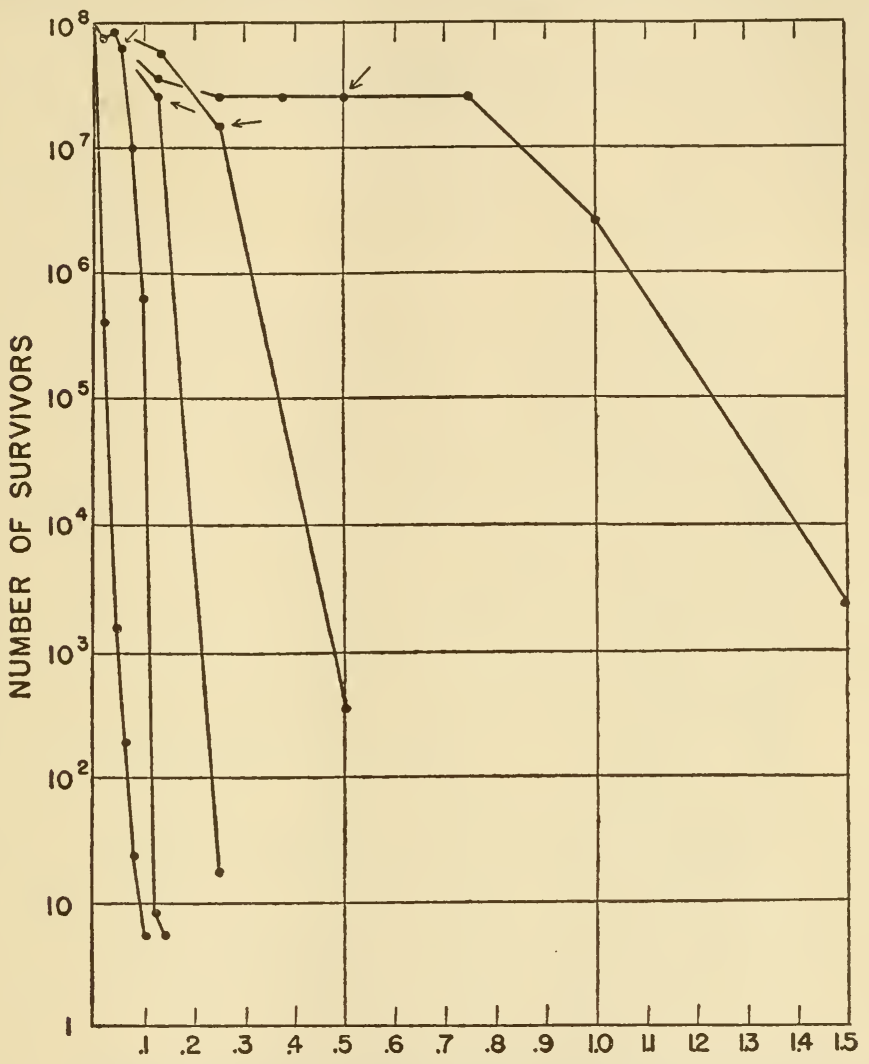

PENICILLIN CONCENTRATION: OXFORD UNITS PER ML

Figure 3. Stepwise build-up of resistance to penicillin in S. aureus. First from left, survival curve of stock culture; second, survival curve of first-step resistant strain isolated from a colony of the stock culture growing on the concentration indicated by the arrow. The other curves are of second-, third-, and fourth-step resistant strains isolated from colonies growing on the concentrations indicated by the arrows.

In the build-up of resistance to streptomycin, resistant strains of the second step, third step, etc., showed behavior similar to the first-step strains; that is, they exhibited a wide range of variability in degree of resistance. By plating bacteria of a low-resistance first-step strain on a concentration of streptomycin that is sublethal for that strain, one can isolate second-step resistant strains that 
vary in degree of resistance from only slightly more resistant than the original strain to very resistant. In fact, there is no difference in degree of resistance between the most resistant strains of the first step and those of the second, third,

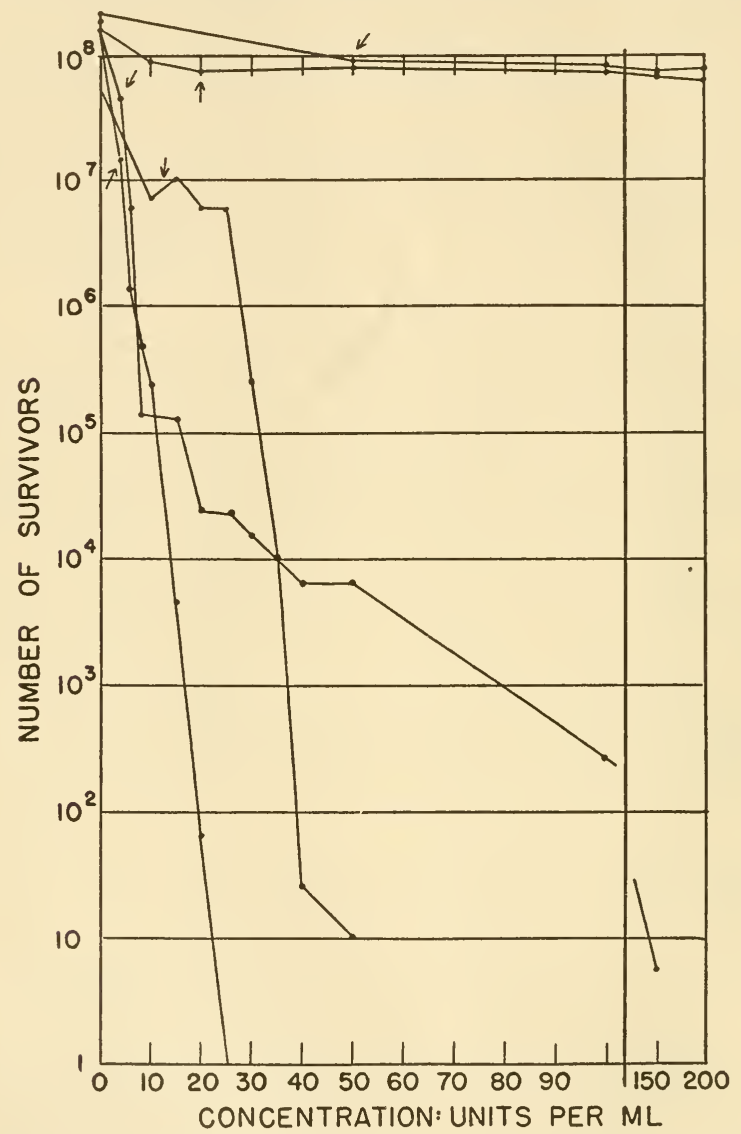

Figure 4. Survival curves of first-step strains of $S$. aureus resistant to streptomycin, showing the great variability in degree of resistance. Each strain was isolated from a colony of the stock culture growing on the concentration of streptomycin indicated by the arrow.

and higher steps. Consequently, strains that are highly resistant to streptomycin may be obtained either in one step, by selection of survivors of very high concentrations, or in several steps, by repeated selection of survivors from bacteria grown on increasingly higher concentrations. 


\section{POSSIBLE MECHANISM OF ORIGIN OF HIGH-DEGREE RESISTANCE}

The experimental evidence available at present indicates that resistance to penicillin and resistance to streptomycin are independent of each other; that such resistance is a heritable property induced by genetic changes comparable to mutations; that first-step penicillin-resistant strains are fairly uniform in their degree of resistance, a highly resistant strain being built up by selection through several steps; and that first-step streptomycin-resistant strains show a great deal of variability in degree of resistance, highly resistant strains being produced either in one step by selection from among first-step resistant mutants or in several steps by repeated selection of strains having higher and higher degrees of resistance.

What mechanism is responsible for the stepwise build-up of resistance, and for the difference between penicillin resistance and streptomycin resistance in this regard? Evidence accumulated by several investigators in recent genetical research with bacteria makes it appear reasonably certain that mutations in bacteria are caused by changes in genes. Granting this assumption, the complexity of behavior observed in studies of resistance to antibiotics indicates that several genes must be involved. Such an inference is not new. Several years ago Demerec and Fano (1945) suggested that the complex situation observed in their study of resistance of $E$. coli to 7 phages pointed to the presence of about 20 distinct mutant types in their material. Since many more than 7 phages affect the strain of coli investigated, and since it is reasonable to assume that extension of the study to these phages would reveal additional mutants, it is evident that the genetic background of resistance to phages is very complex indeed, and that it involves a considerable number of genes.

If a like situation exists in respect to resistance to antibiotics, then it can be assumed that many genes are instrumental in determining resistance to the two antibiotics used in these experiments, and that the genes affecting resistance to penicillin are different from those affecting resistance to streptomycin. If any one of these genes should mutate, the bacterium in which such a mutation occurred and the strain developed from that bacterium would be more resistant to the respective antibiotic than was the original parent strain. Such a strain would be what we have called a "first-step resistant strain."

The fact that first-step penicillin-resistant strains are fairly uniform in degree of resistance (Demerec, 1945b, figure 2) is consistent with the assumption that all genes affecting resistance to penicillin have a similar potency, so that the effect of mutation is the same regardless of which of the genes happens to mutate. According to this hypothesis, there is still present in a first-step resistant strain a number of unmutated genes that affect resistance. Mutation of any of these produces a second-step resistant strain, which possesses a higher degree of resistance than the first-step strain. Similarly, by mutation of another gene in a second-step resistant strain, a still higher degree of resistance is attained, characteristic of the third-step resistant strain. From this, by further mutations, highly resistant fourth- and fifth-step strains may be obtained. The curves in figure 3 indicate that the increase in degree of resistance with each step is ex- 
ponential. This means that the effect of two or more mutants together is considerably greater than would be expected from the added values of the effects of single mutants.

No attempt has been made to determine the frequency with which genes affecting resistance to penicillin mutate. It may be estimated from survival curves, however, that the mutation rate is low, in the neighborhood of $1 \times 10^{-8}$. With such a low mutation rate, it is evident that the increase in resistance must occur in successive steps, and that the chance that one step will be skipped is very slight, the chance that two steps will be skipped practically nil. (A step would be skipped when mutations in two genes occurred simultaneously in the same bacterium; and two steps would be skipped if three mutations occurred simultaneously in a single cell. The chance of two simultaneous mutations is $10^{-8} \times$ $10^{-8}=10^{-16}$, and of three simultaneous mutations, $10^{-8} \times 10^{-8} \times 10^{-8}=10^{-24}$. Since the volume of an $S$. aureus cell is about one cubic micron, it would be expected that one double mutant, on the average, would be found in ten liters of bacteria, and one triple mutant in one million cubic meters.)

The observed behavior of resistance to streptomycin also can be explained by assuming the existence of several genes determining such resistance. Unlike the genes for penicillin resistance, however, these differ greatly from one another in potency. If a gene of low potency mutates, the first-step resistant strain will have a low degree of resistance, but if mutation occurs in a highly potent gene, the first-step resistant strain will be highly resistant. Consequently, considerable variation in degree of resistance is to be expected between first-step strains; and for the same reason a highly resistant strain may be obtained either in one step, by selection of a highly resistant first-step mutant, or in several steps, by selection of mutants of low resistance values.

\section{CLINICAL CONSIDERATIONS}

A major consideration in the clinical use of antibiotics is how to avoid the development of resistant strains, since the usefulness of an antibiotic is closely related to the number of resistant pathogens and to their incidence in infections. For this reason, analysis of the mechanism of origin of resistance to penicillin and streptomycin has an important bearing on the clinical application of these antibiotics.

From the clinical standpoint, the situation in regard to penicillin is relatively simple and well defined. Since resistance develops in steps, and it is very unlikely that a step will be skipped in the process, the clinician can avoid development of resistant pathogens by using initial doses that are adequate for the elimination of first-step resistant individuals. Fortunately, most of the common pathogenic strains that have been investigated (North and Christie, 1945; Meads et al., 1945) are very sensitive to penicillin, so that large doses are not required in clinical use. It is equally important for the clinician to maintain the effective concentration in treatment as long as the infection persists, because decrease of the concentration below the effective level will permit the accumulation of first-step resistant bacteria, which may increase to a point that will allow the occurrence of 
second-step mutants. These are difficult to control. If there is any suspicion that a pathogen may be more resistant to penicillin than the usual strains, it is advisable to determine the degree of resistance before starting treatment, and to adjust the concentration accordingly. If adequate precautions are taken against the development of second-step resistant bacteria, there should be no danger from resistant pathogens in penicillin treatment. It is particularly important to avoid the indiscriminate use of penicillin, however, especially for applications where it can scarcely be of any help (for example, as a mouth wash), because there is positive danger that such use may stimulate the development of resistant strains.

In the clinical use of streptomycin, the situation can be controlled to a much smaller extent. Since highly resistant bacteria are found among the first-step mutants, treatment with high concentrations is not effective in eliminating the whole population of bacteria present in an infection. What it does accomplish is a reduction of the number of bacteria to a level with which the organism is capable of dealing. If for some reason the organism cannot do this, the chances for the development of a resistant strain are exceedingly good. Therefore, it must be expected that pathogenic strains resistant to streptomycin will frequently develop, in the course of time replacing sensitive strains in communities where streptomycin is used and rendering this antibiotic ineffective. This eventuality can be postponed by restricting the use of streptomycin to serious infections which cannot be controlled in any other way.

It may be appropriate to mention here the most effective way, theoretically, of preventing the origin of resistant strains of bacteria. This is the use in clinical treatment of a mixture of two antibiotics, when such are available, that affect the same pathogen but are independent in their actions. The evidence of independence is that bacterial strains that have developed resistance to one antibiotic are still sensitive to the other, and vice versa. If such a mixture of two antibiotics is used, then only bacteria that are resistant to both can survive the treatment and form first-step resistant strains. Such bacteria would be exceedingly rare. For example, if first-step resistant bacteria for each of two antibiotics should be found in a large population with a frequency of $1 \times 10^{-7}$, then the expected frequency of bacteria resistant to both these antibiotics would be $1 \times 10^{-14}$.

\section{SUMMARY}

A method is described that has been used to determine whether resistance to streptomycin is induced by interaction of the compound with bacteria or originates by gene mutation. Data are presented indicating that mutations are responsible for the origin of streptomycin resistance in Staphylococcus aureus. These agree with previously published data regarding the origin of penicillin resistance in the same organism.

The stepwise increase of resistance to penicillin by selection is explained by assuming that mutations in several equally potent genes are effective in inducing resistance, and that the slight degree of resistance characteristic of the first step is due to a mutation in one of these genes, the higher degrees of resistance of subsequent steps to successive mutations in other genes. 
The increase in resistance to streptomycin also can be explained by the assumption that several genes are instrumental in the process. These genes vary greatly in their potency, however, and consequently a mutation in a highly potent gene will be responsible for a high degree of resistance, a mutation in a less potent gene for a low degree of resistance.

From the knowledge gained concerning the mechanism of origin of resistance, it is concluded that in treatment with penicillin the development of highly resistant strains can be avoided by application of the penicillin in doses sufficiently large to prevent survival of first-step resistant mutants. In treatment with streptomycin, however, the development of highly resistant strains cannot be prevented; effective treatment does not eliminate all bacteria, but it probably reduces their number to a level at which the organism is able to eliminate them.

\section{REFERENCES}

Demerec, M. 1945a Production of Staphylococcus strains resistant to various concentrations of penicillin. Proc. Natl. Acad. Sci. U. S., 31, 16-24.

Demerec, M. $1945 b$ Genetic aspects of changes in Staphylococcus aureus producing strains resistant to various concentrations of penicillin. Ann. Missouri Botan. Garden, $32,131-138$.

Demerec, M., ANd Fano, U. 1945 Bacteriophage-resistant mutants in Escherichia coli. Genetics, 30, 119-136.

LuRIA, S. E., AND DelbRÜcK, M. 1943 Mutations in bacteria from virus sensitivity to virus resistance. Genetics, 28, 491-511.

Meads, M., Ory, E. M., Wilcox, C., and Finland, M. 1945 Penicillin sensitivity of strains of six common pathogens and of Hemophilus hemolyticus. J. Lab. Clin. Med., $30,725-729$.

North, E. A., ANd Christie, R. 1945 Observations on sensitivity of staphylococci to penicillin. Med. J. Australia, 2, 44-45.

OAKBerg, E. F., ANd LuRiA, S. E. 1947 Mutations to sulfonamide resistance in Staphylococcus aureus. Genetics, 32, 249-261. 


\section{Chloromycetin Resistance in E. coli, a Case of Quantitative Inheritance in Bacteria}

DRUG resistance in bacteria has been investigated genetically in several cases, since Demerec began with penicillin resistance in Staphylococcus aureus ${ }^{1}$. The proposal to distinguish two classes of drug resistance-those in which the top level of resistance can be obtained in a single step, and those in which it can be acquired only gradually-has clearly a practical importance; its theoretical meaning has not been entirely elucidated, since genetical analysis has several limitations in bacteria. In fact, while mutational patterns are open to investigation in most cases, crossings ean only be regularly undertaken, as in Lederberg and Tatum's original discovery ${ }^{2}$, in a few strains of $E$. coli. In one of these, $E$. coli $K-12$, some work on the genetics of drug resistance has been carried out, the most extensive investigation relating to streptomycin. Full resistance in $E$. coli is developed in a single step $\mathrm{p}^{3,4}$.

Besides resistance, dependence is known, whereby some resistant mutants fail to grow in absence of streptomycin. Full streptomycin-resistance and dependence $^{4,5}$ seem to be due to a single locus (or closely linked loci). Further, Lederberg introduced resistance to azide ${ }^{b}$ as a genetic marker; here a single step brings about a moderate degree of resistance, and the determining factor has been mapped, not only in this laboratory but also independently in two other laboratories, and found to be about midway between $V_{1}$ and $T L$ on the 'chromosome map' of $E$. coli $K \cdot 12$. Nitrogen mustard resistance was found to be gradual or abrupt in increase in different experiments, only a moderate degree of resistance being acquired, which made a detailed analysis difficult. In $E$. coli $K-12$ nitrogen mustard resistance is not accompanied by higher resistance to radiations, as in the case of $E$. coli B.

Completely 'gradual' increase of resistance has been found in the case of chloromycetin in $E$. coli $K-12$. Selecting for chloromycetin resistance by serial transfers in liquid cultures containing increasing concentrations of the drug (on a geometric scale), a rapid and relatively smooth increase of resistance is observed. Starting from a tolerated level of 5-10 $\mathrm{ugm} . / \mathrm{ml}$., as is usual with strains derived from the original $E$. coli $K \cdot 12$, levels of resistance as high as $1,280 \mathrm{gm}$. $/ \mathrm{ml}$. (which is not far from the solubility limit of chloromycetin) can be obtained. The increase

[Reprinted by permission of MacMillan \& Co. Ltd. from Nature 166 : 991, December 9 , 1950] 
in resistance is twofold or lower, on the average, at every transfer. Resistance is relatively stable on repeated subculture in the absence of chloromycetin.

The smoothness of increase of resistance might constitute a prima facie case for non-genetical adaptation; if, on the other hand, adaptation is genetical, a relatively large number of genes should be found affecting chloromycetin resistance, assuming that it is to be accounted for by known genetical mechanisms.

A cross was made between a $W 677$ strain $\left(T-L-B_{1}-L a c_{1}-V_{1}{ }^{r} \mathrm{Mal}_{1}-\mathrm{Gal}_{1}-\mathrm{X} y \mathrm{l}-\mathrm{Ara}-\mathrm{Mtl}-\right)$ which had been grown with $640 \mu \mathrm{gm} . / \mathrm{ml}$. chloromycetin in broth, and a fully sensitive 58-161 strain $(B-M-)$. Crosses were made with the usual technique introduced by Lederberg and Tatum². By this technique, recombinants are selected out of a mixture of parental cells using 'fixed' recombination markers, in this case capacity of growth in absence of given growth factors. Strain 58-161 can grow in the absence of threonine, leucine, vitamin $\mathrm{B}_{1}$, which are needed by $W 677$ for growth; strain $W 677$ can grow in absence of biotin and methionine which are needed for growth by 58-161. Plating the mixture of the two strains on minimal medium, where such growth factors are not supplied, parental cells will not develop, while recombinants of the right type can develop into visible colonies. The two strains differ by a number of other markers, lactose fermentation (absent in $W 677$, present in 58-161), reaction to virus $T_{1}\left(V_{1}{ }^{r}\right.$ indicating resistance in $W 677$, while $58-161$ is sensitive), etc., as indicated by the other symbols.

Such differences can be used as 'free' recombination markers in that they are not directly selected for or against in the minimal agar plates, and their segregation in the recombined colonies is used to build a "chromosome map"? The relative order of the genes which have been 'mapped' satisfactorily, and which are relevant for this and other crosses conducted luring the present work, is as follows: $B_{1}-(B, M)-V_{8}-L a c_{1}-V_{1}-A z-(T, L)$, where $V_{8}$ refers to virus $T_{\theta}$ resistance, $A z$ to azide resistance.

Recombinants between the chloromycetin-resistant $W 677$ and sensitive 58-161 showed a scatter from full sensitivity to nearly full resistance, the resistance of each recombinant being roughly proportional, on the average, to the length of the chromosome segment which-as could be inferred from recombination markers-given recombinants got from the resistant parent. A closer analysis shows one and possibly two loci for resistance in the segment between $B M$ and $L a c$; one locus at least between $L a c$ and $\nabla_{1}-$ 
there is evidence that other loci, too, must take part in building full resistance. These data are therefore consistent with the hypothesis of many loci with cumulative action.

First-step resistants, obtained by both plate and test-tube selection methods, were analysed in crosses of sensitive with resistant and resistant with resistant, and they showed a clear-cut segregation of sensitivity or resistance, within experimental error. Mapping loci of first-step resistance, the loci:s most frequently met so far was closely linked to $V_{6}$. In this way, a cross with four free markers between $B M$ and $T L$ (fixed recombination markers) was made, involving chloromycetin resistance, $V_{8}, L a c$ and $V_{1}$, giving a relatively satisfactory test of linearity of the chromosome region between $B M$ and $T L$. First-step resistants were also found which gave no resistant recombinants when crossed to sensitive, probably because of close linkage with fixed recombination markers ( $B M$ in this case). The interactions in resistance between different loci are under investigation at present. In fact, this case seems to offer unusual possibilities of a detailed analysis of the interactions between genes in quantitative inheritance. The stability of the drug is a factor of no little importance in facilitating quantitative work with chloromycetin.

The K-12 strains used in this research have been kindly sent by Dr. J. Lederberg. One of us (G. A. M.) has been supported during this work by a grant from the Rockefeller Foundation.

L. L. Cavalui

G. A. Macoaonio

Department of Genetics,

Whittingehame Lodge, 44 Storey's Way,

Cambridge.

Aug. 15.

1 Demerec, M., Ann. Miss. Bot. Gard., 82, 131 (1945).

- Lederberg, J., and Tatum, E. L., Nature, 158, 558 (1946).

Newcombe, H. B., and Hawirko, R., J. Bact., 57, 565 (1949).

' Demerec, M., Amer. Nat., 84, 5 (1950).

- Newcombe, H. B. (unpublished).

- Lederberg, J., J. Bact., 59, 211 (1950).

- Jederherg. J., Genetic8, 32, 505 (1947). 


\title{
Studies on Nutritionally Deficient Bacterial Mutants Isolated by Means of Penicillin
}

\author{
By Bernard D. Davis², New York, N.Y.
}

Studies on the mode of action of chemotherapeutic agents have not shown any direct effect on the known ratabolic processes of bacteria. There is good reason, by exclusion, to suppose that they act by means of interfering with anabolic reactions, about which much less is known. In the last decade, BE.ADLE and his school have made striking contributions to this area in biochemistry, as well as to genetics, by the use of nutritionally deficient ("biochemical") mutants of the mold, Neurospora-mutants which have lost one or another specific enzymic activit ${ }^{3}$. More recently, bacteria have been found to yield mutants of the same sort ${ }^{4}$. These microbial mutants offer a promising approach to the major theoretical problems of chemotherapy: the mechanism of chemotherapeutic action and the biochemical alterations responsible for drugresistance in microorganisms. Furthermore, the phenomenon of drug-resistance presents a direct problem in microbial genetics. Finally, just as the nutritional requirements of various wild-type microorganisms have been of tremendous value in isolating the known vitamins, so the extension of this approach to the induction of artificial nutritional requirements offers promise as a method for detecting metabolites peculiar to microorganisms - metabolites of special promise as models for the construction of inhibitory analogues. For these several reasons this laboratory, interested in chemotherapy, has felt warranted in undertaking an excursion into microbial genetics.

It is a simple matter to isolate, from huge microbial populations, rare mutants that can survive or proliferate in a medium which suppresses the parent strain. Examples include mutations to drug-resistance, to bacteriophage-resistance, or to decreased nutritional requirements. Comparable techniques have not been available for the nutritionally deficient mutants, though these are in some respects more interesting. In

1 Based on material delivered in a-symposium on The Relation of Genetics to Biochemistry at the annual meeting of the American Chemical Society in San Francisco, Cal., March $30,1949$.

2 I. S. Public Health Service, Tuberculosis Research Laboratory, Cornell University Medical College, New York 21, N. Y. - This paper is in the hands of the editor since June 16 th, 1949.

3 G. W. Beadle, Physiol. Rev. 25,643 (19.15); Chem. Rev. $37,15(1945)$

4 E.I..TAтU M, Cold Spring Harhor Symp. Quant.13iol, I I,278 (1946). the early investigations, deficient mutants were isolated by testing of spores or colonies selected at random a tedious process, since the total frequency of recognizable mutants, even after optimal irradiation, rarely exceeds 1 or 2 per cent. Improved techniques of isolation have recently been introduced for Neurospora ${ }^{1}$ and other molds ${ }^{2}$, and for bacteria ${ }^{3}$. In the case of the bacteria, the techniques depended upon delayed or limited enrichment of the medium, with consequent production of small colonies of mutants which could be distinguished from the larger colonies of the parent strain. Since the colonies must not be too crowded, these techniques were limited to a few hundred colonies per Petri dish.

\section{The penicillin method}

For the isolation of rare mutants, a method permitting selection from much larger populations would be desirable. The possibility of such a method suggested itself on the basis of the report that penicillin has the remarkable property of sterilizing ("killing") bacteria only under conditions which permit growth ${ }^{4}$. We have confirmed and extended this conclusion. In a minimal medium containing glucose, lactate, ammonia, and sulfate as sole sources of carbon, nitrogen, and sulfur, various amino acid-requiring mutants of $E$. coli are completely resistant to the bactericidal action of penicillin. The addition of the required amino acid however, renders the mutant as sensitive to penicillin as its parent wild-type strain. In applying this method to the isolation of new mutants, a suspension of $E$. coli was irradiated with ultraviolet light, further cultivated in an enriched medium, washed, and then incubated, in inocula of suitable size, with penicillin in.minimal medium for 24 hours. Up to 100 p. c. of the large num. ber of survivors, recovered by plating in agar media supplemented with casein hydrolysate or yeast extract were found to be nutritionally deficient mutants. The penicillin method has been independently developed in

1 J. I.eiN, H. K. Mitchell, and M. 13. HoUlahan, J'toc. Nat. Acad. Sci. 34, 435 (1948).

2 N. Fries, Nature $159,199(1947)$.

3 I. Lederberg, and E. L. TAtum, J. Biol. Chem. 165, $3 \times 1$ (1916). - B. D. Davis, Arch. Biochem. 20, 166 (1949).

4 G. L. Новвr, li. Meyer, and E. Chaffee, Proc. Soc. Exp. Biol. Med. So, 281 (1942).

[Reprinted by permission of Verlag Birkhauser, Basel, from Exirkilntia 6 : (2) 41-50, 1950] 
this laboratory ${ }^{1}$ and by LEDERBERG and Z1NDER ${ }^{2}$. After isolation, the growth requirement of a mutant can be sinply determined by distributing tiny drops of solutions of nutrilites on the surface of a heavily seeded pour plate ${ }^{3}$ (BEIJFR1NCK's " auxanography”).

Since the publication of this method, a modification has been introduced which substantially improves its efficiency: Instead of inoculating the washed organisms directly into minimal medium containing penicillin, the inocula are placed in a medium lacking nitrogen. After + to 6 hours of incubation, during which dissimilation of glucose promotes the exhaustion of stored metabolites, the minimal medinm is completed by addition of ammonium sulfate. Penicillin is added, and the procedure is continued in the usual way. The period of dissimilation particularly improves the recovery of certain vitamin-requiring mutants.

Although the penicillin method provides a marked improvement in the efficiency of isolating mutants, it nevertheless has serious limitations. The population density cannot be indefinitely large, as is possible with the drug-resistant mutants, for at population densities above $10^{5}$ to $10^{7}$ cells per $\mathrm{ml}$, the non-mutants release enough of various metabolites ("syntrophism"; cf. ${ }^{4}$ ) to permit certain mutants to grow slightly and hence be sterilized by penicillin. In addition, even at low population densities the recovery of mutants is not quantitative. Finally, the use of this method for estimating mutation rates is limited by the requirement of a stage of intermediate cultivation between the irradiation and the exposure to penicillin, in order to permit phenotypic expression of the induced mutation. During this cultivation, the distribution of the population will undoubtedly be distorted.

\section{Delayed phenotypic expression}

The requirement of a certain amount of growth for the phenotypic expression of an induced mutation is itself an interesting point, and has also been reported for phage-resistance ${ }^{5}$. The explanation, at least with the nutritionally deficient mutants, appears to be that the cell must undergo some growth, possibly several generations, before the premutational products of the inutated gene (enzymes; possible intermediates between genes and enzrmes) are exhausted. Only then will the pattern of enzrmes in the cell correspond to the new pattern of genes. For this particular mechanism of delayed phenotypic expression we have proposed the term "plienomic delay" 6 , the "phenome" being

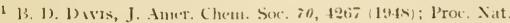
Aead. Sici. 35,1 (1!) 191$)$.

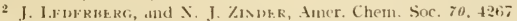
$(1 ! 1)$,

3 J. Lenerberg, J. Bact. jz, ju3 (1:4ti). - (3. Poxifcorvo, I. (ien. Nirrobiol. 3, $122(1949)$

4. Leverberg, J. Bact. 52, 503 (191ti)

5 i. Di:MEREC, and R. L.ITARJET, Cold Spring Harbor Symp Quant. Biol. 11, 3N (1916).

6 I3. I) Divis, Proc, Sat, Acacl Sci. 35, 1 (191! defined as the total non-self-reproducing part of the cell, under the control of the self-reproducing genes. The occurrence of the same phenomenon in mutations in the reverse direction will be discussed later.

Other possible explanations of the requirement of intermediate cultivation in the penicillin method include segregation of mutant and non-mutant nuclei from a multinucleate cell, and a sintrophic effect of the non-viable irradiated bacteria, which would promote sterilization of mutants by penicillin. Evidence will be published elsewhere that neither of these mechanisms furnishes an adequate explanation, while the phenomic delay accounts for all the available facts. A similar conclusion was reached by Newcombe in a thorough analysis of delayed phenotypic expression of phage resistance ${ }^{1}$.

\section{Biochemical advantages of bacteria}

Bacteria are less easily studied genetically than molds such as Neurospora, which ean be made to multiply sexually or asexually at will. While genetic recombination in bacteria has recently been demonstrated by TATLY and LEDERBERG ${ }^{2}$, it apparently occurs in only a few strains and a tiny proportion of the population. For biochemical investigation, however, bacteria appear to have several advantages. Not only can a variety of mutants be isolated relatively quickly: but it is possible to demonstrate very simply, by the syntrophic interaction of adjacent streaks on solid media, the instances in which one mutant accumulates a metabolic intermediate which is utilized as a nutrilite by another mutant. Metabolite accumulations, when present, are extremely useful in analysing biosynthetic pathways ${ }^{3}$.

A further advantage of bacteria is the uniformly dispersed growth of certain species in liquid media, which permits simple and precise quantitative experiments, using colony counts for low population densities and turbidimetry for high densities. On solid media, the production of uniform colonies has made possible a variety of experiments involving prolonged cultivation, without risk of confusion fron back-mutants, which are readily distinguished from the rest of the population. In addition, slight variations in colony size and syntrophism have made it possible to recognize unexpected phenomena which might easily have gone unnoticed in a mycelial mat. Finally, in relation to chemotherapy, the metabolism of bacteria is of particular interest. Although some of the problems to be described here are still under investigation, it seems desirable to illustrate at this time the trpes of phenomena that can be revealed by these primitive techniques, especially. by the test for syntrophism. This effect has been long

I H, L. Newcombe, Geneties 33,447 (1!14

2 E. I. TATLM, and J. LEDFRBERG, J. Bilct. 5.7, $1773(1947)$. J. LEDERBERG, Genetics 32,505 (1917).

3 X. H. Hortswirz, J. Biol. Chem. 162, 412 (1916). 
known in microbiology as the satellite phenomenon; systematically employed, it has been our vade-mecum.

\section{Mutants obtained}

Mutants of E. coli ("Waksman" strain", Amer. Type Culture Collection 9637) have been obtained with requirements for all the naturally occurring amino acids except alanine, aspartic acid, and hydroxyproline. A number of mutants respond to either serine or glycine; thus far, none of our strains has been specific for either of these interconvertible amino acids. The sulfurdeficient mutants, which are a very common class, respond to cystine or less rapidly to methionine, and are blocked in the reduction of sulfate to sulfite or of sulfite to thiosulfate or sulfide. In addition, there are mutants, unresponsive to thiosulfate or sulfide, with specific requirements for cystine and others for methionine. Besides specific proline-requiring mutants, there are others which respond to either proline or glutamic acid or $\alpha$-ketoglutaric acid (but not ornithine).

Many of these types of mutants have already been isolated from $E$. coli by earlier techniques ${ }^{2}$. In addition, we have isolated strains with more complex requirements which should throw light on certain metabolic relationships. Alternative requirements exist for lysine or threonine, and, in another mutant, for $\alpha$-amino butyric acid or isoleucine (or, curiously, D-threonine but not L-threonine). One peculiar mutant responds either to methionine or to thiamine: to methionine in the concentrations of several micrograms per ml usual for amino acid mutants; to thiamine or its pyrimidine in the concentrations, one thousandth as great, required by other thiamine mutants. Another mutant similarly requires, under special conditions, either methionine or vitamin B-12. Finally, there are several mutants with a multiple requirement apparently due to a single genetic block: isoleucine plus valine; phenylalanine plus tyrosine; phenylalanine plus tyrosine plus tryptophan; and these three aromatic amino acids plus $p$-amino benzoic acid. No peptiderequiring mutants have been obtained; although much of our isolation work has been done with tryptic casein hydrolysate, which contains many peptides, all the mutants isolated from this enrichment have grown on a mixture of known amino acids. One serine or glycine mutant and one methionine mutant, like several reported Neurospora mutants, are temperature sensitive, with an absolute requirement at $37^{\circ} \mathrm{C}$, and none at $25^{\circ} \mathrm{C}$.

With yeast extract or hydrolysed yeast nucleic acid, mutants have been obtained with requirements

\footnotetext{
1 The initiation of this work with the "Waksman" strain has leen accidental. For any new program, it would undoubtedly be preferable to use the K-12 strain of $\mathrm{K}$. coli, with which genetic recombination can be studied ${ }^{3}$

2 E. I. Tatu M, Cold Spring Harbor Sy'mp. Quant. Biol. 11, 278 (15.16). - 13. I). DAvis, Arch. Biochem. 20, 166 (1949)

3 E. I.. TATUM, and J. L.EDFRnFR, J. Bact. 53, 673 (1947)
}

for purines or pyrimidines. The purine mutants however, have all responded to adenine or guanine or hypoxanthine or their ribosides or nucleotides, while several have also responded to xan thine ; the pyrimidine mutant has responded to cytosine or thymine or uracil or their ribosides or nucleotides. Because of this nonspecificity, this group has not been further studied.

Among the vitamins, mutants have been obtained with individual requirements for thiamine, nicotinamide or nicotinic acid, pyridoxin or its amine or aldehyde, $p$-amino benzoic acid (PABA), pantothenic acid, and biotin. In spite of a number of attempts, none have been obtained with requirements for riboflavin, inositol, choline, or hemin. The reason for these failures is not apparent. One mutant requiring an unknown factor in yeast extract has been obtained twice.

\section{Microbiological assay}

We have not engaged in extensive studies on the use of these mutants for microbiological assay. Since the medium employed is so simple, mutants might be expected to have some advantage over the wild-type species with complex requirements which are generally used. In addition, turbidimetric assay of bacterial growth is more convenient than the measurement of dry weight of pellicle which is required with Neurospora mutants ${ }^{1}$. The advantage of the mutants would be largely lost, however, if a heavily enriched medium were required in order to prevent other substances present in the material under assay from altering the quantitative response to the required nutrilite. Since secondary effects of other substances have been observed in many instances with wild-type organisms, it would be necessary with each mutaint to determine the conditions for assay. Furthermore, the instability of most of the mutants requires caution. With several mutants of ordinary stability (phenylalanine, tyrosine) we have been able to obtain satisfactory growth curves, without significant appearance of reversions, at 24 hours, but by 48 hours irregular increases in turbidity due to reversions had appeared.

\section{Syntrophism}

The syntrophic technique is illustrated with three arginine-requiring mutants which are shown in Fig. 1 to be blocked at different stages in the well-known Krebs-Henseleit scheme: ornithine $\rightarrow$ citrulline $\rightarrow$ arginine. Sets of mutants of both Neurospora and $E$. coli, blocked at these stages, had previously been reported ${ }^{2}$, having been recognized by their response to these precursors of arginine, In the case of growth factors whose precursors are unknown, however, and which

1 F. J. Ryan, Feder. Proc. 5, 366 (19.16).

2 A. M. SRB, and N. H. Horowitz, J. Biol. Chem. 154, 129 (1944).

R. R. ROEPKE, quoted by E. L. TATUM, Cold Spring Harbor Symp. Quant. Biol. 11, 278 (1946). 
are therefore the most interesting, this technique of substituting precursors cannot be used, except by guesswork, to recognize differences in the site of genetic blocks. For this reason, the alternative technique of syntrophism is particularly useful. This

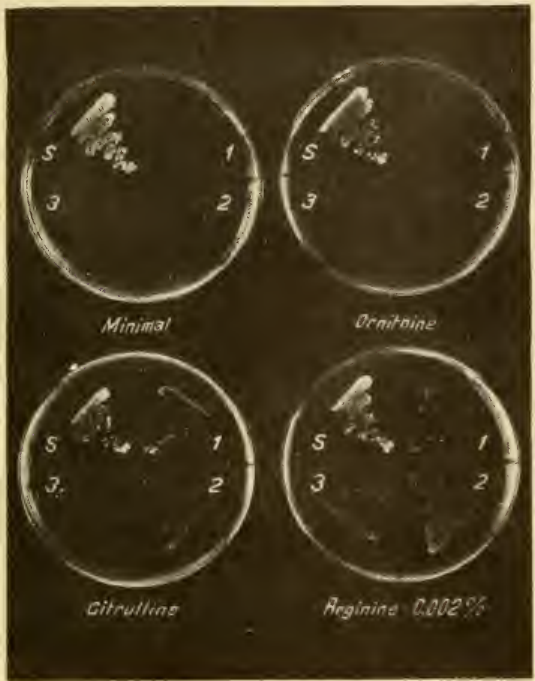

Fig. 1. - Growth of mutants and wild-type ( $S$ stock) on minimal medium enriched with ornithine, citrulline, or arginine. It is seen that one mutant responds to any of the three related compounds, another to eitber citrulline or arginine, the third to arginine only.

technique is shown in Fig. 2, in which the mutant specifically requiring arginine is seen to excrete a factor, presumably citrulline, which stimulates the growth of the two mutants blocked earlier; while the citrulline mutant in turn feeds the ornithine-requiring mutant. The gradient of observed growth reflects the gradient of diffusion through the agar.

Similar relationships, involving unknown precursors, have been observed among proline and among histidinerequiring mutants and among certain mutants requiring aromatic amino acids. Syntrophic accumulation of precursors appears to be quite widespread with $E$. coli and has been observed among sets of mutants (e. g. arginine) which fail to show accumulation in Neurospora. Its absence between two mutants with a common requirement, however, does not prove that they are blocked at the same enzymic site, since precursors may fail to accumulate because of instability, diversion along an alternative path, or the inability of the cell to build up a concentration adequate for excretion.

\section{Causes of syntrophism}

Since syntrophism leads to recognition of the accumulation of a precursor whose subsequent isolation and identification will contribute to the analysis of a biosynthetic chain, it becomes important to be aware of other possible sources of this phenomenon. Several have so far been observed.

(1) Accumulation of the precursor of a genetically blocked reaction. This mechanism is the one discussed above.

(2) Excretion of metabolites by wild-type E. coli. It is known that bacteria not only remove nutrilites from the medium, but contribute metabolic products to it. In order to find whether these include growth factors for any available mutants, the wild-type strain was streaked on minimal medium adjacent to mutants with single requirements for each of the factors listed above. After 48 hours, three of the mutants were so heavily fed as to produce maximal growth. These were the strains requiring biotin, PABA, and pantothenic acid. In addition, the nicotinamide-less mutant was moderately fed. The other available vitamin-requiring mutants (thiamine and pyridoxin), as well as a purine, a pyrimidine, and all the amino acid-requiring mutants, showed that the amounts of their required factors excreted by wild-type were negligible, supporting at most only microscopic growth after 2 to 5 days. It is concluded that this strain of $E$. coli is economical in its synthesis of all the growth factors that could be tested except four vitamins. It is of interest to note, however, that the amounts of the other factors excreted, at

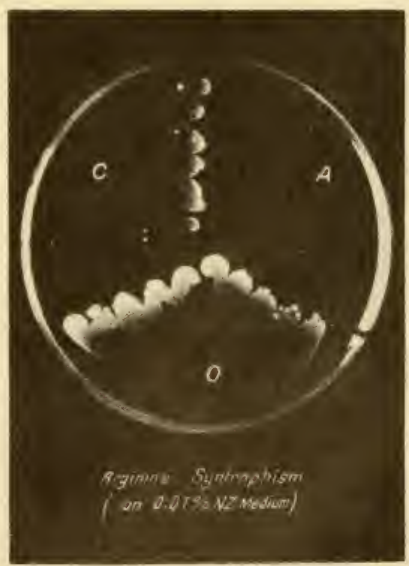

Fig. 2, - Syntrophism among arginine-requiring mutants of Fig. 1. Mutant $O$ responds to ornithine or citrulline or arginine; $C$ responds to citrulline or arginine; $A$ responds to arginine only. 48 hours of growth $\left(37^{\circ} \mathrm{C}\right)$ on medium enriched with very small amount of casein hydrolysate ("NZ Case"). 
least in the presence of penicillin are sufficient to limit the permissible population density in the penicillin method of mutant isolation; this procedure is apparently much more sensitive to traces of syntrophism than is the technique of adjacent streaks.

(3) ionversion of a precursor. It has been observed that wild-type, growing on an excess of the keto acid precursor of isoleucine, will feed a mutant that responcis to isoleucine but not to its keto acid. The same conversion and excretion is carried out by a mutant, blocked earlier, which can use this precursor as well as isoleucine itself. It has also been observed with wildtype acting on certain other precursors. In some reactions, however, such as the conversion of ornithine or citrulline to arginine, it has not been possible with an excess of either precursor to stimulate the excretion of arginine by wild-type or by a mutant. It is evident that the organism possesses more than one type of mechanism for determining the rate of synthesis of an amino acid; in the case of isoleucine, the capacity for amination of the keto acid exceeds the requirement. and hence cannot be the rate-governing mechanism

$$
\begin{aligned}
& \mathrm{A} \rightarrow \text { specific thiazole } \\
& \mathrm{B} \rightarrow \text { specific pyrimidine } \\
& \mathrm{C} \rightarrow \text { pantonine } \rightarrow \text { pantoic acid }>\text { thiamine } \\
& \mathrm{D} \rightarrow \text {-alanine }
\end{aligned}>\rightarrow \text { pantothenic acid }
$$

Fig. 3. - Synthesis of thiamine and pantothenic acid. Blocked arrows represent sites of genetic blocks.

(4) Excretion of a lone conjugant. Thiamine is composed of two moieties, a substituted thiazole and a substituted pyrimidine (Fig. 3). Thiamine-less mutants were obtained which respond respectively to the pyrimidine, to the thiazole, and to neither (implying a deficiency in the conjugation of the two); a fourth type. whose site of genetic deficiency is not readily interpreted, responds to the specific thiazole plus pyrimidine, as well as to thiamine itself, but not to either moiety alone. Tests for syntrophism showed that the thiazole-less mutant freds the pyrimidine-less mutant, but not vice versa. Mutants with similar blocks and accumulations have been observed with Neurospora ${ }^{1}$.

Another vitamin, pantothenic acid, also consists of two components, pantoic acid and $\beta$-alanine (Fig. 3). IV. K. MA.As in this laboratory has isolated various pantothenic acid-less mutants which respond respectively to pantoic acid or pantonine; to $\beta$-alanine; to pantothenic acid only; and to a mixture of pantoic acid and $\beta$-alanine. The pantoic-less mutant feeds the $\beta$-alanine-less mutant, but not vice versa.

In both these instances of syntrophism, the cause of the accumulation of the intermediate is not absence of

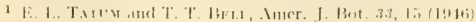

the enzyme concerned with its further conversion, but inability of that enzyme to perform the conversion in the absence of the second conjugant.

(5) Release of physiological brake on a synthetic process. One of the most interesting observations to turn up is mutual sintrophism between certair: tyrosine-less and phenylalanine-less mutants. Paper chromatography confirmed the inference that the block in phenylalanine synthesis resulted in excretion of tyrosine itself, rather than a precursor, while a block in tyrosine synthesis caused excretion of phenylalanine. Feeding of a tyrosine-less mutant by culture filtrates of a phenylalanine-less mutant has also been observed with another strain of $E$. coli ${ }^{1}$.

From the presence of mutants with a double repuirement for these two compounds, as well as others with triple and quadruple requirements for these plus other aromatic compounds, all resulting from single mutations, it had been inferred that phenylalanine and tyrosine arose from a common precursor (Fig. 4). A possible explanation for the mutual syntrophism therefore appeared to be the diversion of this precursor in one rather than the normal two directions, with resultant excretion of the excess of phenylalanine or tyrosine. Further study, however, showed this explanation to be inadequate, as the amount of phenylalanine (or tyrosine) excreted was much larger than the amount of tyrosine (or phenylalanine) consumed. Since the requirements of mutants of $E$. coli for these two compounds are of the same order of magnitude, simple diversion could not account for the large production.

An alternative explanation has been developed, stimulated largely by the interesting work of BONNER ${ }^{2}$. He showed that a single block in isoleucine synthesis, between the $\alpha$-keto and the amino acid, accounted for the double requirement of a Neurospora mutant for isoleucine and valine. Apparently the accumulated isoleucine precursor competes as a structural analogue with the corresponding compound in the valine-chain, causing a requirement for this amino acid as well:

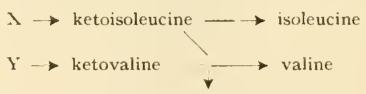

Recently AdELBERG ${ }^{3}$ has found that the compound accumulated by this mutant is not ketoisoleucine, but is rather the $\alpha$ - $\beta$-dihydroxy acid. The principle of internal inhibition by a normal metabolite, however remains unchanged.

This evidence of inhibition of a normal reaction by increased concentration of a normal metabolite has seemed to us to point to the possibility of a general mechanism of integration of various parallel sequences

1 S. SHMMoNbs and J. S. FrtTon, personal communication.

2 D. Bonner, J. Biol. Chem, 166, 5.15) (19.16).

3 1:. A. AnF 13k RG, personal comumunication 
of biosynthesis. If the accumulation of normal metabolite $\mathrm{A}$ in excessive concentration completely blocks a certain enzymic reaction in the production of compound $\mathrm{B}$, then the normal concentration of metabolite A might exert a governing effect on that reaction, while a complete absence of metabolite $\mathrm{A}$, resulting from a genetic block, might permit excessive synthesis of compound B, out of proportion to the remainder of the metabolites being synthesized, with resulting excretion of compound $\mathrm{B}$.

To apply this concept to the present problem, we would postulate a tyrosine precursor which interferes with phenylalanine synthesis, and vice versa. The excretion of plienylalanine would therefore be due to a block early enough in the synthetic chain of tyrosine to cause absence of that tyrosine precursor which normally governs the rate of phenylalanine synthesis, and the same consideration would apply to the phenylalanine mutant which secretes tyrosine. On the other hand, there is one tyrosine mutant which fails to feed phenylalanine; its block would occur after the governing intermediate ( $E$ in Fig. 4). This scheme is strongly supported by the fact that the excretion of phenylalanine by the tyrosine mutant is prevented in the presence of an excessive concentration of tyrosine; the same is true of the phenylalanine mutant whose excretion of tyrosine is inhibited by an excess of phenylalanine or phenylpyruvic acid, which can be substituted for phenylalanine as a growth factor for this mutant. Presumably the excess of the amino acid causes reversal of the normal processes of synthesis, restoring from without the governing compound whose synthesis is genetically blocked.

This scheme for explaining the output of phenylalanine and tyrosine is on speculative grounds, since the intermediates in the synthesis of these amino acids (except for phenylpyruvic acid) are as yet unknown. The concept of normal physiological interaction among separate biosynthetic paths, however, is further strengthened by returning to the better documented isoleucine-plus-valine case. We have isolated a mutant of this type with $E$. coli whose mechanism appears to be identical with that of the similar Neurospora mutant. In addition, another $E$. coli mutant has been isolated which responds equally well to isolencine, its $\boldsymbol{\alpha}$-keto acid, or $\boldsymbol{\alpha}$-amino butyric acid. The doublerequiring mutant, which cannot use ketoisoleucine, is known to accumulate an inhibitor of valine sinthesis : the single-requiring mutant, which can use ketoisoleucine, and which is fed by the double, must be blocked earlier; it would therefore follow, from the reasoning outlined above, that the single-requiring mutant should not form the isolencine intermediate that governs valine synthesis, and hence might be expected to excrete valine. On testing, this mutant was indeed found to feed a valine mutant heavily, and paper chromatography on the culture filtrate showed a dense spot corresponding to valine. While in this case, as in those of tyrosine and phenylalanine, neither microbiological assay nor paper chromatography alone furnishes complete proof that the compound excreted is the amino acid rather than a related compound, it seems unlikely that the non-specificity of these two techniques would overlap to give a false answer when used together.

In several instances, therefore, interference with synthesis of one amino acid leads to excessive synthesis of another. This evidence encourages us to feel that the mutants lend themselves not only to determining the normal steps in biosynthesis, but also to unravelling the mechanisms of integration by which the normal cell determines that its metabolites should be synthesized in proper proportions and not wasted. Recent experiments have shown that this economy of the organism is upset in diverse ways by a single mutation. A tyrosine mutant, for example, not only excretes phentlalanine, but also, to a smaller degree, feeds the mutants requiring tryptophan, lysine, valine, and leucine. This type of study has just begun, but it is already clear that the interrelations between various biosynthetic paths are complex (Fig. 4).

\section{l'recursors of aromatic compounds}

The mechanism of biosynthesis of aromatic compounds has long been a subject of speculation. The availability of mutants with multiple aronatic requirements (Fig. 4) offers an opportunity to obtain definite information on this matter. A wide variety of aromatic compounds, with single and multiple sub-

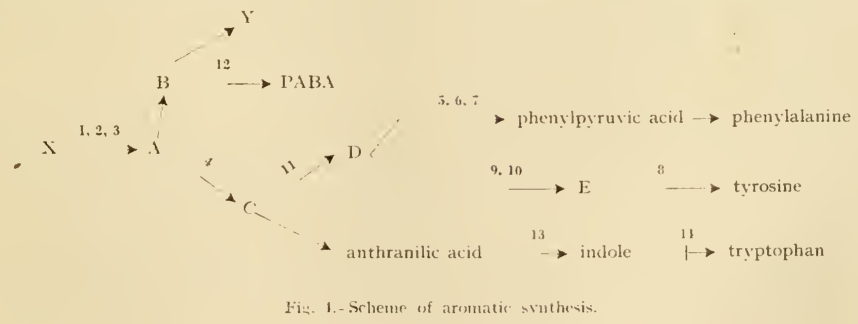


stitutions of carboxyl, amino, and phenolic groups, were tested for their capacity to substitute for the multiple requirements of the aromatic-less mutants. None of these was effective. Similarly cyclohexane carboxylic acid, cyclohexanol, and inositol were not utilized. Shikimic acid, however (suggested and furnished by R. STANiER) was used by aromatic-less mutants with quadruple, but not with triple or double requirements.

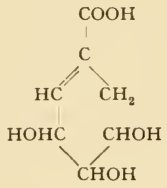

shikimic acid

It therefore appears that the precursor of the benzene ring is at least partly saturated. These mutants do not use quinic acid, in which the double bond of shikimic acid is hydrated.

It will be noted that Fig. 4 contains an unknown "end-product", presumably aromatic, labelled Y. The evidence for the existence of this postulated compound is as follows. (a) The aromatic-less mutants with quadruple requirements require more $\mathrm{PABA}$ than does the PABA mutant, and their growth is accelerated by high concentrations of PABA, suggesting that the synthesis of PABA may be reversed, to yield a precursor common to PABA and an unknown compound. (b) Growth of the aromatic-less mutants on large amounts of the four aromatic compounds is slow; similarly, growth on shikimic acid alone is slow. But shikimic acid plus the three amino acids yields much faster growth, practically as fast as wild-type. These results suggest that shikimic acid may occupy position $B$, rather than $A$, serving rapidly as a precursor of $\mathrm{Y}$ plus PABA (for which PABA alone serves only slowly), but serving only slowly, by reversal of the normal process, as precursor for the amino acids.

The scheme of Fig. 4 must be considered quite tentative. Certain phenomena are difficult to explain, such as the fact that mutants with a double aromatic requirement (tyrosine plus phenylalanine) heavily feed the quadruples, while the triple neither feeds the quadruples nor is fed by the doubles. One must therefore consider more seriously the possibility, always theoretically present, that some of these multiple requirements may depend on internal inhibition, as with isoleucine and valine, rather than on block at an early stage of synthesis.

\section{Partial back-mutants}

Almost all the mutants are detectably unstable; that is to say, spontaneous reversions to nutritional independence (prototrophs) occur with a high enough frequency $\left(10^{-7}-10^{-8}\right)$ to give rise after several days to a few large colonies of back-mutants in a streak on a medium which is sufficiently enriched to permit limited growth (cf. Fig. 2). For this reason practically all of our experiments, even on quantitative response, are carried out on solid media; in tubes of liquid media the greater precision of measurement by turbidimetry is accompanied by greater difficulty in distinguishing growth of back-mutants from that of parent mutants. Maintaining transfer cultures of mutants in liquid media, however, has caused no difficulty provided selection of back-mutants is avoided by the presence of an excess of the nutrient requirement.

Our study of back-mutants had a casual origin which is pointed out here since it illustrates the possibility, based on the uniform colonial growth of bacteria, of encountering interesting phenomena by simple observations on plates. Two presumptive back-mutant colonies from a PABA-less mutant were isolated and streaked on minimal medium, along with wild-type, to verify their nutritional independence. One of these grew as rapidly as wild-type; the other was by chance observed at an early time (18 hrs.) to form slightly smaller colonies than wild-type. Further study showed that the slightly slow prototroph grew as rapidly as wildtype in a medium supplemented with PABA; it apparently had recovered the capacity to synthesize PABA, but not rapidly enough to permit optimal growth.

Since PABA is one of the factors excreted by wildtype in large amounts, this hypothesis was easily tested. The two PABA back-mutants were compared with wild-type for their output of PABA on minimal medium, by pouring plates of minimal medium containing few cells of the prototroph and many PABArequiring cells. (This technique is more sensitive to slight differences in syntrophism than is the technique of adjacent streaks.) As expected, the wild-type colonies were surrounded by an extensive halo of satellites, while the slow prototroph had none. This fact confirmed the earlier conclusion that its growth rate was limited by its rate of synthesis of PABA; it had none to spare. Unexpectedly, however, the other back-mutant, previously indistinguishable from wildtype, was surrounded by a smaller halo of satellites than was wild-type. Apparently its recovered capacity to synthesize PABA was not as great as that of wildtype.

Following this a dozen different back-mutant strains, spontaneous and ultraviolet induced, were isolated from a PABA mutant; among these 6 different rates of growth in the absence of PABA were recognized. In addition, all were more susceptible than wildtype to sulfonamide inhibition, which is not surprising since all synthesized PABA at a lower rate than wildtype. Some of the slower strains had their growth rate 
restored to optimal by the addition of PABA; others did not. Similar studies were carried out with mutants requiring 6 amino acids, purines, biotin, and pantothenic acid. In all cases but one amino acid, a variety of degrees of back-mutation were observed; in only a fraction of the strains were slow growth rates restored to optimal by addition of the parent's growth requirement.

In the absence of the required genetic techniques it has not been possible to demonstrate whether these various degrees of restoration of a nutritional deficiency represent quantitative alleles of the same gene, or mutations in other genes which modify the deficiency. In the cases where the back-mutant is slow either with or without the growth factor, an allelic change seems much less likely than does a mutation of another gene which imposes a rate-limiting alteration of metabolism at the same time that it restores the deficiency. In any event, it is clear that back-mutants, which have lent themselves to quantitative genetic studies, represent a genetically very heterogeneous class. Indeed, it is not evident that any of the back-mutants are truly identical with wild-type. Perhaps they should be understood as "backward" mutants rather than true back-mutants or reversions, having mutated in the direction, but not necessarily to the precise position, of wild-type. One study of back-mutants of Neurospora ${ }^{1}$ has failed to reveal such frequent and varied partial restorations of a deficiency. Incidentally, in this connection, it should be noted that mutations from wild-type to partial deficiencies of various factors are frequent, with Neurospora as well as with bacteria; they are not often mentioned in the literature since they have not seemed to lend themselves to genetic or biochemical analysis as well as mutants with absolute requirements.

\section{Reversion of multiple requirements}

Another genetic question, more essential for biochemical investigation, is whether certain multiple requirements arise from a single mutation and hence presumably from a change in a single enzyme. While we have not tried to solve this problem directly by recombination techniques, it has been possible to circumvent it by use of back-mutations ${ }^{2}$. In several instances it has been easy to obtain spontaneous prototrophs, with no growth requirements, from mutants with multiple requirements (e. g. quadruple and double aromatic), and has not been possible to obtain split reversions, with loss of only part of the requirements. From these results it is inferred that a single mutation has occurred and then reverted. In another instance, requiring histidine and the three

1 N. H. Giles, JR., and E. Z. Lederberg, Amer. J. Bot. 35, 150 (1948).

2 R. R. RoEpke, quoted by E. L. TATUM, Cold Spring Harbor Symp. Quant. Biol. 11, 278 (1946). aromatic amino acids, it has been possible, in appro. priate media (limited histidine, excess of the other requirements) to lose the histidine requirement alone, but it has not been possible to lose all four requirements in one step. It is therefore concluded that this mutant had been altered in two separate steps. Nonallelic reversions from mutant to apparent wild-type in higher organisms have been described in which a mutation of a second gene at a different locus (modifier gene) restores the normal condition. In the absence of a test for allelism it cannot be decided whether our reverse mutations involve a change of the same gene or of another one. It is conceivable that a mutation of a modifier gene might suppress two independent growth requirements. But whatever the genetic mechanism of the reversions may be, one would expect with a double mutant to recover the single backmutants as well as the prototrophs. Complete failure to isolate the single reversions, together with isolation of the total reversions, is therefore considered excellent evidence for a single mutation producing a multiple requirement. On the other hand, it should be pointed out that the recovery of split reversions is not conclusive evidence for the presence of independent mutations, unless it is accompanied by failure to isolate any total reversions. With the isoleucine-plus-valine mutant, for example, one back-mutant was obtained with a relative requirement for valine (slow growth without it), but no requirement for isoleucine. Since many completely prototrophic back-mutants were also obtained from this strain, a probable mechanism for the split reversion would be incomplete restoration of the isoleucine enzyme, leaving enough residual accumulation of the intermediate to inhibit valine synthesis.

This stress on the importance of negative as well as positive results is warranted only because of the great efficiency, essentially $100 \%$, with which reversions to nutritional independence can be isolated from huge bacterial populations.

\section{Delayed phenotypic expression of back-mutation}

The number of spontaneous back-mutant (prototroph) colonies observed on a minimal medium plate is proportional to the size of the inoculated population. In a medium with limited enrichment, in contrast, the inoculum grows until the population reaches a size which is limited by the amount of enrichment; the mutants observed are therefore largely "platemutants" (i.e., those arising during generations occurring on the plate), and their number is, as a first approximation, a function of the enrichment rather than the inoculum size. Following ultraviolet irradiation, however, an entirely different situation was encountered.

Ultraviolet irradiation increases the frequency of back-mutants among the survivors by as much as many 
thousanelfold. Cunder these ircumstances, the number of plate-mutants in a heavily inesulated plate with limited enrichment shomld be only a negligible fraetion of the number of mutants inosulated. The number of altraviolet indued prototrople colonies observed would therefore be evpected to be relativels inelependent of the enrichusent. To our surprise, with certain mutants the dependence wats found to be extremse. With a washed inoculum of a mutant, almost no ultravioletineluced protetrouhs coukd be detected unless the medium was enriched with a trace of the factor re(fuired by the parent strain. To look at one instance: an inoenlum of $10^{*}$ alls of a tryptephan-repuiring mutant vickeded 1 to 3 visible back-mutant colonies after there days on minimal agar, and 1 te 6 , colomics on agar slightly enriched with 0.011 to $0.5 \% \mathrm{ml}$ of tryptephan. The suspension was irradiated until about zand 51$)^{\prime \prime}$ " of the eells survive(l, and the same volumes were innewlated. On minimal asar, 0 to 20 colonies developed; on slightly enriched agar, 2(1) to fone)! (of these, 25 were picked at ranclom, ranging from the large to the microscopic. All were prototrophs, growing on minimal agar, though many of the smallest grew very slowly: Qualitatively similar observations have been made with several amino arid, purine, and vitamin refuiring mutants.

It appears that a certain amount of growth by the parent strains is necessary to permit the ultravioletinduced back-mutants to get started. It is known. however, that small inocula of various bacteria often fail to initiate growth in a medium which is adeguate for larger inocula; the small inocula are presumably. unable to accumulate enough ( $\mathrm{CO}_{2}$ or other esiential metabolites. To rule out the possibility that the growth of the parent strain was promoting the appearance of back-mutant colonies by some such nonspecifie mechanism, varionsly mriched plates were inoculated with an irracliated strain, an unirradiated strain with a lifferent repuirement, and a mixture of the two. Prototrophs appeared in large numbers only from the irradiated strain, and when the medium was supplemented with its growth fac tor rather than that of the companion unirradiated strain.

We are therefore bromght back to the consideration of the phenomic lag, introluced into this work at the outset by the initial failures of the penicillin method. It now appears that a lag occurs not only in exhausting premutational enzymes, but also in buileling up new cnzxmes which the mutated cell is capable of comstruct ing; to build up the new enzome the rell resuires a complete set of building blocks, inchuling the product of the previombly defirient enzyme. In other words, to start the erele, the pump must first be primed. This phenomenom stamele in contrast to. the behavion of certain adajtive fermentative enzmes, which are reported to be formed by bacteria or yeasts in a nit rogenfree merlium, in which no net growth can take plater.

\section{I'se of mutants in studying mechanisms of bacterial inhibition}

1)-herine exerts a marked inhibitery effect on the growth of our wild-type strain of $l$ : coll; manx amino acicls are able to antagonize the inhibition, but aspartic aciel, in contrast, enhances the effect, although by itself it has no influence on growth ${ }^{1}$. MERXER M.s.s, further studving this problem in this laboratory, found that the inhibition is orercome by extremely low concentrations of pantothenic acid, and by somewhat higher concentrations of $\beta$-alanine. The antagonistic action was apparently non-competitive with pantothenate and competitive with $\beta$-alanine. The results suggested that $\beta$-alanine is the substrate and pantothenate the product of the inhibited reaction. This trpe of inhibition analysis has been wilely used to determine whether a given antagonist serves as substrate, precursor, or proluct of the inhibited reaction. For several reasons, huwever, which are diseussed elsewhere 2 , it seems difficult to draw rigorous conclusions as to the site of the inhibition, especially in those cases where only a narrow range of concentration of the inhibitor is possible before other reactions become affected. Witl appropriate mutants, on the other hand, it is pessible to dissect ont the system under investigation and proluce more direct evidence of the site of inhibition. In adelition, mutants shew whether an apparent product of the inhibited reartion, acting non-competitively on wikl-type, has done so in "physiological" concentratiuns.

Mutants blocked at varions stages in the sronthesis of pantothenic acicl were therefore isolated (lig. 3). A mutant blocked in the sinthesis of $\beta$-alanine showed a competitive relation between $\beta$-alanine and 1$)$-serine: a mutant blocked in the synthesis of pantoic acicl showed inhibition by $\mathrm{D}$-serine when grown on pantoic acid, but none when grown on pantothenic acid; and at mutant blocked in the formation of pantothenie aciel grew in proportion to the amount of pantothenic acid present, regardless of the presence or absence of $n$ serine. It has therefore been demonstrated, more conclusively than would be possible with wild-type alone. that $v$-serine (or a product of it) interferes with the conversion of $\beta$-alanine to pantothenic acil'2. Similarly, salicylic aciel, which is known to be antagonized by pantothenic acid ${ }^{3}$, has been found $\mathrm{l}^{4}$ to interfere with the synthesis rather than the utilization of pantoic acicl. Furthermore, several of the amino aciols which antagonize 1 -serine inhibition of wild-type were tested against $n$-serine inhibition of a mutant unable to synthesize $\beta$-alanine. No antagonism of the inhibition

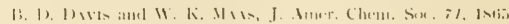
$1: 11: 11$

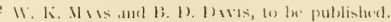

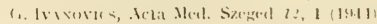

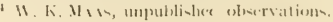


was observed. This result inclieates that these amino acids antagonize 1)-serine only indirestly, acting via the final common path of $\beta$-alanine synthesis. Finally, serine-resistant and salicylate-resistant mutants have been isolated and are uncler investigation. We feel that the pitfalls in interpreting the mode of action of antibacterial agents can sometimes be avoided by the combination of biochemical studies with the use of appropriate mutants.

In connection with the studies on pantothenic acid, certain pantothenate-requiring mutants lave been observed to respond to either pantoic acid or to pantonine (the $\boldsymbol{x}$-amino analogue of the $\boldsymbol{x}$-hydroxy acid, pantoic acid); others respond to pantoic acid only (Fig. 3). It may be inferred that pantonine is either a normal precursor of pantoic acid, or is convertible to such a precursor.

A number of the problems described above are as yet only partly solved; yet so closely intertwined are the varions metabolic sequences that each step toward a solution has revealed unexpected new problems. Herein lies much of the fascination of this field. It has been the main purpose of the present paper to illustrate the ease with which a variety of problems can be revealed and solved, up to a point, by simple methods the isolation of desired bacterial mutants by the penicillin method, followed by direct observation of their behavior, especially with respect to syntrophism and back-mutation, on solid media. Some of the studies described in this paper will be publislied elsewhere in more detail.

It is a pleasure to acknowledge the expert technical assiotance of Mrs. Harlean Cort and Mrs. Elizabetir Minciol.l. We are grateful

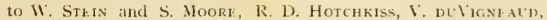

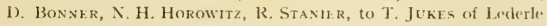
Jaboratories, and to K. FoLkERs, M. TisH!.ER, and IE. F. How if Merck and $\mathrm{Co}$. for generous gifts of chemicals.

\section{Zusammenfassung}

In dieser Arbeit wird eine Methode besehrieben, die es gestattet, in größerer Zahl Stoffwechsel-MInusmutanten von Bakterien zu isolieren. Sie beruht auf der bemerkenswerten Eigenschaft des Penicillins, aus- sehticlilich wathemle und sich vermehrende Bakterien ab)utriten. Nird elem\%ufolge eine grobe Bakterienkultur, dic versehiedene, durch vorangegangene Lltravioletthestrablung herbeigeführte Jutiunten enthält, 1: cinem eben geniigenden Nïhrmedium der Wirkung von P'enicillin ausgeset\%t, so wird die Mrhrzahl der Kultur. die aus den normalen Bakterien des stammes besteht. abgetötet, wälırend die Minusmutanten, die nicht zu wachsen vermögen, selektiv ïberleben.

Mit Hilfe dieser Technik wurden Mutanten ron $l$ : coli mit individuell verschiedenen Anspriehen auf nahezu sämtliche Aminosäuren, V'itamine, P'urine und Pyrimidine isoliert. Einzelne Mutanten mit altornativen oder multiplen Stoffwechsellsedïrfnissen orlaubten Einblicke in den Gang $x$ on Biosynthesen. So wachsen eine Anzahl von "ringfreien" IIutanten, die gleichzeitig Tyrosin, Phenylalanin, Tryptophan und l'araaminobenzoesäure benötigen, auch in alleiniger Gegenwart ron shikimisäure, einem teilweise dehydrierten Derivat der (yelohexanearbonsäure. Dies läibt vermuten, daß im Organismus aromatische Verbin dungen aus wenigstens teilweise gesättigten Ringverbindungen entstehen können. Ferner ist bekannt, daB der Gang einer Biosvinthese durch die Isolierung von Zwischenprodukten analysiert werden kann, die von gewissen Mutanten angestapelt werden. Derartige An. sammlungen lassen sich technisch mit Hilfe von Bakterien leicht nachweisen, wemn versehicdene Mutanten, die sich gegenseitig ernähren, nebeneinander ausgestrichen werden. Nicht nur werden so Stoffwechselvorstufen (precursors) angestapelt; in gewissen Fällen führt das Lnvermögen, eine bestimmte Aminosäure (z. B. Tyrosin, Phenylalanin, Isoleucin) zu synthetisieren, zu Anlänfungen anderer Aminosäuren. Diese Beobachtung leitet zur Annahme, daß normale \%wischenstufen einer Verbindung einen regulierenden linfluß auf die Synthese anderer Stoffe ausüben.

Ferner lassen sich diese Mutanten gut verwenden, um die Wirkungsweise bestimmter antibakterieller Wirkstoffe endgültig festzulegen. lis komnte nachgewiesen werden, daß 3 die $[$ mwandlung von $\beta$-Alanin in l'antothensäure durch $\mathrm{D}$-Serin verhindert wird, während Salicylsäure anf die Bildung der Pantoinsäure störend einwirkt.

Dureh Litraviolettbestrahlung hervorgerufene Mutanten, sowohl Stoffwechsel-Minusvarianten als Rück. mutierungen zur Norm, brauchen eine bestimmte Zeit, bis sie phänotypisch erkennbar sind, was darauf schließen läßt, daß sich die Fermentzusammensetzung erst nach einer Einstellungsphase der neuen Genzusammensetzung angleicht (phenomic lag). 


\title{
THE DEMONSTRATION OF NON-SPECIFIC COM- PONENTS IN SALMONELLA PARATYPHI A BY INDUCED VARIATION ${ }^{1}$
}

\author{
D. W. BRUNER AND P. R. EDWARDS \\ Department of Animal Pathology, Kentucky Agricultural Experiment Station, \\ Lexington, Kentucky
}

Received for publication February 21, 1941

Phases induced by growth in immune serum have been demonstrated in a number of enteric bacilli. Kauffmann (1936) found a second phase in Eberthella typhosa after the organism was cultivated in agglutinating serum derived from the Muenchen type. While Kauffmann found this variant to be irreversible, Edwards and Bruner (1939) reverted similar forms to the original phase by cultivation in serum derived from the variants. Induced phases were described by Kauffmann and Tesdal (1937) in the Schleissheim type and by Gard (1938) in Salmonella abortus-canis. Both the latter types are monophasic under ordinary conditions of culture. Gnosspelius (1939) induced variants with altered flagellar antigens in the naturally diphasic types Stanley and Hvittingfoss. These forms also were irreversible even when cultivated in homologous immune serums. It is significant that all the phases described above were "artificial" phases; that is, they possessed no antigenic relationships to the naturally occurring antigens of the genus Salmonella.

The cultivation of Salmonella strains in immune serum does not always lead to the isolation of artificial phases, as witness the results obtained in the study of the "totally and permanently non-specific" Salmonella types. Thus, through cultivation in immune serum, Scott (1926) isolated a specific phase from a nonspecific culture of the Thompson type and Gard isolated specific

1 The investigation reported in this paper is in connection with a project of the Kentucky Agricultural Experiment Station and is published by permission of the Director.

[Reprinted by permission of The Williams \& Wilkins Company from Journal of BaCterlOLOGY 42: (4) 467.478, October, 1941] 
phases identical with that of Salmonella cholerae-suis from cultures of S. cholerae-suis var. kunzendorf. Bruner and Edwards (1939) isolated specific phases from Salmonella typhi-murium var. binns, Salmonella typhi-suis var. voldagsen, $S$. cholerae-suis var. kunzendorf and the Berlin and Puerto Rico types by cultivation in immune serums. In each instance the specific phases so isolated were identical with those that occur naturally in the diphasic counterparts of the variants. Edwards and Bruner (1939a) working with the naturally monophasic Salmonella abortus-equi isolated two additional phases by cultivation of the organism in various immune serums. One of these was an "artificial" phase closely related to the induced phase of the Schleissheim type described by Kauffman and Tesdal. The second induced phase, on the contrary, was the antigen a of the Kauffmann-White classification and was identical with the flocculating antigen of Salmonella paratyphi $A$.

The purpose of the present work is to describe the isolation of phases closely related to the naturally occurring non-specific phases of the genus Salmonella from S. paratyphi A. It is realized that the serology of the Salmonellas is already sufficiently complicated and that work such as that presented here may seem on first thought only to confuse the classification of the bacilli. However, the demonstration of inapparent components of the bacilli adds to our knowledge of the genetic relationships and hereditary tendencies in the genus.

\section{MATERIALS AND METHODS}

Sixteen cultures of $S$. paratyphi $A$ were used in the study. The sources and designations of the strains were as follows:

1015, Marta, HA1, HA6, Durazzo-From Dr. F. Kauffmann, International Salmonella Center, Copenhagen.

38250, 37407-From Dr. Ruth Gilbert, New York State Department of Health.

Fried, Boyd, 17, W, WB39-From Dr. Ralph Muckenfuss, New York City Department of Health.

228, HR, GV-From Dr. L. F. Rettger, Yale University.

Cal. 49-From Dr. W. R. Hinshaw. Stock culture from Medical School, University of California. 
All the cultures possessed the biochemical and serological properties generally attributed to $S$. paratyphi $A$ with the following exceptions: The strain Durazzo lacked antigen I of the Kauffmann-White classification and produced large amounts of hydrogen sulphide. In these respects it conformed to the description of Kauffmann (1937). The remainder of the strains produced smaller amounts of hydrogen sulphide, as evidenced by the blackening of lead acetate papers suspended over cultures in 2 per cent Bacto-peptone water. In this connection it should be stated that the observations of the writers confirm the conclusions of Hunter and Crecelius (1938) that differences observed in hydrogen sulphide production by different members of the genus Salmonella are quantitative and not qualitative. It is probable that all Salmonella strains produce hydrogen sulphide and that the results obtained in testing for its production depend upon the methods employed for the detection of the substance.

The methods employed to induce variation were largely the same as those used by Edwards and Bruner (1939a) in the study of $S$. abortus-equi. The organisms were grown in semi-solid agar to which was added sufficient agglutinating serum to immobilize the bacilli. The medium was inoculated by stabbing at one side of the tube. Outgrowths from the line of stab occurred only when the flagellar antigens were altered. The spreading growth was transferred successively in tubes of the same medium until the serological reactions indicated that the induced phase was pure. The culture was then plated and isolations made from single colonies for further study. Serum derived from phase 1 of the Bispebjerg type was used to induce variations in the normal phase of the cultures. As additional phases were isolated agglutinating serums were prepared from them. These were used in the study of induced phases and in forcing the bacilli to produce further alterations in the flagellar antigens. Serum derived from 'S. cholerae-suis var. kunzendorf was used in the reversion of nonspecific phases. In many instances, it was found necessary to use combinations of serums to force variation in the desired direction or to revert induced phases to the original phase. When the serums contained $\mathrm{O}$ or $\mathrm{H}$ agglutinins which might interfere with 
the migration of the organisms, such agglutinins were removed by absorption with appropriate bacilli. The serums were preserved by the addition of small amounts of chloroform. The chloroform effectively sterilized the serums and did not affect the growth or motility of the bacilli.

\section{RESULTS}

It should be emphasized that the changes described below involved only the $\mathrm{H}$ or flagellar antigens; that is, the antigens which are affected in the phase variation of normally diphasic types. The heat-stable $\mathrm{O}$ antigens remained unchanged throughout the experiments. On the whole the cultures were quite stable and it was more difficult to induce variation in $S$. paratyphi $A$ than in Eberthella typhosa, S. abortus-equi or the "totally and permanently" non-specific types. In some instances it was necessary to transfer the organisms repeatedly in the presence of agglutinating serum before variation was observed.

From cultures GV and $\mathrm{W}$ no induced phases were isolated. Strains 17 and 37407 each yielded two induced phases (phases 3 and 4) neither of which was closely related to any of the naturally occurring antigens of the genus. All the remaining cultures yielded three induced phases. The normal phase of the bacilli (antigen a of the Kauffmann-White classification) was designated as phase 1 . The induced antigens were denoted as phases 2,3 and 4 . Phase 2 is closely related to the non-specific phases of the diphasic Salmonella types. Phases 3 and 4 are "artificial" phases which closely resemble none of the natural antigens. The relationships of the phases to each other and to the antigens of other species are given in table 1. Only one culture of S. paratyphi $A$ is included in the table since the phases isolated from other strains reacted similarly. Phase 2 is agglutinated in high dilution by Kunzendorf and non-specific Sendai serums. Likewise serum derived from phase 2 agglutinates Kunzendorf and the non-specific phase of Sendai to the titre of the serum. Phase 3 is slightly related to the normal phase 1 , but has little relationship to the other phases or to the natural antigens of the genus. It is agglutinated in low dilution by all the serum of all types that 
contain antigen a. This agglutination probably represents a slight residue of the normal flagellar antigens of the species. Phase 4 shows evidence of a slight serological relationship to phase 3 and to the non-specific phases of other types, otherwise it is unrelated to the known antigens of the Salmonella.

The phases were further examined by agglutinin absorption. The results of these tests confirmed the agglutination tests and need not be given in detail. When absorbed serums were used the cross reactions between the four phases were no longer evident.

TABLE 1

Agglutination tests with phases of S. paratyphi A

\begin{tabular}{|c|c|c|c|c|c|c|c|}
\hline \multirow[b]{2}{*}{ ANTIGENS } & \multicolumn{7}{|c|}{ SERUMS } \\
\hline & $\begin{array}{c}\text { Para- } \\
\text { typhi } \\
\text { A 228 } \\
\text { Phase 1 }\end{array}$ & $\mid \begin{array}{c}\text { Bispe- } \\
\text { bjerg } \\
\text { Phase } 1\end{array}$ & $\begin{array}{c}\text { Para- } \\
\text { typhi } \\
\text { A 228 } \\
\text { Phase } 2\end{array}$ & $\mid \begin{array}{c}\text { Para- } \\
\text { typhi } \\
\text { A 228 } \\
\text { Phase 3 }\end{array}$ & $\begin{array}{c}\text { Para- } \\
\text { typhi } \\
\text { A 228 } \\
\text { Phase 4 }\end{array}$ & $\begin{array}{l}\text { Sendai } \\
\text { Phase 2 }\end{array}$ & $\begin{array}{c}\text { Kunzen } \\
\text { dorf }\end{array}$ \\
\hline $\begin{array}{l}\text { Paratyphi A } 228: \\
\text { Phase } 1 \ldots \ldots \ldots \ldots\end{array}$ & & & & & & & \\
\hline Phase $2 \ldots \ldots \ldots$ & 0 & 0 & 40,000 & 0 & 1,000 & 10,000 & 20,000 \\
\hline Phase $3 \ldots . .$. . & 500 & 500 & 0 & 10,000 & 200 & 0 & 0 \\
\hline Phase $4 \ldots \ldots \ldots \ldots$ & 0 & 0 & 1,000 & 0 & 20,000 & 0 & 0 \\
\hline Abortus equi-Phase $1 \ldots$ & 20,000 & 40,000 & 0 & 1,000 & 0 & 0 & 0 \\
\hline Bispebjerg-Phase $1 \ldots$ & 20,000 & 40,000 & 0 & 1,000 & 0 & 0 & 0 \\
\hline Sendai-Phase $2 \ldots$ & 0 & 0 & 40,000 & 0 & 500 & 10,000 & 20,000 \\
\hline Kunzendorf...... & 0 & 0 & 40,000 & 0 & 500 & 10,000 & 20,000 \\
\hline
\end{tabular}

Figures indicate highest dilution at which agglutination occurred. " 0 " indicates no agglutination at dilution of 1 to 200 .

Furthermore, the absorption of serum derived from one phase by organisms of another did not result in an appreciable reduction of the titre of the serum for the homologous phase. Absorption of serum derived from phase 3 of one strain by phase 3 organisms of a second strain resulted in a complete removal of agglutinins from the serum. This was also true of phase 4. The non-specific components (phase 2) derived from all the cultures were closely related but not identical. This will be discussed below.

While the variants isolated from the various cultures of $S$. paratyphi $A$ were similar, the variational tendencies of the cultures differed. When the organisms were inoculated into semi-solid 
agar which contained serum derived from phase 1 of the Bispebjerg type, culture HR yielded phase 2, 228 yielded a mixture of phases 2 and 4 , while the remainder of the organisms yielded phase 3 or a mixture of phases 3 and 4 . For this reason mixtures of serums were used to force variation in the desired direction. The variations induced in one culture, strain 228 , are given in

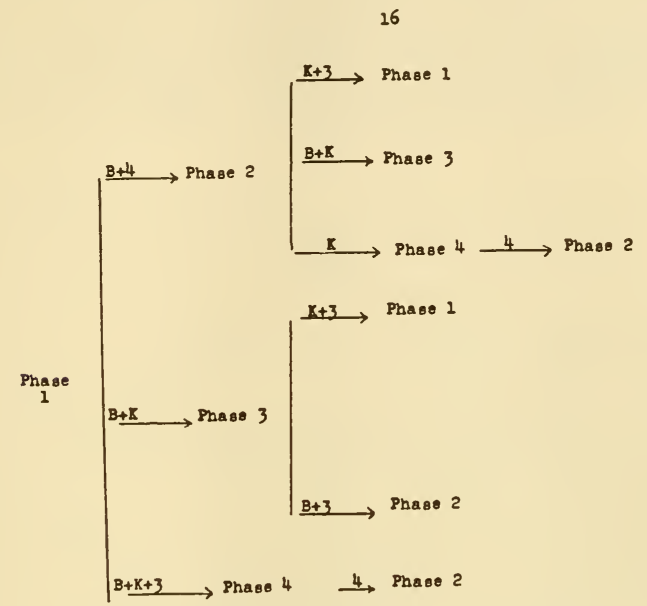

Fig. 1. Changes Induced in S. Paratyphi A 228

Symbols on arrows indicate what serums were added to medium.

$B$, serum derived from phase 1 of Bispebjerg type.

$\mathrm{K}$, serum derived from $S$. cholerae suis var. kunzendorf.

3 , serum derived from $S$. paratyphi $A$, phase 3 . The serum was absorbed with phase 1 before use.

4 , serum derived from $S$. paratyphi $A$, phase 4. The serum was absorbed with phase 3 before use.

figure 1. The changes produced in other cultures differed slightly but they were all quite similar.

When once isolated the induced phases of $S$. paratyphi $A$ are quite stable. No changes in the phases were noted during the two years they were maintained in the laboratory. In this respect they resemble the induced phases of E. typhosa and S. abortus-equi and differ from the normal phases of the diphasic Salmonella 
types. The latter display phase variation under normal conditions of culture. As shown in figure 1, it is possible to produce changes in the induced phases of $S$. paratyphi $A$ by cultivation in appropriate immune serums. The reversibility of the phases is illustrated in figure 2. Phases 1, 2 and 3 were completely interchangeable and reversible. The changes illustrated in these phases were accomplished repeatedly with cultures isolated from single colonies. Phase 4 , on the contrary, was more stable. It was readily converted to phase 2 but not to phase 1 or phase 3 . Efforts to convert phase 4 to phase 1 or phase 3 resulted in the

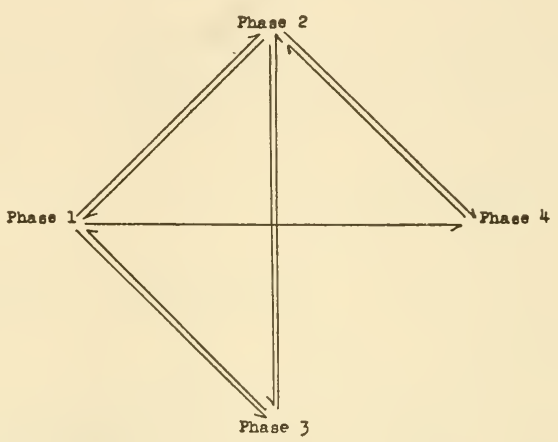

Fig. 2. Reversibility of Phases of S. paratyphi A 228 Arrows indicate direction in which variation occurred

production of a series of ill-defined, serologically-related variants. This observation confirms the work of Gnosspelius (1939), who concluded that through proper manipulation it was possible to isolate an endless number of antigenic components from Salmonella strains.

It is known that "artificial" phases that display little or no relationships to the normal antigens of the genus can be isolated from a number of Salmonella types by induced variation. The isolation of components closely related to the naturally occurring antigens is much more unusual. The isolation of non-specific 
components from a normally monophasic-specific species has not been reported previously. For that reason the characteristics of the non-specific components (phase 2) of S. paratyphi $A$ are given in more detail than are the characteristics of the "artificial" phases 3 and 4 . The agglutinative relationships between the non-specific components of S. paratyphi $A$ and the non-specific phases of diphasic types are given in table 2 .

It is apparent that the non-specific phases of $S$. paratyphi $A$ are more closely related to the $1,5 \ldots$ phases than to the $1,2 \ldots$, $1,6 \ldots$ or $1,7 \ldots$ phases. The organisms were tested with ab-

TABLE 2

Agglutination of non-specific phases of $S$. paralyphi A by non-specific serums of other types

\begin{tabular}{|c|c|c|c|c|c|c|c|}
\hline \multirow[b]{2}{*}{ ANTIGENS } & \multicolumn{7}{|c|}{ BERUMS } \\
\hline & $\begin{array}{c}\text { Para- } \\
\text { typhi B } \\
(1,2 \ldots)\end{array}$ & $\mid \begin{array}{l}\text { Newport } \\
(1,2 \ldots)\end{array}$ & $\begin{array}{c}\text { Kunzen- } \\
\text { dorf } \\
(1,5 \ldots)\end{array}$ & $\begin{array}{c}\text { Para- } \\
\text { typhi } \\
\text { A 228 } \\
\text { Phase 2 }\end{array}$ & \begin{tabular}{|} 
Para- \\
typhi \\
AWB39 \\
Phase 2
\end{tabular} & $\begin{array}{l}\text { Anatum } \\
(1,6 \ldots)\end{array}$ & $\begin{array}{l}\text { Nyborg } \\
(1,7 \ldots)\end{array}$ \\
\hline Paratyphi B $(1,2 \ldots)$. & 10,000 & 10,000 & 5,000 & 1,000 & 500 & 2,000 & 2,000 \\
\hline Newport $(1,2 \ldots) \ldots \ldots$ & 5,000 & 40,000 & 5,000 & 1,000 & 1,000 & 2,000 & 2,000 \\
\hline Kunzendorf $(1,5 \ldots)$. & 2,000 & 10,000 & 20,000 & 40,000 & 40,000 & 5,000 & 2,000 \\
\hline Paratyphi A $228 \ldots \ldots$ & 2,000 & 2,000 & 10,000 & 40,000 & 20,000 & 5,000 & 1,000 \\
\hline Paratyphi A WB39. & 2,000 & 2,000 & 10,000 & 40,000 & 40,000 & 5,000 & 1,000 \\
\hline Sendai $(1,5 \ldots) \ldots \ldots$ & 2,000 & 5,000 & 10,000 & 40,000 & 20,000 & 5,000 & 1,000 \\
\hline Anatum $(1,6 \ldots)$. & 2,000 & 2,000 & 5,000 & 10,000 & 5,000 & 10,000 & 1,000 \\
\hline Nyborg $(1,7 \ldots)$. & 5,000 & 5,000 & 2,000 & 200 & 200 & 2,000 & 10,000 \\
\hline
\end{tabular}

Figures indicate highest dilution at which agglutination occurred.

sorbed serums containing agglutinins for non-specific factors 2 , $3,5,6$ and 7 , respectively. The preparation of these serums was described by Bruner and Edwards (1941). The non-specific components of $S$. paratyphi $A$ were flocculated only by serums containing agglutinins for factors 3 and 5 . In this respect they resembled the Kunzendorf, Berlin and Sendai types. Phase 2 of $S$. paratyphi $A$, therefore, may be expressed as $1,5 \ldots$

As mentioned above, the non-specific phases derived from cultures of $S$. paratyphi $A$ were not identical. The differences in the phases were discernible only in agglutinin absorption tests. It was possible to divide the phases into two groups whose non- 
specific components were identical. In one group were cultures 228, HR and Fried; while 38250, Marta, Cal. 49, 1015, HA1, HA6, WB39, Boyd and Durazzo were included in the second. The differences between these two groups were evidenced only by a slight residue of agglutinins remaining when the serum derived from a member of the second group was absorbed with a member of the first group. These residues of agglutinins did not exceed 2 per cent of the original titres of the serums. The differences in the non-specific components of $S$. paratyphi $A$ were no more pronounced than those that exist in the non-specific phases of different diphasic species in which the second phase is expressed as $1,5 \ldots$ The phases denoted as $1,5 \ldots$ in the Kauffmann-White classification are quite complex and their exact relationships cannot be expressed without the use of further symbols. In this connection it should be remembered that the classification was designed by Kauffmann as a diagnostic schema and that it does not give a complete antigenic delineation of the bacilli.

The relationships of the non-specific components of $S$. paratyphi $A$ to the naturally occurring $1,5 \ldots$ phases were studied by agglutinin absorption tests. It was found that serums derived from 228, HR or Fried were completely exhausted of agglutinins by absorption with the non-specific components of 38250, Marta, Cal. 49, 1015, HA1, HA6, WB39, Boyd, Durazzo or Sendai. The serums of the latter group were not completely absorbed by 228, HR, Fried or Sendai. None of the non-specific phases of $S$. paratyphi $A$ removed all the agglutinins from Sendai serum. The relationships of the non-specific components of $S$. paratyphi $A$ and Sendai were appreciably closer than were their relationships with the non-specific phases of any other type.

\section{DISCUSSION}

While earlier workers realized that the Salmonella group was composed of a mosaic of interrelated antigens, Bruce White (1926) was the first to identify these antigens by symbols and thus express the relationship and divergences of different species in graphic form. In addition he was the first to discuss the 
genetic relations of the various forms with reference to antigenic composition and to propose a theory of Salmonella phylogeny. White believed that the Salmonella species as we know them today arose from a primitive diphasic ancestral stock by variation in $\mathrm{O}$ and $\mathrm{H}$ antigens. He regarded the monophasic state, as seen in S. paratyphi $A, S$. abortus-equi and E. typhosa, as an acquired characteristic which developed through loss variation. The demonstration by Edwards and Bruner that antigen a could be isolated from $S$. abortus-equi revealed that the organism was more closely related to diphasic forms having the antigens a-enx than was previously realized. They suggested that in monophasic types, the antigens present in the original diphasic state were not lost but merely suppressed. The isolation of non-specific components from $S$. paratyphi $A$ confirms this view. Both observations strengthen the theory of White that monophasic types have evolved from a diphasic ancestry.

White emphasized the close relationship that existed between $S$. paratyphi $A$ and Sendai, the only two types then known which possessed antigen a. He used these two types to illustrate the identity of the $\mathrm{H}$ antigens of monophasic types and the specific antigens of diphasic types. The isolation of non-specific phases from $S$. paratyphi $A$ which are all but identical with the nonspecific phase of Sendai reinforces the relationship between the specific phases of the two types and demonstrates the keen appreciation of White of the evolutionary tendencies in the genus.

With the exception of the recovery of specific phases from "totally and permanently" non-specific types, the isolation of antigen a from $S$. abortus-equi and of non-specific components from $S$. paratyphi $A$ constitute the only instances in which antigens resembling the naturally occurring phases have been induced in monophasic cultures. The stability of these induced antigens has been mentioned. It is in direct contrast to the instability of the phases of the diphasic types. These results indicate that once an organism has lost the power of phase variation, it cannot resume this function, even when suppressed components become dominant. Only under the influence of a stimulant, such as specific immune serum, does phase variation occur. 
It has been stated that the third and fourth phases induced in S. paratyphi $A$ showed little relationship to the natural antigens of the genus. However, they do display relationships to the induced phases of several other Salmonella types. As mentioned by Edwards and Bruner (1939a) these induced phases follow a recurring pattern, just as do the naturally occurring antigens. Whether they are artifacts produced under the influence of immune serum or whether they represent components that have been suppressed in the evolution of known species is not clear at present. The writers are inclined to the latter view.

\section{SUMMARY}

1. Through cultivation in agglutinating serums three induced phases were isolated from cultures of Salmonella paratyphi $A$. One of the phases was closely related to the non-specific phases of diphasic types. The other two bore little resemblance to the normal Salmonella antigens.

2: Non-specific components were isolated from 12 of 16 cultures studied. These non-specific antigens were expressed as $1,5 \ldots$ and were more closely related to the non-specific phase of the Sendai type than to any of the other non-specific phases.

3. The induced phases were stable under ordinary conditions of culture and could be reverted only by the addition of appropriate serums to the medium.

4. The isolation of non-specific antigens from $S$. paratyphi $A$ was cited as additional evidence that monophasic Salmonella types are derived from multiphasic types through suppression of phases.

\section{REFERENCES}

Bruner, D. W., ANd Edwards, P. R. 1939 A note on the monophasic nonspecific Salmonella types. J. Bact., 37, 365-370.

Bruner, D. W., and Edwards, P. R. 1941 Microorganisms of group E of the genus Salmonella with special reference to a new Salmonella type. Am. J. Hyg., In press.

Edwards, P. R., AND Bruner, D. W. 1939 Reversibility of the alpha and beta phases of Salmonella typhi. Proc. Soc. Exptl. Biol. Med., 41, 223-224.

Edwards, P. R., ANd Broner, D. W. 1939a The demonstration of phase variation in Salmonella abortus-equi. J. Bact., 38, 63-72. 
GARD, S. 1938 Ein neuer Salmonella-Typ (S. abortus-canis). Z. Hyg. Infektionskrankh., 121, 139-141.

Gnosspelius, A. 1939 Ueber künstliche Veränderungen des H-Antigens in der Salmonella-Gruppe. Z. Hyg. Infektionskrankh., 121, 529-532.

Hunter, C. A., ANd Crecelius, H. G. 1938 Hydrogen sulfide studies. I. Detection of hydrogen sulfide in cultures. J. Bact., 35, 185-196.

Kauffmann, F. 1936 Ueber die diphasische Natur der Typhusbacillen. Z. Hyg. Infektionskrankh., 119, 104-118.

Kadffmann, F. 1937 Salmonella Probleme. Z. Hyg. Infektionskranhk., 120, 177-197.

Kadffmann, F., and Tesdal, M. 1937 Ueber zwei neue Salmonellatypen mit $\alpha-\beta$-Phasenwechsel. Z. Hyg. Infektionskrankh., 120, 168-176.

Scoтt, W. M. 1926 The "Thompson" type of Salmonella. J. Hyg., 25, 398-405.

White, P. B. 1926 Further studies of the Salmonella group. Med. Research Council (Brit.) Special Rept. Series No. 103. 


\title{
THE NATURAL OCCURRENCE OF PHASE 2 OF SALMONELLA PARATYPHI A ${ }^{1}$
}

\author{
P. R. EDWARDS
}

Laboratory Division, Communicable Disease Center, Public Health Service, Federal Security Agency, Atlanta, Georgia,

AND

\section{A. BARNES AND MARY C. BABCOCK}

Bacteriology Division, Naval Medical Research Institute, Bethesda, Maryland

Received for publication October 24, 1949

By a modification of the Gard technique, Bruner and Edwards (J. Bact., 42, 467, 1941) obtained from typical cultures of Salmonella paratyphi A (I, II, XII:a) a variant having the formula I, II, XII:1, 5. This experimental evidence supported the hypothesis of White (Med. Research Council, Brit., Special Rept. Series, No. 103, 1926) that S. paratyphi A, as well as other monophasic Salmonella types, was a loss variant of an originally diphasic ancestral form. In the isolation of the 1,5 phase and its reversion to the original form, two "artificial" phases that did not correspond to any of the known naturally occurring antigens of the genus were produced. These were designated as $z_{5}$ and $z_{11}$, respectively. The natural occurrence of phase 2 (I, II, XII:1,5) has not been reported heretofore.

In 1948 a culture isolated by the United States Naval Medical Research Unit No. 3 in Egypt from the urine of a patient affected with enteric fever of 49 days' duration was forwarded to the Naval Medical Research Institute and subsequently to the Communicable Disease Center for study. Examination revealed that the bacterium was a motile rod that possessed the tinctorial and cultural properties of Salmonella.

The organism reduced nitrates but iailed to utilize citrate or D-tartrate. Acid and gas were formed in the butt of triple-sugar iron agar slants; the medium was not blackened. Indole was not formed in peptone water. Glucose, mannitol, arabinose, rhamnose, maltose, and trehalose were fermented with the production of acid and gas within 24 hours; dulcitol was fermented in 48 hours. Xylose, cellobiose, lactose, sucrose, raffinose, inositol, and salicin were not attacked. The biochemical properties described above are typical of $S$. paratyphi A.

Upon serological examination it was found that the organism was agglutinated strongly by $\mathrm{O}$ serum derived from $S$. paratyph $i \mathrm{~A}$ and possessed somatic antigens I, II, XII. In absorption tests it removed all somatic agglutinins from two serums derived from $S$. paratyphi $\mathrm{A}$. When the $\mathrm{H}$ antigens were examined, it was found

1 The opinions or assertions contained herein are the private ones of the writers and are not to be construed as official or reflecting the views of the Navy Department or the Naval Service at large.

[Reprinted by permission of The Williams \& Wilkins Company from JournaL of BACTERIOLOGY 59: (1) 135-136, January, 1950] 
that the culture was not flocculated by $S$. paratyphi A serum (a) but reacted with serum derived from the "nonspecific" phases of the genus (1,2:1, 5:etc.). When tested for single factors $2,3,5,6$, and 7 , it was agglutinated only by 5 serum. In absorption tests it removed all agglutinins from serums prepared from 1,5 phases of $S$. paratyphi A. Thus the culture was identical with the 1,5 phases previously induced by growing $S$. paratyphi $\mathrm{A}$ in homologous serum.

The culture was monophasic and was immobilized when placed in semisolid medium to which had been added various 1,5 serums. After repeated transfers, bulbs extending from the site of inoculation appeared. When these bulbs were transferred to additional tubes of the same medium, the culture migrated rapidly through the agar. From the spreading growth was isolated a form that was agglutinated to 20 per cent of the titer of $\mathrm{H}$ serum of $S$. paratyphi A and that reacted to approximately the same degree with serums produced from the "artificial" phases $z_{5}$ and $z_{11}$. When the culture was grown in semisolid medium to which $z_{5}$ and $z_{11}$ serums as well as 1,5 serum had been added, no spreading occurred although the serums were carefully absorbed to remove interfering factors and the organism was carried through a number of transplants extending over a period of 6 months. It should be emphasized that it was very difficult to cause reversion of induced 1,5 forms of $S$. paratyphi A to the original form and that attempts to cause reversion failed in most instances.

The observations outlined above provide the first report of the natural occurrence of phase 2 of $S$. paratyphi A having the antigenic formula I, II, XII:1,5. Such a variant is not, therefore, merely a curiosity produced by artificial manipulation, although it was biochemically and antigenically identical with strains developed by cultivation of typical cultures in agglutinating serums. The findings emphasize further the importance of the study of phylogenetic and evolutionary trends of the Enterobacteriaceae in the identification of aberrant cultures. 


\title{
CHANGES INDUCED IN THE O ANTIGENS OF SALMONELLA ${ }^{1}$
}

\author{
D. W. BRUNER AND P. R. EDWARDS \\ Department of Animal Pathology, Kentucky Agricultural Experiment Station, \\ Lexington, Kentucky
}

Received for publication December 11, 1947

It is possible to induce profound changes in the flagellar antigens of Salmonella through growth in media containing $\mathrm{H}$ antisera. These changes were recently summarized by Edwards and Moran (Proc. Soc. Exptl. Biol. Med., 61, 242). Hitherto, the only changes reported in the $\mathrm{O}$ antigens were those associated with variation between smooth and rough forms and those occurring in natural form variation. In this laboratory attempts to transform $\mathrm{O}$ antigens by the method of Boivin et al. (Experientia, 2, 139) and by growth in various combinations of $\mathrm{S}$ and $\mathrm{R}$ antisera and $\mathrm{S}$ vaccines have been unsuccessful. However, by a modification of the method of Gard (Z. Hyg., 120, 615) in which absorbed O antiserums were used in high concentration, it was possible to bring about certain changes.

When S. anatum (III,X,XXVI: e,h-l,6) was cultivated in semisolid medium containing III,X,XXVI serum that had been absorbed with a type having $\mathrm{O}$ antigens III,XV, the organisms gradually spread through the medium. From the spreading growth was isolated a form that was indistinguishable from $S$. newington (III,XV:e,h-1,6) by agglutination and absorption tests. Absorption of the III,X,XXVI serum by S. newington, S. cambridge (III,XV: e,h-l,w), or $S$. new-brunswick (III,XV: $1, \mathrm{v}-\mathrm{l}, 7$ ) gave the same results. The induced III,XV: e,h-1,6 form was then reverted to a typical $S$. anatum strain by cultivation in absorbed III,XV serum. Similarly, S. meleagridis (III,X,XXVI: e,h-l,w) was changed to a form indistinguishable from $S$. cambridge (III,XV: e,h-l,w).

Although filtrates similar to those employed by Boivin were not used in the experiments, it must be remembered that the serums were absorbed with very large doses of bacteria and probably contained dissolved antigens as well as metabolic products. Thus the principle that induced the changes may be the same as that involved in Boivin's work with Escherichia coli. Further, attention should be called to the fact that the changes described are only transformations between subgroups of the same $\mathrm{O}$ group. Whether similar experiments will pcrmit transformation between distinct $O$ groups or whether they will lead only to rough variation remains to be determined.

${ }_{1}^{1}$ The investigation reported in this paper is connected with a project of the Kentucky Agricultural Experiment Station and is published by permission of the Director. It was supported in part by a research grant from the U. S. Public Health Service.

[Reprinted by permission of The Williams \& Wilkins Company from Journal of BACTFRIOLOOYY 55 : 449, 1948] 


\title{
MUTATIONS IN ESCHERICHIA COLI INDUCED BY CHEMICAL AGENTS1
}

\author{
EVELYN M. WITKIN
}

For over two decades geneticists have been interested in the possibility of inducing mutations with chemicals. Particularly, it has been hoped that mutagenic compounds might be discovered which, through their specificity of action, would lead to some understanding of the chemical basis of mutation, and ultimately of the structure and organization of the gene.

The first clearly successful attempt to induce genetic changes chemically was described by Auerbach and Robson (1944), who produced mutations and chromosomal abberrations in Drosophila by exposing the flies to mustard gas and related compounds. More recently, Demerec (1947 and unpub.) has shown that certain carcinogenic hydrocarbons $(1,2,5,6$-dibenzanthracene, methylcholanthrene, beta naphthylamine, benzpyrene) are also effective in inducing mutations in Drosophila. These agents, like radiations, appear to be entirely nonspecific in the sense that the affected loci are distributed at random along the treated chromosomes. Nitrogen mustard has been shown to induce genetic alterations in Neurospora (Tatum, unpub.; Horowitz, Houlahan, Hungate and Wright, 1946), and in bacteria (Tatum, 1946; Bryson, unpub.), and at least one of the carcinogens, methylcholanthrene, is effective in Neurospora (Tatum, unpub.). These successful results seem to be the opening guns in what Muller called, a few years ago, "the coming chemical attack on the nature of the gene" (Muller, 1947). They suggest the need for a systematic survey to determine the distribution of mutagenic compounds among various chemical groups, and to lay the groundwork for subsequent analysis of their mode of action. This paper will deal with preliminary results obtained in tests of 4 substances, the first of a series to be investigated in an extensive survey.

Two methodological factors are of critical importance in an attempt to examine large numbers of compounds for mutagenic activity: the basis upon which the chemicals are selected for test, and the choice of biological material. Concerning the method of selecting chemicals, one sober if somewhat unimaginative approach is an indiscriminate raid on the nearest chemical shelf, which has the advantage of objectivity and avoidance of the hazards of premature preconceptions. On the other hand, it is

\footnotetext{
1 This work was done under an American Cancer Society fellowship recommended by the Committee on Growth of the National Research Council.
}

far more tempting to extend oneself on the basis of present ideas concerning the possible organization of genic material, and to select chemicals which might reasonably be expected to affect, or fail to affect, the projected hereditary units. The approach used in these experiments has been to assume that nucleoproteins are somehow centrally involved in the genetic system, and, as a starting point, to investigate chemicals known to have some more or less well-defined chemical or physical effect on nucleoproteins or nucleic acids. It must be emphasized that this approach has no greater justification on $a$ priori grounds than many others, and that the basis of selection may prove to be entirely spurious, since none of the chemicals tested thus far is specific in its action on nucleoproteins or nucleic acids.

The choice of biological material is obviously very important in this type of investigation. The primary requirements are 1) the availability of techniques for treating the organism with chemicals so as to be reasonably certain that they will reach and penetrate the critical sites, and 2) the availability of clear-cut genetic methods for detecting induced mutations. The penetration problem has been the most serious difficulty in the use of Drosophila for chemical induction, and although improved methods of treatment have been developed, the possibility remains that negative results may be due to the failure of some chemicals to penetrate the germ cells in sufficient concentration. The genetic techniques for detecting induced mutations in Drosophila are unparalleled in many respects, but for purposes of an extensive survey of the mutagenic action of chemicals, they are extremely laborious and slow. The problem of penetration is much less serious in microorganisms. Until recently, however, genetic methods analogous to the $\mathrm{ClB}$ and similar techniques in Drosophila have not been available for bacteria. At the present time, Escherichia coli provides promising material for a survey of the mutagenic activity of chemicals, and for detailed analysis of certain aspects of their mode of action.

Luria and Delbrück (1943) described mutants of strain B of $E$. coli which are resistant to one or more bacteriophages to which the parent strain is sensitive. These mutants arise spontaneously in cultures of the $\mathrm{B}$ strain at a rate of about $10^{-8}$ mutations per bacterium per generation, and can be detected easily by plating out samples of the culture in the presence of an excess of bacteriophage. The

[Reprinted from Cold SPring, Harbor Simposia on Quantitative Biology $12: 256-269$, published by the Long Island Biological Assn.] 
sensitive bacteria are quickly lysed, while mutants resistant to the particular bacteriophage applied appear as distinct colonies after suitable incubation.

Demerec (1946) and Demerec and Latarjet (1946) studied the effect of ultraviolet and X-rays in inducing mutations to resistance to one bacteriophage, $\mathrm{T} 1$, and the techniques used by them have been adapted in these experiments to the investigation of chemical mutagens. These authors showed that a certain proportion of the mutations induced by radiations are expressed immediately, before the treated bacteria undergo division. These mutations were called "zero points," as opposed to the "endpoint" mutations which are also induced by the radiation, but which are expressed phenotypically only after a number of cell divisions. In the investigations to be discussed here, the effect of four chemicals on the induction of zero point mutations to resistance to bacteriophage T1 was studied.

\section{MATERIAL}

Strain $\mathrm{B} / \mathrm{r}$ of $E$. coli, a radiation-resistant mutant of the B strain (Witkin, 1947), was used exclusively throughout these experiments. This strain was employed by Demerec (1946) and by Demerec and Latarjet (1946) in their studies of radiationinduced mutations, and it seemed desirable to use the same material in these investigations, in order to simplify comparison of the mutagenic action of radiation and chemicals.

The bacteriophage used to isolate resistant mutants was T1, sometimes known also as alpha, or $\mathrm{P} 28$. Mutants of strain $\mathrm{B} / \mathrm{r}$ resistant to phage $\mathrm{T} 1$ are referred to as $B / r / 1$.

Difco nutrient agar was used for all platings, and cultures were grown initially in a synthetic medium known as M-9, having the following composition per $1,000 \mathrm{ml}$. of distilled water:

$\mathrm{KH}_{2} \mathrm{PO}_{4} \ldots \ldots .3$ g. $\quad \mathrm{NH}_{4} \mathrm{Cl} \ldots \ldots \ldots 1$ g. $\mathrm{MgSO}_{4} \ldots \ldots \ldots \ldots .2 \mathrm{~g} . \quad \mathrm{Na}_{2} \mathrm{HPO}_{4} \cdot 12 \mathrm{H}_{2} \mathrm{O} 15 \mathrm{~g}$. $\mathrm{NaCl} \ldots \ldots \ldots \ldots .5 \mathrm{~g}$. Dextrose ...... 4 g.

\section{Methods}

The method used to determine the number of mutants resistant to bacteriophage $\mathrm{T} 1$, in both control and experimental cultures, was the standard procedure of coating the surface of agar plates with a suspension containing about $10^{9}$ particles of $\mathrm{T} 1$, and then spreading $0.1 \mathrm{ml}$. of the undiluted bacterial culture on the phage-coated plate with a sterile glass rod. The plates are incubated for 48 hours at $37^{\circ} \mathrm{C}$, after which time the colonies of resistant mutants are counted. The total number of bacteria per ml. of the culture is determined by plating suitable dilutions on agar, and making colony counts. The frequency of $B / r / 1$ mutants is expressed throughout as the number per $10^{8}$ bacteria.

The procedure for testing the mutagenic activity of chemicals has been standardized as far as possible. Variations in technique are required to allow for the peculiar properties of each chemical, and will be described in connection with the specific experiments. The basic procedure used in preliminary tests may be outlined as follows:

\section{(1) The preparation of suitable bacterial cultures}

Ten to 20 cultures are usually started at one time, each with a small inoculum (about 100 cells) from a stock slant of strain $\mathrm{B} / \mathrm{r}$. The cultures are grown for 48 hours at $37^{\circ} \mathrm{C}$, with aeration, in a volume of $40 \mathrm{ml}$. of the synthetic medium, M-9, described above. Each culture is assayed to determine the number of bacteria per $\mathrm{ml}$., and the number of $\mathrm{B} / \mathrm{r} / 1$ mutants per $10^{8}$ bacteria. The number of mutants per $10^{8}$ bacteria in a fully grown, untreated culture is called the "background" number, and must be subtracted from the number of mutants per $10^{8}$ survivors in the same culture after treatment with a possible mutagenic chemical, to determine the number of induced mutations. It is desirable, therefore, to use cultures having the lowest possible background number, since a high background may obscure a positive effect, particularly where the mutagenic activity is weak. Cultures found to contain more than 10 background mutants per $10^{8}$ bacteria are discarded, and the remaining cultures are combined to form a stock pool, which is stored at $5^{\circ} \mathrm{C}$, and serves to provide samples for experiments for a period of about a week.

\section{(2) Toxicity tests}

The optimum concentration of a chemical to be tested depends largely upon its toxicity for the bacteria. In general, for preliminary tests, a combination of concentration and time of exposure was used so as to kill about $99 \%$ of the bacteria. The high killing is an indication that the substance is penetrating the cell and reacting with its components, and $1 \%$ survival is usually just enough to permit the detection of mutants among the survivors even if no mutations are induced. The concentration or time of exposure may be adjusted later, depending upon the results of the preliminary tests. For convenience, an arbitrary time, usually 2 or 3 hours, was chosen, and the concentration of the chemical that would kill $99 \%$ of the bacteria in this period of time was determined. The following method was used.

A concentrated solution of the chemical in distilled water or a suitable buffer was prepared, and a series of widely spaced dilutions of the stock solution were made. A number of centrifuge tubes, each containing the same volume of bacterial culture, was set up and centrifuged for 20 minutes at 4,000 RPM. The clear supernatant was decanted from each tube to eliminate the nutrient medium. The bacterial pellets were then resuspended, so as to have one tube for each dilution of the chemical, and one 
tube in buffer or distilled water to serve as the control. The volume of liquid in each tube was the same. The tubes were incubated for the arbitrarily chosen time, usually 2 or 3 hours, at $37^{\circ} \mathrm{C}$, and then assayed to determine the number of bacteria per ml. in each of the tubes. Sometimes a second toxicity test was required, using dilutions between two of the original steps, to determine the proper concentration more precisely.

\section{(3) Test for mutagenic activity}

Knowing the concentration of the chemical that will kill $99 \%$ of the bacteria in 2 or 3 hours, a preliminary test is run to determine the effectiveness of the chemical under these conditions in inducing zero point mutations. Since sample experiments will be described in detail in the experimental section, only a general outline of the procedure will be given here:

A sample of a stock low-background culture is divided into two equal parts, centrifuged, and the nutrient medium decanted. One pellet is resuspended in the proper concentration of the chemical, and the other in distilled water or buffer as a control. The tubes are incubated for the length of time required to kill $99 \%$ of the bacteria in the experimental tube, and then the cultures are assayed to determine the number of viable bacteria per $\mathrm{ml}$., and the number of $\mathrm{B} / \mathrm{r} / 1$ mutants per $10^{8}$ bacteria. The number of mutants per $10^{8}$ bacteria in the control, the background number, is subtracted from the number of mutants per $10^{8}$ survivors in the treated culture. As the background number of a given culture is extremely constant in independent determinations, seldom differing by more than 2 or 3 mutants per $10^{8}$, an increase of 10 mutants per $10^{8}$ over the background number is considered to be an indication of a positive effect.

Since the effect of chemicals on zero point mutations is under consideration, it is important to establish conditions under which division of bacteria during treatment will not occur. The use of a non-nutrient medium, and a concentration of bacteria at least as great as, and often 10 to 20 times greater than, the concentration reached after maximal growth, were among the precautions taken to prevent division during treatment. In addition, the exposure time was usually well below the normal lag, even for small inocula in fresh nutrient medium. Microscopic total counts revealed no measurable increase in cell number under the conditions described, over periods as long as 72 hours.

\section{(4) Differential survival test}

Whether the preliminary test for mutagenic activity is positive or negative, it is important to eliminate the possibility of a selective action of the chemical. If $\mathrm{B} / \mathrm{r} / 1$ mutants are, for some reason, less sensitive to the toxic effects of the chemical, the proportion of mutants among the survivors of the treated culture will be higher than in the control, and a mutagenic action might be erroneously ascribed to the compound. If the mutants should be more sensitive than the nonmutants, it is possible that a positive mutagenic effect could be masked by the differential killing of mutants. Thus, to be certain that selection for or against the mutants is not responsible for an apparent positive or negative effect, it is necessary to compare the sensitivity of mutants and nonmutants to each of the chemicals tested. The standard procedure used in these experiments involved the following steps:

A stock of $\mathrm{B} / \mathrm{r} / 1$ was established by inoculating a small amount of growth from about ten representative $B / r / 1$ colonies on a control plate into a tube of M-9. A new stock was made up for each experiment, and the colonies which were isolated always came from a plating of the control tube of an experiment designed to test the mutagenic action of the chemical in question. Thus, the $B / r / 1$ stock isolated in this way was representative of the mutants present in an experimental culture at the start of the exposure to the chemical. The culture derived from these colonies was streaked on agar to eliminate contaminating particles of bacteriophage, and another culture was started with an inoculum from at least ten colonies. This culture was used as a source of $\mathrm{B} / \mathrm{r} / 1$ for the selective killing test.

To compare the sensitivity of $B / r$ and $B / r / 1$ to a given chemical, 48-hour aerated M-9 cultures of the two strains were grown, and equal volumes of the two cultures were mixed together, to give a culture containing approximately half mutants and half nonmutants. Two tubes were set up, each containing the same volume of the mixed culture, centrifuged, and the nutrient medium poured off. One pellet was resuspended in the standard concentration of the chemical, and the other in buffer or distilled water. The tubes were incubated for the standard time, and assayed to determine the proportion of mutants in each culture. If mutants and nonmutants are killed at the same rate, there should be no difference in the proportion of mutants between the control and experimental tubes. A sample experiment of this type will be described below.

\section{(5) Test of mutant colonies}

In all cases where a positive effect was obtained, samples of the mutant colonies obtained after treatment with the chemical were isolated, and tested carefully to establish the fact that they were true $\mathrm{B} / \mathrm{r} / 1$ mutants. In experiments where the total number of colonies obtained was small, and where a few contaminants could distort the results, every colony was isolated and tested. The colonies were examined for resistance to $\mathrm{T} 1$, and also to another phage, $\mathrm{T} 2$, to which strain $\mathrm{B} / \mathrm{r}$ is sensitive. Colonies showing resistance to $\mathrm{T} 1$ and sensitivity to $\mathrm{T} 2$ were regarded as $B / r / 1$ mutants. Colonies showing resistance to phages were regarded as contaminants. 


\section{EXPERIMENTAL RESULTS}

Since the effect of chemicals in inducing zero point mutations is to be investigated, it is important to know to what extent mutations occur spontaneously, if at all, in resting cells. Luria and Delbrück (1943) have shown that mutations to resistance to $\mathrm{T} 1$ do not occur spontaneously in other compounds tested in these experiments. It is a highly reactive compound, forming addition compounds known as choleates with a wide variety of organic substances, including fatty acids, ether, xylol and certain carcinogenic hydrocarbons (Wieland and Sorge, 1916; Fieser and Newman, 1935). Alloway (1933) used sodium desoxycholate in his

Table 1. Number of B/r/1 Mutants in Resting Culture of B/r Suspended in Distilled Water for 24 Hours at $37^{\circ} \mathrm{C}$.

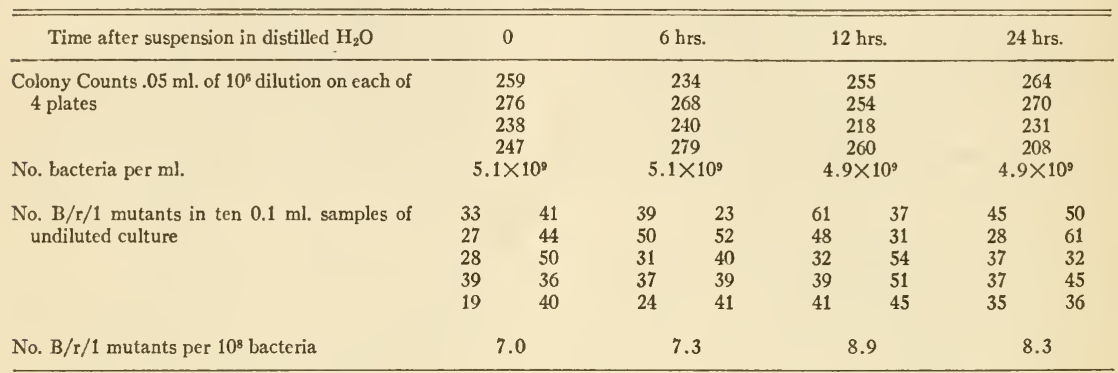

resting bacteria, and our own observations have confirmed this. Table 1 shows the results of an experiment in which resting bacteria, suspended in distilled water and incubated for a period of 24 hours at $37^{\circ} \mathrm{C}$, were periodically assayed to determine the number of $\mathrm{B} / \mathrm{r} / 1$ mutants per $10^{8}$ cells. The number of mutants per $10^{8}$ bacteria remains extremely constant under these conditions, and any mutations induced in resting cells must be compared with a baseline of zero.

\section{Sodium Desoxycholate}

Sodium desoxycholate is a salt of the bile acid desoxycholic acid, the molecular structure of which is shown in Fig. 1, along with the structures of the

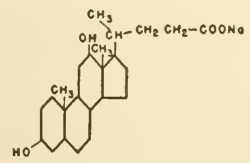

(1) SODIUM DESOXYCHOLATE

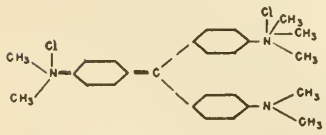

(3) METHYL GREEN

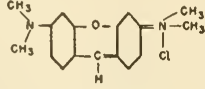

(2) PYRONIN Y

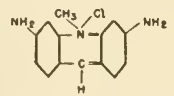

(4) NEUTRAL ACRIFLAVINE
Fic. 1. Molecular structure of Sodium Desoxycholate, Pyronin; Methyl Green and Acriflavine. early work on type transformation, and it is used by Avery and his coworkers in the preparation of transforming principle (1944), where its function is to dissolve the desoxyribose nucleoprotein complex of the pneumococcus. Mirsky and Pollister (1946) have shown that a $.5 \%$ solution of sodium desoxycholate dissolves their preparations of thymus chromosin fibers, and can be used to extract the desoxyribose nucleoprotein complex from minced thymus. While this solvent action of desoxycholate on nucleoproteins served as a basis for selecting this compound for a test of its mutagenic activity, there is no doubt that it is a nonspecific effect.

Sodium desoxycholate dissolves easily in water above $\mathrm{pH} 6.5$, but solutions at $\mathrm{pH} 6.5$ to 7.5 form a gel at high concentrations. Since the reaction of desoxycholate in distilled water is about 7.7 , unbuffered solutions of the compound in distilled water were used. Fresh solutions were made up for each experiment, and sterilized by immersion in a boiling water bath for 15 minutes.

Toxicity. Preliminary toxicity tests showed that $99 \%$ of the exposed bacteria were killed in 3 hours by a $5 \%$ solution, or in 48 hours by a $0.3 \%$ solution. All of the experiments reported here were done using the $5 \%$ solution.

Test for mutagenic activity. To illustrate in detail the method used in testing chemicals for ability to induce mutations, a typical experiment done with sodium desoxycholate will be described.

Ten-ml. aliquots of a stock low-background culture of $\mathrm{B} / \mathrm{r}$ were pipetted into each of 4 centrifuge tubes, which were centrifuged for 20 minutes at 4,000 RPM. The supernatant was then decanted to 
eliminate the nutrient buffered medium. The bacterial pellets in two of the tubes were resuspended in $2 \mathrm{ml}$. of a $5 \%$ solution of sodium desoxycholate, and the bacteria in the remaining two tubes were resuspended in $2 \mathrm{ml}$. of distilled water to serve as controls. In some experiments, a phosphate buffer of $\mathrm{pH} 7.7$ was used for the control, but distilled water was found to be equally satisfactory. In neither case was there any change in the number of viable bacteria or in the background number during the course of any experiment. The initial centrifugation, in addition to eliminating the nutrient mutants and $50 \%$ nonmutants. Ten ml. of the mixed culture was pipetted into each of two centrifuge tubes, centrifuged in the usual way, and the pellets resuspended in $2 \mathrm{ml}$. of liquid, $5 \%$ desoxycholate for the experimental tube and distilled water for the control. The tubes were incubated for three hours at $37^{\circ} \mathrm{C}$. Thus, these cultures received the same treatment throughout as the cultures used to test for mutagenic activity. At the end of the 3-hour incubation, the tubes were assayed to determine the proportion of mutants in the control and experimental cultures. This was done by plating the final

Table 2. Zero-Point Mutations Induced by 3-Hour Exposure to 5\% Sodium Desoxycholate

1 and 2 are duplicate control tubes. 3 and 4 are duplicate experimental tubes. a and b are independent dilutions of each tube.

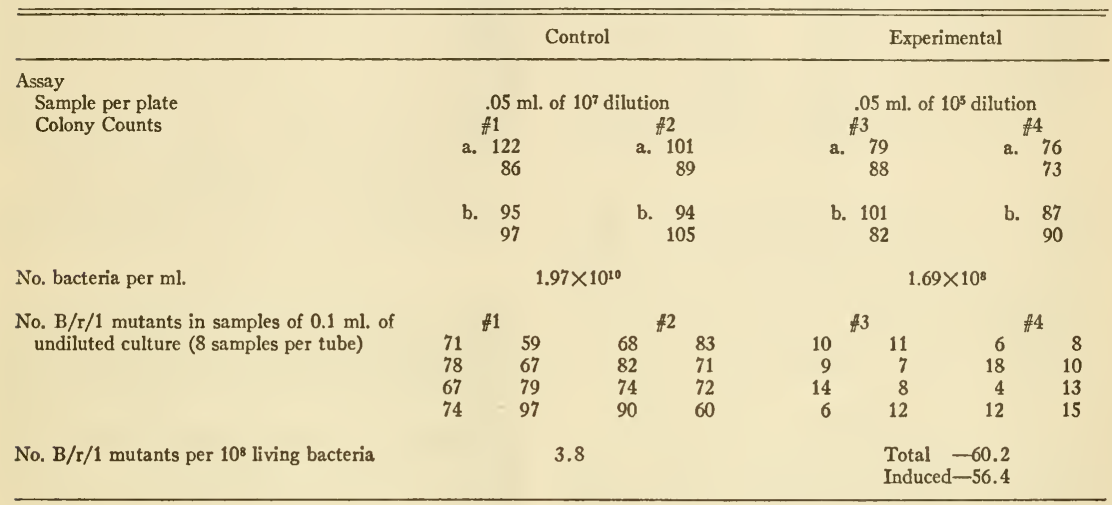

medium, served to concentrate the bacteria by a factor of 5 (in some experiments by as much as a factor of 20), thus facilitating the detection of mutants among the survivors.

The control and experimental tubes were incubated for 3 hours at $37^{\circ} \mathrm{C}$, and were then assayed to determine the number of viable bacteria per ml., and the number of $\mathrm{B} / \mathrm{r} / 1$ mutants per $10^{8}$. The results are shown in Table 2. These results indicate the proportion of mutants among the survivors of the exposure to desoxycholate was increased from 3.8 per $10^{8}$, the background number, to 60.2 per $10^{8}$. Subtracting the background, the increase in mutants per $10^{8}$ survivors is 56.4 .

Differential Survival test. In order to test the possibility that the observed increase in the proportion of mutants among the survivors of desoxycholate-treated bacteria is due to simple selection, the following experiment was done.

Cultures of $B / r$ and $B / r / 1$ were grown with aeration in M-9 for 48 hours, and equal volumes of the two cultures were combined in a single tube, to give a mixed culture containing approximately $50 \%$ dilution of each culture on two series of plates, one consisting of ordinary agar plates, and one consisting of plates which had been coated previously with a heavy suspension of bacteriophage Tl. The ratio of the colony counts on phaged plates to that on unphaged plates gives the proportion of mutants in the mixture. The results are given in Table 3 . In the control culture, the number of colonies developing on phaged plates was $48.9 \%$ of the number appearing on unphaged plates. In the experimental culture, in which about $99 \%$ of the bacteria were killed, the proportion of mutants among the survivors was $51.6 \%$. Thus, it appears that mutants and nonmutants are equally sensitive to the toxic effects of sodium desoxycholate. These tests were made on mixed cultures rather than on pure cultures of the two strains in view of the possibility that competition phenomena might be involved in selective killing.

Tests of Mutant colonies. Ninety-four colonies appearing on phaged plates after treatment with desoxycholate were isolated and tested for resistance to T1 and T2. All but three proved to be 
resistant to $\mathrm{T} 1$ and sensitive to $\mathrm{T} 2$, and were therefore considered to be true $\mathrm{B} / \mathrm{r} / 1$ mutants. Two colonies were resistant to both phage strains, and were regarded as contaminants (in both cases the colony morphology alone rendered them suspect). The third colony was sensitive to both $\mathrm{T} 1$ and $\mathrm{T} 2$,

Table 3. Test for Differential Killing of Strain $B / r$ AND $B / r / 1$ By 3-Hour Exposure to $5 \%$ Sodrum Desoxycholate

Control $=$ mixed culture in distilled water Experimental $=$ mixed culture in $5 \%$ Sodium Desoxycholate

\begin{tabular}{|c|c|c|c|c|}
\hline & \multicolumn{2}{|c|}{ Control } & \multicolumn{2}{|c|}{ Experimental } \\
\hline Sample per plate & \multicolumn{2}{|c|}{$\begin{array}{c}.05 \mathrm{ml} . \text { of } \\
5 \times 10^{6} \text { dilution }\end{array}$} & \multicolumn{2}{|c|}{$\begin{array}{l}.05 \mathrm{ml} \text {. of } \\
5 \times 10^{4} \text { dilution }\end{array}$} \\
\hline Colony Counts & $\begin{array}{l}\text { plated } \\
\text { without } \\
\text { phage }\end{array}$ & $\begin{array}{l}\text { plated } \\
\text { with } \\
\text { excess of } \\
\text { phage }\end{array}$ & $\begin{array}{l}\text { plated } \\
\text { without } \\
\text { phage }\end{array}$ & $\begin{array}{l}\text { plated } \\
\text { with } \\
\text { excess of } \\
\text { phage }\end{array}$ \\
\hline & $\begin{array}{l}462 \\
377 \\
468 \\
408\end{array}$ & $\begin{array}{l}192 \\
226 \\
198 \\
224\end{array}$ & $\begin{array}{l}441 \\
443 \\
482 \\
493\end{array}$ & $\begin{array}{l}233 \\
240 \\
246 \\
239\end{array}$ \\
\hline
\end{tabular}

No. bacteria

per $\mathrm{ml}$.

\begin{tabular}{ccc}
$\begin{array}{c}\mathrm{B} / \mathrm{r}+\mathrm{B} / \mathrm{r} / 1 \\
\mathrm{~B} / \mathrm{r} / 1\end{array}$ & $4.29 \times 10^{10}$ & $4.65 \times 10^{8}$ \\
$\% \mathrm{~B} / \mathrm{r} / 1$ & $2.1 \times 10^{10}$ & $2.4 \times 10^{8}$ \\
& 48.9 & 51.6 \\
\hline
\end{tabular}

and since this colony appeared on the edge of the plate, it is likely that it arose from a sensitive bacterium which escaped lysis due to faulty spreading of the phage suspension.

Relation between the number of induced mutations and time of exposure to $5 \%$ desoxycholate. Demerec and Latarjet (1946), in their studies of radiation-induced mutations to phage-resistance, investigated the relation between dosage of radiation and the number of mutations induced. They found that the number of zero point mutations to $\mathrm{B} / \mathrm{r} / 1$ is directly proportional to X-ray dose, and bears a more complicated exponential relation to ultraviolet dose. In the present investigations, experiments were conducted to determine the analogous relation between the number of induced mutations and time of exposure to a $5 \%$ solution of sodium desoxycholate.

Fig. 2 shows a survival curve obtained with sodium desoxycholate, in which the percentage of surviving bacteria is plotted against time of exposure to the standard concentration. Fig. 3 shows the number of zero point mutations, corrected for the background number, as a function of time of exposure to $5 \%$ desoxycholate. The data upon which this curve is based were obtained by essen- tially the same methods as those described in the sample experiment above. Wherever possible, a single experimental tube was incubated and sampled over the entire 10-hour period, since the use of

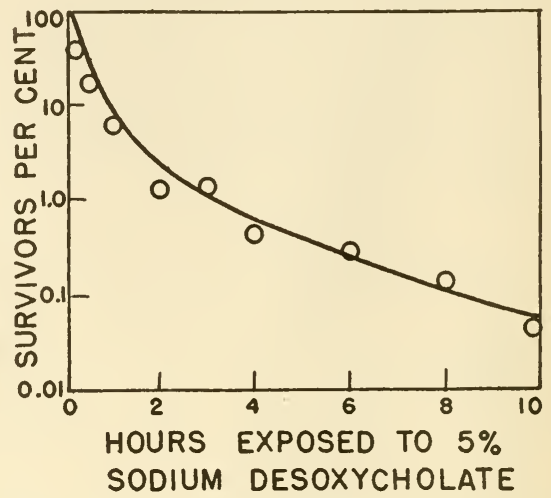

FIG. 2. Survival curve of resting bacteria exposed to $5 \%$ Sodium Desoxycholate.

separate tubes for each determination gave somewhat more variable results. The linear relation between induced mutations and exposure time can be compared directly with the X-ray dosage-effect

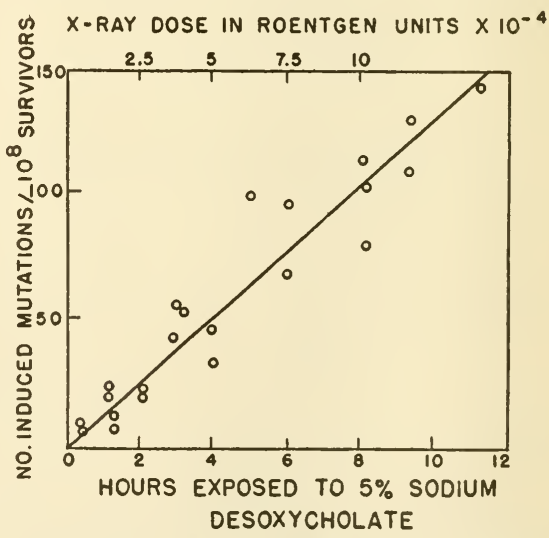

Fic. 3. Relation between the number of zero point mutations induced and time of exposure to $5 \%$ sodium desoxycholate. The upper abscissa represents X-ray dose in roentgen units, on a scale required to make this curve coincide with the curve obtained by Demerec and Latarjet (1946) for the relation between $\mathrm{X}$-ray dose and number of zero point mutations. 
curve obtained by Demerec and Latarjet, and differs fundamentally from their analogous ultraviolet curve. The upper abscissa in Fig. 3 represents X-ray dose in $r$ units, on a scale required to make the desoxycholate curve coincide with the $\mathrm{X}$-ray curve of Demerec and Latarjet. It will be noted that $100,000 \mathrm{r}$ units correspond, in terms of the number of induced zero point mutations, to an 8-hour exposure to $5 \%$ sodium desoxycholate.

On the basis of these positive results obtained with desoxycholate as a mutagenic agent in bacteria, Demerec (unpub.) has tested the ability of this compound to induce lethal mutations in Drosophila. The proportion of lethals among the tested sperm was about $1.5 \%$, as compared with $0.24 \%$ in controls, an effect of the same order of magnitude as that obtained with nitrogen mustard by the same technique. These results with Drosophila suggest that sodium desoxycholate may prove to be a nonspecific mutagen, like radiations and mustard. No tests have been made to investigate the possibility of specific effects on the particular mutation used as an index of mutagenic activity, namely, resistance to bacteriophage T1. For this purpose it will be necessary to investigate the action of desoxycholate on other bacterial mutations.

\section{Pyronin and Methyl Green}

Basic dyes, which stain chromatin by virtue of their affinity for nucleic acids, were considered as a class of compounds worthy of investigation for possible mutagenic activity. Pyronin and methyl green, the components of the Unna-Pappenheim mixture currently of interest in cytochemical research, were the first dyes to be tested. Brachet (1940) has described the usefulness of this mixture as a means of differentiating cytochemically between ribose and desoxyribose nucleic acids, by virtue of the selective affinity of pyronin for the ribose type of nucleic acid. Although there is good evidence for the specificity of the pyronin-methyl green mixture under certain conditions, it must be pointed out that the treatment of living bacteria, without recourse to the procedures of fixation and differentiation which are standard in cytological work, may very well present entirely different conditions. Thus pyronin may be taken up by fatty acids, or other basophilic elements ordinarily removed in cytological preparations. There is no basis, therefore, for assuming that these dyes are specific, under the conditions of these experiments, in acting exclusively upon nucleic acids, or in differentiating between the two types of nucleic acid.

Pyronin was tested by essentially the same techniques described in connection with desoxycholate. One difficulty encountered, however, was the great variability of the toxic effects of a given concentration in different experiments, or in different tubes in the same experiment. One possible source of the variability was thought to be the precipitate which forms in the presence of bacteria at high concentrations of the dye. Low concentrations, in which there is no precipitate, were tried, and in some experiments bacteria were spread on the surface of

Table 4. Zero Point Mutations Induced by Pyronin Y

\begin{tabular}{lclcc}
\hline $\begin{array}{c}\text { Conc. of } \\
\text { Pyronin Y } \\
(\%)\end{array}$ & $\begin{array}{c}\text { Time } \\
\text { of } \\
\text { Exposure }\end{array}$ & $\begin{array}{c}\text { Method } \\
\text { of } \\
\text { Treatment }\end{array}$ & $\begin{array}{c}\text { Survival } \\
(\%)\end{array}$ & $\begin{array}{c}\text { No. In- } \\
\text { duced Mu- } \\
\text { tations } / 10^{8} \\
\text { Survivors }\end{array}$ \\
\hline 0.5 & $20 \mathrm{~min}$. & in agar & 43.0 & 10.6 \\
0.01 & $5 \mathrm{hr}$. & in liquid & 32.2 & 22.4 \\
0.075 & $1 \mathrm{hr}$. & in liquid & 18.6 & 14.0 \\
0.75 & $1 \mathrm{hr}$. & in agar & 18.1 & 22.8 \\
0.5 & $2 \frac{1}{2} \mathrm{hr}$. & in agar & 12.5 & 33.7 \\
0.05 & $3 \mathrm{hr}$. & in liquid & 3.9 & 36.0 \\
2.0 & $30 \mathrm{~min}$. & in agar & 2.5 & 41.3 \\
0.5 & $15 \mathrm{~min}$. & in agar & 0.16 & 62.7 \\
0.75 & $2 \mathrm{hr}$. & in agar & 0.11 & 150.0 \\
0.5 & $2 \mathrm{hr}$. & in agar & 0.03 & 100.7 \\
0.025 & $4 \mathrm{hr}$. & in liquid & 0.01 & 1080 \\
\hline
\end{tabular}

agar containing pyronin, were washed from the surface, and a concentrate of the wash was assayed. The variability of the results was not overcome by these modifications in technique, and the basis of the difficulty is not yet understood. The results obtained with pyronin, at various concentrations, and under various conditions of treatment, are sum-

Table 5. Number of $\mathrm{B} / \mathrm{r} / 1$ Mutants in Cultures EXPOSED to $1 \%$ Methyl Green

\begin{tabular}{lccccr}
\hline \hline Cul- & $\begin{array}{c}\text { Time of } \\
\text { Ex- } \\
\text { ture } \\
\text { posure } \\
\text { to 1\% }\end{array}$ & $\begin{array}{c}\text { Experiment } \# 1 \\
\text { Sur- } \\
\text { vival } \\
(\%)\end{array}$ & $\begin{array}{c}\text { No. } \\
\text { Mut. } \\
\text { per 10 } 10^{8}\end{array}$ & $\begin{array}{c}\text { Experiment } \\
\text { Sur- } \\
\text { vival } \\
(\%)\end{array}$ & $\begin{array}{c}\text { No. } \\
\text { Mut. } \\
\text { per } 10^{8}\end{array}$ \\
\hline Control & - & - & 7.7 & - & 10.2 \\
& & & & & \\
Exp. & $1 \mathrm{hr}$. & 40 & 5.8 & 34 & 10.1 \\
Exp. & $2 \mathrm{hr}$. & 11 & 8.1 & 7 & 8.6 \\
Exp. & $3 \mathrm{hr}$. & 1.1 & 7.2 & 1.1 & 11.2 \\
Exp. & $4 \mathrm{hr}$. & 0.2 & 6.9 & 0.1 & 9.9 \\
\hline
\end{tabular}

marized in Table 4. All results obtained were consistent in indicating a higher proportion of mutants among the survivors of treated bacteria than among controls. Tests for selective killing of mutants showed no difference in the sensitivity of $B / r$ and $B / r / l$ to pyronin. Although these results are to be regarded as preliminary and tentative, they suggest that pyronin is active as a mutagenic agent. The problem of variability will have to be solved before any detailed quantitative analysis can be made.

Pyronin has not yet been tested on Drosophila, and there is no evidence as to its specificity in inducing mutations.

Toxicity tests with methyl green showed that 
$99 \%$ of the exposed bacteria were killed in about three hours by a $1 \%$ solution in distilled water. Table 5 gives the results of two experiments with methyl green, in which the survival was as low as $0.2 \%$, with no increase in the proportion of mutants among the treated bacteria. These results were confirmed by repeated tests. Within the limits of the sensitivity of the technique, which permits the detection of induced mutants under conditions resulting in the destruction of $99.9 \%$ of the treated cells, methyl green seems to show no mutagenic activity.

\section{Acriflavine}

Neutral acriflavine, or euflavine, is an acridine dye used therapeutically as a bacteriostatic agent. McIlwain (1941) has shown that the inhibition of bacterial division caused by acriflavine can be reversed by adding polymerized yeast or thymus nucleic acids. He also showed, in vitro experi-

Table 6. Zero Point Mutations Induced by Acriflavin

\begin{tabular}{cccc}
\hline $\begin{array}{c}\text { Conc. } \\
\text { of } \\
\text { Acriflavin }\end{array}$ & $\begin{array}{c}\text { Time } \\
\text { of } \\
\text { Exposure }\end{array}$ & $\begin{array}{c}\text { Survival } \\
(\%)\end{array}$ & $\begin{array}{c}\text { No. of } \\
\text { Induced Mu- } \\
\text { tations } / 10^{8} \\
\text { Survivors }\end{array}$ \\
\hline .02 & $3 \mathrm{hr}$. & 50.4 & 8.0 \\
.01 & $2 \frac{1}{\mathrm{hr}}$. & 12.8 & 16.5 \\
.01 & $4 \mathrm{hr}$. & 6.5 & 54.1 \\
.05 & $2 \mathrm{hr}$. & 2.5 & 24.7 \\
.05 & $2 \mathrm{hr}$. & 1.1 & 60.6 \\
.05 & $3 \mathrm{hr}$. & 0.4 & 51.3 \\
.05 & $4 \mathrm{hr}$. & 0.08 & 400 \\
.05 & $4 \frac{1}{2} \mathrm{hr}$. & 0.02 & 420 \\
.05 & $4 \mathrm{hr}$. & 0.005 & 1540 \\
.05 & $4 \mathrm{hr}$. & 0.0007 & 4000 \\
\hline
\end{tabular}

ments, that stable complex salts are formed by acriflavine and nucleates. There is again no basis for assuming specificity in these effects, since a number of other compounds, including certain amino acids, were also found to be effective in reversing the inhibition brought about by acriflavine.

Table 6 gives a summary of results obtained with acriflavine. Although reproducible results can be obtained if all factors are carefully standardized, acriflavine presents certain difficulties which stand in the way of quantitative investigation. In the presence of large numbers of bacteria, some of the dye precipitates at high concentrations, and the precipitate goes gradually is:to solution as the tubes are incubated. Thus it is likely that the effective concentration changes during the exposure. This difficulty can be overcome to some extent by using low concentrations and longer periods of exposure. Results with acriflavine consistently indicate an increase in the proportion of mutants among survivors of treated cultures, and suggest that this compound is active in inducing mutations.
Demerec et al (1946) tested the action of acriflavine on Drosophila, prior to the experiments reported here, and obtained negative results. More recently, Demerec (unpub.) has retested acriflavine, using an improved method of administering the chemical, and has found that it is active in inducing lethal mutations in Drosophila.

\section{Discussion}

The experiments described above have indicated that exposure to three out of the four chemicals tested, at concentrations sufficiently toxic to kill all but a small fraction of the treated bacteria, results in a heightened proportion of mutants among the survivors. Since $B / r / 1$ was found to be no more resistant than the nonmutant strain $\mathrm{B} / \mathrm{r}$ to each of the chemicals, it has been concluded that simple selection is not responsible for this effect. Sodium desoxycholate, pyronin and acriflavine are therefore regarded as mutagenic chemicals, although the results for the latter two compounds are only preliminary. Methyl green is apparently unable to induce mutations to phage-resistance.

Although a single specific phenotype, resistance to bacteriophage $\mathrm{T} 1$, was the genetic character used in these experiments, it is likely that at least two separate mutations were involved, since $B / r / 1 \mathrm{mu}-$ tants are known to fall into two distinct classes, differentiated by such secondary characters as colony size, cross resistance to another bacteriophage and growth factor requirements. No attempt was made to separate the two mutant types in these experiments, and the frequencies observed are probably the sums of the two independent mutations.

Because of the lack of specificity in the chemical action of the compounds tested, no attempt can be made at present to relate their effectiveness as mutagenic agents to the properties for which they were selected. The high incidence of positive results obtained, in 3 out of 4 compounds examined, is also difficult to interpret at the present time. Further experiments are required to determine whether these results are due to a particularly fruitful or fortunate basis of selecting chemicals, or, as appears more likely, whether mutagenic action may prove to be more common among biologically active compounds than has hitherto been believed.

Although bacteria are becoming increasingly useful as material for genetic investigations, it is still necessary to be cautious in generalizing results obtained exclusively in bacterial studies. Thus, the fact that sodium desoxycholate and acriflavine appear to induce mutations in Drosophila as well as in $E$. coli is an important contribution toward the validation of the techniques used in these experiments. In addition to confirmation provided by tests on other organisms, the use of other mutations in $E$. coli, entirely independent of the phage-resistance system, would constitute a valuable check on results obtained with nonspecific mutagens. Experiments to 
determine the effects of chemicals on the frequency of reverse mutations from biochemically deficient mutants of $E$. coli are being planned with this need in mind.

The use of phage-resistance in E. coli as material for the investigation of chemically induced mutations offers certain unique possibilities, in addition to its value as a screening test. The analysis of dosage-effect relations, as well as the quantitative investigation of the delayed expression of induced mutations characteristic of radiations and mustard gas, can be carried out with this material. It may also be possible to approach another interesting aspect of induced mutations, the comparison of the effectiveness of mutagenic chemicals on resting and dividing cells. It must be remembered, however, that bacteria are relative newcomers to the laboratory of the geneticist, and that a longer and more intimate acquaintance may be required to establish the reliability of these organisms as tools for the study of the broader problems of heredity and mutation.

\section{SUMMARY}

Four compounds were tested for mutagenic activity in $E$. coli. The techniques used involved suspending resting bacteria in solutions of the chemicals, under conditions resulting in the death of about $99 \%$ of the treated cells. The number of mutants resistant to a bacteriophage, T1, per $10^{8}$ survivors of a treated culture was compared with the number per $10^{8}$ untreated bacteria from the same culture. Only mutations expressed phenotypically before division of the exposed individuals were detected.

The number of mutants per $10^{8}$ survivors was found to be higher in cultures treated with sodium desoxycholate, pyronin and acriflavine than in untreated samples of the same cultures. No such increase was obtained with methyl green. Since mutants and nonmutants were shown to be equally sensitive to the toxic action of each of the compounds, it has been concluded that selection is not responsible for these results. Sodium desoxycholate, pyronin and acriflavine are considered, therefore, to ve mutagenic, while methyl green is not.

The number of mutations induced by sodium desoxycholate is directly proportional to the time of exposure to a $5 \%$ solution of this compound.

\section{ACKNOWLEDGMENTS}

The author wishes to acknowledge the helpful suggestions of Dr. M. Demerec, and the efficient assistance of Miss Marion Crippen in many of these experiments.

\section{REFERENCES}

Alloway, J. L., 1933, Further observations on the use of pneumococcus extracts in effecting transformation of types in vitro. J. exp. Med. 57: 265-278.

Auerbach, C., and Robson, J. M., 1944, Production of mutations by allyl isothiocyanate. Nature, Lond. 154: 81-82.
Avery, O. T., MacLeod, C. M., and McCarty, M., 1944, Studies on the chemical nature of the substance inducing transformation of pneumococcal types. Induction of transformation by a desoxyribonucleic acid fraction isolated from pneumococcus Type III. J. exp. Med, 79: 137-158.

BRACHET, J., 1940, La detection histochimique des acides pentosesnucléiques. C. R. Soc. Biol., Paris 133: 88-90.

Demerec, M., 1946, Induced mutations and possible mechanisms of the transmission of heredity in Escherichia coli. Proc. Nat. Acad. Sci., Wash. 32: 36-46.

1943, Mutations in Drosophila induced by a carcinogen. Nature, Lond. 159: 604.

Demerec, M., and Latarjet, R., 1946, Mutations in bacteria induced by radiation. Cold Spring Harbor Symp. Quant. Biol. 11: 38-50.

Demerec, M., Latarjet, R., Luria, S. E., Oakberg, E. F., and Witkin, E. M., 1946, The gene. Yearb. Carn. Inst. 45: 143-157.

Fieser, L. F., and Newnian, M. S., 1935, The choleic acids of certain carcinogenic hydrocarbons. J. Amer. Chem. Soc. 57: 1602-1604.

Horowitz, N. H., Houlahan, M. B., Hungate, M. V., and Wriger, B., 1946, Mustard gas mutations in Neurospora. Science 104: 233-234.

Luria, S. E., and Deibrück, M., 1943, Mutations of bacteria from virus sensitivity to virus resistance. Genetics 28 : 491-511.

McIlwaIN, H., 1941, A nutritional investigation of the antibacterial action of acriflavin. Bio-chem. J. 35: 13111319.

Mirsky, A. E., and Pollister, A. W., 1946, Chromosin, a desoxyribose nucleoprotein complex of the cell nucleus. J. gen. Physiol. 30: 117-148.

Muller, H. J., 1947, The gene. (Pilgrim Trust Lecture). Proc. Roy. Soc. Lon. (B) 134: 1-37.

TAtuM, E. L., 1946, Induced biochemical mutations in bacteria. Cold Spring Harbor Symp. Quant. Biol. 11: 278284.

WIELAND, H., and SoRge, H., 1916, Untersuchungen über die Gallensäuren. II Mitteilung. Zur Kenntnis der Cboleinsäure. Hoppe-Seyl. Z. 97 : 1-27.

Witxin, E. M., 1947, Genetics of resistance to radiation in Escherichia coli. Genetics 32: 221-248.

\section{Discussion}

Hотсикіss: In examining the most interesting results of Dr. Witkin, I have been struck by the fact that the actual recovery of mutants from a fixed number of original cells, which is the count actually observed in the laboratory, is maximal in the high survival range and decreases markedly as the percentage of total survivors drops. Since the number of mutants does not however decrease to the extent that the total population does, it tends to rise rather considerably, if calculated over to the basis of survivors. It has been recognized by the author that to view this latter calculated value as a "mutation rate" involves the assumption that the phageresistant mutants do not have increased survival value in solutions of the chemical mutagens used. Indeed for this reason, trouble was taken to demonstrate that stock phage-resistant mutants do not survive longer than non-mutants in desoxycholate. 
It appears, however, that the actual mutants isolated after desoxycholate, pyronin or acriflavine treatment were not tested for resistance to these chemicals. It should be pointed out that only a modest degree of chemical-resistance in only a portion of the isolated mutant clones would allow the observed increased proportion of mutants among the survivors.

To bring out this point more clearly I may be permitted perhaps to recalculate one of the tables from the lantern slide dealing with acriflavinetreated cultures. The other tables would give very similar results. From the figures given it is possible to recalculate the actual yield of mutants observed from each $10^{8}$ bacteria of the original culture. While this column has to be calculated from another in this instance the point must be stressed that it was originally an observed part of the experimental data and tabulating it involves no particular assumptions.

$\begin{array}{ccc}\begin{array}{c}\text { Per cent } \\ \text { survival }\end{array} & \begin{array}{c}\text { Mutants per } \\ 10^{8} \text { survivors }\end{array} & \begin{array}{c}\text { Corrected for } \\ \text { estimated } \\ \text { "background" }\end{array} \\ 100 & 0 & 5 \\ 50 & 8 & 13 \\ 6.5 & 51 & 56 \\ 1 & 61 & 66 \\ 0.02 & 420 & 425 \\ 0.005 & 1540 & 1545 \\ 0.0007 & 4000 & 4000\end{array}$

It will be noted that, considered from this point of view, the most striking production of mutants was in the low-killing range where an increase of some $30 \%$ was observed. Detected as it presumably was in counts of some one or two hundred colonies, this increase represents at present the only positive indication, free of assumptions, that new mutants have been produced. The particular figure is of course rather dependent upon the value taken for the background content of mutants; what was understood to be an average figure was used here. In any case, where the killing has been greater, the "yield" of mutants has also decreased well below the background value.

Although several theories or explanations come to mind to account for the findings, it should be pointed out that these comments arise as it were from experimental considerations only and are suggested mainly as a more conservative way of expressing the data. When it has been demonstrated to what degree, if any, representative mutants recovered from the chemical mutagen solutions are resistant to that mutagen, then it will be more safe to calculate the actual yield of mutants, dead and alive, produced from $10^{8}$ original cells.

Finally, it may be pointed out that there is no reason to suppose that mutations induced by chemicals need show randomness with repect either to the individual cells affected within a population or to the characters, genetic or somatic, affected within a given cell. Once a particular cell, by virtue of its permeability, physiological state, internal $\mathrm{pH}$, or other inherent or transient property, has accumulated in its interior sufficient chemical mutagen to suffer a mutation at one locus, we would indeed expect that many other changes might become manifest. This picture would furnish one plausible basis for the conservative viewpoint that only a moderate amount of chemically-induced mutation has taken place, and that this has been to some degree associated with temporary or inheritable resistance to the chemicals, a finding not to be expected to any great extent in spontaneous or irradiation-induced mutants.

Witkin: Dr. Hotchkiss has emphasized the fact that the absolute recovery of mutants falls off, except in the range of high survival, as the exposure to the chemical is extended. This is true not only

$\begin{aligned} & \text { Yield of mutants } \\ & \text { per } 10^{8} \text { original } \\ & \text { cells } \\ & 5 \\ & 6.5 \\ & 3.6 \\ & 0.7 \\ & 0.085 \\ & 0.077 \\ & 0.028\end{aligned}$

Per cent of Yield expressed as original mutants

72

14

1.7

1.5

0.6

for the chemicals studied in these experiments, but for radiations as well. An absolute increase of mutants is to be expected only in the case of nontoxic mutagens, or in the case of agents whose mutagenic activity is relatively much greater than its toxicity. No such case is known as yet, and it seems unlikely, at least for mutagens acting in an aspecific manner, that such cases are theoretically possible, since induced lethal mutations may be expected to exceed, as a class, any single phenotype under consideration. Nevertheless, the fact that absolute increases are not observed, except where survival is high, does create the necessity for caution in interpreting the results.

First of all, it can be pointed out that, for each of the three chemicals considered to be mutagenic, absolute increases in the recovery of mutants were observed under special circumstances. In the case of acriflavin and pyronin, the actual number of mutants recovered from a given number of original bacteria increases significantly when the killing does not exceed $50 \%$ to $60 \%$. In the case of sodium desoxycholate, it is possible to use low concentrations where the maximal killing is reached by 24 hours, and there is no further killing between 24 and 48 hours of exposure. The number of mutants, however, does increase during this period, resulting in actually higher mutant counts on the experimental 
plates at 48 hours, starting with a sample of the same size. Thus, for each chemical it is possible to show that new mutants do actually appear.

Dr. Hotchkiss suggests that the induced mutants may differ from the spontaneous mutants in resistance to the chemical used to induce them. In the case of desoxycholate, where there is a linear relation between the number of induced mutations and the time of exposure to the chemical, in spite of the fact that the rate of killing changes markedly during the period of exposure, it is difficult to see how selection can be involved. If the increase in number of mutants were due in large measure to the selective survival of a few mutants induced early in the treatment, surely the rate of increase in the proportion of mutants with time could not be totally independent of the survival curve.

Dr. Hotchkiss suggests that the mutant colonies actually obtained after treatment with the chemical should be compared with the nonmutant stock with respect to resistance to the chemical. It seems to me that this would not constitute a critical test of the hypothesis Dr. Hotchkiss has raised. First of all, a certain amount of the chemical is carried over onto the plates, and then mutant colonies develop in the presence of a very low concentration of the chemical, negligible in terms of toxic or mutagenic activity, but possbily sufficient to permit selection during the growth of the colony of spontaneous variants better able to withstand the action of the substance. Thus, to find that the mutant colonies obtained after exposure to the chemical are more resistant to its toxic action than the untreated nonmutant stock, is not a fair test of the situation before the development of the colony on the plate. Should the mutant colonies prove no more resistant to the action of the chemical than the untreated non-mutant stock, the possibility of a temporary resistance, as Dr. Hotchkiss has suggested, can be raised. I cannot at present see any way of testing this possibility critically, since it is based on the state of a recently induced mutant, during treatment with the chemical, and a transient resistance to the chemical can be postulated. One possible way to avoid considerations of this kind might be to start out by developing a strain which is maximally resistant to the chemical to begin with, and use this strain throughout.

The best evidence that new mutations are in fact appearing throughout the exposure, to my mind, will be based upon comparison of survival curves and curves of induced mutations as a function of exposure time. If, as has been found for desoxycholate, the proportion of mutants increases at a constant rate, regardless of changes in killing rate, the possibility that recently induced mutants survive selectively will become quite remote.

KURNICK: Dr. Witkin has expressed some surprise at the fact that 3 out of 4 tested chemicals proved mutagenic in her experiments and suggested that it will be found that mutagenicity is a common property of chemical reagents. This appears to be a not unlikely prognostication. While not wishing to deny the possibility of direct chemical action to induce mutations, I should like to present an alternative hypothesis here.

Dr. Witkin searches only for a specific mutationresistance to $\mathrm{T} 1$ phage - not random mutations. She observes that this mutation is present spontaneously in her controls in 5 to 10 per $10^{8}$ bacilli. It is not improbable that this mutant contains a specific mutagenic substance similar to that isolated by Avery for pneumococcic strains and by Boivin for a colon bacillus strain. Dr. Witkin has observed that the mutation effect of a given chemical corresponds more nearly to its killing effect than to any other factor, such as concentration of the chemical or the time of exposure. It is obvious that if, as she has shown, the mutant and the original strains are equally sensitive to the lethal effect of the drugs used, the higher the percentage of lethality, the greater will be the chance of destruction of some of the 5 to 10 spontaneous mutants per $10^{8}$ bacteria. The autolysis of the killed mutant (or penetration through its wall whose permeability is apt to differ from the living cell) will free the specific mutator substance (for $\mathrm{T} 1$ phage resistance) into the medium where it may cause the specific, directed mutation of other organisms. The nearer the killing rate approaches $100 \%$, the higher will be the concentration of this mutator substance, and so the higher the mutation rate.

Dr. Witkin has observed that after about $99.9 \%$ of the bacteria have been killed, the killing rate approaches zero, but the production of mutations continues. She suggests that this may mean that the lethal and mutagenic properties of her chemicals are unrelated, and that the $0.1 \%$ of surviving cells, while no longer susceptible to the lethal effect are still susceptible to the mutagenic effect of the drug. If the hypothesis presented herein were correct, we could readily account for the continued occurrence of mutations in the surviving bacteria by the fact that they are continuously exposed to the liberated mutator of the killed spontaneous mutants and so continue to undergo directed mutation.

Of the 4 chemicals studied by Dr. Witkin, only methyl green was inert as a "mutagen," although its lethality in the concentrations used was comparable to that of the others. Now if, in fact, as is indicated by Boivin and Avery, desoxyribonucleic acid is an essential component (perhaps the only component, but this argument is not germaine to this discussion), a chemical which combined specifically with this nucleic acid might inactivate the mutator substance. Methyl green is such a compound (as opposed to pyronin, for example). Thus, we may account for the lethality of this compound without the expected production of mutations.

On the other hand, some chemicals, which might 
themselves not inactivate the mutator substance, might nevertheless render it ineffective by altering the cell surface of the bacteria so as to deny access of the mutator substance to the interior of the cell. Such a reagent may be chloroform, which Dr. Beale has found ineffective as a mutagen despite its lethal effect.

Thus, the induction of mutations by chemicals which do not themselves directly interact with the gene would be a function of their lethality, the degree of interaction between the chemical reagent and the liberated mutator substance, and the degree to which the permeability of the cell wall is altered. In this manner we may account for differences in "mutagenicity" of compounds with identical killing rates.

This hypothesis is susceptible of experimental test in several ways. I shall suggest two such experiments. One would be to test (a) the effect of lethal reagents which do not induce mutations (particularly methyl green) on the mutator substances of Boivin and Avery before addition to the culture substrates to determine whether inactivation does in fact occur, and (b) the effect of adding the chemical (particularly chloroform) to the appropriate culture before adding Boivin's or Avery's substance to determine whether a reagent which does not itself inactivate the mutator substance but nevertheless fails to produce mutations, prevents the effect of the mutator substance by its action in the otherwise susceptible cells. Another would be to centrifuge off the surviving bacteria after $99.9 \%$ had been killed, wash them several times in water and then resuspend them in a medium containing the chemical under investigation (let us say pyronin). Controls would consist of bacteria left in the original medium and bacteria resuspended in water. If the bacteria resuspended in a fresh solution of the reagent and those resuspended in water show a significantly lower rate of continued mutation than those permitted to remain in the original suspension (provided the killing rate remained near zero in all samples), one could conclude that the mutagenic factor was not the chemical reagent, but rather some material which was present in the first supernate alone-this could only be derived from the cultures themselves. If, on the other hand, mutations continued to occur in all three test samples at the same rate, the conclusion would again be that the mutagenicity was probably not a direct function of the chemical (since one suspension is resuspended in water), but that the mutator substance was adsorbed to the washed bacteria or had a significant lag time between its action on the bacteria and the emergence of the mutant. The third result which might occur, namely that the sample resuspended in a fresh solution of the chemical showed a continued mutation rate equal to that of the original suspension and greater than that of the suspension in water, would disprove the hypothesis
I have proposed above and demonstrate the capacity of the chemicals themselves to produce mutations, presumably by direct attack on the gene.

I do not by any means, I repeat, wish to imply that chemical mutations due to the direct action of a chemical reagent on the gene do not occur. Quite the contrary, there is good reason to believe that such effects are possible. I wish only to interject a word of caution in interpreting the induction of mutations by a considerable number of reagents, particularly when working with such material as bacteria where the opportunity for interaction between large numbers of organisms exists. This pitfall may be avoided by suitable controls, as suggested above, to distinguish between direct interaction with the gene and the indirect effect due to autolysis.

Witkin: It is, of course, possible that phageresistant mutants of strain $\mathrm{B} / \mathrm{r}$ contain specific mutator substances, similar to the transforming factors of Avery and Boivin. The addition of filtrates of heat-killed cultures of $B / r / 1$, however, does not increase the number of resistant mutants in cultures of $\mathrm{B} / \mathrm{r}$. Numerous other attempts to detect evidence for transformation in this system have failed. This, in addition to the fact that the number of induced mutants obtained is independent of the background number, over a range of one to fifty per $10^{8} \mathrm{bac}$ teria, seems to me to render Dr. Kurnick's hypothesis unlikely.

BRYSON: In the event that we continue to find numerous and unrelated chemicals that are able to induce mutation, it may be of particular interpretive value to study with care those substances that are not mutagenic. It would also be desirable that more simple chemical substances such as inorganic salts be surveyed as mutagens, even as Drs. Greenstein, Carter and Chalkley have surveyed them for effect on enzymatic degradation of nucleic acid. One might then bring to bear what is known of the effects of ions on cells, and compare mutagenesis with what has been established about permeability and relative toxicity of various materials whose fate as reactants in living systems has already been the subject of extensive biochemical investigations. The problem of toxicity itself presents a difficulty in the classification of chemical mutagens since toxicity may act as a limiting factor in exploring mutagenic potential. For example, we have found that the induction of mutations of phage resistance in $E$. coli by bis-betachloroethylamine hydrochloride is most readily performed on a strain of cells that has been through twelve consecutive exposures to the inducing agent with repeated selection of survivors for resistance to the chemical. The number of zero point mutations induced by nitrogen mustard in stock $\mathrm{B}_{12} / \mathrm{M}$ at a survival level of $0.007 \%$ is 240 per $10^{8}$. It is always possible that a chemical like methyl green that Dr. Witkin has described as negatively mutagenic could be made to induce mutations in a strain of cells 
selected for resistance to its toxic effects and therefore capable of surviving in relatively high concentrations.

No attempt has been made to approach a comparable killing value with another mutagenic agent (Zephiran chloride) because this quaternary ammonium compound shows a selective toxic effect against $\mathrm{B} / 1$ at high concentrations, a process which incidentally would tend to make the detection of mutations a more difficult process. An experiment using 1:14,000 aq. Zephiran chloride (a non-selective concentration) is shown in Table 1. A definite mutagenic effect is observed if periodic sampling of the same culture is performed.

Table 1. Periodic Assay of Cells Incubated with Zephiran Chlorde at $37^{\circ} \mathrm{C}$. And Tested for 0 Polnt Mutations to Phage Resistance

\begin{tabular}{ccccc}
\hline \hline $\begin{array}{c}\text { Time of } \\
\text { Assay }\end{array}$ & $\begin{array}{c}\text { Bacteria } \\
\text { per cc. }\end{array}$ & $\begin{array}{c}\text { B/1 per } 10^{8} \\
\text { bacteria }\end{array}$ & $\begin{array}{l}\text { Percent } \\
\text { survival }\end{array}$ & $\begin{array}{c}\text { Zero } \\
\text { point } \\
\text { mutants }\end{array}$ \\
\hline 0 & $5.6 \times 10^{10}$ & $4.2 / 10^{8}$ & - & - \\
3 min. & $2.2 \times 10^{10}$ & $8.1 / 10^{8}$ & $39 \%$ & $3.9 / 10^{8 *}$ \\
$10 \mathrm{~min}$. & $2.0 \times 10^{10}$ & $8.6 / 10^{8}$ & $36 \%$ & $4.4 / 10^{8}$ \\
$30 \mathrm{~min}$. & $1.1 \times 10^{10}$ & $13.3 / 10^{8}$ & $20 \%$ & $9.1 / 10^{8}$ \\
$100 \mathrm{~min}$. & $6.4 \times 10^{9}$ & $21.1 / 10^{8}$ & $11 \%$ & $16.9 / 10^{8}$ \\
\hline
\end{tabular}

$8.1 / 10^{8}-4.2 / 10^{8}$.

In another experiment yielding $19 \%$ survival the zero point mutations numbered 24 per $10^{8}$.

The intergeneric variation in sensitivity of microorganisms to toxic agents is perhaps of more general importance than experimentally induced interstrain differences as a factor to be kept in mind when screening of possible mutagens is contemplated. Unless one adopts the relatively extreme position that cell death in the presence of chemicals is the consequence of induced lethals it is not unduly speculative to assume that cells highly resistant to deleterious chemical effects on extragenic processes will be best suited for a demonstration of mutagenesis. If analysis of toxicity is to be performed as a differential survival test it may also be necessary with some chemicals to extend the tests over the killing range included within limits of the experiment.

As Dr. Witkin has observed, the span of our experience with induced phage resistance as a genetic tool has not been of sufficient length to evaluate its place in the general scheme of experimentally induced mutations in more complex biological systems. Investigators using the method may find themselves in the paradoxical situation that each new success contributes to a failure, that is, to the widening of a gap between mutagenesis of microorganisms and of higher forms of life. This would inevitably decrease the value of bacterial studies in interpreting mutation among higher organisms. Un- til other mutations in bacteria have been studied by similar methods and until the growing list of chemical mutagens has been used on more familiar genetic material and placed within some kind of quantitative and qualitative limits, it will be impossible to judge the real significance of Dr. Witkin's most stimulating and capable study.

Herskowitz: The work of Dr. Witkin demonstrates a facile bacterial method for identifying certain groups of chemicals as mutagens. She has already pointed out that by using this technique negative mutagenic action by a chemical is not conclusively demonstrated. However, it may well be that those chemicals screened out by the bacterial technique as being non-mutagenic will be important in the long run. Therefore, it seems advisable to supplement this technique with others which may permit a more sensitive test for mutagenic action.

For the detection of the action of a chemical on nucleic acids and nucleoproteins the use of Drosophila sperm seems most suitable. There are several reasons for this. In spermatozoa there are a minimum of cytoplasmic substances which might interfere with a chemical affecting genic nucleoprotein. Moreover, even though a chemical is not specific for nucleoprotein it may yield positive results more readily in sperm than in other cells. There is excellent evidence from genetic and irradiation experiments that genic nucleoprotein may be drastically changed without killing the sperm cell; this permits the utilization of such sperm for fertilization with the subsequent detection of lethal genes and chromosome rearrangements in addition to other types of inherited changes. In Drosophila it is also possible accurately to localize inherited changes in the chromosomes. Dr. Demerec no doubt had these advantages in mind when he developed the aerosol technique.

As Dr. Witkin has mentioned, there is no conclusive proof that aerosols always reach the spermatozoa in the testes. Again, negative mutagenic results with a chemical may be misleading. Therefore, a technique which would directly treat Drosophila sperm with chemicals is highly desirable. Accordingly, an investigation was initiated to discover if chemicals could be injected into the vagina of adult females, which would then copulate, and thereby expose the sperm to the respective chemicals.

A vaginal douche technic was successfully worked out, and is described in detail in a recent published note (Herskowitz, Evolution 1: 111-112, 1947). The method was tried with methyl bis amine hydrochloride at concentrations of $0.2 \%$ and $10.0 \%$, with positive results.

The data establish the practicality of the vaginal douche technique for the detection of chemical mutagens. There are two advantages of this method. Low concentrations of mutagens may be effective 
because of the direct contact of the chemical with the sperm; high concentrations of chemicals can be used without killing the organism, since a localized part of the body rather than the whole individual is treated. These advantages point to the possibility of a chemical analysis of the processes leading to gene changes as well as the analysis of the processes involved in direct changes in genic nucleoprotein. With such objectives in mind, the search for and study of the mutagenic activity for many compounds would most efficiently be investigated by using the bacterial or fungus method first, then the use of the aerosol method, and finally, if necessary, the vaginal douche technic.

ZAMENHof: I wonder if Dr. Witkin would be willing to comment on qualitative differences between spontaneous mutations and mutations induced by chemicals, in addition to the quantitative ones. As Dr. Witkin has pointed out, the spontaneous mutations do not seem to occur in the non-dividing cells (see also Zamenhof, Genet. Soc. Rec. 13: 41-42, 1944). The mutation ratio in this case can therefore be defined as the number of mutations per cell division. On the other hand, the mutations induced by chemicals seem to take place even in the absence of cell divisions; in this case the mutation ratio can be defined as the number of mutations per cell per unit of time. Thus the two phenomena seem to be different. What is your opinion on this subject?

Witkin: The fact that mutations can be induced in resting bacteria by radiations and chemicals, whereas spontaneous mutations seem to occur only in dividing cells, certainly suggests an important difference between induced and spontaneous mutations, although the end product of the two processes, the mutated genes, need not necessarily differ in a fundamental way. Chemical or mechanical errors of duplication, or slips in some metabolic cycle inactive in resting cells, may be the only natural circumstances capable of bringing about mutations. Thus, the failure of spontaneous mutation to occur in resting cells may result from the relatively inert and quiescent state of the genic material, and of the metabolic activities associated with cell division, rather than to a greater degree of inherent stability. Zero point mutations may be due to the direct action of chemicals or radiations on the gene, or more indirectly, to the stimulation of cellular activities normally involved in the production of spontaneous mutations. A careful comparison of the properties of mutants arising spontaneously and by induction may throw some light on this important question. 


\title{
THE EFFECT OF METABOLITES UPON GROWTH AND VARIATION OF BRUCELLA ABORTUS
}

\author{
ROBERT J. GOODLOW, LEONARD A. MIKA, AND WERNER BRAUN \\ Camp Detrick, Frederick, Maryland
}

Received for publication June 22, 1950

\begin{abstract}
In previous work with Brucella abortus (Braun, 1946) it has been demonstrated how mutation and selection can account for the progressive establishment of nonsmooth types in smooth broth cultures frequently observed during prolonged periods of growth. Evidence was presented that indicated that such population changes, common to most bacterial species and often referred to as dissociation (Braun, 1947a), involve the spontaneous occurrence of a small number of undirected variants (mutants) and their subsequent establishment within a population under the control of inherent and environmental factors governing population dynamics. It was also illustrated how competition between spontaneously arising mutants with different selective values can produce the appearance of apparently cyclic, successive, orderly changes (Braun, 1947b).
\end{abstract}

In the earlier studies it was recognized that variants do not establish themselves within growing populations until competitive conditions exist. Such competitive conditions presumably occur when the number of viable cells (plate counts) reaches a maximum, whereas the total number of cells (direct cell counts) continues to increase. However, the factors responsible for the limitation of the size of the viable population (i.e., the number of cells that retained the ability to multiply), or the "M concentration" of Bail (1929), have remained obscure. A paucity of information also exists regarding the possible influence of metabolites produced by one cell type upon the establishment of variant types and the role that such "association factors" (Braun, 1947a) play in population changes. Zamenhof (1946) and especially Ryan and Schneider (1949) obtained data in their studies with Escherichia coli that suggested an important role for such postulated factors in bacterial variation. Etinger-Tulczynska (1932), Neufeld and Kuhn (1934), and Mohr (1934) are among the earlier workers who assumed such effects but were unable to demonstrate their metabolic nature. Similarly, in studies with complex media, Braun (1946) was unable to demonstrate any effect of old culture filtrates upon the establishment of variants in $B$. abortus cultures. In contrast, in recent studies with $B$. abortus, in which paper partition chromatography and a simple synthetic medium containing DL-asparagine as the only amino acid source were utilized, the effect of specific cell metabolites of one type upon the establishment of variant types became clearly evident, and the manner in which such metabolites restrict the " $M$ concentration" of the cells that produce them became obvious. The following data will describe these observations.

[Reprinted by permission of The Williams \& Wilkins Company from Journal of Bactiriology 60 : (3) 291-300, September, 1950] 


\section{METHODS AND MATERIALS}

Smooth and rough clones isolated from the $\mathrm{CO}_{2}$-requiring strain 6232 of $B$. abortus were used throughout. For the preparation of inocula the growth from a 24-hour modified tryptose agar slant culture (tryptose agar supplemented with glucose, thiamine, and $\mathrm{Fe}-\mathrm{McCullough}$ et al., 1947) was washed off with sterile Gerhardt and Wilson (1948) synthetic medium (G-W medium) and adjusted in the Coleman spectrophotometer to 70 per cent light transmittance. To each of a series of flasks containing $100 \mathrm{ml}$ of $\mathrm{G}-\mathrm{W}$ medium was added 1.0 $\mathrm{ml}$ of the adjusted suspension of organisms, and the cultures were then incubated at $37 \mathrm{C}$ under approximately 10 per cent $\mathrm{CO}_{2}$. At regular intervals throughout the periods of observation samples for the determination of viable cell counts were removed from the cultures, and serial dilutions, prepared in tryptose saline ( 0.1 per cent tryptose, 0.5 per cent $\mathrm{NaCl}$ ), were mixed with modified tryptose agar. In addition, samples were streaked on "2-1" agar plates ${ }^{1}$ for the determination of the percentage of nonsmooth variants (Braun, 1946).

In the determination of the effects of the addition of old culture filtrates to freshly inoculated smooth cultures, Seitz filtrates were employed. Ten $\mathrm{ml}$ of such filtrates were added to $90 \mathrm{ml}$ of G-W medium, inoculated with $1.0 \mathrm{ml}$ of a tryptose-saline suspension of smooth Brucella abortus, and incubated at $37 \mathrm{C}$ under 10 per cent $\mathrm{CO}_{2}$.

One-dimensional ascending paper chromatograms of Seitz filtrates were prepared according to the technique used by Horne and Pollard (1948). The strips were suspended in 82 per cent distilled phenol for 24 hours and then developed with 0.1 per cent ninhydrin solution in 85 per cent butanol.

\section{RESULTS}

The growth of smooth B. abortus, 6232, in G-W synthetic medium (figure 1) was characterized by a steady increase in viable cell counts until the fourth day of incubation, after which the number of viable organisms steadily decreased for a period of 6 to 7 days. After the tenth day of incubation a second rise in viable cells occurred. This increase reached its peak on the twentieth day. The second peak usually exceeded that of the initial maximum viable level. Examination of 2-1 agar plates revealed that during the first 10 days of incubation the population was smooth. Coincidentally with the second rise in viable cells, there was a steady increase of nonsmooth variants (predominantly rough types), which, at the time of the second peak of increase in viable cells, frequently constituted over 90 per cent of the population (table 1).

The effects of adding to freshly inoculated smooth cultures Seitz filtrates of 24-day-old cultures of $B$. abortus, in which the original smooth population had been replaced by nonsmooth variants, were then investigated. The addition of 10 per cent filtrate was found to produce a marked effect on the subsequent

${ }^{1}$ Composition of 2-1 agar: 2.5 per cent Difco purified agar; 1 per cent Difco peptone; 0.5 per cent $\mathrm{NaCl} ; 0.5$ per cent $\mathrm{Difco}$ beef extract; 2 per cent glycerol; and 1 per cent glucose. Adjusted to $\mathrm{pH} 7.4$ before autoclaving. 


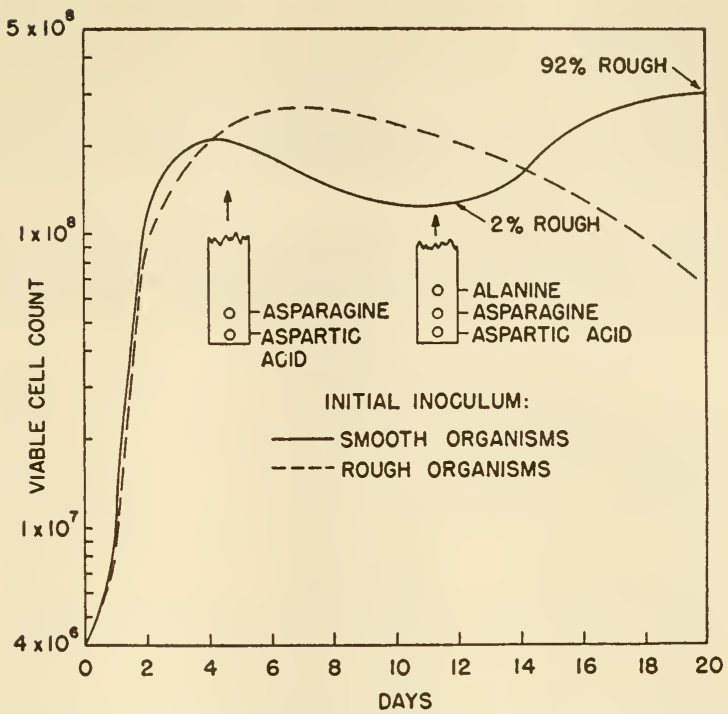

Figure 1. Growth (viable cell counts) of smooth and rough B. abortus in synthetic me dium.

TABLE 1

Growth and variation of smooth B. abortus in synthetic medium

\begin{tabular}{c|c|c}
\hline DAY OF GROWTH & VIABLE CELI COUNT & \% NONSMOOTH VARIANTS $\dagger$ \\
\cline { 2 - 3 } 1 & $8.4 \times 10^{6}$ & 0 \\
2 & $6.9 \times 10^{6}$ & 0 \\
3 & $2.8 \times 10^{7}$ & 0 \\
4 & $11.3 \times 10^{7}$ & 0 \\
5 & $13.5 \times 10^{7}$ & 0 \\
6 & $11.8 \times 10^{7}$ & 0 \\
7 & $11.9 \times 10^{7}$ & 0 \\
8 & $10.2 \times 10^{7}$ & 0 \\
9 & $8.6 \times 10^{7}$ & 0 \\
11 & $6.0 \times 10^{7}$ & 0 \\
14 & $9.1 \times 10$ & 2 \\
19 & $12.4 \times 10$ & 6 \\
23 & $19.6 \times 10$ & 76 \\
\hline
\end{tabular}

* Averages of 6 plates.

$\dagger$ Predominantly rough type. 
growth and variation of smooth cultures. The increase of the viable smooth organisms was repressed, and an earlier and more rapid establishment of nonsmooth variants occurred (table 2). When such filtrates were added to freshly inoculated rough $B$. abortus cultures, only a slight inhibitory effect on the growth of the rough organisms was observed. Subsequent experiments demonstrated that the smooth-suppressing factor in filtrates was dialyzable and heat-resistant (100 C for 5 minutes).

Paper chromatograms were then prepared daily on filtrates of actively growing smooth cultures in G-W medium. Until the eighth or tenth day of growth these chromatograms showed no spots, other than those of asparagine and aspar-

TABLE 2

Growth and variation of smooth B. abortus in synthetic medium after addition of old culture filtrate

\begin{tabular}{c|c|c}
\hline DAX OF GROWTH & VIABLE CELI COUNT & \% NONSMOOTH VARIANTS $\dagger$ \\
\cline { 2 - 3 } 0 & $8.8 \times 10^{*}$ & 0 \\
1 & $9.2 \times 10^{6}$ & 0 \\
2 & $5.1 \times 10^{7}$ & 0 \\
3 & $5.3 \times 10^{7}$ & 0 \\
4 & $6.3 \times 10^{7}$ & $<.01$ \\
5 & $6.7 \times 10^{7}$ & 0 \\
6 & $6.5 \times 10^{7}$ & 1 \\
7 & $5.8 \times 10^{7}$ & 2 \\
8 & $5.0 \times 10^{7}$ & 2 \\
9 & $6.8 \times 10^{7}$ & 10 \\
11 & $16.2 \times 10^{7}$ & 64 \\
14 & $16.7 \times 10^{7}$ & 91 \\
19 & $16.4 \times 10^{7}$ & 91 \\
23 & & 90 \\
\hline
\end{tabular}

* Averages of 6 plates.

$\dagger$ Predominantly rough type.

tic acid. After that period another spot with the Rf value of glutamine, threonine, or alanine made its appearance. The use of collidine-lutidine ${ }^{2}$ solvent subsequently indicated that this spot was probably due to alanine. Further observations showed that this spot became more intense during the subsequent 10 days of growth. After this time no further differences in intensity were visible.

Because of the interesting correlation between the appearance in the culture medium of this amino acid and the simultaneous increase in the establishment of nonsmooth variants, the effects of adding glutamine, threonine, or alanine to freshly inoculated smooth cultures were tested. Neither the addition of $0.5 \mathrm{mg}$ per $\mathrm{ml}$ glutamine nor $0.015 \mathrm{mg}$ per $\mathrm{ml}$ of DL-threonine resulted in any appreciable change in growth and variation when compared with control cultures. The addition of $0.5 \mathrm{mg}$ per $\mathrm{ml}$ DL-alanine resulted, however, in a marked suppression

${ }_{2}^{2}$ Thirty-five per cent collidine (mixed), 35 per cent 2,6-lutidine, and 30 per cent distilled water. 
of viable smooth organisms and enhanced the earlier and more rapid establishment of nonsmooth variants. The curves of increase in viable cells of cultures

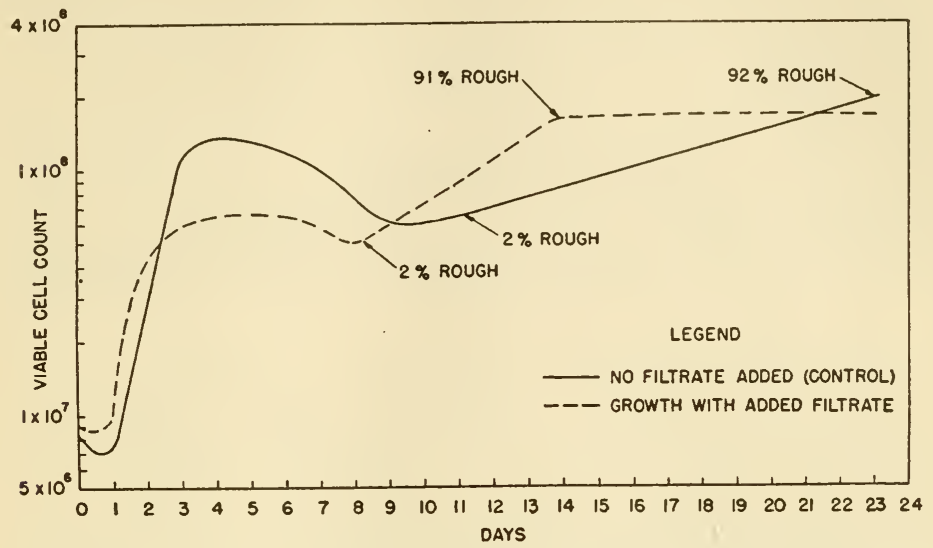

Figure 2. Growth (viable cell counts) of smooth B. abortus in synthetic medium supplemented with filtrates from old cultures.

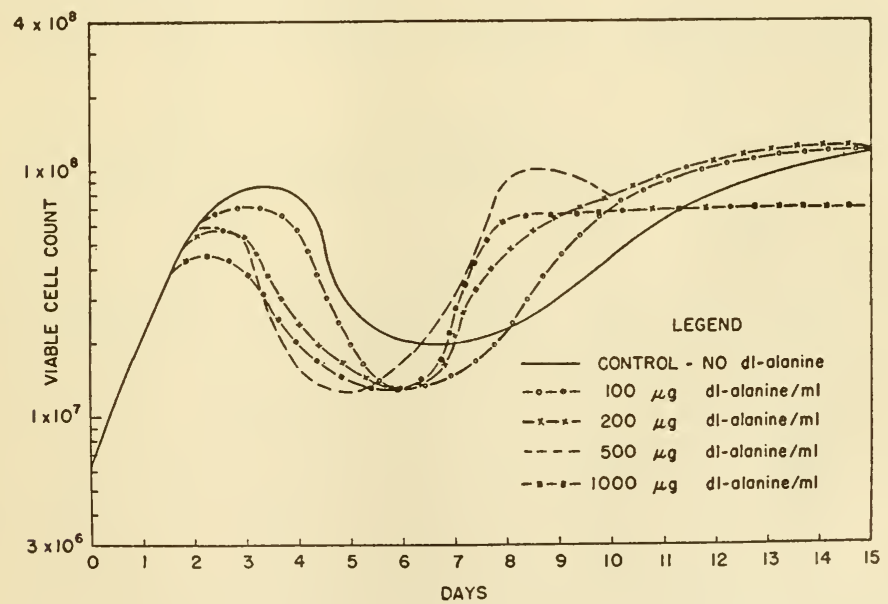

Figure 3. Growth (viable cell counts) of smooth B. abortus in the presence of different concentrations of DL-alanine.

to which alanine had been added were strikingly similar to those of cultures to which filtrates had been added (figures 2 and 3 ).

In order to determine a possible differential resistance of smooth and rough 
types of $B$. abortus to the toxicity of alanine a series of flasks of $\mathrm{G}-\mathrm{W}$ medium $(100 \mathrm{ml})$ were inoculated with smooth or rough organisms. To each culture was added sufficient DL-alanine to yield final concentrations of $100 \mu \mathrm{g}, 200 \mu \mathrm{g}$,

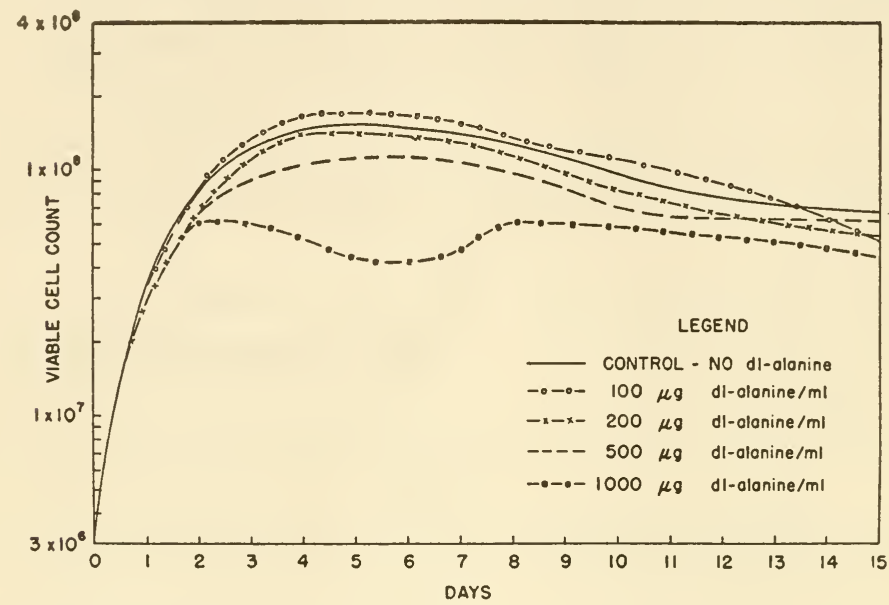

Figure 4. Growth (viable cell counts) of rough B. abortus in the presence of different concentrations of DL-alanine.

TABLE 3

Growth and variation of smooth B. abortus in synthetic medium containing different concentrations of DL-alanine

\begin{tabular}{|c|c|c|c|c|c|c|c|c|c|c|}
\hline \multirow[b]{2}{*}{ DAY } & \multicolumn{2}{|c|}{$\begin{array}{c}\text { CONTROL } \\
\text { (NO ALANINE) }\end{array}$} & \multicolumn{2}{|c|}{$\begin{array}{c}100 \mu \mathrm{G} \text { DL- } \\
\text { ALANINE/ML }\end{array}$} & \multicolumn{2}{|c|}{$\begin{array}{c}200 \mu \mathrm{G} \text { DL- } \\
\text { ALANINE } / M \mathrm{LL}\end{array}$} & \multicolumn{2}{|c|}{$\begin{array}{c}500 \mu \mathrm{G} \text { DL- } \\
\text { ALANINE } / \mathrm{ML}\end{array}$} & \multicolumn{2}{|c|}{$\begin{array}{l}1,000 \mu \mathrm{G} \text { DL- } \\
\text { ALANINE/ML }\end{array}$} \\
\hline & $\begin{array}{c}\text { Viable } \\
\text { count } \\
\left(\times 10^{6}\right)\end{array}$ & $\begin{array}{c}\% \text { non- } \\
\text { smooth } \\
\text { vari- } \\
\text { ants } \dagger\end{array}$ & $\begin{array}{c}\text { Viable } \\
\text { count } \\
\left(\begin{array}{ll}\times & 10^{6}\end{array}\right)\end{array}$ & $\mid \begin{array}{c}\% \text { non- } \\
\text { smooth } \\
\text { vari- } \\
\text { ants }\end{array}$ & $\begin{array}{l}\text { Viable } \\
\text { count } \\
\left(\times 1^{6}\right)\end{array}$ & $\mid \begin{array}{c}\% \text { non- } \\
\text { smooth } \\
\text { vari- } \\
\text { ants }\end{array}$ & $\begin{array}{l}\text { Viable } \\
\text { count } \\
\left(\begin{array}{lll}\times & 10^{6}\end{array}\right)\end{array}$ & $\begin{array}{c}\% \text { non- } \\
\text { smooth } \\
\text { vari- } \\
\text { ants }\end{array}$ & $\begin{array}{c}\text { Viable } \\
\text { count } \\
\left(\times 10^{6}\right)\end{array}$ & $\begin{array}{c}\% \text { non- } \\
\text { smooth } \\
\text { vari- } \\
\text { ants }\end{array}$ \\
\hline 0 & 6.2 & 0 & 6.3 & 0 & 6.7 & 0 & 6.7 & 0 & 6.5 & 0 \\
\hline 2 & 60.0 & 0 & 61.0 & 0 & 54.0 & 0 & 58.0 & 0 & 45.0 & 0 \\
\hline 4 & 79.0 & 0 & 57.0 & 1 & 24.0 & 2 & 14.0 & 3 & 19.0 & 5 \\
\hline 6 & 20.0 & 3 & 13.0 & 6 & 13.0 & 19 & 17.0 & 61 & 13.0 & 54 \\
\hline 8 & 22.0 & 11 & 22.0 & 14 & 63.0 & 36 & 101.0 & 63 & 69.0 & 57 \\
\hline 10 & 44.0 & 22 & 72.0 & 43 & 70.0 & 83 & 80.0 & - & 65.0 & 88 \\
\hline 15 & 117.0 & 42 & 120.0 & 57 & 121.0 & 86 & - & - & 71.0 & 82 \\
\hline
\end{tabular}

* Averages of 6 plates.

$\dagger$ Predominantly rough type.

$500 \mu \mathrm{g}$, or $1,000 \mu \mathrm{g}$ per $\mathrm{ml}$ of medium. Upon repeated sampling of these cultures during incubation it was found that alanine in a concentration of $100 \mu \mathrm{g}$ per $\mathrm{ml}$ or more exhibited a significant growth-depressing effect on the smooth type, whereas the rough variant was unaffected until a concentration of $1,000 \mu \mathrm{g}$ of 
alanine per $\mathrm{ml}$ of medium was reached (tables 3 and 4 , figure 4 ). The resistance of the rough variant to the toxicity of alanine was, therefore, approximately 10 times that of the smooth type.

It was also observed that during prolonged incubation of cultures in which rough variants had become established alanine accumulation continued that led to an eventual second decline of the viable count. This decline was then followed, approximately 50 days after the start of the originally smooth cultures, by a renewed increase in viable cells and a simultaneous establishment of another smooth variant. This highly alanine-resistant variant, labeled $\mathbf{S}^{\prime}$, proved to be similar antigenically to the original smooth type (acriflavine test and aglutinin absorption test), but when $\mathrm{S}^{\prime}$ was plated in mixtures with the original $\mathrm{S}$ a distinct difference in colony morphology was detectable.

TABLE 4

Growth of rough B. abortus in synthetic medium containing different concentrations of DL-alanine*

\begin{tabular}{|c|c|c|c|c|c|}
\hline \multirow{2}{*}{ DAY } & $\begin{array}{l}\text { CONTROL } \\
\text { (NO ALANINE) }\end{array}$ & $\begin{array}{l}100 \mu \text { G DL-ALA- } \\
\text { NINE/MC }\end{array}$ & $\begin{array}{l}200 \mu \mathrm{G} \text { DL-ALA- } \\
\text { NINE/ML }\end{array}$ & $\begin{array}{l}500 \mu \text { G DL-ALA- } \\
\text { NINE/ML }\end{array}$ & $\begin{array}{l}\text { 1,000 } \mu \mathrm{G} \text { DL-ALA- } \\
\text { NINE/ML }\end{array}$ \\
\hline & $\begin{array}{c}\text { Viable count } \dagger \\
\left(\times 10^{6}\right)\end{array}$ & $\begin{array}{c}\text { (Viable count } \\
\left(\times 10^{6}\right)\end{array}$ & $\begin{array}{l}\text { Viable count } \\
\left(\times 10^{6}\right)\end{array}$ & $\begin{array}{l}\text { Viable count } \\
\left(\times 10^{6}\right)\end{array}$ & $\begin{array}{l}\text { Viable count } \\
\left(\times 10^{6}\right)\end{array}$ \\
\hline 0 & 3.7 & 3.3 & 3.3 & 3.1 & 3.6 \\
\hline 2 & 83.0 & 83.0 & 67.0 & 67.0 & 62.0 \\
\hline 4 & 146.0 & 168.0 & 140.0 & 100.0 & 52.0 \\
\hline 6 & 145.0 & 118.0 & 108.0 & & 42.0 \\
\hline 8 & 132.0 & 130.0 & 114.0 & 107.0 & 63.0 \\
\hline 10 & 96.0 & 111.0 & 83.0 & 65.0 & 56.0 \\
\hline 15 & 66.0 & 51.0 & 54.0 & 62.0 & 44.0 \\
\hline
\end{tabular}

* No variants were observed in these cultures up to 15 days.

$\dagger$ Averages of 6 plates.

\section{DISCUSSION}

These data demonstrate that the accumulation of a specific metabolite, alanine, limits the increase in viable smooth cells and at the same time creates an environment favorable for the progressive establishment of nonsmooth (e.g., $\mathrm{R}$ ) types with greater resistance to this toxic metabolite. It is interesting to note that prior to population changes in a closed system (test tube) the parent type creates conditions that may be termed suicidal and thereby sets up specific environmental effects that will favor the establishment of metabolite-resistant mutants. In a way, then, the parent type actually influences the direction in which population changes may occur since its metabolic products may determine which of the many possible spontaneously arising mutants will have the greatest survival value at any given time of a culture's growth. It is easy to visualize how the continual quantitative and qualitative alteration of metabolite concentration may create a constantly changing environment that successively favors different mutants and thereby apparently causes progressive population changes as long as reproduction continues. 
The reasons for past failures to detect this phenomenon in nonsynthetic media are still obscure. That the complexity of such media may be responsible is indicated by the recent results of Schuhardt, Rode, and Oglesby (1949), who demonstrated that many amino acids, including alanine, are toxic to Brucella, whereas other amino acids may neutralize their effects. Whether such interactions may have masked the influence of filtrates when complex media were used remains questionable since population changes similar to those in simple media regularly occur in complex media, even though they remain unaffected by the addition of filtrates from old cultures.

A neutralization of the toxic effects of alanine accumulation in Brucella cultures, possibly with the help of specific antagonists, should be capable of preventing population changes in smooth cultures during prolonged periods of growth in liquid media. Actually, such interference with the accumulation of metabolites favoring the establishment of mutants may have been practiced unwittingly in prior experiments that were concerned with specific selective agents that suppressed the establishment of nonsmooth types in smooth populations. Thus it has been demonstrated (Braun, 1949) that the addition of small amounts of normal serum from Brucella-susceptible hosts to smooth broth cultures will prevent the establishment of nonsmooth types. Preliminary data suggest that in such serum cultures the accumulation of alanine is actually reduced or that in addition to alanine a different amino acid, possibly antagonistic to alanine, may accumulate. Similarly, the prevention of population changes in smooth Brucella cultures containing 0.03 M sodium pyrophosphate (Braun, 1950) may involve an interference with enzymatic processes leading to alanine production through the removal of trace metals that may act as catalysts for these reactions. This possibility is further supported by the fact that the addition of $\mathrm{Mn}^{++}$or $\mathrm{Mg}^{++}$to smooth cultures results in a considerable enhancement of the establishment of nonsmooth types (Cole and Braun, 1950).

Attempts are being made to determine the pathways that may be involved in alanine production. At least three mechanisms may be suggested, namely: (1) There may be transamination between aspartic and pyruvic acids yielding oxalacetic acid and alanine. The pyruvic acid may be formed as an end product in the oxidation of lactic acid initially present in the medium, whereas the hydrolysis of asparagine, also initially present, would yield aspartic acid and free ammonia. (2) There may be direct amination of pyruvic acid with the free ammonia to yield alanine. (3) There may be a combination of pathways (1) and (2).

It has been ascertained that the accumulation of alanine in smooth cultures and the simultaneous establishment of alanine-resistant nonsmooth mutants described for $B$. abortus occurs in cultures of $B$. suis, B. melitensis, and strain 19 of $B$. abortus as well. This phenomenon, therefore, appears to be of general significance for brucellae. To what extent it may also apply to other bacterial species remains to be determined. However, there are a number of observations, reported in the recent literature, which indicate the metabolic accumulation of specific amino acids and their toxicity for certain cell types. If tested by methods 
analogous to those used in the present study, these metabolites may prove to have similar effects on population changes. For example, Dubos (1949) has reported that virulent types of Mycobacterium tuberculosis are highly susceptible to DL-alanine and serine, whereas avirulent variants are more resistant to these amino acids; Dagley, Dawes, and Morrison (1950) determined with the help of chromatography that certain amino acids, including alanine, accumulated when $E$. coli was grown in a simple synthetic medium; Linggood and Woiwod (1949) have described parallel increases between alanine accumulation and toxin production in cultures of Corynebacterium diphtheriae; and Gordon and Gordon (1947) noted the development of alanine- and glycine-resistant strains of Shigella dysenteriae.

Finally, these results suggest the possibility of controlling population changes of pathogenic bacteria within infected hosts, such as enhancing the establishment of less virulent nonsmooth types in vivo through the administration of alanine, which in turn may result in therapeutic effects. Studies in this direction are now under way.

\section{SUMMARY}

The application of paper chromatography to studies of the growth and variation of smooth Brucella abortus in Gerhardt and Wilson's synthetic medium revealed a striking correlation between the accumulation of certain amino acids in the medium and the appearance of nonsmooth variants.

The role of one of the amino acids, alanine, in favoring the establishment of nonsmooth variants, was verified by the addition of filtrates of old cultures or of alanine alone to freshly inoculated smooth cultures in synthetic medium. Under both conditions, a more rapid and enhanced establishment of nonsmooth variants was observed, and it was found that alanine markedly suppressed the viable count of smooth cells but failed to exhibit a similar marked effect on nonsmooth types.

It thus appears that the accumulation of alanine as a metabolite of smooth cells creates an environment favorable for the establishment of spontaneously occurring nonsmooth variants.

The possible metabolic pathways involved and the relation of these data to general phenomena of population changes have been discussed.

\section{REFERENCES}

BAIL, O. 1929 Ergebnisse experimenteller Populationsforschung. Z. Immunitäts., 60, $1-22$.

Braun, W. 1946 Dissociation in Brucella abortus : a demonstration of the role of inherent and environmental factors in bacterial variation. J. Bact., 61, 327-349.

Braun, W. 1947a Bacterial dissociation. Bact. Revs., 11, 75-114.

BRAUN, W. 1947b The production of apparent cycles in bacterial variation. J. Bact., 63, $250-251$.

BrauN, W. 1949 Studies on bacterial variation and selective environments. II. The effects of sera from Brucella-infected animals and from normal animals of different species upon the variation of Brucella abortus. J. Bact., 58, 299-305. 
BraUn, W. 1950 Variation in the genus Brucella. In Brucellosis. AAAS Symposia, 10, $26-36$.

Cole, L., And Braun, W. 1950 The effect of ionic Mn and Mg on the variation of Brucella abortus. J. Bact., 60, 283-289.

Dagley, S., Dawes, E. A., And Morrison, G. A. 1950 Production of amino-acids in synthetic media by Escherichia coli and Aerobacter aerogenes. Nature, 165, 437-438.

Dubos, R. J. 1949 Toxic effects of $d l$-serine on virulent human tubercle bacilli. Amer. Rev. Tuberc., 60, 385.

Etinger-Tulcznyska, R. 1932 Úber bakterien Antagonismus. Z. Hyg. Infektionskrankh., 113, 762-780.

Gerhardt, P., and Wilson, J. B. 1948 The nutrition of brucellae: growth in simple chemically defined media. J. Bact., 56, 17-24.

Gordon, J., And Gordon, M. 1947 Development of resistance of Shigella shigae to glycine. J. Path. Bact., 59, 445-451.

Horne, R. E., and Pollard, A. L. 1948 The identification of streptomycin on paper chromatograms. J. Bact., 55, 231-234.

LiNGGOOD, F. V., AND Worwod, A. J. 1949 The application of paper partition chromatography to the production of diphtheria toxin; two-dimensional chromatography. Brit. J. Exptl. Path., 30, 93-100.

McCullough, W. G., Mills, R. C., Herbst, E. J., Roessler, W. G., and Brewer, C. R. 1947 Studies on the nutritional requirements of Brucella suis. J. Bact., 53, 5-15.

Монг, W. 1934 Untersuchungen über antagonistische Vorgänge zwischen Varianten desselben Stammes. Z. Hyg. Infektionskrankh., 116, 288-294.

NeUfELd, F., AND KUHN, H. 1934 Untersuchungen über “direkten" Bacterienantagonismus. Z. Hyg. Infektionskrankh., 116, 95-110.

Ryan, F. J., AND Schneider, L. K. 1949 The consequences of mutation during the growth of biochemical mutants of Escherichia coli. J. Bact., 58, 201-213.

Schuhardt, V. T., Rode, L. J., ANd Oglesbx, G. 1949 The toxicity of certain amino acids for brucellae. J. Bact., 58, 665-674.

ZamenhoF, S. 1946 Studies on bacterial mutability: the time of appearance of the mutant of Escherichia coli. J. Bact., 51, 351-361. 


\title{
GENE RECOMBINATION AND LINKED SEGREGATIONS IN ESCHERICHIA COLI ${ }^{1}$
}

\author{
JOSHUA LEDERBERG ${ }^{2}$ \\ Department of Botany and Microbiology, Osborn Botanical Laboratory, \\ Yale University, New Haven, Conn.
}

Received August I, 1947

$\mathrm{T}$ HE occurrence of factor recombination in the bacterium, Escherichia coli, has been described in previous reports (LEDERBERG and TATUM, I 946 b, c, TATUM and LEDERBERG, I947). In an attempt to elucidate further the genetic structure of this organism, these studies have been extended to crosses involving several characters, and to the quantitative enumeration of various recombination classes. The results described in this paper provide evidence supporting the sexual basis of factor recombination and of the existence of an organized array of genes comparable to that of higher forms.

\section{MATERIALS AND METHODS}

The parent "wild-type" strain, K-I 2, of $E$. coli used in these experiments and the production and behavior of biochemical mutants have been described (Gray and Tatum, 1944, Lederberg and Tatum, i946a, Roepke, LibBy, and SMall, I944, TATUM, I945). Specific requirements, notation, and other data pertinent to the biochemical mutants are summarized in tables $I$ and 2 . In general, a biochemical deficiency resulting from mutation is designated by the initial of the substance required (e.g. $B^{-}$for biotinless), while the wild type alternative is written with a " + " $\operatorname{sign}$ (e.g. $B^{+}$to emphasize the alternative to $B^{-}$). The term "prototroph" (Ryan and LEDERBERG, I946) has been devised for strains exhibiting the nutritional behavior of the wild type, which for $E$. coli implies independence of any specific growth factors. Prototroph is, however, not synonymous with "wild type" since it refers (a) only to the phenotypic appearance of a culture and (b) only to nutritional and not to other possible mutant characteristics.

$\mathrm{K}-\mathrm{I} 2$ as a coliform is capable of fermenting, or producing acid, from a variety of sugars, including glucose, galactose, maltose, lactose and mannitol; however, it ferments glycerol only weakly, and sucrose even less so. Because of the ease of scoring and their biochemical specificity, mutants unable to ferment various sugars have been looked for. Particular attention was paid to the isolation of "lactose-negative" or " $\mathrm{Lac}^{- \text {" }}$ mutants, because of the taxonomic significance which has been attached to this character.

1 Abstracted from a dissertation offered in partial fulfillment of requirements for the degree of Doctor of Philosophy at Yale University.

2 Fellow of the Jane Coffin Childs Memorial Fund for Medical Research. This work has been supported by the Jane Coffin Childs Memorial Fund for Medical Research. The author's present address is: Department of Genetics, University of Wisconsin, Madison, Wis

[Reprinted by permission from GeNFtics $32: 505-525$, September, 1947] 
The detection of fermentation mutants is readily accomplished by the use of indicator media. The medium "EMB-lactose" used in routine bacteriological work was found to be highly useful. It consists of the following (in $\mathrm{g} / \mathrm{l}$ ): peptone (or "N-Z-Case") Io, yeast extract I, lactose Io, agar I5, eosin Y 0.4, methylene blue 0.06 , sodium chloride 5 , dipotassium phosphate 2. On this medium, colonies of bacteria which can ferment lactose (or any other sugar added in its place) rapidly turn a deep purple color, while colonies of nonfermenting organisms remain white or pink but may slowly turn light blue.

$\mathrm{Lac}^{-}$mutations have been recovered in two instances. Among ${ }_{15}, 000$ colonie $_{\mathrm{S}}$

TABLE I

Symbols used for various loci.

I. Nutritional requirements. Allele for requirement of a given substance is designated by the superscript " ${ }^{-\eta}$; independence by "+» . E.G., $\mathrm{B}^{-}$is biotinless; $\mathrm{B}^{+}$is biotin-independent.

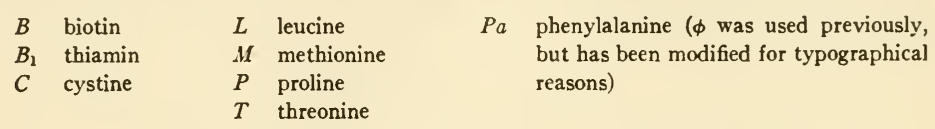

2. "Sugar" fermentations. The ability to ferment is designated "+"; the inability "-".

Lac lactose

Gly glycerol

3. Bacteriophage resistance. Resistance is designated by the superscript " $r$; sensitivity by $u_{\diamond}$.

E.G. $V_{1}$.

$V_{1} \quad$ resistant to $T_{I}, T_{5}$

$V_{1 a}$ resistant to $T_{I}$; sensitive to $T_{5}$

$V_{1 b} \quad$ resistant to $T_{I}$; mucoid colonies

$V_{6} \quad$ resistant to $T 6$.

4. Resistance to chemical agents. Resistance and sensitivity " $r$ " and " $s$ " respectively, as $\mathrm{Cla}^{*}$.

$\mathrm{Cla}$ sodium chloroacetate

A sodium azide

of strain Y-Io $\left(T^{-} L^{-} B_{1}^{-}\right)$obtained by spreading a culture previously treated with ultraviolet light on EMB-lactose agar, a single pink colony was noted. It proved to be the same, nutritionally, as $\mathrm{Y}$-Io and was therefore regarded as a $\mathrm{LaC}^{-}$mutant; this stock is labelled $\mathrm{Y}-53$. Among 30,000 colonies of $\mathrm{Y}-40$ $\left(B^{-} M^{-} V_{1}{ }^{r}\right)$ a single $L^{-} c^{-}$was recovered following treatment with nitrogenmustard (TATUM, 1946), and was designated as Y-87. Tests showing that these independent mutations are probably allelic will be described in a later section (see table 5). Strains $\mathrm{Y}-53$ and $\mathrm{Y}-87$ differ in the rate at which the $\mathrm{Lac}^{-}$character reverts to the $\mathrm{Lac}^{+}$condition, but whether this is due to different allelic states or to differences at other loci, cannot be definitively asserted.

Attempts to obtain maltose, mannitol, and galactose-negative mutants were not successful, presumably because the populations tested were too small. A glycerol-negative strain has been obtained, but the wild type ferments this polyalcohol so poorly to begin with that accurate scoring is difficult; studies on this character will not be further reported here. 
Mutations for resistance to specific bacteriophages or bacterial viruses have proven to be exceedingly useful. They are readily obtained as spontaneous mutants by plating a large number of sensitive bacteria with the particular virus in question; only resistant mutants escape lysis and may be recovered as "secondary" colonies (fig. I). Resistant mutants are readily freed from residual virus by serial single colony isolation. Resistance to a given virus may be

TABLE 2

A summary of the mutants used.

\begin{tabular}{|c|c|c|c|c|}
\hline STRAIN NO. & GENOTYPE & ORIGIN & GENOTYPE & AGENT \\
\hline $\mathrm{K}-\mathrm{I} 2$ & prototroph. & $\begin{array}{l}\text { Original wild } \\
\text { strain }\end{array}$ & & \\
\hline 58 & $B^{-}$ & $\mathrm{K}-12$ & $B^{+}$ & X-ray \\
\hline $5^{8-161}$ & $B^{-} \mathrm{I}^{-}$ & $5^{8}$ & $\mathrm{~B}^{-} \mathrm{M}^{+}$ & $\mathrm{X}$-ray \\
\hline $5^{8-278}$ & $\mathrm{~B}^{-} P a^{-}$ & $5^{8}$ & $\mathrm{~B}^{-} \mathrm{Pa}^{+}$ & X-ray \\
\hline$Y-24$ & $\mathrm{~B}^{-} \mathrm{Pa}^{-} \mathrm{C}^{-}$ & $5^{8-278}$ & $\mathrm{~B}^{-} \mathrm{Pa}^{-} \mathrm{C}^{+}$ & ultra-violet \\
\hline 679 & $T^{-}$ & $\mathrm{K}-12$ & $T^{+}$ & X-ray \\
\hline $679-680$ & $T^{-} L^{-}$ & 679 & $T^{-} L^{+}$ & X-ray \\
\hline $\mathrm{Y}$-10 & $T^{-} L^{-} B_{1}^{-}$ & $679-680$ & $T^{-} L^{-} B_{1}+$ & X-ray \\
\hline$Y-46$ & $T^{-} L^{-} B_{1}{ }^{-} V_{1}^{r}$ & Y-10 & $T^{-} L^{-} B_{1}-V_{1}^{s}$ & selection \\
\hline$Y-53$ & $T^{-} L^{-} B_{1}^{-} L_{a c}^{-}$ & $Y$-10 & $T^{-} L^{-} B_{1}^{-} L_{a c}^{+}$ & ultra-violet \\
\hline$Y-64$ & $T^{-} L^{-} B_{1}-L_{a c}^{-} V_{1}^{r}$ & $Y-53$ & $T^{-} L^{-} B_{1}^{-} L^{-} c^{-} \mathrm{V}_{1}{ }^{8}$ & selection \\
\hline$Y-40$ & $B^{-} U^{-} V_{1}^{r}$ & $5^{8-16 I}$ & $B^{-} M^{-} V_{1}^{8}$ & selection \\
\hline$Y-87$ & ${ }^{*} B^{-} M I^{-} V_{1}^{r} L a c^{2-}$ & $Y-40$ & $B^{-}-I^{-} V_{1}^{r} L a c^{+}$ & nitrogen mustard \\
\hline$Y-24^{-} V_{1}^{r}$ & $B^{-} P a^{-} C^{-} V_{1}^{r}$ & $Y-24$ & $B^{-} P a^{-} C^{-} V_{1}^{s}$ & selection \\
\hline $679-183$ & $T^{-} P^{-}$ & 679 & $T^{-} P^{+}$ & $\mathrm{X}$-ray \\
\hline Y-88 & $T^{-} L^{-} B_{1}{ }^{-} \mathrm{Lac}^{-} \mathrm{Cla}^{r}$ & $\mathbf{Y}-53$ & $T^{-} L^{-} B_{1}^{-} \mathrm{Lac}^{-} \mathrm{Cla}^{s}$ & selection \\
\hline $\mathrm{Y}-80$ & $B^{-} \mid I^{-} V_{1}^{r}$ Gly & $Y-40$ & $B^{-}-I^{-} V_{1}^{r} G^{\prime} y^{+}$ & nitrogen mustard \\
\hline$Y-91$ & $B^{-} M^{-} V_{1}^{r} C l a^{r}$ & $\mathrm{I}-40$ & $B^{-} M^{-} V_{1}^{r} C l a^{s}$ & selection \\
\hline$Y-92$ & $B^{-} M^{-} V_{1}^{r} A z^{r}$ & $Y-40$ & $B^{-} \mid I^{-} V_{1}^{r} A z^{s}$ & selection \\
\hline$Y-94$ & $T^{-} L^{-} B_{1}^{-} L^{-1 a c^{-}} V_{6}{ }^{r}$ & $\mathbf{Y}-53$ & $T^{-} L^{-} B_{1}-L_{a c}^{-} I_{6}{ }^{s}$ & selection \\
\hline$Y-100$ & $T^{-} L^{-} B_{1}^{-} L_{a c}^{-I_{1 a^{r}}^{r}}$ & $Y-53$ & $T^{-} L^{-} B_{1}-L_{a c}^{-} I_{10}$ & selection \\
\hline$Y-86$ & $T^{-} L^{-} B_{1}-L a c^{-} I_{1 b^{r}}^{r}$ & $Y-53$ & $T^{-} L^{-} B_{1}-L_{a c}^{-I}{ }_{1 b^{8}}$ & selection \\
\hline
\end{tabular}

* $\mathrm{Lac}^{2-}$ in mutant $\mathrm{Y}-87$ differs from $\mathrm{Lac}^{-}$in mutant $\mathrm{Y}-53$ and its derivatives in the greater reverse-mutability of the latter. $\mathrm{Lac}^{-}$and $\mathrm{Lac}^{2-}$ are otherwise similar, and allelic.

scored by streaking a loopful of bacteria on an EMB or nutrient agar plate at right angles to a previous streak of the virus suspension (DEMEREC and FANo, I 945 , see fig. I of the present report.)

It was found, however, that mutations for resistance to a given virus are not entirely specific, but that resistant mutants display "cross-resistance," i.e., are also resistant to other viruses. For example, most $T_{I \text {-resistant types }}$ are also resistant to $T_{5}$. (For the nomenclature of the bacterial viruses used in this investigation, and a detailed account of the cross-resistance patterns of another strain, E. coli $B$, see DEMEREC and FANo, I945). The cross-resistance patterns of K-I 2 are similar to those of $E$. coli $B$ with the exception that $T_{I-}$ resistant mutants which are sensitive to $T_{5}$ are not tryptophaneless, as has 
been reported by ANderson (1946) for the corresponding mutants of $E$. coli $B$. In this paper, the designation $V_{1}^{r}$ will be used for the more frequent $T_{1}$-resistant mutant, which is also resistant to $T_{5}$. The symbol $V_{1 a}{ }^{r}$ is reserved for the $T_{5}$-sensitive, $T_{I}$-resistant mutant, but the evidence that distinct loci are involved will be presented in extenso in another place.

In addition to $V_{1}{ }^{r}$ and $V_{1 a^{r}}$, just mentioned, a third type of "secondary colony" has been found among populations treated with the virus $T_{I}$. This type, $V_{I_{b}}$ is characterized by an exceedingly slimy or mucoid colony confor-

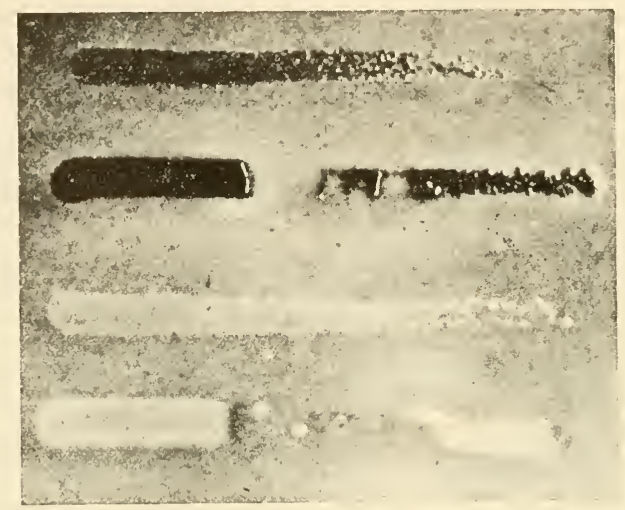

Figure I.-The phenotypes of the four combinations of $L a c$ and $V$ are illustrated. In order they are: $\mathrm{Lac}^{+} \mathrm{V}_{1}{ }^{r} ; \mathrm{Lac}^{+} \mathrm{I}^{r}{ }_{1} ; \mathrm{Lac}^{-} \mathrm{V}_{1}^{r} ; \mathrm{Lac}^{-} \mathrm{V}_{1}{ }^{s}$. An EMB-lactose agar plate was first streaked vertically with the virus $T_{\mathrm{r}}$. Subsequently, each of the bacterial types was streaked, from left to right, perpendicularly across the virus streak. After 16 hours incubation, both the Lac and $V_{1}$ phenotypes are well developed. Developing in the zone where $\mathrm{Lac}^{-} V_{1^{s}}$ has been lysed can be seen two colonies of resistant mutants: $\operatorname{Lac}^{-} V_{1}{ }^{r}$.

mation. Recombination studies on this mutant are complicated by its genetic instability; $V_{1 b^{r}}$ rapidly reverts to the wild type, and in addition may also be strongly selected against in competition with $V_{1 b^{s}}$. However, the locus of $V_{1 b}$ can be distinguished from the locus of the other $V_{1}$ mutants by the demonstration of a different recombination frequency with Lac. These data are summarized in order to emphasize the importance of genetic tests to insure the allelic identity of phenotypically similar mutants.

It is particularly fortunate that resistance tests can be conducted on EMB agar, since this allows the characterization of a strain with respect to virusresistance and to lactose fermentation with a single streaking (see fig. I).

Mutants resistant to sodium chloroacetate $\left(\mathrm{Cla}^{r}\right)$ were obtained by streaking a large number (about $\mathrm{Io}^{7}$ ) of bacteria on nutrient agar to which filter-sterilized chloroacetate has been added to make a final concentration of $2 \mathrm{mg} / \mathrm{ml}$. At this concentration, the wild type is substantially inhibited, while resistant mutants grow luxuriously. This mutation is accompanied by deficiencies in 
the metabolism of pyruvic and acetic acids, which will be described in more detail elsewhere. Independent mutations to other inhibitors, including iodoacetate, azide, streptomycin, streptothricin, mercuric chloride, and Brilliant Green, can be secured in a similar fashion, but genetic analysis of these mutations has not been completed.

Morphological variation has occasionally been noted (exceedingly rough or very mucoid colonial form) but is relatively unsuitable for genetic work because the presumably random choice of prototroph recombinants may be influenced.

In addition to the EMB agar already described, a number of other natural or "complete" media have been used. The Difco froduct "Penassay Broth" has been used most extensively, and is satisfactory for the preparation of inocula, except that it must be supplemented with cystine for the growth of cystineless organisms, such as strain Y-24. Other satisfactory media include a broth consisting of : peptone 5 , glucose 5 , yeast extract $3, \mathrm{~g} / \mathrm{l}$, as well as Difco Nutrient Broth, and diverse concoctions containing peptone or casein hydrolysates and meat or yeast extract.

The synthetic or minimal medium contains, in $\mathrm{g} / \mathrm{l}: \mathrm{NH}_{4} \mathrm{Cl} 5, \mathrm{NH}_{4} \mathrm{NO}_{3} \mathrm{I}$, $\mathrm{Na}_{2} \mathrm{SO}_{4} 2, \mathrm{~K}_{2} \mathrm{HPO}_{4} 3, \mathrm{KH}_{2} \mathrm{PO}_{4} \mathrm{I}$, glucose 5 , asparagine I.5, $\mathrm{MgSO}_{4}$ ○. I, trace elements (Gray and TAtuM I944), and $\mathrm{CaCl}_{2}$, a trace. The medium is made solid by the addition of agar in a concentration of $\mathrm{I} .5$ percent.

To avoid flocculation when used with agar, the glucose and agar in solution should be autoclaved separately, and mixed with the other components just before using. Unwashed agar (Difco) is sufficiently free of the growth factors under consideration to be satisfactory for many experiments; the use of washed agar, however, is recommended for the cleanest results.

The detection of recombinants is based upon the inability of biochemical mutant bacteria to proliferate in the absence of their specific growth substances. Plating in minimal agar, therefore, has the effect of a sieve for prototroph cells. To insure against contamination with prototrophs derived by reverse mutation, which has been noticed at certain loci, it has been desirable to use multiple biochemical mutants as the parental stocks in recombination studies. Coincidental reversion at two or more loci is theoretically improbable, and experimentally undemonstrable (Ryan, I946, TATUM and LEDERBERG, I947). For example, plating either $B^{-} M^{-} T^{+} L^{+} B_{1}{ }^{+}$or $B^{+} M^{+} T^{-} L^{-} B_{1}-$ separately into minimal agar did not lead to the appearance of prototrophs, $B^{+} M^{+} T^{+} L^{+}$$B_{1}{ }^{+}$. When, however, a mixture of these cell types was so "sieved," one prototroph was found for about each $10^{7}$ cells inoculated. These have been assumed to arise from the recombination of "+" alleles to form the prototroph.

In previous experiments, the two multiple mutants were inoculated together into a complete medium and allowed to grow in mixed culture before plating into minimal agar. This method is not satisfactory for present purposes because it allows possible selective differentials to alter the relative frequencies of different recombination classes. A modified procedure has been developed, which will now be described in detail.

The mutant stocks are maintained on "complete" agar slants, transferred 
at intervals of 6-8 weeks. They are inoculated ser arately into test-tubes containing about ten $\mathrm{ml}$ of liquid com lete medium and incul ated overnight at $30^{\circ} \mathrm{C}$ with gentle shaking. The following morning, an additional ten $\mathrm{ml}$ of the same medium is added to each culture, and the tubes are incubated in the same manner for an additional three to five hours. These cultures contain from $\mathrm{I}^{-4} \mathrm{X}$ $10^{9}$ cells per $\mathrm{ml}$. They are then washed in the following manner: the cotton plugs are replaced with sterile corks which have been kept in 95 percent alcohol and the alcohol flamed off just before using. The cultures are then centrifuged at about 2500 r.p.m. for 20 minutes, which suffices to I ack the cells in the bottom of the test tubes. The sup ernatant medium is carefully foured off, and the tube is rinsed with about Io ml sterile distilled water, care being taken not to disturb the pellet. The cells are then resusfended in an additional $15^{-20} \mathrm{ml}$ sterile water, and recentrifuged. The supernatant wash water is decanted and replaced with an equal volume of fresh sterile water, in which the cells are suspended. In the meantime, minimal agar plates are prepared. A bottom layer of about ${ }_{5} 5 \mathrm{ml}$ minimal agar is poured into each Petri plate and allowed to solidify. Cell suspensions of different mutant stocks are mixed at this time and measured quantities (usually about $10^{8}-10^{9}$ cells) are pipetted onto the agar surface. At this time also, one may add such growth factor supplements as are desired to permit the growth of recombination types other than prototrophs. The cell susf ensions are then mixed into a layer of about ten $\mathrm{ml}$ molten minimal agar $\left(\right.$ at $45^{-} 50^{\circ} \mathrm{C}$ ) which is poured onto the plates. After the agar hardens, the plates are incubated at $30^{\circ} \mathrm{C}$ for a period of 48 hours. At this time prototroph colonies will be found distributed throughout the plate, many of them at or near the surface and accessible to picking for further characterization.

The procedure may be varied in several ways. It is important however that the inoculum consist of "young" cells, since cultures of 24 hours or older have given quite inconsistent results. It is possible to store the inoculum in distilled water for at least twenty-four hours without appreciably affecting the yield, which suggests that the aggregation of genetic types leading to the recombination process occurs in the molten or the solidified agar. This occurrence must, however, take place within a few hours, since the recombinant prototrophs are not appreciably slower to appear than wild type cells in a similar physiological state which may be streaked on the surface of the plates. Presumably, therefore, one could increase the yield of prototrophs by making conditions more favorable for the free contact of the cells, as by packing them together in a centrifuge tube in minimal liquid medium. However the complication of proliferation of prototrophs already formed would interfere with the interpretation of such an experiment. Many physiological factors may interfere with the recombination process, and, for example, the yield may be reduced markedly by inoculating too heavily, or by omitting an under-layer of agar into which, presumably, deleterious metabolic products may diffuse. Instead of mixing the cells in semisolid agar, it is possible to streak the mixture on the surface of slightly dried minimal agar plates. Under these conditions, however, the rototroph colonies are likely to be more heavily contaminated with the residual parental mutant types. 
For most purposes, however, this contamination may be ignored, as will be shown in a later section. Prototroph colonies are then fished and streaked directly on EMB plates, or otherwise tested, to classify them with respect to other factors that may be segregating.

\section{RESULTS AND CONCLUSIONS}

In most organisms inheritance is studied by the examination of zygotes carrying the gene alternatives determining a character. The segregants are chosen at random, and factor linkage is recognized by deviations in the frequency of parental and new couplings of a series of characters. In the absence of a random method of separating zygotes in $E$. coli, one is limited here to the members of specific recombination classes, namely the prototrophs. It is however, possible to introduce other factor differences into the biochemical mutants from which prototrophs are obtained, and to determine how such factors segregate into this recombination class. It was hoped in this way to obtain information concerning the haploid or diploid condition of the bacterial cell, and to determine whether factors segregated at random, or according to specific, perhaps linear chromosomal laws.

The first factor pair to which this approach was applied was $V_{1}{ }^{r} / V_{1}{ }^{\circ}$ (LEDERBERG and TATUM, I946b). In the cross $B^{-} M^{-} P^{+} T^{+} V_{1}{ }^{r} \times B^{+} M^{+} P^{-} T^{-} V_{1}{ }^{8}$, ten $B^{+} M^{+} P^{+} T^{+}$were isolated. Eight proved to be $V_{1}{ }^{r}$ while two were $V_{1} s$. This at once suggested that the vegetative cell of $E$. coli is haploid, since segregation could be observed in the first filial generation clone. It was noted also at that time that the "reversed" cross: $B^{-} M^{-} P^{+} T^{+} V_{1}{ }^{8} \times B^{+} M^{+} P^{-} T^{-} V_{1}{ }^{r}$ gave quite a different ratio of $r / s$ in the prototrophs, namely $3: 7$. Results on so small a sample are of doubtful significance, but they suggested the technique by which the basis of this character "segregation" could be elucidated. For this reason, the study of "reversed" crosses was extended to include numerically more data, using various combinations of mutants, and involving in addition to $V_{1}^{r} / V_{1}^{s}, L a c^{+} / L_{a c}$. The information which was obtained is summarized in tables 3 and 5 . The data show clearly that neither of the factor alternatives $V_{1}^{r} / V_{1}$ or $\mathrm{Lac}^{+} / \mathrm{Lac}^{-}$segregates at random into the prototroph recombination class. However, the occurrence of all factor combinations, albeit with different frequencies, is evident, at least with respect to $L a c$ and $V_{1}$. It seemed clear that there are only two alternative explanations for the unequal frequencies with which alternative alleles are manifested in the prototrophs: (a) that the alleles were characterized by some differential physiological property, such as dominance, or preferential segregation, or (b) that the nonrandom segregation was due purely to the mechanics of factor recombination. which is to say a linkage system.

The results of "reversed crosses" have a distinct bearing on this problem. If nonrandom segregation into protctrophs were due to some physiological property of the allele concerned, its particular coupling in the parent in which it is introduced should have no great effect on the segregation frequency; if on the other hand, the effect were purely mechanical, the segregation would reflect entirely the couplings of the parents, and the substitution of one allele for 
another in the parents (as in reversed crosses) should lead to a corresponding inversion in the ratios with which that allele is found in the prototrophs. The tables cited show that in every case there is no agreement between the ratios found in reversed crosses, unless the comparison is made with one of the ratios inverted, in which case there is reasonably good agreement. This result is in accord with the hypothesis that the genes in $E$. coli are arranged in one or more linkage groups, and is in disagreement with the fostulation of a diploid con-

TABLE 3

Comparisons of $V_{1}^{r}$ segregations when introduced with alternative parents. ${ }^{*}$

\begin{tabular}{|c|c|c|c|c|c|}
\hline \multirow{2}{*}{\multicolumn{3}{|c|}{ PARENTS }} & \multicolumn{3}{|c|}{ PROTOTROPHS $\left[\mathrm{B}^{+} \mathrm{MI}^{+} \mathrm{Pa}^{+} \mathrm{C}^{+} \mathrm{T}^{+} \mathrm{L}^{+} \mathrm{B}_{1}{ }^{+} \mathrm{P}^{+}\right]$} \\
\hline & & & \multirow[t]{2}{*}{$V_{1}{ }^{r}$} & \multirow[t]{2}{*}{$V_{1^{s}}$} & \multirow[t]{2}{*}{$\% V_{1}^{r}$} \\
\hline$B^{-} \mathrm{Pa}^{-} \mathrm{C}^{-} \mathrm{T}^{+} \mathrm{P}^{+}$ & & $B^{+} P^{+} C^{+} T^{-} P^{-}$ & & & \\
\hline$\cdots V_{1}^{r}$ & $x$ & $\cdots V_{1}^{8}$ & 76 & 6 & 92 \\
\hline$\cdots V_{1}$ & $x$ & $\cdots V_{1}^{r}$ & 30 & 107 & 22 \\
\hline$B^{-} \mathrm{Pa}^{-} \mathrm{C}^{-} \mathrm{T}^{+} \mathrm{L}^{+} \mathrm{B}_{1}{ }^{+}$ & & $B^{+} P^{+} C^{+} T^{-} L^{-} B_{1}^{-}$ & & & \\
\hline$\cdots V_{1}^{r}$ & $x$ & $\cdots V_{1}$ & 80 & 23 & 77 \\
\hline$\cdots V_{1}^{\circ}$ & $x$ & $\cdots V_{1}^{r}$ & 53 & 133 & 28 \\
\hline$B^{-} M^{-} T^{+} P^{+}$ & & $B^{+} M^{+} T^{-} P^{-}$ & & & \\
\hline$\cdots V_{1}^{r}$ & $x$ & $\cdots V_{1}^{s}$ & 49 & 8 & 86 \\
\hline$\cdots V_{1}$ & $x$ & $\cdots V_{1}^{r}$ & 5 & 19 & $2 \mathrm{I}$ \\
\hline
\end{tabular}

* See Lederberg (1947) for a statistical analysis of tables 3, 5, and 6.

dition, or with a state of indefinite "ploidy" which would be characteristic of a system of cytoplasmic inheritance.

The results of these experiments seemed sufficiently secure that one could adopt the existence of a linkage system as a working hypothesis and on this foundation, an attempt has been initiated to "map" a number of markers in E. coli. It was hoped at first that there might be found linkage groups which would be independent of one another, so that recombination between biochemical markers in one group could be used to detect recombinants, yet not interfere with the segregations in the other group(s). There was, however, no immediate prospect that these relationships could be found initially, so it was decided to study linkage relationships in a single pair of mutant stocks, and their derivatives. The stocks which were selected for this study were $5^{8-16} \mathrm{I}$ $\left(B^{-} M^{-}\right)$and Y-53 $\left(T^{-} L^{-} B_{1}^{-} L a c^{-}\right)$and their $V_{1}^{r}$ mutants. Since Lac and $V_{1}$ could be so readily scored, using only a single streak from each prototroph colony which appeared, it was hoped that the collection of an adequate volume of data could be accomplished with greater facility than if biochemical markers only were used.

It was, however, necessary to determine the relationships of the biochemical mutant loci of which at least four must be used to obtain recombinants. Mixtures were, therefore, plated into minimal medium supplemented with a single 
nutritional requirement, i.e., either biotin, methionine, threonine, leucine, or thiamin, allowing the proliferation of the corresponding single mutant as well as the prototrophic type. Colonies were then picked at random and scored according to their nutritional requirements. The results are summarized in table 4. Unfortunately, it was found that the addition of methionine to the minimal medium allowed excessive growth of $B^{-} M^{-}$, presumably because of a degree of contamination of the methionine with biotin. This datum is, however, not essential for the argument. In general, it will be seen that the + classes are markedly and significantly more frequent than the single mutant types, with

TABLE 4

Relative frequency of various biochemical recombination classes in the cross.

$B^{-} M^{-} T^{+} L^{+} B_{1}{ }^{+} \times B^{+} M^{+} T^{-} L^{-} B_{1}^{-*}$

\begin{tabular}{|c|c|c|c|c|c|c|c|}
\hline \multirow{2}{*}{$\begin{array}{c}\text { FROM } \\
\text { PLATES } \\
\text { SUPPLEMENTED } \\
\text { WITH }\end{array}$} & \multirow{2}{*}{\begin{tabular}{l} 
NUMBER \\
\multicolumn{1}{c}{ OF } \\
COLONIES \\
TESTED
\end{tabular}} & \multicolumn{4}{|c|}{ RECOMBINATION CLASSES FOUND } & \multirow{2}{*}{ RATIO } & \multirow{2}{*}{$\chi^{2}$} \\
\hline & & TYPE & NUMBBER & TYPE & NUMBER & & \\
\hline Biotin & 70 & $B^{-}$ & I0 & $B^{+}$ & 60 & 0.17 & 36 \\
\hline Threonine & 46 & $T^{-}$ & 9 & $T^{+}$ & 37 & 0.24 & I 7 \\
\hline Leucine & 56 & $L^{-}$ & 5 & $L^{+}$ & $5 \mathrm{I}$ & 0.096 & 38 \\
\hline Thiamin & 87 & $B_{1}^{-}$ & 79 & $B_{1}^{+}$ & 8 & 9.88 & 56 \\
\hline
\end{tabular}

* Cells of the parental types were mixed and plated into agar supplemented with the growth factor indicated. On this medium, the two recombination classes indicated on each line of the table could form colonies. Contrasting alleles only are specified; other loci, unless otherwise specified, have the "+" configuration. The $\chi^{2}$ for the ratio of single bixchemically deficient types to prototrophs is calculated for a comparison with the $1: 1$ expectation of a random segregation. As can be seen from the $\chi^{2}$ values, the probability that the deviations are due solely to chance is, in each case, less than .00I.

the exception of $B_{1}{ }^{-}$which is nearly ten times as frequent as $B_{1}{ }^{+}$. Writing the cross as $B^{-} M^{-} T^{+} L^{+} B_{1}+\times B^{+} M^{+} T-L^{-} B_{1}^{-}$, these results may be interpreted as follows:

I. $B^{+} M^{+} T^{+} L^{+} B_{1}{ }^{+}$more frequent than $B^{-} M^{+}$. Therefore $B$ and $M$ are linked.

2. $T^{+} L^{+} B^{+} M^{+} B_{1}+$ more frequent than either $T^{-} L^{+}$or $T^{+} L^{-}$. Therefore $T$ and $L$ are linked.

3. $B_{1}-B^{+} M^{+} T^{+} L^{+}$more frequent than $B_{1}{ }^{+} B^{+} M^{+}$. Therefore $B_{1}$ is linked to $B$ and $M$, but probably not between them.

One may therefore map these five loci onto not more than two linkage groups, according to the scheme in fig. 2a. In all that follows, the $[B-M]$ and $[T-L]$ combinations will be regarded as single units, since conclusive information as to their relative order has not been obtained. These data so far do not allow any conclusion to be drawn as to whether the regions $B_{1}-[B M]$ and $[T L]$ are linked or are indefendent of each other, since a recombination between them is a necessary requirement for a detectable tyfe. 
TABLE 5

Segregation of Lac and $V_{1}$ into prototrophs issuing from various parental combinations. ${ }^{*}$

\begin{tabular}{|c|c|c|c|c|c|c|}
\hline \multicolumn{2}{|c|}{ PARENTS } & \multirow[b]{2}{*}{$B^{+} I^{+} T^{+} L^{+}$} & \multicolumn{4}{|c|}{ RECOMBINATIONS } \\
\hline$B^{-} M^{-} T^{+} L^{+} B_{1}{ }^{+}$ & $B^{+} M I^{+} T^{-} L^{-} B_{1}^{-}$ & & $\operatorname{Lac}^{-V_{1}^{r}}$ & $\operatorname{Lac}^{-} V_{1}$ & $\mathrm{Lac}^{+} \mathrm{V}_{1^{r}}$ & $\operatorname{Lac}^{+} V_{1}$ \\
\hline $\operatorname{Lac}^{+} \mathrm{V}_{1}$ & $\mathrm{Lac}^{-\mathrm{I}}$, & $\begin{array}{l}\cdots B_{1}^{+} \\
\cdots B_{1}^{-* * *} \\
\cdots B_{1}^{-* *}\end{array}$ & $\begin{array}{l}602 \\
{ }^{13}[45.8] \\
\\
244 \\
{[45]} \\
\text { (D) }\end{array}$ & 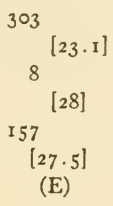 & $\begin{array}{c}387 \\
829.4] \\
{[28]} \\
159 \\
{[27.9]} \\
\text { (C) }\end{array}$ & $\begin{array}{l}{ }^{22}[\mathrm{I} \cdot 7] \\
0{ }^{[0]} \\
10 \\
{[\mathrm{I} \cdot 9]} \\
\text { (triple) }\end{array}$ \\
\hline $\operatorname{Lac}^{+} V_{1}^{\circ}$ & $\operatorname{Lac}^{-} V_{1}^{\top}$ & $\begin{array}{l}\cdots B_{1}^{+} \\
\cdots B_{1}^{-* *}\end{array}$ & $\begin{array}{l}107 \\
I 34{ }^{[33 \cdot 2]} \\
(\mathrm{E})\end{array}$ & $\begin{array}{l}\text { I45 } \\
\text { I } 51 \\
{[45.0]} \\
\text { (D) }\end{array}$ & $\begin{array}{c}{ }^{9}[2.8] \\
9{ }^{[}{ }^{2.4]} \\
\text { (triple) }\end{array}$ & $\begin{array}{l}{ }^{6 I} \\
80^{[19.0]} \\
\text { (C) }{ }^{[2 \mathrm{r} \cdot 4]}\end{array}$ \\
\hline $\operatorname{Lac}^{-} V_{1}^{r}$ & $\mathrm{Lac}^{+} V_{1^{\circ}}$ & $\begin{array}{l}\cdots B_{1}^{+} \\
\cdots B_{1}^{-* *}\end{array}$ & $\begin{array}{l}28 \\
1023.9] \\
{[25.4]} \\
(\mathrm{C})\end{array}$ & $\begin{array}{l}{ }^{6} \\
\quad[5 \cdot I] \\
\quad[1 \cdot 7] \\
\text { (triple) }\end{array}$ & ${ }_{201}^{46} \begin{array}{l}{[39 \cdot 3]} \\
{[50 \cdot 1]} \\
\text { (D) }\end{array}$ & $\begin{array}{ll}37 & \\
91 & {[31.6]} \\
& {[22.7]} \\
& (E)\end{array}$ \\
\hline$\dagger \operatorname{Lac}^{+} V_{1}{ }^{r}$ & $\operatorname{Lac}^{-} V_{1}^{r}$ & $\cdots B_{1}^{-* *}$ & I 28 & $\circ$ & 33 & $\circ$ \\
\hline$\dagger \operatorname{Lac}^{-} V_{1}{ }^{r}$ & $\left.{ }_{7}\right)^{\operatorname{Lac}^{-} V_{1^{\circ}}}(Y-$ & $3)^{3} \cdots B_{1}^{-* *}$ & I34 $\operatorname{Lac}^{-} ; \mathrm{n}$ & ot scored fo & $r V_{1}$ & \\
\hline
\end{tabular}

* Cell mixtures of the indicated composition were plated into minimal agar plates or into plates supplemented with thiamin. $B_{1}{ }^{+}$types refer to scores of prototrophs picked at random from minimal plates.

${ }^{* *} B_{1}-$ refers to colonies picked at random from thiamin supplemented plates. Although predominantly $B_{1}{ }^{-}$they contain $B_{1}{ }^{+}$colonies in the proportion 1 : 10 as may be seen from table 4 .

${ }^{* * * *} B_{1}^{-}$. In this series, colonies were scored as to $B_{1}$, and only the $B_{1}^{-}$are recorded.

The letters (C), (D), (E), refer to crossover types corresponding to the regions $[B M]$ ] $L a c$; $L a c-V_{1}$; and $V_{1}-[T L]$ respectively, according to the map of Fig. 2 d.

$\dagger$ Test for allelism.

On the basis of table 5 , the factors $V_{1}$ and Lac may be brought into the argument. In addition to the joint segregations of these factors, the effect of the $B_{1}$ segregation was studied in the following way. It would be uneconomical, in view of the relative paucity of $B_{1}+$ types, to separate these from the $B_{1}-$ by nutritional testing of colonies which appear on thiamin supplemented agar. Instead, the entire sample was regarded as $B_{1}$ - with the proviso that it might be contaminated to the extent of ten percent with $B_{1}+$. However, it has been found that the distribution of $L a c$ and $V$ on colonies picked from thiamin 
supplemented agar is homogeneous with the distribution in prototrophs, so that the segregation of these factors is not influenced by the $B_{1}$ segregation.

The data in table 5 show that $L a c$ is inclined not to separate from $B M$, and is therefore regarded as linked to it, while there is a similar linkage of $V_{1}$ to $T L$. Since the recombination of $L a c$ with $B M$ is not influenced by the interchange between $B_{1}$ and $B M$, they are on opposite sides of $B M$ as suggested by map 2 b. Finally, a scrutiny of the interaction between the Lac and $V$ segregations shows that these are not independent of each other, particularly because of the rarity of the least frequent class. This suggests, then, that the two linkage groups of fig. $2 \mathrm{~b}$ be combined to give the map of fig. $2 \mathrm{c}$. (The locus of $V_{6}$ on this map is obtained from additional data.) According to this interpretation, the rarity of the least frequent $L a c-V$ combination stems from the fact that a triple-crossover is necessary for its production. In fig. $2 \mathrm{~d}$, the cross $\mathrm{Y}-40 \times \mathrm{Y}-53$ is interpreted according to the map, with a table citing the regions in which interchange must take place to yield the given types.

That the first seven factors to be investigated should fall in the same linkage group leads to the inference that there is only a single chromosome in E. coli. This inference is supported by incomplete analyses of the segregations of 8 other markers referred to in table r. None of these factors has been found to segregate independently of the factors which have already been described as belonging to a single linkage group. The possibility that segregation interactions may, in some cases, be based upon an inter-chromosomal type of interference (compare Steinberg and Fraser, I944), has not been ruled out, however.

The distances recorded in fig. $2 \mathrm{c}$ are derived from the recombination totals in tables 5 and 6 . However, the distance between $[B M]$ and $[T L]$ cannot be estimated directly, but only the partition of that distance among the regions $B M-L a c, L a c-V_{1}$, and $V_{1}-T L$. The relative frequency of the "triple-interchange" type can be used to estimate the absolute map distances, if it is assumed that there is no interference. This frequency, about 2.I percent, is readily calculated to be consistent with a map length of between 75 and 80 units altogether either in a two-strand or a four-strand system (LEDERBERG, 1947). These values must be regarded as rough approximations, because they are extremely sensitive to error in the estimation of the proportion of the "triple" types.

\section{Linearity}

In constructing a map, and calculating distances, it has been taken for granted that there is in $E$. coli a system of linear linkage, such as has been demonstrated quite conclusively in Drosophila, and inferred in all higher organisms. What direct evidence may one bring to bear on this question?

The method which one is forced to em loy in hybridizing this Lacterium introduces certain complications. The classical proof of linearity is based on the additive character of distances, expressed in morgans, Letween loci occurring within the same linkage group. The determination of map distances is based upon a comparison between parental and new combinations of linked 
genes, as determined in the progeny of zygotes selected at random. In E. coli, on the other hand, one is limited to the recovery of that recombination class in which there has necessarily been an interchange between certain biochemical loci, in the cases here discussed, betwen $[B M]$ and $[T L]$. For this reason, it is not possible to obtain a direct measure of the absolute distance between factors which are located within this critical region, and any argument in favor of linearity which is based on the segregations of such factors may have the
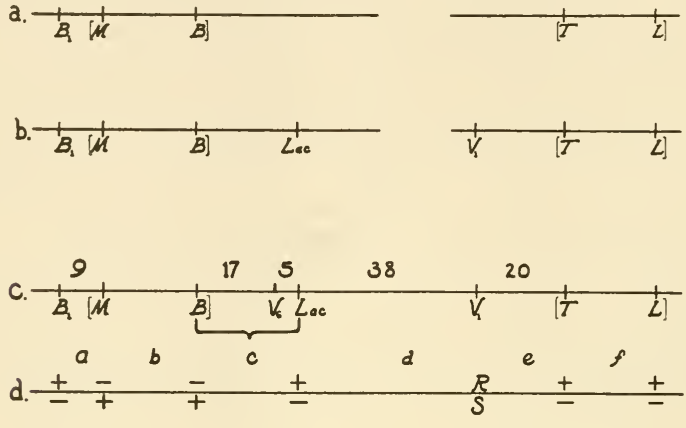

Crossovers

$(c, d$, or $e)$

(a) $(c, d$, or $e)$

Recoverable type

(a) (b)

(a) $(f)$

(a) $(f)(c, d$, or $e)$

$B_{1}-M+B+T+L+$

$B_{1}+M+B+T+L+$

(a) $(c)$

(a) $(d)$

$B_{1}+M+B-T+L+$

$B_{1}+M+B+T-L+$

$B_{1}+M+B+T+L-$

(a) $(c)(d)(e)$

$\begin{aligned} B_{1}+M+B+T+L+ & \cdots \operatorname{Lac}^{+} V_{1}{ }^{r} \\ & \cdots \operatorname{Lac}^{-} V_{1} \\ & \cdots \operatorname{Lac}^{-} V_{1} \\ & \cdots \operatorname{Lac}^{+} V_{1}\end{aligned}$

Figure 2.- $a, b$, and $c$. Mapping of genetic factors. $d$. The cross $B_{1}{ }^{+} M T^{-} B^{-} L a c^{+} V_{1}{ }^{r} T^{+} L^{+} \times B_{1}{ }^{-}$ $M^{+} B^{+} \operatorname{Lac}^{-} V_{1} T^{-} L^{-}$and some of the recoverable crossover classes. (See table 5.)

flavor of circular reasoning. It would be preferable to study the segregations of factors which are assigned to loci distal to the biochemical factors whose recombination is the basis of the detection of sexual offspring. The stocks with which this might be accomplished are not yet available, but it is hoped that they will be for future work.

That there does exist some sort of linkage system is made highly credible by the results of the "reverse crosses" tabulated in tables 3 and 5 . The chief difficulty in proving that this system is linear has been to formulate the feasible alternatives, so that critical experiments, the results of which could discriminate between linearity and a given alternative, might be set up. Certain types of "linkage" can be disqualified by the data already at hand. For example, one might postulate that genes of bacteria are embedded in a two-dimensional 
matrix, and there occasionally occurs a gene-for-gene interchange. This is equivalent to the "Konversion" theory once proposed by WinkLER (r932), to account for interchanges in Drosophila. While this type of arrangement would account for a tendency to preserve the parental configuration, it fails to explain either quantitative linkage intensities, or the interaction of segregations which is revealed by the data on $L a c$ and $V$ in table 5 . Naturally, one could further modify the "Konversion" theory to take these exigencies into account, but in so doing one would be elaborating an exceedingly complicated theory which would, in fact, be a re-expression of a mechanical theory of linkage.

The interaction of the $L a c$ and $V$ segregations is perhaps the most critical datum with which a genetic system for $E$. coli can be formulated. The interaction may be expressed as follows: the frequency of interchanges between $[B M]$ and $L a c$ is dependent upon the interchanges between $[B M]$ and $V$. Specifically, in the cross $B^{+} M^{+} T^{-} L^{-} B_{1}-L a c^{-} V_{1}{ }^{8} \times B^{-} M^{-} T^{+} L^{+} B_{1}{ }^{+} L a c^{+} V_{1}{ }^{r}$, one finds in the $B^{+} M^{+} T^{+} L^{+} B_{1}{ }^{+}$the following distribution of classes: $L a c^{-} V_{1}{ }^{8} 23$ percent, $L a c^{+} V_{1}^{r} 29$ percent (for the parental combinations) and $L a c^{-} V_{1}^{r}{ }_{4}^{6}$ percent, $\mathrm{Lac}^{+} V_{1}^{s} 2$ percent (for the new combinations). With reference to $\left[\mathrm{B}^{+} \mathrm{M}^{+}\right], \mathrm{Lac}^{-}$is the parental, $\mathrm{Lac}^{+}$the interchange type. The proportion of $V_{1}^{r}$ (representing an interchange between $V_{1}$ and $[B M]$ ) is different in the $L a c^{-}$ and $\mathrm{LaC}^{+}$segregations: namely $46: 23=2: \mathrm{I}$ and $29: 2=14 \cdot 5: \mathrm{I}$ respectively. This interaction between interchanges is most simply explained by the assumption that factors are located on a linear segment, so that interchanges between proximal factors also lead to the crossing over of more distal factors, barring the occurrence of additional interchanges.

Additional support for the theory of linear arrangement has been found in the segregation of $V_{6}$, summarized in table 6 . It will be noted that the segregations of $L a c, V_{1}$, and $V_{6}$ are quite congruous in the $B_{1}{ }^{-}$and $B_{1}{ }^{+}$classes. In the totals, one finds the ratios, for each factor separately, of $\mathrm{Lac}^{-} 78$ percent; $V_{6}{ }^{r} 82$ percent; $V_{1}{ }^{8} 36$ percent; indicating that the first two are both linked to $[B M]$ while the latter is linked to $[T L] . V_{6}$ cannot, however, be to the left of $[B M]$ because it does not interact with $B_{1}$. If, therefore, there is a linear order of genes, $V_{6}$ must be to the right of $[B M]$, and because of its greater linkage intensity, nearer $[B M]$ than is $L a c$. This arrangement is indicated in the map in table 6 , and in fig. $2 \mathrm{c}$. The agreement of the data with the hypothesis can be examined at several points. In the first place, the single exchange types, as indicated in the table, should be the most frequent. Secondly, barring multiple exchanges, an interchange between $V_{6}$ and $L a c$ should lead also to an interchange between $V_{6}$ and $V_{1}$. That is to say, the $\mathrm{Lac}^{+} V_{6}{ }^{r}$ class should be more of ten $V_{1}^{r}$ than $V_{1}^{s}$. Finally, in view of the similarity in linkage intensities to $[B M], L a c$ and $V_{6}$ must be closely linked. Although the "triple-interchange" types would seem to be rather frequent, reference to the table may suggest that these conditions are fulfilled. In particular, it will be noted that among the $\mathrm{Lac}^{-}$, the ratio of $\mathrm{V}_{6}{ }^{r}: V_{6}{ }^{8}$ is $94: 3$, or $3 \mathrm{r}: \mathrm{I}$, while among the $\mathrm{Lac}^{+}$, this same ratio is $10: 29$, or $1: 3$. This difference is interpreted to mean that $L a c$ and $V_{6}$ are linked to each other, as demanded by the theory of linearity.

It is not, of course, proven that the gene order is not branched at some other 
point. The most economical hypothesis at this time, however, is that there is a single unbranched chromosome as the physical basis of inheritance in E. coli.

\section{Attempts to Induce Aberrations}

Using a chromosomal theory as a working hypothesis, it was hoped that some verification could be found by the study of types in which the normal order of genes was disturbed. Since there is only one chromcsome (from the

TABLE 6

Segregation of Lac, $V_{1}$ and $V_{6}$. $B^{-} M^{-} T^{+} L^{+} B_{1}{ }^{+} L_{a c}+V_{1}^{r} V_{0^{\prime}} \times B^{+} M^{+} T^{-} L^{-} B_{1}-L a c^{-} V_{1} V_{\theta^{r}}$

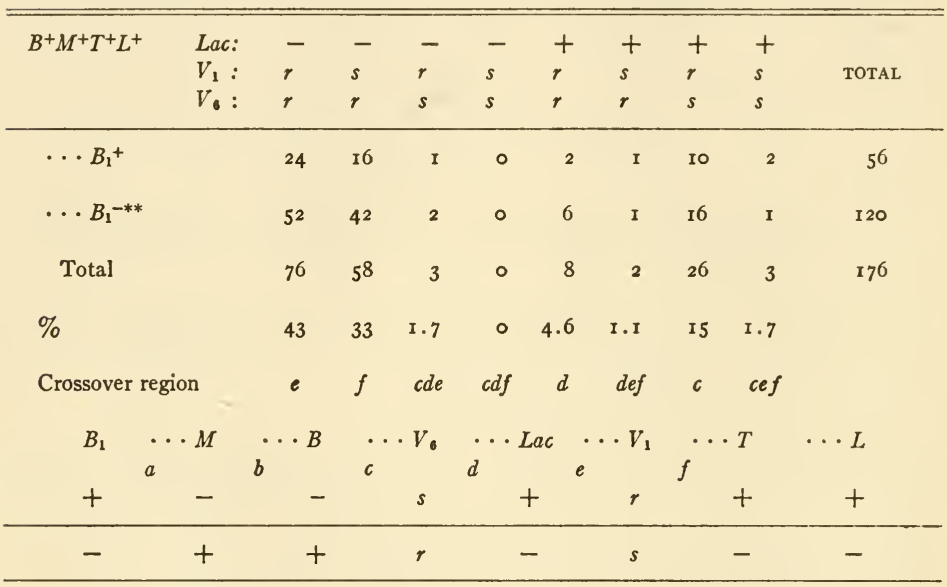

** See footnote to table 4 .

genetic evidence), the only types of rearrangements would be changes leading to a series of inversion-transposition types. It was thought that such types might be detected by genetical procedures by virtue of their effect on crossing over. In particular, the occurrence of an inversion in the region $B_{1} \cdots[M B]$ would be expected to have the effect of eliminating the recombination classes involving interchanges in this region. In the cross $B-M^{-} T^{+} L^{+} B_{1}+$ $\times B^{+} M^{+} T-L^{-} B_{1}-$ this would be equivalent to the suppression of prototroph recombinants; $B_{1}-$ types, however, would be recoverable, and allow the investigation of the extent of the changes.

Preliminary attempts to find such aberration types have, to date, been unsuccessful. The procedure was as follows:

Following treatment with nitrogen mustard (TATUM, 1946) or $20,000 \mathrm{r}$ of $\mathrm{X}$-rays, cells of $\mathrm{Y}-40$ and of $\mathrm{Y}-53$ were incubated separately for 24 hours, to allow the separation of cells or nuclei that might have been associated at the time of treatment. The cultures were then streaked out on nutrient agar 
plates. Single colonies of $\mathrm{Y}-40$ were picked and streaked across a nutrient agar plate. Streaks of similarly treated $\mathrm{Y}-53$ colonies were made from the opposite direction, so that in the center of the plate, cells of the two types were mixed, treated colony by treated colony. The plates were incubated for 24 hours, the mixed growth scraped from the plates, suspended in sterile water and plated into minimal agar. The occurrence of colonies which would not interact to produce prototrophs, as detected by plating into minimal medium, would be an indicator that the combination was heterogeneous for an aberration. Since in these experiments, both "parents" were exposed to treatment, each plating was equivalent to the testing of two chromosomes for the occurrence of an aberration. No marked variation in the yield of prototrophs was noted in tests involving I2I mustard- and $28 \mathrm{x}$-ray-treated chromosomes. This can scarcely be regarded as an adequate sample in view of the stringent selection imposed by the technique, which might be expected to eliminate any aberration types which are even slightly less vigorous than the normal. This consideration is especially relevant in view of the "hemizygous" condition of any aberrations in the probably haploid vegetative cells. These studies will be continued.

\section{How Many Segregants per Zygote?}

In the experiments detailed in this paper, recombinants were obtained from different cell types which were exposed to each other in an agar medium. Therefore each prototroph recombinant colony seen by the experimenter marks the site of formation of a zygote. The question may immediately be raised whether there are at that site other recombination classes which, by virtue of their biochemical deficiencies, remain dormant within the prototroph colony on the minimal selective medium. This is equivalent to inquiring whether there is but a single viable product of meiosis (as in megasporogenesis in many higher plants) or more than one, as in the ascomycetes. The solution to this problem would be of special interest in relation to the possible occurrence of four-strand crossing over. In addition, if an appreciable profortion of prototroph colonies consisted of two distinct segregation types, it would be necessary to isolate these types for the collection of segregation data.

There are at least three ways in which a zygote might yield more than one haploid recombinant. Firstly, the zygote might be capable of proliferation in the diplophase (or sporophyte), leading to the concurrence of several diploid cells, each of which might undergo meiosis independently, and by chance yield several segregation types. Secondly, a single zygote might produce, after meiosis, in addition to the prototroph, the complementary multiple mutant class. Thirdly, in a system of four-strand crossing-over, there might be two supplementary prototroph recombinants differing in the segregation of factors such as $L a c$ and $V_{1}$ for which the diploid was heterozygous.

Obviously, the proper investigation of these possibilities requires that one stringently avoid contamination of one colony with another. For this reason, the cell suspensions used were diluted so as to yield only about five to ten recombination colonies per plate. 
Crosses were made between $\mathrm{Y}-40$ and $\mathrm{Y}-53\left(B^{-} M^{-} T^{+} L^{+} B_{1}{ }^{+} L a c^{+} V_{1}{ }^{r} \times B^{+}-\right.$ $\left.M^{+} T^{-} L^{-} B_{1}-L a c-V_{1}{ }^{8}\right)$ on $B_{1}$-containing minimal agar medium. As already noted, about 90 percent of the colonies from such a cross are $B^{+} M^{+} T^{+} L^{+} B_{1}{ }^{-}$. The theoretical complementary class would be $B^{-} M^{-} T^{-} L^{-} B_{1}+$. Because of its nutritional deficiencies, it could not be expected to proliferate on the minimal medium even had it been produced after meiosis. The possibility remains, however, that a few cells of this constitution might still be present among the $10^{8}$ or so $B_{1}{ }^{-}$cells of the predominant type in a colony. By plating such colonies into medium lacking $B_{1}$ but containing biotin, methionine, threonine and leucine, the $B_{1}^{-}$cells would be suppressed, while the postulated multiple mutant type could form colonies and be recovered.

The experiment just described was carried out, testing 52 colonies for their content of other cell types. In general, a thiaminless colony could be shown to contain from ro-100 cells capable of forming colonies on the $B, M, T, L$ medium. However, in each case investigated these have been shown to be indistinguishable from the $\mathrm{Y}_{-4}$ o parental $B-M^{-}$type, and must be presumed tc arise from a surprisingly low degree of contamination of the colony with these cells from the heavily seeded plate. A few colonies were found which could be characterized as reversions from $B_{1}{ }^{-}$to $B_{1}{ }^{+}$. These experiments are then, inconclusive with respect to the occurrence of complementary genotypes in the same colony. With appropriate stocks, not as yet available, it should eventually be possible to manipulate the situation so that the complementary type could be recovered selectively, excluding both parents and the predominant recombination class.

A search for supplementary types was conducted with the same crosses, except that colonies appearing on $B_{1}$ agar were streaked out directly on EMBlactose agar to determine whether any of them were heterogeneous for Lac. In some cases, a number of isolated colonies from each EMB-test plate were then also tested for homogeneity with respect to $T_{I}$-resistance. About 90 colonies were so tested; only one colony was found containing both $\mathrm{Lac}^{+}$and $\mathrm{Lac}^{-}$cells. It is impossible to be certain that, with this low frequency, the single colony which was picked was not actually derived from two distinct zygotes. These experiments cannot be considered as bearing critically on the question of the occurrence of two- or four-strand crossing over because of the absence of information concerning (a) the viability of more than one meiotic product and (b) chiasma interference. The results do, however, justify the technique of picking the prototroph colonies directly, and testing them without further purification for the collection of segregation data.

\section{A Comparison of Sexual Recombination and Transformation}

The occurrence of recombination types has been interpreted by us (LEDERBERG and TATUM I946c, TATUM and LEDERBERG I947) as a consequence of cell fusion, "karyogamy" and meiosis with crossing over. This is, however, not the only allowable interpretation of the general phenomenon of the occurrence of new character combinations. By analogy with the systems which have been described in pneumococci (Avery, MacLeod and McCarty 1944) and other 
strains of $E$. coli (BoIvin and VENDRELEy I946) one might postulate that genotypically distinct cells interact not through cell fusion, but through the release of "transforming substances" diffusing through the medium. Such transforming substances would have the property of inducing or directing mutational changes in the cell receiving them so as to lead to what appear to be recombination types. Our inability to separate such postulated transforming substances from the cells themselves is not proof of their absence but could be due to their lability in our hands.

In previous publications, certain reasons were given for the rejection of the transformation hypothesis in favor of a picture of cell fusion, and so forth. It was not our intention thereby to state, with clairvoyant insight, that no investigator will be able to duplicate the results which we have reported, using instead of living cells extracts specially prepared. It is, rather, our view that since we have been able to demonstrate no appreciable point of difference between the features of gene exchange in this strain of $E$. coli and in the classical materials of Mendelian experimentation, the most economical conclusion is that the mechanisms involved are also similar. In the absence of more detailed information on the behavior of transforming systems, a critic would be free to impute to such systems all of the properties which have been found to characterize the genetic system of $E$. coli, $\mathrm{K}-\mathrm{I} 2$. While this would be tailoring the cloth to suit the customer, it cannot be disputed that the only conclusive method by which it could be shown that cell fusion underlies gene recombination would be a direct cytological demonstration. The rarity with which the presumed zygote occurs, however (as indicated by the low frequency of effective recombination types) is very discouraging to attempts to find and characterize the "fusion-cell," at least in the present material.

Certain genetic experiments were performed in an attempt to characterize further the behavior of this system. On the transformation hypothesis, one must attribute the rarity of the imputed transformations primarily to restricted conditions for susceftibility to the transforming factors released into the milieu. Otherwise, one would expect to find "transformations" for single factors much more frequent than those involving more than two factors. A glance at tables 4 and 6 illustrates that certain "multiple transformed" types are much more frequent than singly transformed classes. Under these conditions, one might also anticipate that genetic materials from two different kinds of cells could mix in the medium and together transform a third. In a mixture of three cell types then, one should find cases where genes from all three have combined. Using $L a c$ and $V_{1}$ as markers, this type of experiment was set up in several different ways, as summarized in table 7. Pairwise, prototrophs can be formed only from biochemically distinct and nonoverlapping parents. Combinations of $B^{-} M^{-}$and of $T^{-} L^{-} B_{1}-$ were arranged so that taken two at a time they were heterozygous either for $L a c$ or for $V_{1}$ but not both. For example, a mixture of $B^{-} M^{-} L a c^{-} V_{1}^{r}, T^{-} L^{-} B_{1}-L a c^{-} V_{1}{ }^{8}$ and $T^{-} L^{-} B_{1}-L a c^{+} V_{1}{ }^{r}$ was plated. Prototrophs could be formed by recombination between either of the two latter and the former types. In one case, only $V_{1}$ would be heterozygous, and the expected types would be $L a c^{-} V_{1}^{r}$ and $\operatorname{Lac}^{-} V_{1}{ }^{8}$. In the other, $L a c$ 
would be heterozygous, and prototrophs carrying the markers $\mathrm{Lac}^{+} V_{1}{ }^{r}$ and $\mathrm{Lac}^{-} V_{1}{ }^{r}$ could be produced. The type $\mathrm{Lac}^{+} V_{1}$ s would not be expected unless, indeed, genetic material from all three types could combine in a sort of ménage à trois. As recorded in table 7 , no instance of such a three-way combination was found in 628 tests, a different class being vacant, as anticipated, in each of the four parts of the experiment. It may be concluded that genetic factors from different cells are not freely miscible, as would be demanded by the most economical version of the interpretation of transformations.

From all the experiments so far cited, it must be concluded that if trans-

TABLE 7

Pairwise occurrence of recombination in mixtures of three components.

\begin{tabular}{|c|c|c|c|c|c|c|}
\hline \multicolumn{2}{|c|}{ PARENTAL TYPES } & \multicolumn{5}{|c|}{$\begin{array}{l}\text { RECOMBINANT PROTOTROPHS* } \\
B^{+} M^{+} T^{+} L^{+} B_{1}^{+} \text {or } B_{1}^{-}\end{array}$} \\
\hline$B^{-} M^{-} T^{+} L^{+} B_{1}^{+}$ & $B^{+} M^{+} T^{-} L^{-} B_{1}^{-}$ & $L a c^{-} V_{1}^{r}$ & $\operatorname{Lac}^{-} V_{1}$ & $\mathrm{Lac}^{+} \mathrm{V}_{1}{ }^{r}$ & $\operatorname{Lac}^{+} V_{1^{\bullet}}$ & TOTAL \\
\hline $\operatorname{Lac}^{-} V_{1}^{r}$ & $\begin{array}{l}\operatorname{Lac}^{+} V_{1}^{r} \\
\operatorname{Lac}^{-} V_{1}\end{array}$ & 173 & 49 & 4 & $\circ$ & 226 \\
\hline $\mathrm{Lac}^{+} V_{1^{r}}$ & $\begin{array}{l}\operatorname{Lac}^{-} V_{1}{ }^{r} \\
\operatorname{Lac}^{+} V_{1^{\circ}}\end{array}$ & 16 & $\circ$ & 7 & 28 & $5 \mathrm{I}$ \\
\hline $\operatorname{Lac}^{+} V_{1}$ & $\begin{array}{l}\operatorname{Lac}^{+} V_{1}^{r} \\
\operatorname{Lac}^{-} V_{1}^{8}\end{array}$ & $\circ$ & 136 & 37 & 40 & 213 \\
\hline \multirow[t]{2}{*}{$\begin{array}{l}\mathrm{Lac}^{+} V_{1} \\
\operatorname{Lac}^{-} V_{1}^{r}\end{array}$} & $\operatorname{Lac}^{-} V_{1^{\circ}}$ & 65 & 48 & $\circ$ & 25 & 138 \\
\hline & & & & & Total & 628 \\
\hline
\end{tabular}

* Mixtures of the three types indicated in each experiment were plated into thiamin-contain ing agar. The prototrophs are therefore a mixture of $B_{1}{ }^{-}$and $B_{1}{ }^{+}$types, as indicated in table 4 , footnote.

forming factors are operating in this system, the diverse factors (or genes) are not independent of one another, but are grouped in separate and immiscible parcels. Such parcels would also be potentially capable of transmitting all of the genetic factors of a cell, so that there seems to be no compelling reason why such a parcel, speaking purely genetically, could not be regarded as a gamete. MULLER (1947) has interpreted the pneumococcus transformation in terms of "still viable bacterial chromosomes or parts of chromosomes floating free in the medium ... these have penetrated the capsuleless bacteria and in part at least, taken root there, perhaps after having undergone a kind of crossing over with the chromosomes of the host." It remains to be seen whether this interpretation will be upheld by further studies on factor interaction in bona fide transforming systems.

Several attempts were made to determine whether "transforming activity" could be separated from the living cell under conditions comparable to the 
platings in minimal agar medium, or after extraction of cells by Borvin's method (Borvin et. al., I946). No activity was found in the supernatant of a suspension of $\mathrm{Y}-40$ and $\mathrm{Y}-53$ together or separately in the same minimal liquid medium to which agar is added for plating experiments. The only manipulation involved here consists of the removal of most of the bacteria by ordinary centrifugation. It could thus be shown that the "activity" was associated with the cells. Equally negative results characterized attempts to reveal transforming activity on culture filtrates and cell autolysates prepared as crude fractions according to Borvin's procedure. Finally, the addition of desoxyribonuclease in a final concentration of $.05 \mathrm{mg} / \mathrm{ml}$ to the mixing and plating medium had no effect on the number of prototrophs which appeared in the cross of $\mathrm{Y}-40$ and $\mathrm{Y}-53$. Tests for the destruction of enzymatic activity under these conditions were, however, not done.

The conclusions which we draw from these experiments are (a) that the existence of transforming factors is exceedingly unlikely and (b) it would be * not worthwhile to go to extreme trouble to attempt to isolate such factors from this system until the study of bona fide transforming systems has progressed sufficiently that the genetical criteria already discussed might be applied.

\section{DISCUSSION}

Regardless of the stand that one takes on the issue of invisible zygotes versus non-extractable transforming factors, it can be asserted that $E$. coli K-I 2 provides a useful tool for genetic analysis. The use of biochemical mutants as parents allows crosses which are nearly as well controlled as in Neurospora. The segregational behavior of mutant factors seems to be closely analogous to that of higher forms, and seems to compel their admission into the same arena as the genes of Drosophila. However, it would be premature to transfer these conclusions to other genetic characters of other microorganisms, each of which must be examined on its own merits.

It may be wondered that the apparent recombination rate is so low. However, this is possibly not to be attributed to any sexual imperfections of $E$. coli, but to the method of enumeration. It seems likely that an analogous comparison of the number of somatic and generative cells in an organism like the oak-tree, or man (especially the female of the species) would give ratios similar to those prevailing in $E$. coli. It is also possible that the optimal conditions for zygote formation or germination have not yet been achieved and that by special procedures the rate of zygote-formation may be accelerated to the level where there might be some hope of finding it in the field of the microscope.

Attempts to detect recombination in two other strains of $E$. coli, B (Demerec and Fano, I945) and L-I 5 (Roepke, Libby, and Small, I944) by analogous methods have been unsuccessful (LURIA, I947, TATUM and LEDERBERG, I947). At least two strains then must be classified with the "Fungi Imperfecti." This dismal conclusion is, however, illuminated by the fact that many heterothallic species have been eliminated from the Fungi Imperfecti with the discovery of the appropriate opposite mating-tyfes. At the present time, one scarcely knows where to begin to look for the bacterial analogy. The 
application of genetic techniques to the elucidation of unusual life-cycles in diverse bacteria (Braun and Elrod, I946, Dienes, I946) cannot fail, however, to be most fruitful.

The evolutionary significance of gene recombination has been made so widely familiar by DoBZHANSKY's book (I94I), and adequately discussed, more recently, by MULLER (1947), that it would be impertinent to do more than simply refer to these papers.

\section{SUMMARY}

The recombination of genetic factors and their segregation into prototroph recombinants of Escherichia coli have been studied. It was found that genetic markers behaved as if they were part of a system of linked genes. Some evidence for linear order of genes was obtained. Each of 15 factors studied fell into the same linkage group. Data are given in detail for the segregation of factors involved in the biosyntheses of biotin, methionine, threonine, leucine, or thiamin; in the fermentation of lactose, and in resistance to bacterial viruses $T_{I}$ and T6. On the basis of these data a tentative 8-point genetic map of the chromosome of $E$. coli is presented.

\section{ACKNOWLEDGEMENTS}

The author is deeply indebted to many of his colleagues and friends, too numerous to mention, for stimulating discussions of the problems discussed in this paper. They are numbered, by and large, among the participants and discussants of the 1946 Cold Spring Harbor Symposium on Quantitative Biology. He is indebted to DR. MACLYN MCCARTy for a generous sample of purified desoxyribonuclease, and to Drs. S. E. LuRIa and M. DEMEREC for cultures of the bacteriophages used in this investigation. He owes much of the genetic analysis to the criticism of DR. K. Mather of the John Innes Horticultural Institution. Above all, he is indebted to Professor E. L. Tatum for the opportunity and much of the stimulus to do these experiments.

\section{LITERATURE CITED}

ANDERson, E. H., I946 Growth requirements of virus-resistance mutants of Escherichia coli strain "B." Proc. Nat. Acad. Sci. 32: I 20-1 28.

Avery, O. T., C. M. MacLeod, and M. McCarty, 1944 Studies on the chemical nature of the substance inducing transformation of pneumococcal types. J. Exp. Med. 79: 137-r 58.

Borvin, A., and R. VENDRELEY, I946 Rôle de l'acide désoxy-ribonucléique hautement polymérisé dans le déterminisme des caractères héréditaires des bactéries. Signification pour la biochemie générale de l'hérédité. Helv. Chim. Acta 29: 1338-1344.

Braun, A. C., and R. P. Elrod, 1946 Stages in the life history of Phytomonas tumefaciens. J. Bact. 52: 695-702.

Demerec, M., and U. FANO, 1945 Bacteriophage-resistant mutants in Escherichia coli. Genetics 30: $119-136$.

Drenes, L., I946 Complex reproductive processes in bacteria. Cold Spring Harbor, Symposia on Quantitative Biology II: 5 I-59.

Dobzhansky, Th., 194I Genetics and the Origin of Species. Rev. Ed. xviii+446 pp. New York: Columbia Univ. Press.

Gray, C. H., and E. L. TAtUm, 1944 X-ray induced growth factor deficiencies in bacteria. Proc. Nat. Acad. Sci. 30: 404-410. 
LEDERBERG, J., 1947 Genetic recombination in Escherichia coli. Dissertation; Yale University.

LederberG, J., and E. L. TATUM, r946a Detection of biochemical mutants of microorganisms. J. Biol. Chem. 165: 381-382.

1946b Novel genotypes in mixed cultures of biochemical mutants of bacteria. Cold Spring Harbor, Symposia on Quantitative Biology II: I13-114.

1946C Gene recombination in Escherichia coli. Nature 158: 558.

LURIA, S. E., 1947 Recent advances in bacterial genetics. Bact. Rev. II : 1-40.

Muller, H. J., 1947 The gene. Proc. Roy. Soc., London Br34: 1-37.

ROEPKE, R. R., R. L. LIBBy, and M. H. SMALI, I944 Mutation or variation of Escherichia coli with respect to growth requirements. J. Bact. 48: 40I-4I 2.

Ryan, F. J., 1946 Back-mutation and adaptation of nutritional mutants. Cold Spring Harbor, Symposia on Quantitative Biology II : 215-226.

RyAN, F. J., and J. LEDERBERG, 1946 Reverse-mutation and adaptation in leucineless Neurospora. Proc. Nat. Acad. Sci. 32: 163-1 73.

StennberG, A. G., and F. C. FrASER, 1944 Studies on the effect of X chromosome inversions on crossing over in the third chromosome of Drosophila melanogaster. Genetics 29: 83-103.

Tatum, E. L., I945 X-ray induced mutant strains of Escherichia coli. Proc. Nat. Acad. Sci. 31: 215-219.

1946 Induced biochemical mutations in bacteria. Cold Spring Harbor, Symposia on Quantitative Biology II : 278-284.

TATUM, E. L., and J. LEDERBERG, 1947 Gene recombination in the bacterium, Escherichia coli. J. Bact. 53: 673-684.

WINKLER, H., 1932 Konversions-Theorie und Austausch-Theorie. Biol. Zentralbl. 52: 163-189 


\title{
ABERRANT HETEROZYGOTES IN ESCHERICHIA COLI*
}

\author{
By Joshua Lederberg
}

Department of Genetics, University of Wisconsin

Communicated by R. A. Brink, February 9, 1949

A mechanism of genetic recombination has been indicated in experiments on Escherichia coli, strain K-12. ${ }^{1}$ A synthetic agar medium was used as a selective sieve to isolate occasional prototroph recombinants which appear in mixed cultures of complementary biochemical mutants. Later, additional genetic factors were introduced, including fermentation and virusresistance mutations, and these factors were found to segregate in characteristic ratios, suggesting linkage. All the cells in a given prototroph colony showed the same combination of characters and were stable on further cultivation. Therefore, it was inferred that segregation had occurred before the initiation of the colony, and that the postulated zygote had a very short life, probably a single cell generation. The life cycle would resemble the ascomycete's, in which haploid nuclei fuse to form a transient diploid zygote which undergoes meiosis without any intervening mitoses.

Exceptions to this rule have now been found in the form of unstable prototroph cultures which continually segregate out various recombination types so as to suggest that they are heterozygous and diploid. But

[Reprinted from Proceedings of the National Academy of Scifnces 35 : (4) 178-184, April, 1949] 
abnormalities in the segregation ratios suggest some sort of chromosomal aberration whose nature has not yet been proved.

Material and Methods.-The cultural and crossing techniques used earlier have generally been followed. Crosses were also made by spreading mixtures of cells on the surface of synthetic medium ${ }^{2}$ to which carbohydrate and an eosin-methylene blue indicator are added. This medium, EMS-, classifies prototrophs as soon as they appear for fermentation of the sugar used. Other media used include EMB-agar, in which the indicator is incorporated in a sugar peptone base. The genetic factors involved in this study are summarized in table 1 .

TABLE 1

\begin{tabular}{|c|c|c|c|}
\hline \multicolumn{4}{|c|}{ SUMMARY OF MUTANTS } \\
\hline Locus & $\begin{array}{l}\text { "TYPE" } \\
\text { ALLELE }\end{array}$ & $\begin{array}{l}\text { MUTANT } \\
\text { ALLELE }\end{array}$ & CHARACTER OP MUTANT \\
\hline B & + & - & Needs biotin for growth \\
\hline M & + & - & Needs methionine \\
\hline$T$ & + & - & Needs threonine \\
\hline $\mathrm{L}$ & + & - & Needs leucine \\
\hline $\mathrm{B}_{1}$ & + & - & Needs thiamin \\
\hline Lac & + & - & Unable to ferment lactose \\
\hline Mal & + & - & Unable to ferment maltose \\
\hline Gal & + & - & Unable to ferment galactose \\
\hline Ara & + & - & Unable to ferment $\mathrm{L}$-arabinose \\
\hline Xyl & + & - & Unable to ferment $\mathrm{D}$-xylose \\
\hline $\mathrm{Mtl}$ & + & - & Unable to ferment mannitol \\
\hline$V_{1}$ & $s$ & $r$ & Resistant to phages $\mathrm{T} 1, \mathrm{~T} 5$ \\
\hline$V_{1 c}$ & $s$ & $r$ & Resistant to $\mathrm{T} 1$; sensitive to $\mathrm{T} 5$ \\
\hline "Het" & .. & .. & Persistent heterozygote factor \\
\hline
\end{tabular}

Crosses Involving "Het."-The first unstable prototroph to be discovered. " $H-1$, , arose in a cross to test the allelism of the virus resistance factors $V_{1}$ and $V_{1 c}$. The parentswere $\mathrm{B}-\mathrm{M}-\mathrm{Lac}+V_{1 c}{ }^{r}$ and $\mathrm{T}-\mathrm{L}-\mathrm{B}_{1}-\mathrm{Lac}-V_{1}{ }^{r}$. Among 200 prototrophs picked and tested on synthetic agar for sensitivity to $\mathrm{T} 1$, only one was an apparent crossover showing a full sensitive reaction. However, when this unique culture was streaked out on EMB - lactose, it gave rise not to typical dark Lac+ or light Lac - colonies, but to highly variegated colonies with intermingled sectors of light and dark cells. The variegated reaction will be referred to as Lacv.

When Lac $v$ colonies were streaked out again on EMB-lactose, pure Lac-, pure Lact and a few Lac $v$ colonies were seen. But when the pure - and + colonies were inoculated on EMS-lactose they failed to grow, showing that they were nutritionally deficient. Furthermore, although they had been derived from a virus-sensitive culture, most of the purified "segregants" were resistant to T1 when tested on EMB-lactose. On the other hand, Lacv colonies gave rise to normal appearing Lact on 
EMS-lactose, which, as before were sensitive to T1. Taken back to EMB lactose, they showed the Lacv result of splitting off pure - and + types. When single colonies were streaked out in series for 15 transfers on EMSlactose, they retained their normal Lac+ appearance, but throughout the experiment they continued to segregate when brought on EMB-lactose, which is a nutritionally "complete" medium. To summarize, $H-1$ was an unstable prototroph culture which was apparently Lac+ and T1sensitive so long as it was maintained on a synthetic medium, but which segregated nutritionally deficient, Lac - and T1-resistants on a "complete" medium.

At this point, three alternatives had to be distinguished. The segregating entities might be $(a)$ associations of intact cells of the original parental mutants collaborating by syntrophism to allow growth on synthetic medium; or $(b)$ heterokaryons comprising intact nuclei of the parents in the same cell; or finally $(c)$ diploid heterozygotes. The phage sensitivity of $H-1$, contrasted to the resistance of the parents, render $(a)$ unlikely, but the segregation might still be from a heterokaryon or a heterozygote. To test these alternatives, it was thought that a heterokaryon should split out each of the two parental genotypes, but no additional combinations. On the other hand, segregation from a heterozygote should sometimes be accompanied by crossing over, and the formation of new combinations of the parental characters.

One hundred thirty-five Lacv colonies were separately streaked out on $\mathrm{EMB}$ - lactose, and a single pure Lac + and Lac - segregant isolated from each of the 135 streakings, to insure that each of the cultures tested was an independent segregant. Among the $135 \mathrm{Lac}-$ tested, there were 121 $\mathrm{T} 5^{r}$ (parentals) and $14 \mathrm{~T}^{s}$ (exchanges); the Lac + included $133 \mathrm{~T} 5^{s}$ (parentals) and $2 \mathrm{~T} 5^{r}$ (exchanges). Therefore, out of 270 tests there were 16 , or $6 \%$, new combinations. Therefore, crossing over occurs during the segregation of $H-1$. This is compatible only with the hypothesis of segregation from a heterozygote.

The following remarks should not obscure this major conclusion. The amount of exchange in the Lac - and Lac + series is not the same $\left(\chi^{2}{ }_{1}=9\right)$. The crossovers in the two series came from different Lac $v$ colonies, but reciprocality cannot be demanded if many different segregations occur during the growth of a single Lacv colony. Even the higher value of $14 / 135$ or $10 \%$ for exchanges between Lac and $V_{1}$ in the Lac - series is lower than the $38 \%$ derived in previous studies on prototroph recombinants. Finally, it was very obvious that the Lac- segregants far outnumbered the Lact. If anything, selective pressures on $\mathrm{EMB}-$ lactose seem to favor Lact, so this finding was most unexpected.

Owing to technical difficulties, tests on nutritional segregations have been less extensive. It proved to be especially difficult to score for the 
biotin requirement in the presence of amino acids, probably due to partial replacement. The classification of the thiamin requirement $\left(B_{1}-\right)$ also requires fastidious attention to clean chemicals and glassware. Most of the Lac+ $\mathrm{T}^{s}$ segregants are the parental $\mathrm{B}-\mathrm{M}-$; $\mathrm{Lac}-\mathrm{T} 5^{\dagger}$ are usually $\mathrm{T}-\mathrm{L}-\mathrm{B}_{1}-$. However, prototrophs and the multiple mutant combinations $\mathrm{M}-\mathrm{T}-\mathrm{L}-$ and $\mathrm{M}-\mathrm{T}-\mathrm{L}-\mathrm{B}_{1}-$ have been encountered. These combinations could not be obtained by previous methods, which necessarily isolated prototrophs.

Unstable heterozygotes like $H-1$ had not been encountered before, although many hundreds of Lac+ prototrophs from crosses heterozygous for Lac had been streaked out on EMB - lactose. The persistence of $H-1$ as a diploid might be a non-genetic accident or perhaps an effect of a spontaneous life cycle mutation. If so, it would be possible to set up crosses to yield diploids heterozygous for additional markers.

Three segregants from $H-1$ have been used in crosses to test for the production of persistent heterozygotes. $(A)(\mathrm{W}-466)$ was $\mathrm{B}-\mathrm{M}-\mathrm{Lac}_{1}-$ $\mathrm{V}_{1}{ }^{s} ;(B)(\mathrm{W}-477) \mathrm{T}-\mathrm{L}-\mathrm{B}_{1}-\mathrm{Lac}_{1}-\mathrm{V}_{1}{ }^{\mathrm{r}}$ and $(C)(\mathrm{W}-478) \mathrm{B}-\mathrm{M}-\mathrm{Lac}+\mathrm{V}_{1}^{s}$. These cultures were crossed on EMS-lactose with appropriate, "standard" complementary stocks $\left(\mathrm{B}-\mathrm{M}-\right.$ or $\mathrm{T}-\mathrm{L}-\mathrm{B}_{1}-$; Lac-orLac + ) of independentorigin. Lac+ prototrophs werestreaked out on $\mathrm{EMB}-$ lactose, and Lac $v$ colonies looked for. In each of these crosses, about $5 \%$ of the Lact prototrophs were heterozygous. Crosses of $A \times C$ gave the same result. If "Het" is located on a chromosome, it is effective whether present in one or both parents.

In further studies still in progress, $C$ has been crossed with multiple fermentation mutant stocks, $\mathrm{T}-\mathrm{L}-\mathrm{B}_{1}-\mathrm{Lac}_{1}-\mathrm{Mal}-\mathrm{Gal}-\mathrm{Ara}-\mathrm{Xyl}-$, and some also $\mathrm{Mtl}-$. Such stocks were developed by irradiations on $\mathrm{EMB}-$ media, in sequence. As before, about 5 to $10 \%$ of the + prototrophs isolated from EMS lactose or xylose plates are heterozygous. However, these cultures are not uniformly heterozygous for all the factors in which the parents differed. It is especially notable that in over a hundred heterozygotes obtained in this way, Mal has never been heterozygous. Usually, the cultures are pure $\mathrm{Mal}-$, sometimes $\mathrm{Mal}+$. Xylv cultures are equally often Lacv or pure for Lac, as well as the converse.

It remains to be decided whether the "pure" characters are homozygous or hemizygous. The Mal locus which, as mentioned, has always been "pure" is probably hemizygous, as shown by reversion studies. If the cultures were homozygous, Mal - would be twice represented, i.e., Mal-/Mal-. Reverse mutations of one of these genes should lead to heterozygosity at the locus. Preliminary tests on reversions for Mal in these stocks have invariably given pure $\mathrm{Mal}+$ cultures, although they still segregated for other factors. This suggests that $\mathrm{Mal}$ is represented only once, that it is hemizygous, and, therefore, that there is deficiency for a 
region including this locus. This tentative conclusion is in accord with the aberrant segregation of Lac (excess of Lac-) observed in $H$-1. The hypothetical deficiency would be lethal in a haploid, and lead to the loss of segregants carrying the alleles linked to it.

Some quantitative studies of segregation and crossing over have been carried out on these complex heterozygotes. H-72 segregates both for lactose and xylose fermentation. Segregation was permitted to occur "enmasse" in heavy cultures in nutrient broth, the cultures diluted and plated on EMB - xylose and EMB - lactose. Of 895 colonies on EMB xylose, 19 were still Xylv; $815 \mathrm{Xyl}-$ and $61 \mathrm{Xyl}+$. The $\mathrm{Xyl}+$ were all Lac-. The segregation ratio is $815-: 61+$ or more than $13: 1$. Similarly, of 753 colonies on EMB-lactose, 23 were still Lacv; 654 Lacand $76 \mathrm{Lac}+$ (all of which were $\mathrm{Xyl}-$ ). Thus, no $\mathrm{Lac}+\mathrm{Xyl}+$ segregants were observed in this sample. The other classes are computed to be $\mathrm{Lac}-\mathrm{Xyl}-83 \%, \mathrm{Lac}-\mathrm{Xyl}+7 \%$ and $\mathrm{Lac}+\mathrm{Xyl}-10 \%$. These figures might be accounted for by mapping:

$$
\mathrm{Xyl}+7.7 \text { Lethal 11.2 Lac }+/ \mathrm{Xyl}-\ldots \mathrm{Lac}-\text {. }
$$

To justify these conclusions, independent mapping of Xyl, Mal, Lac will be necessary.

Four hundred ten segregants were tested both on EMB-lactose and on EMB - xylose. Segregation was always complete; i.e., no cultures were found which had segregated for one factor, and not for the other. This eliminates interpretations based on new segmental losses, or on some forms of autogamy.

It may be argued that the unequal segregations are due to selection. This factor cannot be excluded, but the dominant segregant differs from diploid to diploid. That is to say, other heterozygotes have been isolated from parallel crosses which gave an excess of Lac + rather than Lacsegregants, or Xyl+ more than $\mathrm{Xyl}$ - or both. It seems more likely that the ratios observed are based upon the genetic constitution of the heterozygote.

The Lac + character of $\mathrm{Lac}_{1}$ heterozygotes shows that $\mathrm{Lac}_{1}+$ is dominant to $\mathrm{Lac}_{1}$-. A number of other loci have been identified, mutations at which lead to the inability to ferment lactose. At two more of these at least, the + allele is dominant. Cultures carrying $\mathrm{Lac}_{2}-$ or $\mathrm{Lac}_{4}-$ were crossed to "Het" stocks carrying $\mathrm{Lac}_{1}-$. A large proportion of the Lac+ prototrophs seen on EMS-lactose turned out to segregate for lactose fermentation. Their variegation, however, was generally periclinal rather than sectorial, with dark, Lac + centers and light, Lac - margins with only occasional streaks of Lact. This difference is to be expected, since each of the chromosomes of the diploid carries a Lac- mutation. Therefore, only those segregants in which there has been a crossover 
between the two Lac loci, bringing the + alleles into coupling phase will be lactose positive. That these cultures are segregating for two Lacfactors has been confirmed by physiological and genetic tests on the segregants.

Heterozygotes from Standard Crosses.-All the heterozygotes so far referred to are the issue of crosses involving the hypothetical " $\mathrm{Het}$ " factor derived from $H-1$. In these crosses, several per cent of the prototrophs are demoistrably heterozygous. Previous and current controls showed that, if they occurred at all in "normal" crosses, they must be very much rarer. In order to make a more thorough test, advantage was taken of the very close linkage (less than $1 \%$ recombination) which has been observed between the $\mathrm{Lac}_{1}$ and $\mathrm{Lac}_{4}$ loci mentioned above. $\mathrm{B}-\mathrm{M}-\mathrm{Lac}_{1}-$ $\mathrm{Lac}_{4}+$ was crossed with $\mathrm{T}-\mathrm{L}-\mathrm{B}_{1}-\mathrm{Lac}_{1}+\mathrm{Lac}_{4}-$. Much less than $1 \%$ of the prototrophs of this cross on EMS-lactoseare Lact. It was reasoned that a Lac+ prototroph might represent either a very rare crossover, or a diploid in which the + factors were carried on opposite chromosomes. Because most haploid recombinants, being $\mathrm{Lac}-$, can be set aside by inspection, this is a fairly efficient way of screening for rare diploids. In this way, heterozygous diploids were also obtained from normal stocks, not carrying "Het," but they constitute only about $0.1 \%$ of the prototrophs. These prototrophs are generally similar to the previous ones, except that they are somewhat more stable. It has not been established whether new "Het" mutations have occurred here. When these diploids are singly heterozygous for various sugar fermentation factors, they also show non-random segregations, but these mutations are not the same as those in the "Het" series, so that a direct comparison is not yet possible.

Discussion.-It is not clear what relationship there is between the exceptional persistence of these diploids, and the abnormalities in their segregation. There may be some direct connection between their heteroploidy, i.e., deficiency for the Ma1 region, and the prolongation of the diplophase. But the very disturbing possibility has not been discounted that these heterozygotes merely reveal a situation which also operates in the formation of haploid prototrophs from transient zygotes. If this is so, the linkage map of $E$. coli $\mathrm{K}-12$ will have to be systematically reexamined, with the use of several unrelated sets of stocks. In any case, the discrepancies affect only the details of chromosome behavior. These heterozygotes, on the other hand, have provided an unexpected confirmation of the sexual basis of genetic recombination in this bacterium.

Although the heteroploidy is somewhat of a limitation, heterozygote formation is a very helpful tool in genetic analysis. Since all types of recombinants, not only prototrophs, can be recovered, it is feasible to extend pedigrees to several generations, and interesting combinations of factors can be put together in a form allowing their use in crosses. For 
example, a $\mathrm{Lac}_{1}-$ mutation occurring in a $\mathrm{T}-\mathrm{L}-\mathrm{B}_{1}-$ stock has been transferred to a combination with $\mathrm{B}-\mathrm{M}-$, allowing it to be compared genetically in crosses with other $\mathrm{Lac}-$ mutants induced in $\mathrm{T}-\mathrm{L}-\mathrm{B}_{1}-$ material.

The experimental production and maintenance of diploids raises questions of dominance and dosage effects. It has already been noted that the normal alleles of several biochemical mutations are dominant, although quantitative comparisons of enzyme competence in heterozygotes, haploids and homozygotes remain to be carried out. The dominance of the type sensitive alleles of genes for phage resistance supports, at least in part, a segregation interpretation for the delayed effects noted by Demerec and Latarjet in induced resistance mutations. ${ }^{3}$ On the other hand, for the study of phenotypic lag in bacteria, ${ }^{4}$ segregation provides a far larger and more reproducible source of material than mutation.

In respect to lactose fermentation, such a lag cannot last more than a few cell generations in this material, in view of the appearance of numerous $\mathrm{Lac}$ - sectors in Lac $v$ colonies on EMB - lactose agar. The masking effects noted ${ }^{5}$ with yeast asci segregating in the presence of the substrate have thus not beeen found here.

Cytological and single cell studies on these strains are in progress.

Summary.-1. Unstable prototrophs have been isolated from certain crosses in $E$. coli $\mathrm{K}-12$, and characterized as segregating heterozygous diploids.

2 . The capacity to produce appreciable numbers of persistent heterozygous diploids is inherited.

3. The segregation of various mutant factors is strongly biased, possibly due to a recessive lethal deletion including a locus affecting maltose fermentation.

4. In the heterozygotes, the type + alleles of factors controlling several fermentations and nutritional requirements are dominant. Sensitivity to bacteriophage $\mathrm{T} 1$ is dominant to resistance.

* Paper No. 391 from the Department of Genetics, College of Agriculture, University of Wisconsin. This work has been aided by a grant from the Wisconsin Alumni Research Foundation. The author is indebted to Professor James F. Crow for his stimulating discussions.

${ }^{1}$ Tatum, E. L., and Lederberg, J., J. Bact., 53, 673-684 (1947). Lederberg, J.. Genetics, 32, 505-525 (1947).

${ }^{2}$ The composition of EMS-, in grams per liter, is: Sodium Succinate 5; $\left(\mathrm{NH}_{4}\right)_{2} \mathrm{SO}_{4}$ 5; $\mathrm{NaCl} 1 ; \mathrm{MgSO}_{4} 1 ; \mathrm{K}_{2} \mathrm{HPO}$ 2; Agar 15; Methylene Blue Hydrochloride 0.065; Eosin Y 0.4. This formula is conveniently stored as a dry mixture of the powdered components.

${ }^{3}$ Demerec, M., and Latarjet, R., Cold Spring Harbor Symposia Quant. Biol., 11, 38-50 (1946).

${ }^{4}$ Newcombe, H. B., Genetics, 33, 447-476 (1948).

'Spiegelman, S., Lindegren, C. C., and Lindegren, G., these Proceedings, 31, 95-102 (1945). 


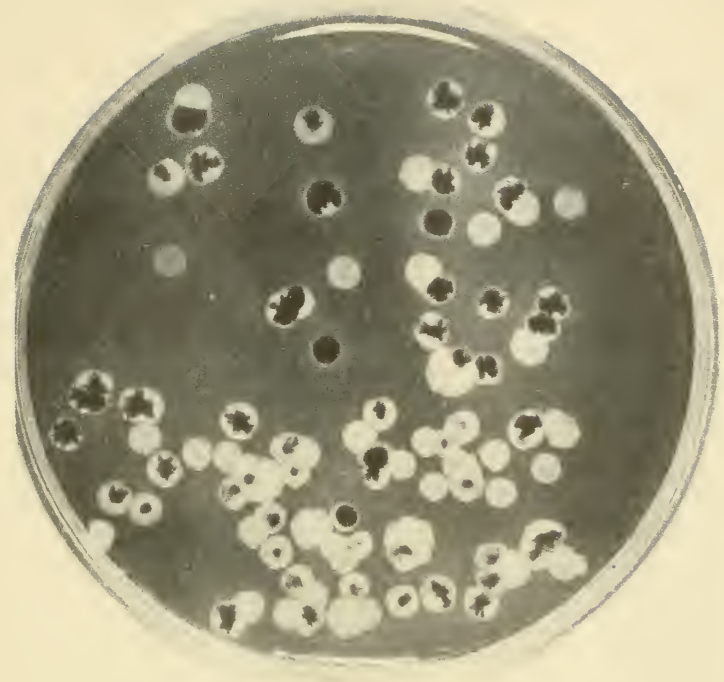

Figure 1.-This figure, also reproduced in reference 142, illustrates the variegated appearance of colonies derived from diploid heterozygous cells as mentioned on p. 179 of the preceding paper. (Reproduced by permission of The Williams \& Wilkins Co., publishers of the Journal of Bacteriology.) 


\title{
CYTOLOGICAL OBSERVATIONS ON BACT. COLI, PROTEUS VUL- GARIS AND VARIOUS AEROBIC SPORE-FORMING BACTERIA WITH SPECIAL REFERENCE TO THE NUCLEAR STRUCTURES
}

\author{
By C. F. ROBINOW*, Strangeways Research Laboratory, Cambridge
}

(With Plates 5-8, and 3 Figures in the Text)

\section{INTRODUCTION}

Discrete Feulgen-positive chromatinic bodies, occurring in regular numbers and going through a regular cycle of division, were first demonstrated convincingly by Stille (1937) and Piekarski (1937) in several well-known species of sporing and non-sporing bacteria. The literature on the subject has already been briefly reviewed in a previous communication (Robinow, 1942) in which the nuclear apparatus in the spores and vegetative cells of $B$. mycoides and various other bacteria was described.

The first part of the present paper deals with the shape and behaviour of the chromatinic bodies of Bact. coli and Proteus vulgaris, and in particular with the numerical relationships and distribution of these bodies in growing bacteria from young cultures. The technique of fixing, staining and observing the material has been described in some detail because scepticism about the chromatinic bodies in bacteria is usually due to the use of inadequate cytological methods and unsuitable optical equipment.

The latter part of the paper is concerned with observations on the cellular structure of bacteria from young cultures. Since de Bary's original deseription of B. megatherium (1884), it has been known that the rod forms of some of the aerobic spore-bearing bacilli are not single units but are composed of several separate cells. The validity of this structural principle for growing bacteria of many species besides $B$. megatherium is not always realized, though it is recognized by some workers (cf. Migula, 1897; Nakanishi, 1901; Knaysi, 1930; and Badian, 1933). My own observations on suitably stained and plasmolysed bacteria of several species provide further evidence in support of the view that rod forms have a composite structure from the earliest growth stages.

\section{MATERIAL}

The following organisms were investigated:

Bact. coli: a strain received from the National Institute of Medical Research, Hampstead;

Proteus vulgaris: No. 5821 Nat. Coll. Type Cultures and a strain isolated from human faeces in the Pathology Department, Cambridge;

and for purposes of comparison:

B. mycoides: No. 16 Nat. Coll. Type Cultures;

$B$. mesentericus: from the collection of Dr W. J Dowson of the Botany School, Cambridge;

* Of the Cancer Department, St Bartholornew's Hospital.
B. megatherium: isolated from the soil of the Laboratory garden and identified by $\mathrm{Dr} \mathrm{T}$. Gibson, Edinburgh;

B. cereus: from the Nat. Coll. Type Cultures.

Although observations were made on only one strain of Bact. coli and two strains of Proteus vulgaris, experience has shown (Piekarski, 1937-40; Neumann, 1941 ; E. Klieneberger, unpublished observations; and Robinow, unpublished observations) that there is such a close similarity between the chromatinic structures in different species that nothing is to be gained by a comparative study of many strains belonging to the same species.

\section{TECHNIQUE}

Nutrient agar (heart) and broth (heart), both from Messrs British Drug Houses Ltd., were used as culture media throughout the investigation.

The chromatinic structures were differentiated from the cytoplasm by treating the osmium-fixed cells with $N / \mathrm{HCl}$ at $60^{\circ} \mathrm{C}$. before staining them with Giemsa solution (Piekarski, 1937; Robinow, 1942). This method gives preparations of uniform elarity and is equally useful for studying nuclear structures in whole mount of yeasts, moulds and blue-green algae. The effect of the treatment with hydrochloric acid is not to make the chromatinic bodies more stainable as is sometimes sup. posed, but merely to remove from the cytoplasm something with a strong affinity for Giemsa's stain which obscures the chromatinic structures in the interior of the cell. The chromatinie structures can be demonstrated without hydrolysis (Badian, 1933; Neumann, 1941) but less clearly and regularly.

The details of the procedures adopted were as follows :

\section{A. Fixation}

\section{(1) Spore-forming organisms}

Air-dried films of spores made on glass cover-slips from cultures on potato agar, were covered with two or three narrow strips of nutrient agar and incubated in a moist chamber at $37^{\circ} \mathrm{C}$. for $2-3 \mathrm{hr}$. For the staining of nuclear structures the agar strips were removed and the cover-slips quickly transferred to a deep dish, well sealed by a greased glass plate, where $5 \mathrm{ml}$. of $2 \%$ osmium tetroxide wetting three layers of glass balls produced a strong concentration of osmic vapour. After 2-3 min. the films were allowed to dry in air, then treated for 1-2 min. in Schaudinn's sublimate alcohol warmed to $45-50{ }^{\circ} \mathrm{C}$, and finally rinsed and stored in $70 \%$ alcohol. The secondary fixation with sublimate, recommended

[Reprinted by permission of the Cambridge University Press from The Journal. of Hygirne

43: (6) 413-423, Septemher, 1944] 
by Giemsa (1912), is not indispensable, but the contrast between chromatin and cytoplasm in the stained preparation is much sharper and the staining is more uniform throughout the preparation than after osmic vapour fixation alone.

For the demonstration of cytoplasmic cell boundaries (vide infra) E. Klieneberger's (1934, 1942) method of fixation through the agar with Bouin's fluid was used in a simplified form. The cover-slips bearing the agar strips were left for $30-45 \mathrm{~min}$. or longer in a large volume of the fixative after which the agar was removed and the preparation stored in $70 \%$ alcohol.

\section{(2) Proteus and Bact. coli}

After many disappointments the making of wet smears was completely abandoned in favour of impression preparations or of dry smears from material fixed in osmic vapour while still on the agar. To make an impression preparation, a square was cut from the agar plate, not larger than could easily be handled by the tip of an iridectomy knife and accommodated on a $\frac{7}{8}$ in. sq. cover-slip. This square was exposed to osmic vapour for 2-3 min. and was then placed face downwards on a clean, dry cover-slip. After removal of the agar, the film of fixed bacteria deposited on the cover-slip was dried, treated with warm sublimate alcohol as described above ( $\$ \mathrm{~A}(1))$, and finally rinsed and stored in $70 \%$ alcohol. Klieneberger's method of fixation through the agar with Bouin's fluid was also used (see § A (1)).

Not only are the bacteria in the impression films broader than those in ordinary smears but all of them are dried within a few seconds which prevents them from becoming arranged in elusters; also they stick to the glass surface more evenly and tend to form well-spaced groups with all their elements at the same level of focus. Impression preparations from plate cultures have the further advantage of providing ample material during the first few hours of incubation, the stage of the growth cycle when much the clearest results are obtained but when there is very little to scrape off an agar slant.

To obtain preparations with many cells in the earliest stages of growth a loopful of bacteria from an 18-24 hr. slant culture, incubated at $37^{\circ} \mathrm{C}$., was emulsified with 2-3 ml. of tap water, and three or four drops of this milky suspension were spread on a freshly poured and dried nutrient agar plate. The plate was again dried, incubated and impression preparations were made in the usual manner from small pieces of the medium, cut out and fixed at suitable intervals; an ' 0 hours' sample was taken immediately the inoculum had dried on the plate.

Impression preparations were found profitable only if there were not more than one layer of bacteria on the agar. Accordingly plates spread with a fow drops from a very rich suspension were used only to study the earliest stages of development and more dilute inocula forming microscopically well-separated colonies, were used for preparations of the growth stages from $4 \mathrm{hr}$. onwards. Films from confluent growths on solid media were obtained by first making a suspension of the material in tap water, then spreading some of it on fresh agar, fixing it immediately after drying and making an im. pression preparation.

Particularly clear and instructive preparations of the chromatinic structures of Proteus vulgaris were obtained both from the earliest growth stages after subculture on a fresh nutrient medium and from dry smears of fixed material from the raised edge of a colony on an agar plate towards the end of one of the periods of 'consolidation' which alternate with periods of rapid swarming (Russ-Muenzer, 1935). Impression preparations of fixed, swarming Proteus filaments show very regular spacing of the chromatinic structures within the filaments, but cytological detail is not very clear.

\section{B. Staining}

Method 1. From $70 \%$ alcohol films were transferred directly to $\mathrm{N} / \mathrm{HCl}$ warmed to $60^{\circ} \mathrm{C}$. and 'hydrolysed' for about $10 \mathrm{~min}$. They were then rinsed in tap water and two changes of distilled water and floated on the staining solution made with 2-3 drops of Gurr's 'improved Giemsa-stain R 66' per ml. of phosphate buffer (Balint, 1926), or a mixture of equal parts of tap water and distilled water. Duration of staining differed according to whether the preparations were to be differentiated and dehydrated in the conventional acetone/xylol mixtures (pure A-A 14 pts, X 6 pts-A 6 pts, X 14 ptsa few seconds in each, followed by three changes of xylol, $10 \mathrm{~min}$. in each), or whether they were to be examined undifferentiated, mounted in water. In the latter case it sufficed to stain for half an hour at $37^{\circ} \mathrm{C}$., whereas several hours were required when the stained preparations were to be dehydrated and mounted in canada balsam. In water the outlines of the bacterial eytoplasm are much more clearly defined than in balsam-mounted preparations, the bacteria appear broader, and in hydrolysed preparations cell boundaries are visible together with the chromatinic structures, whereas most of the boundaries become invisible during the process of dehydration which precedes mounting in balsam. Photomicrographs too are often more satisfactory in their contrasts and richer in detail when taken from water-mounted material, particularly where very small elements are concerned such as the cells of old eultures. Sealed with wax, water-mounted preparations will keep their colour contrasts for several days; finally they may be stained once more, dehydrated and mounted in canada balsam.

Method 2. A quick way to obtain passably well-defined pictures of chromatinic structures is to dip unfixed, airdried impression preparations for $5 \mathrm{sec}$. into boiling $N / 5$ $\mathrm{HCl}$, rinse and mount in $0.1 \%$ erystal violet in water.

Method 3. To stain the cell boundaries, Bouin-fixed films were floated on Giemsa stain of the same strength as that used for nuclear structures, for not more than 3 min., rinsed and taken through 70,96 and $100 \%$ alcohol (5-10 sec. in each) to xylol and canada balsam. When examining such preparations an orange light filter was often used.

Method 4. The Feulgen reaction was carried out according to the prescriptions given for ordinary cytological work, except in staining B. megatherium for which the optimal conditions of hydrolysis were found to be $10-15$ min. at 40 instead of $60^{\circ} \mathrm{C}$.

Method 5. To demonstrate the cell wall a plasmolysislike effect was produced in $B$. megatherium by dipping air-dried, unfixed vegetative cells grown from spores for $2-21 \mathrm{hr}$. under agar strips (see $\S \mathrm{A}(1))$ into boiling $2 \cdot 5-8.0 \% \mathrm{NaOH}$ for $15-30 \mathrm{sec}$. The films were then rinsed in water and mounted in a $0.5 \%$ watery solution 
of erystal violet. The shrunk cytoplasm stains deep blue to purple and the cell wall a light pink. The sodium hydroxide solution was prepared by dissolving one pellet of the reagent in $10-30 \mathrm{ml}$. of water.

\section{Optical methods}

In all staining operations much 'trial and error' can be avoided by using a water immersion lens before the preparations are mounted. Economy in the use of fixing and staining reagents ean be achieved by employing cover-slips instead of slides. The cover-slips should be mounted on slides of a thickness not greater than that tolerated by oil-immersed achromatic substage condensers of high numerical aperture. The light source should be minute, at the correct distance from the microscope and fitted with a field stop. For work on the bench the Koehler principle of illumination has been found convenient and has been used throughout. The immersion objectives should be of a quality permitting the use of a 15 times eyepiece, since the magnification of about a 1000 times, commonly employed in bacteriological routine, is not high enough for a detailed study of the chromatinic structures.

\section{RESULTS}

(1) The chromatinic structures of the cells of young cultures of Bact. coli and Proteus vulgaris

Impression preparations made from agar plates during the first few hours of growth show masses of transparent bacteria containing brilliantly stained, sharply defined and regularly spaced ehromatinic structures.

The 18-24 hr. slant cultures from which the plate cultures are seeded consist chiefly of three different types of bacteria. Small coccoid elements, short plump bacilli and small slender rods. This diversity of form persists during the first few hours of growth on the plate (Pl. 5, figs. 1, 2).

The division processes of the chromatinic structures during the development of the coccoid forms (type 1) of Bact. coli into the familiar rod-shaped bacteria follow the simple pattern previously outlined for $B$. mycoides (Robinow, 1942) (Text-fig. 1). The first visible changes in the chromatinie structures of coccoid elements from I8 to -4 hr. slant cultures awakening on a fresh nutrient medium are: increase in size, in the depth of staining and in the intensity of the Feulgen reaction. Next the chromatinic structure divides into two closely contiguous dumbbells. The daughter dumbbells increase in width, their contours become asymmetrical and often before completing their separation, they in turn proceed to split longitudinally (Pl. 5, figs. 1, 2). A small round granule or strands of some faintly coloured or of some chromatinic material often persist for some time between separating dumbbell bodies (Pl. 5, figs. $1 e, f, 2 b, c, 9 a$ ). After the first division of the original chromatinic struc. ture, the cell may divide directly by constriction into two seprarate ovoid daughter cells. Usually, however, division of the young bacterium does not take place until after the second or third division of the chromatinic bodies. The early development of coccoid forms of Proteus vulgaris, whether from an $18 \mathrm{hr}$. agar slant or from the first belt of confluent growth proximal from the zone of swarming in an agar plate culture, is essentially the same as that of Bact. coli (Pl. 5, figs. 10-12). In the two strains of Proteus used in this study awakening of the cells was, however, much more protracted than in Bact. coli, and consequently a higher proportion of cells with single chromatinic bodies were found in young subcultures.

If the early growth stages of Bact. coli and Proteus are compared with those of $B$. mycoides, there is found to be a close similarity (compare Pl. 5, figs. 1, 2 with Pl. 7 , figs. 25-27). The smallest elements of Bact. coli and Proteus resemble the first vegetative generation of

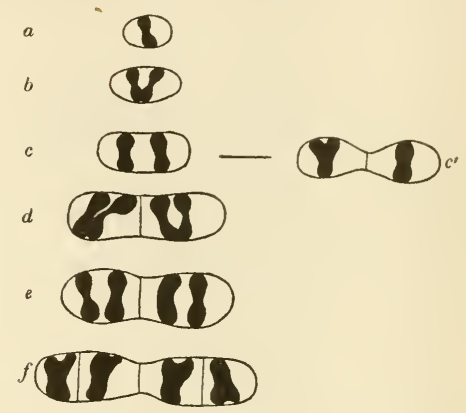

Text-fig. 1. Diagram of successive division stages of the chromatinic bodies from the beginning of the lag phase, after transfer to a fresh nutrient medium, to the first division of the growing bacterium. The diagram is based mainly on preparations of Bact. coli but applies equally well to the early development of Proteus vulgaris. $c-c^{\prime}$ and $c-f$ are alternative modes of developinent, $c-f$ being that most commonly followed.

Compare:

Stage $a, b \ldots$ with smallest elements in ... Pl. 5, figs. 1. 2, 10

Stage $c \quad \ldots \quad$ with $\quad \ldots(c)$ in Pl. 5, figs. 2,12 Stage $d \quad \ldots \quad$ with $\quad \ldots$ Pl. 5, fig. 4

$\begin{array}{llll}\text { Stage } e \quad \ldots & \text { with } & \text {... Pl. 5, figs. 5-7 }\end{array}$

Stage $f \quad \ldots \quad$ with $\quad \ldots$ dividing bacterium (c) in right-hand bottom corner of PI. 6, fig. 20, and $(d)$ Pl. 7, fig. 24

Compare stage $c^{\prime}$ with constricted bacterium $(c)$ near the top in Pl. 5, fig. 11.

Cell boundaries in $(f)$ inferred from Bouin-Giemsa preparations.

B. mycoides in their ovoid shape and in the double structure and variable position in the cell of the chromatinic body. In all three species the mode of division of the chromatinic body is essentially the same and the products of one division frequently split precociously for the next division before separation is complete $B$. mycoides (as well as $B$. megatherium) differs from Proteus and Bact. coli, however, in so far as division of the bacterium does not occur until after the third division of the chromatinic bodies (stage of four double chromatinic structures), whereas in the two non-sporing organisms, 
as stated above, it may already occur after the first division of the chromatinic body.

When plump bacillary forms (type 2) are reactivated by transference to a fresh nutrient medium the two, three or four chromatinic structures which each contains first stretch and enlarge, and then divide into dumbbell bodies which become symmetrically distributed along the bacillus. In this way the same type of bacterium with two or four resting or dividing chromatinic bodies is produced, as that derived from the coccoid forms. .

lihen the slender rod forms (type 3 ) are about to resume their growth activities, their chromatinic structures are at first arranged in a long, almost homogeneous eore (Pl. 5, figs. 1, 2, and fig. 9, top corners). During the first $2 \mathrm{hr}$. after subculture, the chromatinic core, which is presumably a column of tightly packed chromatinic elements, breaks up and forms groups of regularly spaced dumbbell bodies between which cytoplasmic boundaries then develop. Eventually large rod forms result which have the same structure and nuclear complement and follow the same development as the rods directly derived from the coccoid elements. For example, in Pl. 5, fig. 1, the very large bacterium, above $(a)$, has probably developed from a rod with three chromatin masses like that at the extreme right edge of the same figure; this would explain the unusual asymmetrical distribution of the chromatinic bodies in the large bacterium.

The redistribution of the chromatinic matter and its differentiation into dumbbell bodies in the slender rod forms of reactivated cultures of Bact. coli and Proteus vulgaris is essentially the same process as that previously described in the vegetative cells from old cultures of $B$. mycoides after transference to fresh nutrient medium (Robinow, 1942).

During the second hour of incubation the bacteria, which are now predominantly rod-shaped (Pl.6, figs. $20,21)$ begin to react differently to Giemsa's stain. The chromatinic bodies in the early stages are stout and very deeply stained (cf. Pl. 5, figs. 1, 2 and 7) and the cytoplasm assumes a reddish colour; in the older bacilli the dumbbell bodies become slender and in between divisions stain comparatively faintly, while the cytoplasm, though it may show a reddish tinge while still in the staining solution, becomes light blue in the acetone-xylol mixtures by which it is now very easily decolorized.

At all stages of growth division of the ehromatinic bodies may result either in two single dumbbells which separate completely before beginning a fresh process of division (Pl. 5, figs. 5-7), or the daughter bodies may start to divide before they have yet completely separated, as described above for the first division of the chromatinic structure. From the second hour onwards, the greater transparency of the cytoplasm renders the differences between faintly stained, slender, resting dumbbells and deeply stained, stout, dividing dumbbells much more distinct than in the earliest growth stages when the cytoplasm is more heavily stained.

When two plump dumbbell bodies of symmetrical build are closely contiguous it is impossible to see whether their long axes are parallel or at right angles to the long axis of the cell. (For an illustration of this difficulty, as well as its solution, in the ease of a large spore-forming bacterium see Pl. 6, figs. 15 and 16.) The latter arrangement is the usual one; Neumann (1941) describes cases, however, in which one or two dumbbell bodies lie near the surface of the bacterium and parallel with the long axis of the cell. As a rare occurrence I have confirmed this observation in my own cultures.

As the culture gets older the bacteria become shorter and narrower and their chromatinic structures increasingly difficult to resolve (Pl. 6, figs. 20, 21); at the edge of isolated colonies, however, bacteria with well-differentiated chromatinic structures can be demonstrated until the growth of the colony ceases.

At the edge of colonies of Proteus vulgaris growing on a suitably moist solid medium, periods of intense reproductive activity alternate with the well-known phenomenon of swarming. The ehromatinic structures in the long filaments of the swarming zone are very regularly spaced (Pl. 6, fig. 14) but the individual elements are less clearly resolved than in the short rods (Pl. 6, fig. 13) which predominate in the marginal zone of a Proteus colony between the periods of swarming. The long filaments may divide by breaking into pieces of equal length (Pl. 6, fig. $14 \mathrm{~b}$ ), but usually fragments of variable length break off from one end. The cause of the periodic changes in the periphery of Proteus plate cultures remains obscure (cf. Russ-Muenzer, 1935).

\section{(2) The composite struciure of rod-shaped bacteria}

The mono- and multinucleate elements described above are really single and multiple forms of a basic building unit possessing a single chromatinie body (Pl. 5, fig. $10 \mathrm{a}$ ). The simplest form of this unit is represented by the coccoid elements found in old cultures and in the marginal zone of Proteus colonies between the periods of swarming and the composite form by the rod-shaped bacteria which predominate in young cultures. Although the rod-shaped bacteria appear homogeneous in ordinary preparations, their composite structure can be clearly demonstrated in the following ways:

(a) Staining (Bouin fixation). When bacteria are fixed through the agar with Bouin's fluid (see p. 414) and stained for not more than a few minutes with Giemsa solution, the nuclear bodies and the cell wall are not revealed but most of the rod-shapedelements show a regular pattern of dark transverse lines and their tips also are deeply stained (Pl. 8, figs. 28, 29; Pl. 7, fig. 24). The lines are absent in the smallest elements though the poles of the cell may be deeply stained (Pl. 8, fig. $28 a$ ).

The relative position of the dark lines and the chromatinic bodies can be determined by comparing bacteria treated by the Bouin-Giemsa method with elements of equal length in preparations made by the $\mathrm{OsO}_{4}-\mathrm{HCl}$ Giemsa technique (cf. Pl. 6, figs. 17, 18). This comparison shows that the dark lines always occupy the space between the chromatinic structures, which explains why the smallest elements with only one chromatinic body lack transverse lines.

Young bacilli in Bouin-Giemsa preparations lave the following appearance (Pl. 8, figs. 28, 29). Usually a very broad und dark double band runs across the middle of the rod with a fine line symmetrically placed on either side of it so that the rod is subdivided into four equal sections. In long bacteria there may be several thick double lines evenly distributed on either side of the broad central band, with a fine line nidway between each pair of thicker ones. 
If the bacteria are studied in the order of gradually increasing length, it is easy to see how the pattern of lines in the long forms is derived from the simple arrangement in the short rods by the transformation of fine lines into broad ones and by the appearance of new fine lines midway between those which are beginning to thicken. The contour of the cytoplasm, which is deeply stained, has many irregularities similar to those observed by Knaysi (1930) in cells of $B$. subtilis: 'The ectoplasm shows thickenings and appendages of various sizes and shapes on its internal surface.'

When the bacterium divides, the plane of division always coincides with the broad central band. The constriction occurs in the middle of this double band which at this stage lias the appearance of a plump $x$ (Pl. 7, fig. $24 c, d, e$ ). This suggests that the eytoplasm in the deeply stained region is concerned with the formation of the new transverse cell wall (cf. bacillus $(a), \mathrm{Pl} .8$, fig. 30, and fig. I of Knaysi, 1930). Since the dark lines are visible long before the bacterium has begun to show signs of constriction it must be assumed that transverse divisions, either cytoplasmic or of the same nature as the outor cell wall, are laid down long before the bacterium has begun to 'divide' in the ac. cepted sense of the term.

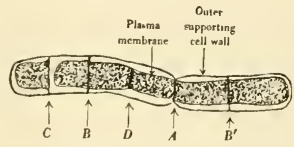

Text-fig. 2. Semi-diagrammatic clrawing of a plasmolysed, almost completely divided specimen of $B$. megatherium from a preparation made as described under Method 5, p. 414. Dimensions: $12 \cdot 4 \times 1 \cdot 5 \mu$. Letters indicate transverse cell walls (septa) in order of decreasing age: $D$ points to a cytoplasmic membrane which resulted from the division of the protoplast $A B$. A transverse septum, continuous with the outer cell wall laid down in the plane of this boundary, would have completer the spatial separation of the two sister protoplasts. Compare with Pl. 8, figs. 31-34.

(b) Plasmolysis. Instudying the chambered structure of bacteria it would, however, be of advantage to see the cell wall clearly stained together with the cytoplasm. Knaysi (1930) obtained this result in B. subtilis by suspending the eells in a drop of $25 \%$ watery solution of $\mathrm{NaCl}$ containing one per mille of crystal violet. Using a different method suggested hy the experiences of Ruhland \& Hoffmann (1925) with Beggiatoa mirabilis and staining with either erystal violet or Giemsa (see Method 5, p. 414) I have obtained preparations of the much larger cells of $B$. megatherium and also of $B$. cereus in which cell wall and eytoplasm were differentially stained and the cytoplasm had shrunk from the cell wall which, as Knaysi (1930) has ulready pointed out, is a great advantage in studying the mode of division of the bacilli. Unfortunately, I was unable to procluce this retraction of the cytoplasm at all regularly in Buct. coli iurd Proteus, so that the observations made by this method refer unost exclusively to $B$. megatherium and $B$. cereus.

Although permanent mounts can be made, freshly stained preparations mounted in water give the sliarpest and most brilliant pictures. In such material the cell walls are pink and the cytoplasm a dense mauve or purple. The rods consist of two, three or four separate portions divided from each other by transverse septa giving the same staining reaction as the cell wall. Owing to the shrinking of the eytoplasm the protoplasts are retracted from both the outer cell wall and the transverse septa, so that the septa are very elearly seen (Text-fig. 2; Pl. 8, figs. 30-34). The youngest cells are nearly cubical; they grow and elongate until their length is about three times their width when a new transverse septum is formed and the growth process repeated. Heavy staining of plasınolysed* films, which is necessary to demonstrate the cell wall, tends to obseure the cytoplasmic boundaries which are the precursors of the transverse septa. Light staining of plasinolysed films, as well as the $\mathrm{O}_{8} \mathrm{O}_{4}-\mathrm{HCl}$ Giemsa and the Bouin-Giemsa technique show that oblong photoplasts, even though they may have retracted as a whole, actually consist of two more or less completely divided cells. Thus had the bacillus at the top of Pl. 8 , fig. 30 been adequately plasmolysed and more heavily stained it would probably have presented the same appearance as the right-hand bacillus in Pl. 8 , fig. 33. Text-fig. 2 gives an example, at $(D)$, of a protoplast that is still only divided by a cytoplasmic membrane, while neighbouring cells in the same bacterium are already separated from this protoplast and from each other by transverse partitions which are continuous with the outer cell wall.

These results confirm de Bary's original description (1884) of the multiple structure of the large Megatherium rods. They also show that the process of annular furrowing by which whole bacteria are seen to divide is really the splitting of a preformed transverse partition (Textfig. 2 A; Pl. 8, fig. 33). In the deseription of bacteria the term 'cell division' should be restricted to the formation of the eytoplasmic membranes-precursors of transverse partitions-which develop in the protoplasts after the separation of recently divided ehromatinic structures.

Although it was not possible to make good plasmolysed preparations of Bact. coli and Proteus with the $\mathrm{NaOH}$ method, the number of separate cells in the plasmolysed bacteria of $B$. megatherium, i.e. two, three or four, corresponds with the number of sections into which the transverse lines seen in Bouin-Giemsa preparations usually divide the rods in young cultures of Bact. coli and Proteus (ef. Pl. 7, fig. 24, and Pl. 8, figs. 30-34).

(c) Chilling. If the average bacterium in a young culture consists of two to four cells as the foregoing results indicate, it was thought that a mildly damaging treatment might not affect all the cells of the same rod equally. To test this, young cultures of Bact. coli were chilled in the refrigerator at $2-4 \mathrm{C}$. for periods ranging from $20 \mathrm{hr}$. to several dars. The expected result was obtained, and annong many hundreds of normal or severcly clamaged bacteria, a few regularly presented the appearance shown in $\mathrm{Pl} .8$, figs. $35-37$.

* In this paper the term 'plasmolysed' is used, for the sake of convenience, in a purely morphological sense and in disregard of the physico-chemical differences between the (irreversible) method of fixation with $\mathrm{NaOH}$ and the reversible response of plant cells to which the term uormally refers. 
In preparations made by the Bouin-Giemsa technique but more heavily stained than for the demonstration of cell boundaries, one-half of such a differentially affected rod is seen to have partly disintegrated and stains faintly pink, while the other half has the deep blue colour characteristic of bacteria which were alive and growing at the time of fixation. The half damaged rods show no sign of a medial constriction but have the usual smooth contour of resting bacteria; yet under the conditions of the experiment each behaves as if consisting of two physiologically independent units. Presumably this independence is not absolute and the fact that most of the affected rods disintegrated as a whole implies some kind of plasmatic connexion between the two cells of which each is composed.

(3) The relationship of the transverse septa to the arrangement of the chromatinic bodies. As stated above, if bacteria stained to show the cytoplasmic boundaries are compared with bacteria of equal length stained for the chromatinic bodies, it is found that the chromatinic bodies lie between the boundaries. This alternation of the two structures can also be demonstrated in the same bacteria by a slight modification of the osmium-HClGiemsa method.

In ordinary hydrolysed preparations of $B$. mega. therium and $B$. mycoides stained with Giemsa's solution, red lines, corresponding in position with the transverse partitions made visible by the sodium hydroxide treatment are often visible in the differentiated, dehydrated films mounted in canada balsam (Pl. 7, fig. 25), but such lines are rare in similar preparations of Bact. coli and $B$. mesentericus. If, however, films of these organisms are mounted in water immediately after staining and without differentiation the lines are clearly seen bisecting the rod-shaped elements and intervening between recently divided dumbbell bodies (Pl. 6, figs. 15, 16, 17). They are more distinct in B. mesentericus than in Bact. coli in which they are usually too thin and faintly stained for good photography (Pl. 5, fig. 9).

Normally cytoplasmic cell boundaries divide both short rods and long winding filaments into a series of cells, each with its own single or multiple, i.e. dividing, chromatinie structure.

If for some reason growth occurs without division of the cytoplasm there is no orderly arrangement of the chromatinic bodies. Long filaments are formed in which no cell boundaries can be demonstrated and in which the distribution of numerous ehromatinic bodies is chaotic. Such filaments (Text-fig. 3) arise spontaneously in cultures of Bact. coli and Proteus, where they were also obsorved by F. Neumann (1941), especially if derived from old stock and they can be produced experimentally (Robinow and Lea, unpublished observations) by certain doses of ionizing radiations which do not interfere with cytoplasmic growth (Lea et al. 1937).

(4) The chromatinic structures of the cells of old cultures. Even in old eultures kept from $18 \mathrm{hr}$. to 4 days at $37^{\circ} \mathrm{C}$., or for 2 days to several months at room temperature, cells with well-developed chromatinic structures are never completely absent, a fact already noted by Neumann (1941).

As stated above, such cultures contain three main types of bacteria: small coccoid forms, short stout rods and small slender rods. The first predominate in old cultures of Proteus vulgaris, while all three are represented in 18-24 hr. slant cultures of Bact. coli. When stained for chromatinie matter by the $\mathrm{OsO}_{4}$-HCl-Giemsa method, old cultures present a variegated appearance. A few colourless and structureless elements are obviously dead remains (cf. cell above (e) in Pl. 5, fig. 2), but most bacteria of all three types contain one or more chromatinic bodies (lower half of Pl. 7, fig. 2). The chromatinic structures are either deeply stained and centrally placed, not touching the periphery, or less densely stained dumbbell forms extending right across the cell (Pl. 7, fig. $22 b$ ). The minute coccoid forms are best studied in preparations mounted in water in which it is possible to see the delicate layer of cytoplasm surrounding the relatively large chromatinic structures; after dehydration and decolorization this marginal cytoplasm is often invisible.

Neumann (1941) has reported a diffuse Feulgen reaction in all the cells of old cultures, I have found examples of both diffuse and localized positive reactions in cells from 18 to $24 \mathrm{hr}$. slant cultures, but these elements are so small that interpretation is very difficult and $I$ have been unable to reach any definite conclusions about the results of the Feulgen test at this stage.

Preparations of old cultures made by the BouinGiemsa method for the demonstration of cell boundaries present a remarkable contrast to similar preparations of young growing eultures. Instead of the bright, pure blue coloration and conspicuous banded or chambered appearance characteristic of the young bacteria (Pl. 7, fig. 24), the great majority of the old bacilli of all three types stain a faint mauve or dull light blue and show little or no sign of the differential staining which indicates division processes (Pl. 7, fig. 23).

These differences in staining reaction reflect the well-known change seen in living bacteria after subculture, when the pale transparent cytoplasm of the old cells acquires a brightly refractile appearanco during the so-called 'lag-phase' (which comprises all but the last developmental stages shown in Text-fig. 1).

Quantitative observations on five slant eultures of Bact. coli incubated for 24-18 hr. at $37^{\circ} \mathrm{C}$, were made from films prepared by the Bouin-Giemsa method for the demonstration of cell boundaries; 500-600 bacteria from each culture were examined. In these five cultures active bacteria with deeply stained and clearly subdivided eytoplasm constituted only $4 \cdot 7,4 \cdot 8,5 \cdot 5,8 \cdot 0$ and $12.0 \%$ respectively of the total count. The remaining bacteria were faintly and uniformly stained and the cell boundaries were either absent or indistinet. On the other hand, osmium-HCl-Giemsa preparations from two of the same cultures showed that $65-67 \%$ of the bacteria contained more than one chromatinic body. Whether all the bacilli in which cell boundaries are indistinguishable are really single cells, or whether the intercellular boundaries are sometimes present but at this stage are unstainable by the methods used, is not certain.

In spite of the diffuse Feulgen reaction and apparent absence of cell boundaries in most bacteria in old $(18-24 \mathrm{hr}$.) cultures, the great majority of these organisms are fully viable. Neumann's view that there is an 'early degeneration of the cells in well-grown cultures' is untenable when the changes induced in the chroma- 
tinic structures by 45-90 $\mathrm{min}$. incubation (i.e. during the lag phase) on a fresh nutrient medium are studied. The results of such observations show that all but a small proportion of the bacteria undergo the growth changes described in the preceding sections.

(5) Atypical forms. To complete this account of the chromatinic structures, three abnormal types of cells must be mentioned which occur in cultures of Bact. coli, Proteus vulgaris and many other species (Text-fig. 3). Of these, the most interesting are bacteria of normal length which apparently are not divided into two cells; they have a faintly stained dumbbell body at each end and a more deeply stained pair of these bodies near the middle.

The second atypical form may be short or several times the normal length and contains a variable number of chromatinic dumbbells compacted into a central deeply staining mass. The third and fourth forms are long filaments with many discrete but irregularly spaced chromatinic bodies. Though always rare, the last two types are more common in young cultures derived from very old (e.g. 4 weeks) material, than in subcultures from slants incubated for only $18 \mathrm{hr}$. Both have been accurately described and illustrated by Neumann, whose inability to determine their function I unfortunately share.

Speculation about the significance of all these enigmatic cells such as their connexion with autogamic processes, is bound to be unprofitable until it is possible to isolate them from normal living material and follow their development separately.

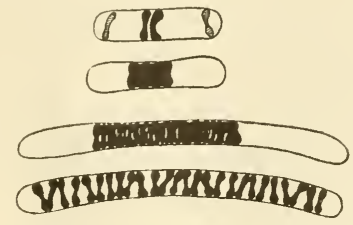

Text-fig. 3. Diagram of certain peculiar types of cells, small numbers of which are regularly found in cultures of Protcus vulgaris and Bact, coli. Tentatively arranged in order of what may be their true genetical relationship. At present any such comexion between the different types is purely hypothetical.

\section{DINCTSSION}

The chromatinic structures described in this communication obviously eorrespond to those which J. Badian, in a series of papers since 1930, has reported in the cells of myxobacteria, actinomycetes and aerobic sporeforming barteria and which were also described in the spores and regetative forms of $B$. mycoides and $B$. mesentericus by Robinow (194:). There is good reason to believe that such, structures oceur very widely annong barteria.

The chromatinic dumbbell bodies are also identical with the 'nucleoids' of Piekarski $(193 \bar{i}-4(1)$. The difference betwoen l'iekarski's and my own description of the nuelear structures is twofold and is due to differences in technique. According to Piekarski the nucleoids are round bodies and each cell has two, one near each end. My observations make it clear that (1) the nucleoids in Piekarski's preparations are optically unresolved dumbbell bodies, or, more often, configurations of these and (2) that the 'cell' or 'Primaerform' of his terminology is actually a two-cell rod with a resting or dividing dumbbell body in the centre of each of its component cells. In other words: the binucleate form which Piekarski puts at the beginning of his sequence of developmental stages is really composite and derived from small, uninucleate coccoid or rod-shaped elements (Piekarski's 'Secundaerform') through growth and cell division. This process is evident during the lag-phase and is less common during the first few hours of a culture's active growth, the stage to which Piekarski paid particular attention.

Neumann's (1941) observations on Bact. coli and Proteus agree with those of Piekarski. Neumann observed chromatinic dumbbell bodies but thought they were stages in the division, hour-glass fashion, of a single chromatinic granule identical with Piekarski's nucleoids. Neumann was not aware of the multiple cell structure of rod-shaped and filamentous bacteria, but apart from these and other, less important, differences my observations on Proteus vulgaris and Bact. coli fully confirm Neumann's detailed description of the distribution of nuclear material in the various growth forms of these organisms.

There is some diversity of opinion about the mode of division of the chromatinic bodies in different groups of bacteria. This problem is important in connexion with the possible function of these bodies in the transmission of hereditary characters.

In the rod-shaped bacteria, my own observations indicate that the dumbbell bodies divide like chromosomes by splitting longitudinally. The compact nuclei which Beebe (1941) describes in the vegetative cells of Myxococcus xanthus n.sp. and which he believes divide amitotically by constriction, are more probably composed of closely contiguous dumbbell bodies. The appearance of a compact nucleus dividing by constriction is easily simulated when, as described in the present communica. tion, the chromatinic dumbbell bodies divide again before they have completely separated after the previous division, and especially if coloured strands persist be. tween two pairs of closely contiguous bodies as they move towards opposite poles (cf. Pl. 5, figs. $1 e, 9 a$; Pl. 7, fig. 27 a). This interpretation of Beebe's results is the more likely since he himself describes the nuclei of cells migrating to the fruiting centres as breaking up into 'four irregularly shaped bodies or chromosomes'. An alternation of amitotic and mitotic division such as this author envisages seems hardly likely.

Direct division of a round nuclear body by elongation and constriction has recently been described by Knyasi $(1942)$ in a new species of yellow coccus. I have previously expressed the view (Robinow, 1942) that the Feulgen positive granules which I noted in most of the cells of two sarcinae were the ends of dumbell bodies which divided by longitudinal splitting. I now find that my photographs are more satisfactorily explained by Knyasi's assumption that the granules divide directly, the smaller cells containing one and the larger cells two. 
On the other hand, Miss E. Klieneberger (umpublished observations) has recently demonstrated unmistakable dumbbell bodies and their $\mathrm{V}$-shaped division stages in large streptococei from a human case of bronchopneumonia, and I have seen the same appearances in a large, colourless unidentified coceus which was encountered as a contaminant on an old agar plate. One or two Feulgen-positive bodies, similar to those which I have previously described in two sareinae are also present in the cells of Staphylococcus aureus and of Streptococcus pyogenes (unpublished observations). I cannot, therefore, agree with the conclusions drawn by Knaysi \& Mudd (1943) from electron micrographs, that these organisms lack discrete nuclear structures and that the organization of nuclear material varies widely among different species of bacteria.

Their small size renders cocci rather difficult objects for the study of the chromatinic bodies and even in the more readily investigated rod-shaped bacteria, resolution of the chromatinic structures into chromosome-like bodies is not always possible (cf. B. mesentericus, PI. 6, figs. 17, 19).

It seems plausible that the chromatinic bodies of bacteria are homologous with the chromosomes of more highly differentiated organisms. The positive Feulgen reaction of these bodies, their special affinities for nuclear dyes, their regular eycle of multiplication co-ordinated with cytoplasmic division and their simple and constant numerical relationships strongly suggest that they are true nuclear structures. Moreover, their mode of division appears well suited for the transmission of hereditary factors in linear array such as occurs in chromosome division, though whether they actually perform this function is of course not yet known.

Also, direct proof that the chromatinic bodies contain nucleic acid is still lacking; to obtain this it will be necessary to determine whether they maximally absorb the same wave-lengths of ultra-violet light as the nucleic acids, an undertaking rendered difficult by the small size of the bacteria. Piekarski's claims (1938) to have obtained a selective absorption of ultra-violet light require confirmation.

Further evidence for the nuclear character of the dumbell bodies in bacteria is afforded, however, by a comparison of these bodies with the nuclear apparatus of veasts. Badian (1937) has studied several species of yeasts and has shown that they possess two chromosomes which in form and size closely resemble the dumbbell bodies of bacteria. I have been able to confirm these observations for the vegetative stages of Saccharomyces cerevisiae and Schizosaccharomyces Pombe. My results will be described fully elsewhere but in the present commexion it is interesting to note that in yeasts delicate strands extend between the two pairs of chromosomes at anaphase and between daughter nuclei, which exactly resemble the strands so often seen between separating chromatinic structures in bacteria.

Statistical evidence that the bacterial cell contains an organ of higher radiosensitivity than the rest of the cytoplasm has been provided by D. E. Lea and his collaborators in a series of radiological investigati. $n=$ $(1937,1940,1941)$. This organ cannot be regenerated by the cell once it has been destroyed by irradiation and is probably identical with the auclear material. 1t is hoped to supplement these observations with morphological data on the effect of ionizing radiations on the chromatinic structures.

The chambered appearance that Giemsa's stain lends to bacteria which have been fixed through the agar with Bouin's fluid, can also be produced by iron alum haematoxylin in the case of the aerobic spore-formers. Using this stain, Guilliermond (1908) has described such boundaries ('cloisons transversales') in B. mycoides. The pattern of aliernating dark and fine transverse markings is also reminiscent of the chambered structure of large spirochaetes as described by Gross (1911) and Dobell (1912) (cp. PI. 8, figs. 28, 29 with figs. 143 and 144 of Dobell's memoir).

The existence of a bacterial cell wall as a structure differing physico-chemically from the cytoplasm has been recognized since the work of A. Fischer (1895) and his contemporaries, but the elear conception of the cell wall, derived from plasmolysing experiments, which prevailed forty years ago has become somewhat obscured by the difficulty of demonstrating it satisfactorily in most of the bacteria which interest the medical bacteriologist.

Knyasi (1930) has described the morphological relationship of the plasma membrane and the outer supporting cell wall in plasmolysed bacteria. My own results with the Bouin-Giemsa and $\mathrm{NaOH}$-crystal violet method fully confirm his findings with regard to this relationship, but differ from them in showing that the aerobic spore-formers have a multiple cell structure from the earliest growth stages onwards.

\section{NOTE}

In the present account observations on the cell wall in $B$. megatherium have been used to suppleinent the description of the chambered cytoplasm in rod forms and filaments of Bact. coli and B. mesentericus, i.e. in two species in which the demionstration of the outer supporting cell wall which surrounds the cytoplasm had not proved successful. Since this article first went to press I have leannt distinctly to stain the cell wall in all three organisms by mordanting Bouin-fixed preparations with , $\%$ tannic acid and staining for $10 \mathrm{sec}$. (!) with $0 \cdot 02 \%$ crystal violet in water. The new preparations further emphasise the distinction bet $\pi$ en eytoplasmic cell boundaries and transverse cill walls (septa) drawn in the present article and have allowed the formation of the transverse septa to be studied in detail. These observations will be published elsewhere.

\section{SUMMARY}

1. The basic observations of Piekarski (1937-40) and F. Neumann (1941) on Feulgen-positive, chromatinic structures, going through a regular cycle of division, in the cells of Bact. coli and Proteus vulgaris are confirmed and the view that these structures are nuclear in nature is accepted.

2 . The chromatinic structures in bacteria from old cultures, although usually distinguishable from the cytoplasm, are too small to be resolved accurately. After transfer to a fresh nutrient medium the chroma. tinic structures increase in size and give rise to short, often dumbbell shaped, rods (ehromosomes) which 
multiply by splitting lengthwise in a plane more or less parallel with the short axes of the bacterium.

3. The chromatinic structures of Bact. coli and of Proteus vulgaris are essentially the same as those previously described in myxobacteria and actinomycetes (Badian, 1930, 1933, 1936) and in various well-known aerobic spore-forming bacteria (Badian, 1933, 1935; Robinow, 1942).

4. A single cell of Bact. coli or Proteus vulgaris contains one chronatinic body or one or two pairs of these, representing primary and secondary division products.

5. Few bacteria from young cultures are single cells. When fixed through the agar with Bouin's mixture and stained briefly with Giemsa's solution, bacteria from young growing cultures of Bact. coli, Proteus vulgaris,
$B$. mesentericus and $B$. megatherium assume a banded appearance indicating that each bacterium consists of two, three or four separate cells.

6. A plasmolysing treatment has been applied to $B$. megatherium which provides direct proof of the composite structure of this bacillus by inducing its component protoplasts to shrink away independently from the outer supporting cell wall.

I wish sincerely to thank Dr H. B. Fell for the hospitality offered to me at the Strangeways Laboratory, for ler sustained interest in this investigation and for her help in preparing the manuscript for the press. I have also pleasure in admitting $\mathrm{my}$ indebtedness to Prof. E. G. Pringsheim for much helpful criticism and advice in matters relating to the bacterial cell wall.

\section{REFERENCES}

Badian, J. (1930). Acta Soc. Bot. Polon. 7, 55.

Badian, J. (1933). Acta Soc. Bot. Polon. $10,361$.

Badian, J. (1935). Acta Soc. Bot. Polon. 12, 69.

Badian, J. (1936). Acta Soc. Bot. Polon. 13, 105.

Badian, J. (1933). Arch. Mikrobiol. 4, 409.

Badiay, J. (1937). Bull. int. Acad. Cracovie, B, 61.

BALINT, A. (1926). Klin. Wschr. 5, 147.

DE BARY, A. (1884). Vergleichende Morphologie und

Biologie der Pilze, etc. Leipzig. English edition,

Oxford, 1887.

BeEbee, J. M. (1941). J. Bact. 42, 193.

Dobell, C. (1912). Arch. Protistenk. 26, 117.

Fischer, A. (1895). Jb. wiss. Bot. 27, 1.

Giemsa, G. (1912). Handbuch der pathogenen Protozoen,

1. Leipzig.

Guilliermond, A. (1908). Arch. Protistenk. 12 , 1.

Gross, F. (1911). Mitt. Zool. Stat. Neapel, $2 a, 188$.

Klieneberger, E. (1934). J. Path. Bakt. 39, 409.

Klieneberger, E. (1942). J. Hyg., Camb., 42, 110

Knaysi, G. (1930). J. Bact. 19, 113.

KNaysi, G. (1938), Bot. Rev. 4. 83-112.
Knaysi, G. \& MudD, s. (1943). J. Bact. 45, 349.

KxAYSI, G. (1942). J. Bact. $42,365$.

LEA, D. E. (1940). Nature, Lond., 146, 137.

Lea, D. E., Haines, R. B. \& Bretscher, E. (1941). J. Hyg., Camb., 41 . 1.

Lea, D. F., Haines, R. B. \& Coulson, C. A. (1937). Proc. Roy. Soc. B, $123,1$.

Migula, IV. (1897). System d. Bakterien, 1. Jena.

Nakanishi, K. (1901). Zbl. Baht. Abt. 1, 30, 97.

Neumann, F. (1941). Zbl. Bakt. Abt. II, Orig. 103, 385.

Piekarski, G. (1937). Arch. Mikrobiol. 8, 428.

Piekarski, G. (1938). Zbl. Bakt. Abt. I, Orig. 142, 69.

Piekarski, G. (1939). Zbl. Bakt. Abt. I, Orig. 144, 140.

Piekarski, G. (1940). Arch. Mikrobiol, 11, 460.

Robinow, C. (1942). Proc. Roy. Soc. B, 130, 299.

Ruhland, W. \& Hofrmany, C. (1925). Arch. wiss. Bot. $1,1$.

Russ-Muenzer, A. (1935). Zbl. Bakt.

Stille, B. (1937). Arch. Mikrobiol. 8, 125.

\section{EXPLANATION OF PLATES 5-8}

All figures on Plates 5 and 6 , with the exception of fig. 18, are of preparations fixed with osmium vapour, treated with $N / 1 \mathrm{HCl}$ at $60^{\circ} \mathrm{C}$. and stained with Giemsa's solution. Unless otherwise described, preparations were photographed mounted in water. A scale of magnification is provided on every plate and is the same for all figures.

\section{Plate 5. Bacterium coli and Proteus vulgaris}

Figs. 1-9. Chromatinic structures in Bact. coli from five nutrient agar plate cultures seeded with material from $18 \mathrm{hr} .37^{\circ} \mathrm{C}$. slant cultures and incubated for $45-90 \mathrm{~min}$. at $37^{\circ} \mathrm{C}$.

Fig. 1. $a-h$ are successive stages in the development of a coccoid element with a central pair of closely contiguous chromatinic bodies into a typical rod-shaped bacterium with four chromatinic structures. $h$ may, of course, also have arisen directly from a plump bacillary form without having gone through the coccoid stage. Note the $\mathrm{V}$-shaped division stage at $d$.
The dumbhell body forming the left limb of the $V$ is so much broader than the right one, that it seems plausible to assume that it is preparing for the next division. Compare this stage with Pl, $\overline{7}$, fig. 26. $e$ a strand of ehromatinic matter is seen connecting recently separated chromatinic structures. Compare with Pl. 7 , fig. 27.

Figs. 2, 3. $a-e$ successive developmental stages. The two deeply stained dumbbell bodies at $c$ are connected at their base by a faintly stained round body similar to that in $f$, fig. 1. Loss of clear-cut dumbbell shape together with asymmetrical broadening of the chromatinic bodies at $d$ indicates impending division. The same applies to the chromatinic structures in the central bacterium in fig. 3 .

Fig. 4. Separation of the products of the 2nd division of the chromatinic bodies is almost complete in the right chromatinic structure in the central bacterium. Stage $d$ of Text-fig. 1 gives an interpretation of these configurations suggested by their appearance under the microscope at different levels of focus. 
Fig. 5 (from a preparation mounted in canada balsam). A typical two-cell bacterium with four chromatinic bodies, three of which are dumbbell-shaped and must be considered as having been in a state of rest at the time of fixation.

In fig. 6 (mounted as in fig. 5) $a$ represents a stage comparable to $c$ in fig. 2, while $b$ is another two-cell bacterium with four dumbbell-shaped chromatinic bodies.

Fig. 7. In the large bacterium below and to the right of the centre the first dumbbell body from the left as well as the fourth are at rest while the greater width and asymmetrical shape of the two middle ones again suggest a process of division (reduplication).

Fig. 8. All four chromatinic structures in the large bacterium are preparing to divide.

Fig. 9. Separation of daughter chromatinic bodies has begun in the two middle ones of the four chromatinic structures in the large bacterium; less densely stained than the chromatinic bodies of fig. 7, they correspond to the stage of fig. 3 , i.e. that of impending separation of the products of a recent longitudinal division. A faintly stained cell boundary traverses the gap between the two pairs of chromatinic configurations. The same type of two-cell rod is also found at $e$ in fig. 2 and at $h$ in fig. 1. At $a$ a filamentous structure connects recently separated, complex chromatinic structures. A slender rod with a long core of chromatinic matter is seen in the right-hand top corner; others are visible in figs. 1 and 2 . The development of these forms is explained in the text.

Figs. 10-12. From a culture of Proteus vulgaris of $1 \mathrm{hr}$. incubation, prepared from the coccoid elements with poorly differentiated chromatinic structures, that form the first belt of confluent growth inside the zone of swarming filaments at the periphery of an agar plate culture. The preparation was photographed mounted in canada balsum.

Figs. 10, 11. $a$, coccoid cell with a single, diagonally placed chromatinic body. $b$, cell with two chromatinic bodies resulting from recent longitudinal division. $c$, division of this bacterium will give rise to two single cells (stage $c^{\prime}$ of Text-fig. 1), in contrast to the more usual mode of division at the four-cell stage, illustrated by the dividing bacterium above $d$ in Pl. 6, fig. 13 .

Fig. 12. In the centre are two young rod forms with clearly defined dumbbell bodies. In the one on the right a rounded faintly stained body is visible between the chromatinic dumbbell bodies, similar to $f$, fig. 1 and $c$, fig. 2, PI. 5 .

\section{Plate 6. Proteus vulgaris, Bact. coli, B. mesentericus}

All figures except fig. 18 are from preparations mounted in water. Fig. 18 is the same as the top part of fig. 29, Pl. 8 and is from a Bouin-Giemsa preparation mounted in canada balsam.

Figs. 13, 14. From one culture. Fig. 13 shows the short normal bacteria from the raised edge of an agar culture shortly before swarming was due to begin.

Fig. 14. From an impression preparation made after swarming had been in progress for $1 \mathrm{hr}$. In fig. 13 particularly clear division stages at $a$ and $b ; c$ and $d$ illustrate further stages in the formation of two-cell bacteria with four chromatinic bodies. In fig. 14 four compact, symmetrically arranged chromatinic configurations are visible in the larger bacterium marked $a$. Many of the chromatinic structures in the long filaments are very broad, which suggests that they may be composed of several pairs of dumbell bodies. At $b$ a large filament is seen to break into two fragments of equal length. A delicate strand of chromatinic matter connects the first two chromatinic structures from the left, in the left daughter bacterium.

Fig. 15. A two-cell rod of $B$. mesentericus containing two pairs of closely contiguous, symmetrically built dumbbell bodies separated by a median cytoplasmic cell boundary. The shadows on top of the bacillus are discarded spore cases.

Fig. 16. An early stage in the separation of the closely contiguous dumbbell bodies. Note the well-defined median cell boundary and compare this photograph with $b$ in $\mathrm{Pl}$. 8, fig. 28. The corresponding stage in the development of Bact. coli is shown in Pl. 5, fig. 4. Fig. 16 should also be compared with stage $h$ of Badian's text-fig. 1 (1933).

Fig. 17. Near the lower edge of the picture a typical composite filament of $B$. mesentericus from the first $2 \mathrm{hr}$. after spore germination. Note that the middle one of the three septa is particularly deeply stained; it is here that a break will take place like that illustrated in fig. 19. There are also clear-cut median cell boundaries in two other bacteria on this picture.

Fig. 18. Comparison with the previous figure shows that the dark bands in the long bacillus correspond to cell boundaries. No chromatinic structures are shown in fig. 18.

Fig. 19. See legend of fig. 17.

Figs. 20, 21. Bact. coli, $4 \mathrm{hr}$. agar slant at $37^{\circ} \mathrm{C} . ; 2 \mathrm{hr}$. in tap water at room temperature, $1 \frac{3}{1} \mathrm{hr}$. on fresh nutrient agar at $37^{\circ} \mathrm{C}$. (Control to a radiation experiment.) The photograph shows slender bacteria typical for growth on erowded plates of more than $2 \mathrm{hr}$. incubation. At $a, b$ and elsewhere two-cell rods with four resting or dividing chromatinic bodies are seen. A slightly constricted two-cell rod with a pair of closely approximated chromatinic bodies in each of its cells is visible in the right-hand bottom corner of fig. 20 . To the left of this bacterium, at $c$, sister bacteria are seen connected by a slender protoplasmic bridge. Compare these two photographs with Pl. 7, fig. 24, where the distribution of cell boundaries is shown.

\section{Plate 7. Brat. coli, B. mycoides}

All figures except figs. 25-27 are from preparations mounted in water.

Fig. 22. Typical sample of the different types of cells in an $18 \mathrm{hr} .37^{\circ} \mathrm{C}$. slant culture. $a$ are coccoid forms with single dumbbell bodies. Compare the poor definition of the chromatinic structures in this picture with the strong contrasts between cytoplasm and chromatinic structures in Pl. 5, figs. 1, 2 .

Fig. 23. Bacteria from an $18 \mathrm{hr}$. slant culture, spread on agar, fixed with Bouin's fluid and stained briefly with Giemsa's solution. Except for the darkly stained bacterium with median constriction in the left-hand 
JOURNAL OF HYGIENE, VOL. 43 , NO. 6

PLATE 5
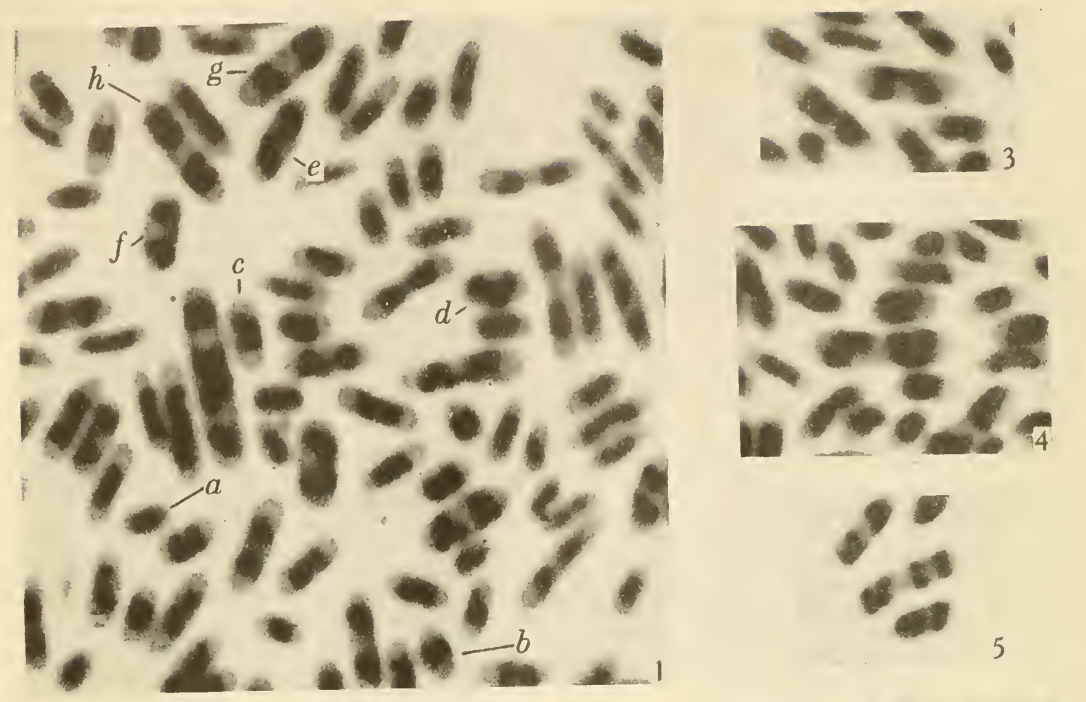

$\operatorname{lov}_{b}^{2} \geq 20$
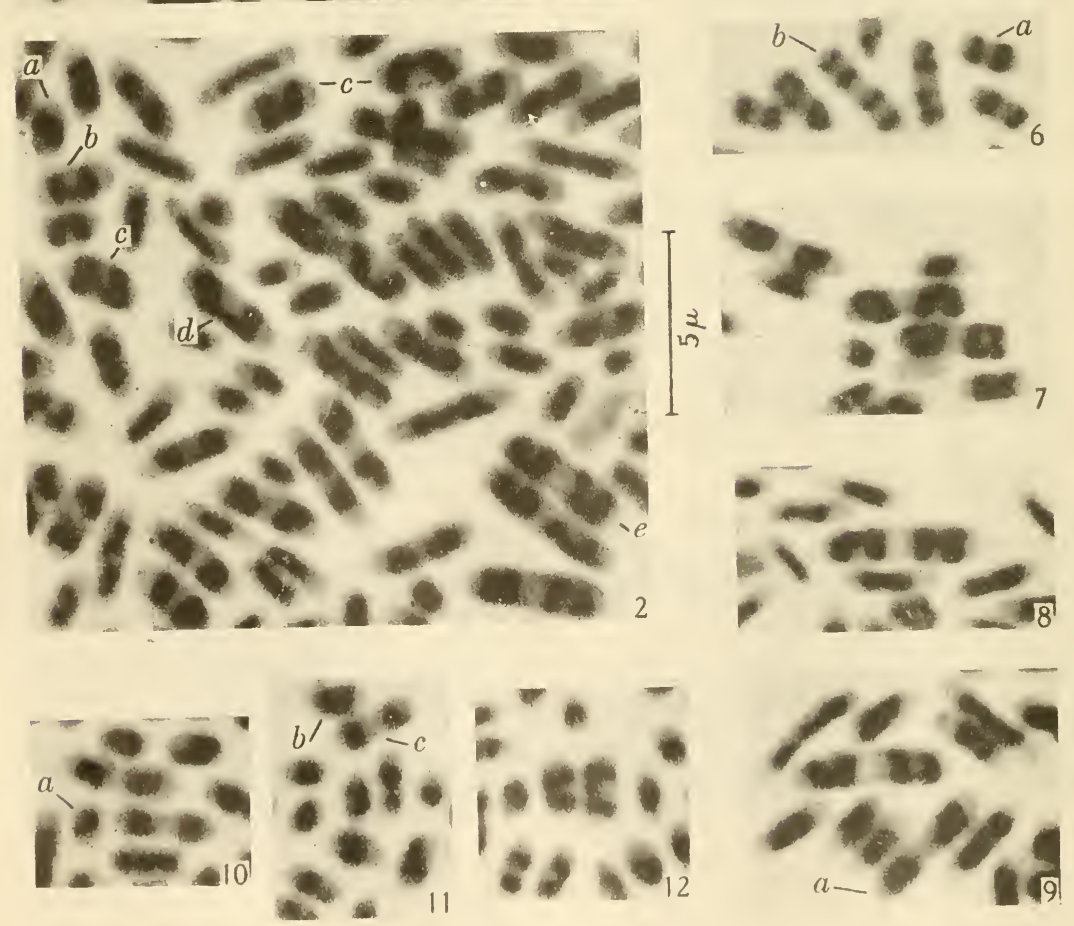


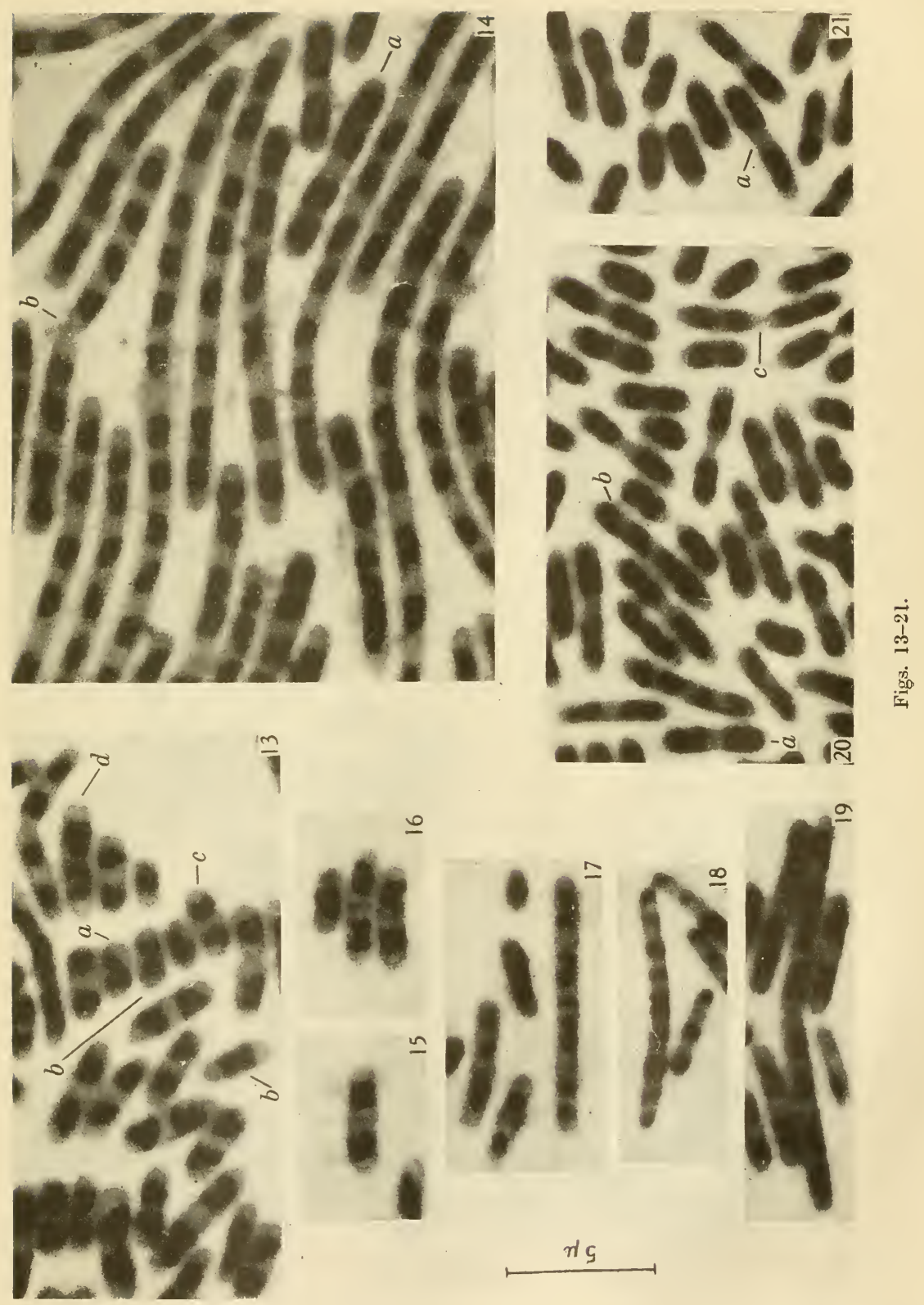


JOURNAL OF HYGIENE, VOL. 43, NO. 6

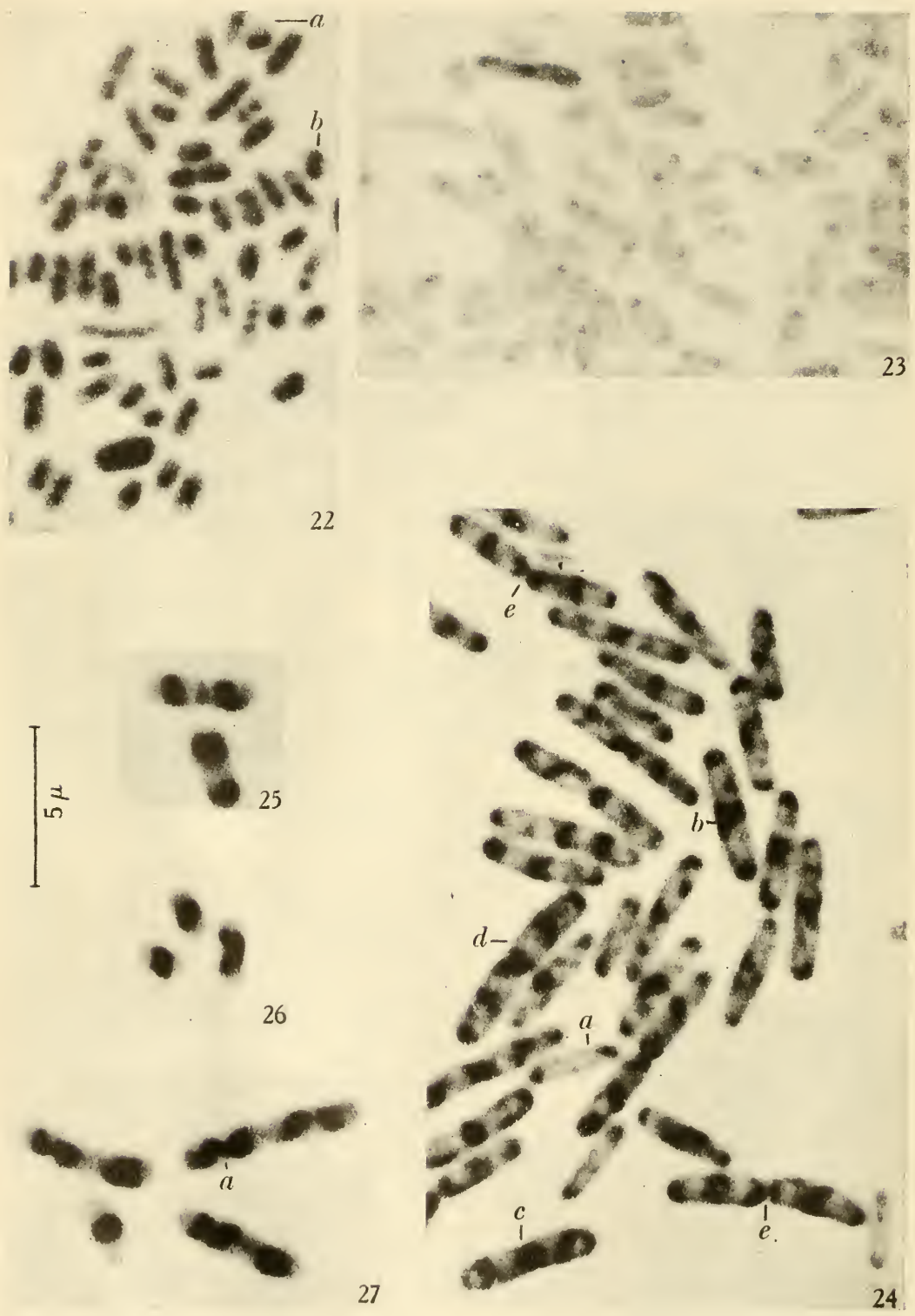

Figs. 22-27. 

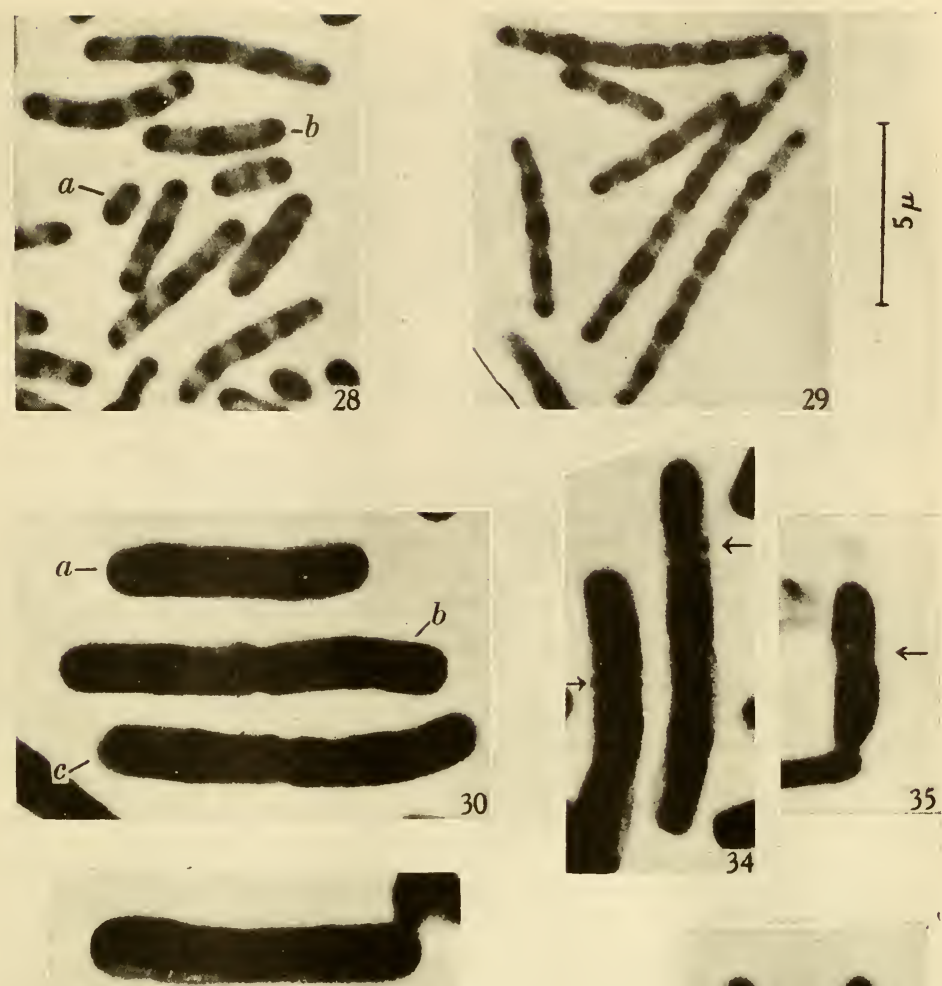

31
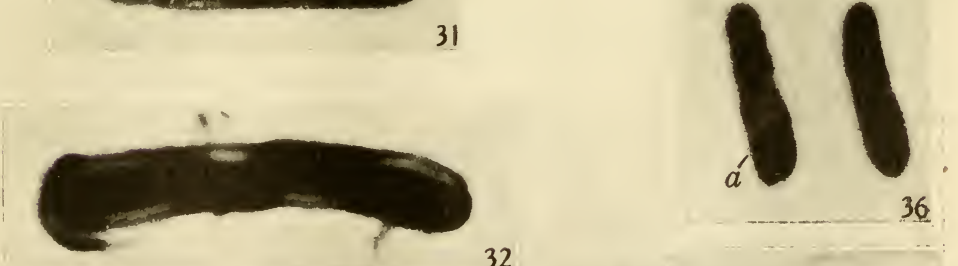

32
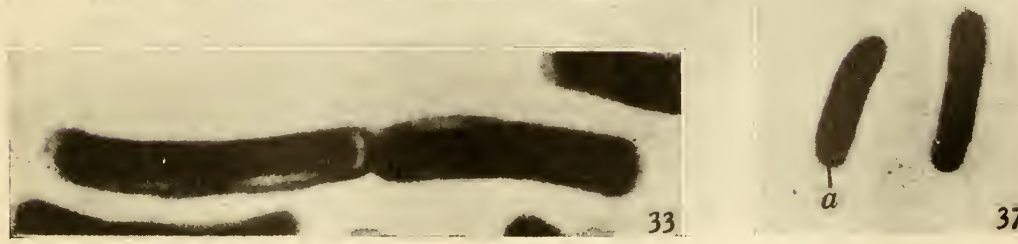

Figs. 28-37. 
top corner the cells in this picture were dormant at the time of fixation.

Fig. 24. The same culture as in fig. 25, incubated for $90 \mathrm{~min}$., fixed and stained in the same way. $a$, a cell of the slender rod type which has not yet begun to develop into a large two-cell bacterium. $b$, a two-cell bacterium. $c$, a slightly constricted four-cell bacterium. $e$, dividing four-cell bacteria.

Figs. 25, 27 are successive stages in the development of $B$. mycoides beginning with spores near the end of the germination period.

Fig. 26 should be compared with the corresponding stages $a, b, d$ in the development of Bact. coli in Pl. 5, figs. 1,2 .

Fig. 26. Demonstrates a clear median cell boundary.

The three bacteria in fig. 27 correspond to those in figs. 8 and 9 of Bact. coli on Pl. 5. At $a$ a strand of chromatinic matter is seen extending between separating chromatinic structures.

\section{Plate 8}

B. mesentericus, figs. 28,29

$B$. megatherium, figs. $30-34$

Bact. coli, figs. 35-37

No nuclear structures are shown in any of the photographs on this plate. The photographs of $B$. megatherium are from water-mounted preparations, the rest from balsam mounts.

Figs. 28, 29. B. mesentericus from spores incubated for $2 \mathrm{hr}$. and $23 \mathrm{hr}$. respectively. $a$ in fig. 28 marks a single-cell stage, all the remaining bacilli in both pictures are composite in nature. Note the dark staining of the tips and of the places where the bacteria will eventually divide by constriction. The 2 . and 4 -cell bacilli in fig. 28 are all 'resting', the two long diagonally placed filaments in fig. 9 show narrow median constrictions. Each consists of at least four cells. Compare with Pl. 7, fig. 24 of Bact. coli and note similarity in structure.

Figs. 30-34. From three different preparations of B. megatherium, treated with boiling $1.3 \% \mathrm{NaOH}$ as deseribed under method 5 . The stain is $0.5 \%$ erystalviolet and the preparations were photographed mounted in water.

Fig. 30. Plasmolysis and differentiation of cytoplasm and cell wall are imperfect but the composite structure of all three bacilli is nevertheless recognizable. $a$, the cytoplasm of the left half is incompletely, that of the right half completely divided by a transverse cytoplasmic partition not yet strengthened by cell-wall material. The specimen might be described as a $3-4$ cell bacillus. Had the plasmolysing treatment been more effective, it is probable that the internal configuration would have resembled that of the right half of the large dividing specimen in fig. $33 . b$, note the retraction of deeply stained cytoplasm from the cell wall in the plane of constriction and from the horizontal part of the adjoining region (under side) of the third cell from the left. There has also been a concave retraction of the cytoplasm in cell no. 4 at the right extremity of the bacillus. (Compare this specimen with the large, darkly stained bacterium to the left of the centre in Pl. 7, fig.24d.) There has been some retraction of the cytoplasm from the cell wall at both poles of the third bacillus (c).

Fig. 31. The two cells on either side of the future plane of constriction in this bacillus have retracted in two continuous portions from the cell wall where the formation of transverse septa, destined to cut into the cytoplasm, can be seen to have begun. In the right-hand top corner is a discarded spore case.

Fig. 32. All four component cells of this young bacillus have retracted independently from the outer cell wall. The third cell from the left has also retracted from the transverse partition separating it from the fourth cell. The left end of the bacillus is still contained in the spore case.

Figs. 33, 34. All-round retraction of the cytoplasm from the cell wall in the bacillus on the right has provicled a clear view of the medium transverse partition. Further examples are seen in the right-hand top corner and near the left margin of fig. 34 .

Figs. 35-37. Bact. coli, incubated for $3 \mathrm{hr}$. on an agar plate at $37^{\circ} \mathrm{C}$. followed by $20 \mathrm{hr} .2-4^{\circ} \mathrm{C}$. Bouin fixation through the agar, Giemsa's stain. Mounted in canada balsam.

Fig. 35. Retrattion of the cytoplasm from a inedian septum. Probably comparable to the condition of the upper half of the right-hand bacillus in the adjoining fig. 34 .

Figs. 36,37 . In both figures the bacterium marked $a$ is composed of one deeply stained and one lightly stained half. Demarcation of the two is particularly clear in fig. 36 . The faint staining is due to disintegration of the cytoplasm in this half of the bacterium. Explanation in the text. 


\title{
STUDIES ON THE CHEMICAL NATURE OF THE SUBSTANCE INDUCING TRANSFORMATION OF PNEUMOCOCCAL TYPES
}

\author{
Induction of Transformation by a Desoxyribonucleic Acid Fraction \\ Isolated from PNeumococcus Type III
}

\author{
By OSWALD T. AVERY, M.D., COLIN M. MACLEOD, M.D., AND \\ MACLYN MCCARTY,* M.D. \\ (From the Hospital of The Rockefeller Institute for Medical Research)
}

Plate 1

(Received for publication, November 1, 1943)

Biologists have long attempted by chemical means to induce in higher organisms predictable and specific changes which thereafter could be transmitted in series as hereditary characters. Among microörganisms the most striking example of inheritable and specific alterations in cell structure and function that can be experimentally induced and are reproducible under well defined and adequately controlled conditions is the transformation of specific types of Pneumococcus. This phenomenon was first described by Griffith (1) who succeeded in transforming an attenuated and non-encapsulated ( $R$ ) variant derived from one specific type into fully encapsulated and virulent (S) cells of a heterologous specific type. A typical instance will suffice to illustrate the techniques originally used and serve to indicate the wide variety of transformations that are possible within the limits of this bacterial species.

Griffith found that mice injected subcutaneously with a small amount of a living $\mathrm{R}$ culture derived from Pneumococcus Type II together with a large inoculum of heat-killed Type III (S) cells frequently succumbed to infection, and that the heart's blood of these animals yielded Type III pneumococci in pure culture. The fact that the $\mathrm{R}$ strain was avirulent and incapable by itself of causing fatal bacteremia and the additional fact that the heated suspension of Type III cells contained no viable organisms brought convincing evidence that the $\mathrm{R}$ forms growing under these conditions had newly acquired the capsular structure and biological specificity of Type III pneumococci.

The original observations of Griffith were later confirmed by Neufeld and Levinthal (2), and by Baurhenn (3) abroad, and by Dawson (4) in this laboratory. Subsequently Dawson and Sia (5) succeeded in inducing transformation in vitro. This they accomplished by growing $\mathrm{R}$ cells in a fluid medium containing anti-R serum and heat-killed encapsulated S cells. They showed that in the test tube as in the animal body transformation can be selectively induced, depending on the type specificity of the S cells used in the reaction system. Later, Alloway (6) was able to cause

\footnotetext{
* Work done in part as Fellow in the Medical Sciences of the National Research Council.
}

[Reprinted by permission of The Rockefeller Institute for Medical Research from The Journal of Experimental Mfedicinf 79 : (2) 137-158, February 1, 1944] 
specific transformation in vitro using sterile extracts of $\mathrm{S}$ cells from which all formed elements and cellular debris had been removed by Berkefeld filtration. He thus showed that crude extracts containing active transforming material in soluble form are as effective in inducing specific transformation as are the intact cells from which the extracts were prepared.

Another example of transformation which is analogous to the interconvertibility of pneumococcal types lies in the field of viruses. Berry and Dedrick (7) succeeded in changing the virus of rabbit fibroma (Shope) into that of infectious myxoma (Sanarelli). These investigators inoculated rabbits with a mixture of active fibroma virus together with a suspension of heat-inactivated myxoma virus and produced in the animals the symptoms and pathological lesions characteristic of infectious myxomatosis. On subsequent animal passage the transformed virus was transmissible and induced myxomatous infection typical of the naturally occurring disease. Later Berry (8) was successful in inducing the same transformation using a heat-inactivated suspension of washed elementary bodies of myxoma virus. In the case of these viruses the methods employed were similar in principle to those used by Griffith in the transformation of pneumococcal types. These observations have subsequently been confirmed by other investigators (9).

The present paper is concerned with a more detailed analysis of the phenomenon of transformation of specific types of Pneumococcus. The major interest has centered in attempts to isolate the active principle from crude bacterial extracts and to identify if possible its chemical nature or at least to characterize it sufficiently to place it in a general group of known chemical substances. For purposes of study, the typical example of transformation chosen as a working model was the one with which we have had most experience and which consequently seemed best suited for analysis. This particular example represents the transformation of a non-encapsulated $R$ variant of Pneumococcus Type II to Pneumococcus Type III.

\section{EXPERIMENTAL}

Transformation of pneumococcal types in vitro requires that certain cultural conditions be fulfilled before it is possible to demonstrate the reaction even in the presence of a potent extract. Not only must the broth medium be optimal for growth but it must be supplemented by the addition of serum or serous fluid known to possess certain special properties. Moreover, the $\mathrm{R}$ variant, as will be shown later, must be in the reactive phase in which it has the capacity to respond to the transforming stimulus. For purposes of convenience these several components as combined in the transforming test will be referred to as the reaction system. Each constituent of this system presented problems which required clarification before it was possible to obtain consistent and reproducible results. The various components of the system will be described in the following order: (1) nutrient broth, (2) serum or serous fluid, (3) strain of R Pneumococcus, and (4) extraction, purification, and chemical nature of the transforming principle. 
1. Nutrient Broth.-Beef heart infusion broth containing 1 per cent neopeptone with no added dextrose and adjusted to an initial $\mathrm{pH}$ of $7.6-7.8$ is used as the basic medium. Individual lots of broth show marked and unpredictable variations in the property of supporting transformation. It has been found, however, that charcoal adsorption, according to the method described by MacLeod and Mirick (10) for removal of sulfonamide inhibitors, eliminates to a large extent these variations; consequently this procedure is used as routine in the preparation of consistently effective broth for titrating the transforming activity of extracts.

2. Serum or Serous Fluid.-In the first successful experiments on the induction of transformation in vitro, Dawson and Sia (5) found that it was essential to add serum to the medium. Anti- $\mathrm{R}$ pneumococcal rabbit serum was used because of the observation that reversion of an $\mathrm{R}$ pneumococcus to the homologous $\mathrm{S}$ form can be induced by growth in a medium containing anti-R serum. Alloway (6) later found that ascitic or chest fluid and normal swine serum, all of which contain $\mathrm{R}$ antibodies, are capable of replacing antipneumococcal rabbit serum in the reaction system. Some form of serum is essential, and to our knowledge transformation in vitro has never been effected in the absence of serum or serous fluid.

In the present study human pleural or ascitic fluid has been used almost exclusively. It became apparent, however, that the effectiveness of different lots of serum varied and that the differences observed were not necessarily dependent upon the content of $\mathrm{R}$ antibodies, since many sera of high titer were found to be incapable of supporting transformation. This fact suggested that factors other than $\mathrm{R}$ antibodies are involved.

It has been found that sera from various animal species, irrespective of their immune properties, contain an enzyme capable of destroying the transforming principle in potent extracts. The nature of this enzyme and the specific substrate on which it acts will be referred to later in this paper. This enzyme is inactivated by heating the serum at $60^{\circ}-65^{\circ} \mathrm{C}$., and sera heated at temperatures known to destroy the enzyme are often rendered effective in the transforming system. Further analysis has shown that certain sera in which $\mathrm{R}$ antibodies are present and in which the enzyme has been inactivated may nevertheless fail to support transformation. This fact suggests that still another factor in the serum is essential. The content of this factor varies in different sera, and at present its identity is unknown.

There are at present no criteria which can be used as a guide in the selection of suitable sera or serous fluids except that of actually testing their capacity to support transformation. Fortunately, the requisite properties are stable and remain unimpaired over long periods of time; and sera that have been stored in the refrigerator for many months have been found on retesting to have lost little or none of their original effectiveness in supporting transformation.

The recognition of these various factors in serum and their rôle in the reaction system has greatly facilitated the standardization of the cultural conditions required for obtaining consistent and reproducible results.

3. The $R$ Strain $(R 36 A)$. - The unencapsulated $\mathrm{R}$ strain used in the present study was derived from a virulent " $S$ " culture of Pneumococcus Type II. It will be recalled that irrespective of type derivation all " $R$ " variants of Pneumococcus are characterized by the lack of capsule formation and the 
consequent loss of both type specificity and the capacity to produce infection in the animal body. The designation of these variants as $\mathrm{R}$ forms has been used to refer merely to the fact that on artificial media the colony surface is "rough" in contrast to the smooth, glistening surface of colonies of encapsulated S cells.

The $\mathrm{R}$ strain referred to above as $\mathrm{R} 36 \mathrm{~A}$ was derived by growing the parent $\mathrm{S}$ culture of Pneumococcus Type II in broth containing Type II antipneumococcus rabbit serum for 36 serial passages and isolating the variant thus induced. The strain R36A has lost all the specific and distinguishing characteristics of the parent $\mathrm{S}$ organisms and consists only of attenuated and non-encapsulated $\mathrm{R}$ variants. The change $\mathrm{S} \rightarrow \mathrm{R}$ is often a reversible one provided the $\mathrm{R}$ cells are not too far "degraded." The reversion of the $\mathrm{R}$ form to its original specific type can frequently be accomplished by successive animal passages or by repeated serial subculture in anti-R serum. When reversion occurs under these conditions, however, the $\mathrm{R}$ culture invariably reverts to the encapsulated form of the same specific type as that from which it was derived (11). Strain R36A has become relatively fixed in the $\mathrm{R}$ phase and has never spontaneously reverted to the Type II S form. Moreover, repeated attempts to cause it to revert under the conditions just mentioned have in all instances been unsuccessful.

The reversible conversion of $\mathrm{S} \rightleftharpoons \mathrm{R}$ within the limits of a single type is quite different from the transformation of one specific type of Pneumococcus into another specific type through the $\mathrm{R}$ form. Transformation of types has never been observed to occur spontaneously and has been induced experimentally only by the special techniques outlined earlier in this paper. Under these conditions, the enzymatic synthesis of a chemically and immunologically different capsular polysaccharide is specifically oriented and selectively determined by the specific type of $\mathrm{S}$ cells used as source of the transforming agent.

In the course of the present study it was noted that the stock culture of R36 on serial transfers in blood broth undergoes spontaneous dissociation giving rise to a number of other $\mathrm{R}$ variants which can be distinguished one from another by colony form. The significance of this in the present instance lies in the fact that of four different variants isolated from the parent $R$ culture only one ( $R 36 \mathrm{~A})$ is susceptible to the transforming action of potent extracts, while the others fail to respond and are wholly inactive in this regard. The fact that differences exist in the responsiveness of different $R$ variants to the same specific stimulus enphasizes the care that must be exercised in the selection of a suitable $R$ variant for use in experiments on transformation. The capacity of this $\mathrm{R}$ strain (R36A) to respond to a variety of different transforming agents is shown by the readiness with which it can be transformed to Types I, III, VI, or XIV, as well as to its original type (Type II), to which, as pointed out, it has never spontaneously reverted.

Although the significance of the following fact will become apparent later on, it must be mentioned here that pneumococcal cells possess an enzyme capable of destroying the activity of the transforming principle. Indeed, this enzyme has been 
found to be present and highly active in the autolysates of a number of different strains. The fact that this intracellular enzyme is released during autolysis may explain, in part at least, the observation of Dawson and Sia (5) that it is essential in bringing about transformation in the test tube to use a small inoculum of young and actively growing $\mathrm{R}$ cells. The irregularity of the results and often the failure to induce transformation when large inocula are used may be attributable to the release from autolyzing cells of an amount of this enzyme sufficient to destroy the transforming principle in the reaction system.

In order to obtain consistent and reproducible results, two facts must be borne in mind: first, that an $\mathrm{R}$ culture can undergo spontaneous dissociation and give rise to other variants which have lost the capacity to respond to the transforming stimulus; and secondly, that pneumococcal cells contain an intracellular enzyme which when released destroys the activity of the transforming principle. Consequently, it is important to select a responsive strain and to prevent as far as possible the destructive changes associated with autolysis.

Method of Titration of Transforming Activity.-In the isolation and purification of the active principle from crude extracts of pneumococcal cells it is desirable to have a method for determining quantitatively the transforming activity of various fractions.

The experimental procedure used is as follows: Sterilization of the material to be tested for activity is accomplished by the use of alcohol since it has been found that this reagent has no effect on activity. A measured volume of extract is precipitated in a sterile centrifuge tube by the addition of 4 to 5 volumes of absolute ethyl alcohol, and the mixture is allowed to stand 8 or more hours in the refrigerator in order to effect sterilization. The alcohol precipitated material is centrifuged, the supernatant discarded, and the tube containing the precipitate is allowed to drain for a few minutes in the inverted position to remove excess alcohol. The mouth of the tube is then carefully flamed and a dry, sterile cotton plug is inserted. The precipitate is redissolved in the original volume of saline. Sterilization of active material by this technique has invariably proved effective. This procedure avoids the loss of active substance which may occur when the solution is passed through a Berkefeld filter or is heated at the high temperatures required for sterilization.

To the charcoal-adsorbed broth described above is added 10 per cent of the sterile ascitic or pleural fluid which has previously been heated at $60^{\circ} \mathrm{C}$. for 30 minutes, in order to destroy the enzyme known to inactivate the transforming principle. The enriched medium is distributed under aseptic conditions in $2.0 \mathrm{cc}$. amounts in sterile tubes measuring $15 \times 100 \mathrm{~mm}$. The sterilized extract is diluted serially in saline neutralized to $\mathrm{pH} 7.2-7.6$ by addition of $0.1 \mathrm{~N} \mathrm{NaOH}$, or it may be similarly diluted in $\mathrm{M} / 40$ phosphate buffer, $\mathrm{pH} 7.4$. $0.2 \mathrm{cc}$. of each dilution is added to at least 3 or 4 tubes of the serum medium. The tubes are then seeded with a 5 to 8 hour blood broth culture of R36A. $0.05 \mathrm{cc}$. of a $10^{-4}$ dilution of this culture is added to each tube, and the cultures are incubated at $37^{\circ} \mathrm{C}$. for 18 to 24 hours. 
The anti- $R$ properties of the serum in the medium cause the $R$ cells to agglutinate during growth, and clumps of the agglutinated cells settle to the bottom of the tube leaving a clear supernatant. When transformation occurs, the encapsulated $\mathrm{S}$ cells, not being affected by these antibodies, grow diffusely throughout the medium. On the other hand, in the absence of transformation the supernatant remains clear, and only sedimented growth of $\mathrm{R}$ organisms occurs. This difference in the character of growth makes it possible by inspection alone to distinguish tentatively between positive and negative results. As routine all the cultures are plated on blood agar for confirmation and further bacteriological identification. Since the extracts used in the present study were derived from Pneumococcus Type III, the differentiation between the colonies of the original $\mathrm{R}$ organism and those of the transformed $\mathrm{S}$ cells is especially striking, the latter being large, glistening, mucoid colonies typical of Pneumococcus Type III. Figs. 1 and 2 illustrate these differences in colony form.

A typical protocol of a titration of the transforming activity of a highly purified preparation is given in Table IV.

\section{Preparative Methods}

Source Material.-In the present investigation a stock laboratory strain of Pneumococcus Type III (A66) has been used as source material for obtaining the active principle. Mass cultures of these organisms are grown in $\mathbf{5 0}$ to $\mathbf{7 5}$ liter lots of plain beef heart infusion broth. After 16 to 18 hours' incubation at $37^{\circ} \mathrm{C}$. the bacterial cells are collected in a steam-driven sterilizable Sharples centrifuge. The centrifuge is equipped with cooling coils immersed in ice water so that the culture fluid is thoroughly chilled before flowing into the machine. This procedure retards autolysis during the course of centrifugation. The sedimented bacteria are removed from the collecting cylinder and resuspended in approximately $150 \mathrm{cc}$. of chilled saline $(0.85$ per cent $\mathrm{NaCl}$ ), and care is taken that all clumps are thoroughly emulsified. The glass vessel containing the thick, creamy suspension of cells is immersed in a water bath, and the temperature of the suspension rapidly raised to $65^{\circ} \mathrm{C}$. During the heating process the material is constantly stirred, and the temperature maintained at $65^{\circ} \mathrm{C}$. for 30 minutes. Heating at this temperature inactivates the intracellular enzyme known to destroy the transforming principle.

Extraction of Heat-Killed Cells.-Although various procedures have been used, only that which has been found most satisfactory will be described here. The heatkilled cells are washed with saline 3 times. The chief value of the washing process is to remove a large excess of capsular polysaccharide together with much of the protein, ribonucleic acid, and somatic " $\mathrm{C}$ " polysaccharide. Quantitative titrations of transforming activity have shown that not more than 10 to 15 per cent of the active material is lost in the washing, a loss which is small in comparison to the amount of inert substances which are removed by this procedure.

After the final washing, the cells are extracted in $150 \mathrm{cc}$. of saline containing sodium desoxycholate in final concentration of 0.5 per cent by shaking the mixture me- 
chanically 30 to 60 minutes. The cells are separated by centrifugation, and the extraction process is repeated 2 or 3 times. The desoxycholate extracts prepared in this manner are clear and colorless. These extracts are combined and precipitated by the addition of 3 to 4 volumes of absolute ethyl alcohol. The sodium desoxycholate being soluble in alcohol remains in the supernatant and is thus removed at this step. The precipitate forms a fibrous mass which floats to the surface of the alcohol and can be removed directly by lifting it out with a spatula. The excess alcohol is drained from the precipitate which is then redissolved in about $50 \mathrm{cc}$. of saline. The solution obtained is usually viscous, opalescent, and somewhat cloudy.

Deproteinization and Removal of Capsular Polysaccharide.-The solution is then deproteinized by the chloroform method described by Sevag (12). The procedure is repeated 2 or 3 times until the solution becomes clear. After this preliminary treatment the material is reprecipitated in 3 to 4 volumes of alcohol. The precipitate obtained is dissolved in a larger volume of saline $(150 \mathrm{cc}$.) to which is added 3 to 5 mg. of a purified preparation of the bacterial enzyme capable of hydrolyzing the Type III capsular polysaccharide (13). The mixture is incubated at $37^{\circ} \mathrm{C}$, and the destruction of the capsular polysaccharide is determined by serological tests with Type III antibody solution prepared by dissociation of immune precipitate according to the method described by Liu and $\mathrm{Wu}$ (14). The advantages of using the antibody solution for this purpose are that it does not react with other serologically active substances in the extract and that it selectively detects the presence of the capsular polysaccharide in dilutions as high as 1:6,000,000. The enzymatic breakdown of the polysaccharide is usually complete within 4 to 6 hours, as evidenced by the loss of serological reactivity. The digest is then precipitated in 3 to 4 volumes of ethyl alcohol, and the precipitate is redissolved in $50 \mathrm{cc}$. of saline. Deproteinization by the chloroform process is again used to remove the added enzyme protein and remaining traces of pneumococcal protein. The procedure is repeated until no further film of protein-chloroform gel is visible at the interface.

Alcohol Fractionation.-Following deproteinization and enzymatic digestion of the capsular polysaccharide, the material is repeatedly fractionated in ethyl alcohol as follows. Absolute ethyl alcohol is added dropwise to the solution with constant stirring. At a critical concentration varying from 0.8 to 1.0 volume of alcohol the active material separates out in the form of fibrous strands that wind themselves around the stirring rod. This precipitate is removed on the rod and washed in a 50 per cent mixture of alcohol and saline. Although the bulk of active material is removed by fractionation at the critical concentration, a small but appreciable amount remains in solution. However, upon increasing the concentration of alcohol to 3 volumes, the residual fraction is thrown down together with inert material in the form of a flocculent precipitate. This flocculent precipitate is taken up in a small volume of saline $(5$ to $10 \mathrm{cc}$.) and the solution again fractionated by the addition of 0.8 to 1.0 volume of alcohol. Additional fibrous material is obtained which is combined with that recovered from the original solution. Alcoholic fractionation is repeated 4 to 5 times. The yield of fibrous material obtained by this method varies from 10 to 25 mg. per 75 liters of culture and represents the major portion of active material present in the original crude extract.

Effect of Temperature.-As a routine procedure all steps in purification were carried 
out at room temperature unless specifically stated otherwise. Because of the theoretical advantage of working at low temperature in the preparation of biologically active material, the purification of one lot (preparation 44) was carried out in the cold. In this instance all the above procedures with the exception of desoxycholate exextraction and enzyme treatment were conducted in a cold room maintained at $0-4^{\circ} \mathrm{C}$. This preparation proved to have significantly higher activity than did material similarly prepared at room temperature.

Desoxycholate extraction of the heat-killed cells at low temperature is less efficient and yields smaller amounts of the active fraction. It has been demonstrated that higher temperatures facilitate extraction of the active principle, although activity is best preserved at low temperatures.

\section{Analysis of Purified Transforming Material}

General Properties.-Saline solutions containing 0.5 to $1.0 \mathrm{mg}$. per cc. of the purified substance are colorless and clear in diffuse light. However, in strong transmitted light the solution is not entirely clear and when stirred exhibits a silky sheen. Solutions at these concentrations are highly viscous.

Purified material dissolved in physiological salt solution and stored at $2-4^{\circ} \mathrm{C}$. retains its activity in undiminished titer for at least 3 months. However, when dissolved in distilled water, it rapidly decreases in activity and becomes completely inert within a few days. Saline solutions stored in the frozen state in a $\mathrm{CO}_{2}$ ice box $\left(-70^{\circ} \mathrm{C}\right.$. $)$ retain full potency for several months. Similarly, material precipitated from saline solution by alcohol and stored under the supernatant remains active over a long period of time. Partially purified material can be preserved by drying from the frozen state in the lyophile apparatus. However, when the same procedure is used for the preservation of the highly purified substance, it is found that the material undergoes changes resulting in decrease in solubility and loss of activity.

The activity of the transforming principle in crude extracts withstands heating for 30 to 60 minutes at $65^{\circ} \mathrm{C}$. Highly purified preparations of active material are less stable, and some loss of activity occurs at this temperature. A quantitative study of the effect of heating purified material at higher temperatures has not as yet been made. Alloway (6), using crude extracts prepared from Type III pneumococcal cells, found that occasionally activity could still be demonstrated after 10 minutes' exposure in the water bath to temperatures as high as $90^{\circ} \mathrm{C}$.

The procedures mentioned above were carried out with solutions adjusted to neutral reaction, since it has been shown that hydrogen ion concentrations in the acid range result in progressive loss of activity. Inactivation occurs rapidly at $\mathrm{pH} 5$ and below.

Qualitative Chemical Tests. - The purified material in concentrated solution gives negative biuret and Millon tests. These tests have been done directly on dry material with negative results. The Dische diphenylamine reaction 
for desoxyribonucleic acid is strongly positive. The orcinol test (Bial) for ribonucleic acid is weakly positive. However, it has been found that in similar concentrations pure preparations of desoxyribonucleic acid of animal origin prepared by different methods give a Bial reaction of corresponding intensity.

Although no specific tests for the presence of lipid in the purified material have been made, it has been found that crude material can be repeatedly extracted with alcohol and ether at $-12^{\circ} \mathrm{C}$. without loss of activity. In addition, as will be noted in the preparative procedures, repeated alcohol precipitation and treatment with chloroform result in no decrease in biological activity.

Elementary Chemical Analysis. ${ }^{1-}$-Four purified preparations were analyzed for content of nitrogen, phosphorus, carbon, and hydrogen. The results are presented in Table I. The nitrogen-phosphorus ratios vary from 1.58 to 1.75 with an average value of 1.67 which is in close agreement with that calculated

TABLE I

Elementary Chemical Analysis of Purified Preparations of the Transforming Substance.

\begin{tabular}{c|c|c|c|c|c}
\hline Preparation No. & Carbon & Hydrogen & Nitrogen & Phosphorus & N/P ratio \\
\cline { 2 - 2 } & per cent & per cent & per cent & per cent & \\
37 & 34.27 & 3.89 & 14.21 & 8.57 & 1.66 \\
42 & - & - & 15.93 & 9.09 & 1.75 \\
44 & 35.50 & 3.76 & 15.36 & 9.04 & 1.69 \\
\hline Theory for sodium & - & - & 13.40 & 8.45 & 1.58 \\
desoxyribonucleate.... & 34.20 & 3.21 & 15.32 & 9.05 & 1.69 \\
\hline
\end{tabular}

on the basis of the theoretical structure of sodium desoxyribonucleate (tetranucleotide). The analytical figures by themselves do not establish that the substance isolated is a pure chemical entity. However, on the basis of the nitrogen-phosphorus ratio, it would appear that little protein or other substances containing nitrogen or phosphorus are present as impurities since if they were this ratio would be considerably altered.

Enzymatic Analysis.-Various crude and crystalline enzymes ${ }^{2}$ have been tested for their capacity to destroy the biological activity of potent bacterial extracts. Extracts buffered at the optimal $\mathrm{pH}$, to which were added crystalline trypsin and chymotrypsin or combinations of both, suffered no loss in activity following treatment with these enzymes. Pepsin could not be tested because

1 The elementary chemical analyses were made by Dr. A. Elek of The Rockefeller Institute.

${ }^{2}$ The authors are indebted to Dr. John H. Northrop and Dr. M. Kunitz of The Rockefeller Institute for Medical Research, Princeton, N. J., for the samples of crystalline trypsin, chymotrypsin, and ribonuclease used in this work. 
extracts are rapidly inactivated at the low $\mathrm{pH}$ required for its use. Prolonged treatment with crystalline ribonuclease under optimal conditions caused no demonstrable decrease in transforming activity. The fact that trypsin, chymotrypsin, and ribonuclease had no effect on the transforming principle is further evidence that this substance is not ribonucleic acid or a protein susceptible to the action of tryptic enzymes.

In addition to the crystalline enzymes, sera and preparations of enzymes obtained from the organs of various animals were tested to determine their effect on transforming activity. Certain of these were found to be capable of completely destroying biological activity. The various enzyme preparations tested included highly active phosphatases obtained from rabbit bone by the method of Martland and Robison (15) and from swine kidney as described by

TABLE II

The Inactivation of Transforming Principle by Crude Enzyme Preparations

\begin{tabular}{|c|c|c|c|c|}
\hline \multirow[b]{2}{*}{ Crude enzyme preparations } & \multicolumn{4}{|c|}{ Enzymatic activity } \\
\hline & Phosphatase & $\begin{array}{l}\text { Tributyrin } \\
\text { esterase }\end{array}$ & $\begin{array}{l}\text { Depolymer- } \\
\text { ase for } \\
\text { desoxyribo- } \\
\text { nucleate }\end{array}$ & $\begin{array}{l}\text { Inactivation } \\
\text { of trans- } \\
\text { forming } \\
\text { principle }\end{array}$ \\
\hline Dog intestinal mucosa........... & + & + & + & + \\
\hline Rabbit bone phosphatase.......... & + & + & - & - \\
\hline Swine kidney " $\quad \ldots \ldots \ldots \ldots \ldots$ & + & - & - & - \\
\hline Pneumococcus autolysates............. & - & + & + & + \\
\hline Normal dog and rabbit serum........... & + & + & + & + \\
\hline
\end{tabular}

H. and E. Albers (16). In addition, a preparation made from the intestinal mucosa of dogs by Levene and Dillon (17) and containing a polynucleotidase for thymus nucleic acid was used. Pneumococcal autolysates and a commercial preparation of pancreatin were also tested. The alkaline phosphatase activity of these preparations was determined by their action on $\beta$-glycerophosphate and phenyl phosphate, and the esterase activity by their capacity to split tributyrin. Since the highly purified transforming material isolated from pneumococcal extracts was found to contain desoxyribonucleic acid, these same enzymes were tested for depolymerase activity on known samples of desoxyribonucleic acid isolated by Mirsky $^{3}$ from fish sperm and mammalian tissues. The results are summarized in Table II in which the phosphatase, esterase, and nucleodepolymerase activity of these enzymes is compared with their capacity to destroy the transforming principle. Analysis of these results shows that irrespective of the presence of phosphatase or esterase only those

${ }^{3}$ The authors express their thanks to Dr. A. E. Mirsky of the Hospital of The Rockefeller Institute for these preparations of desoxyribonucleic acid. 
preparations shown to contain an enzyme capable of depolymerizing authentic samples of desoxyribonucleic acid were found to inactivate the transforming principle.

Greenstein and Jenrette (18) have shown that tissue extracts, as well as the milk and serum of several mammalian species, contain an enzyme system which causes depolymerization of desoxyribonucleic acid. To this enzyme system Greenstein has later given the name desoxyribonucleodepolymerase (19). These investigators determined depolymerase activity by following the reduction in viscosity of solutions of sodium desoxyribonucleate. The nucleate and enzyme were mixed in the viscosimeter and viscosity measurements made at intervals during incubation at $30^{\circ} \mathrm{C}$. In the present study this method was used in the measurement of depolymerase activity except that incubation was carried out at $37^{\circ} \mathrm{C}$. and, in addition to the reduction of viscosity, the action of the enzyme was further tested by the progressive decrease in acid precipitability of the nucleate during enzymatic breakdown.

The effect of fresh normal dog and rabbit serum on the activity of the transforming substance is shown in the following experiment.

Sera obtained from a normal dog and normal rabbit were diluted with an equal volume of physiological saline. The diluted serum was divided into three equal portions. One part was heated at $65^{\circ} \mathrm{C}$. for 30 minutes, another at $60^{\circ} \mathrm{C}$. for 30 minutes, and the third was used unheated as control. A partially purified preparation of transforming material which had previously been dried in the lyophile apparatus was dissolved in saline in a concentration of $3.7 \mathrm{mg}$. per cc. $1.0 \mathrm{cc}$. of this solution was mixed with $0.5 \mathrm{cc}$. of the various samples of heated and unheated diluted sera, and the mixtures at $\mathrm{pH} 7.4$ were incubated at $37^{\circ} \mathrm{C}$. for 2 hours. After the serum had been allowed to act on the transforming material for this period, all tubes were heated at $65^{\circ} \mathrm{C}$. for 30 minutes to stop enzymatic action. Serial dilutions were then made in saline and tested in triplicate for transforming activity according to the procedure described under Method of titration. The results given in Table III illustrate the differential heat inactivation of the enzymes in dog and rabbit serum which destroy the transforming principle.

From the data presented in Table III it is evident that both dog and rabbit serum in the unheated state are capable of completely destroying transforming activity. On the other hand, when samples of dog serum which have been heated either at $60^{\circ} \mathrm{C}$. or at $65^{\circ} \mathrm{C}$. for 30 minutes are used, there is no loss of transforming activity. Thus, in this species the serum enzyme responsible for destruction of the transforming principle is completely inactivated at $60^{\circ} \mathrm{C}$. In contrast to these results, exposure to $65^{\circ} \mathrm{C}$. for 30 minutes was required for complete destruction of the corresponding enzyme in rabbit serum.

The same samples of dog and rabbit serum used in the preceding experiment were also tested for their depolymerase activity on a preparation of sodium desoxyribonucleate isolated by Mirsky from shad sperm. 
A highly viscous solution of the nucleate in distilled water in a concentration of 1 mg. per cc. was used. $1.0 \mathrm{cc}$. amounts of heated and unheated sera diluted in saline as shown in the preceding protocol were mixed in Ostwald viscosimeters with $4.0 \mathrm{cc}$.

TABLE III

Differential Heat Inactivation of Enzymes in Dog and Rabbit Serum Which Destroy the Transforming Substance

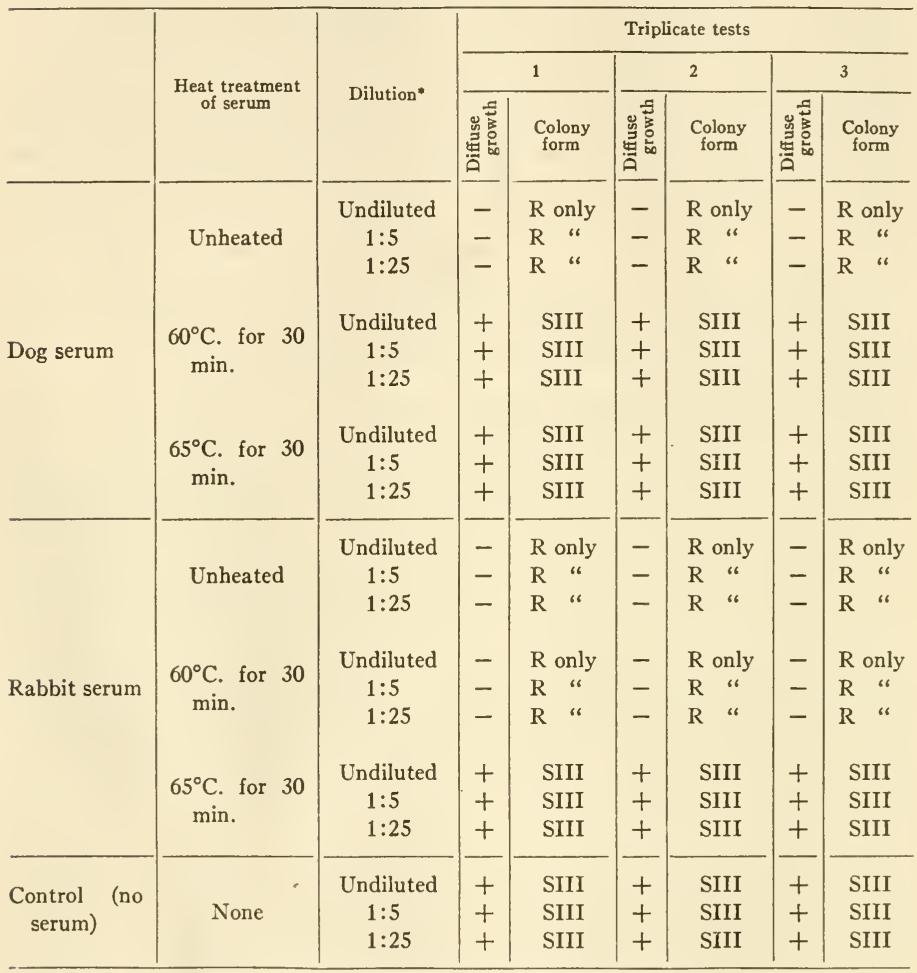

* Dilution of the digest mixture of serum and transforming substance.

of the aqueous solution of the nucleate. Determinations of viscosity were made immediately and at intervals over a period of 24 hours during incubation at $37^{\circ} \mathrm{C}$.

The results of this experiment are graphically presented in Chart 1 . In the case of unheated serum of both dog and rabbit, the viscosity fell to that of water in 5 to 7 hours. Dog serum heated at $60^{\circ} \mathrm{C}$. for 30 minutes brought about 
no significant reduction in viscosity after 22 hours. On the other hand, heating rabbit serum at $60^{\circ} \mathrm{C}$. merely reduced the rate of depolymerase action, and after 24 hours the viscosity was brought to the same level as with the unheated serum. Heating at $65^{\circ} \mathrm{C}$., however, completely destoyed the rabbit serum depolymerase.

Thus, in the case of dog and rabbit sera there is a striking parallelism between the temperature of inactivation of the depolymerase and that of the enzyme which destroys the activity of the transforming principle. The fact that this difference in temperature of inactivation is not merely a general property of all enzymes in the sera is evident from experiments on the heat inactivation of

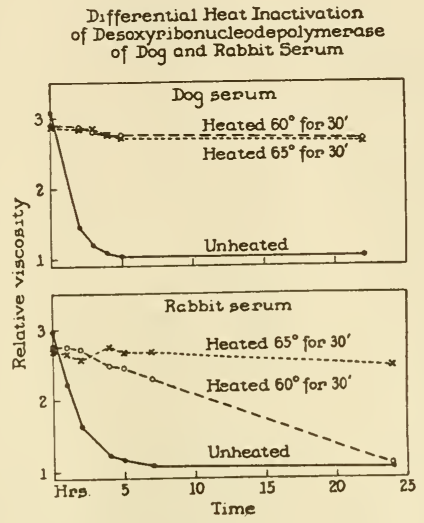

Chart 1

tributyrin esterase in the same samples of serum. In the latter instance, the results are the reverse of those observed with depolymerase since the esterase of rabbit serum is almost completely inactivated at $60^{\circ} \mathrm{C}$. while that in dog serum is only slightly affected by exposure to this temperature.

Of a number of substances tested for their capacity to inhibit the action of the enzyme known to destroy the transforming principle, only sodium fluoride has been found to have a significant inhibitory effect. Regardless of whether this enzyme is derived from pneumococcal cells, dog intestinal mucosa, pancreatin, or normal sera its activity is inhibited by fluoride. Similarly it has been found that fluoride in the same concentration also inhibits the enzymatic depolymerization of desoxyribonucleic acid.

The fact that transforming activity is destroyed only by those preparations containing depolymerase for desoxyribonucleic acid and the further fact that 
in both instances the enzymes concerned are inactivated at the same temperature and inhibited by fluoride provide additional evidence for the belief that the active principle is a nucleic acid of the desoxyribose type.

Serological Analysis. - In the course of chemical isolation of the active material it was found that as crude extracts were purified, their serological activity in Type III antiserum progressively decreased without corresponding loss in biological activity. Solutions of the highly purified substance itself gave only faint trace reactions in precipitin tests with high titer Type III antipneumococcus rabbit serum. ${ }^{4}$ It is well known that pneumococcal protein can be detected by serological methods in dilutions as high as 1:50,000 and the capsular as well as the somatic polysaccharide in dilutions of at least 1:5,000,000. In view of these facts, the loss of serological reactivity indicates that these cell constituents have been almost completely removed from the final preparations. The fact that the transforming substance in purified state exhibits little or no serological reactivity is in striking contrast to its biological specificity in inducing pneumococcal transformation.

Physicochemical Studies. ${ }^{5}-$ A purified and active preparation of the transforming substance (preparation 44) was examined in the analytical ultracentrigue. The material gave a single and unusually sharp boundary indicating that the substance was homogeneous and that the molecules were uniform in size and very asymmetric. Biological activity was found to be sedimented at the same rate as the optically observed boundary, showing that activity could not be due to the presence of an entity much different in size. The molecular weight cannot be accurately determined until measurements of the diffusion constant and partial specific volume have been made. However, Tennent and Vilbrandt (20) have determined the diffusion constant of several preparations of thymus nucleic acid the sedimentation rate of which is in close agreement with the values observed in the present study. Assuming that the asymmetry of the molecules is the same in both instances, it is estimated that the molecular weight of the pneumococcal preparation is of the order of 500,000 .

Examination of the same active preparation was carried out by electrophoresis in the Tiselius apparatus and revealed only a single electrophoretic component of relatively high mobility comparable to that of a nucleic acid. Transforming activity was associated with the fast moving component giving the

${ }^{4}$ The Type III antipneumococcus rabbit serum employed in this study was furnished through the courtesy of Dr. Jules T. Freund, Bureau of Laboratories, Department of Health, City of New York.

${ }^{5}$ Studies on sedimentation in the ultracentrifuge were carried out by Dr. A. Rothen; the electrophoretic analyses were made by Dr. T. Shedlovsky, and the ultraviolet absorption curves by Dr. G. I. Lavin. The authors gratefully acknowledge their indebtedness to these members of the staff of The Rockefeller Institute. 
optically visible boundary. Thus in both the electrical and centrifugal fields, the behavior of the purified substance is consistent with the concept that biological activity is a property of the highly polymerized nucleic acid.

Ultraviolet absorption curves showed maxima in the region of $2600 \AA$ and minima in the region of $2350 \AA$. These findings are characteristic of nucleic acids.

Quantitative Determination of Biological Activity.-In its highly purified state the material as isolated has been found to be capable of inducing transformation in amounts ranging from 0.02 to $0.003 \mu \mathrm{g}$. Preparation 44, the purification of which was carried out at low temperature and which had a nitrogen-phosphorus

TABLE IV

Titration of Transforming Activity of Preparation 44

\begin{tabular}{|c|c|c|c|c|c|c|c|c|c|}
\hline \multirow{2}{*}{\multicolumn{2}{|c|}{$\begin{array}{l}\text { Transforming principle } \\
\text { Preparation } 44^{*}\end{array}$}} & \multicolumn{8}{|c|}{ Quadruplicate tests } \\
\hline & & \multicolumn{2}{|c|}{1} & \multicolumn{2}{|c|}{2} & \multicolumn{2}{|c|}{3} & \multicolumn{2}{|c|}{4} \\
\hline Dilution & $\begin{array}{l}\text { Amount } \\
\text { added }\end{array}$ & $\begin{array}{l}\text { Diffuse } \\
\text { growth }\end{array}$ & $\begin{array}{l}\text { Colony } \\
\text { form }\end{array}$ & $\begin{array}{l}\text { Diffuse } \\
\text { growth }\end{array}$ & $\begin{array}{l}\text { Colony } \\
\text { form }\end{array}$ & $\begin{array}{l}\text { Diffuse } \\
\text { growth }\end{array}$ & $\begin{array}{l}\text { Colony } \\
\text { form }\end{array}$ & $\begin{array}{l}\text { Diffuse } \\
\text { growth }\end{array}$ & $\begin{array}{c}\text { Colony } \\
\text { form }\end{array}$ \\
\hline $10^{-2}$ & $\begin{array}{l}\mu g . \\
1.0\end{array}$ & + & SIII & + & SIII & + & SIII & + & SIII \\
\hline $10^{-2.5}$ & 0.3 & + & SIII & + & SIII & + & SIII & + & SIII \\
\hline $10^{-3}$ & 0.1 & + & SIII & + & SIII & + & SIII & + & SIII \\
\hline $10^{-3.5}$ & 0.03 & + & SIII & + & SIII & + & SIII & + & SIII \\
\hline $10^{-4}$ & 0.01 & + & SIII & + & SIII & + & SIII & + & SIII \\
\hline $10^{-4.5}$ & 0.003 & - & $\mathrm{R}$ only & + & SIII & - & $\mathrm{R}$ only & + & SIII \\
\hline $10^{-5}$ & 0.001 & - & R “ & - & $\mathrm{R}$ only & - & R “ & - & $\mathrm{R}$ only \\
\hline Control & None & - & $\mathrm{R}$ “ & - & $\mathrm{R}$ “ & - & $\mathrm{R}$ “ & - & $R$ " \\
\hline
\end{tabular}

* Solution from which dilutions were made contained $0.5 \mathrm{mg}$. per cc. of purified material. $0.2 \mathrm{cc}$. of each dilution added to quadruplicate tubes containing $2.0 \mathrm{cc}$. of standard serum broth. $0.05 \mathrm{cc}$. of a $10^{-4}$ dilution of a blood broth culture of R36A is added to each tube.

ratio of 1.58 , exhibited high transforming activity. Titration of the activity of this preparation is given in Table IV.

A solution containing $0.5 \mathrm{mg}$. per cc. was serially diluted as shown in the protocol. $0.2 \mathrm{cc}$. of each of these dilutions was added to quadruplicate tubes containing $2.0 \mathrm{cc}$. of standard serum broth. All tubes were then inoculated with $0.05 \mathrm{cc}$. of a $10^{-4}$ dilution of a 5 to 8 hour blood broth culture of R36A. Transforming activity was determined by the procedure described under Method of titration.

The data presented in Table IV show that on the basis of dry weight 0.003 $\mu \mathrm{g}$. of the active material brought about transformation. Since the reaction system containing the $0.003 \mu \mathrm{g}$. has a volume of $2.25 \mathrm{cc}$., this represents a final concentration of the purified substance of 1 part in $600,000,000$. 


\section{DISCUSSION}

The present study deals with the results of an attempt to determine the chemical nature of the substance inducing specific transformation of pneumococcal types. A desoxyribonucleic acid fraction has been isolated from Type III pneumococci which is capable of transforming unencapsulated $R$ variants derived from Pneumococcus Type II into fully encapsulated Type III cells. Thompson and Dubos (21) have isolated from pneumococci a nucleic acid of the ribose type. So far as the writers are aware, however, a nucleic acid of the desoxyribose type has not heretofore been recovered from pneumococci nor has specific transformation been experimentally induced in vitro by a chemically defined substance.

Although the observations are limited to a single example, they acquire broader significance from the work of earlier investigators who demonstrated the interconvertibility of various pneumococcal types and showed that the specificity of the changes induced is in each instance determined by the particular type of encapsulated cells used to evoke the reaction. From the point of view of the phenomenon in general, therefore, it is of special interest that in the example studied, highly purified and protein-free material consisting largely, if not exclusively, of desoxyribonucleic acid is capable of stimulating unencapsulated $\mathrm{R}$ variants of Pneumococcus Type II to produce a capsular polysaccharide identical in type specificity with that of the cells from which the inducing substance was isolated. Equally striking is the fact that the substance evoking the reaction and the capsular substance produced in response to it are chemically distinct, each belonging to a wholly different class of chemical compounds.

The inducing substance, on the basis of its chemical and physical properties, appears to be a highly polymerized and viscous form of sodium desoxyribonucleate. On the other hand, the Type III capsular substance, the synthesis of which is evoked by this transforming agent, consists chiefly of a non-nitrogenous polysaccharide constituted of glucose-glucuronic acid units linked in glycosidic union (22). The presence of the newly formed capsule containing this type-specific polysaccharide confers on the transformed cells all the distinguishing characteristics of Pneumococcus Type III. Thus, it is evident that the inducing substance and the substance produced in turn are chemically distinct and biologically specific in their action and that both are requisite in determining the type specificity of the cell of which they form a part.

The experimental data presented in this paper strongly suggest that nucleic acids, at least those of the desoxyribose type, possess different specificities as evidenced by the selective action of the transforming principle. Indeed, the possibility of the existence of specific differences in biological behavior of nucleic acids has previously been suggested $(23,24)$ but has never been experimentally demonstrated owing in part at least to the lack of suitable biological methods. 
The techniques used in the study of transformation appear to afford a sensitive means of testing the validity of this hypothesis, and the results thus far obtained add supporting evidence in favor of this point of view.

If it is ultimately proved beyond reasonable doubt that the transforming activity of the material described is actually an inherent property of the nucleic acid, one must still account on a chemical basis for the biological specificity of its action. At first glance, immunological methods would appear to offer the ideal means of determining the differential specificity of this group of biologically important substances. Although the constituent units and general pattern of the nucleic acid molecule have been defined, there is as yet relatively little known of the possible effect that subtle differences in molecular configuration may exert on the biological specificity of these substances. However, since nucleic acids free or combined with histones or protamines are not known to function antigenically, one would not anticipate that such differences would be revealed by immunological techniques. Consequently, it is perhaps not surprising that highly purified and protein-free preparations of desoxyribonucleic acid, although extremely active in inducing transformation, showed only faint trace reactions in precipitin tests with potent Type III antipneumococcus rabbit sera.

From these limited observations it would be unwise to draw any conclusion concerning the immunological significance of the nucleic acids until further knowledge on this phase of the problem is available. Recent observations by Lackman and his collaborators (25) have shown that nucleic acids of both the yeast and thymus type derived from hemolytic streptococci and from animal and plant sources precipitate with certain antipneumococcal sera. The reactions varied with different lots of immune serum and occurred more frequently in antipneumococcal horse serum than in corresponding sera of immune rabbits. The irregularity and broad cross reactions encountered led these investigators to express some doubt as to the immunological significance of the results. Unless special immunochemical methods can be devised similar to those so successfully used in demonstrating the serological specificity of simple non-antigenic substances, it appears that the techniques employed in the study of transformation are the only ones available at present for testing possible differences in the biological behavior of nucleic acids.

Admittedly there are many phases of the problem of transformation that require further study and many questions that remain unanswered largely because of technical difficulties. For example, it would be of interest to know the relation between rate of reaction and concentration of the transforming substance; the proportion of cells transformed to those that remain unaffected in the reaction system. However, from a bacteriological point of view, numerical estimations based on colony counts might prove more misleading than enlightening because of the aggregation and sedimentation of the $\mathrm{R}$ cells ag- 
glutinated by the antiserum in the medium. Attempts to induce transformation in suspensions of resting cells held under conditions inhibiting growth and multiplication have thus far proved unsuccessful, and it seems probable that transformation occurs only during active reproduction of the cells. Important in this connection is the fact that the R cells, as well as those that have undergone transformation, presumably also all other variants and types of pneumococci, contain an intracellular enzyme which is released during autolysis and in the free state is capable of rapidly and completely destroying the activity of the transforming agent. It would appear, therefore, that during the logarithmic phase of growth when cell division is most active and autolysis least apparent, the cultural conditions are optimal for the maintenance of the balance between maximal reactivity of the $\mathrm{R}$ cell and minimal destruction of the transforming agent through the release of autolytic ferments.

In the present state of knowledge any interpretation of the mechanism involved in transformation must of necessity be purely theoretical. The biochemical events underlying the phenomenon suggest that the transforming principle interacts with the $\mathrm{R}$ cell giving rise to a coordinated series of enzymatic reactions that culminate in the synthesis of the Type III capsular antigen. The experimental findings have clearly demonstrated that the induced alterations are not random changes but are predictable, always corresponding in type specificity to that of the encapsulated cells from which the transforming substance was isolated. Once transformation has occurred, the newly acquired characteristics are thereafter transmitted in series through innumerable transfers in artificial media without any further addition of the transforming agent. Moreover, from the transformed cells themselves, a substance of identical activity can again be recovered in amounts far in excess of that originally added to induce the change. It is evident, therefore, that not only is the capsular material reproduced in successive generations but that the primary factor, which controls the occurrence and specificity of capsular development, is also reduplicated in the daughter cells. The induced changes are not temporary modifications but are permanent alterations which persist provided the cultural conditions are favorable for the maintenance of capsule formation. The transformed cells can be readily distinguished from the parent $\mathrm{R}$ forms not alone by serological reactions but by the presence of a newly formed and visible capsule which is the immunological unit of type specificity and the accessory structure essential in determining the infective capacity of the microorganism in the animal body.

It is particularly significant in the case of pneumococci that the experimentally induced alterations are definitely correlated with the development of a new morphological structure and the consequent acquisition of new antigenic and invasive properties. Equally if not more significant is the fact that these changes are predictable, type-specific, and heritable. 
Various hypotheses have been advanced in explanation of the nature of the changes induced. In his original description of the phenomenon Griffith (1) suggested that the dead bacteria in the inoculum might furnish some specific protein that serves as a "pabulum" and enables the $\mathrm{R}$ form to manufacture a capsular carbohydrate.

More recently the phenomenon has been interpreted from a genetic point of view $(26,27)$. The inducing substance has been likened to a gene, and the capsular antigen which is produced in response to it has been regarded as a gene product. In discussing the phenomenon of transformation Dobzhansky (27) has stated that "If this transformation is described as a genetic mutation-and it is difficult to avoid so describing it-we are dealing with authentic cases of induction of specific mutations by specific treatments. ..."

Another interpretation of the phenomenon has been suggested by Stanley (28) who has drawn the analogy between the activity of the transforming agent and that of a virus. On the other hand, Murphy (29) has compared the causative agents of fowl tumors with the transforming principle of Pneumococcus. He has suggested that both these groups of agents be termed "transmissible mutagens" in order to differentiate them from the virus group. Whatever may prove to be the correct interpretation, these differences in viewpoint indicate the implications of the phenomenon of transformation in relation to similar problems in the fields of genetics, virology, and cancer research.

It is, of course, possible that the biological activity of the substance described is not an inherent property of the nucleic acid but is due to minute amounts of some other substance adsorbed to it or so intimately associated with it as to escape detection. If, however, the biologically active substance isolated in highly purified form as the sodium salt of desoxyribonucleic acid actually proves to be the transforming principle, as the available evidence strongly suggests, then nucleic acids of this type must be regarded not merely as structurally important but as functionally active in determining the biochemical activities and specific characteristics of pneumococcal cells. Assuming that the sodium desoxyribonucleate and the active principle are one and the same substance, then the transformation described represents a change that is chemically induced and specifically directed by a known chemical compound. If the results of the present study on the chemical nature of the transforming principle are confirmed, then nucleic acids must be regarded as possessing biological specificity the chemical basis of which is as yet undetermined.

\section{SUMMARY}

1. From Type III pneumococci a biologically active fraction has been isolated in highly purified form which in exceedingly minute amounts is capable under appropriate cultural conditions of inducing the transformation of unencapsulated $R$ variants of Pneumococcus Type II into fully encapsulated cells of the 
same specific type as that of the heat-killed microorganisms from which the inducing material was recovered.

2. Methods for the isolation and purification of the active transforming material are described.

3. The data obtained by chemical, enzymatic, and serological analyses together with the results of preliminary studies by electrophoresis, ultracentrifugation, and ultraviolet spectroscopy indicate that, within the limits of the methods, the active fraction contains no demonstrable protein, unbound lipid, or serologically reactive polysaccharide and consists principally, if not solely, of a highly polymerized, viscous form of desoxyribonucleic acid.

4. Evidence is presented that the chemically induced alterations in cellular structure and function are predictable, type-specific, and transmissible in series. The various hypotheses that have been advanced concerning the nature of these changes are reviewed.

\section{CONCLUSION}

The evidence presented supports the belief that a nucleic acid of the desoxyribose type is the fundamental unit of the transforming principle of Pneumococcus Type III.

\section{BIBLIOGRAPHY}

1. Griffith, F., J. Hyg., Cambridge, Eng., 1928, 27, 113.

2. Neufeld, F., and Levinthal, W., Z. Immunitätsforsch., 1928, 55, 324.

3. Baurhenn, W., Centr. Bakt., 1. Abt., Orig., 1932, 126, 68.

4. Dawson, M. H., J. Exp. Med., 1930, 51, 123.

5. Dawson, M. H., and Sia, R. H. P., J. Exp. Med., 1931, 54, 681.

6. Alloway, J. L., J. Exp. Med., 1932, 55, 91; 1933, 57, 265.

7. Berry, G. P., and Dedrick, H. M., J. Bact., 1936, 31, 50.

8. Berry, G. P., Arch. Path., 1937, 24, 533.

9. Hurst, E. W., Brit. J. Exp. Path., 1937, 18, 23. Hoffstadt, R. E., and Pilcher, K. S., J. Infect. Dis., 1941, 68, 67. Gardner, R. E., and Hyde, R. R., J. Infect. Dis., 1942, 71, 47. Houlihan, R. B., Proc. Soc. Exp. Biol. and Med., 1942, 51, 259.

10. MacLeod, C. M., and Mirick, G. S., J. Bact., 1942, 44, 277.

11. Dawson, M. H., J. Exp. Med., 1928, 47, 577; 1930, 51, 99.

12. Sevag, M. G., Biochem. Z., 1934, 273, 419. Sevag, M. G., Lackman, D. B., and Smolens, J., J. Biol. Chem., 1938, 124, 425.

13. Dubos, R. J., and Avery, O. T., J. Exp. Med., 1931, 54, 51. Dubos, R. J., and Bauer, J. H., J. Exp. Med., 1935, 62, 271.

14. Liu, S., and Wu, H., Chinese J. Physiol., 1938, 13, 449.

15. Martland, M., and Robison, R., Biochem. J., 1929, $23,237$.

16. Albers, H., and Albers, E., Z. physiol. Chem., 1935, 232, 189.

17. Levene, P. A., and Dillon, R. T., J. Biol. Chem., 1933, 96, 461.

18. Greenstein, J. P., and Jenrette, W. Y., J. Nat. Cancer Inst., 1940, 1, 845. 
19. Greenstein, J. P., J. Nat. Cancer Inst., 1943, 4, 55.

20. Tennent, H. G., and Vilbrandt, C. F., J. Am. Chem. Soc., 1943, 65, 424.

21. Thompson, R. H. S., and Dubos, R. J., J. Biol. Chem., 1938, 125, 65.

22. Reeves, R. E., and Goebel, W. F., J. Biol. Chem., 1941, 139, 511.

23. Schultz, J., in Genes and chromosomes. Structure and organization, Cold Spring Harbor symposia on quantitative biology, Cold Spring Harbor, Long Island Biological Association, 1941, 9, 55.

24. Mirsky, A. E., in Advances in enzymology and related subjects of biochemistry, (F. F. Nord and C. H. Werkman, editors), New York, Interscience Publishers, Inc., 1943, 3, 1.

25. Lackman, D., Mudd, S., Sevag, M. G., Smolens, J., and Wiener, M., J. Immunol., 1941, 40, 1.

26. Gortner, R. A., Outlines of biochemistry, New York, Wiley, 2nd edition, 1938, 547.

27. Dobzhansky, T., Genetics and the origin of the species, New York, Columbia University Press, 1941, 47.

28. Stanley, W. M., in Doerr, R., and Hallauer, C., Handbuch der Virusforschung, Vienna, Julius Springer, 1938, 1, 491.

29. Murphy, J. B., Tr. Assn. Am. Physn., 1931, 46, 182; Bull. Johns Bopkins Bosp., $1935,56,1$. 


\section{EXPLANATION OF PLATE 1}

The photograph was made by Mr. Joseph B. Haulenbeek.

FIG. 1. Colonies of the $\mathrm{R}$ variant (R36A) derived from Pneumococcus Type II. Plated on blood agar from a culture grown in serum broth in the absence of the transforming substance. $\times 3.5$.

Fig. 2. Colonies on blood agar of the same cells after induction of transformation during growth in the same medium with the addition of active transforming principle isolated from Type III pneumococci. The smooth, glistening, mucoid colonies shown are characteristic of Pneumococcus Type III and readily distinguishable from the small, rough colonies of the parent $\mathrm{R}$ strain illustrated in Fig. $1 . \quad \times 3.5$. 
THE JOURNAL OF EXPERIMENTAL MEDICINE VOL. 79

PLATE 1

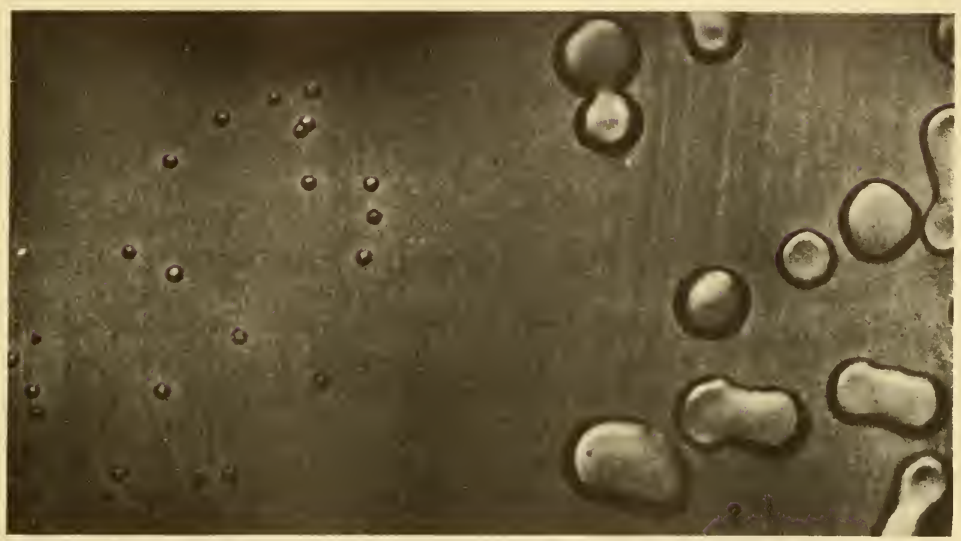

(Avery et al.: Transformation of pneumococcal types) 


\title{
INDUCED LYSOGENICITY AND MUTATION OF BACTERIOPHAGE WITHIN LYSOGENIC BACTERIA ${ }^{1}$
}

by

\author{
F. M. BURNET AND DORA LUSH \\ (From The Walter and Eliza Hall Institute, Melbourne).
}

(Submitted for publication 29th November, 1935.)

In the course of work on staphylococcal bacteriophages, we have encountered a curious relation between two phages, one of which was apparently a mutant derivative of the other. The only essential difference seemed to be that the original phage produced resistant cultures with extraordinary facility while the presumed mutant behaved in the normal fashion of any moderately active bacteriophage. When we attempted to elucidate the nature of this difference, attention was first concentrated on finding correlated differences between the two phages. No such differences were found. The nature of the resistant cultures then became the centre of interest. It was found that all the resistant forms appearing after the action of the original phage were lysogenic, producing at first phage of the "original" type. As the culture aged, the "mutant" phage made its appearance along with the "original"

Such relationships between phages, in the clear-cut form of this example, are unique in our experience. Similar, if less striking phenomena, however, have been recorded for other phages and bacteria, and the data obtained in the present work may perhaps be of value to some of those interested in the interactions between phage and susceptible bacterium.

The phages in question, $\mathrm{C}$ and $\mathrm{C}^{\prime}$, were described by Burnet and McKie in 1929 , but the nature of their difference was not then investigated. Phage $\mathrm{C}$ was obtained from rodent faeces and lysed only a white coccus SF, isolated from the same source. The phage produced very little clearing in broth eulture, though filtrates gave fairly good plaque count titres. The plaques were highly characteristic in appearance, very large for a coceal phage, about $34 \mathrm{~mm}$. in diameter and of annular form, the centre being invariably oceupied by a heavy growth of bacteria, and the clear ring only a little more than a millimetre across. An unusual but constant feature of the plaques is that they extend their diameter when the plate is incubated for periods beyond 24 hours. To some extent the central bacterial growth also expands, but this lags relatively to the lysis, and the sum effect

1 This work was carried out under a grant for work on virus diseases, from the Commonwealth Government Department of Health, and the Rockefeller Foundation.

[Reprinted from The Australian Journal of Experimental Biologiy and Medical Science $14: 27 \cdot 38,1936]$ 
is chiefly a widening of the ring of lysis. Phage $\mathrm{C}^{\prime}$ made its appearance after the pliage had been under study for some months, in the form of certain plaques identical with the normal ones except for the absence of the central growth of bacteria (Fig. 1). The plaques when isolated bred true, and phage $\mathrm{C}^{\prime}$ has maintained this characteristic since.

At the time of its appearance, no large plaque staphylococcal phage other than C had been isolated in the laboratory, so that the possibility of eontamination can be ruled out. The two phages are serologically identical (Burnet and Lush 19:35) and the further relations to be discussed below will make it clear that there is a true genetic relation between them : $\mathrm{C}^{\prime}$ must be regarded as a mutant of $\mathrm{C}$.

\section{The Type of Resistant Cultures Produced by the Two Phages.}

There is no difficulty in obtaining resistant cultures after the action of these two phages. If two plates are spread with a young culture of SF, and when this has dried a few drops of $\mathrm{C}$ and $\mathrm{C}^{\prime}$ spread on respective plates, after incubation the first will show a thin line of lysis marking the edge of the area on which the phage was spread (see Fig. 2), but over the rest of the area there is a confluent sheet of apparently normal culture. This central growth, however, is completely resistant to phage $\mathrm{C}$ and retains its character on subculture : it will be referred to as $\mathrm{SF} / \mathrm{C}$. The plate spread with $\mathrm{C}^{\prime}$ shows the usual appearance obtained after lysis with a moderately strong phage. There is an area of complete clearing on which are scattered a few well-developed resistant colonies and an occasional semilysed "nibbled" colony (Fig. 3). The intact colonies breed true and retain their resistance : they will be referred to as $\mathrm{SF} / \mathrm{C}^{\prime}$.

The properties of $\mathrm{SF}, \mathrm{SF}^{\prime} / \mathrm{C}$, and $\mathrm{SF} / \mathrm{C}^{\prime}$ may now be compared briefly. All show a similar type of growth on broth or agar, and the colonies are indistinguishable from one another by inspection. The effect of various phages lysing SF on the two variants is shown in Table 1 , which shows both the lytic effect on agar and the absorptive power of killed bacteria for the phages.

'TAISLE 1.

Characteristic's of Initial and I'hage-resistant Cultures.

\begin{tabular}{|c|c|c|c|c|c|c|c|c|c|c|c|c|}
\hline \multirow{5}{*}{$\begin{array}{l}\text { Culture. } \\
\text { SF }\end{array}$} & & & & & & & \multicolumn{6}{|c|}{$\begin{array}{l}\text { Absorption of phages (' and (") } \\
\text { by dead cultures. }\end{array}$} \\
\hline & \multicolumn{6}{|c|}{ Lytie action of phages shown } & \multicolumn{3}{|c|}{ Phage C. } & \multicolumn{3}{|c|}{ Phage $\mathrm{C}^{\prime}$} \\
\hline & A & B & (') & $\mathrm{C}^{\prime \prime}$ & D & $\mathrm{Au} \mathrm{I}$. & 2 & 20 & 200 & 2 & 20 & 200 \\
\hline & + & + & + & + & + & + & $100 \%$ & $60 \%$ & 0 & $80 \%$ & $40 \%$ & 0 \\
\hline & + & + & - & - & + & + & 0 & 0 & 0 & 0 & 0 & 0 \\
\hline $\mathrm{SF} / \mathrm{C}^{\prime}$ & + & - & - & - & + & + & 0 & 0 & 0 & 0 & 0 & 0 \\
\hline
\end{tabular}

Absorption of phages by bacteria was tested according to the technique previously described (Burnet and Lush 19:35), serial dilutions of heat-killed broth cultures being added to small constant amounts of phage, and the mixtures plated 
after 4 hours at $37^{\circ} \mathrm{C}$. The percentage reduction in plaque count is shown in the table.

The essential point to be noted is that $\mathrm{SF} / \mathrm{C}$ is also resistant to the action of phage $\mathrm{C}^{\prime}$. SF $/ \mathrm{C}^{\prime}$ is resistant to both $\mathrm{C}$ and $\mathrm{C}^{\prime}$ but differs from $\mathrm{SF} / \mathrm{C}$ in being also resistant to phage $\mathrm{B}$. These reactions are paralleled by the phage absorption results obtained with dead bacteria. Serologically no difference could be detected between SF and SF/C by cross absorption tests, but SF/C' is serologically distinguishable from SF by the same technique (Burnet and McKie 1929).

Most of the experiments to be described depend on the fact that organisms of SF/C type are never produced by phage $\mathrm{C}^{\prime}$, but when present can multiply normally in the presence of excess of this phage. This makes it possible to study fairly closely the influence of phage $\mathrm{C}$ in converting $\mathrm{SF}$ organisms into the heritable modification SF/C.

\section{The Production of $S F / C$ by Phage $C$.}

It is obvious from the appearance of its plaques that phage $\mathrm{C}$ provokes resistance with the greatest of ease. This can be established quantitatively in a number of ways. If one plates a series of dilutions of SF culture on normal agar plates and on plates spread with concentrated phage $\mathrm{C}$, the number of colonies is identical within the limits of experimental error in the two series, but on the phage spread plates the colonies are all of SF/C type.

In another type of experiment we have plated serial dilutions of $\mathrm{C}$ with SF as in a plaque titration, but, instead of allowing plaques to form, the areas are respread with strong phage $\mathrm{C}^{\prime}$ within half an hour of mixing $\mathrm{C}$ and SF. After incubation we find that the number of resistant colonies on each area is roughly proportional to the concentration of phage $\mathrm{C}$, and in some experiments approximates to the number of plaques obtained in a normal phage titration. A typical protocol is as follows:

Experiment 1. Plates of nutrient agar were spread with 0.1 c.c. of a 4 hour broth culture of SF. When these had dried, 0.02 c.e. quantities of serial dilutions of phage $\mathrm{C}$ in broth were spread on sectors according to the usual technique in phage titrations. Fifteen minutes later each sector was spread with a drop of undiluted phage $C^{\prime}$ to cover the whole of the area on which the $\mathrm{C}$ phage dilution had been placed. The plates were again allowed to dry, and incubated over night.

TABLE 2.

Relation between Concentration of Phage C and the Numbers of Resistant Colonies induced.

Concentration of phage C.

10-2

$10-3$

$10-4$

$10^{-5}$

Nil
Colonies appearing in the presence of excess of phage $\mathrm{C}^{\prime}$

Semi-confluent growth.

$500 \pm$

120,151

26,28

18,12 
TABLE 2A.

Type of Resistant Colonies Produced.

\begin{tabular}{|c|c|c|c|c|c|c|}
\hline \multirow{2}{*}{$\begin{array}{l}\text { Sources of colonies } \\
\text { (see Table II).. }\end{array}$} & \multirow{2}{*}{$\begin{array}{l}\text { Number of } \\
\text { colonies. }\end{array}$} & \multicolumn{4}{|c|}{ Lytic action of phages. } & \multirow{2}{*}{$\begin{array}{l}\text { Type of } \\
\text { resistant culture }\end{array}$} \\
\hline & & B & C & $C^{3}$ & $\mathrm{D}$ & \\
\hline Phage $C 10^{-}-$, excess $C^{\prime}$ & 11 & + & 一 & - & + & $\mathrm{SF} / \mathrm{C}$ \\
\hline Phage C $10^{-5}$, excess $C^{\prime}$ & 9 & - & 一 & - & + & $\mathrm{SF} / \mathrm{C}^{\prime}$ \\
\hline Excess phage $C^{\prime}$ alone & 10 & - & - & - & + & $\mathrm{SF} / \mathrm{C}^{\prime}$ \\
\hline
\end{tabular}

Table 2 shows the number of colonies which developed on each sector. In the absence of phage $\mathrm{C}$ about 15 colonies resistant to $\mathrm{C}^{\prime}$ appeared, and we can assume that on each of the other sectors about that number are also due directly to the influence of $\mathrm{C}^{\prime}$, the remainder representing the number of organisms which were rendered resistant to phage $\mathrm{C}$ before they came into effective contact with phage $\mathrm{C}^{\prime}$. This assumption could be tested directly as far as it applied to the sectors spread with $10^{-5}$ dilution of $\mathrm{C}$. Table $2 \mathrm{~A}$ shows that of 20 colonies tested from these sectors, approximately half were of the $\mathrm{SF} / \mathrm{C}$ type, the others being $\mathrm{SF} / \mathrm{C}^{\prime}$. In the absence of phage $\mathrm{C}$ all the colonies were as was to be expected of the $\mathrm{SF} / \mathrm{C}^{\prime}$ type. At a dilution of $10^{-5}$ then, phage $\mathrm{C}$ produces under these conditions 13 or 14 resistant bacteria. The same quantity of phage would give rise to 20-50 plaques as judged by previous titrations, but this was not actually determined in this experiment.

Several other experiments were carried out to determine the ratio between the number of plaques produced under normal conditions and the number of resistant colonies obtained in the present type of experiment. Table 3 shows the results of four experiments using the same batch of phage $C$, and essentially the same technique as in Experiment 1 . Certain variations were made in the time and conditions of contact between phage $\mathrm{C}$ and the sensitive culture before $\mathrm{C}^{\prime}$ was added, but they seemed to have no significant effect, and will not be discussed. In general the results show that allowing for the Dreyer and Campbell Renton effect the number of resistant colonies is roughly proportional to the amount of phage present, and, that as a rule, about $10-20$ p.c. of phage particles capable of giving rise to a plaque will, under these conditions, induce the appearance of a resistant colony.

\section{TABLE 3.}

Relation between Plaque Count and Number of Resistant Colonies.

\begin{tabular}{|c|c|c|c|c|c|}
\hline \multirow{2}{*}{$\begin{array}{c}\text { Phage } \mathrm{C} \\
\text { concentration. }\end{array}$} & \multirow{2}{*}{$\begin{array}{l}\text { Plaque } \\
\text { count. }\end{array}$} & \multicolumn{4}{|c|}{$\begin{array}{l}\text { Average number of colonies in each experiment. } \\
\text { Phage } C^{\prime} \text { being added } 15 \text { minutes after phage } C \text {. }\end{array}$} \\
\hline & & 1 & 2 & 3 & 4 \\
\hline $10-3$ & - & 158 & 320 & 185 & \pm 450 \\
\hline $10^{-4}$ & \pm 500 & 60 & 38 & 18 & 54 \\
\hline $10^{-\overline{-}}$ & 78 & 14 & $5 \cdot 5$ & $2 \cdot 0$ & 11 \\
\hline $2 \times 10^{-6}$ & 16 & - & $3 \cdot 0$ & $2 \cdot 6$ & $6 \cdot 0$ \\
\hline $\mathrm{Nil}$ & - & 4 & $1 \cdot 25$ & 0.5 & $3 \cdot 25$ \\
\hline
\end{tabular}


There is no necessity that phage $\mathrm{C}^{\prime}$ should be added subsequent to the action of C. Figures 2-4 show plates spread with a 1:100 dilution of a broth culture of $\mathrm{SF}$ and, subsequently, spread over a central area with (Fig. 2) phage C (Fig. 3) phage $\mathrm{C}^{\prime}$ and (Fig. 4) a mixture of the two. It will be seen that there are about 100 times as many colonies arising on (4) as on (3). Even if phage $\mathrm{C}$ is added 15 minutes after $\mathrm{C}^{\prime}$ there is a large increase in the number of resistant colonies over those produced by $\mathrm{C}^{\prime}$ alone.

In considering the time relationship in these experiments, it must be remembered that neither phage is very actively adsorbed to the susceptible organism, either when killed by heat (see Table 1) or in the living state. Even in the presence, as in all these experiments, of excess of bacteria it may be an hour or longer before any given phage particle makes effective eontact with a susceptible organism. During this period the bacterial population is also of course increasing in density, so that the conditions are too complex for any strictly quantitative interpretation of the results.

Certain points are, however, clearly established; the most important is that the appearance of $\mathrm{SF} / \mathrm{C}$ resistant colonies is due to the direct positive effect of phage $\mathrm{C}$ on the coccus SF and not to a selection of pre-existent variants. This latter alternative is in most examples of resistant production by phage very difficult to exclude, and is often apparently the most important factor (Burnet 1929). A glance at Figs. 3 and 4 show at once that, while the colonies on 4 (mixed phages) are obviously resistant to $\mathrm{C}^{\prime}, 99$ p.c. of the organisms from which they arose were not initially resistant to $\mathrm{C}^{\prime}$, since they fail to produce colonies on the plate sprear with this phage only.

The change from SF to SF/C under the influence of phage C must in some cases at least occur quite rapidly, probably within an hour, if the descendants are to be protected against the action of undiluted $C^{\prime}$ phage. Further, the experiments indicate that a remarkably high proportion of effective contacts between phage and bacteria must result in the induction of resistance rather than lysis. The difficulties associated with the slow adsorption of phage to bacteria made it impossible to give any unmerical estimate of this proportion, but it seems very unlikely that it is less than 5 p.c. On the evidence that when SF is plated on agar covered with strong phage $\mathrm{C}$ just as many resistant colonies appear as susceptible ones on ordinary agar, we were at first inclined to consider the possibility that 100 p.c. of contacts resulted in the appearance of resistant forms, phage multiplication taking place by some other process than the usual one. Probably a majority of the authors who have studied the question consider that in the initial stages of the action of phage on a susceptible culture, multiplieation of the lytic agent proceeds in the absence of any bacterial lysis. Bronfenbrenner (1928) in fact claims that there is a distinet growth-stimulating effect in the early stages. One of us (Burnet 1934) has argued that there is no experimental basis for this point of view, and 


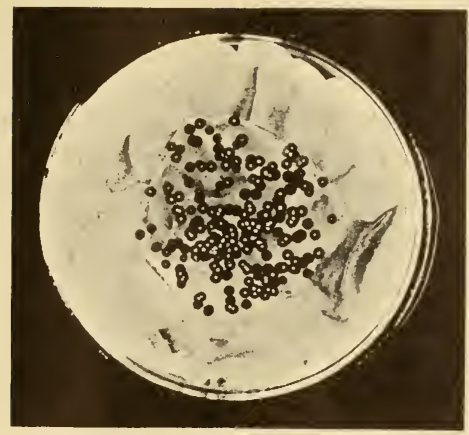

Fig. 1.

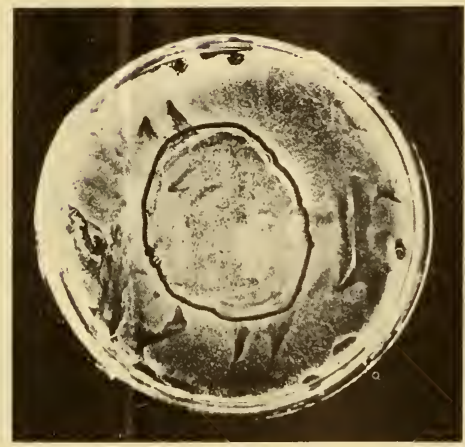

Fig. 2.

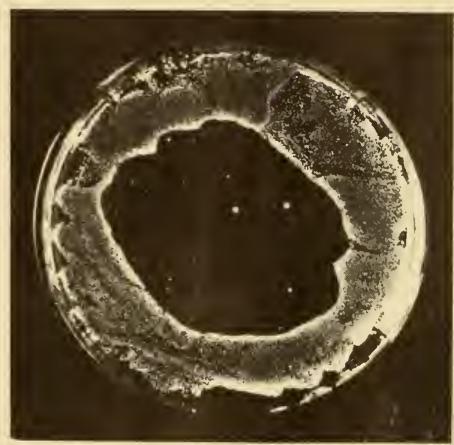

Fig. 3.

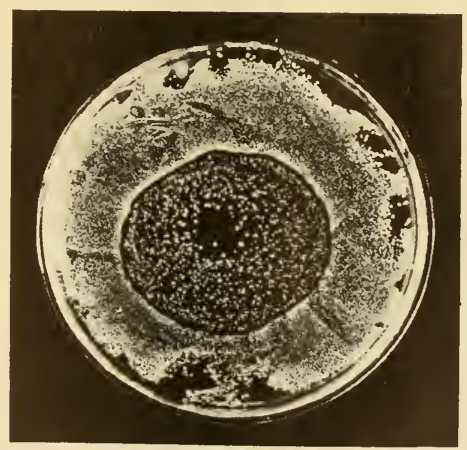

Fig. 4. 
that all the published experimental data are compatible with the hypothesis that increase in amount of phage results only from the disruption of infected bacteria. $A$ priori it seemed likely that if there were any exceptions to this rule the action of phage $\mathrm{C}$ on SF would be one of them. Phage $\mathrm{C}^{\prime}$ on the other hand was obviously a typical bacteriophage-producing only about one resistant colony per million bacteria lysed-and was known from previous experiments to multiply in normal fashion. A series of experiments was therefore carried out on the functional activities of phage $\mathrm{C}$, mostly in comparison with $\mathrm{C}^{\prime}$, in order to discover whether it showed any fundamental peculiarities of behaviour.

In their general intrinsic qualities the two phages are practically identical; serologically they are indistinguishable. Both are relatively resistant to the photodynamic action of methylene blue and to inactivation by strong urea solution, and both are unaffected by citrate in the medium. Functionally, too, there is extremely little difference between the two phages, apart from the greater readiness of $\mathrm{C}$ to produce resistant variants. On agar, plaques of each type can just be distinguished about 2 hours after spreading. The two types develop at the same rate, and the central bacterial growth of $\mathrm{C}$ is only clearly visible after about 5 hours. When lysis by the two phages is studied by direct microscopic observation on the agar surface, in each case first lysis is observed about $1 \frac{1}{4}-1 \frac{1}{2}$ hours after mixing. With $\mathrm{C}$, many more bacteria give rise to microcolonies than with $\mathrm{C}^{\prime}$, but typical disappearance of the bacterium by lysis is readily seen, and appears to be quite similar to that induced by $\mathrm{C}^{\prime}$.

Multiplication of phage $\mathrm{C}$ in broth follows the ordinary course, with, however, a longer initial lag than is found with dysentery phages.

Experiment 2. A tube containing a growing broth culture of SF was seeded with sufficient phage to give 2 or 3 plaques when 0.02 c.c. was plated. It was maintained at $37^{\circ} \mathrm{C}$.; and at suitable intervals eight such volumes were plated on agar. The plaque counts were as tabulated (Table IV), each figure being the average of eight counts.

TABLE 4.

Multiplication of Phage C in Broth at $37^{\circ} \mathrm{C}$.

$\begin{array}{ccccccc} & \text { Immediately. } & 30 \mathrm{~min} . & 45 \mathrm{~min} . & 60 \mathrm{~min} . & 75 \mathrm{~min} . & 90 \mathrm{~min} . \\ \text { Average plaque count. } & 2.0 & 1.75 & 2.4 & 2.25 & 23.0 & 160\end{array}$

Using the method of single particle multiplication (Burnet 1934) the same explosive increase occurred as is characteristic of all phages which have been

EXPLANATION OF TEXT FIGURES.

Fig. 1. Plate inoculated with SF and spread with a dilute mixture of phages $\mathrm{C}$ and $\mathrm{C}^{\prime}$. The plaques produced by phage $\mathrm{C}$ show central resistant growth while those of phage $\mathrm{C}^{\prime}$ have a clear centre.

Figs. 2, 3, and 4. Plates spread with 0.1 c.c. of 24 hour broth culture of SF diluted $1: 100$. After drying, 0.02 c.c. of the phages shown was spread over the central area. Fig. 2. Phage C undiluted stock filtrate. Fig 3. Phage $C^{\prime}$ undiluted stock filtrate. Fig. 4. A mixture of equal parts of phages $\mathrm{C}$ and $\mathrm{C}^{\prime}$. 
studied by this technique. The best of three consistent experiments gave the plaque counts shown in Table $4 \mathrm{~A}$ with phage C. In all experiments the first inerease indicated that $80-150$ phage particles were liberated from each infected bacterium, lysis occurring between 70 and 90 minutes after mixing. A suitable modification of this experiment showed that a similar explosive multiplication took place on the surface of agar, although in this instance the first increase appeared later, between 90 and 105 minutes.

TABLE $4 \mathrm{~A}$.

To Show Explosive Multiplication of Phage C.

Time of plating after phage and culture mixer.

$60-65$ minutes
$65-70 \quad$,
$70-75 \quad "$
$75-80 \quad "$
$80-85 \quad "$
$85-90 \quad "$
$90-95 \quad "$

\begin{tabular}{rrrrrrrr}
\multicolumn{7}{c}{ Plaque eounts obtained from each tube. } \\
0 & 1 & 0 & 0 & 0 & 3 & 0 & 0 \\
0 & 1 & 1 & 0 & 0 & 2 & 1 & 0 \\
2 & 0 & 0 & 1 & 0 & 0 & 1 & 1 \\
1 & 1 & 0 & 0 & 1 & 0 & 1 & 2 \\
1 & 0 & 1 & 1 & - & - & - & - \\
1 & 0 & 1 & 154 & 1 & 0 & 1 & 136 \\
0 & 0 & 186 & 0 & 0 & 79 & 0 & 2
\end{tabular}

Lysogenesis of $S F / C$.

The resistant culture SF / C which is so readily induced by the action of phage $\mathrm{C}$ is consistently lysogenic, producing in young eulture phage of $\mathrm{C}$ type, but in older cultures it gives rise to plaques of both $C$ and $C^{\prime}$ types. Both types like the original ones breed true after isolation and passage on SF.

A series of cultures of a strain of $\mathrm{SF} / \mathrm{C}$ which had been re-isolated three or four times since its induction by phage $\mathrm{C}$, was set up in broth $(\mathrm{pH} 8.0)$ and kept at $37^{\circ} \mathrm{C}$. Each day the lysogenicity was tested by filtering portion of a culture through a gradocol membrane (A.P.D. $0 \cdot 8 \mu$ ) and plating both filtrate and unfiltered culture on plates spread with SF. Details of one such experiment are given in Table 5.

TABLE 5.

\section{Lysogenesis of $S F / C$ Cultures of Different Ages.}

$\begin{array}{llc}\text { Time. } & \text { Unfiltered SF/C. } & \text { Gradocol membrane filtrate SF/C. } \\ 1 \text { day } & 1 \times 10^{6}, \text { all C type } & - \\ 2 \text { days } & 1 \times 10^{5} \text {, all } \mathrm{C} \text { type } & 3 \times 106, \text { all C type } \\ 4 \text { days } & 2 \times 10^{5}, 1 \% \mathrm{C}^{\prime} \text { type } & 2 \times 10 \mathrm{C}^{\prime}, 2 \% \mathrm{C}^{\prime} \text { type } \\ 5 \text { days } & 5 \times 10^{7}, 0.5 \% \mathrm{C}^{\prime} \text { type } & 2 \times 108,50 \% \mathrm{C}^{\prime} \text { type } \\ 6 \text { days } & 2 \times 10^{\circ}, 50 \% \mathrm{C}^{\prime} \text { type } & - \\ 7 \text { days } & 5 \times 10^{7}, 1 \% \mathrm{C}^{\prime} \text { type } & \end{array}$

In the majority of experiments $C^{\prime}$ appeared somewhat earlier, usually on the third day, a maximum mutation being reached on the fourth or fifth day. The test shown in Table 5 was carried over a longer period of time, and a marked drop in the amount of $\mathrm{C}^{\prime}$ present was noted on the seventh day. 
Apart from the change in type of the liberated phage with ageing of the culture, the strain $\mathrm{SF} / \mathrm{C}$ behaved as regards lysogenesis in the same fashion as any classical lysogenic strain, e.g. the enteritidis strain BTM described by Burnet and McKie (1929). Lysis of the strain by an unrelated phage failed to liberate any detectably greater amount of phage. In these experiments phage Au1 was used (Burnet and Lush 1935), and after lysis titration of the $\mathrm{C}$ or $\mathrm{C}^{\prime}$ phage present was carried out in the presence of excess of antiphage serum specific for phage Au1. The strain also resembled BTM in that when it was rendered resistant to another phage (in this case phage B) it still retained its characteristic lysogenicity, nor could the lysogenic quality be removed by successive subculture of $\mathrm{SF} / \mathrm{C}$ in the presence of an antiphage serum highly active against $\mathrm{C}$ and $\mathrm{C}^{\prime}$.

Some differences were observed in the time taken for phage $\mathrm{C}^{\prime}$ to appear from different $\mathrm{SF} / \mathrm{C}$ cultures, and with one the change in type failed to occur. A number of experiments were made in an attempt to control the rate of appearance of the variant phage, but no significant results were obtained.

The transformation of the incorporated $\mathrm{C}$ phage into $\mathrm{C}^{\prime}$ which occurs as the culture ages is a phenomenon of great interest, but very difficult to interpret. A clue is perhaps provided by the fact that in the later platings one frequently encounters plaques which show both $\mathrm{C}$ and $\mathrm{C}^{\prime}$ characteristics. In every case such double plaques indicate that from what was originally a $\mathrm{C}$ plaque a $\mathrm{C}^{\prime}$ variant has appeared and given rise to a sector of $\mathrm{C}^{\prime}$ clearing in the plaque. Change from $\mathrm{C}^{\prime}$ to $\mathrm{C}$ does not occur. It is reasonable to assume that the $\mathrm{C} \rightarrow \mathrm{C}^{\prime}$ change in this instance is via the resistant $\mathrm{SF} / \mathrm{C}$ form which is developing in the centre of the growing plaque. The phage particle which initiates the appearance of such a composite plaque is clearly different from those present in stock phage C filtrates or liberated from young cultures of SF/C in virtue of this greater ease with which the $\mathrm{C} \rightarrow \mathrm{C}^{\prime}$ transformation occurs. In some sense at least it must be regarded as an intermediate unstable form.

There are indications from two different directions that the liberation of phage $\mathrm{C}^{\prime}$ from ageing cultures of $\mathrm{SF} / \mathrm{C}$ is always associated with the destruction of the coccus from which it is derived. Unless this were the case, platings from unfiltered cultures on the susceptible strain SF would almost certainly show some $\mathrm{C}^{\prime}$ plaques in which there was a distinct single colony of $\mathrm{SF} / \mathrm{C}$. If we consider a single coccus lodging on the plate and within its first 3 or 4 generations giving rise to phage $\mathrm{C}^{\prime}$ the appearance resulting would be a typical $\mathrm{C}^{\prime}$ plaque with a single central colony, not the irregular accumulation of secondary colonies visible with a lens in the central region of a $\mathrm{C}$ plaque. Such plaques were carefully looked for but never seen. It is probable therefore that when the phage within a given coceus has developed the potentiality of giving rise to a $\mathrm{C}^{\prime}$ plaque, it destroys the coccus by lysis as soon as the latter is placed in or on fresh media in which growth can occur.

In the second place if we could convert the lysogenic strain SF/C by some 
means not involving the use of phage (" into a variant showing the same type of resistance as is shown by the natural non-lysogenic resistant strain sF ("), one might expect to ammul the produetion of phage (" if its liberation were dependent on actual lysis of the bacterim by internally appearing phage (". Referenee to Table 1 will show that by the aetion of phage li on s's (Y a variant having sF (" characters can be obtained. Two such variants SF/ ( $/ 3$ wele prepared and broth enltures tested over a period of 8 days. In neither instance did any (" type platues appear. ()n one oceasion a single plaque of a new type was found. This showed a clear centre, then a very narrow ring of secondary growth, then the usual elear outer zone. Phage clerived from this plaque bred true to type, consistently giving playnes of similar character. In general this strain $C^{\prime \prime}$ more elosely resembled (" than (", giving resistant variants of the SF (" type whieh were lysogenie, producing phage of (" type. The point to be stressed is that in the doubly resistant strain produced by the action of phage $B$ on $\mathbf{S} \mathbf{F}^{\prime / C}$, no mutation of phage $C$ to $C^{\prime}$ was observerl. It seems as if the rexistance indueed by $\mathrm{B}$ on $\mathrm{SF} / \mathrm{C}$ (or the almost similar besistance of the non-lysogenic sF/ (" obtained by the direct action of (" on the original $\mathrm{SF}^{\mathrm{F}}$ (enlture) is of a more fundamental character than the resistanee to (" shown by SF/C. The most reasonable interpretation is that sF/ ( resists (" becanse of a surface change preventing effective attachment of the phage, but is incapable of preventing its lytie aetion should it develop within the bacterimu from

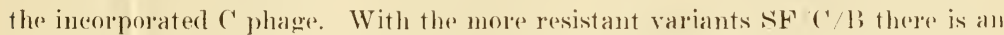
intrinsie resistanee to ("irresperetive of whethere attack is flom without or within or altermatively the change ( to (" is completely inhibiterl.

\section{DISCTRSTON.}

This investigation arose ont of a desire to elucidate the striking difference in appearance between the plaques of plagee ("and its mutant (", and has led us considerably further than we had anticipated. The actual form taken by the phemonena is almost migue as far as can be judged from the literatme and our own experience, and any defuctions are not immediately applieable to any oflere types of bacteriophage activity. In the eourse of the investigation. however, a number of points were established which may be of sufficient general interest to workers with bacteriophages to warrant publication.

In the first place the extraorelinary ease with which plage (" produeed resistant variants suggested strongly that if any phages were capable of multiplieation withont lysis of the sensitive baeterim this should be one of them. The experiment illustrated by 'Table ta shows conelusively that like erery other phage that has been tested, phage ('multiplies explosively, 80 or more fresh phage particles being liberated from a dispupted bacterium from 60 to 90 mimutes after phage was adeled to the culture. This provides a fresh eonfirmation of the correctness of d'Herelle's original view of the natme of phage multiplication. 
Secondly, the production of the resistant lysogenic strain $\mathrm{SF} / \mathrm{C}$ provides a clear-cut example of the direct positive induction of change in bacterial character by the aetion of a baeteriophage. In this instanee the alternative of seleetion by phage from pre-existent variants in the population submitted to lysis is definitely exeluded. The rapidity with which the ehange is induced is noteworthy. Within an hour of contact with phage $\mathrm{C}$ the surface of the bacterium has ehanged so that it no longer adsorbs either phage $\mathrm{C}^{\prime}$ or $\mathrm{C}^{\prime}$ and becomes insuseeptible to their action. This changed charaeter is then transmitted indefinitely to its deseendants. It is not possible to say whether this surface ehange results from an altered genetic constitution of the bacterium or is directly indueed by the associated phage at each generation. Aceording to Wollmann's hypothesis the distinction between the two alternatives would disappear, the phage being regarded as a gene re-introduced into the genetie make-up of the organism.

Finally, we have the demonstration of a definite mutation of a bacteriophage oecurring in the eourse of lysogenesis. Most aceounts of bacteriophage mutations are open to the same objections as can be raised against the elaims to have produced phage from normal baeterial cultures. In the present instance the mutation appears to affect only one aspect of the phage-that which determines the relative proportion of effective eontacts whieh give rise to lysis and resistance respectively - the other reeognizable eharacteristies remaining unehanged. Change in the character of a phage incorporated in a lysogenie strain has recently been described by d'Herelle and Rakieten (1934) and, although there is rarely any evidence of increase in vigour in these cases, the $\mathrm{C}^{\prime}$ to $\mathrm{C}^{\prime}$ change cannot be regarded as unique.

\section{SUMMARY.}

A bacteriophage (C) acting on the non-pathogenic white coceus $\mathrm{SF}$ is eharacterized by the unusual frequency with which it provokes the appearance of resistant forms. Each plaque shows a eentral growth of resistant culture and suitable experiments indicate that under the usual conditions of growth 10 or 20 p.c. of effective contacts between phage and susceptible bacterium result in the appearance of resistant lysogenic variants, the remainder initiating lysis in classical fashion. The resistant eulture shows no gross evidence of lytic aetion on agar, but in broth culture liberates considerable amounts of phage. In young eultures this phage is of the original type, but as the culture ages a variant phage $\mathrm{C}^{\prime}$ makes its appearance. This produces plaques of ordinary appearance without the central resistant growth, and gives only a small number of predominantly non-lysogenic resistant colonies after lysis of agar cultures. In all other respects, serological character, for example, the variant phage is indistinguishable from the parent phage C. Certain aspects of these phenomena are briefly discussed. 


\section{REFERENCES.}

Bronfenbrenner, J. J. (1928): "Filterable Viruses" (Ed. T. M. Rivers), Baltimore, p. 398. Burnet, F. M. (1929) : Brit. J. Exp. Path., 10, p. 109.

Burnet, F. M. (1932) : J. Path. Bact., Lond., 35, p. 851.

Burnet, F. M. (1934): Biol. Rev., 9, p. 332.

Burnet, F. M. and Lush, Dora (1935) : J. Path. Bact., Lond., 40, p. 455.

Burnet, F. M. and McKie, Margot (1929): Austral. J. exp. Biol., 6, p. 21, p. 277.

d'Herelle, F. and Rakieten, T. L. (1934) : J. infect. Dis., 54, D. 313 


\title{
MUTATIONS OF BACTERIAL VIRUSES AFFECTING THEIR HOST RANGE
}

\author{
S. E. LURIA \\ Indiana Universily, Bloomington, Indiana
}

Received July 8, 1944

\section{INTRODUCTION}

WHEN susceptible bacteria are spread on a solid culture medium with a large amount of a bacterial virus, complete lysis occurs after incubation, except for the appearance in some cases of colonies consisting of virus-resistant cells. These are the descendants of bacteria that had undergone a mutation from virus-sensitivity to virus-resistance prior to the action of the virus (LURIA and DELBR ÜCK 1943). The virus-resistant bacteria do not adsorb the virus. Their resistance is generally specific, not extending to unrelated viruses. Conversely, when a virus is plated with a suspension of bacteria resistant to its action, it generally dow not affect the bacteria; a uniform layer of bacterial growth results. We observed, however, that plating very large amounts of a virus with a resistant bacterial mutant strain occasionally results in the formation of a few clear "plaques"-that is, of a few virus colonies. From these plaques a new virus strain may be isolated that is active on the bacterial mutant resistant to the normal virus. A study of the origin of the new virus proved that it arises by mutation from the normal virus. A mutation of the virus can thus compensate for a mutation of the bacterial host. The present paper is concerned with the study of these virus mutations and of their relation to bacterial mutations.

Mutations affecting characters of bacterial viruses have been described before (Gratia i 936a; Burnet and Lush 1936). In i929, Sertic clearly recognized the occurrence of true breeding variants of bacterial viruses capable of attacking bacterial strains resistant to the original virus. He appears to have considered such variants as the result of an adaptation of the virus when in the presence of resistant bacteria.

\section{EXPERIMENTAL}

\section{Material and basic findings}

The material for the experiments described in this paper consisted originally of a strain of Escherichia coli B and of two viruses, $\alpha$ and $\gamma$, witive on strain B (DelbRÜCK and LURIA 1942). Virus $\alpha$ gives large plaques, virus $\gamma$ small plaques when plated with $\mathbf{B}$ on solid media. From strain B, a series of mutant strains can be isolated, some sensitive to virus $\alpha$ and resistant to virus $\gamma$, others sensitive to virus $\gamma$ and resistant to virus $\alpha$. These strains are obtained as secondary growths after lysis of B by virus $\gamma$ or virus $\alpha$. They are easily purified by repeated streak platings, and their resistance to one of the two viruses is

I Aided by a grant from the Dazian Foundation for Medical Research.

[Reprinted by permission from Genktics $30: 84-99$, January, 1945] 
generally found to be complete: the virus is not adsorbed by the bacterial cells and does not grow in their presence.

We have adopted in this paper the convention of naming the mutant bacterial strains by the Roman letter corresponding to the original strain, followed by the Greek letter corresponding to the virus in the presence of which they have developed as secondary growth after lysis. For example, strain $\mathbf{B} \alpha$ indicates a mutant from $\mathbf{B}$ isolated as secondary growth after $\mathbf{B}$ was lysed by virus $\alpha$. This mutant will generally be resistant to virus $\alpha$ (unless otherwise specified). Strains isolated in the presence of the same virus, but different in some properties (colonial morphology, range of sensitivity, etc.), are distinguished by sub-indexes: $\mathbf{B} \alpha_{1}, \mathbf{B} \alpha_{2}, \cdots$ Mutants obtained from other mutants are named by adding the Greek letter corresponding to the virus in the presence of which the new mutant has been isolated: $\mathrm{B} \alpha \gamma$, for example, will be a mutant from $\mathbf{B} \alpha$ isolated after lysis of $\mathbf{B} \alpha$ by virus $\gamma$, and will generally be resistant to both viruses $\alpha$ and $\gamma$. This notation offers the advantage that the name of each mutant strain mirrors its previous history, indicating the strain of origin and the virus, or viruses, whose selective action has brought about its isolation.

From strain $\mathbf{B}$, one can easily isolate two mutants, $\mathbf{B} \alpha_{1}$ and $\mathbf{B} \alpha_{2}$, differing in some growth characteristics but both resistant to virus $\alpha$ and sensitive to virus $\gamma$. Very exceptionally, one obtains from $\mathbf{B}$ a mutant $\mathbf{B} \gamma$, resistant to virus $\gamma$ and sensitive to virus $\alpha .^{2}$

Plating very large amounts of virus $\gamma$ with a suspension of cells $\mathbf{B} \gamma$ often produces some small, clear plaques, similar to the regular $\gamma$-plaques produced on B. The ratio of the number of plaques thus produced to the number of plaques produced by the same virus suspension when plated with strain $\mathbf{B}$ is generally very small (of the order of $10^{-7}$, occasionally up to $10^{-5}$ ). For a given suspension of virus, this ratio remains constant, at least for several months. The plaques produced on $\mathbf{B} \gamma$ being clear, they cannot be attributed to non-homogeneity of the bacterial suspension, with presence of some $\gamma$-sensitive cells: the action of a virus on a bacterial mixture partially sensitive results in the formation of plaques of turbid appearance, due to the growth of the resistant cells.

If some of the contents of one of the few plaques produced by a suspension of virus $\gamma$ on $\mathbf{B} \gamma$ are picked up and immediately plated again with a suspension of $\mathbf{B} \gamma$, a large number of plaques are obtained. This confirms the expectation that the plaque contained a large number of virus particles attacking $B \gamma$. By means of repeated one-plaque isolations and platings with $\mathrm{B} \gamma$, a pure virus strain is obtained which can be grown in liquid cultures of $\mathbf{B} \gamma$. This pure virus strain we called $\gamma^{\prime}$; its properties will be described in the following section. ${ }^{3}$

It may be stated here that the presence of virus $\gamma^{\prime}$ has been found in concen-

\footnotetext{
${ }^{2}$ The strains $\mathbf{B} \gamma$ and $\mathbf{B} \alpha_{1}$ have been called $\mathrm{A}$ and $\mathrm{C}$ in previous papers (DELBR $\ddot{C} \mathrm{CK}$ and LURIA 1942), in which the interest was focused on their use as indicator strains for plating mixed virus suspensions rather than on their mutational origin.

${ }^{3}$ Several of the experiments with virus $\gamma^{\prime}$ were done using a strain $\mathbf{B} \gamma \alpha_{2}$ instead of strain $\mathbf{B} \gamma$, because our stock of strain B $\gamma$ (obtained from strain B in I94I) had recently, in the course of subculturing, become partially sensitive to virus $\gamma$. Strain $\mathbf{B} \gamma \alpha_{2}$, derived from $\mathbf{B} \gamma$ before this occurred, behaved toward viruses $\gamma$ and $\gamma^{\prime}$ exactly like the original strain $\mathbf{B} \gamma$.
} 
trated suspensions of virus $\gamma$ prepared directly from one $\gamma$-particle (one $\gamma$ plaque transferred to a liquid culture of $\mathbf{B}$, incubated until lysis, lysate filtered). This proves that virus $\gamma^{\prime}$ does not represent an initial non-homogeneity of the stock of virus $\gamma$, but arises from normal particles of virus $\gamma$.

We have said that at least two different mutants, $\mathrm{B} \alpha_{1}$ and $\mathrm{B} \alpha_{2}$, can be isolated after lysis of B by virus $\alpha$. Plating any amount of virus $\alpha$ with bacteria $B \alpha_{1}$ does not result in any effect on the bacterial growth. We found, however, that plating very concentrated suspensions of virus $\alpha$ with cells of strain $\mathbf{B} \alpha_{2}$, characterized by slow and limited growth on nutrient agar, often results in the formation of a few clear plaques. These plaques contain a true breeding virus $\alpha^{\prime}$, active on strains $\mathrm{B}$ and $\mathrm{B} \alpha_{2}$, but not on $\mathrm{B} \alpha_{1}$. The properties of this virus $\alpha^{\prime}$ will be discussed in a later section.

The culture media used in this study were: nutrient broth +0.5 per cent $\mathrm{NaCl}$ for liquid cultures; the same plus I.I per cent powdered agar for platings. All experiments were performed in water-baths or incubators at $37^{\circ} \mathrm{C}$. The technique for grow th curves of bacteria, grow th experiments of the viruses, and experiments on interference between viruses have been described in detail in a previous paper (DELBRÜCK and LURIA 1942). The meaning of certain terms used hereafter, however, may be recalled: constant period = minimum time between infection of a bacterium by virus and its lysis with virus liberation; burst size = average yield of virus particles per lysed bacterium; infective cen$t e r=$ anything that produces one plaque when plated with sensitive bacteria. An infective center, therefore, can be either a free particle of virus or a bacterium infected by the virus; both of them will give just one plaque when plated with sensitive bacteria; efficiency of plating = the ratio between the number of plaques produced by a virus suspension in a given plating and the maximum number of plaques which that suspension can give when plated under optimum conditions. The latter has been shown to correspond very closely to the actual number of active virus particles present (DELBRÜCK and LURIA 1942).

\section{Properties of virus $\gamma^{\prime}$}

Each one of the plaques obtained by plating a suspension of virus $\gamma$ with $\mathrm{B} \gamma$ is capable of yielding a strain of virus $\gamma^{\prime}$, which can be purified by repeated platings with $\mathbf{B} \gamma$ and one-plaque isolations, since normal virus $\gamma$ does not grow on bacteria $\mathbf{B} \gamma$. After isolation and purification, virus $\gamma^{\prime}$ can be grown in liquid cultures of either bacteria $\mathrm{B}$ or $\mathrm{B} \gamma$; filtrates of such cultures yield stable stocks. Whether grown on strain B or on strain B $\gamma$, the particles of virus $\gamma^{\prime}$ show exactly the same properties, as hereafter described.

The plaques produced by virus $\gamma^{\prime}$ on B are small, sharp edged, and indistinguishable from those produced by virus $\gamma$. Those produced on $\mathrm{B} \gamma$ can be distinguished with some experience mainly because of their slightly larger size.

An interesting feature of virus $\gamma^{\prime}$ is that of giving a smaller number of plaques when plated with strain $\mathbf{B} \gamma$ than with $\mathbf{B}$. The efficiency of plating on $\mathbf{B} \gamma$ varies from 0.2 to 0.6 of that on $\mathbf{B}$. It seemed important to investigate whether or not this difference in the efficiency of plating was due to non- 
homogeneity of the particles of virus $\gamma^{\prime}$. It could be imagined that the $\gamma^{\prime}$-particles in reproducing gave origin to a certain proportion of particles of type $\gamma$, and therefore active on B only. This was excluded by experiments of the following type: Virus $\boldsymbol{\gamma}^{\prime}$ was plated with cells $\mathbf{B}$ in amounts that gave a few hundred plaques. After incubation, the contents of each of 20 plaques were picked up with a needle, inoculated into separate samples of a suspension of cells $\mathbf{B} \gamma$, and immediately plated. All the plates showed numerous plaques, proving that all the plaques produced by the suspension of virus $\gamma^{\prime}$ on $\mathbf{B}$ actually contained virus $\gamma^{\prime}$.

Another conceivable possibility was that a certain proportion of the particles in a suspension of virus $\gamma^{\prime}$ produced virus $\gamma^{\prime}$ when growing on $\mathbf{B}$, but were not themselves capable of attacking cells of type $\mathbf{B} \gamma$. This possibility was excluded by experiments of the following kind, directed to prove that all particles in a suspension of virus $\gamma^{\prime}$ can attack B $\gamma$ in liquid media: A definite amount of virus $\boldsymbol{\gamma}^{\prime}$, after titration with both bacteria $\mathbf{B}$ and $\mathbf{B} \gamma$, was added to an excess of growing cells $\mathbf{B} \gamma$. After allowing a few minutes for adsorption (ro minutes, that is, much less than the constant period; see below), the mixture was divided into two portions. One portion was centrifuged for titration of the free virus in the supernatant, the other portion was tested for the number of infective centers, by plating separately with $\mathbf{B}$ and with $\mathbf{B} \gamma$. Since no liberation of new virus had yet taken place, each infective center represented either a free virus particle or an infected bacterium. If those particles of virus $\gamma^{\prime}$ that give plaques on $\mathbf{B}$ and not on $\mathbf{B} \gamma$ are actually unable to attack $\mathbf{B} \gamma$, they will remain free and not appear as infective centers on $\mathbf{B} \gamma$. If, on the contrary, they can be adsorbed by $\mathbf{B} \gamma$, they will account for a part of the infected bacteria. As such, they are likely to appear as infective centers on $\mathbf{B} \gamma$, because each infected bacterium later liberates a large number of virus particles, thereby increasing the chances of plaque formation. This will result in an increase of the number of infective centers relative to the original input of virus as measured by plating with $\mathbf{B} \gamma$. Table I shows the results of three such experiments.

It is seen that the number of infective centers on $\mathbf{B} \gamma$ after adsorption by $\mathbf{B} \gamma$

TABLE I

The number of infective centers of virus $\gamma^{\prime}$ after adsorption by cells $\mathbf{B} \gamma$.

\begin{tabular}{|c|c|c|c|c|c|c|}
\hline & \multicolumn{2}{|c|}{$\begin{array}{c}\text { EXPERIMENT } \\
\text { NO. } 13\end{array}$} & \multicolumn{2}{|c|}{$\begin{array}{l}\text { EXPERIMENT } \\
\text { NO. I4 }\end{array}$} & \multicolumn{2}{|c|}{$\begin{array}{l}\text { EXPERIMENT } \\
\text { NO. } 16\end{array}$} \\
\hline & $\begin{array}{l}\text { PLATING } \\
\text { WITH B }\end{array}$ & $\begin{array}{l}\text { PLATING } \\
\text { WITH B } \gamma\end{array}$ & $\begin{array}{l}\text { PLATING } \\
\text { WITH B }\end{array}$ & $\begin{array}{l}\text { PLATING } \\
\text { WITH Br }\end{array}$ & $\begin{array}{l}\text { PLATING } \\
\text { WITH B }\end{array}$ & $\begin{array}{l}\text { PLATING } \\
\text { WITH By }\end{array}$ \\
\hline Virus input per cc & $27 \times 10^{6}$ & $7 \times 10^{6}$ & $35 \times 10^{6}$ & $7 \times 10^{6}$ & II $X 10^{6}$ & $3 \times 10^{6}$ \\
\hline Free virus per cc & $8 \times 10^{6}$ & $2.7 \times 10^{6}$ & $8 \times 10^{6}$ & $2.5 \times 10^{6}$ & $2.7 \times 10^{6}$ & I. I X $10^{6}$ \\
\hline $\begin{array}{l}\text { Infective centers after adsorption } \\
\text { per cc }\end{array}$ & $28 \times 10^{6}$ & $27 \times 10^{6}$ & $35 \times 10^{6}$ & $28 \times 10^{6}$ & $1 \mathrm{I} .5 \times 10^{6}$ & $10.5 \times 10^{6}$ \\
\hline
\end{tabular}


Growth of virus $\gamma^{\prime}$ on bacterial strains $\mathrm{B}$ and $\mathrm{B} \gamma$ in broth at $37^{\circ} \mathrm{C}$.

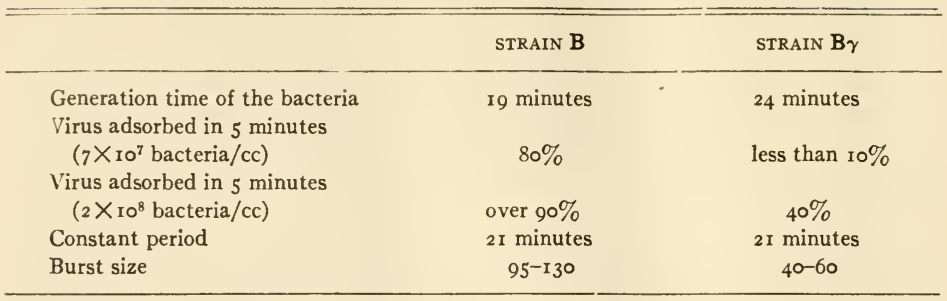

is almost as high as the number of infective centers on $\mathbf{B}$. The differences are partly accountable for by the free virus, partly by sampling errors. These experiments prove that all particles in a suspension of virus $\gamma^{\prime}$ are capable of attacking cells $\mathbf{B} \gamma$ - that is, they are all $\gamma^{\prime}$-particles.

The low efficiency of plating of virus $\gamma^{\prime}$ with $\mathbf{B} \gamma$ must, therefore, be due to some reason other than those considered above. The most likely explanation is that it is a result of the very low adsorption rate of the virus by these cells, as shown below. Many particles of virus may either remain unadsorbed on the agar or be adsorbed by bacteria too late to produce a fully developed, visible plaque. Any such explanation is bound to be tentative, in view of our incomplete knowledge of the process of nlaque formation.

The interaction of virus $\gamma^{\prime}$ with bacteria $\mathbf{B}$ and $\mathbf{B} \gamma$ was studied by means of adsorption experiments and "one-step grow th" experiments (DELBRÜCK I942). The results are given in table 2. The interaction of virus $\gamma^{\prime}$ with bacteria of strain B is in every respect similar to that of virus $\gamma$ (DELBRÜCK and LURIA I942). Strain $B \gamma$ adsorbs virus $\gamma^{\prime}$ much more slowly than strain $B$. In order to obtain measurable adsorption, the experiments with $\mathbf{B} \gamma$ had to be done with older cultures, containing more cells than those used for $\mathbf{B}$. The cells in such cultures are likely to be of smaller size, and this makes the values for the adsorption rates and also for the burst size not strictly comparable. An experiment with cells $\mathbf{B}$ grown to reach the same concentration as used for $\mathbf{B} \gamma$, however, showed a burst size of 95 - that is, not so low as for $\mathbf{B} \gamma$.

It is interesting to notice that, whereas the bacterial strain $\mathbf{B} \gamma$ has a longer generation time than $B$, virus $\gamma^{\prime}$ grows on $B \gamma$ with the same constant period $d \mathrm{~s}$ on $\mathbf{B}$. The burst size, however, is smaller. The mutation $\mathbf{B} \rightarrow \mathbf{B} \gamma$ involves, besides the change in virus sensitivity, changes in the rate of bacterial division and in the yield of virus $\gamma^{\prime}$ per cell.

\section{Interference of virus $\gamma$ with the growth of virus $\gamma^{\prime}$}

Interference between different bacterial viruses growing on the same host has been described and found to conform to the general rule that one cell liberates only virus of one type (DELBRÜCK and LURIA I942; DELBRÜCK 1944). It was concluded previously that interference also occurs between particles of the same virus strain: when a cell is attacked by several particles of the same strain 
(multiple infection), the result is the same as with single infection, as if only one particle could grow in a cell. Direct proof of this "self-interference" was difficult to obtain, since the offspring of a virus particle is indistinguishable from that of another particle of the same virus. Interference with the growth of virus $\gamma$ by an excess of ultraviolet inactivated virus $\gamma$ (LURIA and DELBRÜCK I942) was an indirect confirmation of the occurrence of self-interference.

The availability of virus $\gamma^{\prime}$, identical with virus $\gamma$ in its behavior toward cells of strain $\mathbf{B}$, but traceable by its activity on strain $\mathbf{B} \gamma$, offered an opportunity for further study of self-interference. The experiments were done by adding both viruses $\gamma$ and $\gamma^{\prime}$, the former in excess, to cells B, then using B $\gamma$ as a plating indicator for virus $\gamma^{\prime}$. If virus $\gamma$ interferes with the growth of virus $\gamma^{\prime}$, a bacterium infected first with virus $\gamma$, then with virus $\gamma^{\prime}$, will not liberate any virus $\gamma^{\prime}$ and will not give a plaque when plated with $\mathbf{B} \gamma$. A loss of infective centers will result.

We give here the data from such an experiment, in which the results were made more clear by the use of anti-virus serum to eliminate the free virus. DELBRÜCK (I 944) has found that anti-virus serum inactivates free virus but generally does not affect the growth of virus particles already adsorbed by bacteria.

A culture of $\mathbf{B}$ was divided into two portions. At time zero one portion received an excess of virus $\gamma$, and one minute later a smaller amount of virus $\gamma^{\prime}$. The other portion received only virus $\gamma^{\prime}$. After five minutes, the cultures were diluted into tubes containing a dilution of anti- $\gamma$ serum sufficient to reduce the amount of free virus (both $\gamma$ and $\gamma^{\prime}$ ) to about I per cent in three minutes (see a later section). At the eighth minute the mixtures were highly diluted into broth to stop the action of serum, and the infective centers of virus $\gamma^{\prime}$ were measured by plating with $\mathbf{B} \gamma$ at $\mathbf{I} 2$ and $\mathrm{I} 7$ minutes - that is, before liberation of new virus had taken place. Other platings were done to determine the amounts of free viruses. The results may be summarized as follows:

\begin{tabular}{|c|c|c|}
\hline & 1 portion & 11 portio \\
\hline Bacteria/cc & $8 \times 10^{7}$ & $8 \times 10^{7}$ \\
\hline Absorbed virus $\gamma / \mathrm{cc}$ & $80 \times 10^{7}$ & \\
\hline Adsorbed virus $\gamma^{\prime} / c c$ & $2 \times 10^{7}$ & $2 \times 10^{7}$ \\
\hline Infective centers $\gamma^{\prime} / \mathrm{cc}$ & $0.2 \times 10^{7}$ & $2 \times 10^{7}$ \\
\hline
\end{tabular}

It is seen that in this experiment about 90 per cent of the bacteria infected with both viruses $\gamma$ and $\gamma^{\prime}$ failed to liberate any virus $\gamma^{\prime}$. Other experiments, in which anti-virus serum was not used, gave results of the same type, although the presence of free virus $\gamma^{\prime}$ made the loss of infective centers less conspicuous. If we are justified in considering virus $\gamma^{\prime}$ as identical to virus $\gamma$ in its action on strain B, we can view these experiments as a direct proof of the occurrence of interference between similar virus particles adsorbed by the same cell.

\section{Properties of virus $\alpha^{\prime}$}

Virus $\alpha^{\prime}$, as isolated from the few plaques obtained by plating virus $\alpha$ with $\mathbf{B} \alpha_{2}$, is indistinguishable from virus $\alpha$ in its activity on bacteria of strain $\mathbf{B}$. The 
plaques are identical, and so is the growth in liquid medium: constant period I 3 minutes, burst size I IO-I 30 in broth at $37^{\circ} \mathrm{C}$. Plated with bacteria of strain $\mathrm{B} \alpha_{2}$, a suspension of virus $\alpha^{\prime}$ gives a smaller number of plaques than with $\mathrm{B}$. The efficiency of plating is $0.25-0.7$. The plaques are of smaller and more variable size. In liquid medium, bacteria $\mathrm{B} \alpha_{2}$ adsorb virus $\alpha^{\prime}$ extremely slowly, so that reliable adsorption measurements are difficult to obtain. The constant period for growth in broth at $37^{\circ} \mathrm{C}$ is $\mathrm{I} 3$ minutes, similar to the growth on $\mathbf{B}$. The burst size is difficult to determine with any degree of accuracy, because the presence of large amounts of unadsorbed virus disturbs the calculation of the number of infected bacteria. From an experiment in which at least a great part of the free virus was eliminated by means of anti-serum, we obtained for the burst size a minimum value of 55 .

There are some indications that strains of virus $\alpha^{\prime}$ independently isolated may differ from one another. The size of the plaques produced on the same bacterial strain is sometimes different. This point has not yet been further investigated.

\section{The mutational origin of viruses $\alpha^{\prime}$ and $\gamma^{\prime}$}

The presence of virus $\alpha^{\prime}$ in suspensions of virus $\alpha$ and that of virus $\gamma^{\prime}$ in suspensions of virus $\gamma$ are demonstrated by plating a large amount of the suspensions with bacteria resistant to the normal virus. We observe only the end result-that is, the appearance of a few plaques containing a new type of virus. Several alternative modes of origin of the new virus are $a$ priori conceivable. Hypothesis I: There is a small finite probability that, when plated with $r e-$ sistant bacteria, a normal virus particle succeeds in attacking one of the resistant cells; when this happens, then the particle will give rise to a virus strain capable of attacking the bacteria resistant to the original virus. Hypothesis 2: There is a small finite probability that a normal virus particle in a culture of virus growing on sensitive bacteria mutates, becoming hereditarily capable of attacking the resistant bacteria. Hypothesis 3 : There are in a sensitive bacterial culture some exceptional, abnormal bacteria which, when infected by a normal virus particle, produce virus of the new type instead of the normal type.

As far as hypotheses $I$ and 2 are concerned, the situation is similar to that encountered in the study of virus-resistant bacteria from virus-sensitive bacterial strains, and analogous considerations apply (LURIA and DELBRÜCK 1943).

According to hypothesis $\mathrm{I}$, on the one hand, the number of wirus particles that succeed in giving plaques on the resistant bacteria should be proportional to the number of virus particles tested. This will be true whether these particles come from the same virus culture or from different cultures, since the particles that produce plaques on the resistant bacteria are normal particles at the time of plating, and the probability of producing a plaque is assumed to be uniform for all particles. If we test a large number of samples each containing the same amount of normal virus, the numbers of plaques produced on the resistant bacteria should show only the fluctuations due to the sampling er- 
ror. These numbers should therefore show a Poisson distribution (variance = mean).

According to hypothesis 2, on the other hand, the new type of virus particles stem from mutations occurring during the growth of normal virus on sensitive bacteria, prior to the test. If a mutation occurs before the growth of the virus is completed, the mutant particle will multiply on the normal bacteria and give rise to a clone of mutant particles. The earlier a mutation occurs, the larger the clone will be. If we test a large number of samples, each containing the same amount of normal virus, the result will be different, depending on whether the samples come from the same virus culture or from different virus cultures. If we test different samples of the same culture, we shall again find a Poisson distribution of the number of plaques produced on resistant bacteria. If, however, we test a series of similar virus cultures, all started with a few sensitive bacteria and a few normal virus particles and all containing the same final amount of virus, the numbers of plaques produced will show a distribution with a variance much higher than the mean, because of the presence of clones of mutant particles.

The situation in the case of bacterial viruses is more complicated than in the case of bacteria. The virus particle multiplies by infecting a sensitive bacterium, which, after a latent period, liberates a hundred or more new particles. The mechanism of multiplication of the virus inside the bacterial cell is not known. If the new type of virus arises by mutation during the growth of the normal virus, the distribution of the number of mutant particles will depend on the modalities of the growth of the virus inside the cell.

As far as hypothesis 3 is concerned, if the new type of virus is produced by some abnormal cells, these may be expected to liberate a full burst of particles of the new type. Therefore, we should find that in each virus culture the particles of the new type occur in clones averaging the burst size.

The three hypotheses thus lead to different predictions regarding the distribution of the numbers of plaques produced on resistant bacteria by a series of similar cultures of virus. Accordingly, experiments were undertaken to determine this distribution. In these experiments, a small number of bacteria of strain B (about $\mathrm{IO}^{3} / \mathrm{cc}$ ) were added to a broth suspension of either virus $\alpha$ or virus $\gamma$ containing $10^{3}-10^{4}$ particles/cc. The mixture was immediately divided into portions of 0.2 or $0.5 \mathrm{cc}$ and these were incubated at $37^{\circ} \mathrm{C}$. Upon incubation, the cultures of virus $\gamma$ always remained clear (complete lysis, due to the rarity of the mutation $\mathbf{B} \rightarrow \mathbf{B} \gamma$ ). Cultures of virus $\alpha$ generally also gave complete lysis; only occasionally a few cultures prepared under such conditions showed secondary growth of resistant bacteria. This is explained by the fact that generally in such cultures complete lysis takes place before the bacteria reach a titer high enough to render the occurrence of mutations $B \rightarrow B \alpha$ likely. If exceptionally a mutation $\mathbf{B} \rightarrow \mathbf{B} \alpha_{1}$ occurs, the bacteria $\mathbf{B} \alpha_{1}$, resistant to both viruses $\alpha$ and $\alpha^{\prime}$, grow to saturation. If a mutation $\mathrm{B} \rightarrow \mathbf{B} \alpha_{2}$ occurs, the mutant cells grow to saturation if no $\alpha^{\prime}$-particle is present. If $\alpha^{\prime}$-particles are present, they are adsorbed by the cells $B \alpha_{2}$ when the concentration of the latter is high enough, and the result is a culture containing a large amount of virus $\alpha^{\prime}$, com- 
TABLE 3

Distribution of the numbers of plaques produced on $\mathrm{B} \alpha_{2}$ by a series of similar cultures of virus $\alpha$.

\begin{tabular}{|c|c|c|c|c|c|}
\hline EXPERIMENT NO. & II 2 & II 3 & II 4 & I $55^{a}$ & II $5 \mathrm{~b}$ \\
\hline Number of cultures tested & 20 & 34 & 87 & II & 40 \\
\hline $\begin{array}{l}\text { Volume of each culture, cc } \\
\text { Virus } \alpha \text { per culture }\end{array}$ & $1.2 \times 10^{8}$ & $\begin{array}{c}.5 \\
7 \times 10^{8}\end{array}$ & $8.9 \times 10^{8}$ & $\begin{array}{c}.5 \\
9 \times 10^{8}\end{array}$ & $\frac{.5}{7.8 \times 10^{8}}$ \\
\hline $\begin{array}{l}\text { NUMBER OF PLAQUES } \\
\text { PRODUCED ON B } \alpha_{2}\end{array}$ & $\begin{array}{l}\text { NUMBER OF } \\
\text { CULTURES }\end{array}$ & $\begin{array}{l}\text { NUMBER OF } \\
\text { CULTURES }\end{array}$ & $\begin{array}{l}\text { NUMBER OF } \\
\text { CULTURES }\end{array}$ & $\begin{array}{l}\text { NUMBER OF } \\
\text { CULTURES }\end{array}$ & $\begin{array}{l}\text { NUMBER OF } \\
\text { CULTURES }\end{array}$ \\
\hline 0 & I6 & 9 & 3 & 2 & 6 \\
\hline I & I & 5 & 2 & 2 & I \\
\hline 2 & 3 & 6 & I & 2 & 2 \\
\hline 3 & $\circ$ & 2 & 2 & I & 4 \\
\hline 4 & $\circ$ & $\mathbf{I}$ & 2 & $\circ$ & I \\
\hline 5 & $\circ$ & $\mathbf{I}$ & 4 & 0 & I \\
\hline 6 & 0 & I & 3 & $\circ$ & I \\
\hline 7 & ० & $\circ$ & 2 & $\circ$ & 2 \\
\hline 8 & $\circ$ & I & I & $\circ$ & I \\
\hline 9 & $\circ$ & I & 2 & $\circ$ & $\circ$ \\
\hline 10 & $\circ$ & $\circ$ & I & $\circ$ & $\circ$ \\
\hline $11-20$ & $\circ$ & 3 & II & I & 9 \\
\hline $2 \mathrm{I}-50$ & $\circ$ & I & 14 & 2 & 10 \\
\hline $5 \mathrm{I}-100$ & $\circ$ & I & 13 & I & I \\
\hline $101-1000$ & $\circ$ & I & $2 \mathrm{I}$ & $\circ$ & I \\
\hline over rooo & $\circ$ & I & 5 & $\circ$ & $\circ$ \\
\hline Average per culture & .35 & 45 & 125 & 13.7 & 15.6 \\
\hline Variance & .57 & 1200 & 45,000 & 570 & 380 \\
\hline
\end{tabular}

parable to its titer of virus $\alpha$. In our experiments all these possibilities were actually realized: we found several cultures with growth of $\mathbf{B} \alpha_{1}$, one culture with growth of $\mathrm{B} \alpha_{2}$, and one culture completely lysed but containing such a large amount of virus $\alpha^{\prime}$ as to suggest that virus $\alpha^{\prime}$ had multiplied on a secondary growth of $\mathbf{B} \alpha_{2}$.

After incubation, the completely lysed cultures were tested for the amount of virus $\alpha$ (or virus $\gamma$ ) and for the number of plaques produced on $\alpha$-resistant (or $\gamma$-resistant) bacteria. For the latter purpose, the whole contents of each culture were plated according to the technique devised by GRATIA (1936b) and by Hershey et al. (1943). The results are given in tables 3 and 4 . The amounts of virus $\alpha$ (or virus $\gamma$ ) represent averages; the individual counts of normal viruses in different cultures within each experiment never vary more than can be accounted for by the sampling errors. In considering the counts from the platings with the resistant bacteria, we must remember that for both virus $\alpha^{\prime}$ and virus $\gamma^{\prime}$ the efficiency of plating is low and slightly variable from one experiment to another. The plaque counts should be too low by a factor corresponding to the efficiency of plating. It is worth recalling that this factor, if constant within one experiment, should not affect the type of distribution if 
TABLE 4

Distribution of the numbers of plaques produced on $\mathbf{B} \gamma$ by a series of similar cultures of virus $\gamma$.

\begin{tabular}{|c|c|c|c|c|c|c|}
\hline EXPERIMENT NO. & 27 & $28 \mathrm{a}$ & $28 \mathrm{~b}$ & $28 \mathrm{c}$ & $28 d$ & 29 \\
\hline $\begin{array}{l}\text { Number of cultures tested } \\
\text { Volume of each culture, cc } \\
\text { Virus } \gamma \text { per culture }\end{array}$ & $\begin{array}{l}20 \\
20 \times 10^{8}\end{array}$ & $\begin{array}{l}9 \\
.2 \\
16 \times 10^{8}\end{array}$ & $\frac{9}{15 \times 10^{8}}$ & $\frac{9}{22 \times 10^{7}}$ & $\begin{array}{c}9 \\
6 \times 10^{7}\end{array}$ & $\begin{array}{c}40 \\
15 \times 10^{7}\end{array}$ \\
\hline $\begin{array}{l}\text { NUMBER OF PLAQUES } \\
\text { PRODUCED ON B } \gamma\end{array}$ & $\begin{array}{l}\text { NUMBER } \\
\text { OF } \\
\text { CULTURES }\end{array}$ & $\begin{array}{l}\text { NUMBER } \\
\text { OF } \\
\text { CULTURES }\end{array}$ & $\begin{array}{l}\text { NUMBER } \\
\text { OF } \\
\text { S CULTURES }\end{array}$ & $\begin{array}{l}\text { NUMBER } \\
\text { OF } \\
\text { CULTURES }\end{array}$ & $\begin{array}{l}\text { NUMBER } \\
\text { OF } \\
\text { CULTURES }\end{array}$ & $\begin{array}{l}\text { NUMBER } \\
\text { OF } \\
\text { CULTURES }\end{array}$ \\
\hline 0 & I & 2 & 3 & 6 & 8 & 25 \\
\hline $\mathbf{I}$ & ० & $\circ$ & ० & $\mathbf{I}$ & 0 & 8 \\
\hline 2 & o & 2 & 4 & 2 & 0 & 2 \\
\hline 3 & I & I & I & ० & 0 & 3 \\
\hline 4 & 2 & 2 & $\circ$ & ० & $\circ$ & 2 \\
\hline 5 & I & 0 & $\circ$ & 0 & 0 & 0 \\
\hline 6 & $\circ$ & $\circ$ & $\circ$ & $\circ$ & ० & 0 \\
\hline 7 & 0 & 0 & ० & 0 & 0 & ० \\
\hline 8 & 2 & ० & $\circ$ & ० & ० & ० \\
\hline 9 & I & $\circ$ & $\circ$ & $\circ$ & 0 & 0 \\
\hline IO & 0 & ० & $\circ$ & ० & o & ० \\
\hline I $I-20$ & 4 & 2 & 0 & ○ & 0 & $\circ$ \\
\hline $2 I-50$ & 3 & $\circ$ & $\circ$ & $\circ$ & 0 & $\circ$ \\
\hline $51-100$ & 2 & $\circ$ & $\circ$ & ० & I & $\circ$ \\
\hline IOI-IOOO & 2 & 0 & $\mathbf{I}$ & ० & 0 & 0 \\
\hline over I000 & I & 0 & 0 & $\circ$ & $\circ$ & 0 \\
\hline Average per culture & 190 & 4.8 & 55 & .55 & 8 & .72 \\
\hline Variance & 215,000 & 24 & 27,000 & .82 & 540 & I . 4 \\
\hline
\end{tabular}

this is a Poisson one. The experimental distribution will be derived from the theoretical one by multiplying the mean by the efficiency of plating and will still be a Poisson distribution.

It is immediately seen in tables 3 and 4 that the fluctuations of the numbers of plaques produced on the resistant bacteria are much higher than could be accounted for by the sampling errors. The variance is generally much higher than the mean, in accord with the expectation from the hypothesis that the new virus arises by mutation from the normal virus.

Another result evident in tables 3 and 4 is the presence of a large proportion of low values, well below the value of the burst size. This suggests that a bacterium may liberate a mixture of normal and mutant viruses and seems to exclude the possibility that the new virus be produced in full bursts by some abnormal bacteria, according to hypothesis 3 . The new virus particles arise by mutation in the course of the multiplication of normal virus inside the cell.

It would now be desirable to compare the experimental distribution of the number of mutant virus particles with the distributions to be expected accord- 
ing to various conceivable mechanisms of multiplication of the virus inside the bacterial cell, thus proving or disproving the correctness of each of these mechanisms. This comparison, however, is hampered by the low efficiency of plating of both the mutant viruses $\alpha^{\prime}$ and $\gamma^{\prime}$. Some of the theoretical distributions are not completely known, and the effect on them of the low efficiency of plating cannot be predicted. This is true, for instance, of the distribution encountered in the study of bacterial mutations (LURIA and DELBRÜCK I943), which would apply with some modifications to our case if the virus multiplied in the cell as bacteria multiply in a culture. Besides, the efficiency of plating may not be exactly constant even within each experiment, and its variations may affect any distribution. Further discussion of this point is postponed until experimental data obtained under conditions in which the efficiency of plating is equal to one will be available. Experiments in this direction are now in progress.

Similar considerations make it pointless to attempt figuring values for the rates of mutation from the data in tables 3 and 4 . Obviously, the correct mode of calculating mutation rates depends on the unknown mechanism of virus multiplication.

\section{Antigenic relation of normal and mutant viruses}

Bacterial viruses injected into the animal body stimulate the production of antibodies, of which the best known property is their specific virus-neutralizing power. Antisera against viruses $\alpha, \gamma$, and $\gamma^{\prime}$ were prepared by repeated injections into white rabbits, until the homologous titer had reached a satisfactory level. Cross-inactivation tests were then performed. It was found that, within the experimental precision, a serum neutralized virus $\alpha$ and virus $\alpha^{\prime}$, or virus $\gamma$ and virus $\gamma^{\prime}$, with the same titer and at the same rate. As an example, we give the values of the fractional rate of neutralization (HERsHey et al. r943) obtained by testing the same dilutions of various sera on normal and mutant viruses:

Serum anti- $\gamma: \mathrm{k}_{\gamma}=\mathrm{r} .3 ; \mathrm{k}_{\gamma^{\prime}}=\mathrm{r}$.0.

Serum anti- $\gamma^{\prime}: \mathrm{k}_{\gamma}=0.8 ; \mathrm{k}_{\gamma^{\prime}}=0.8$ (approximate values).

Serum anti- $\alpha: \mathrm{k}_{\alpha}=0.3 ; \mathrm{k}_{\alpha^{\prime}}=0.35$.

No cross-inactivation was found between viruses $\alpha$ and $\alpha^{\prime}$ on the one hand and viruses $\gamma$ and $\gamma^{\prime}$ on the other hand. For virus $\gamma^{\prime}$, it was found that both its activities on $\mathbf{B}$ and on $\mathbf{B} \gamma$ were neutralized at the same rate.

These results clearly show that, whatever structural differences exist between a virus and a mutant derived from it, they are not revealed by serological tests, at least of this rather simple nature.

\section{The virus mutations in relation to the bacterial mutations}

Arising by mutation from viruses $\alpha$ and $\gamma$, viruses $\alpha^{\prime}$ and $\gamma^{\prime}$ acquire the ability to attack bacterial strains that by mutation have lost their sensitivity to the original virus. The virus mutations compensate for the bacterial mutations. The mutation $\gamma \rightarrow \gamma^{\prime}$ is complementary to the mutation $\mathbf{B} \rightarrow \mathbf{B} \gamma$; the $m u$ tation $\alpha \rightarrow \alpha^{\prime}$ is complementary to the mutation $\mathbf{B} \rightarrow \mathbf{B} \alpha_{2}$. That the compensation 
is not complete is shown by the fact that the mutant viruses are adsorbed by the mutant bacteria more slowly than by the normal bacteria. One may say that the affinity of the mutant viruses for the mutant bacterial strains is poorer than for strain $\mathbf{B}$.

Strain $\mathbf{B} \gamma$ is sensitive to virus $\alpha$ and to virus $\gamma^{\prime}$. Does the mutation $\gamma \rightarrow \gamma^{\prime}$ involve the acquisition by the virus of the same structural configuration that enables virus $\alpha$ to attack strain B $\boldsymbol{B}$ ? If so, mutants from strain $\mathrm{B} \gamma$ which are resistant to virus $\alpha$ should also be resistant to virus $\gamma^{\prime}$. It was easy to isolate from $\mathbf{B} \gamma$ several $\alpha$-resistant mutants, called $\mathbf{B} \gamma \alpha$; all of them proved perfectly sensitive to virus $\gamma^{\prime}$ (fig. I). Sensitivity to viruses $\alpha$ and $\gamma^{\prime}$ cannot, therefore, be due to the same structural configuration.

It was more difficult to isolate bacterial mutants stably resistant to virus $\gamma^{\prime}$. Eventually, we obtained from strain $\mathrm{B} \alpha_{1}$ a mutant strain $\mathrm{B} \alpha_{1} \gamma^{\prime}$ that proved resistant to virus $\gamma$ and to virus $\gamma^{\prime}$, and, surprisingly enough, sensitive to virus $\alpha$. The sensitivity of strain $B \alpha_{1} \gamma^{\prime}$ to virus $\alpha$ was unexpected, since this strain was a mutant from strain $B \alpha_{1}$, which was completely resistant to virus $\alpha$. The mutation $\mathrm{B} \alpha_{1} \rightarrow \mathrm{B} \alpha_{1} \gamma^{\prime}$, therefore, involved loss of sensitivity to viruses $\gamma$ and $\gamma^{\prime}$, but gain of sensitivity to virus $\alpha$.

It may be added that from strain $\mathrm{B} \alpha_{1} \gamma^{\prime}$ a mutant $\mathrm{B} \alpha_{1} \gamma^{\prime} \alpha$ can easily be obtained, resistant to all three viruses $\alpha, \gamma$, and $\gamma^{\prime}$ (fig. I).

Let us now consider the situation concerning virus $\alpha^{\prime}$. The mutation $\alpha \rightarrow \alpha^{\prime}$ involves gain of ability to attack the $\alpha$-resistant mutant $\mathbf{B} \alpha_{2}$. This ability is not due to the same configuration which enables virus $\gamma$ to attack $B \alpha_{2}$, as proved by the fact that virus $\alpha^{\prime}$ also attacks a mutant strain $\mathrm{B} \gamma \alpha_{2}$, resistant to virus $\gamma$. Differences in the configurations of virus $\alpha^{\prime}$ and virus $\gamma^{\prime}$ are brought out by their relations to the mutants $\mathrm{B} \gamma \alpha_{1}$ and $\mathrm{B} \alpha_{1} \gamma^{\prime}$ (fig. I). The remarkable point here is that the mutation $\alpha \rightarrow \alpha^{\prime}$ compensates for only certain mutations of $\alpha$-sensitive bacteria-namely, $\mathbf{B} \rightarrow \mathbf{B} \alpha_{2}$ and $\mathbf{B} \gamma \rightarrow \mathbf{B} \gamma \alpha_{2}$, but not for the mutations $\mathrm{B} \rightarrow \mathrm{B} \alpha_{1}$ or $\mathbf{B} \gamma \rightarrow \mathrm{B} \gamma \alpha_{1}$. It is worth mentioning that both strains $\mathbf{B} \alpha_{2}$ and $\mathbf{B} \gamma \alpha_{2}$ belong to the group of "small colony" mutants that grow poorly and slowly on nutrient agar, whereas $B \alpha_{1}$ and $B \gamma \alpha_{1}$ are normal colony formers. This is an example of a modification of the cultural, physiological properties of the bacterial cells correlated with the mutational change to virus-resistance.

The results summarized in figure $I$ indicate that various bacterial mutations leading to resistance to unrelated viruses are generally independent of each other. For instance, the same two mutations (sub-I and sub-2) leading to resistance to virus $\alpha$ (with or without resistance to virus $\alpha^{\prime}$ and change in cultural characters) can occur either in strain $\mathbf{B}$ or in strain $\mathbf{B} \gamma$, in spite of the fact that the latter has already undergone a mutation toward resistance to virus $\gamma$.

Figure I also shows the occurrence of $\alpha^{\prime}$-resistant strains, all of which are also resistant to virus $\alpha$.

The results of this section show that some, if not all, mutational changes in virus sensitivity by our bacterial strains can be compensated for by complementary mutations of the viruses. It is possible that in cases of bacterial mutations for which complementary virus mutations have not been found (for ex- 
S. E. LURIA

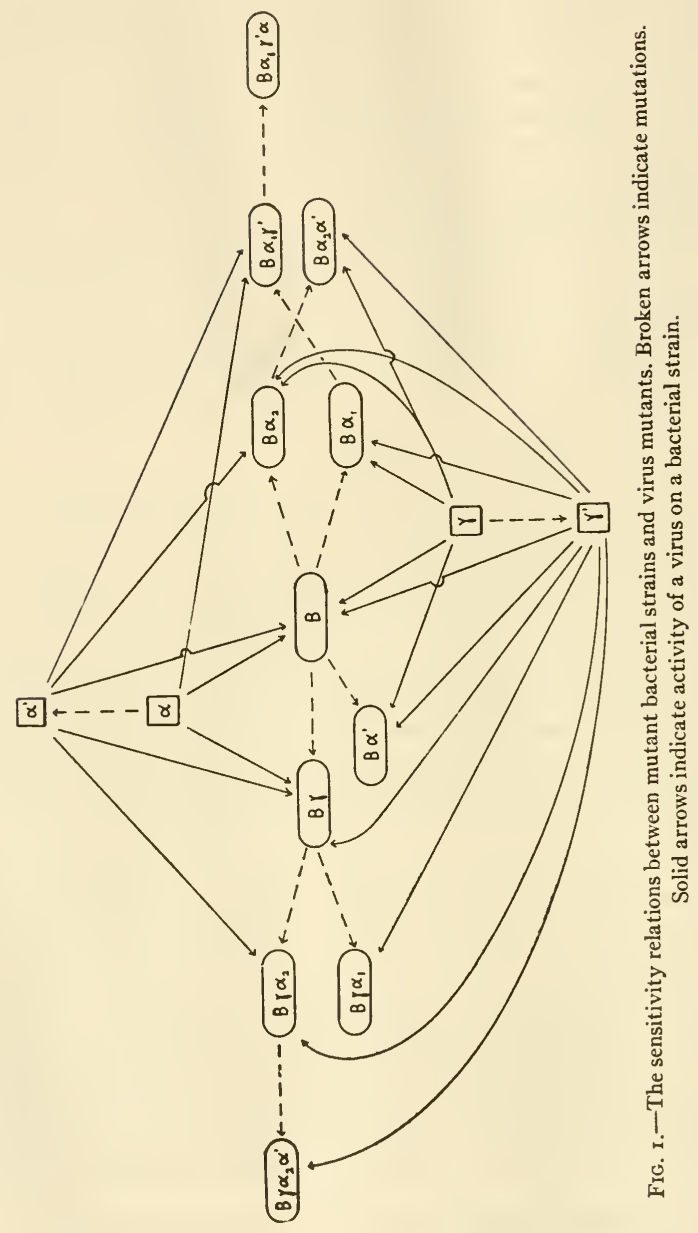


ample, $\left.\mathbf{B} \rightarrow \mathbf{B} \alpha_{1}\right)$, the virus mutations occur with a frequency too low to make them detectable in virus suspensions of the usual titers.

It is clear from the results obtained with viruses $\alpha$ and $\alpha^{\prime}$ that loss of sensitivity to a given virus can be brought about, in the same bacterial strain, by different mutations, leading to differences in sensitivity to another virus closely related to the first.

\section{DISCUSSION}

The results described above demonstrate that, whereas bacteria can be altered by mutaiion in their susceptibility to bacterial viruses, the latter can in turn acquire by mutation the ability to attack new bacterial strains. The reverse change, by which a virus particle would lose by mutation the capacity of attacking a certain bacterium, might conceivably occur, but would be difficult to demonstrate, except in cases where it occurred very frequently.

The changes in virus properties are here called "mutations" because of their apparently spontaneous and random occurrence, of their transmission to the offspring, and of their stability. The same may be said of the bacterial mutations affecting virus-sensitivity. In making any analogy with the process of gene mutation in plants and animals, we should not forget the lack of any direct evidence of the presence, in bacteria or viruses, of "genes" in the sense of discrete material units, whose existence in higher organisms is proved by linkage studies.

As for the structural changes involved in the virus mutations, we have seen that the serological tests failed to reveal any difference between original and mutant strains. For the time being, our only basis for attempting to understand the structural changes involved in the mutations is the infectivity of the viruses for different bacterial strains.

The virus-host interaction involves as first step a process of specific adsorption. The specificity of the adsorption of a virus by a bacterium is generally conceived as due to the presence of "receptors" for the virus on the bacterial surface (see BURNET 1930). Adsorption is conditioned by complementarity of the surface structures of the receptor and of the virus, which enables them to fit together. Since the mutant bacteria resistant to a virus do not adsorb that virus, we may assume that the bacterial mutation causes a change in the receptors. It has long been known (BURNET 1930) that changes in virus-sensitivity of bacterial strains are often accompanied by changes in the antigenic make-up of their surface, and some of the antigens have been supposed to be responsible for virus adsorption.

The fact that a change in the surface structure of a bacterium can be compensated for by an independent change in the virus particle suggests that the changes involved are relatively small, possibly limited to simple stereochemical rearrangements, suppressing or restoring the complementarity of the surface structures. The fact that normal and mutant viruses are serologically indistinguishable speaks in favor of this conception. It is interesting to recall that STANLEy (I943) and his collaborators found that strains of tobacco mosaic virus, supposed to be closely related to each other, showed serological relation- 
ship, common structural pattern in X-ray diffraction studies, and similarity of gross chemical composition, but showed differences in finer chemical composition.

The low rate of adsorption of the mutant bacterial viruses by their new hosts may be attributed to a less satisfactory fitting of the surface structures of virus and bacterium, lowering their affinity.

A possible alternative explanation must be mentioned-namely, that the acquired ability of a mutant virus to be adsorbed by a new host may be due to a change in only one out of a number of surface structures of the virus particle. This would explain the smaller adsorption rate of the mutant viruses by the mutant bacteria, while the rate of adsorption by the normal bacteria remains the same as for normal viruses.

The evolutionary implications that the results discussed above present for the system bacterium-virus are of some interest. Because of the lytic activity of most bacterial viruses, a bacterial strain is doomed to destruction once it has come in contact with a virus active upon it. The only chance of survival of the bacterial strain is the occurrence of mutations to virus-resistance. As we have seen, however, this occurrence does not necessarily protect the strain, because its mutation to virus-resistance may be compensated for by an independent complementary mutation of the virus. In certain cases (SERTIC I929) parasitism may be maintained by two parallel series of complementary mutations in the host and the parasite.

Mutations of bacterial viruses enlarging their host range need not always be limited to activity upon closely related bacterial strains, but may conceivably render a virus active on strains belonging to different species. It is interesting, from the standpoint of bacterial taxonomy, that while bacterial viruses may be active on species belonging to different genera, chiefly within the family Enterobacteriaceae, no virus has ever been found to be active on members of different families.

\section{SUMMARY}

From two bacterial viruses, $\alpha$ and $\gamma$, two new viruses $\alpha^{\prime}$ and $\gamma^{\prime}$ were isolated, differing from $\alpha$ and $\gamma$ by their ability to attack bacterial strains which by mutation had become resistant to virus $\alpha$ or to virus $\gamma$.

An analysis of the distribution of the particles of a new virus in a series of similar cultures of normal virus proved that the new virus arises by mutation from the particles of normal virus in the course of their growth on sensitive bacteria.

Each mutant is indistinguishable from its parent virus in serological properties and in its activity on the common bacterial host. The latter property was utilized to study the interference between similar virus particles. The results confirmed the conclusion that only one of the infecting particles succeeds in growing in each bacterial cell.

The mutant viruses are poorly adsorbed by their new hosts. Their growth on these was investigated. 
The sensitivity of a series of bacterial mutants to viruses $\alpha, \alpha^{\prime}, \gamma$, and $\gamma^{\prime}$ was studied. It was found that resistance to virus $\alpha$ may be brought about in the same bacterial strain by different mutations. These lead to differences in sensitivity to the related virus $\alpha^{\prime}$ and in other physiological properties.

Bacterial mutations leading to resistance to a mutant virus lead also to resistance to the parent virus.

Bacterial mutations leading to resistance to unrelated viruses generally prove independent; the same mutation can occur in strains with a different history of previous mutations. One exception to the independence of various mutations was found. A bacterial strain resistant to viruses $\alpha$ and $\alpha^{\prime}$ reverted to sensitivity as a consequence of a mutation to resistance to viruses $\gamma$ and $\gamma^{\prime}$.

\section{ACKNOWLEDGMENTS}

Some of the experiments reported in this paper were done in the summer of I944 in the Department of Biology, Vanderbilt University, Nashville, Tennessee. The author expresses his thanks to VANDERBILt University for the hospitality and for a grant-in-aid, and to DR. M. DELBRÜCK for many helpful discussions.

\section{LITERATURE CITED}

BURNeT, F. M., I930 Bacteriophage activity and the antigenic structure of bacteria. J. Path. Bact. 33: 647-664.

BURNET, F. M., and D. Lush, 1936 Induced lysogenicity and mutation of bacteriophage within lysogenic bacteria. Aust. J. Exp. Biol. Med. Sci. 14: 27-38.

DELBRÜCK, M., I942 Bacterial viruses (Bacteriophages). Advances in Enzymology 2: 1-32. I944 Unpublished experiments.

DeLBRÜCK, M., and S. E. LURIA, 1942 Interference between bacterial viruses. I. Arch. Bioch. I : I I I-I 4 I.

Gratia, A., I936a Mutation d'un bactériophage du Bacillus megatherium. C. R. Soc. Biol. 123: I 253-1 255 .

1936b Des relations numériques entre bactéries lysogènes et particules de bactériophage. Ann. Inst. Pasteur 57:652-676.

Hershey, A. D., G. Kalmanson, and J. Bronfenbrenner, 1943 Quantitative methods in the study of the phage-antiphage reaction. J. Immunol. 46: $267-279$.

Luria, S. E., and M. DELBRÜCK, I942 Interference between bacterial viruses. II. Arch. Bioch. I: $207-218$.

I943 Mutations of bacteria from virus sensitivity to virus resistance. Genetics 28: 4915 II.

SERTIC, V., 1929 Procédé d'obtention de variantes du bactériophage adaptées à lyser des formes bactériennes secondaires. C. R. Soc. Biol. roo: 61 2-6r 4 .

Stanley, W. M., I 943 Chemical structure and the mutation of viruses. In: Virus diseases. Ithaca: Cornell University Press. 


\title{
GENETIC RECOMBINATION BETWEEN HOST-RANGE AND PLAQUE-TYPE MUTANTS OF BAC'TERIOPHAGE IN SINGLE BACTERIAL CELLS ${ }^{1,2}$
}

\author{
A. D. HERSHEY AND RAQUEL ROTMAN ${ }^{3}$ \\ Department of Bacteriology and Immunology, Washington University Medical School,
} St. Louis, Missouri

Received June 28, 1948

W

E HAVE previously shown that any two of several independently arising plaque-type $(r)$ mutants of the bacterial virus $T 2 H$ interact with each other, in bacterial cells infected with both, to give rise to wild type and double mutant genetic recombinants (HERSHEy and RotMan 1948). In this paper we describe comparable interactions between host-range and $r$ mutants of the same virus. The experiments furnish new information because it has proved possible to count the numbers of all four types of virus found in yields from the mixedly infected bacteria.

\section{MATERIALS AND METHODS}

The types of viral mutant to which we shall refer in this paper may be summarized in terms of the mutational pattern illustrated in fig. 1. In this diagram, $h$ refers to a host-range mutant, $r$ to any one of the rapidly lysing mutants (Hershey and Rotman 1948), and $m$ ("minute") to a mutant not previously described which is characterized by a very small haloless plaque. The $h$ mutant is one which forms plaques identical in appearance and number on typically sensitive strains of Escherichia coli, and on an indicator strain (No. $2 \mathrm{~B} / 2 \mathrm{H}, 2 \mathrm{~K}$ ) resistant to $h^{+}$forms of the virus (Hershey 1946a). All the steps indicated in the diagram by arrows can be observed either as spontaneous mutations, or by making the appropriate crosses. Only one example of the mutant $m$, obtained by crossing wild type with an $r m$ arising in a stock of the mutant $r 13$, has been studied. The plaques of $m$ and $r m$ are different, but are not easily distinguishable, as shown in the photograph (fig. 2).

In principle, the experimental technique we have to describe is very similar to that of genetic crossing, and will be referred to in this paper in genetic terms. One starts with a pair of mutants, each corresponding to a mutant haploid germ cell differing from wild type by a different unit change. Bacterial cells are infected with both members of the pair, and during viral growth the pair interact to produce viral progeny corresponding to germ cells of a new

' Aided by a grant from the U. S. Pubi.ic Health Service.

${ }^{2}$ The manuscript was prepared while the senior author held a temporary appointment in the Department of Biology of the Cal.rfornia Instunere of 'Tecunology. It is a pleasure for him to acknowledge material and intellectual aid received from members of the staff of that department.

${ }^{3}$ P'resent address: University of Minnesota, Minneapolis, Minn.

[Reprinted by permission from GFNFTics 34 : 44-71, January, 1949] 
generation, but now including some individuals differing from wild type by both unit changes, and other individuals differing from wild type not at all.

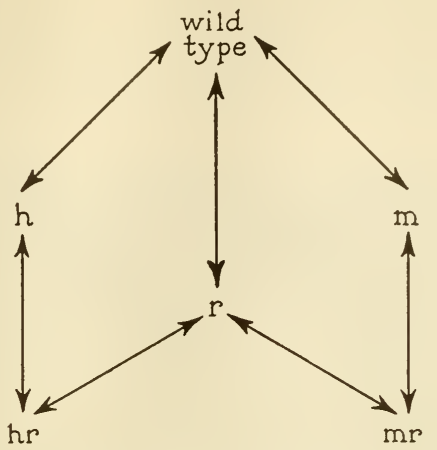

Figure 1.-Mutational pattern of the bacterial virus $T 2 H$.

The analogy to other genetic recombinations is obvious, and it is natural to look for a common mechanism.

The procedure of making a cross consists essentially in infecting a measured number of growing bacteria with larger measured numbers of two kinds of virus, diluting the culture before lysis begins to prevent readsorption of viral progeny to bacteria not yet lysed, and plating samples of the total yield of

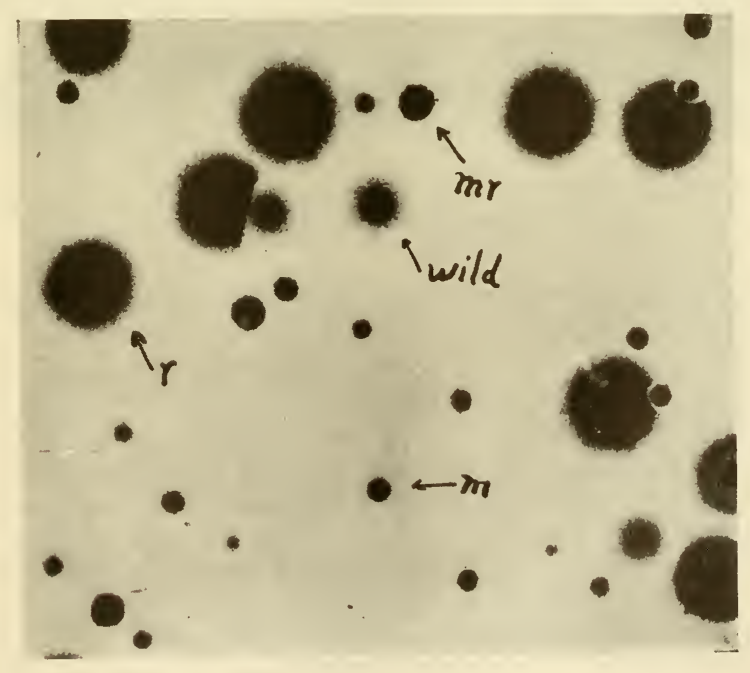

FIGURE 2.-Progeny of the cross $m \times r 1$. 
virus for a differential count of its component types. This procedure was first used, for another purpose, by DELBRÜCK and LURIA (1942).

The advantage of the $h \times r$ cross is that all the genetic types of virus to which it gives rise can be recognized in a single plating on a mixture of bacterial strains (fig. 3). This makes possible the analysis of viral yields from single bacterial cells. For this purpose the procedure already described is modified

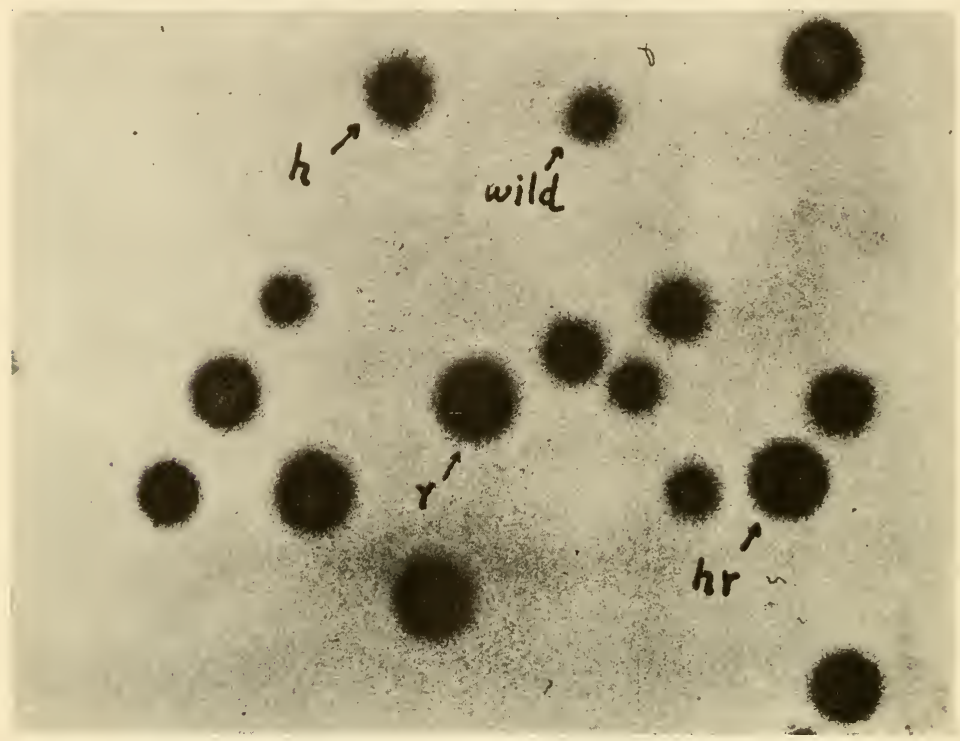

FIGURE 3.-Progeny of the cross $h \times_{r 1}$ plated on mixed indicator. The acentric clearings in the $h^{+} r$ plaques result from secondary $h$ mutations.

by increasing the factor of dilution to obtain only one infected bacterium to about three $\mathrm{ml}$ of nutrient broth. The culture is then divided up into samples of one ml, most of which will contain either no bacteria at all, or a single one. A large number of these samples are plated out after the bacteria lyse, and the elementary yields, averaging about 500 particles of virus in our experiments, are analyzed in toto. Both the mixed indicator method for differential counting of viral mixtures, and the single burst technique as here employed, were first used by DELBRÜCK (1945a, b).

The remaining portion of this discussion of materials and methods is of technical interest only.

Viral stocks are prepared by seeding nutrient broth cultures of $E$. coli strain $\mathrm{S}$ with material taken directly from a single plaque. Stocks of wild type and $h$ mutant usually have titers exceeding $10^{10} / \mathrm{ml} ; r$ mutant titers are always a 
little less. Many of the stocks, particularly of the $r$ mutants, contain unknown substances inhibiting adsorption of the virus if the dilution into bacterial suspension is less than 1:100 or so. There is no difference in the adsorption of different stocks at high dilutions of the virus. If a mixed infection is attempted with an $r$ stock which contains inhibitor, and an $h$ or wild type stock which does not, the adsorption of both viruses in the mixture is prevented equally. No undesirable disturbance of the relative multiplicity of infection in mixtures is therefore encountered. To obtain satisfactory levels of adsorption, all $r$ mutant stocks are sedimented in a refrigerated International centrifuge with Multispeed head, and resuspended in a solution containing 1 percent Bactopeptone and 0.5 percent $\mathrm{NaCl}$. Very little loss of virus occurs during sedimentation, and probably no permanent aggregation, since sedimented mixtures of $r$ and wild type virus do not yield mixed plaques. The resuspended virus is stable for at least several weeks.

To make a cross, a two hour culture of $E$. coli strain $\mathrm{H}$ in nutrient broth, containing $2 \times 10^{7}$ bacteria per $\mathrm{ml}$, is infected at $37^{\circ} \mathrm{C}$ in an aerated culture tube (the "adsorption tube") with $\frac{1}{10}$ volume of a mixture of diluted viral stocks containing $2 \times 10^{9}$ plaque forming particles of each kind per ml. After five minutes, during which equal numbers (about 50 percent) of each virus are adsorbed, a $10^{4}$ dilution is made into broth (the "growth tube") for further incubation, and a second diluted sample is spun for the assay of unadsorbed virus. Sixty minutes after infection, an assay from the growth tube gives the average yield of virus from about 40,000 mixedly infected bacteria.

For the single burst experiments, an additional dilution from the adsorption tube into antiserum to neutralize the unadsorbed virus (DELBRÜCK 1945c), is made at the end of the adsorption period. Five minutes later, a further dilution from the antiserum tube is made into broth to contain about one infected bacterium per three $\mathrm{ml}$. Before the 20 th minute after infection, samples measuring one $\mathrm{ml}$ are distributed into a series of small tubes. The virus yields in these tubes are assayed by plating $0.3 \mathrm{ml} 60$ minutes or more after infection. The remainder of each sample, excepting those containing no virus or unmixed yields, is plated on two additional plates the next day. About 10 percent of each sample is mechanically lost. An important feature of these experiments is the guard against contamination of materials provided by the fact that about $\frac{3}{4}$ of the samples contain no virus, whereas the remainder contain more than 100 particles.

Viral yields from the growth tube are plated on sensitive bacteria (strain $\mathrm{S})$, on the indicator strain $(\mathrm{No} .2 \mathrm{~B} / 2 \mathrm{H}, 2 \mathrm{~K}$ ), and on a mixture containing one volume $\mathrm{S}$ and two volumes indicator (day-old broth cultures). On the mixed indicator all four types of virus can be recognized (fig. 3), and their sum equals the count on S. Mixed indicators plates always show a few doubtful plaques which can be identified only by sampling and retesting, but their number is too small to be of importance, and mixtures of pure stocks can be counted with satisfactory accuracy. The counts on the single indicator, giving only the $h$ mutants, are also satisfactory with mixtures of pure stocks. These counts tend to be low, however, for mixed yields of $h$ and $h^{+}$virus. The cause 
of this "mixed indicator effect," similar to that observed by DELBRÜCK and BAILEy (1946), remains obscure. It does not appear to be the result of segregation of multiple $h$ factors, because no intermediate genetic types can be found in crosses between $h$ and wild type. In $h \times r$ crosses, it affects equally counts of parental and recombinant virus.

The validity of the mixed indicator count itself rests on three lines of evidence. First, plaques sampled and retested always conform to the genetic type deduced from inspection. Second, the ratio of $h$ to $h^{+}$virus in the yield following mixed infection, measured by the mixed indicator count, is the same as the corresponding ratio of infecting viruses, with minor exceptions to be mentioned below. Third, the yield of the two recombinants in either $h \times r$ or $h r \times$ wild type crosses is very nearly equal, and the slight bias (actually of doubtful significance) correlates not with the $h$, but with the $r$ pair of alleles.

For these reasons, and because of its statistical efficiency, only the mixed indicator plating is used for counting single bursts. The reproducibility of the counts so obtained may be judged from the examples shown in table 1 .

TABLE 1

\section{Mixed Indicator Counts of Viral Types in the First Eight Bursts Examined from the Cross $h \times r 7$.}

The counts shown are for 3 aliquots of $0.3 \mathrm{ml}$ from each tube. The volumes are not measured very accurately, owing to the effort made to plate the entire sample. In computing results, it was assumed that totals of counts for each tube represented 90 percent of the actual virus content, 10 percent of the fluid being lost mechanically.

\begin{tabular}{crrrrrrrrr}
\hline \hline \multirow{2}{*}{ TUBE } & $h^{+} r^{+}$ & $h r^{+}$ & $h^{+} r$ & $h r$ & TUBE & $h^{+} r^{+}$ & $h r^{+}$ & $h^{+} r$ & $h r$ \\
\hline \multirow{2}{*}{2} & 9 & 57 & 46 & 19 & 35 & 21 & 80 & 71 & 23 \\
& 9 & 77 & 56 & 20 & & 24 & 74 & 65 & 18 \\
& 11 & 50 & 47 & 18 & & 14 & 68 & 39 & 15 \\
10 & 18 & 17 & 95 & 13 & 36 & 9 & 136 & 30 & 9 \\
& 10 & 14 & 73 & 8 & & 7 & 100 & 22 & 7 \\
& 10 & 22 & 118 & 15 & & 14 & 120 & 18 & 9 \\
26 & 9 & 84 & 51 & 8 & 38 & 9 & 139 & 30 & 9 \\
& 10 & 124 & 53 & 5 & & 9 & 130 & 19 & 13 \\
34 & 8 & 78 & 34 & 7 & & 4 & 102 & 31 & 4 \\
& 2 & 20 & 58 & 17 & 40 & 1 & 24 & 54 & 1 \\
& 6 & 26 & 78 & 32 & & 3 & 38 & 83 & 10 \\
& 6 & 33 & 55 & 16 & & 3 & 24 & 66 & 7 \\
\hline
\end{tabular}

The nutrient broth referred to above is composed of Bacto-peptone $10 \mathrm{~g}$, Bacto-beef extract $3 \mathrm{~g}, \mathrm{NaCl} 5 \mathrm{~g}$, glucose $1 \mathrm{~g}$, per liter distilled water. The $\mathrm{pH}$ (unadjusted) is about 6.8. An occasional batch of broth prepared according to this formula proves unfavorable to the adsorption of the virus.

Nutrient agar plates are poured with a minimum of $35 \mathrm{ml}$ per $9 \mathrm{~cm}$ Petri dish of Bacto-agar $10 \mathrm{~g}$, Bacto-Tryptose $10 \mathrm{~g}, \mathrm{NaCl} 8 \mathrm{~g}$, sodium citrate crystals $2 \mathrm{~g}$, glucose $1 \mathrm{~g}$, per liter distilled water. The $\mathrm{pH}$ of the agar is adjusted to 6.8 to 7.0. Contrary to an early experience, we have recently found that Bacto-Tryptone can be substituted for Tryptose. Poured plates are stored in 
the refrigerator. Agar for layer plating has the same nutrient composition, but contains only 0.5 to 0.6 percent agar, the optimal concentration depending on the age of the plates and on other variables.

All platings of virus are made by the agar layer method, by adding an aliquot of virus measuring 0.1 to $0.5 \mathrm{ml}$ and about $0.1 \mathrm{ml}$ of a day old unaerated broth culture of bacteria, to $2 \mathrm{ml}$ of melted soft agar at $45^{\circ} \mathrm{C}$, and pouring the mixture over the surface of an agar plate at room temperature. The plates are incubated 18 to 24 hours at $37^{\circ} \mathrm{C}$ without inverting, during which time fluid collects on the agar surface without undesirable effects if conditions are optimal.

Bacterial counts are made by spreading $0.1 \mathrm{ml}$ aliquots on the surface of dried agar plates.

\section{THE LINKAGE SYSTEM}

The mutants $m$ and $h$ (minute plaque and host-range modification, respectively) have been crossed with wild type, with the mutants called $r 1, r 7$, and $r 13$, and with each other. The crosses with wild type yield only the parental types of virus, confirming that the mutants are unit modifications. The crosses with $r$ mutants, and the intercross, extend the linkage system previously described (Hershey and Rotman 1948) as shown in fig. 4. The $h$ locus is closely linked to the locus $r 13$; the $m$ locus belongs in a third linkage group C.

It should be understood that the diagram of fig. 4 is only a convenient representation of linkage relations whose structural basis remains to be elucidated (Hershey and Rotman 1948). The question of linear structure will be returned to in the discussion of this paper. At this point we insert an experiment which supports the general interpretation in terms of linkage.

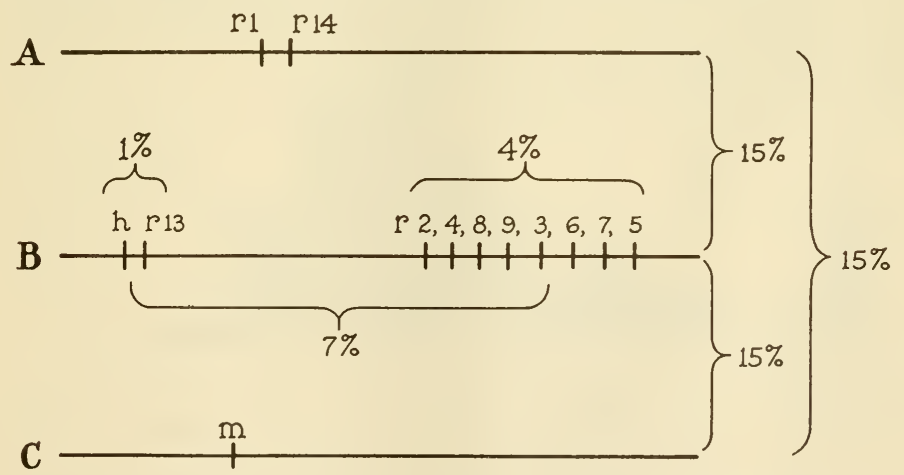

FIGURE 4.-Linkage relations among mutants of $T 2 H$. The percentages indicate yields of wild type in two factor crosses.

The double mutants $h r 7$ and $h r 13$ were isolated by making the respective crosses $h \times r 7$ and $h \times r 13$. The three crosses $r 7 \times r 13, h r 7 \times r 13$, and $h r 13 \times r 7$ were then compared with respect to the yield of $r^{+}$virus, classified without 
respect to the host-range character. In each case the yield was the same within experimental error, seven percent of the total virus. This experiment shows that the small yield of recombinants (one percent of wild type) in the cross $h \times r 13$ cannot be attributed to suppression of a hypothetical conjugation, which would affect the interaction of $h r 7$ with $r 13$ as well, but indicates some kind of linkage between the genetic factors concerned.

\section{AVERAGE YIELDS OF VIRUS IN CROSSES BETWEEN HOST-RANGE AND $r$ MUTANTS}

Six crosses have been studied by the single burst technique; namely, $h \times r 1$, $h \times r 7, h \times r 13$ and the corresponding reverse crosses, $h r \times$ wild type. Each cross was made three to five times for the collection of the single burst data, and each time the viral yield was examined also from a culture tube containing about 40,000 mixedly infected bacteria. The average yields from the culture tubes are summarized in table 2 . They show that the $h$ locus is very closely linked to $r 13$ (less than one percent of wild type), and that the linkage relations to $r 1$ and $r 7$ are approximately what would have been predicted from this fact, respectively 12 and 6 percent of wild type. According to arguments previously given (HERSHEy and RotMan 1948), the factors $h, r 7$, and $r 13$ are

TABLE 2

\section{Average Percent Distribution of Viral Types in Vields from about 40,000 Mixedly Infected Bacteria}

The results shown are from the same experiments for which single burst data are also reported, except that one growth tube was lost among the crosses $h r 7$ by wild type. The total multiplicity of infection is about five of each type per bacterium; the adsorption period is five minutes; the total incubation period one hour. The distribution of viral types is computed from the results of mixed indicator platings. The column headed eop(h) gives the efficiency of plating of $h$ virus on single indicator as compared with mixed indicator, and illustrates the mixed indicator effect mentioned in Methods. The column headed $\mathrm{p}(\mathrm{h})$ gives the percent of virus containing the $h$ allele, and shows the effect of selection during growth.

\begin{tabular}{|c|c|c|c|c|c|c|c|c|}
\hline CROSS & $\begin{array}{l}\text { No. OF } \\
\text { EXPTS. }\end{array}$ & $h^{+} r^{+}$ & $h r^{+}$ & $h^{+} r$ & $h r$ & $\begin{array}{l}\text { BURST } \\
\text { SIZE }\end{array}$ & $\operatorname{eop}(\mathrm{h})$ & $\mathrm{p}(\mathrm{h})$ \\
\hline \multirow[t]{2}{*}{$h \times r 1$} & \multirow[t]{2}{*}{5} & input 0 & 53 & 47 & 0 & & 1.0 & 53 \\
\hline & & yield 12 & 42 & 34 & 12 & 630 & 0.8 & 54 \\
\hline \multirow[t]{2}{*}{$h r l \times++$} & \multirow[t]{2}{*}{3} & input 57 & 0 & 0 & 43 & & 1.0 & 43 \\
\hline & & yield 44 & 14 & 13 & 29 & 680 & 0.7 & 43 \\
\hline \multirow[t]{2}{*}{$h \times r 7$} & \multirow[t]{2}{*}{4} & input 0 & 49 & 51 & 0 & & 1.1 & 49 \\
\hline & & yield 5.9 & 56 & 32 & 6.4 & 650 & 0.8 & 62 \\
\hline \multirow[t]{2}{*}{$h r 7 \times++$} & \multirow[t]{2}{*}{2} & input 49 & 0 & 0 & 51 & & 1.0 & 51 \\
\hline & & yield 42 & 7.8 & 7.1 & 43 & 690 & 0.8 & 51 \\
\hline \multirow[t]{2}{*}{$h \times r 13$} & \multirow[t]{2}{*}{3} & input 0 & 49 & 51 & 0 & & 1.1 & 49 \\
\hline & & yield 0.74 & 59 & 39 & 0.94 & 510 & 0.8 & 60 \\
\hline \multirow[t]{2}{*}{ hr13X++ } & \multirow[t]{2}{*}{4} & input 52 & 0 & 0 & 48 & & 0.9 & 48 \\
\hline & & yield 50 & 0.83 & 0.76 & 48 & 590 & 0.7 & 49 \\
\hline
\end{tabular}


linked to each other, but $r 1$ is probably situated on an independently exchanging structure.

The results of table 2 show further that the two recombinants appear in equal numbers in any one cross, and that pairs of reverse crosses yield equal numbers of recombinants. It is these relations, which increase the resemblance to simple types of Mendelian segregation, that we wish to examine by the single burst technique. In the remainder of the experimental part of this paper we describe the results of this examination, but limit our comments chiefly to the technical problems encountered. The general implications of the data will be considered in the discussion.

\section{VARIATIONS IN YIELDS OF PARENTAL TYPES OF \\ VIRUS AMONG INDIVIDUAL BACTERIA}

Yields of virus from single bacteria show large fluctuations in size (DELBRÜCK 1945b) and, in our experiments, variations in relative yields of the two infecting viruses. The variations in total yield are shown in fig. 5 which includes the complete data for six experiments in which the proportion of multiple bursts is small (100 bursts out of 484 tubes, or 11 probable multiples). The burst sizes range from 150 to several thousand, with a mean of 520 , or 470 corrected for probable multiples. The distribution is the same for the mixed bursts, and for the bursts containing only one viral type. Owing to these variations, it is convenient to describe the individual bursts in terms of the fractional yield of the several viral types.

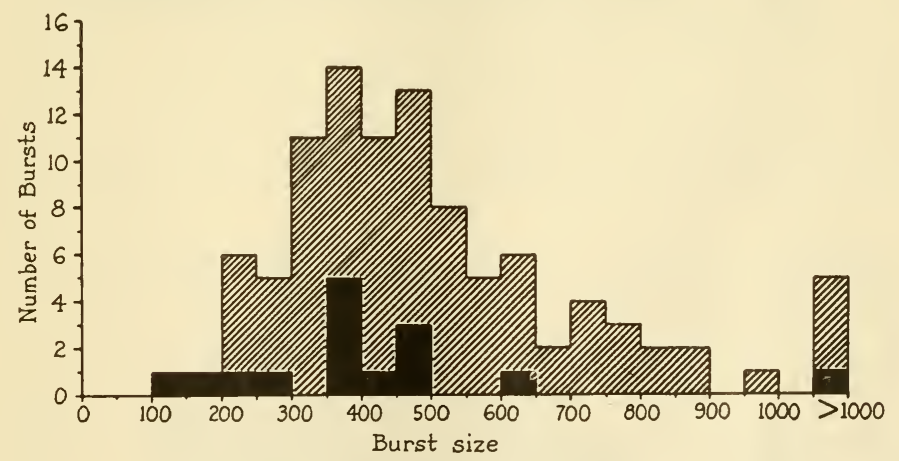

FIgURE 5.-Distribution of total viral yields among 100 single bursts from mixedly infected bacteria. The solidly shaded areas refer to unmixed yields.

Each cross yields four types of virus, wild type, the single mutants $h$ and $r$, and the double mutant $h r$, of which two are parental and two are recombinant types. In order to examine the variations in relative yields of parental types independently of variations in yields of recombinants, it is convenient to express the former in terms of the proportion of virus containing a specified allele, viz: 


$$
\begin{aligned}
& \mathrm{p}(h)=\frac{\mathrm{n}\left(h r^{+}\right)+\mathrm{n}(h r)}{\mathrm{n}(\text { total })} \\
& \mathrm{p}(r)=\frac{\mathrm{n}\left(h^{+} r\right)+\mathrm{n}(h r)}{\mathrm{n}(\text { total })}
\end{aligned}
$$

where $\mathrm{n}$ indicates the number of the specified viral types, $\mathrm{p}(h)$ is the proportion of the yield containing the $h$ allele, and $1-\mathrm{p}(h), \mathrm{p}(r)$, and $1-\mathrm{p}(r)$ are respectively the proportions containing the $h^{+}, r$, and $r^{+}$alleles. The fraction $\mathrm{p}(h)$ is equal to $1-\mathrm{p}(r)$ if the numbers of the two recombinant types are the same, or approximately equal if these numbers are small. A fair idea of the complete distribution of alleles is therefore given by the distribution of $\mathrm{p}(h)$ alone. Two examples of this distribution, showing the proportions of the $h$ allele in different single bursts for the crosses $h \times r 1$ and $h \times r 7$, are given in fig. 6 .
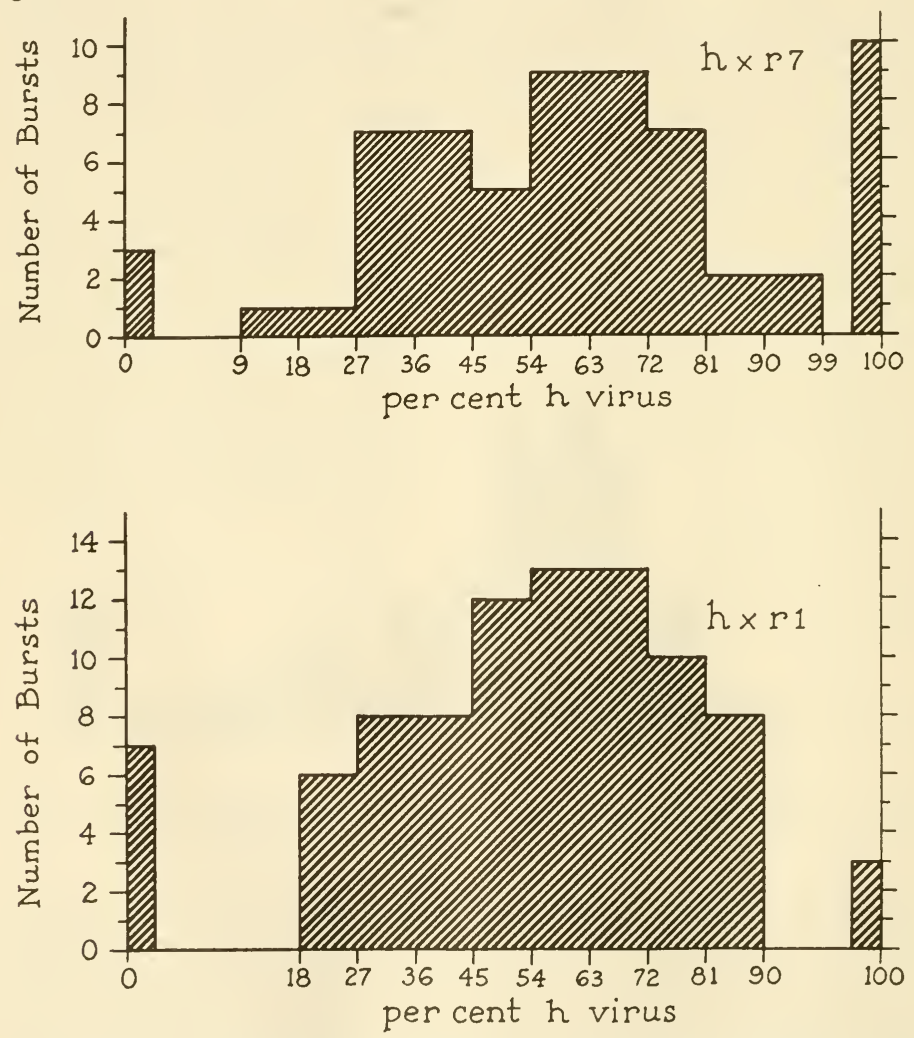

FIGURE 6.-Distribution of proportions of $h$ virus in yields from single bacteria. 
The variations shown in fig. 6 are evidently due in part to variations in relative numbers of the two kinds of virus adsorbed, and in part to variations in viral growth. For purposes of comparison, the variation in relative multiplicity has been computed on the assumption of a random distribution of two types of virus over a population of bacteria receiving on the average five particles of each kind. The distribution for this case is approximately that shown in table 3.

TABLE 3

Ideal Distribution of Multiplicities in Mixed Infection

The proportions of bacteria falling into the specified groups classified with respect to relative multiplicity of infection with two kinds of virus have been calculated for random adsorption with average multiplicity of five each of the two kinds.

\begin{tabular}{cc}
\hline \hline $\begin{array}{c}\text { NUMBERS OF FIRST KIND } \\
\text { AS PERCENT OF TOTAL VIRUS } \\
\text { ADSORBED }\end{array}$ & PERCENT OF BACTERIA \\
\hline 0 or 100 & 0.7 (exact) \\
$1-9$ or $91-99$ & 0.2 (approximate) \\
$10-18$ or $82-90$ & 2.0 " \\
$19-27$ or $73-81$ & 5.1 \\
$28-36$ or $64-72$ & 10.8 \\
$37-45,46-54$, or $55-63$ & 18.2 \\
total & 92.2 \\
\hline
\end{tabular}

The distributions of yields actually found (fig. 6) differ from the theoretical distribution of multiplicities in showing a considerably broader spread, and a significant excess of yields containing only one kind of virus. These effects could be due in part to an inhomogeneity of the bacteria with respect to adsorbing power for virus; otherwise they suggest that a bacterium infected with two viruses is somewhat less likely to liberate a given one, than a bacterium infected with that one alone. Dulbecco (1949) has shown that the latter is, in fact, the case.

Another possible contribution to the variations described is connected with the relatively long period (five minutes) allowed for adsorption of virus, which permits some bacteria to be infected with one or more particles of one type of virus considerably in advance of infection by the second. Owing to the slow adsorption of the virus $\mathrm{T} 2 \mathrm{H}$ and its mutants, the adsorption time cannot be much reduced without reducing the total multiplicity of infection, or introducing excessive amounts of virus. This contribution to the variation in composition of viral yields has not, therefore, been assessed.

\section{COMPETITION BETWEEN VIRAL MUTANTS}

The competition between viral mutants expresses itself in two ways; first, by the complete suppression of one virus or the other in mixedly infected bacteria, and second, by excessive growth of one of the two types in bacteria liberating both. These effects are slight among mutants of $T 2 H$ so far examined, 
and probably do not influence appreciably the yields of recombinants in genetic crosses, as the following discussion will show.

The two distributions shown in fig. 6 illustrate the competitive relations encountered. In the cross $h \times r 7$, most of the unmixed yields contain the $h$ rather than the $r$ parental type. Corresponding to this, there is a tendency for the mixed yields to contain an excess of $h$ virus. The combined effect is to cause a definite increase in yield of $h$ virus at the expense of $r$, as compared with the input proportions. The cross $h \times r 13$ also shows these characteristics, the effects being evident in table 2 .

In the cross $h \times r 1$, on the other hand, the unmixed yields of each kind are approximately equal in number, and the mean proportion of $h$ virus in the mixed bursts, in the total yield, and in the input mixture of viruses is the same. The crosses between wild type and $h r 1, h r 7$, and $h r 13$ are like $h \times r 1$ in this respect, as shown in table 2.

It might be supposed that the suppression of one virus by a second is favored by an excess of the second. This is true only in a special sense, as Dulbecco (1949) has shown, and we have confirmed. An excess of one virus tends to suppress a minority type completely in some bacteria, but there is a compensating excess of this type among the mixed bursts, so that the average proportion of the minority virus in the yield averaged over many bacteria is the same as in the input mixture. This identity has been established with considerable precision for proportions of $r 1$ between 7 and 50 percent in mixed infection with wild type. The nature of this relationship, which is at first sight perplexing in the case of unequal multiplicity, has been explained by Dulbecco (1949) in terms of a limitation to the number of viral particles which can participate in growth in a single bacterium. If all those viral particles in excess of a certain number attached to the same bacterium fail to grow, and if the excluded ones are chosen at random, the result will be precisely the one described, provided there is no selection during the growth of the successful particles.

It is apparent that with certain viral pairs, the excluding mechanism does not operate at random, or there is continuing selection during growth. Thus $h$ mutant slightly suppresses $r 7$ or $r 13$, but not $r 1$ or wild type. Wild type suppresses $r 13$, but not $r 1$ or $r 7$ (Hershey and Rotman 1948). There is no selection with respect to either $h$ or $r$ factors when wild type is crossed with $h r 1, h r 7$, or $h r 13$ (table 2).

The competitive relations discussed above are of immediate interest only in the negative sense that they probably do not influence the yields of recombinant virus in crosses. The evidence for the latter conclusion, drawn from data presented elsewhere in this paper, may be summarized as follows: (1) the linkage relations deduced from average yields of virus are the same as those deduced from single bursts selected for equality of yields of the two infecting viruses; (2) in the reverse crosses $h \times r 7$ and $h r 7 \times$ wild type, one gets within experimental error equal numbers of all four recombinants in spite of the fact that in one case the infecting pair, and in the other the recombinant pair, have unequal excluding power; (3) in all crosses, the distribution of yields of recombinants among single bursts does not show one peak at zero and another 
above the mean, as is the case with the yields of a minority infecting type, but shows a single mode slightly less than the mean.

The last two lines of evidence cited seem to show that the principle of limited participation (Dulbecco 1949) referred to above, operates only during the initial stages of infection, or at any rate does not influence the yields of genetic recombinants arising within the mixedly infected bacteria. They suggest further that the $h$ mutant is superior to $r 7$ or $r 13$ in excluding power only, not as a competititor during actual multiplication.

\section{YIELDS OF GENETIC RECOMBINANTS FROM SINGLE \\ MIXEDLY INFECTED BACTERIA}

In order to study the variations in yields of recombinants intrinsic to the recombination process, one would like to exclude as many as possible of the accessory sources of variation. The most important of these are variations in burst size, and variations in the relative numbers of the two infecting viruses adsorbed to individual bacteria. It will be seen presently that effects of variations in burst size can be avoided by the simple expedient of computing proportionate yields of recombinants, these being independent of burst size. The effect of variations in relative multiplicity could be minimized either by going to very small or very large total multiplicities. Low multiplicities are uneconomical, because at multiplicities sufficiently small so that most of the mixedly infected bacteria receive only one viral particle of each type, very few of the test cultures will yield a mixed burst. High multiplicities also introduce difficulties (DulBecco 1949). We have chosen to use total multiplicities between 10 and 20, within which range the yield of recombinants is constant.

As previously described, the elementary viral yields vary considerably in the relative numbers of the two parental types of virus and, as expected, these variations influence in turn the yields of recombinants. A correction for this source of variation was devised as follows. Assuming that the genetic interaction occurs between unlike viral pairs, and that the composition of the viral yield provides a direct measure of the composition of the intracellular viral population during growth, one computes an interaction coefficient

$$
\mathrm{k}=\mathrm{p}(h)[1-\mathrm{p}(h)]
$$

in which $\mathrm{p}(\mathrm{h})$ is given by (1), and $\mathrm{k}$ expresses the influence of the composition of the population on the number of unlike viral pairs present in the cell, neglecting effects of genetic recombination.

The coefficient $\mathrm{k}$ has a maximum of 0.25 when half the viral yield contains the $h$ allele. Dividing the proportions of recombinants by $4 \mathrm{k}$ serves therefore as a correction for inequality of yields of the parental viruses. This correction is ambiguous only for bursts in which the yields of the two recombinants are large and unequal, and bursts from which either recombinant is absent.

A summary of the single burst data is given in table 4 , which includes the mixed bursts only. The bursts have been separated into the classes $k \geqq 0.21$ and $\mathrm{k} \leqq 0.20$, to show the effect of the correction described above. It will be seen that the uncorrected mean proportion of recombinants is larger for the 
bursts with the larger $\mathrm{k}$, and that the effect of dividing each proportion by $4 \mathrm{k}$ is to make the results homogenous. These facts justify the use of the correction. Its theoretical significance is clarified in the discussion.

TABLE 4

Single burst data for $h \times r$ crosses

$k=a$ measure of disproportion between yields of parental types (Eq. 3).

$\overline{\mathrm{x}}=$ average yield of the $r^{+}$recombinant as percent of total virus.

$\bar{y}=$ average yield of the $r$ recombinant as percent of total virus.

$\overline{\mathrm{n}}=$ average burst size.

$r(x, y)=$ coefficient of correlation between proportionate yields of the two recombinants.

$r(n, x+y)=$ coefficient of correlation between burst size and sum of proportions of the two recombinants.

The variations of $x, y$ and $n / 100$ shown are standard deviations within the sample. The standard errors of the means are obtained by dividing these by the square root of the number of bursts.

The correction referred to is described in the text.

\begin{tabular}{|c|c|c|c|c|c|c|c|c|c|}
\hline cross & $\begin{array}{c}\text { NO. OF } \\
\text { BURSTS }\end{array}$ & k & $4 \overline{\mathrm{k}}$ & & $\overline{\mathbf{x}}$ & $\overline{\mathbf{y}}$ & $\overline{\mathbf{n}} / 100$ & $r(x, y)$ & $r(n, x+y)$ \\
\hline$h \times r I$ & $\begin{array}{l}25 \\
52\end{array}$ & $\begin{array}{l}\leqq 0.20 \\
\geqq 0.21\end{array}$ & $\begin{array}{l}0.65 \\
0.94\end{array}$ & $\begin{array}{l}\text { uncorr. } \\
\text { corr. } \\
\text { uncorr. } \\
\text { corr. }\end{array}$ & $\begin{array}{r}8.9 \pm 3.8 \\
13.9 \pm 5.3 \\
15.5 \pm 6.9 \\
16.6 \pm 6.3\end{array}$ & $\begin{array}{l}12.1 \pm 6.2 \\
19.0 \pm 9.2 \\
17.1 \pm 7.1 \\
18.1 \pm 7.2\end{array}$ & $\begin{array}{l}5.5 \pm 2.4 \\
5.8 \pm 3.2\end{array}$ & $\begin{array}{r}0.09 \\
-0.01\end{array}$ & $\begin{array}{r}-0.25 \\
0.15\end{array}$ \\
\hline$h r l \times++$ & $\begin{array}{l}19 \\
36\end{array}$ & $\begin{array}{l}\leqq 0.20 \\
\geqq 0.21\end{array}$ & $\begin{array}{l}0.67 \\
0.94\end{array}$ & $\begin{array}{l}\text { uncorr. } \\
\text { corr. } \\
\text { uncorr. } \\
\text { corr. }\end{array}$ & $\begin{array}{l}13.6 \pm 5.9 \\
20.1 \pm 7.4 \\
16.5 \pm 5.8 \\
17.5 \pm 6.2\end{array}$ & $\begin{array}{l}13.2 \pm 4.4 \\
20.8 \pm 8.5 \\
17.1 \pm 5.6 \\
18.2 \pm 6.0\end{array}$ & $\begin{array}{l}5.1 \pm 1.9 \\
5.4 \pm 3.0\end{array}$ & $\begin{array}{r}-0.27 \\
0.16\end{array}$ & $\begin{array}{l}-0.26 \\
-0.11\end{array}$ \\
\hline$h \times r 7$ & $\begin{array}{l}13 \\
35\end{array}$ & $\begin{array}{l}\leqq 0.20 \\
\geqq 0.21\end{array}$ & $\begin{array}{l}0.64 \\
0.93\end{array}$ & $\begin{array}{l}\text { uncorr. } \\
\text { corr. } \\
\text { uncorr. } \\
\text { corr. }\end{array}$ & $\begin{array}{l}5.7 \pm 3.5 \\
8.8 \pm 4.8 \\
6.8 \pm 4.2 \\
7.2 \pm 4.3\end{array}$ & $\begin{array}{l}6.6 \pm 4.6 \\
9.9 \pm 5.9 \\
9.2 \pm 4.5 \\
9.8 \pm 4.8\end{array}$ & $\begin{array}{l}6.2 \pm 1.9 \\
5.9 \pm 3.2\end{array}$ & $\begin{array}{l}0.65 \\
0.53\end{array}$ & $\begin{array}{r}-0.43 \\
0.16\end{array}$ \\
\hline$h r 7 \times++$ & $\begin{array}{l}17 \\
26\end{array}$ & $\begin{array}{l}\leqq 0.20 \\
\geqq 0.21\end{array}$ & $\begin{array}{l}0.63 \\
0.95\end{array}$ & $\begin{array}{l}\text { uncorr. } \\
\text { corr. } \\
\text { uncorr. } \\
\text { corr. }\end{array}$ & $\begin{array}{r}6.4 \pm 3.1 \\
10.0 \pm 4.3 \\
6.7 \pm 3.2 \\
7.1 \pm 3.6\end{array}$ & $\begin{array}{l}4.8 \pm 2.9 \\
8.0 \pm 5.0 \\
8.9 \pm 5.4 \\
9.3 \pm 5.6\end{array}$ & $\begin{array}{l}6.4 \pm 7.8 \\
5.8 \pm 3.7\end{array}$ & $\begin{array}{l}0.12 \\
0.47\end{array}$ & $\begin{array}{r}-0.45 \\
0.02\end{array}$ \\
\hline$h \times r 13$ & $\begin{array}{l}29 \\
29\end{array}$ & $\begin{array}{l}\leqq 0.20 \\
\geqq 0.21\end{array}$ & $\begin{array}{l}0.61 \\
0.94\end{array}$ & $\begin{array}{l}\text { uncorr. } \\
\text { uncorr. }\end{array}$ & $\begin{array}{l}0.5 \pm 0.5 \\
0.9 \pm 0.9\end{array}$ & $\begin{array}{l}0.8 \pm 0.8 \\
1.1 \pm 0.9\end{array}$ & $\begin{array}{l}5.2 \pm 2.1 \\
5.1 \pm 2.3\end{array}$ & $\begin{array}{r}0.07 \\
-0.05\end{array}$ & $\begin{array}{r}0.35 \\
-0.30\end{array}$ \\
\hline$h \cdot 13 \times++$ & $\begin{array}{l}21 \\
46\end{array}$ & $\begin{array}{l}\leqq 0.20 \\
\geqq 0.21\end{array}$ & $\begin{array}{l}0.69 \\
0.94\end{array}$ & $\begin{array}{l}\text { uncorr. } \\
\text { uncorr. }\end{array}$ & $\begin{array}{l}0.6 \pm 0.7 \\
0.8 \pm 0.7\end{array}$ & $\begin{array}{l}0.6 \pm 0.9 \\
0.7 \pm 0.6\end{array}$ & $\begin{array}{l}4.6 \pm 1.4 \\
4.9 \pm 2.3\end{array}$ & $\begin{array}{l}0.15 \\
0.21\end{array}$ & $\begin{array}{l}0.22 \\
0.04\end{array}$ \\
\hline
\end{tabular}

The data of table 4 for mixed single bursts confirm fairly well the average data of table 2, except that the yields of recombinants are somewhat greater owing to the exclusion of the unmixed bursts, and that the yields corrected for unequal growth of parental viruses are higher still.

The chief point of interest is the question of the correlation between yields of the two recombinants in single bursts. This has been measured in terms of the correlation coefficient $r$ (RIDER 1939). This measure varies between -1 and +1 , a value near 0 indicating independence of variates, and values near unity indicating negative or positive correlation, respectively. The data of table 4 show clearly that there is no significant correlation between the proportions of the two recombinants in single bursts except for the crosses $h \times r 7$ and $h r 7 \times$ wild type. Even for these crosses the correlation is weak and not entirely convincing, especially since the data are not completely unselected (see below). 
The correlation between the uncorrected proportions of the two recombinants is shown in the form of scatter diagrams in fig. 7. These data might be expected to show some degree of spurious correlation owing to the fact that bursts with disproportionate yields of the two parental types tend to contain diminished numbers of both recombinants. This tendency can be seen in the
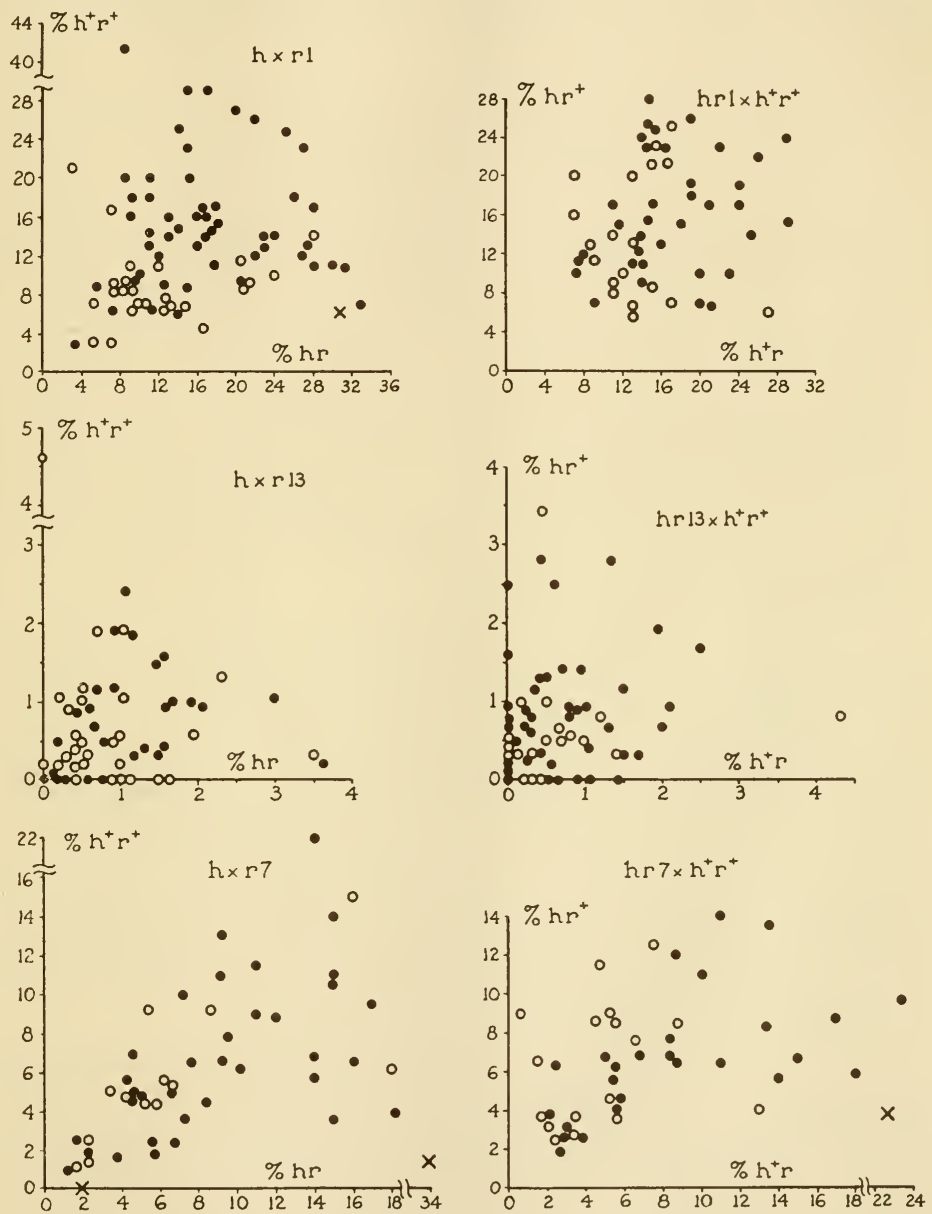

FIGURE 7.-Correlation between proportionate yields of the two recombinants in single bursts. The open circles indicate bursts with disproportionate yields of the two parental viruses. Crosses indicate yields omitted from the data of table 4. 
diagrams in which the disproportionate yields $(\mathrm{k} \leqq 0.20)$ are indicated by open circles. Actually this effect is of minor importance, since the variations due to unknown causes are so much greater than those due to variations in $\mathrm{k}$. Consequently, the uncorrected data lead to the same conclusions as the corrected data; namely, that the proportionate yields of the recombinants are uncorrelated in the crosses $h \times r 1, h \times r 13$, and the corresponding reverse crosses, but that there is a weak positive correlation for the crosses $h \times r 7$ and $h r 7 \times$ wild type.

As mentioned earlier, the correlation data in table 4 are not unselected. The diagrams of fig. 7, however, show all the mixed bursts for the respective experiments, those omitted from the table being indicated by crosses in the diagram. It is plausible that some of the discrepant bursts came from bacteria infected with a spontaneous mutant present in one of the parental stocks of virus. In fact, two bursts from the cross $h \times r 7$ were found to contain a large proportion of mutants, $m$ in one case and weak inhibitor (HERSHEy 1946b) in the other, which almost certainly arose in this way. The $h$ stock used in these crosses contained about 0.1 percent of $h r$ virus, so that in crosses with $r$ at least one bacterium in 200 was infected with three types of virus, and would be likely to yield an excess of one of the recombinant phenotypes. Unfortunately the recombinant progeny in the exceptional bursts were not checked by crossing with the parental stocks, which should be done in any further experiments of this type. For the present these bursts throw some doubt on the significance of the positive correlation between proportions of recombinants in the crosses $h \times r 7$ and $h r 7 \times$ wild type.

The results of the cross $h \times r 13$ are of special interest because of the small yield of recombinants. The distribution of the bursts with respect to absence of recombinants is shown in table 5 . Nine of the 125 mixed bursts fail to show either recombinant, and 31 more lack one recombinant or the other. One can test the hypothesis that the two sister recombinants arise independently as follows. About 20 percent of the bursts fail to show a specified one of the two possible recombinants. If the absence of the one were independent of the absence of the other, about $(0.2)^{2}$ or four percent of the bursts should show neither recombinant. The number found, $9 / 125$, is larger than this, but not significantly so. Moreover, bursts lacking one recombinant do not show less than the average proportion of the other (table 5). The data evidently fail to exclude the hypothesis of independent origin of the two recombinants, but do not, of course, rule out the hypothesis of reciprocal exchange.

Another question that arises in connection with the data is concerned with the number of genetic exchanges per bacterium. For explicitness, we consider separately the hypotheses of reciprocal and non-reciprocal exchange. If exchanges are reciprocal, the bursts lacking a single recombinant are the result of failure to recognize the few plaques of either type, of losses in the ten percent of each culture not examined, and of unspecified biological accidents. As previously computed, four percent or 5 of the 125 bursts fail to show either recombinant for one or another of these reasons, leaving only 4 without recombinants possibly owing to failure of exchange. This number is too small 
if some of the single absences result from erroneous recognition of one recombinant, but is otherwise subject only to its sampling errors. Taken as a measure of failure of reciprocal exchange, the fraction $4 / 125$ implies an average of 3.4 exchanges per bacterium. If, on the other hand, exchanges are not reciprocal, the fraction (20 percent) of the bursts lacking any given recombinant corresponds to 1.7 genetic transfers per bacterium.

These estimates may be too low if conditions vary from bacterium to bacterium in such a way that genetic exchange is suppressed in some bacteria.

TABLE 5

Distribution of Bursts with Respect to Absence of Recombinants

See legend table 4 . The proportions of recombinants have not been corrected for disproportionate yields of parental types of virus.

\begin{tabular}{|c|c|c|c|c|c|c|}
\hline CROSS & CLASS & $\begin{array}{l}\text { NO. OF } \\
\text { BURSTS }\end{array}$ & $\bar{x}$ & $\bar{y}$ & $\overline{\mathbf{n}}$ & $\overline{\mathrm{k}}$ \\
\hline \multirow[t]{4}{*}{$h \times r 13$} & both absent & 2 & 0.0 & 0.0 & 390 & 0.20 \\
\hline & $h^{+} r^{+}$absent & 10 & 0.0 & 0.81 & 460 & 0.19 \\
\hline & $h r$ absent & 2 & 2.4 & 0.0 & 390 & 0.19 \\
\hline & total & 58 & 0.72 & 0.97 & 520 & 0.20 \\
\hline \multirow[t]{4}{*}{$h r 13 \times++$} & both absent & 7 & 0.0 & 0.0 & 340 & 0.21 \\
\hline & $h r^{+}$absent & 8 & 0.0 & 0.66 & 360 & 0.21 \\
\hline & $h^{+} r$ absent & 11 & 0.75 & 0.0 & 380 & 0.22 \\
\hline & total & 67 & 0.73 & 0.64 & 480 & 0.22 \\
\hline
\end{tabular}

Yields with small $\mathrm{k}$ and small burst size must have this effect, but are evidently not very important in the data of table 5 , since the different classes are very similar with respect to $\mathrm{k}$ and burst size. In short, it is necessary to conclude that there are at least two or three genetic exchanges per bacterium, independently of the mechanism by which recombinants arise.

A different kind of estimate of the number of exchanges per bacterium is obtained from the number of recombinants actually found. The data are summarized in table 6 in the form of distributions of numbers of the several recombinant types. One finds on the average 3.4 recombinants of any one kind per bacterium. This evidently furnishes an upper limit to the number of exchanges per bacterium, insofar as exchanges yield viable and countable progeny. This result, taken in conjunction with the preceding estimates, leads to several remarkable conclusions.

First, since the two methods of estimation, one minimal and one maximal, yield about the same result, there must be in fact only two or three exchanges per bacterium in the crosses between closely linked factors.

Second, the recombinants must undergo little multiplication after they arise in the cell.

Third, the conditions of viral growth in different bacterial cells must be equally favorable to genetic recombination; otherwise a larger proportion of bursts would fail to show recombinants. 
These conclusions are substantially confirmed by the distributions of numbers of recombinants shown in table 6, which are essentially of the Poisson type, with variance only moderately greater than the mean. In other words, the individual particles of any one recombinant must often arise independently of each other in the same bacterial cell, and with equal probability in different bacterial cells. The deviations from the Poisson distribution are nevertheless significant, and can be attributed to a moderate amount of growth of recombinants.

TABLE 6

\section{Distributions of Numbers of Recombinants in Single Bursts from the Crosses $h \times r 13$ and $h$ r13 $\times$ wild type}

The Poisson distributions show the numbers of tubes in the various classes expected if there were no growth of recombinants. The distribution with mean 3.4 is appropriate to the hypothesis of reciprocal exchange, and the distribution with mean 1.7 to the hypothesis of non-reciprocal genetic interaction.

\begin{tabular}{ccccccc}
\hline \hline $\begin{array}{c}\text { NO. OF RE- } \\
\text { COMBINANTS } \\
\text { PER TUBE }\end{array}$ & \multicolumn{3}{c}{ NO. OF TUBES FOUND } & \multicolumn{2}{c}{$\begin{array}{c}\text { POISSON } \\
\text { DISTRIBUTIONS }\end{array}$} \\
\cline { 2 - 5 } & $h^{+} r^{+}$ & $h r$ & $h r^{+}$ & $h^{+} r$ & & \\
\hline 0 & 12 & 4 & 15 & 18 & 2.1 & 11.5 \\
1 & 11 & 11 & 11 & 14 & 7.2 & 19.6 \\
2 & 9 & 8 & 8 & 6 & 12.2 & 16.7 \\
3 & 6 & 9 & 11 & 9 & 13.8 & 9.5 \\
4 & 3 & 7 & 6 & 4 & 11.7 & 4.0 \\
5 & 7 & 3 & 4 & 2 & 8.0 & 1.4 \\
6 & 1 & 2 & 3 & 6 & 4.5 & 0.4 \\
7 & 3 & 6 & 0 & 2 & 2.2 & 0.1 \\
8 & 2 & 2 & 3 & 3 & 0.9 & 0.0 \\
9 & 1 & 2 & 2 & 1 & 0.3 & 0.0 \\
$10-18$ & 3 & 2 & 3 & 2 & 0.1 & 0.0 \\
$19-27$ & 0 & 1 & 1 & 0 & 0.0 & 0.0 \\
$28-36$ & 0 & 1 & 0 & 0 & 0.0 & 0.0 \\
\hline No. of tubes & 58 & 58 & 67 & 67 & 63 & 63 \\
Mean per tube & 3.1 & 4.5 & 3.3 & 2.8 & 3.4 & 1.7 \\
Variance & 9.2 & 27 & 16 & 10 & 3.4 & 1.7 \\
\hline
\end{tabular}

The conclusion that genetic recombination is not suppressed in some of the bacteria is also supported by the results of the other crosses, in which no bursts yielding both parental viruses and lacking both recombinants were found. Only one burst, from the cross $h \times r 7$, failed to show one of the recombinants; it contained 95 percent of the $h$ parent.

INDEPENDENCE BETWEEN PROPORTION OF RECOMBINANTS AND BURST SIZE

The data of table 4 do not show any significant correlation between burst size and proportion of recombinants, which means that the number of recombinants must be very nearly proportional to the total yield of virus in single bursts.

This conclusion must be qualified in view of the following considerations. 
Some of the tubes contain one or more mixed bursts plus one or more unmixed bursts. Such tubes would tend to show less than average values of $\mathrm{k}$ and less than average yields of recombinants together with greater than average burst size. The number of such tubes is about five percent of the total in our experiments and these tend to be concentrated in the class $\mathrm{k} \leqq 0.2$. This probably explains the negative correlation between proportions of recombinants and burst size among tubes selected for small $\mathrm{k}$.

A larger proportion of the tubes, between 10 and 20 percent in our experiments, contain two or more mixed bursts. These tubes would tend to have greater than average $\mathrm{k}$ and greater than average burst size, but will not show exceptional proportions of recombinants. These tubes have the effect of weakening any correlation that may exist between burst size and proportion of recombinants, especially among the class $k \geqq 0.21$. The data previously considered do not, therefore, exclude the possibility of a weak positive correlation between proportion of recombinants and size of bursts.

Advantage was taken of the finding that high $\mathrm{pH}$ reduces size of bursts, to examine the relation between burst size and yield of recombinants in another

TABLE 7

Small Bursts from the Cross $h \times r 7$ in Broth of $p H 9.0$

See legend table 4 . The proportions of recombinants have not been corrected for disproportionate yields of parental types of virus. The individual cultures contain an average of about $\mathbf{1 . 4}$ mixed bursts.

\begin{tabular}{cccccccc}
\hline $\begin{array}{c}\text { No. OF } \\
\text { BURSTS }\end{array}$ & $\mathrm{k}$ & $4 \overline{\mathrm{k}}$ & $\mathrm{x}$ & $\overline{\mathrm{y}}$ & $\overline{\mathrm{n}}$ & $\mathrm{r}(\mathrm{x}, \mathrm{y})$ & $\mathrm{r}(\mathrm{n}, \mathrm{x}+\mathrm{y})$ \\
\hline 20 & $\leqq 0.20$ & 0.61 & $6.3 \pm 3.8$ & $10.7 \pm 6.2$ & $99 \pm 50$ & 0.53 & -0.34 \\
34 & $\geqq 0.21$ & 0.93 & $7.4 \pm 3.5$ & $10.5 \pm 4.6$ & $123 \pm 73$ & 0.61 & 0.35 \\
54 & all & 0.81 & $7.0 \pm 3.7$ & $10.6 \pm 5.2$ & $114 \pm 66$ & 0.56 & 0.12 \\
\hline
\end{tabular}

way. Since increased $\mathrm{pH}$ was found also to cause some of the bacteria to fail to liberate virus, the single burst technique was chosen.

Bacteria were infected in the usual way with mutants $h$ and $r 7$ in broth of $\mathrm{pH}$ 6.8. At the end of the five minute adsorption period, and without treatment with antiserum, single burst cultures were prepared after diluting in broth of $\mathrm{pH}$ 9.0. Preliminary experiments showed that the yield of virus was complete under these conditions within one hour, and than no inactivation occurred during two additional hours at $37^{\circ} \mathrm{C}$, or overnight in the refrigerator. Entire samples were plated on single mixed indicator plates. One successful experiment yielded 66 bursts, of which 54 were mixed, among 104 tubes receiving on the average 1.4 bacteria per tube. Evidently most of the bacteria liberated some virus. The average burst size, after subtracting the virus carried over from the input (totalling 36 particles per tube), was only 114 per tube, or about 70 per bacterium corrected for the probable multiples. The average proportion of recombinants was nevertheless of normal size (table 7).

It will be noticed also that the correlation between the numbers of the two recombinants in these bursts is exceptionally good. The correlation is, however, 
slightly exaggerated owing to the fact that the 54 tubes contain on the average mixed yields of virus from about 1.4 bacteria per tube.

THE EFFECT OF A SHORT PERIOD OF ADSORPTION AND LOW MULTIPLICITY

OF INFECTION ON THE DISTRIBUTION OF RECOMBINANTS

The following experiment shows that when the multiplicity of infection in the cross $h \times r 7$ is reduced from five to about one of each viral type per bacterium, the distribution of recombinants among single mixed bursts is little if any altered.

Six crosses were made in the usual way, except that the period allowed for adsorption was reduced to one minute, without reducing the total input of virus. The amount of virus adsorbed was too small to be measured, but the multiplicity of infection can be estimated from the data given below.

The single burst cultures collected from the six crosses are sufficiently similar to be considered together. The mean number of bacteria per tube for the six sets, determined by colony counts from the growth tubes immediately before adding virus, is 0.23 . The mean number of infected bacteria per tube determined by plaque counts of samples taken before lysis is 0.20 . The mean number of bursts per tube calculated from the proportion, 141 out of 720 , of tubes containing virus is 0.22 . The 141 tubes therefore contained about 157 infected bacteria.

From the distribution of viral types among the tubes, namely, 69 containing $h$ only, 22 containing $r$ only, 50 containing both, and 579 containing neither, one finds the probable distribution with respect to bacteria to be 80 infected with $h$ only, 28 with $r$ only, 49 both, and 46 neither. The multiplicity of infection is therefore about 1.0 with respect to $h$, and 0.48 with respect to $r$. One can estimate further that about seven of the tubes contained one or more mixed bursts plus one or more unmixed; and that about three contained both $h$ and $r$ bursts without any mixed bursts. Also, among the mixedly infected bacteria, 45 percent were infected with one particle only of each viral type.

In making the above computations we have neglected the probability that the $h$ mutant suppresses the growth of $r$ in some bacteria adsorbing both types of virus. The apparent inequality of infection is probably due in some part to this effect. However, in other experiments with low multiplicity of infection with $h$ and $r 7$ designed to check this point, the split into $h, r$, and mixed yielders was nearly equal. It seems likely, therefore, that in the experiments reported here the two viral types were unequally adsorbed for unknown reasons.

The tubes containing only one viral type may be dismissed by saying that their average content of virus did not differ significantly from that of the mixed yields, and that the $h$ and $r$ yields per bacterium were the same. There was one exceptional burst containing only $h r^{+}$and $h r$ phenotypes. The characteristics of the remaining 49 cultures containing $h$ and $r$ virus are summarized in table 8. The data show no unusual features excepting the small burst size, which is a direct effect of the low multiplicity of infection, and the somewhat 
small proportion of recombinants, probably due to the appreciable number of superimposed unmixed bursts.

\section{TABLE 8}

Single Bursts from the Cross $h \times r 7$ with Low Multiplicity of Infection

See legend table 4 . The proportion of recombinants have not been corrected for disproportionate yields of parental types of virus. Adsorption time one minute. Multiplicity $1.0 \mathrm{~h}$ and $0.5 r$ per bacterium.

\begin{tabular}{cccccccc}
\hline \hline $\begin{array}{c}\text { No. of } \\
\text { BURSTS }\end{array}$ & $\mathrm{k}$ & $4 \overrightarrow{\mathrm{k}}$ & $\overline{\mathrm{x}}$ & $\overline{\mathrm{y}}$ & $\overline{\mathrm{n}} / 100$ & $\mathrm{r}(\mathrm{x}, \mathrm{y})$ & $\mathrm{r}(\mathrm{n}, \mathrm{x}+\mathrm{y})$ \\
\hline 17 & $\leqq 0.20$ & 0.59 & $5.3 \pm 4.0$ & $4.5 \pm 3.7$ & $2.9 \pm 1.6$ & 0.38 & -0.17 \\
32 & $\geqq 0.21$ & 0.95 & $4.4 \pm 3.1$ & $6.1 \pm 5.4$ & $3.5 \pm 2.0$ & 0.20 & -0.33 \\
49 & all & 0.84 & $4.7 \pm 3.5$ & $5.7 \pm 4.9$ & $3.2 \pm 1.9$ & 0.24 & -0.30 \\
\hline
\end{tabular}

The principal point to be made here is that the variation in yields of recombinants from tube to tube is not exceptional. In this connection it must be mentioned that 5 of the 49 tubes contained $h$ and $r$ virus without any recombinants. Of these, one was exceptional in containing only 24 viral particles, and another for the extreme disproportion of parental types ( 88 percent $h$ ). The remaining three, each containing from 450 to 570 particles, are probably superimposed unmixed $h$ and $r$ bursts.

It should be noted also that the correlation between the yields of the two recombinants in this set is not significant, since the observed correlation is exaggerated by the tubes containing unmixed $h$ and $r$ bursts without recombinants. Whether the poor correlation is accidental, or an effect of the low multiplicity of infection, remains to be determined. The negative correlation between burst size and proportion of recombinants can, however, be ascribed to the superimposed mixed and unmixed bursts, as well as to the unmixed $h$ and $r$ bursts, in some of the tubes.

\section{IDENTITY OF RECOMBINANTS WITH THE CORRESPONDING ANCESTRAL TYPES}

According to any simple hypothesis of factorial recombination, one expects the recombinant virus arising in crosses not to differ genetically from the corresponding ancestral type. Two kinds of test indicate that this is so. In the first kind of test (HERSHEy and Rotman 1948) stocks of the phenotypic wild type arising from the cross between two different $r$ mutants were backcrossed to authentic wild type. No $r$ mutants appeared in such crosses, and it was concluded that the stocks were genetically identical.

The second kind of test is the following. The double mutant $h r 7$, itself obtained by crossing the two single mutants, was crossed with wild type and the recombinants $h$ and $r$ were re-isolated. These were then tested by making the homologous (parental $h$ by recombinant $h$ and parental $r$ by recombinant $r$ ) and heterologous (parental $h$ by recombinant $r$ and parental $r$ by recombinant h) back crosses. In both cases, the homologous cross yielded only one type of 
virus, and the heterologous cross yielded recombinants in the same proportion as found with the parental stocks. These tests show not only that the recombinants contain the same genetic markers as the corresponding progenitive types, but also that the region between the markers is unchanged.

\section{MIXED YIELDS CONTAINING ONLY ONE PARENTAL TYPE OF VIRUS}

Only four mixed bursts lacking one of the parental types of virus were found among the experiments reported in this paper. One, from the cross $h \times r 1$, contained 86 percent $h^{+} r$ and 14 percent $h r$. A second, from the cross $h \times r 7$ contained 99.6 percent $h r^{+}$and 0.4 per cent $h r$. A third, from the same cross with low multiplicity of infection, contained 83 percent $h r^{+}$, and 17 percent $h r$. The fourth, from the cross $h r 7 \times$ wild type, contained 23 percent $h r^{+}$and 77 percent $h r$. In this case the yield of $h r^{+}$, which appeared to be homogenous, formed atypical plaques and proved on isolation to differ from any known mutant of $T 2 H$. It seems reasonable to suppose that these exceptional bursts contained progeny stemming from mutants contaminating the parental stocks of virus. On the other hand, the one exceptional burst following low multiplicity of infection, together with the failure to find similar bursts among the crosses involving $h$ and $r 13$, suggest that a different interpretation should be looked for. Genetic tests which might have clarified this point are lacking. For the present we conclude, as a first approximation, that recombinants arise only in those bacteria in which both parental types of virus succeed in multiplying.

It may be added here, because the question arises in connection with these exceptional bursts, that no correlation can be seen between the proportion in mixed bursts of the total virus containing the $h$ allele, and the proportion of the recombinant virus containing the $h$ allele. We have therefore omitted this datum from the tables.

\section{DISCUSSION}

In collecting and analyzing the data just described, we have had in mind the following questions. Does genetic exchange occur in the course of matings between viral particles, or is it the expression of a mechanism of growth such as that visualized by LURIA (1947), according to which the multiplying units in the cell are not phage particles, but simpler structures derived from them? Can the linkage relations represented in fig. 1 be interpreted in terms of linear chromosome-like structures? Are the genetic exchanges reciprocal, as one expects for simple cases of crossing over, or must one look for an alternative mechanism more intimately connected with the mode of reproduction of the virus?

It was soon apparent that the data for crosses between linked and unlinked factors tended to give different answers to these questions, and we were led to consider a model based on two distinct mechanisms of exchange. The necessity for this arises from the following facts.

First, the linkage data indicate a limitation at about seven percent to the proportion of wild type found in crosses between linked factors, which is dif- 
ficult to reconcile, in terms of a single mechanism, with the existence of a second class of crosses yielding about 15 percent of wild type.

Second, the correlation between proportions of the two recombinants in the cross $h \times r 7$, and the lack of a corresponding correlation in the cross $h \times r 1$, is incompatible with a single mechanism for the two crosses. It must be recalled, however, that the correlations found are too weak to be wholly convincing.

Third, LURIA's (1947) evidence for a mechanism of independent multiplication and transfer of subunits of the virus, and ours for a system of linkage, require dissimilar types of interpretation.

The model to which these considerations seem to lead is described below, but we do not consider that we have decisive answers to any of the questions originally posed. The remainder of this discussion is of value only insofar as it clarifies the questions, and systematizes the experimental results so far obtained.

The two linkage structures bearing the markers $r 1$ and $h$, respectively, are assumed to be examples of the class of independently multiplying subunits of the virus whose minimal number LuRIA and DulBecco (1949) estimate at about 25. The reconstitution of virus from these units must be regulated in such a way that each particle receives one representative of each kind of unit. In the cross $h \times r 1$ the choice between $h$ and $h^{+}$, and between $r$ and $r^{+}$, is decided nearly at random to yield on the average 37 percent of recombinants and 63 percent of the parental types in bacteria yielding equal numbers of the two parents. The deficit of recombinants below 50 per cent is unexplained, but may be thought of as an effect of incomplete mixing between neighboring clones of multiplying virus in the cell.

According to this hypothesis one expects from the cross $h \times r 1$ proportionate yields of the two recombinants in a single burst to be:

$$
\begin{aligned}
\mathrm{p}\left(h^{+} r^{+}\right) & =\mathrm{m}\left(\frac{\mathrm{n}\left(h^{+}\right)}{\mathrm{n}\left(h^{+}\right)+\mathrm{n}(h)}\right)\left(\frac{\mathrm{n}\left(r^{+}\right)}{\mathrm{n}\left(r^{+}\right)+\mathrm{n}(r)}\right) \\
\mathrm{p}(h r) & =\mathrm{m}\left(\frac{\mathrm{n}(h)}{\mathrm{n}\left(h^{+}\right)+\mathrm{n}(h)}\right)\left(\frac{\mathrm{n}(r)}{\mathrm{n}\left(r^{+}\right)+\mathrm{n}(r)}\right)
\end{aligned}
$$

where the expressions on the left refer to proportions of recombinant virus, the corresponding expressions on the right refer to the intra cellular yields of the respective unit linkage structures, and the coefficient $\mathrm{m}$ expresses the fraction of the intracellular virus which may be regarded as a random mixture of the two parental types, the remainder being considered unmixed. If the structures carrying the markers $h, h^{+}, r$, and $r^{+}$grow independently in the cell, their yields will fluctuate independently, and no correlation will be expected between the numbers of the two recombinants in sufficiently small yields of virus. This expectation is borne out by the data for viral yields from single bacteria. The average yields of the two recombinants are equal, however, showing that the several unit structures grow at equal rates. According to equations (4) and (5), the proportionate yield of recombinants should not be 
influenced by burst size unless the latter has an effect on the fraction $\mathrm{m}$. According to the data it does not, and $\mathrm{m}$ is a parameter having the average value $37 / 50$. This interpretation requires that 26 percent of either kind of parental virus in the cell should multiply in effective isolation from the other parent.

It will be noticed that the expressions (4) and (5) reduce to $\mathrm{mk}$ if one makes the approximations mentioned in connection with equation (3). This provides a theoretical basis for the correction we have applied to yields of recombinants in crosses between unlinked factors. An analogous justification for its use in crosses between linked factors will appear in the discussion to follow.

The predictions for the cross $h \times r 7$ are different from the preceding case, because here the markers are situated on homologous linkage structures, so that recombination requires something like crossing over, which in turn requires something like synapsis. The expected fractional yields of recombinants are

$$
\mathrm{p}\left(h^{+} r^{+}\right)=\mathrm{p}(h r)=\frac{1}{2} \mathrm{msc},
$$

in which $\mathrm{m}$ has been defined previously, $\mathrm{s}$ is a fraction independent of $\mathrm{m}$ expressing frequency of pairing, and $\mathrm{c}$ is a crossover frequency. In order to make $\mathrm{c}$ independent of $\mathrm{m}$ and $\mathrm{s}$ it is evidently sufficient to define the product $\mathrm{ms}$ as the fraction of the viral yield made up of particles in which the marked unit has descended from an unlike synapsed pair. The application of (6) leads to ambiguity if exchanges can occur between descendants of unlike synapsed pairs (Hershey and Rotman 1948). In what follows this difficulty does not appear to be very serious, but no rigorous analysis has yet been attempted.

For the cross $h \times r 7$, if exchanges are reciprocal, one expects the correlation between proportions of the two recombinants to be disturbed only by fluctuations in relative growth of the two exchange products, in contrast to the cross $h \times r 1$, where the correlation is subject to fluctuations in the growth of four independent units. That these fluctuations are individually considerable is shown by the variations in relative and total viral yields in mixedly infected bacteria. A weak but probably significant correlation between proportions of the two recombinants is nevertheless visible in the cross $h \times r 7$. No such correlation can be seen in the cross $h \times r 1$. If the mechanism of exchange for these two crosses were the same, the greater correlation would be expected in the cross $h \times r 1$, which gives the larger yield of recombinants.

The predictions for the cross $h \times r 13$ are the same as for $h \times r 7$ except as modified by the much smaller yield of recombinants, presumably owing to a smaller frequency of crossing over. We have shown that in this cross the recombinants come from two or three individual exchanges per bacterium, and that there is little growth of recombinants subsequent to exchange. These circumstances ought to be favorable for testing the hypothesis of reciprocal exchange. The data are nevertheless inconclusive of this point.

The experiments provide information about the sequence of events in the cell. A mechanism of exchange limited to an initial phase of multiplication is ruled out by the following consideration. If exchange occurred at a time when there were few replicas in the cell, any cross yielding a small average number of 
recombinants would show some individual bursts containing no recombinants and others containing a very large proportion, especially at low multiplicity of infection. Instead one finds a comparatively uniform yield of recombinants, and the distribution of their proportions is not affected by the multiplicity of infection.

Also if exchanges occurred freely throughout the period of multiplication of the virus, one would expect considerably greater variations in yields of recombinants than we have found. For instance, in the cross $h \times r 13$, in which there are only two or three exchanges per bacterium, the variations in yields of recombinants are not much greater than those expected to result from a random variation in the number of exchanges alone. Moreover, most of the bacteria yield only a few recombinants, so that little growth can have occurred subsequent to exchange. The conclusion is unavoidable that the exchanges are limited to the terminal phase of multiplication, or at any rate that recombinants are prevented from multiplying appreciably in most of the bacteria.

It is remarkable that the variations in proportions of recombinants are so little dependent on the degree of linkage (as between $h \times r 7$ and $h \times r 13$ ), or on the postulated mechanism of exchange (as between the above and $h \times r 1$ ). The coefficients of variation in proportions of individual recombinants among single bursts are, for $h \times r 1$, about 40 percent; for $h \times r 7$, about 60 percent; and for $h \times r 13$, about 100 percent. This circumstance also supports the inference that the exchanges are limited to a late phase of multiplication.

The hypothesis stated permits one to examine further the structure of the linkage units. Since crosses between $r 13$ and any of the mutants belonging to the group closely linked to $r 7$ yield about the same proportion, seven percent, of wild type (Hershey and Rotman 1948), it might be supposed that one crossover between the distant markers is always accompanied by several others, so that 50 percent of the progeny of synapsed pairs of the units $r 7$ and $r 13$, for example, would be recombinant types. If this supposition is correct the terms of equation (6) can be evaluated by setting $\mathrm{c}=0.5$ and $\mathrm{p}\left(h^{+} r^{+}\right)=0.07$. This gives 0.28 for the average fraction $\mathrm{ms}$ of virus descending from unlike synapsed pairs. If this fraction is assumed to be the same in other crosses involving the same linkage structure (the cross $h r 7 \times r 13$ reported in this paper suggests that it is), one can write for them

$$
\mathrm{p} \text { (wild type) }=0.14 \mathrm{c} \text {, }
$$

where $\mathrm{c}$ is the appropriate crossover frequency and the proportion of wild type is experimentally measured. The data for the three point crosses involving $r 2, r 3$, and $r 6$ (HERSHEY and RotMAN 1948) are examined from this point of view in table 9 . The proportions of wild type have been calculated for random crossing over between unit linear structures, using the crossover frequencies given by (7). It will be seen that the data are entirely compatible with the hypothesis tested. Additional tests of this kind are needed, however.

It will have been noticed that the average yield of recombinants in crosses between distant linked factors is very nearly half that found for unlinked factors. DR. M. DELBRÜCK has pointed out to us that this relationship can be 
understood in terms of random pairing between homologous structures. In the simplest case one visualizes unrepeated pairing, that is, pairing limited to a phase in which there is no multiplication, and during which no structure finds more than one partner. For this case, the frequency of synapsis in equation (6) is simply the ratio of the number of unlike homologous pairs to the total number of homologous pairs. This ratio can be written

$$
s=\frac{2 a b}{(a+b)(a+b-1)} .
$$

where $\mathrm{a}$ and $\mathrm{b}$ are the respective numbers of the two unlike homologous structures. Inspection of (8) shows that this ratio is essentially $2 \mathrm{k}$ as given by equation (3) when $a+b$ is large compared to unity. Instead of equation (6) we have, therefore,

$$
\mathrm{p}(\text { wild type })=\mathrm{mkc} \text {, }
$$

from which the proportion of either recombinant expected in crosses between distant linked factors can be computed as follows.

The parameter $\mathrm{m}$, taken to be the average fraction of virus randomly mixed in the cells at the time of reconstitution of virus from subunits, was found to

\section{TABLE 9}

\section{Three Point Linkage Tests of Linear Structure}

The symbol $r 2,3$ refers to the double mutant containing $r$ alleles at the loci $r 2$ and $r 3$, etc.

$c_{1}$ is the crossover frequency for the region between $r 2$ and $r 3$.

$c_{2}$ is the crossover frequency for the region between $r 3$ and $r 6$.

The locus $r 3$ is assumed to lie between $r 2$ and $r 6$.

\begin{tabular}{|c|c|c|c|}
\hline CROSS & \multicolumn{2}{|c|}{ FRACTIONAL YIELD OF WILD TYPE } & COMPUTATION \\
\hline$r 2 \times r 3$ & - & 0.020 & $c_{1}=0.020 / 0.14=0.14$ \\
\hline$r 3 \times r 6$ & - & 0.014 & $c_{2}=0.014 / 0.14=0.10$ \\
\hline$r 2 \times r 6$ & 0.030 & 0.024 & $0.14\left[c_{1}\left(1-c_{2}\right)+c_{2}\left(1-c_{1}\right)\right]$ \\
\hline$r 2,3 \times r 6$ & 0.012 & 0.008 & $0.14 c_{2}\left(1-c_{1}\right)$ \\
\hline$r 3,6 \times r 2$ & 0.018 & 0.014 & $0.14 c_{1}\left(1-c_{2}\right)$ \\
\hline$r 2,6 \times r 3$ & 0.002 & 0.003 & $0.14 c_{1} c_{2}$ \\
\hline
\end{tabular}

The factor 0.14 is explained in the discussion.

be $37 / 50$ in crosses between unlinked factors. In (9) we require the corresponding fraction at the time of pairing, and assume this to be the same. The average of $\mathrm{k}$ for bacteria giving mixed viral yields (table 4) is 0.21 , or 0.19 if one includes the ten percent of bacteria yielding only one type of virus. If $k$ and $m$ vary independently, their mean product is the same as the product of means, or 0.14 averaged over all bacteria. The average yield of either recombinant from equation (9), for factors sufficiently far apart so that $c=0.5$, is accordingly seven percent, computed solely from the data for crosses between unlinked factors. This is the maximum actually found in crosses 
between linked factors (Hershey and Rotman 1948). Equation (9) also predicts, in agreement with the data for single bursts, proportionality between yields of recombinants and $\mathrm{k}$.

The agreement supports the inferences previously drawn that the markers $r 7$ and $r 13$ are attached to the same linkage unit, and that the frequency of crossing over between them is 0.5 . It suggests that the pairing itself is complete without appreciable repetition, and occurs at random except that about 26 percent of the units of each kind are effectively segregated from their opposite numbers. The measure of this segregation, $m$, is on this view the same for crosses between linked and unlinked factors. On the other hand, this interpretation cannot be rigorously correct, because one can show by multiple factor crosses (HERSHEY and RotMAN 1948) that repeated exchanges, or exchanges among three viral particles, occur. An estimate of the amount of repeated pairing has not yet been attempted, except that the considerations just offered suggest either that it is small, or that random pairing is limited to a small proportion of the population.

It follows from equation (9) that the interpretation in terms of orderly pairing accords with the fact, otherwise very puzzling, that the proportion of recombinants is not affected by size of burst even in crosses between linked factors.

It has been seen that the linkage data support fairly well the idea of linear structure, but independent evidence for crossing over is meagre. According to any simple model of reciprocal exchange, a correlation between proportions of sister recombinants in individual bursts would be expected. This expectation has been only partially realized, and the question arises whether the linkage data themselves require the crossover hypothesis. The following model, suggested by Dr. A. H. Sturtevant, shows that they do not, and also shows that the question of reciprocity is closely connected with the question whether the exchanges are material transfers.

Suppose that the replication of linear structures occurs zipperwise along the pattern from one end to the other, but that the partners separate prematurely to yield fragmentary replicas. Additions to the fragments are subsequently possible only after pairing with the same or another homologous structure, which in mixedly infected bacteria could belong either to the same or a different parental line. Genetic recombination in a two factor cross will depend, then, on the contingency that the two marked regions of a given replica be laid down one after the other on homologous structures from the two unlike parents. With simple assumptions, all the consequences of the crossover hypothesis (equation (6)) follow from this model, except that the independent origin of the two recombinants provides an additional source of independent variation in their numbers.

The complications peculiar to this model have to do principally with the evidence that exchanges occur only during the terminal phase of growth. These complications are not very serious if one assumes that during early stages of growth the probability is great that a fragment will be started and completed on patterns belonging to the same parental line; that is, that the 
mixing of the cell contents is relatively incomplete, and the distance between unlike clones relatively great, for small total populations. It has to be stipulated further that the terminal mixing is independent of the final concentration of virus in the cell, to account for the lack of dependence of proportion of recombinants on burst size. Some hypothesis of this sort may prove useful if further experiments fail to strengthen the present evidence for reciprocal exchange.

It is notable that two very different lines of evidence, ours and that of LURIA (1947), have led to the idea of independently multiplying subunits of the virus. Our results differ from LURIA's only in calling for a system of linkage superimposed on the set of independent units. It remains to be seen whether a combination of genetic and radiological techniques bears out the present conclusions, and perhaps leads to an identification of the radiation-sensitive units with the linkage structures.

\section{SUMMARY}

Genetic recombination between two viruses differing by two mutational steps has been studied by infecting bacteria with the pair, and counting the numbers of the four types of virus found in yields from single bacteria. The crosses so examined include $h \times r 1$ (unlinked), $h \times r 7$ (linked), and $h \times r 13$ (closely linked), where $h$ refers to a mutant of altered host range, and $r 1, r 7$, and $r 13$ are different mutations producing the same alteration in type of plaque. The reverse crosses, $h r \times$ wild type, were also studied. The results may be summarized as follows.

Nearly all mixedly infected bacteria yield both parental types of virus and two recombinants, according to the scheme $h+r=h r+$ wild type. The ten percent or so of bacteria yielding only one of the parental types seldom or never yield any recombinants. The rest of the bacteria always yield two recombinants, except for the occasional absence of one or both in the crosses between closely linked factors.

The average yields of the two recombinants in any one cross are the same, and are independent of the direction of exchange, so that reverse crosses involving the same pair of mutant factors yield the same number of recombinants. The proportionate yields of recombinants from individual bacteria are independent of burst size, and of the total multiplicity of infection, but depend on the relative yields of the two parental types. The effect of the latter is not marked, however, and the variations from bacterium to bacterium must be chiefly the result of variations in the number of genetic exchanges and in the growth of recombinants subsequent to exchange. These variations may be described by saying that one finds a moderately skewed distribution, with mode less than the mean, and with mean and standard deviation dependent on the linkage relations as follows: for $h \times r 1,15 \pm 6$, for $h \times r 7,7 \pm 4$, for $h \times r 13,1 \pm 1$, expressed in round numbers as percent of either recombinant in the total yield of virus.

A weak but moderately convincing correlation between the proportionate 
yields of the two recombinants in individual bacteria is discernible in the cross $h \times r 7$ and its reverse, but not in the other crosses.

In the cross $h \times r 13$ only two or three genetic exchanges occur during the multiplication of the virus in a single bacterial cell. These exchanges take place near the end of the period of multiplication of the virus.

A hypothesis is outlined which is compatible with the genetic data and with the results of LURIA concerning reactivation of irradiated virus in bacteria receiving two or more individually noninfective particles. The hypothesis is an extension of that of LURIA, according to which one visualizes genetic interaction not between two viral particles, but between two sets of independently multiplying chromosome-like structures. Genetic exchange occurs either by reassortment of these structures, or by something like crossing over between homologous pairs, depending on the structural relation between the genetic factors concerned. The interpretation made brings the linkage relations into superficial agreement with the requirements of linear structure, but there is little evidence that the genetic exchanges are reciprocal, and accordingly little evidence that they are material exchanges.

\section{IITERATURE CITED}

DelBrÜcr, M., 1945a Interference between bacterial viruses III. The mutual exclusion effect and the depressor effect. J. Bact. 50: 151-170.

1945b The burst size distribution in the growth of bacterial viruses. J. Bact. 50: 131-135. 1945c Effects of specific antisera on the growth of bacterial viruses. J. Bact. 50: 137-150.

DelbrücK, M., and W. T. BAILEY, Jr., 1946 Induced mutations in bacterial viruses. Cold Spring Harbor Symp. Quant. Biol. 11 : 33-37.

DELBRÜCK, M., and S. E. LURIA, 1942 Interference between bacterial viruses I. Interference between two bacterial viruses acting upon the same host, and the mechanism of virus growth. Arch. Biochem. 1: 111-141.

Dulbecco, R., 1949 The number of particles of bacteriophage $T 2$ that can participate in intracellular growth. Genetics 34: (In press).

Hershey, A. D., 1946a Mutation of bacteriophage with respect to type of plaque. Genetics 31: $620-640$.

1946b Spontaneous mutations in bacterial viruses. Cold Spring Harbor Symp. Quant. Biol. 11: 67-77.

Hershey, A. D., and R. Rotman, 1948 Linkage among genes controlling inhibition of lysis in a bacterial virus. Proc. nat. Acad. Sci. 34: 89-96.

LURIA, S. E., 1947 Reactivation of irradiated bacteriophage by transfer of self-reproducing units. Proc. nat. Acad. Sci. 33: 253-264.

LURIA, S. E., and R. Dulbecco, 1949 Genetic recombinations leading to production of active bacteriophage from ultraviolet inactivated bacteriophage particles. Genetics 34: (In press).

Rmer, Paul R., 1939 An introduction to modern statistical methods. ix +220 pp. New York: Wiley \& Sons. 
GENETIC RECOMBINATIONS LEADING TO PRODUCTION OF ACTIVE BACTERIOPHAGE FROM ULTRAVIOLET INAC'TIVATED BACTERIOPHAGE PARTICLES ${ }^{1}$

\author{
S. E. LURIA AND R. DULBECCO \\ Department of Bacteriology, Indiana University, Bloomington, Indiana
}

Received June 28, 1948

$T$ $\mathrm{HE}$ potentialities of bacteriophage genetics have been revealed by the discovery of genetic recombinations among related phage particles infecting the same bacterial cell (DELBRÜCK and BAILEy 1946). The complexities of these genetic systems have been further illustrated by the work of HERSHEY and Rotman (1948) on a number of different genetic determinants involved in the determination of the alternative phenotypes $r^{+}$and $r$ in phage $T 2 H$. A different approach to the genetic mechanisms of bacteriophages originated from the chance observation by DELBRÜCK and BAILEY that, after exposure to ultraviolet light, some phages gave variable plaque counts, depending on the relative concentrations of phage lysates and host cells at the time of assay. In investigating this phenomenon, one of us (LURIA 1947) discovered a mechanism of phage reactivation by interaction among inactive particles in the course of intracellular growth. The detailed investigation of this phenomenon has indicated new possibilities for a quantitative analysis of the genetic structure of these viruses and has suggested a possible mechanism for their reproduction.

A preliminary discussion of some of the results reported in this paper has appeared (LURIA 1947); their implications for a number of problems have been discussed in a forthcoming publication (LuRIA 1948). The present article is intended to present the results in detail, and, by describing techniques and methods of analysis, to serve as a background for future publications on this topic.

The analysis of the results presented in the following pages is based on the hypothesis that inactivation of bacteriophage particles by ultraviolet light is due to production of discrete alterations in individual portions of genetic material. Although the internal evidence in support of this hypothesis, as presented in this paper, is quite satisfactory, it must be said that satisfactory external evidence from other lines of attack is not yet available. The conclusions reached in this article must be considered for the time being as working hypotheses for further investigation.

\title{
MATERIAL AND GENERAL METHODS
}

The system of phages T1-T7, their $r$ mutants, and their common host Escherichia coli strain B have repeatedly been described, as well as the use of

${ }^{1}$ This work was done under an AMERICAN Cancer Society grant recommended by the Committee on Growth of the National Research Council. We wish to acknowledge the able assistance of MRS. J. P. HFADDY.

[Reprinted by permission from Genetics 34 : 93-122, March, 1949] 
bacterial mutants resistant to one or more phages as indicators for one phage in the presence of another (see DELBRÜCK 1946). Plate counts for viable bacteria, and plaque counts in agar layer for active phage were used throughout, employing 1.1 percent agar in "Difco" nutrient broth plus 0.5 percent $\mathrm{NaCl}$. All plates were incubated at $37^{\circ} \mathrm{C}$. Experimental bacterial cultures in the logarithmic phase of growth were grown with aeration at $37^{\circ} \mathrm{C}$ from standard inocula.

The phage stocks were lysates in glucose +ammonia (or lactate+ammonia) medium. These media give negligible absorption of the ultraviolet light used in this work. High titer phage lysates (over $1 \times 10^{11}$ particles per ml) might give some ultraviolet screening effect because of bacterial debris and of phage itself. Whenever possible, therefore, phage was irradiated after a dilution 1:5 or higher in the same medium. The source of ultraviolet was a General Electric Company germicidal bulb, 15 watts, alimented through a stabilizer. At a distance of $50 \mathrm{~cm}$ from the center of this bulb, the flux-measured with a Westinghouse SM-200 meter with tantalum phototube WL-775-is about 7 erg $\times \mathrm{mm}^{-2} \mathrm{sec}^{-1}$. The beam contains mainly radiation of wavelength $2537 \AA$. Samples were irradiated in a thin layer (not over $0.4 \mathrm{~mm}$ ) in open Petri dishes rocked during exposure.

The technique of "one-step growth" experiment in its various forms has been described in detail previously (DELBRÜCK and LURIA 1942).

\section{EXPERIMENTAL}

\section{Inactivation and reactivation of bacteriophages}

Plaque counts on phage suspensions exposed to ultraviolet for various lengths of time generally give survival ratios whose logarithms are proportional to the dose, that is, to the time of exposure (see LATARJET and WAHL 1945, and figure 1). The logarithmic rate indicates a one-hit mechanism of inactivation (LEA 1947), and we can assume that the hit consists of the successful absorption of one quantum. The probability that one quantum produces inactivation is, however, very small: for phage $T 2$, for example, one inactivating hit is produced by a dose corresponding to almost $10^{4}$ quanta absorbed per particle (M. ZELle, personal communication). Only one absorption in $10^{4}$ on the average is, therefore, effective, the others probably producing excitations that do not lead to the inactivating effect.

When the average number of effective hits per particle is $r$, the proportion of active to total phage will be $\mathrm{e}^{-r}$. For $r=1, \mathrm{e}^{-r}=0.37$; the corresponding dose is the "inactivation dose" in LEA's terminology (1947). If doses are expressed in multiples of the inactivation dose, their values give directly the average number of hits per particle.

Phage particles inactivated by ultraviolet light are adsorbed by bacteria (LURIA and DeLBRÜCK 1942). This is detected because adsorption of one particle by a bacterium causes death of the latter. One can, therefore, measure the rate of adsorption of inactive particles from the survival of bacteria in mixtures containing bacteria and irradiated phage in known proportions. If, on the average, $x$ particles are adsorbed per bacterium, a fraction $\mathrm{e}^{-x}$ of the 


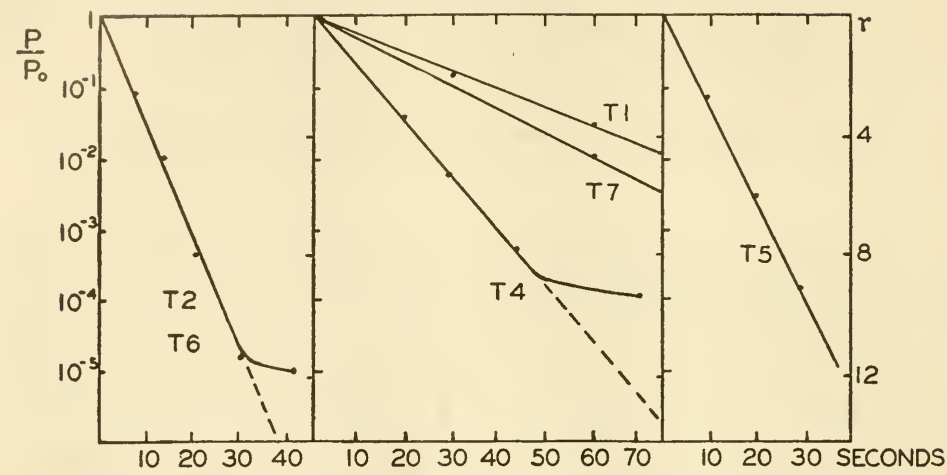

FIGURe 1.-Inactivation of various phages by ultraviolet light. $P / P_{0}=$ proportion of active phage particles after irradiation. $r=\ln P_{0} / P=$ average number of lethal hits per phage particle. The doses are expressed in seconds of exposure. The deviations for high doses in the curves for $T 2, T 4$, and $T 6$ are due to reactivation occurring on the assay plates (see text). The broken lines represent extrapolations from the logarithmic portions of the curves.

bacteria should receive no particle and survive. By this means, we could establish that for irradiated phages $T 2, T 4, T 5$, and $T 6$, even for high doses, the rate of adsorption is the same as for unirradiated phage. An experiment of this type is shown in table 1. Only for very high doses a slight reduction occurs in the ability of phage to kill bacteria. This reduction is never such as to require important corrections in the analysis presented in later sections.

With phages $T 2, T 4, T 5$, and T6 (the "large particle" phages) the plaque counts on irradiated samples are not independent of the mode of assay. They depend on the concentration of the samples when first mixed with bacteria, in a way illustrated in table 2 . In these experiments, bacteria were mixed with various concentrations of irradiated phage. Before lysis and phage liberation

TABLE 1

Killing of bacteria by irradiated phage

$0.9 \mathrm{ml}$ of a bacterial culture was mixed with $0.1 \mathrm{ml}$ of each of five suspensions of phage $T 2 r$ that had received various doses of radiation. After 10 minutes, samples were diluted and plated for viable bacterial count.

\begin{tabular}{|c|c|c|c|c|c|c|c|}
\hline \multirow{2}{*}{$\begin{array}{l}\text { EXPERI- } \\
\text { MENT } \\
\text { NO. }\end{array}$} & \multirow{2}{*}{$\begin{array}{l}\text { PHAGE } \\
\text { INPUT, } \\
\text { PARTICLES } \\
\text { PER ML }\end{array}$} & \multirow{2}{*}{$\begin{array}{l}\text { BACTERIAL } \\
\text { INPUT, } \\
\text { CELLS } \\
\text { PER ML }\end{array}$} & \multicolumn{2}{|c|}{ DOSE OF RADIATION } & \multirow{2}{*}{$\begin{array}{c}\text { SURVIVING } \\
\text { BACTERIA } \\
\text { PER ML }\end{array}$} & \multirow{2}{*}{ 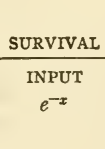 } & \multirow{2}{*}{$\begin{array}{c}\text { PHAGE } \\
\text { ADSORBED } \\
\text { PER BAC- } \\
\text { TERIUM } \\
x\end{array}$} \\
\hline & & & SECONDS & $\begin{array}{c}\text { HITS } \\
\text { PER } \\
\text { PARTICLE }\end{array}$ & & & \\
\hline \multirow{5}{*}{129} & \multirow{5}{*}{$2 \times 10^{9}$} & \multirow{5}{*}{$1.15 \times 10^{9}$} & 0 & 0 & $2.3 \times 10^{8}$ & 0.20 & 1.60 \\
\hline & & & 60 & 18 & $2.4 \times 10^{8}$ & 0.21 & 1.56 \\
\hline & & & 70 & 21 & $2.2 \times 10^{8}$ & 0.19 & 1.66 \\
\hline & & & 80 & 24 & $1.6 \times 10^{8}$ & 0.14 & 1.96 \\
\hline & & & 100 & 30 & $2.7 \times 10^{8}$ & 0.23 & 1.47 \\
\hline
\end{tabular}


took place, dilutions were made to bring the total dilution of the irradiated sample to a constant value, and an aliquot plated for plaque count. The plaque counts represent infected bacteria that liberate active phage. Although the total dilution of the irradiated phage on all plates is the same, it is seen that the plaque counts are higher when bacteria have first been placed in contact with a more concentrated phage lysate.

This means that bacteria may produce active phage if they pick up the ir-

TABLE 2

Dependence of plaque counts on irradiated phage Tor on the concentration of the phage sample that is mixed with bacteria

A sample of phage $T 6 r$ containing $1.5 \times 10^{10}$ particles $/ \mathrm{ml}$ was irradiated for 20 seconds. The bacterial suspension (B) contained $2 \times 10^{9}$ cells $/ \mathrm{ml}$. Each plate received $0.05 \mathrm{ml}$ of phage dilution and $0.2 \mathrm{ml}$ of suspension (B).

\begin{tabular}{|c|c|c|c|c|c|}
\hline $\begin{array}{l}\text { EXPERI- } \\
\text { MENT } \\
\text { NO. }\end{array}$ & $\begin{array}{l}\text { MIX- } \\
\text { TURE } \\
\text { NO. }\end{array}$ & PROCEDURE & $\begin{array}{c}\text { DILUTION } \\
\text { OF PHAGE } \\
\text { WHEN } \\
\text { FIRST } \\
\text { MIXED } \\
\text { WITH (B) }\end{array}$ & $\begin{array}{c}\text { TOTAL DILU- } \\
\text { TION FROM } \\
\text { THE ORIGINAL } \\
\text { PHAGE TO THE } \\
\text { SUSPENSION } \\
\text { FROM WHICH } \\
\text { SAMPLES ARE } \\
\text { PLATED }\end{array}$ & $\begin{array}{c}\text { PLAQUE } \\
\text { COUNT } \\
\text { (SUM OF } \\
\text { TWO } \\
\text { PLATES) }\end{array}$ \\
\hline \multirow{4}{*}{5} & 1 & $\begin{array}{l}0.1 \mathrm{ml} T 6 r \rightarrow 0.9 \mathrm{ml}(\mathrm{B}) ; \text { kept } 10 \mathrm{~min} \text {. } \\
\text { at } 37^{\circ} \mathrm{C} \text {; diluted } 1: 10^{3}, 0.05 \mathrm{cc} \text { plated }\end{array}$ & $1: 10$ & $1: 10^{4}$ & 1318 \\
\hline & 2 & $\begin{array}{l}0.1 \mathrm{ml}(T 6 r 1: 10) \rightarrow 0.9 \mathrm{ml}(\mathrm{B}) ; \text { kept } \\
10 \mathrm{~min} \text {. at } 37^{\circ} \mathrm{C} \text {; diluted } 1: 10^{2}, 0.05 \\
\mathrm{ml} \text { plated }\end{array}$ & $1: 10^{2}$ & $1: 10^{4}$ & 474 \\
\hline & 3 & $\begin{array}{l}0.1 \mathrm{ml}\left(\text { Tor } 1: 10^{3}\right) \rightarrow 0.9 \mathrm{ml}(\mathrm{B}) ; \mathrm{kept} \\
10 \mathrm{~min} \text {. at } 37^{\circ} \mathrm{C} \text {; diluted } 1: 10,0.05 \\
\text { ml plated }\end{array}$ & $1: 10^{4}$ & $1: 10^{4}$ & 250 \\
\hline & 4 & $0.05 \mathrm{ml}\left(\right.$ T6r $\left.1: 10^{4}\right)$ plated & $\begin{array}{l}\text { Less than } \\
\qquad 1: 10^{5} \\
\text { (on plate) }\end{array}$ & $1: 10^{4}$ & 57 \\
\hline
\end{tabular}

radiated particles from a concentrated phage suspension, but not from a dilute one. The immediate explanation is that from a concentrated lysate the bacteria receive some other "factor," which, inside the bacterium, somehow reactivates an "inactive" particle and which is not present in dilute lysates. An "inactive" particle can be defined as one that has lost the ability to initiate production of active phage unless adsorbed by a bacterium together with the unknown "factor."

Reactivation still occurs after storage of irradiated phage for weeks in an ice-box. Reactivation gives rise to fully active phage particles. This can be proved either by sampling phage from the plaques or by letting the bacteria, in which reactivation occurs, lyse in liquid and then testing the lysate for active particles. 
The occurrence of reactivation for certain phages accounts for deviations from the logarithmic inactivation rate found for these same phages (see figure 1). As the dose of radiation increases beyond a certain point, the survival, as determined by plaque count, appears to diminish less rapidly. This is due to an unavoidable partial reactivation. In a phage titration, we mix approximately $5 \times 10^{7}$ bacteria with an amount of phage suspension such as to give approximately 100 plaques, and pour the mixture on an agar plate. On the one hand, if we are dealing with a fully active sample, the bacteria only come in contact with 100 active particles. On the other hand, in assaying an irradiated suspension containing, for example, one active particle in $10^{6}$, we expose the bacteria to $10^{8}$ inactive particles plus a correspondingly large amount of other lysate constituents, besides the residual 100 active particles. Conditions permitting reactivation, therefore, obtain in these plating mixtures, and reactivation disturbs and often completely obscures the count of the residual active phage. For this reason, the survival of fully active phage for high doses must be obtained by extrapolation from the logarithmic part of the curve, a necessarily inefficient procedure. If deviations in the survival rate for high doses occurred, this extrapolation would not be justified. One possible cause of error - screening of some phage particles from radiation by components of the lysate itself - was excluded by irradiating concentrated phage $T 6$ mixed with phage $T 1$, and testing for the inactivation rate of the latter, which is not disturbed by reactivation phenomena. The inactivation rate of $T 1$ remains the same as in the absence of $T 6$ up to doses that correspond to 100 hits per particle of phage $T 6$.

\section{Identification of the reactivating factor}

What is the "factor" present in irradiated stocks of phages $T 2, T+, T 5$, or T6, which, if acting on bacteria that have adsorbed inactive phage particles, allows production of active phage? Since phage stocks are lysates produced by lysis of the common host $E$. coli B, the factor might be either of phage or of bacterial origin.

The factor was identified as being phage itself, inactive or active, in that reactivation occurs in bacteria that adsorb either more than one inactive particle of a given phage, or one inactive particle of one phage plus some active or inactive particles of a related phage. The evidence for this conclusion, which is illustrated in part by the data in table 3 , can be summarized as follows.

(a) Addition of an excess of supernatant from a heavy bacterial culture to a mixture of dilute irradiated phage plus bacteria gives no increase in plaque count. The factor in the lysates is not a normal bacterial secretion.

(b) Concentrated lysates of phages $T 1, T 5$, or $T 7$ added to a mixture of bacteria and dilute irradiated $T 2$ (or $T 4$, or $T 6$ ) do not cause reactivation. No heterologous lysate causes reactivation of $T 5$. The test for cross-reactivation is done by plating the mixtures, before lysis, with bacterial indicator strains sensitive to the phage whose reactivation is tested, but not to the others. Since all phage stocks are lysates of common host cells, the factor is not an unspecific bacterial product liberated upon lysis. 
TABLE 3

Reactivation of phage $T 2$ under various conditions

Phage $T 2$, containing $2 \times 10^{10}$ particles per $\mathrm{ml}$, was irradiated for 35 seconds. Phages $T 6$ and $T 4$, containing $4 \times 10^{10}$ particles per $\mathrm{ml}$, were irradiated for 30 seconds. Phages $T 1$ and $T 5$ contained $3 \times 10^{10}$ particles per $\mathrm{ml}$. The bacterial suspensions $(\mathrm{B})$ and $(\mathrm{B} / 6)$ contained $10^{9}$ cells per ml.

\begin{tabular}{|c|c|c|c|c|}
\hline $\begin{array}{l}\text { MIXTURE } \\
\text { NO. }\end{array}$ & CONTENTS & AFTER 10 MINUTES & $\begin{array}{l}\text { PLAQUE } \\
\text { COUNT } \\
\text { (SUM OF } \\
\text { TWO } \\
\text { PLATES) }\end{array}$ & $\begin{array}{l}\text { REACTIVA- } \\
\text { TION }\end{array}$ \\
\hline 1 & $0.1 \mathrm{ml}$ Phage $T 2$ dil. $1: 500+1.9 \mathrm{ml}(\mathrm{B})$ & $0.05 \mathrm{ml}$ plated with $B$ & 22 & - \\
\hline 2 & $0.1 \mathrm{ml}$ Phage $T 2$ dil. $1: 500+1.9 \mathrm{ml}(\mathrm{B} / 6)$ & $0.05 \mathrm{ml}$. plated with $\mathrm{B} / 6$ & 20 & - \\
\hline 3 & $0.1 \mathrm{ml}$ Phage $T 2$ pure $+1.9 \mathrm{ml}(\mathrm{B})$ & $\begin{array}{l}\text { dil. } 1: 500 \text {, } \\
0.05 \mathrm{ml} \text { plated with } B\end{array}$ & & \\
\hline 4 & $0.1 \mathrm{ml}$ Phage $T 2$ pure $+1.9 \mathrm{ml}(\mathrm{B} / 6)$ & $\begin{array}{l}\text { dil. } 1: 500 \\
0.05 \mathrm{ml} \text { plated with } \mathrm{B} / 6\end{array}$ & 3000 & + \\
\hline 5 & $\begin{array}{l}0.1 \mathrm{ml} \text { Phage T2. dil } 1: 500+0.1 \mathrm{ml} \text { irrad. } \\
T 6+1.8 \mathrm{ml}(\mathrm{B})\end{array}$ & $0.05 \mathrm{ml}$ plated with $\mathrm{B} / 6$ & $\begin{array}{r}3000 \\
670\end{array}$ & $\begin{array}{l}+ \\
+\end{array}$ \\
\hline 6 & $\begin{array}{l}0.1 \mathrm{ml} \text { Phage } T 2 \text { dil. } 1: 500+0.1 \mathrm{ml} \text { unirrad. } \\
T 6+1.8 \mathrm{ml}(\mathrm{B})\end{array}$ & $0.05 \mathrm{ml}$ plated with $B / 6$ & 190 & + \\
\hline 7 & $\begin{array}{l}0.1 \mathrm{ml} \text { Phage } T 2 \text { dil. } 1: 500+0.1 \mathrm{ml} \text { irrad. } \\
T 4+1.8 \mathrm{ml}(\mathrm{B})\end{array}$ & $0.05 \mathrm{ml}$ plated with $\mathrm{B} / 4$ & 661 & + \\
\hline 8 & $\begin{array}{l}0.1 \mathrm{ml} \text { Phage } T 2 \text { dil. } 1: 500+0.1 \mathrm{ml} \text { irrad. } \\
T 6+1.8 \mathrm{ml}(\mathrm{B} / 6)\end{array}$ & $0.05 \mathrm{ml}$ plated with $\mathrm{B} / 6$ & 10 & - \\
\hline 9 & $\begin{array}{l}0.1 \mathrm{ml} \text { Phage } T 2 \text { dil. } 1: 500+0.1 \mathrm{ml} \text { unirrad. } \\
T 6+1.8 \mathrm{ml}(\mathrm{B} / 6)\end{array}$ & $0.05 \mathrm{ml}$ plated with $\mathrm{B} / 6$ & 12 & - \\
\hline 10 & $\begin{array}{l}0.1 \mathrm{ml} \text { Phage } T 2 \text { dil. } 1: 500+0.1 \mathrm{ml} \text { irrad. } \\
T 4+1.8 \mathrm{ml}(\mathrm{B} / 6)\end{array}$ & $0.05 \mathrm{ml}$ plated with $\mathrm{B} / 4$ & 883 & + \\
\hline 11 & $\begin{array}{l}0.1 \mathrm{ml} \text { Phage } T 2 \text { dil. } 1: 500+0.1 \mathrm{ml} \text { unirrad. } \\
T 1+1.8 \mathrm{ml} \text { (B) }\end{array}$ & $0.05 \mathrm{ml}$ plated with $\mathrm{B} / 1,5$ & 18 & - \\
\hline 12 & $\begin{array}{l}0.1 \mathrm{ml} \text { Phage } T 2 \text { dil. } 1: 500+0.1 \mathrm{ml} \text { unirrad. } \\
T 5+1.8 \mathrm{ml}(\mathrm{B})\end{array}$ & $0.05 \mathrm{ml}$ plated with $\mathrm{B} / 1,5$ & 17 & - \\
\hline
\end{tabular}

(c) Concentrated lysates of phages $T 2$ (or $T 4$, or $T 6$ ), whether fully active or irradiated, can reactivate irradiated particles of any other T-even phage. These phages are morphologically, serologically, and probably genetically related, whereas $T 5$ belongs to a fully separate group (DELBRücK 1946). The "factor" in the lysates appears to carry the same pattern of relatedness. It is not produced by irradiation, since its presence can be proved in unirradiated lysates by the technique of cross-reactivation.

(d) Reactivation is independent of contact between phages prior to infection of the host: a mixture of bacteria and phages gives the same amount of reactivation independently of how long the phages have been together before adding the bacteria.

(e) Phage $T 4 r$ purified by fast centrifugation (kindly supplied by $\mathrm{DR}$. T. F. ANDERSON) shows both self-reactivation as a function of concentration in the mixtures, and ability to reactivate phages $T 2$ or $T 6$. The factor, therefore, is present in such a purified phage suspension.

(f) Reactivation of phage $T 2$ by lysates of $T 6$, for example, only occurs in presence of bacteria capable of adsorbing both phages. Inactive phage $T 2$ in presence of bacteria $B / 6$, by which it is adsorbed, is not reactivated by $T 6$, which is not adsorbed, but is reactivated by $T 4$, which is adsorbed. This proves 
that the "factor" has the same host specificity as the phage in whose lysate it is found.

The last point, particularly, was considered crucial in showing that production of active phage from an inactive particle was actually due to infection of the same bacterial cell with other particles, either of the same or of a different but genetically related phage, active or inactive. Further confirmation came from the experiments discussed below, which showed that in a mixture of inactive phage and bacteria the number of bacteria yielding active phage is never greater than the number of bacteria that adsorb two or more inactive particles, and in some cases actually equals it. The same holds true for reactivation of an inactive phage, for instance $T 2$, by a related one, for instance $T 4$; the number of bacteria liberating active $T z$ is lower than-or equal to-the number of bacteria receiving at least one particle of each phage. The analysis of cross-reactivation between these different wild-type phages will not be discussed further in this paper, but will form the subject of a future publication. ${ }^{2}$

(g) All bacteria which, after infection with inactive particles, do not liberate active phage also fail to lyse. This was proved by mixing bacteria and inactive phage under conditions in which some reactivation occurs, plating a sample of the mixture for plaque count, and another sample for direct microscopic observation of lysis on agar. The results of such experiments proved that the fraction of bacteria that are lysed is the same as the fraction of bacteria that liberate active phage. The other infected bacteria fail to grow and divide, and can be seen still apparently unchanged 24 hours later.

\section{Reactivation and genetic transfer}

The limitation of cross-reactivation to the $T$-even phages immediately brought out a similarity between this phenomenon and that of genetic transfer described by DeLBR ÜCK and BAILEy (1946). In the latter case, bacteria simultaneously infected with the phages $T 2 r^{+}$and $T 4 r-$ the $r$ character being the result of mutation from the wild-type, which can be designated as $r^{+}$-liberate a mixture of particless of the four types, $T 2 r^{+}, T 2 r, T 4 r^{+}$, and $T 4 r$, among which the second and third represent new types. These must owe their origin to some sort of recombination involving the genetic determinants for the alternative $r^{+}$and $r$ phenotypes. Evidence for the discrete nature of these determinants has since been reported by Hershey and Rotman (1948).

We assumed then, as a working hypothesis for the analysis of the reactivation phenomenon, that inactivation by ultraviolet light resulted from "lethal mutations" in a number of discrete genetic determinants among inactive particles in the same bacterium to reconstitute fully active particles. This hypothesis can be formulated quantitatively in terms of measurable

\footnotetext{
${ }^{2}$ Cross-reactivation between $T$-even phages has the limitation that the individual phages are distinguishable only by test of differential properties such as ability to grow on different hosts and rate of inactivation by different antisera. Cross-reactivation can only be defined as the production, upon mixed infection, of active particles having the distinctive properties of an inactive parent particle.
} 
quantities by making a number of simple assumptions. We shall first develop this simple theory and then describe the experiments by means of which it was tested.

\section{Theory}

We shall assume that in each particle of a given phage there exist $n$ "units" (or "loci") each capable of undergoing a lethal mutation when exposed to ultraviolet light. Since one effective hit is sufficient to inactivate a phage particle, a lethal mutation can be defined as an effective hit, that is, as an alteration of one unit which makes the phage particle unable to initiate by itself the production of active phage in a bacterium. A particle may undergo more than one lethal mutation, and mutations will be distributed at random and independently among the different units, the distribution depending only on the sensitivity of each unit.

We shall now make the assumption that the sensitivity of all units is the same, and show later that this assumption, if incorrect, only requires a numerical correction which does not invalidate the applicability of the theory.

Our next assumption is that actize phage cannot be produced in a bacterium unless the infecting particle or particles, taken as a group, contain at least one copy of each unit in non-lethal form. This assumption is an essential feature of the theory, and corresponds to treating each unit as a discrete, material, independent hereditary unit endowed with genetic continuity and individuality. An inactive unit cannot be replaced by copies of different units. This assumption implies that production of active units cannot result from the cooperation of two or more lethal units, but only from actual reproduction of active units.

When a population consisting of $M$ phage particles is irradiated with a given dose, there will be produced in each particle, on the average, $r$ lethal mutations, or a total of $M \times r$ mutations in the whole population. Since $M$ particles contain $M \times n$ units, each unit will receive on the average $M r / M n=r / n$ lethal mutations.

A given unit, taken at random, will have a probability $\mathrm{e}^{-r / n}$ of not having a lethal mutation, and a probability $\left(1-\mathrm{e}^{-r / n}\right)$ of having at least one.

We ask next: what is the probability that each of the $n$ units is present in at least one non-lethal copy in a group of $k$ particles that enter a bacterium? According to our assumptions, this probability should represent an upper limit for the probability that a bacterium produces active phage.

If a bacterium is infected by $k$ particles, the probability that a given unit is lethal in all of them is: $\left(1-\mathrm{e}^{-r / n}\right)^{k}$, and the probability that it is non-lethal in at least one of them is: $1-\left(1-\mathrm{e}^{-r / n}\right)^{k}$.

The probability that at least one non-lethal copy of each of the $n$ units is present in the $k$ particles is the product of the probabilities referred to the individual units. Since we have assumed equal sensitivity for all units-that is, $r / n$ constant for all units for each value of $r$ - the product will be

$$
\left[1-\left(1-\mathrm{e}^{-r / n}\right)^{k}\right]^{n} \text {. }
$$

The expression (1) represents the probability that a bacterium infected by $k$ particles receives at least one full non-lethal complement of the $n$ units. 
In a mixture of phage with bacteria, however, there is a distribution of the number of phage particles infecting individual bacteria. With relatively good approximation--see Appendix-this distribution can be considered as a Poisson distribution. If $x$ is the average number of particles adsorbed per bacterium, the fraction of bacteria with $k$ particles is: $x^{k} \mathrm{e}^{-x} / k$ !

The fraction of bacteria receiving $k$ particles which carry a full complement of non lethal units is then:

$$
\frac{x^{k} \mathrm{e}^{-x}}{k !}\left[1-\left(1-\mathrm{e}^{-r / n}\right)^{k}\right]^{n} .
$$

Finally, the fraction of the total bacterial population which receives all units in a non-lethal form is the sum of the expression (2) for all possible values of $k$ :

$$
Z=\sum_{k=0}^{\infty} \frac{x^{k} \mathrm{e}^{-x}}{k !}\left[1-\left(1-\mathrm{e}^{-r / n}\right)^{k}\right]^{n} .
$$

This expression embodies the following consequences of our hypothesis:

(a) No full complement of active units can be present in uninfected bacteria $(k=0)$.

(b) Of the bacteria with one phage particle $(k=1)$, only those with an active particle fulfill the requirement for active phage production $\left(Z=x \mathrm{e}^{-x} \mathrm{e}^{-r}\right)$.

(c) Any bacterium that receives at least one active particle fulfills the requirement for active phage production, whether it also receives inactive particles or not. For cach unit of that particle, $1-\mathrm{e}^{-r / n}=0$; hence, $\left(1-\mathrm{e}^{-r / n}\right)^{k}=0$, and $\left[1-\left(1-\mathrm{e}^{-r / n}\right)^{k}\right]^{n}=1$.

(d) For any given value of $r>0, Z$ increases with increasing $x$, that is, the probability of having a full complement of active units increases as the number of particles adsorbed per bacterium increases.

(e) For any given value of $x, Z$ diminishes with increasing $r$, that is, the probability of having all active units diminishes as the dose of radiation increases.

For the purpose of comparison with data from different experiments, it is more convenient to eliminate from the computation those bacteria that receive either zero or one phage particle, since they are not expected to contribute to reactivation. This is done by using instead of $Z$ the expression

$$
z=\sum_{k=2}^{\infty} \frac{x^{k} \mathrm{e}^{-x}}{k !}\left[1-\left(1-\mathrm{e}^{-r / n}\right)^{k}\right]^{n} .
$$

$z$ represents the fraction of bacteria in the total population that have two or more phage particles, which together contain a full complement of active units. Since the fraction $m$ of bacteria with two or more phage particles ("multipleinfected bacteria") is

$$
m=1-(x+1) \mathrm{e}^{-x}
$$

the multiple-infected bacteria receiving a full complement of active units represent a fraction 


$$
y=z / m=\frac{\sum_{k=2}^{\infty} \frac{x^{k} \mathrm{e}^{-x}}{k !}\left[1-\left(1-\mathrm{e}^{-r / n}\right)^{k}\right]^{n}}{1-(x+1) \mathrm{e}^{-x}} .
$$

The expression $y$ thus obtained is a function of $x$ (average number of phage particles adsorbed per bacterium), of $r$ (average number of lethal hits per particle), and of $n$ (number of units per particle).

We shall call $w$ the ratio between the number of bacteria that actually liberate active phage (plaque count) and the number of multiple-infected bacteria. For each mixture of bacteria and irradiated phage, we can determine experimentally $r, x$, and the plaque count, and obtain from these the values of $m$ and $w$. We can then compare the experimental values of $w$ with the calculated values of $y$ for several different values of $n .^{3}$

The function $y=F(r, x, n)$ was tabulated numerically for a range of values of $r$ (between 3 and 50), of $x$ (between 0.05 and 20), and of $n$ (between 10 and $60)$. We used for $k$ those ranges of values for which the contributions of the corresponding classes were relevant. The corresponding curves were drawn for $y=F(r)$ ( $x$ and $n$ constant), and for $y=F(x)$ ( $r$ and $n$ constant).

Before comparing the experimental results with the curves, it is useful to discuss briefly what we may expect from the comparison. If reactivation only occurs in bacteria with more than one inactive particle, $w$ should never be greater than unity. If among the requirements for reactivation there are those stated in the assumptions of our theory, the ratio $w / y$ should never be greater than unity. Finally, if the requirements stated in our assumptions are necessary and sufficient for reactivation, the ratio $w / y$ should be unity, that is, active phage should be produced in all those bacteria that receive a full complement of the hypothetical units in non-lethal form. Should this obtain, it would then be possible to calculate the value of $n$ for each phage from the experimental values of $w$.

It is important to keep in mind that the assumptions of our theory, up to this point, do not contain any implication as to the nature, properties, or mechanism of transfer of the postulated units. They only assert that each unit has genetic individuality and can be made lethal by radiation as a result of one photochemical reaction, which is of the "all or none" type and independent of other reactions of the same type in other units of either the same or other phage particles. Production of active phage is conditioned by the presence in one bacterium of one active copy of each unit, this copy not being replaceable by any number of inactive copies.

The theory does not imply that all phage particles receive the same number of lethal hits, but that the lethal hits are distributed at random among the units of all phage particles.

${ }^{3}$ In preliminary reports (LURIA 1947, 1948) we used the symbol $y$ both for the theoretical and experimental probabiiities of reactivation. We also gave values of $1 / y$ instead of $y$ (or $w$ ). The present notation, while consistent with the previous one, makes the presentation mcre logical. 
Comparison of theory with experiment

Quantitative experiments consisted of testing mixtures of bacteria and irradiated bacteriophage for the number of bacteria that liberate active phage. Only phages $T 2, T 4$, and $T 6$ were studied in detail.

In a typical experiment, such as the one described in detail in table 4 , a standard culture of bacteria, grown to a titer of either $10^{8}$ cells per $\mathrm{ml}$ or $10^{9}$ cells per ml, was chilled by immersion in a water bath at $5-6^{\circ} \mathrm{C}$. This treatment interrupts multiplication without changing the ability to resume immediate multiplication and to support normal growth of phage upan return to $37^{\circ} \mathrm{C}$. In experiments with bacteria grown to a titer of $10^{9}$ (latest part of logarithmic growth phase), immersion in ice-water is not necessary, since multiplication stops almost immediately upon interruption of aeration and transfer to room temperature.

Ten minutes after interrupting multiplication, a sample of the culture is diluted and assayed for viable count. If several mixtures of bacteria and phage are to be prepared, the culture may have to be used for one or two hours, in which case at least one other similar assay is made at the end of the experiment to make sure that no proliferation has occurred. At intervals, undiluted samples of the culture are placed into test tubes, and a constant volume of irradiated (or control) phage variously diluted is added. In this way, we know for each mixture the input of bacteria and of phage per ml. For irradiated samples, the input of active phage is determined from one or more assays done at very high dilution. When this is impossible - for high doses of radiation-the amount of active phage is determined by extrapolation from the first part of the inactivation curve.

Adsorption is interrupted by heavy dilution after a short time (generally five or ten minutes). The amount of adsorption is determined either by determination of free phage in the supernatant of a centrifuged sample from a mixture containing active phage, assuming similar adsorption in all other mixtures (see table 1), or by determining the bacterial survival in samples from various mixtures. With the $T$-even phages, 80 to 95 percent of the phage is adsorbed in ten minutes. The value for the multiplicity of infection, $x$, for each mixture is used in calculating the fraction $m$ of bacteria with two or more phage particles: $m=1-(x+1) \mathrm{e}^{-x}$.

Before lysis begins, a suitably diluted sample of the mixture is plated with an excess of sensitive bacteria for plaque count. The plaque count-corrected, when necessary, for the active phage by subtracting the value corresponding to the latter-is divided by $m$ to obtain the experimental value $w$ for that mixture (see table 4 ).

A large number of experiments, yielding a total of over 1000 values of $w$, for various doses of radiation and for different multiplicities, were done with the $T$-even phages. Phage $T 5$ was only partially investigated, because of difficulties in obtaining reproducible results in view of the low and irregular adsorption rate for this phage.

The data for phages $T$-even include those for several of their $r$ mutants, 

年 औे क्षै

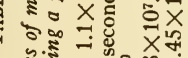
. 会 ․ํㅀ

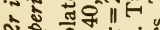
సิ स्षे

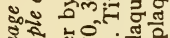
इ कर

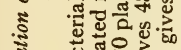

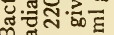
की 금ำ

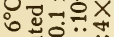
군 ㄷํ유: 궁 늘ㅋㅀㅇㅠ

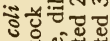

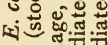

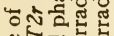
널을

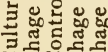

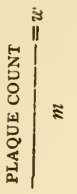

影 出造

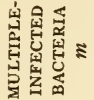

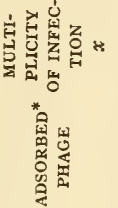

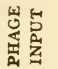

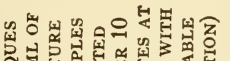

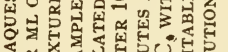

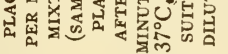

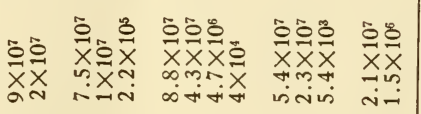

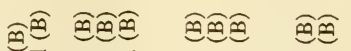

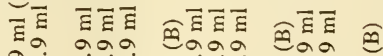

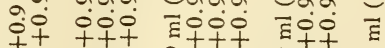

กิ กิㅇำ

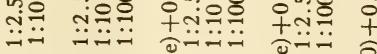

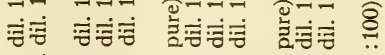

نुّ

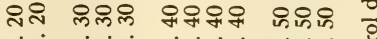

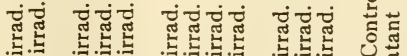

$0 \%$ है

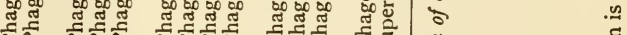

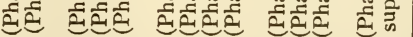

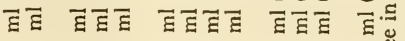

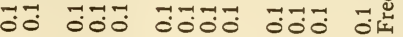

离密完 
TABLE 5

Probability of reactivation for various phages as a function of dose and of multiplicity of infection

Values of the ratio $w$ between bacteria that yield active phage and multiple-infected bacteria.

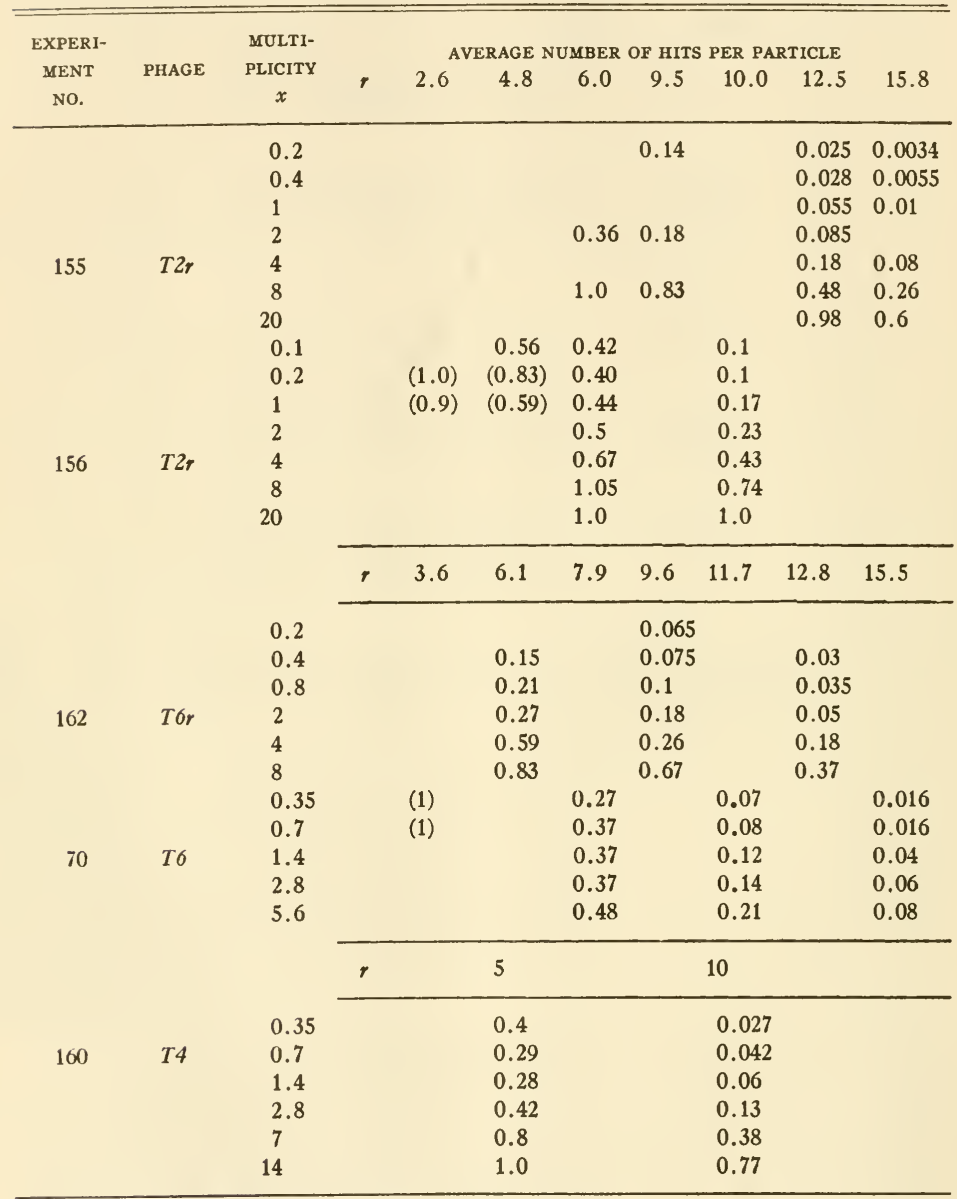

The values in parentheses are from separate experiments. 
which were found to have the same probability of reactivation as the respective wild types. The results cover ranges of values of $r$ from 2.5 to over 30 , and of $x$ from 0.02 to 20 .

The individual values of $w$, and those of the variables, $r$ and $x$, are obtained from the following actual measurements: 1) titer of phage; 2 ) total number of bacteria; 3) survival of phage; 4) survival of bacteria and/or assay of free phage; 5) plaque count from the mixture. Each of these measurements involves an error of estimation due to dilution and sampling errors. Several of these determinations, however, are the same within each experiment. The results from individual experiments are, therefore, more consistent than those from different experiments, as shown in table 5 .

The only graphic representation that could show all values of $w$ for each phage and allow of comparison with the calculated values of $y$ would be a tri-dimensional plot of $w$ as a function of $r$ and of $x$. As second best choice, we plotted the values of $w$ as a function of $r$ for several values of $x$ taken as constant, and as a function of $x$ for several values of $r$ taken as constant. Individual values of $w$ fluctuate rather widely, but the data as a whole make it possible to draw curves, which represent averages and which can be considered as the curves for $w$ as a function of $r$ and of $x$. A number of such plots using all the experimental points for the corresponding values of the variables, are presented in figures 2,3 , and 4 (multiplicity of infection as variable) and figures 5,6 , and 7 (dose of radiation as variable).

The trend of these plots is similar to that of the theoretical curves for $y$, and it is possible to find for each phage a constant value of $n$ (number of units) such that the corresponding values of $y$ become very similar to those of $w$ for low values of $x$ and for any value of $r$. That is, it is possible for each phage to determine a constant number of units for which the experimental probability of reactivation equals the theoretical one for any dose of radiation provided the multiplicity of infection is low. The corresponding theoretical curves for $y$ have been drawn in the plots of the values of $w$. For phage $T 2$, the best fit is for $n=25$; for $T 4, n=15$; for $T 6, n=30$ (see especially figures 5,6 , and 7 ).

The main feature emerging from the curves in figures 2,3 , and 4 is that the values of $w$ tend to unity for increasing multiplicity of infection. In several cases, the number of cells that liberate phage actually reaches the number of multiple-infected cells, but in no case does it go beyond it, proving that reactivation does not occur in single-infected cells.

The curves in figures 5,6,7, for $w$ as a function of $r$, are of the multiple-hit type, indicating that suppression of phage production depends on damage in a number of elements. The values of $w$ tend to unity for low doses, again showing that reactivation potentially can take place in every multiple-infected cell.

Comparison in figures $2-4$ with the curves for $y$, chosen to fit the experimental curves for low values of $x$, shows that the general similarity is limited by a systematic deviation. As the multiplicity increases, both $w$ and $y$ tend asymptotically to unity, but $w$ increases more slowly. This means that, as the number of phage particles per bacterium increases, the probability of reacti- 


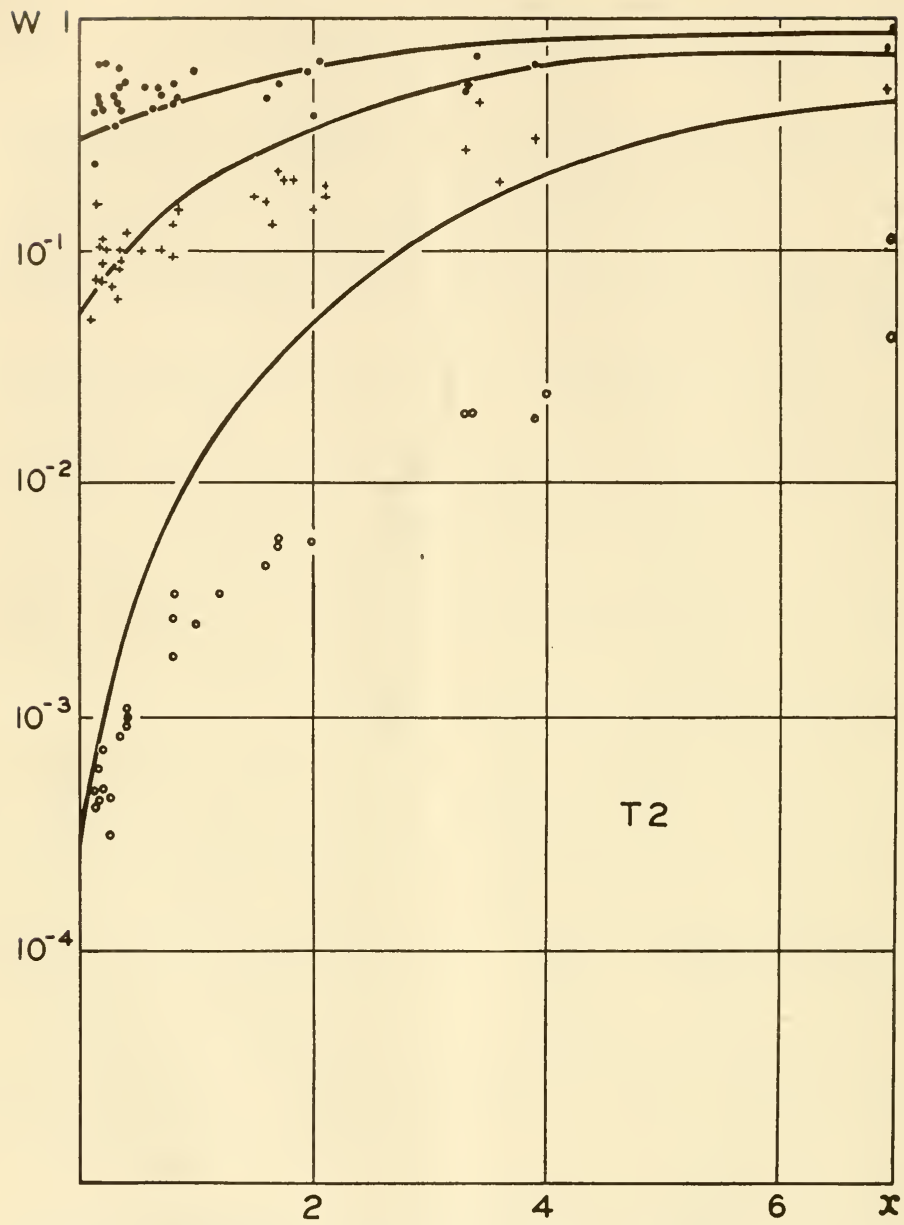

FIGURE 2.-The probability $w$ of reactivation for irradiated phages $T 2$ and $T 2 r$ as a function of the multiplicity of infection $x$, for several doses of radiation. Abscissae: values of $x$. Ordinates: values of $w$.

values of $w$ for $r=6$ hits per particle.

+ values of $w$ for $r=10$ hits per particle.

values of $w$ for $r=20$ hits per particle.

Solid lines: theoretical curves for $y$ a a function of $x$ for $n=25$, and for the values of $r$ given above. 


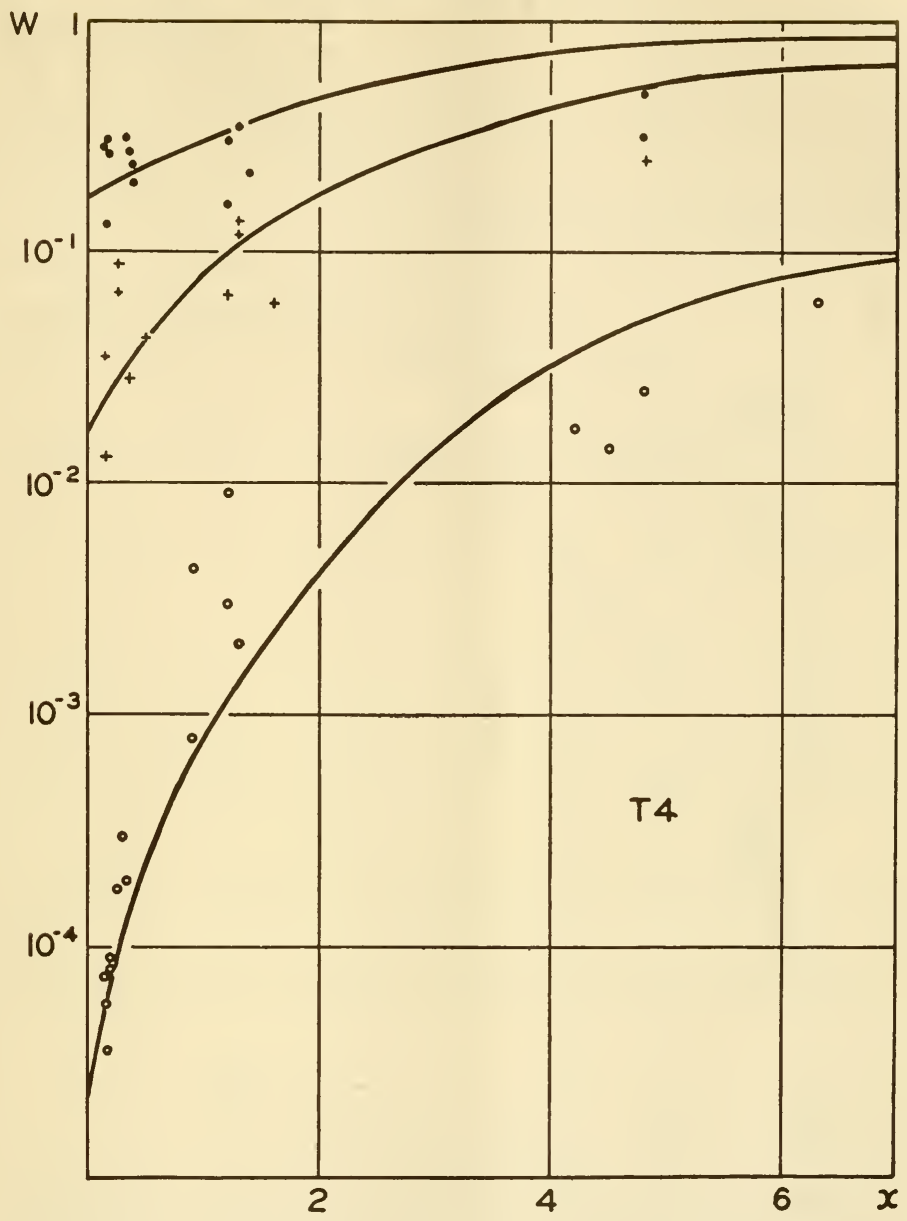

Figure 3.-Same as figure 2, for phages $T 4$ and $T 4 r$. The theoretical curves for $y$ correspond to $n=15$.

vation, while steadily increasing, does not keep pace with the theoretical function $y$. The cooperation within groups consisting of more than two particles is not as successful in bringing about reactivation as required by the simple theory, whereas pairs of particles apparently collaborate with an efficiency of one hundred percent.

The deviation for higher multiplicities is reflected in the curves of figures 5-7. The curves for $w$ as a function of the dose are very close to the theoretical 
curves for low multiplicities, up to $x=0.5$; for higher multiplicities they fall below the corresponding curves for $y$ calculated for the same number of units. We can actually find, for each value of $x$, a curve for $y$-for the same $n$ but corresponding to a lower $x$-which fits the experimental curve. The corresponding theoretical curves are drawn in figures 5-7. This indicates that groups of more than two particles collaborate in reactivation as if they consisted of a lower but definite number of particles.

It is interesting to notice that the systematic deviation from theory for

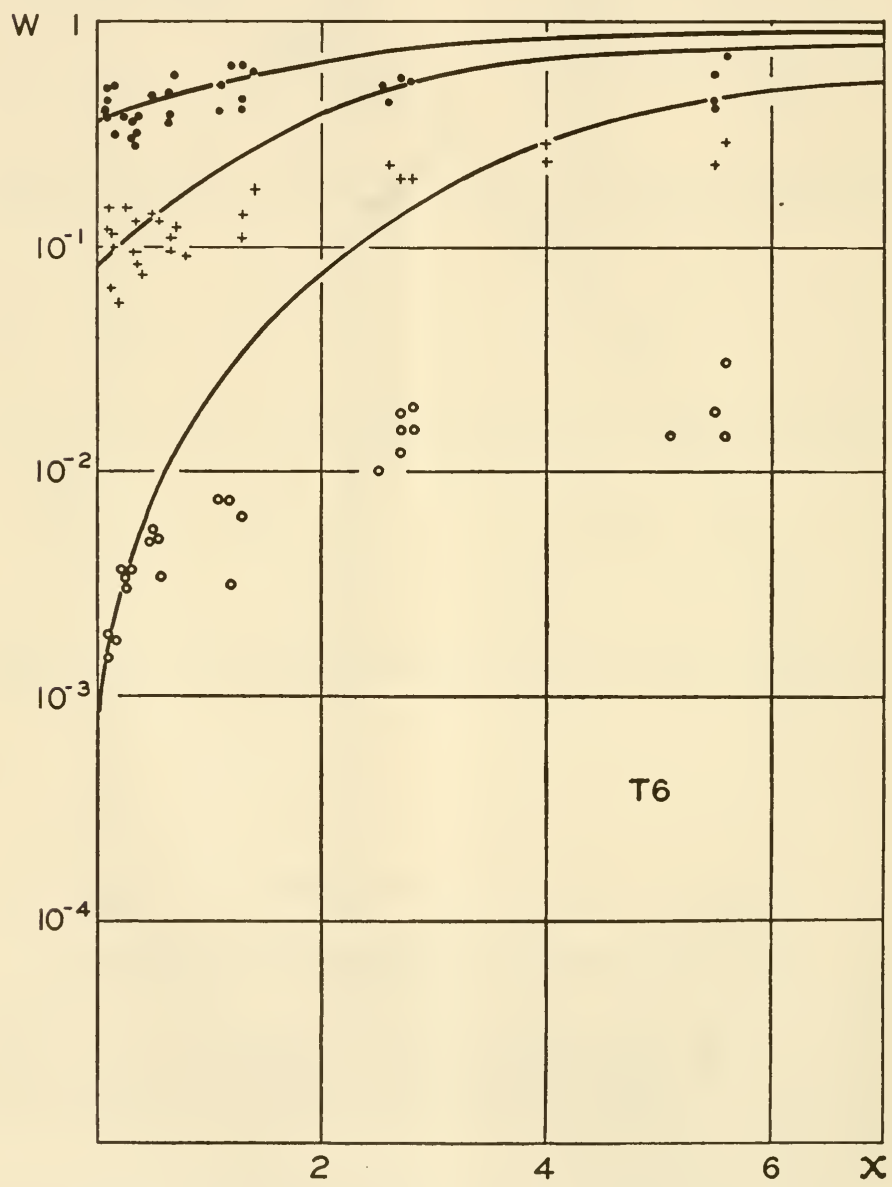

FGURE 4.-Same as figure 2, for phages $T 6$ and $T 6 r$. The theoretical curves for $y$ correspond to $n=30$. 


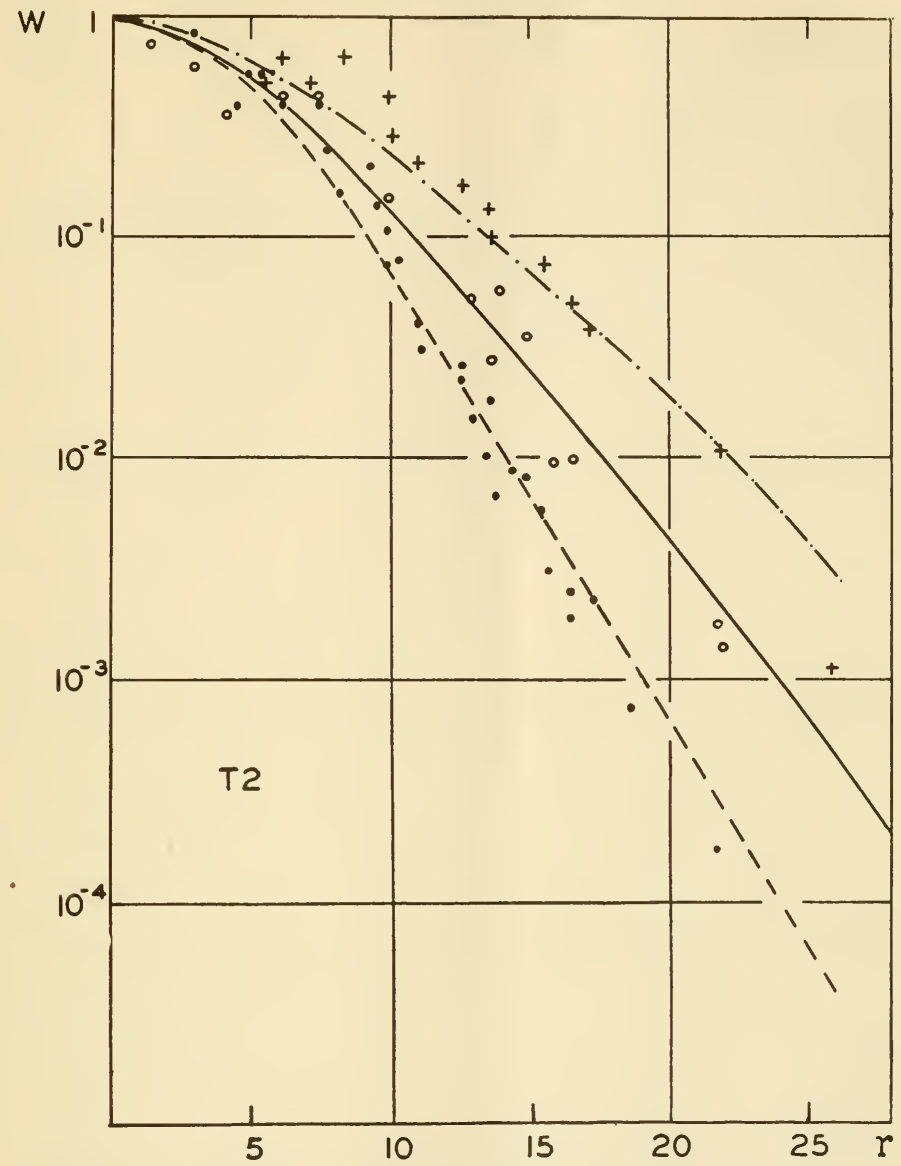

FIGURE 5.-The probability $w$ of reactivation for phages $T 2$ and $T 2 r$ as a function of the dose of irradiation $r$ (in hits per particle) for several multiplicities. Abscissae: values of $r$. Ordinates: values of $w$.

$$
\begin{aligned}
& \text { values of } w \text { for } x=0.1-0.2 \text {. } \\
& +\quad \text { values of } w \text { for } x=0.8-1.5 \text {. } \\
& +\quad \text { values of } w \text { for } x=2.5-4.0 \text {. }
\end{aligned}
$$

Broken line: theoretical zurve for $y$ as a function of $r$ for $n=25, x=0.15$. Solid line: theoretical curve for $y$ as a function of $r$ for $n=25, x=0.6$. Broken and dotted line: theoretical curve for $y$ as a function of $r$ for $n=25, x=1.3$. 


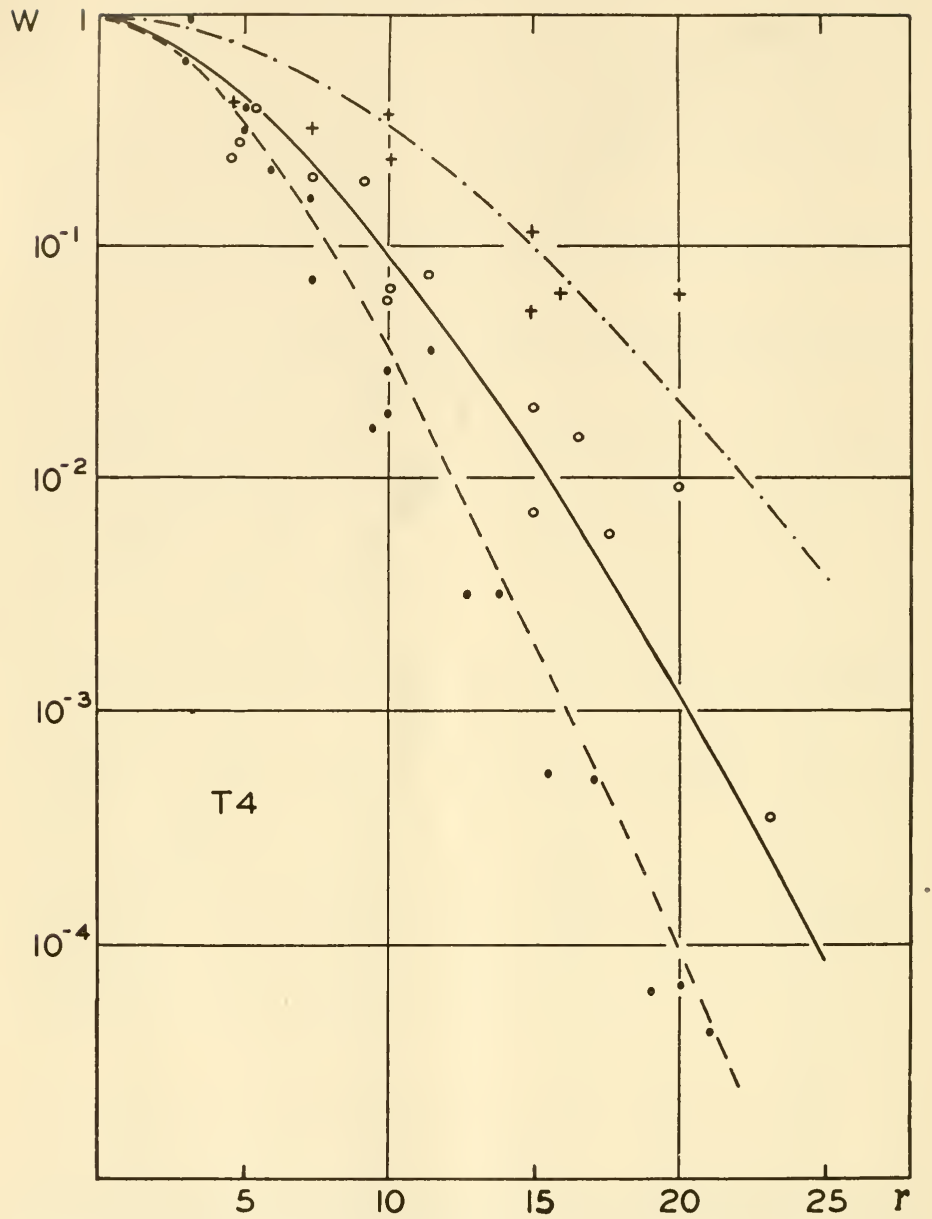

Figure 6.-Same as figure 5, for phages $T 4$ and $T 4$ r.

- values of $w$ for $x=0.1-0.2$.

values of $w$ for $x=0.8-1.5$.

+ values of $w$ for $x=4-7$.

Broken line: curve of $y$ for $n=15, x=0.15$. Solid line: curve of $y$ for $n=15, x=1.1$. Broken and dotted line: curve of $y$ for $n=15, x=4.0$. 


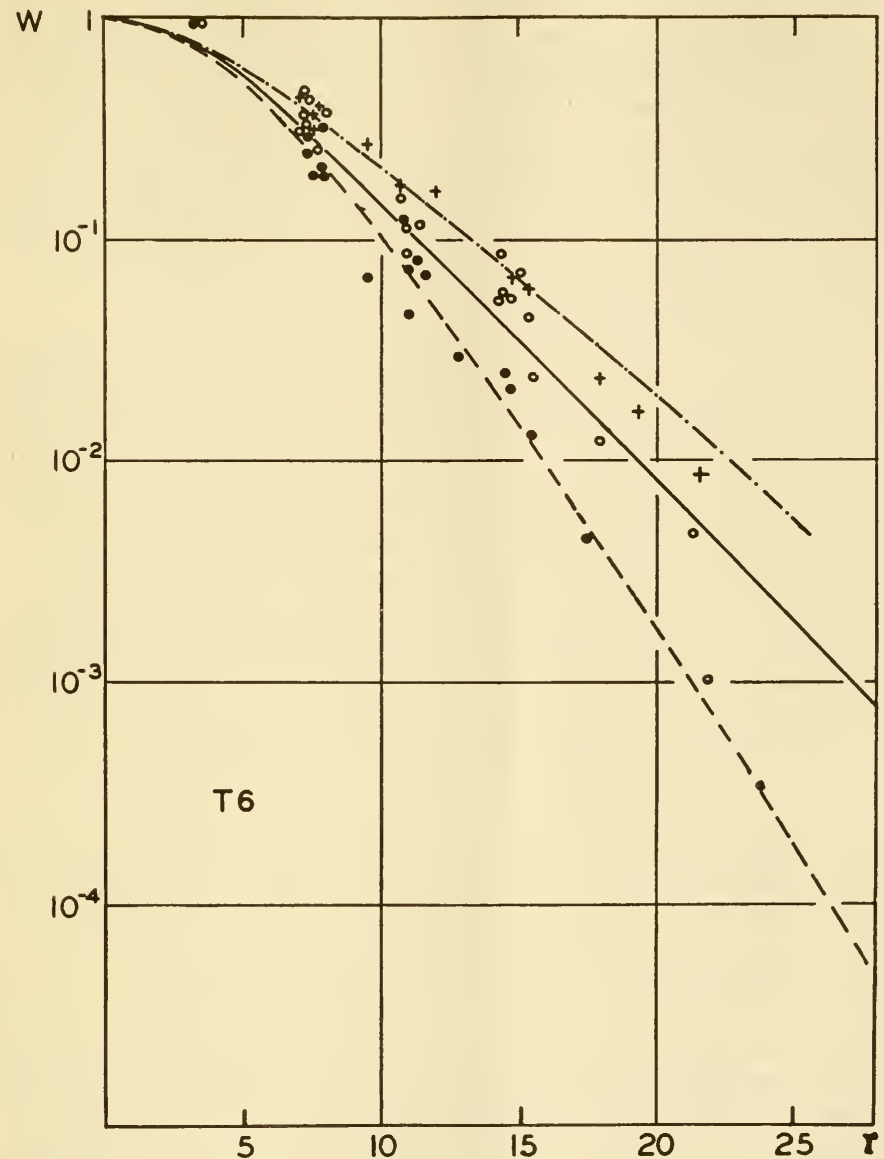

Figure 7.-Same as figure 5, for phages $T 6$ and $T 6 r$.

- values of $w$ for $x=0.1-0.2$.

values of $w$ for $x=0.8-1.5$.

+ values of $w$ for $x=2.5-4.0$.

Broken line: curve of $y$ for $n=30, x=0.15$. Solid line: curve of $y$ for $n=30, x=0.5$. Broken and dotted line: curve of $y$ for $n=30, x=0.9$. 
increasing multiplicities is less evident for phages $T 2$ than for $T 6$, and still less for T4, diminishing in the same order as the calculated number of units.

To summarize, the results show that the probability of production of active phage from inactive particles depends on the dose of radiation and on the multiplicity of infection in a way similar to the one predicted by the simple theory, which assumes lethal mutations in discrete transferable units of equal radiation sensitivity and a hundred percent efficient recombination of active units to reconstitute active particles. All deviations can be accounted for by a limitation in the efficiency of recombination when the active units derive from more than two inactive particles.

Several explanations may be offered for this limitation; some of them have been tested experimentally. Lysis from without - failure to liberate phage due to excessive multiplicity of infection (DELBRÜCK 1940)-was found not to take place for multiplicities of the order of those for which the deviations from theory occur. Limitations in the number of particles of a given phage that can participate in phage growth were looked for and found (see DuLBEcco 1949a), but their magnitude cannot account for the differences between $w$ and $y$.

The process of reactivation must involve complex mechanisms of transfer of genetic material among phage particles. Whatever these mechanisms, it is reasonable to expect that they will work less efficiently as the number of phage particles increases. Limitations may conceivably be caused by steric reasons - shape of the particles, position in the bacterium - or by physiological reasons-limited number of units of some catalyst, competition for substrates.

\section{Estimation of the number of units}

We have compared our results with the theoretical curves for $y$ calculated for different values of $n$, the unknown number of transferable units per particle. The curves that best fit the results for phages $T 2$ and $T 2 r$ are those for $n=25$; for $T 6$ and $T 6 r, n=30$; for $T 4$ and $T 4 r, n=15$. For phage $T 5$, no accurate estimate of $n$ was obtained, but $n$ appears to be lower than for T4.

If the interpretation of the results based on the simple theory is justified, we must consider the values thus obtained for $n$ as minimum estimates of the number of radiation-sensitive, transferable units per phage particle. The estimates are minima because of the assumption of equal sensitivity of all units. Should there be units more sensitive than others, they would be hit more often, and in order to obtain the correct probability of reactivation we should assume more units of the less sensitive type. For example, if one unit were twice as sensitive as the average of the others, one locus of the average sensitivity should be added to our estimate in order to distribute the probability of inactivation over all units in such a way that the reactivation probability remains the same.

In our preliminary report (LURIA 1947) we calculated $n$ in a different manner, by assuming that for low multiplicities and low doses, where the probability of reactivation appeared to be approximately constant as a function of $x$, we could consider all multiple-infected bacteria as double-infected. This corre- 
sponded to putting $k=2$ in formula (6) and to comparing the experimental values of $w$ with the values of $y$ for $x=0$. Upon closer analysis, this method proved incorrect, because the contribution of higher multiple infection cannot be neglected, even for low multiplicities. Analysis of more data showed that the probability of reactivation is in fact not constant for low values of $x$, but appears to be so for low doses, because the differences are small and of the order of the experimental errors. The use of the wrong approximation made our previous estimates of $n$ too high.

\section{Yield of active phage from bacteria in which reactivation occurs}

The yield of active phage following reactivation was studied systematically for phages $T 2 r$ and $T 4$. After infection, bacteria were diluted and allowed to lyse in liquid, as in a typical "one-step growth" experiment. The latent period before lysis is somewhat longer than for active phage (about 26 minutes instead of 21 for T2, 30 minutes instead of 25 for T4), and the rise in phage titer upon liberation somewhat slower. All yields were calculated from plaque counts after the titer had reached a steady level. The results, shown in table 6, indicate that the yields are generally somewhat lower than those from bacteria infected with active phage particles. No clear relation of yield to dose of radiation or to probability of reactivation was detected. For $T 2 r$ irradiated with high doses, there is a certain tendency toward higher yields for higher multiplicities.

\section{Mixed infection with active and inactive phage}

Transfer of genetic material involved in reactivation must occur between active and inactive phage particles, since, as we saw before, an active particle of a $T$-even phage can reactivate an inactive particle of another $T$-even phage. If transfer occurred by reciprocal exchanges of genetic material, we should expect that upon mixed infection with active and inactive particles of the same phage some of the active particles would receive inactive units and, therefore, be inactivated. This possibility was tested for phages $T 2$ and $T 4$ by experiments of the following type.

Bacteria are added to mixtures containing various proportions of active phage and of phage of the same strain irradiated with different doses. For each mixture, the average numbers of active and of inactive particles adsorbed per bacterium are calculated and, hence, the number of bacteria receiving both active and inactive phage. A plaque count before lysis gives the number of bacteria that liberate phage, while a plaque count after lysis gives the yielci of phage per bacterium. In this manner, we can determine whether inactive phage suppresses production of active phage from bacteria that also adsorb an active particle, or possibly affects the yield.

The results of these tests can be listed as follows:

(a) a bacterium receiving an active particle plus one inactive particle of the same phage-no matter how many hits the latter has received-never fails to liberate active phage;

(b) part of the bacteria that adsorb one active particle plus several inactive 
ones (the latter carrying enough lethal hits, so that reactivation does not occur among them) fail to liberate active phage. This suppression of active phage production is evident for multiplicities 5 or higher of inactive phage $T 4$, and for multiplicities 8 or higher of inactive phage T2. The suppression is independent of the time allowed for adsorption of the phages. It may in part be

TABLE 6

The yield of active phage from bacteria in which reactivation takes place

\begin{tabular}{|c|c|c|c|c|c|c|}
\hline \multirow{2}{*}{ PHAGE } & \multirow{2}{*}{$\begin{array}{l}\text { MULTIPLICITY } \\
\text { OF INFECTION }\end{array}$} & \multicolumn{5}{|c|}{ DOSE } \\
\hline & & HITS & 0 & 6 & 12 & 18 \\
\hline \multirow{7}{*}{$T 2 r$} & $0.2-0.25$ & & 114 & 51 & 122 & \\
\hline & $0.5-0.6$ & & 50 & 42 & 22 & \\
\hline & 2.9 & & 89 & & 100 & 29 \\
\hline & 7.7 & & 59 & & & 60 \\
\hline & 9 & & 100 & 70 & 94 & \\
\hline & $10-11$ & & 73 & 120 & 59 & 130 \\
\hline & & HITS & 0 & 7.5 & 15 & 18 \\
\hline \multirow{6}{*}{$T 4$} & 0.65 & & 260 & 120 & 87 & 200 \\
\hline & 1.1 & & 200 & 87 & 162 & \\
\hline & 1.6 & & 275 & & 140 & 140 \\
\hline & 2.7 & & 212 & & 197 & \\
\hline & $7-8$ & & & & 135 & 200 \\
\hline & $14-15$ & & & & 250 & 85 \\
\hline
\end{tabular}

accounted for by the limitation phenomenon described by the junior author (Dulbecco 1949a);

(c) for those bacteria that liberate active phage, the yield per bacterium is not affected by the presence of inactive particles, but remains the same as in controls without the inactive phage;

(d) when bacteria are infected with several heavily irradiated particles that do not give reactivation, and a few minutes later with one active particle, suppression of phage production occurs in a proportion of bacteria that increases with the interval between infections. Suppression is evident with an interval of 2.5 to 4 minutes and practically complete after 10 minutes. These time intervals between infections are, of course, averages, since infection may occur earlier or later for individual bacteria in the same mixture. The yield from those bacteria that liberate phage still remains normal.

The suppression of active phage reproduction by inactive phage in excess indicates the existence of some type of "mutual exclusion" between particles of the same phage. Such exclusion is also indicated by the experiments of Dulbecco (1949a).

More detailed analysis of the interaction between active and inactive phage, using genetic markers, will be reported in future papers. Our results 
discussed above are in agreement with earlier observations (LURIA and DELBRÜCK 1942) on interference by a large excess of irradiated phage $T 2$ with the growth of active phage $T 2$ when the inactive phage was mixed with bacteria one minute and a half before the active one.

\section{Cross-reactivation between phage particles differing by one character}

Only one group of experiments will be discussed here, because of its bearing on the analysis of the data presented in this article. Bacteria were infected with

TABLE 7

Cross-reactivation between one inactive particle of phage T2 and one inactive particle of phage $\mathrm{T} 2 \mathrm{r}$

Compare column (3) with column (1)

\begin{tabular}{|c|c|c|c|c|c|c|}
\hline $\begin{array}{l}\text { EXPERI- } \\
\text { MENT } \\
\text { NO. }\end{array}$ & $\begin{array}{c}\text { DOSE OF } \\
\text { RADIATION, } \\
\text { HITS }\end{array}$ & $\begin{array}{l}\text { MULTIPLIC- } \\
\text { ITY OF } \\
\text { INFECTION } \\
\text { FOR T2 }\end{array}$ & $\begin{array}{l}\text { MULTIPLIC- } \\
\text { ITY OF } \\
\text { INFECTION } \\
\text { FOR } T 2 r\end{array}$ & $\begin{array}{c}\text { (1) } \\
\text { FRACTION OF } \\
\text { BACTERIA RE- } \\
\text { CEIVING one } \\
\text { INACTIVE PAR- } \\
\text { TICLE T2 AND } \\
\text { one INACTIVE } \\
\text { PARTICLE T2r } \\
\text { AMONG THE } \\
\text { BACTERIA THAT } \\
\text { LIBERATE AC- } \\
\text { TIVE PHAGE, } \\
\text { CALCULATED }\end{array}$ & $\begin{array}{c}(2)^{*} \\
\text { OTHER BAC- } \\
\text { TERIA THAT } \\
\text { COULD GIVE } \\
\text { MOTTLED } \\
\text { PLAQUES (AS } \\
\text { FRACTION OF } \\
\text { THE BACTERIA } \\
\text { THAT LIBERATE } \\
\text { ACTIVE PHAGE), } \\
\text { CALCULATED }\end{array}$ & $\begin{array}{l}\text { FRACTION } \\
\text { OF MOTTLED } \\
\text { PLAQUES, } \\
\text { FOUND }\end{array}$ \\
\hline $1 \mathrm{~A}$ & 2.9 & 0.055 & 0.055 & 0.19 & 0.045 & 0.14 \\
\hline $2 \mathrm{~A}$ & 4.6 & 0.055 & 0.049 & 0.335 & 0.053 & 0.16 \\
\hline $3 \mathrm{~A}$ & 6.2 & 0.055 & 0.055 & 0.42 & 0.074 & 0.155 \\
\hline $4 \mathrm{~A}$ & 3.4 & 0.053 & 0.053 & 0.24 & 0.046 & 0.14 \\
\hline $5 \mathrm{~A}$ & 5.0 & 0.053 & 0.053 & 0.355 & 0.068 & 0.20 \\
\hline $7 \mathrm{~A}$ & 3.9 & 0.05 & 0.05 & 0.28 & 0.046 & 0.14 \\
\hline $8 \mathrm{~A}$ & 6.5 & 0.05 & 0.05 & 0.19 & 0.087 & 0.21 \\
\hline
\end{tabular}

* The values in this column include all bacteria with two or more particles of one type and one or more of the other type, plus all bacteria with an active particle of one type and an inactive particle of the other. The values are upper limits, since only a fraction of these bacteria will actually give mottled plaques.

phages $T 2$ and $T 2 r$, both irradiated ( $r=5$ or 6 ). Low multiplicities were used, so that a large proportion of the infected bacteria only received one inactive particle, and, of those that received two, a great proportion received one particle of each type. The infected bacteria were plated before lysis, and the plaques examined for the proportion of "mottled plaques," that is, of plaques containing both $T 2$ and $T 2 r$ active phages. Such plaques can only arise from bacteria infected with both phages. It is seen from the data shown in table 7 that more than half the bacteria infected with one inactive particle of each of the two phages actually liberate a mixture of active particles of both types.

This proves that recombination cannot result from reciprocal exchanges of 
units between the infecting particles bringing together into one particle all the active units before multiplication begins. Evidently, this particle should be either $T 2$ or $T 2 r$, and could not give rise to active particles of both types. This conclusion will be analyzed further in the discussion. Quantitative analysis of the number and contents of mixed yields from mixed infection with $T 2$ and $T 2 r$ shall be the subject of future publications.

\section{Phages inactivated by $X$-rays or nitrogen muslard}

In a preliminary article (LURIA 1947) it was stated that no reactivation had been detected for $T$-even phages inactivated by hard X-rays, using the same technique employed for ultraviolet. The same was found in our laboratory by Miss M. E. WiLLIs for phages $T 2$ and $T 6$ inactivated by a nitrogen mustard (methyl bis ( $\beta$-chloroethyl) amine hydrochloride).

Experiments by MR. J. WATson, still in progress in our laboratory, have recently shown, however, that phage $T 2$ inactivated by hard $\mathrm{X}$-rays can take part in reactivation, but this reactivation occurs with such a low probability that special techniques are required for its detection. In part, the low probability of reactivation of X-ray inactivated phage is due to reduced rate of adsorption. This work will be reported by Mr. WATSON in a future publication.

It seems possible that phages $T 1$ and $T 7$, for which no reactivation was detected after ultraviolet inactivation, may also be found by similar techniques to give some reactivation. It is clear that the probability of reactivation will be low if the number of transferable units is small, or if each lethal mutation involves several units. Its detection will be difficult whenever the number of bacteria in which reactivation occurs is small in comparison with the number of bacteria that receive residual active phage. ${ }^{4}$

\section{DISCUSSION}

The experiments described above have given results consistent with the hypothesis that inactivation of several and possibly all bacteriophages by ultraviolet light is to be attributed to lethal mutations in discrete units of genetic material. A genetic basis for inactivation of viruses and bacteria by radiation has often been postulated either on statistical grounds or by analogy (see RAHN 1929; LEA 1947). Our results bring new support to this view, and suggest that most, if not all, the inactivating effect of ultraviolet light ( $2537 \AA)$ on certain phages is due to the production of localized lethal mutations.

The hypothesis of inactivation by lethal mutations and reactivation by transfer of genetic material following multiple infection, as developed in this paper, has been useful in suggesting a quantitative analysis of the reactivation phenomena and has led to fairly accurate predictions of the experimental results. The following discussion assumes the correctness of this working hypothesis.

${ }^{4}$ While this paper was in press, the senior author found that some reactivation by multiple infection takes place with phage $T 1$ inactivated by ultraviolet light. For equal multiplicity of infection and equal number of hits, the frequency of reactivation is much lower with $T 1$ than with any of the $T$-even phages and with $T 5$. Assuming that the type of analysis presented in this paper applies to the results with $T 1$, a value of $n$ smaller than 5 would be obtained. 
According to our analysis, active phage is reconstituted from inactive by reincorporation of active units derived, directly or indirectly, from the inactive particles in a kind of hybridization. As in hybridization, the possibility of recombinations between particles of different wild-type phages suggests the existence of common genetic determinants and the absence of complete incompatibility. It is possible that various interference phenomena among different phages may result from such an incompatibility.

Reassembly of material from inactive particles into active ones is a remarkably efficient process. Genetic material from several inactive particles may be brought together, although the relative efficiency of cooperation diminishes as the number of particles involved in this pluriparental reproduction increases.

We have given estimates for the minimum number of transferable units per particle for several phages. It is interesting to notice that phage $T 4$, more resistant than the related phages $T 2$ and $T 6$ to ultraviolet light (figure 1), appears to have fewer units. This may indicate absence of a portion of genetic material present in the other $T$-even phages.

LEA and Salaman (1946), analyzing the dependence of the rate of inactivation of phages by $\mathrm{X}$-rays as a function of the density of ionization, and assuming that the radiosensitive material consisted of spherical units, arrived at the conclusion that a large phage contained 14 such units, whereas a small phage contained one only. Although the hypotheses involved were probably oversimplifications, the conclusion receives qualitative support from our results.

We must consider next the possible mechanisms of genetic transfer. We may divide the mechanisms in to two groups, those in which the reproducing element is supposed to be at all times the phage particle as a whole, and those in which the reproducing elements are assumed to be component parts of the particle.

The simplest hypothesis of the first group would be that reactivation results from pairing (or grouping) or the initial infecting particles, followed by reciprocal exchanges such as occur in chromosomal crossing-over; if these exchanges lead to formation of an active particle, the latter proceeds to multiply. This simple hypothesis can easily be disproved. The high efficiency of reactivation would require very large numbers of successive reciprocal exchanges to bring together all active genetic material before multiplication takes place. Mixed infection with one active and one inactive particle should also lead to the occasional loss of active phage, since we know that genetic recombinations occur between active and inactive particles. Finally, the fact that infection with one particle each of inactive $T 2$ and inactive $T 2 r$ yields a mixture of active $T 2$ and active $T 2 r$ disproves this hypothesis, since any number of reciprocal exchanges between the original particles before multiplication could never lead to formation of active particles of both types.

Another interpretation based on reciprocal exchanges would be that inactive particles reproduce, and that exchanges occur at various stages of the reproduction among the original particles or their inactive offspring. These exchanges should be numerous enough to make the probability of incorporation of all active units into an active particle close to unity, and an ac- 
tive particle, once formed, should be favored in multiplication. Without completely disproving it, our results make this explanation very unlikely. The fact that the probability of reactivation increases greatly by increasing (for example, from 8 to 20) the multiplicity of infection with heavily irradiated particles would require a very high number of exchanges. To obtain high yields of active phage in these cases we should assume, moreover, that the exchanges take place early, and that the active particles, once formed, multiply with little or no interference from the inactive particles in large excess. This seems contradicted by the fact already mentioned that active particles can actually undergo interchanges with inactive ones. Altogether, there is strong evidence that inactive units have less chance than active units of entering the final particles, even when the active units derive from inactive particles.

In search for a mechanism that could selectively bring together the active units, the senior author (LURIA 1947) suggested the hypothesis of independent reproduction of individual units to form a "gene pool," from which the new active particles could be derived. Inactive units were considered to be those that cannot reproduce and that have, therefore, little chance of incorporation into the final particles. The tendency to reduction in yield may be due to occasional incorporation of some of the original inactive units. No hypothesis is made as to how the units reproduce or reassemble. The last step is the most difficult to visualize, and we incline to the belief that the original particles may play a role in it, possibly by supplying a framework for reassembly. This is suggested by the limited efficiency of collaboration among large groups of particles, which indicates a certain tendency of the units to remain together with their original companions.

One may ask whether, inside bacteria in which reactivation does not take place, the active units present in the infecting particles reproduce or not. CoHEN (1948) states that no desoxyribose nucleic acid is synthesized in bacteria infected with particles of $T 2$ exposed to doses of ultraviolet light much higher than those employed in our study. Preliminary cytological evidence, collected with the collaboration of DR. C. F. RoBINow in our laboratory, indicates that in infected bacteria, in which reactivation does not take place, there is no accumulation of stainable material supposedly representing desoxyribose nucleotides. If this evidence is confirmed and found to apply to the conditions of our experiments, it might then suggest, either that there is no reproduction of active units when they are not all present (which might altogether invalidate the hypothesis of independent reproduction), or that at least part of the reproduction of the active units may take place without increase in desoxyribose nucleotides.

The hypothesis of a "gene pool," although by no means the only possible one, ${ }^{5}$ fits all results of reactivation. Its validity may soon be amenable to

${ }^{5}$ Another possibility, suggested by $D_{R}$. A. H. Sturtevant, would be a process of zipperwise replication of the various units of a phage particle. When in this process an inactive unit was reached, replication could only continue if the partial replica came in contact with another phage particle in which that unit was active. The process would then continue by addition of replicas of the active units of the second phage particle. If repeated several times, such a mechanism would provide for selective recombination of all active units. 
critical test. In postulating a phase in phage growth in which particles, as we know them in the extracellular phase, are not present, the hypothesis accounts for the repeated failures to obtain active phage by premature artificial disruption of infected bacteria. It agrees with the observation made by FOSTER (1948) in our laboratory that in the presence of proflavine the reactions leading to production of active phage proceed normally for a part of the latent period, but, upon lysis, no active particle is liberated. According to FosTer (1948) active phage particles are present in the infected bacterium after the proflavine sensitive stage is passed-beginning 12 to 14 minutes after infection for phages $T 2$ or T6. A similar conclusion was reached by A. H. Doermann on the basis of experiments on the effect of other inhibitors on the growth of $T 3$ and $T 4 r$ (Doermann 1948). That viruses multiplying inside the host cell may not have the same organization as in the extracellular form has been suggested before for certain animal viruses (see BLAND and RoBINow 1939).

Our theory does not assume any degree of linkage among units, although linkage may be compatible with the theory. Each group of strongly linked units would behave as one unit, possibly as a particularly sensitive one. Weakly linked units would probably reduce the probability of reactivation for high doses, when lethal units present in one group would hinder the utilization of the linked active units for reactivation.

In their work on the $r$ and $h$ mutants of phage $T 2 H$, Hershey and RotMAN $(1948,1949)$ found evidence for a series of determinants exhibiting various degrees of linkage, from those apparently unlinked (high frequency of independent transfer, no correlation between the frequencies of complementary recombinant types in the yield) to others rather strongly linked. For the latter ones, the authors considered that their results suggested the possibility of reciprocal exchanges.

As a working hypothesis we may assume, together with Hershey and RotMAN (1949) that unlinked determinants may be located in different reactivation units, possibly transferred by a gene pool mechanism, while linked determinants may be located in the same reactivation unit. If reciprocal exchanges were found to occur, then homologous units or groups of units should be supposed to pair or group together at some stage in the growth process. Techniques recently developed in our laboratory should soon permit a study of the inactivation of individual genetic determinants and a solution of some of these problems. It appears, therefore, advisable to refrain from further discussion at the present time.

The formation of active phage from inactive by transfer of discrete units requires some revision of the interpretation of experiments on irradiation of phage inside infected bacteria with ultraviolet light (LuRIA and LATARJET 1947). In case of multiple infection, the survival curves for phage-producing ability immediately after infection indicated suppression by damage of a number of centers, with the sensitivity of individual centers lower than that of extracellular phage particles. It now seems clear that what was measured was the rate of inactivation of individual units rather than of whole particles. The curves for suppression of the phage-producing ability of multiple-infected 
bacteria immediately after infection are similar to the curves for the probability of reactivation for a comparable group of irradiated particles. For example, the suppression curve given by LURIA and LATARJET (1947) for bacteria infected by five particles of phage $T 2$ is very similar to the curve for the probability of reactivation $w$ as a function of $r$ for $x=5$. It is clear that in interpreting experiments on inactivation of intracellular phage it will be necessary to take into account the occurrence of genetic transfers. ${ }^{6}$

\section{SUMMARY}

Coli-bacteriophages $T 2, T 4, T 5$, and $T 6$ inactivated by ultraviolet light are still adsorbed by sensitive bacteria. Bacteria infected by only one inactive phage particle are not lysed and do not yield active phage. Infection of bacteria with more than one inactive particle leads to lysis and production of active phage in a fraction of the bacteria. This fraction diminishes with increasing doses of radiation and increases with increasing numbers of particles adsorbed per bacterium. The assumption is made that inactivation is due to lethal mutations in a number of genetic "units" of the phage particle, and that production of active phage from inactive is due to recombination of non-lethal units to form active particles. The values of the probability of active phage production calculated from these assumptions agree with the experimental results with certain limitations. In order to explain the very high frequency of recombination, the hypothesis is proposed that phage growth occurs by independent reproduction of each unit followed by reassembly of the units into complete phage particles. The minimum number of units per particle is estimated for various phages.

\section{LITERATURE CITED}

Bland, J. O. W., and C. F. Robinow, 1939 The inclusion bodies of vaccinia and their relationship to the elementary bodies studied in cultures of the rabbit's cornea. J. Path. Bact. 48: 381-403.

CoHEn, S. S., 1948 The synthesis of bacterial viruses. I. The synthesis of nucleic acid and protein in Escherichia coli B infected with $\mathrm{T}^{+} r^{+}$bacteriophage. J. Biol. Chem. 174: 281-293.

Delbrück, M., 1940 The growth of bacteriophage and lysis of the host. J. Gen. Physiol. 23: 643-660.

1946 Bacterial viruses or bacteriophages. Biol. Rev. Cambridge Phil. Soc. 21: 30-40.

Delbrǘck, M., and W. T. BAILEY, JR., 1946 Induced mutations in bacterial viruses. Cold Spring Harbor Symp. Quant. Biol. 11: 33-37.

DelbrücK, M., and S. E. LuRIA, 1942 Interference between bacterial viruses. I. Interference between two bacterial viruses acting upon the same host, and the mechanism of virus growth. Arch. Biochem. 1: 111-141.

DoermanN, A. H., 1948 Intracellular growth of bacteriophage. Carnegie Instn. Wash. Yearb. (in press).

${ }^{6}$ While this paper was in press, one of us (DuLBecco 1949b) discovered that ultraviolet irradiated phages can be reactivated by exposure to visible light of short wave length in presence of bacterial cells. This "photoreactivation" differs from reactivation by multiple infection in many of its features. Photoreactivation does not take place to any appreciable extent under the conditions in which the experiments reported in this paper were performed. A series of experiments of the type exemplified in table 4 , but carried out in dim yellow light- - under conditions that completely avoid photoreactivation - gave results undistinguishable from those of the earlier experiments, in which no precaution had been taken to control illumination. 
Dulbecco, R., 1949a The number of particles of bacteriophage $T 2$ that can participate in intracellular growth. Genetics 34: (in press).

1949b Reactivation of ultraviolet inactivated bacteriophage by visible light. Nature (in press).

Foster, R. A. C., 1948 An analysis of the action of proflavine on bacteriophage growth. J. Bact. 56: 795-809.

Hershey, A. D., and R. Rotman, 1948 Linkage among genes controlling inhibition of lysis in a bacterial virus. Proc. nat. Acad. Sci. 34: 89-96.

1949 Genetic recombination between host-range and plaque-type mutants of bacteriophage in single bacterial cells. Genetics 34: 44-71.

LATARJET, R., and R. WAHL, 1945 Précisions sur l'inactivation des bactériophages par les rayons ultraviolets. Ann. Inst. Pasteur 71: 336-339.

LEA, D. E., 1947 Actions of radiations on living cells. xii +402 pp. Cambridge: University Press.

LeA, D. E., and M. H. Salaman, 1946 Experiments on the inactivation of bacteriophage by radiations, and their bearing on the nature of bacteriophage. Proc. roy. Soc., B, 133: 434-444.

LURIA, S. E., 1947 Reactivation of irradiated bacteriophage by transfer of self-reproducing units. Proc. nat. Acad. Sci. 33: 253-264.

1948 Bacteriophage mutations and genetic interactions among bacteriophage particles inside the host cell. A.A.A.S. Symposium on Genetics of Microorganisms (in press).

LURIA, S. E., and M. DELBRÜCK, 1942 Interference between bacterial viruses. II. Interference between inactivated bacterial virus and active virus of the same strain and of a different strain. Arch. Biochem. 1: 207-218.

LURIA, S. E., and R. LATARJET, 1947 Ultraviolet irradiation of bacteriophage during intracellular growth. J. Bact. 53: 149-163.

RAHN, O., 1929 The size of bacteria as the cause of the logarithmic order of death. J. gen. Physiol. 13: 179-205. 

BIBLIOGRAPHY

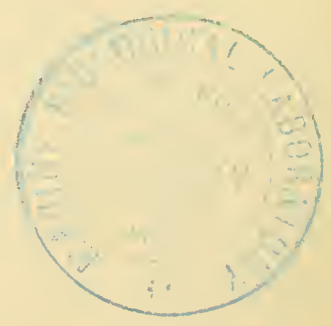



THE REPRINTED papers are numbered from I to 20 ; citations to the literature at large are numbered from 21 to 142 , alphabetized by authors' names. Choice of citations has been dictated by the same general considerations as were applied to the reprints, and many interesting examples of specific mutant types have necessarily been omitted. Many reviews are available which provide more detailed and balanced accounts of specific subjects; these are listed with an asterisk $\left({ }^{*}\right)$.

1. Luria, S. E., and Delbrück, M., 1943 Mutations of bacteria from virus sensitivity to virus resistance. Genetics $28: 491 \cdot 511$.

2. Lea, D. E., and Coulson, C. A., 1949 The distribution of the numbers of mutants in bacterial populations. J. Genet. $49: 264-285$.

3. Newcombe, Howard B., 1949 Origin of bacterial variants. Nature 164 : 150.

4. Newcombe, Howard B., 1948 Delayed phenotypic expression of spontaneous mutations in Escherichia coli. Genetics 33 : 447-476.

5. Demerec, M., 1948 Origin of bacterial resistance to antibiotics. J. Bact. 56 : 63-74.

6. Cavalli, L. L., and Maccacaro, G. A., 1950 Chloromycetin resistance in $E$. coli, a case of quantitative in. heritance in bacteria. Nature 166 : 991-992.

7. Davis, Bernard D., 1950 Studies on nutritionally deficient bacterial mu. tants isolated by means of penicillin. Experientia $6: 41 \cdot 50$.

8. Bruner, D. W., and Edward, P. R., 1941 The demonstration of nonspecific components in Salmonella paratyphi $\mathbf{A}$ by induced variation. J. Bact. $42: 467.478$.

9. Edwards, P. R., Barnes, L. A., and Babcock, Mary C., 1950 The nat. ural occurrence of phase 2 of Salmonella paratyphi A. J. Bact. $59: 135-136$.

10. Bruner, D. W., and Edwards, P. R., 1948 Changes induced in the $O$ antigens of Salmonella. J. Bact. 55 : 449.

11. Witkin, Evelyn M., 1947 Mutations in Escherichia coli induced by chemical agents. Cold Spring Harlor Symposia on Quantitative Biology $12: 256-269$.

12. Goodlow, Robert J., Mika, Leonard A., and Eraun, Werner, 1950 The effect of metabolites upon growth and variation of Brucella abortus. J. Bact. 60 : 291-300.

13. Lederberg, Joshua, 1947 Gene recombination and linked segregations in Escherichia coli. Genetics 32 : 505-525.

14. Lederberg, Joshua, 1949 Aberrant heterozygotes in Escherichia coli. Proc. nat. Acad. Sci. U.S. 35 : 178 184.

15. Robinow, C. F., 1944 Cytological observations on Bact. coli, Proteus vulgaris and various aerobic sporeforming bacteria with special reference to the nuclear structures. J. Hyg. 43 : $413-423$.

16. Avery, Oswald T., MacLeod, Colin M., and McCarty, Maclyn, 1944 Studies on the chemical nature of the substance inducing transforma. tion of pneumococcal types. J. Exp. Med. $79: 137-158$.

17. Burnet, F. M., and Lush, Dora, 1936 Induced lysogenicity and mutation of bacteriophage within lysogenic bacteria. Austr. J. Exp. Biol. Med. Sci. $14: 27-38$.

18. Luria, S. E., 1945 Mutations of bacterial viruses affecting their host range. Genetics $30: 84-99$.

19. Hershey, A. D., and Rotman, Raquel, 1949 Genetic recombination between host-range and plaque-type mutants of bacteriophage in single hacterial cells. Genetics $\$ 4$ : 44-71.

20. Luria, S. E., and Dulbecco, R., 1949 Genetic recombinations leading to production of active bacteriophage from ultraviolet inactivated bacteriophage particles. Genetics $34: 93$ 122.

-21. Adams, M. H., 1950 Methods of study of bacterial viruses in "Methods in Medical Research." Vol. II, The Year Book Publishers, Chicago.

22. Alexander, H. E., and Leidy, G., 1951 Determination of inherited traits 


\section{BIBLIOGRAPHY}

of $H$. influenzae by desoxyribonucleic acid fractions isolated from type-specific cells. J. Exp. Med. 93: 345-359.

23. Anderson, E. H., 1946 Growth requirements of virus-resistant mutants of a bacterial strain. Proc. nat. Acad. Sci., U.S. 32 : 120-128.

-24. Anderson, T. F. 1949 The reactions of bacterial viruses with their host cells. Bot. Rev. 15 : 464.505.

25. Atwood, K. C., Schneider, L. K., and Ryan, F. J., 1951 Periodic selection in Escherichia coli. Proc. Nat. Acad. Sci., U.S. 37 : 146-155.

-26. Auerbach, C., 1949 Chemical mutagenesis. Biol. Rev. 24 : $955-391$.

27. Austrian, R., and Macleod, C. M. 1949 Acquisition of $\mathrm{M}$ protein by pneumococci through transformation reactions. J. Exp. Med. 89 : 451-460.

28. Barer, G. R., 1951 The action of streptomycin on Bacterium lactis aerogenes. J. Gen. Microbiol. 5 : 1 17.

-29. Beadle, G. W., 1945 Biochemical genetics. Chem. Rev. 37 : 15-96.

30. Beadle, G. W., and Tatum, E. L. 1945 Neurospora II. Methods of producing and detecting mutations concerned with nutritional requirements. Amer. J. Bot. 32 : 678-686.

31. Boivin, A., 1947 Directed mutation in colon bacilli, by an inducing principle of desoxyribonucleic nature: its meaning for the general biochemistry of heredity. Cold Spring Harbor Symp. Quant. Biol. $12: 7 \cdot 17$

-32. Braun, W., 1947 Bacterial dissociation. Bact. Rev. 11 : 75-114.

33. Bryson, V., 1948 Effects of nitrogen mustard on Escherichia coli. J. Bact. $56: 423-433$.

34. Burnet, F. M., 1929 "Smooth-Rough" variation in bacteria and its rela. tion to bacteriophage. J. Path. Bact. 32 : 15.42.

*35. Burnet, F. M., 1945 Virus as organism. Harvard University Press, Cambridge, Mass.

- 96. Burnet, F. M., and Fenner, F., 1948 Genetics and immunology. Heredity $2: 289-324$.

37. Burnet, F. M., and Lind, P. E., 1951 Recombination of characters between the influenza virus $A$ strain NWS and strains of different serological subtypes. J. Gen. Microbiol. $5: 67-82$.

38. Byatt, P. H., Jann, G. J., and Salle, A. J., 1948 Variation in pigment production in Staphylococcus aur. eus. J. Bact. 55 : 787-792.

39. Coleman, M. F., and Reid, J. J., 1949 The conversion of strains of Alcali. genes radiobacter and Phytomonas tumefaciens in the " $S$ " phase to the " $M$ " phase of the heterologous species. Phytopath. $39: 182-190$.

40. Davis, B. D., 1950 Nonfiltrability of the agents of genetic recombination in Escherichia coli. J. Bact. $60: 507$. 508.

-41. Delaporte, B., 1950 Ohservations on the cytology of bacteria. Advances in Genetics 3: 1-32.

42. Delbrück, M., 1945 Spontaneous mutations of bacteria. Ann. Mo. bot. Gdn. 32 : 223-233.

-43. Delbrück, M., 1946 Bacterial viruses or bacteriophages. Biol. Rev. 21 : 30-40.

-44. Delbrück, M., (ed.), 1950 Viruses 1950. California Institute of Technology, Pasadena, California.

-45. Demerec, M. (ed.), 1946 Heredity and variation in microorganisms (Cold Spr. Harbor Symp. Quant. Biol. XI), L. I. Biol. Assn., Cold Spring Harbor, N. Y.

46. Demerec, M., 1950 Reaction of populations of unicellular organisms to extreme changes in environment. Am. Nat. $84: 5-16$.

-47. Demerec, M. (ed.), 1951 Genes and mutations. (Cold Spring Harbor Symp. Quant. Biol., XVI), L.I. Biol. Assn., Cold Spring Harlor, N. $Y$.

48. Demerec, M., and Fano, U., 1945 Bacteriophage-resistant mutants in Escherichia coli. Genetics $30: 119$. 136.

49. Demerec, M., Wallace, B., Witkin, E. M., and Bertani, G., 1949 The gene. Carnegie Institution (Wash.) Y'r. Book 48: 154-166.

50. Dienes, L., 1942 The significance of the large bodies and the development of $\mathbf{L}$ type of colonies in bacterial cultures. J. Bact. 44 : $37-73$.

51. Dienes, L., 1949 The development of Proteus cultures in the presence of penicillin. J. Bact. $57: 529-546$.

52. Dienes, L., and Smith, W. E., 1944 The significance of pleomorphism in Bacteroides strains. J. Bact. 48 : 125-153.

53. Doudoroff, Michael, Hassid, W. Z., Putman, E. W., Potter, A. L., and Lederberg, J., 1949 Direct utiliza. tion of maltose by Escherichia coli. J. Biol. Chem. $179: 921.934$.

-54. Dubos, R. J., 1945 The bacterial cell. Harvard University Press, Cambridge, Mass.

55. Dulbecco, R., 1949 Reactivation of UV-inactivated bacteriophage by visible light. Nature 163: 949 .

56. Dulbecco, R., 1950 Experiments on photoreactivation of UV-inactivated bacteriophage. J. Bact. 59 : $329-347$. 


\section{BIBLIOGRAPIHY}

-57. Dunn, L. C. (ed.), 1951 Genetics in the 20th century. MacMillan, New York.

58. Englesberg, E., 1951 Formaldehyde as a mutagenic agent in the bacteria. Bacteriol. Proc. $2: 49$.

-59. Fredericq, P., 1948 Actions antibiotiques reciproques chez les Enterobacteriaceae. Rev. belge Path. Med. exp. 19 (Supplement 4) : 1-107.

-60. Giese, A. C., 1950 Action of ultra. violet radiation on protoplasm. Physiol. Rev. 30 : 431.458.

61. Goebel, W. F., 1950 Studies on bac. teriophage. J. Exper. Med. 92 : 527 543.

-62. Gordon, F. B., 19:30 Genetics of viruses. Ann. Rev. Microbiol. 4: 151 . 168.

63. Grav, C. H., and Tatum, E. L., 1944 $\mathrm{X}$-ray induced growth factor requirements in bacteria. Proc. nat. Acad. Sci.. I.S. 30 : 404-410.

64. Griffith, F.. 1928 The significance of pneumococcal types. J. Hyg. 27 : 113.159.

65. Haas, F. L., Clark, J. B., Wyss, O. and Stone, W. S., 1950 Mutations and mutagenic agents in bacteria. Ain. Nat. $84: 261-274$.

-66. Harrison, J. A., 1947 Antigenic variation in protozoa and bacteria. Ann. Rev. Microbiol. 1 : 19-42.

67. Hinshelwood, C. N., 1946 The chemical kinetics of the bacterial cell. Oxford.

68. Horowitz, N. H., 1945 On the evolution of biochemical syntheses. Proc. nat. Acad. Sci. U.S. 31 : 153-157.

69. Horowitz, N. H., 1950 Biochemical genetics of Neurospora. Advances in Genetics 3: 33-71.

70. Jackson, S., and Hinshelwood, C. N., 1950 An investigation of the na. ture of certain adaptive changes in bacteria. Proc. roy. Soc., London B $136: 562-576$.

-71. Kauffmann, F., 1950 The diagnosis of Salmonella types. C. C. Thomas, Springfield, III.

72. Kelner, A., 1949 Effect of visible light on the recovery of Streptomyces griseus conidia from ultraviolet irradiation injury. Proc. nat. Acad. Sci., U.S. 35 : 73-79.

73. Kelner, A., 1949 Photoreactivation of ultraviolet-irradiated Escherichia coli, with special reference to the dose-reduction principle and to ultraviolet-induced mutation. J. Bact. 58 : 511.522.

74. Klieneberger-Nobel, E., 1945 Changes in the nuclear structure of bacteria, particularly during spore formation. J. Hyg. 44 : 99-108.

75. Klieneberger-Nobel, E., 1949 On Streptobacillus moniliformis and the filtrability of its L-form. J. Hyg. 47 : $393-395$.

76. Klieneberger-Nobel, E., 1949 Origin, development and significance of $L$. forms in bacterial cultures. J. Gen. Microbiol. 3 : 434-443; 1951 Filterable forms of bacteria. Bact. Rev. $15: 77-103$.

-77. Knaysi, G., 1949 Cytology of bac. teria. II. Bot. Rev. 15 : 106-151.

-78. Knight, B. C. J. G., 1945 Growth factors in microbiology. Vitamins and Hormones $3: 105-228 \mathrm{~b}$.

-79. Kunkel, L. O., 1947 Variation in phytopathogenic viruses. Ann. Rev. Microbiol. 1 : 85-100.

-80. Lea, D. E., 1947 Actions of radiations on living cells. Mac.Millan, New York.

-81. Lederberg, J., 1948 Problems in microbial genetics. Heredity $2: 145$ 198.

-82. Lederberg, J., 1949 Bacterial varia. tion. Ann. Rev. Microbiol. 3 : 1-22.

83. Lederberg, J., 1950 The selection of genetic recombinations with bacterial growth inhibitors. J. Bact. $59: 211-215$.

84. Lederberg, J., and Tatum, E. L., 1946 Gene recombination in Escherichia coli. Nature $158: 558$.

85. Lincoln, R. E., 1947 Mutation and adaptation of Phytomonas stewar. tii. J. Bact. $54: 745-757$.

86. Loveless, A., 1951 Qualitative aspects of the chemistry and biology of radiomimetic (mutagenic) sub. stances. Nature $167: 338-342$.

-87. Luria, S. E., 1947 Recent advances in bacterial genetics. Bact. Rev. 11 : 1.40 .

88. Luria, S. E. (ed.) Genetics of microorganisms. In Methods of Medica] Research, Volume III, Yearbook Publishers, Chicago.

-89. Lwoff, A., 1943 L'evolution physiologique. Etude des pertes de fonctions chez les microorganismes. Paris : Masson.

90. Lwoff, A., and Gutman, A., 1950 Recherches sur un Bacillus mega. terium lysogène. Ann. Inst. Pasteur 78 : 711.739 .

91. Macleod, C. M., and Krauss, M. R., 1947 Stepwise intratype transformation of pneumococcus from $\mathbf{R}$ to $S$ by way of a variant intermediate in capsular polysaccharide production. J. Exp. Med. 86 : 439-453.

92. Malmgren, B., and Heden, C. G., 1947 Studies of the nucleotide metabolism of bacteria. Acta pathologica $24: 417.504$.

-93. McCarty, M., 1946 Chemical nature and biological specificity of the sub. stance inducing transformations of pneumococcal types. Bact. Rev. 10 : 63-71. 


\section{BIBLIOGRAPHY}

-94. Miles, A. A., and Pirie, N. W. (ed.), 1949 The nature of the bacterial surface. C. C. Thomas, Springfield, III.

-95. Miller, C. P., and Bohnhoff, M., 1950 The development of bacterial resistance to chemotherapeutic agents. Ann. Rev. Microbiol. 4 : 201-222.

-96. Monod, J., 1942 Recherches sur la croissance des cultures bacteriennes. Herrman \& Cie., Paris.

-97. Monod, J., 1947 The phenomenon of enzymatic adaptation. Growth $11: 223-289$.

98. Monod, J., 1950 Adaptation, mutation, and segregation in the formation of bacterial enzymes. Biochem. Soc. Symp. $4: 51-58$.

99. Mudd, S., and Smith, A. G., 1950 Electron and light microscopic studies of bacterial nuclei. I. J. Bact. $59: 561.573$.

100. Newcombe, H. B., and Hawirko, R., 1950 Spontaneous mutation to streptomycin resistance and dependence in Escherichia coli. J. Bact. 57 : 565-572.

101. Newcombe, H. B., and Nyholm, M. H., 1950 The inheritance of streptomycin resistance and dependence in crosses of Escherichia coli. Genetics $35: 603-611$.

102. Newcombe, H. B., and Nyholm, M. H., 1950 Anomalous segregation in crosses of Escherichia coli. Amer. Nat. $84: 457.465$.

103. Newcombe, H. B., and Scott, G. W. 1949 Factors responsible for the delayed appearance of radiationinduced mutants in Escherichia coli. Genetics $34: 475-492$.

104. Newcombe, H. B., and Whitehead, H. A., 1951 Photoreversal of ultraviolet-induced mutagenic and lethal effects in Escherichia coli. J. Bact. 61 : 243-251.

105. Novick, A., and Szilard, L., 1949 Experiments on light-reactivation of ultra-violet inactivated bacteria. Proc. nat. Acad. Sci., U. S. 35 : 591 600.

106. Novick, A., and Szilard, L., 1950 Experiments with the chemostat on spontaneous mutations of bacteria. Proc. nat. Acad. Sci., U.S. 36 : 708 . 719.

107. Oakberg, E. F., and Luria, S. E., 1947 Mutations to sulfonamide resistance in Staphylococcus aureus. Genetics 32 : 249-261.

108. Oparin, A. I., 1938 The origin of life. Translated by $S$. Morgulis, MacMillan, New York.

109. Page, L. A., Goodlow, R. J., and Braun, W., 1951 The role of threonine levels in bacterial variation and modification of virulence of
Salmonella typhimurium. Bact. Proc. 2: 647 (abstract).

110. Peterson, W. H., and Peterson, M. S. 1945 Relation of bacteria to vitamins and other growth factors. Bact. Rev. 9 : 49-109.

111. Roblin, R. O., 1946 Metabolite antagonists. Chem. Rev. 38 : 255 377.

112. Roepke, R. R., Libby, R. L., and Small, M. H., 1944 Mutation or variation in Escherichia coli with respect to growth requirements. J. Bact. 48 : 401.412 .

113. Roepke, R. R., and Mercer, F. E., 1947 Lethal and sublethal effects of X-rays on Escherichia coli as related to the yield of biochemical mutants. J. Bact. $54: 731-743$.

114. Ryan, F. J., 1948 On the stability of nutritional mutants of bacteria. Proc. nat. Acad. Sci., U. S. $34: 425$. 435.

115. Ryan, F. J., and Schneider, L. K., 1948-49 The consequences of mutation during the growth of biochemical mutants of Escherichia coli. I-IV. J. Bact. 56 : 699-708; 58 : 181-189; 58 : 191-200; 58 : 201-213.

116. Ryan, F. J., and Schneider, L. K., 1949 Mutations during the growth of biochemical mutants of Escherichia coli. Genetics 34 : 72-91.

117. Skoog, F. (ed.), 1951 Plant growth substances. University of Wisconsin Press, Madison.

118. Smith, A. G., 1950 Electron and light microscopic studies of bacterial nuclei. II. J. Bact. 59 : 575-587.

119. Sonneborn, T. M., 1950 The cyto. plasm in heredity. Heredity $4: 11$. 36.

120. Spiegelman, S., Lindegren, C. C., and Hedgecock, L., 1944 Mechanisms of enzymatic adaptations in generally controlled yeast populations. Proc. nat. Acad. Sci., U. S. 30 : 13-23.

121. Stanier, R. Y. 1951 Adaptation in bacteria. Ann. Rev. Microbiol. 5 : 35-56.

122. Stempen, H., 1950 Demonstration of the chromatinic bodies of Escherichia coli and Proteus vulgaris with the aid of the phase contrast micro. scope. J. Bact. $60: 81 \cdot 87$.

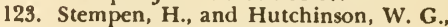
1951 The formation and develop. ment of large bodies in Proteus vulgaris OX-19. J. Bact. 61 : 321. 335; $397-344$.

124. Stocker, B. A. D., 1949 Measurements of rate of mutation of flagel. lar antigenic phase in Salmonella typhi-murium. J. Hyg. 47 : 398-413.

125. Swenson, P. A., and Giese, A. C., 19:1 Photoreactivation of galactozymase formation in yeast. J. Cell. Comp. 


\section{BIBLIOGRAPHY}

Physiol. 36 : 369-380.

126. Tatum, E. L., $1945 \mathrm{X}$-ray induced mutant strains of Escherichia coli. Proc. nat. Acad. Sci., U. S. 31 : 215. 219.

127. Tatum, E. L., 1949 Amino acid metabolism in mutant strains of microorganisıns. Feder. Proc. 8 : 511-517.

128. Tatum, E. L., and Lederberg, J. 1947 Gene recombination in the bacterium Escherichia coli. J. Bact. $53: 673-684$

129. Tatum, E. L., and Perkins, D. D., 1950 Genetics of microorganisms. Ann. Rev. Microbiol. 4 : 129-150.

130. Taylor, H. E*, 1949 Additive effects of certain transforming agents from some variants of pneumococcus. J. Exp. Med. 89 : 399-424.

131. Taylor, H. E., 1949 Transformations reciproques des formes $R$ et $\mathbf{E R}$ chez le pneumocoque. C. R. Acad. Sci. Paris 228 : 1258-1259.

132. Tulasne, R., 1949 Existence of Lforms in common bacteria and their possible importance. Nature 164 : 876-877.

133. Weil, A. J., and Binder, M., 1947 Experimental type transformation of Shigella paradysenteriae (Flexner). Proc. Soc. exp. Biol. N.Y. 66 : 349-352.

134. Wells, P. H., and Giese, A. C., 1950 Photoreactivation of ultraviolet light injury in gametes of the seaurchin Stongylocentrotus purpura. tus. Biol. Bull. 99 : 163-172.

*135. Welsch, M., 1950 De la résistance nicrobiènne aux agents chimiothérapeutiques. Rev. med. Liege $5: 487-500$.

*136. Welsch, M., 1950 Recherches sur l'origine de la résistance microbiènne à la streptomycine. Bull. Acad. roy. Med. Belg. 15 : 454-471.

137. Williams-Smith, H., 1948 Investigations on the typing of staphylococci by means of bacteriophage. J. Hyg. 46 : 74-81; 82-89.

138. Witkin, E. M., 1947 Genetics of resistance to radiation in Escherichia coli. Genetics 32:221-248.

139. Wollman, E., 1947 Essai d'analyse des besoins en tryptophane de mutants d'Escherichia coli resistants au bacteriophage. Ann. Inst. Pasteur $73: 1082-1088$.

140. Work, T. S., and Work, E., 1948 The basis of chemotherapy. Interscience, New York.

141. Zalokar, M., 1948 The p-aminobenzoic acid requirement of the "sulfonamide-requiring" mutant strain of Neurospora. Proc. nat. Acad. Sci., U.S. 34 : 32-36.

142. Zelle, M. R., and Lederberg J., 1951 Single cell isolations of diploid heterozygous Escherichia coli. J. Bact. 61 : 351-355. 




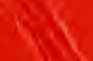

.

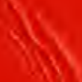

N5.

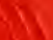

ato

1.

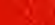

Ny 
